9366

IV 


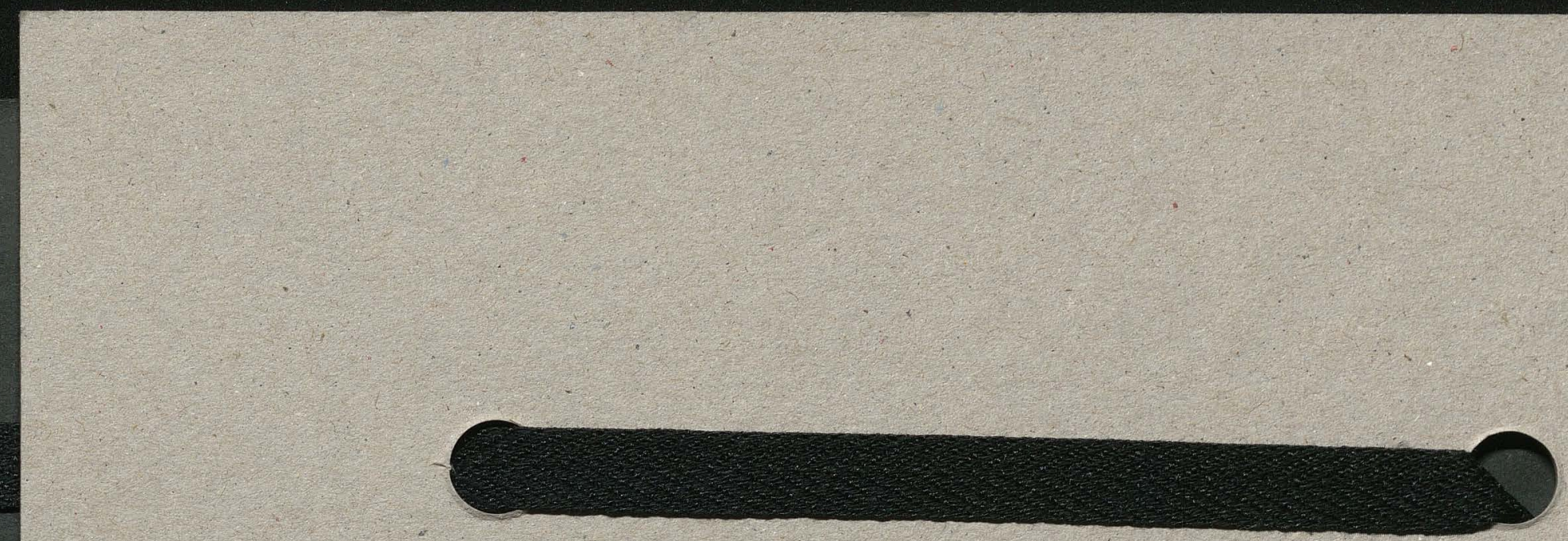


9366

N

M. Smoluchonsk

Versuch einer mathematischen. Theovie der Kongula tionskinetik Kolloider Lösungen. 


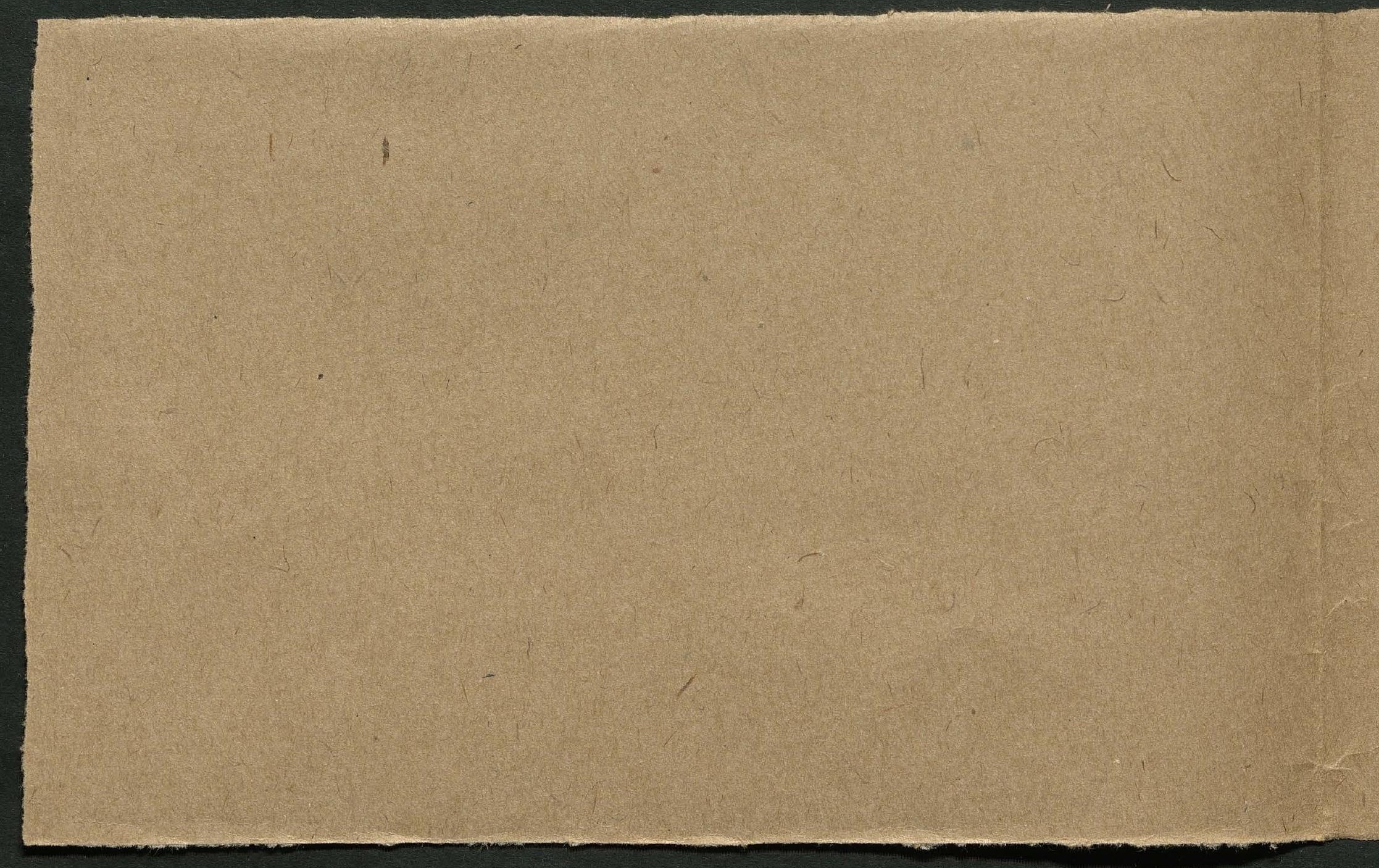


100 Sourrab dinike!

Versuch ciner mathematrichm Theorie der toagulations Kinatik tollevider Losungm.

m

M.r. Surh cho ostei

Zimelaufer an 8. Jept. 1916 .

I. Sinlecting.

So sche anch fis hunte dix Literatur iber Toogulation trollsider Lisingen

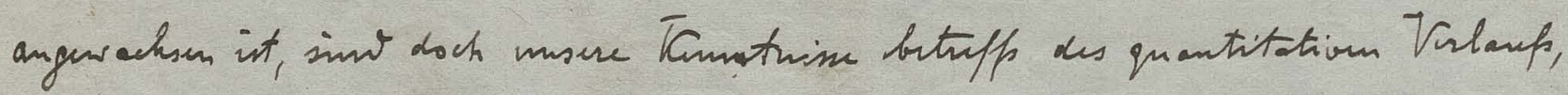
inni betriff des Mechantsuns des Toagulations pusesses anstust mangelhaft.

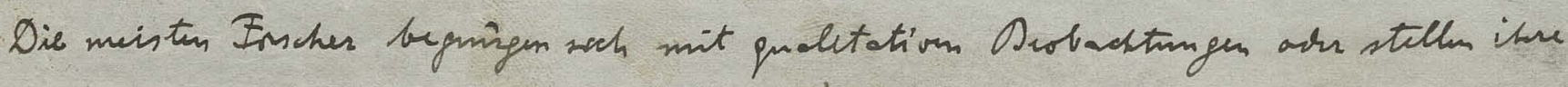

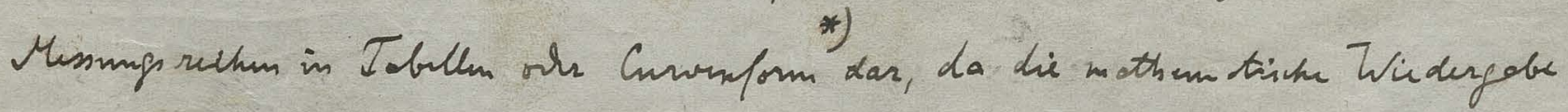

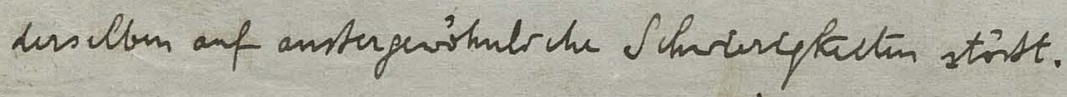

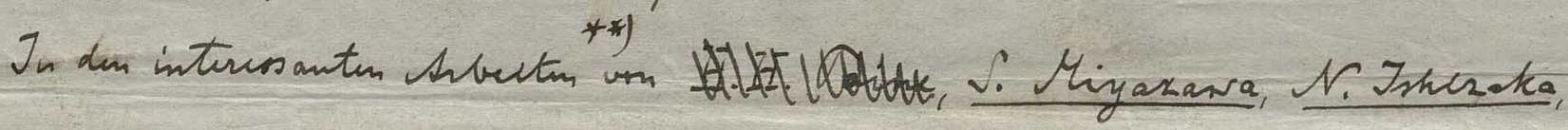

I. Fremmreh J.A. Sann sin allerdings inc

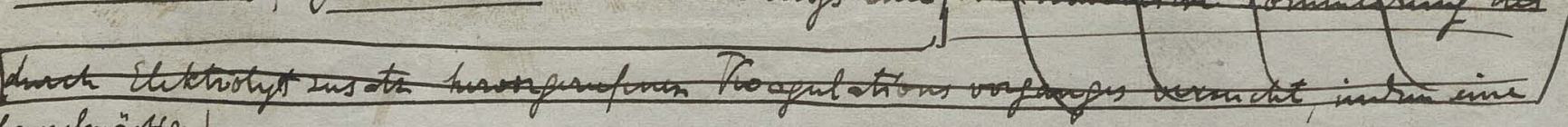

formilmaingige

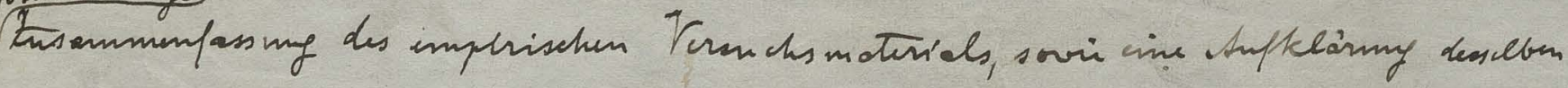

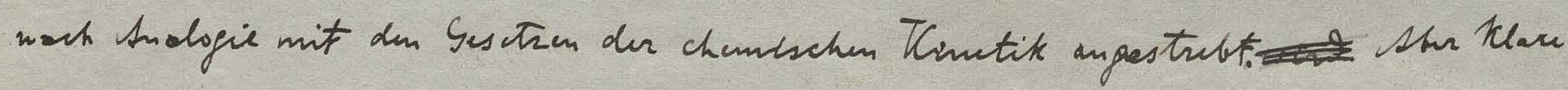

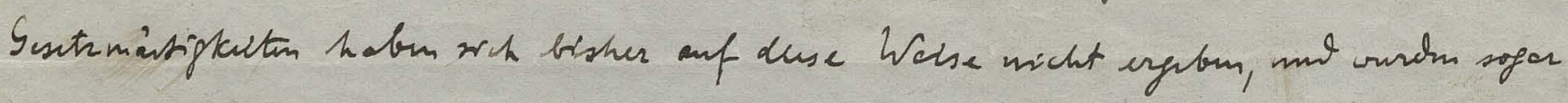

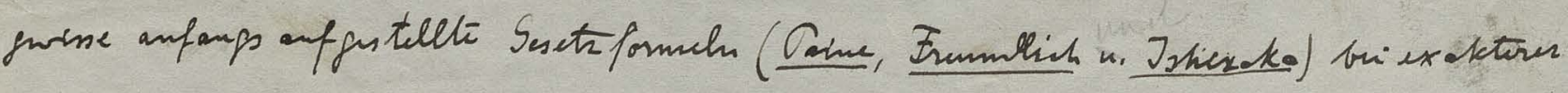

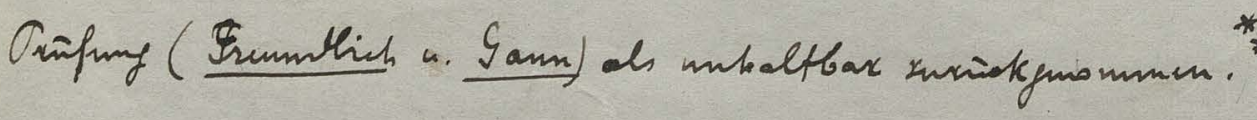

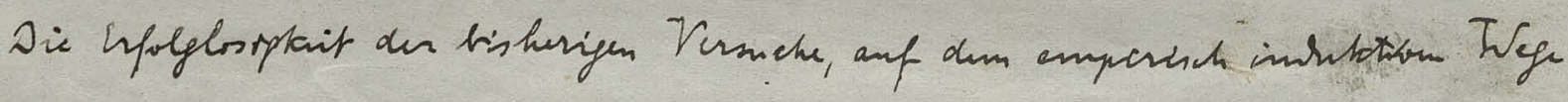

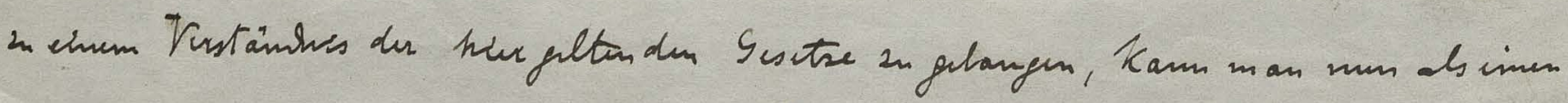

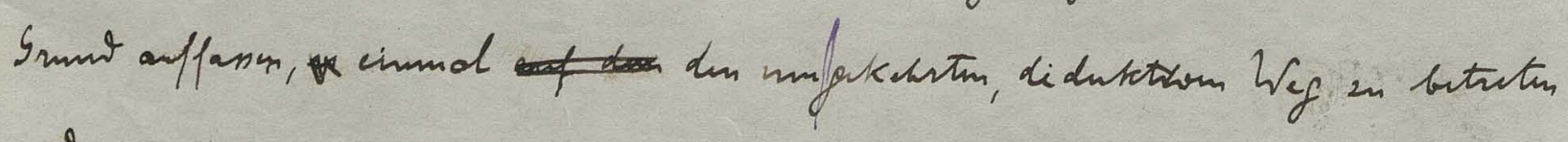

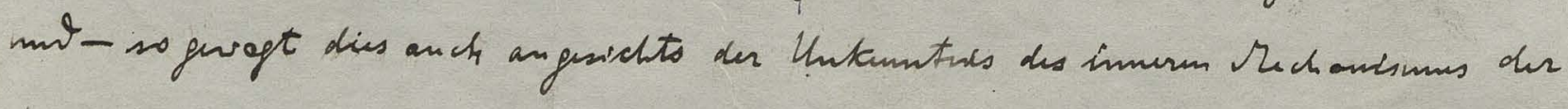

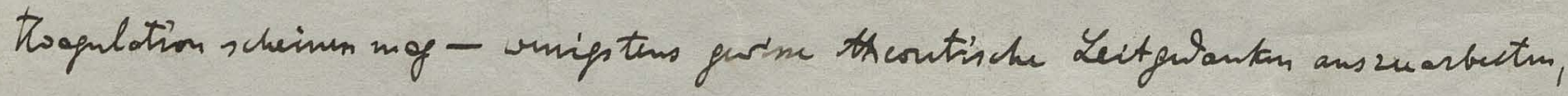

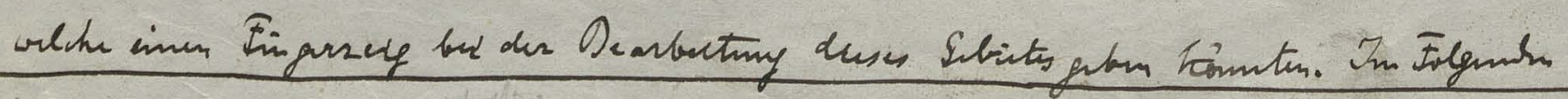

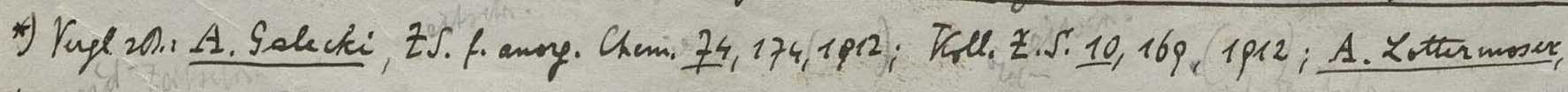

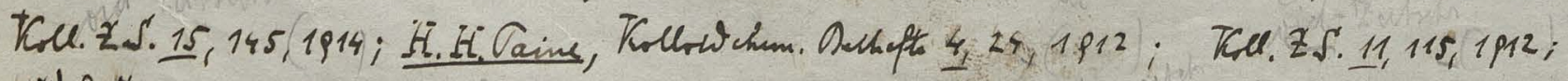

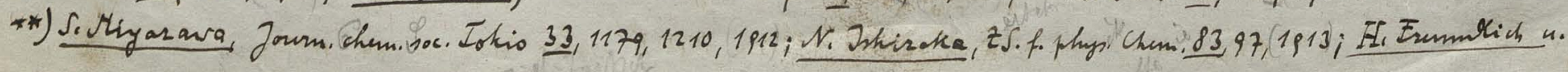

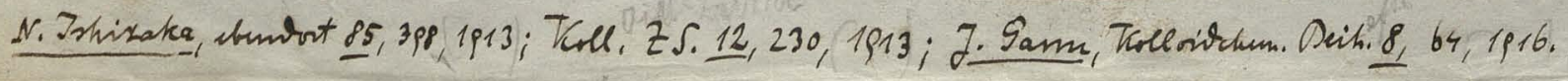




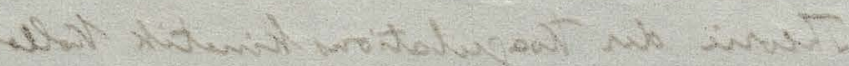

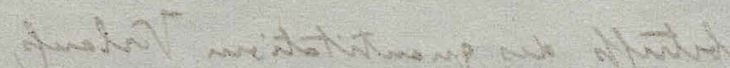

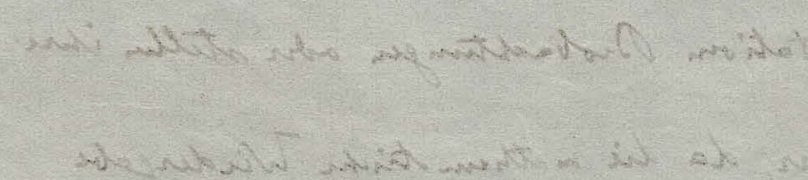

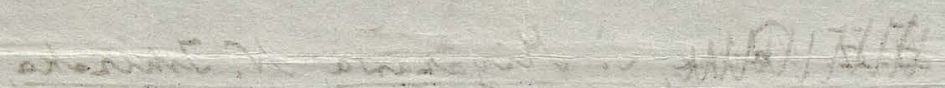

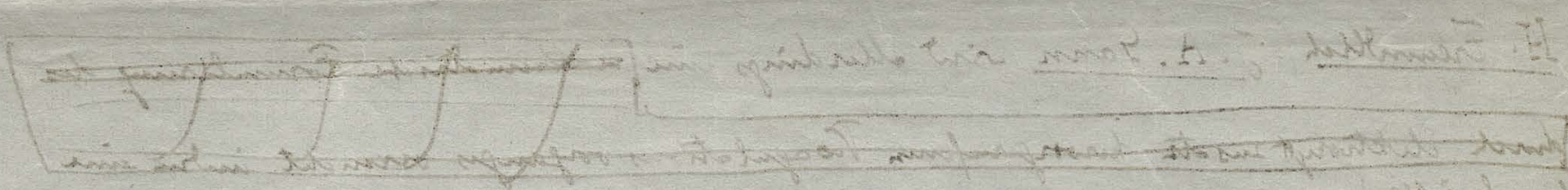

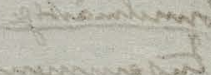

wast

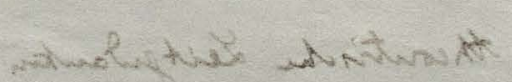

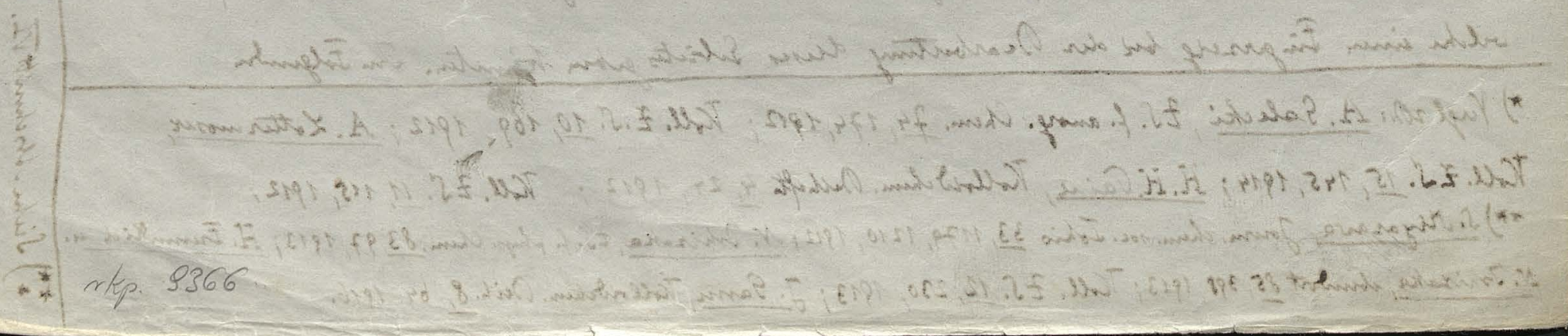




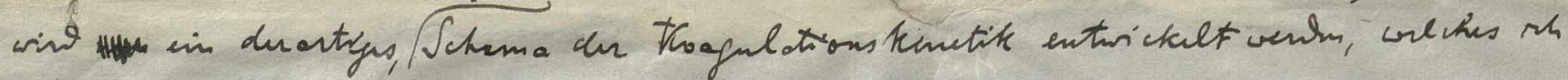

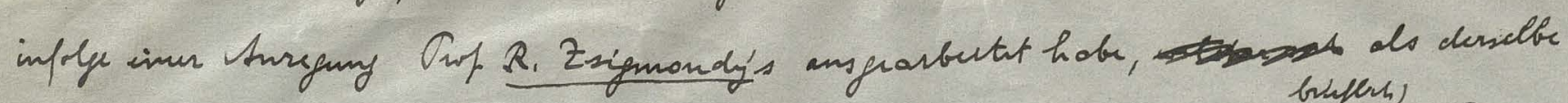

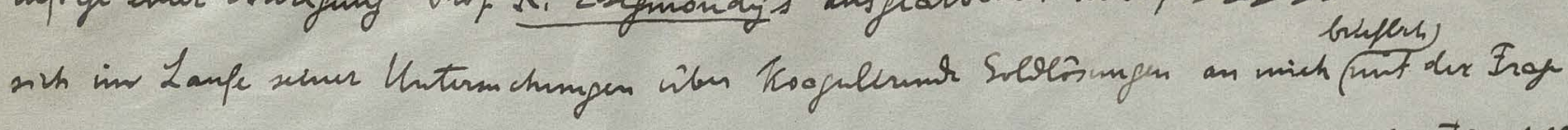

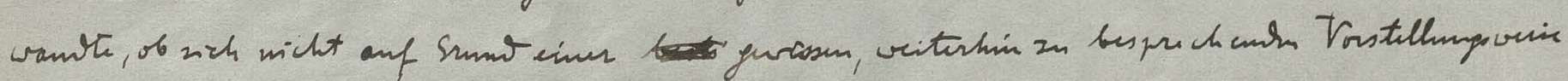

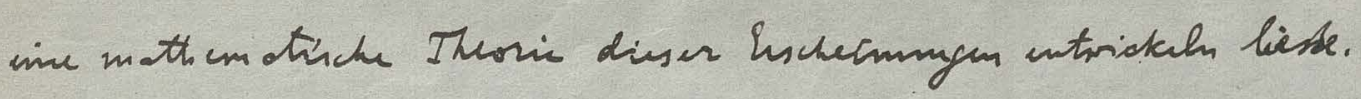

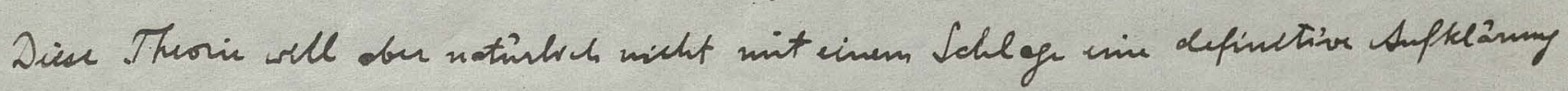

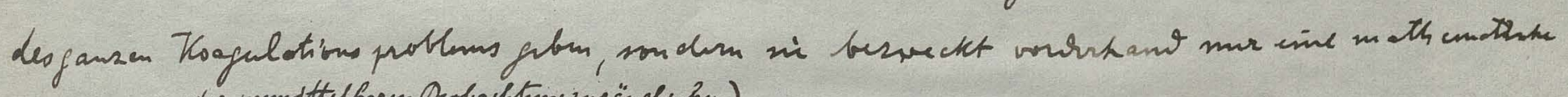

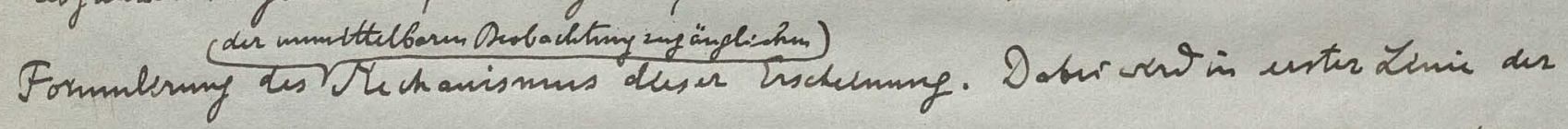

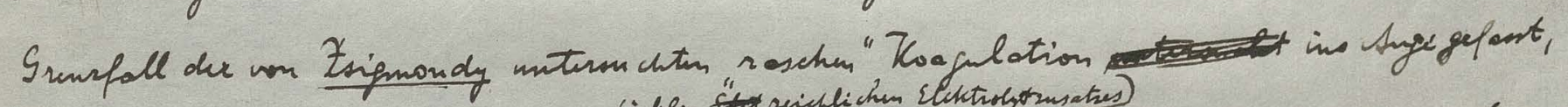

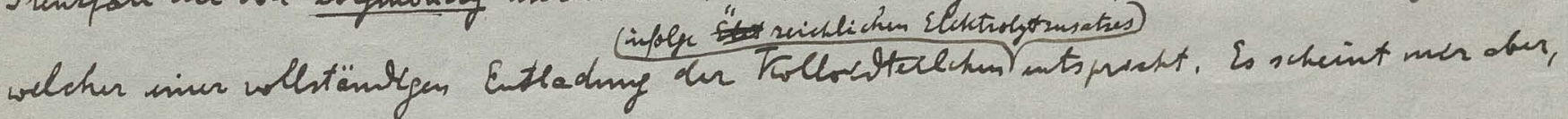

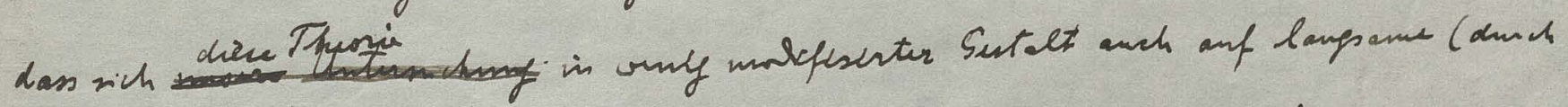

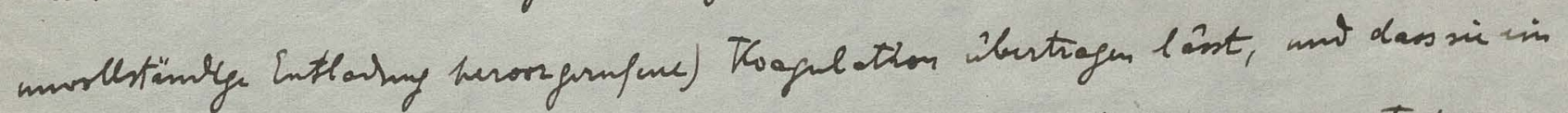

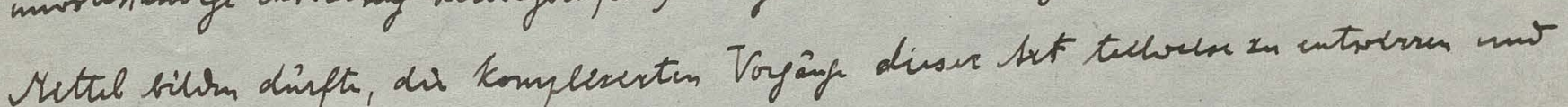

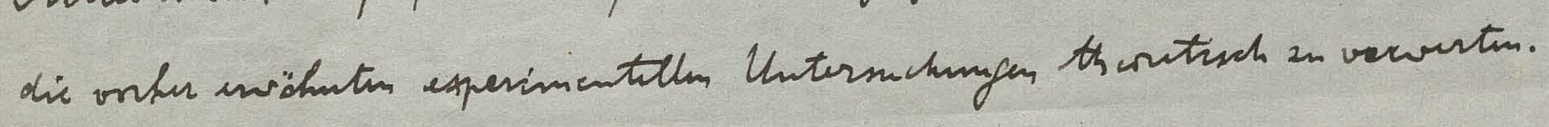

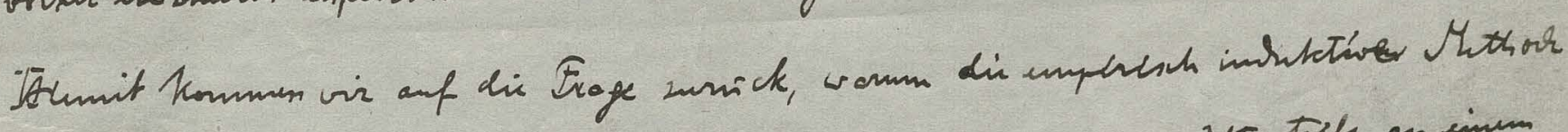

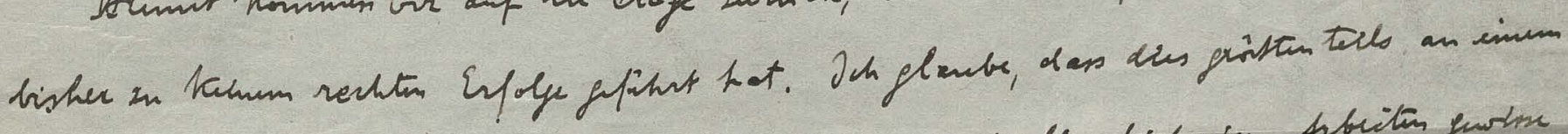

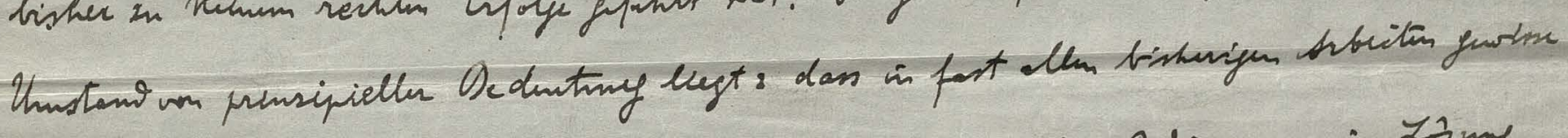

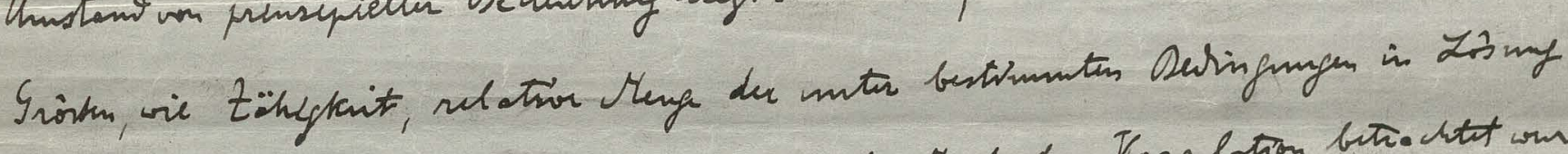

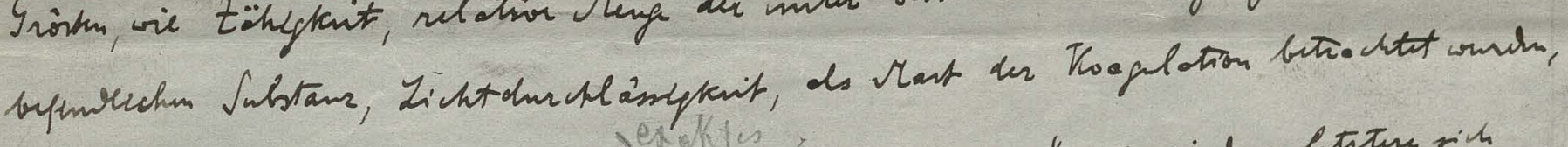

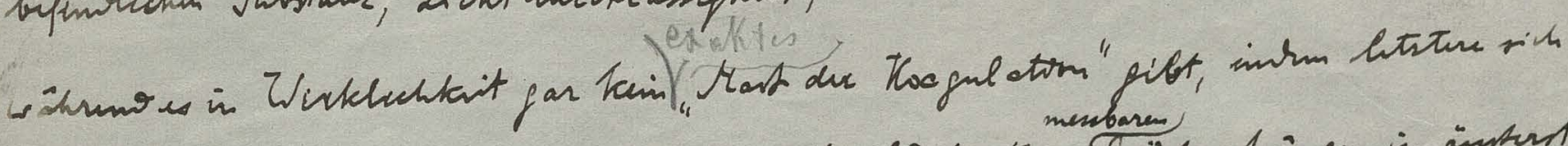

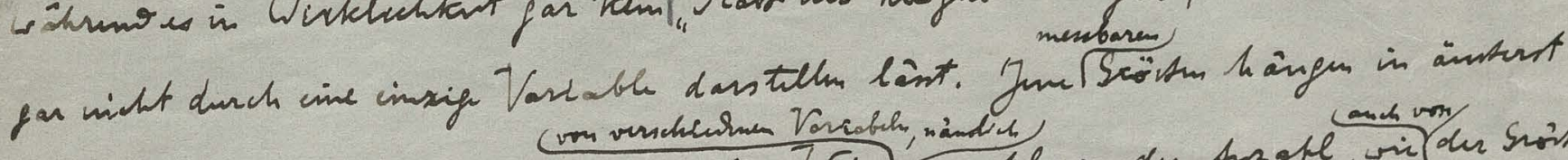

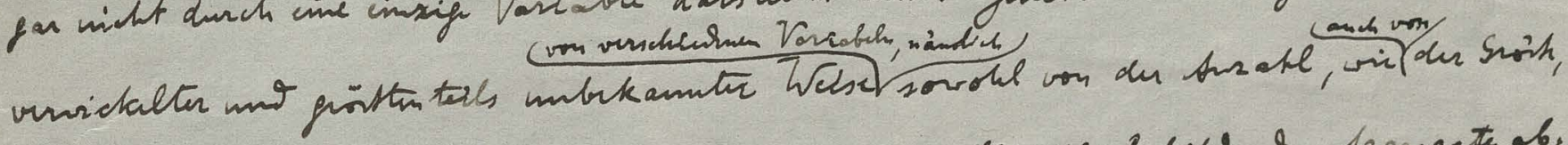

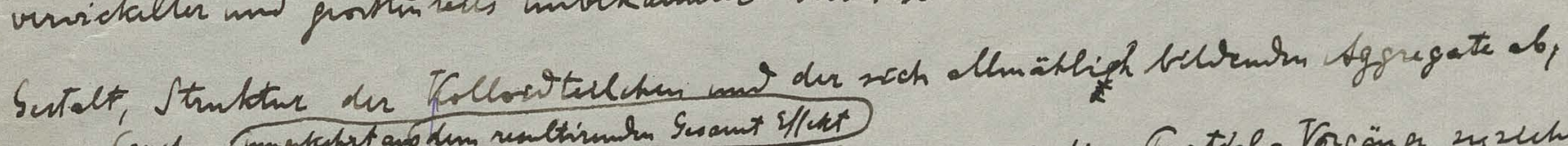

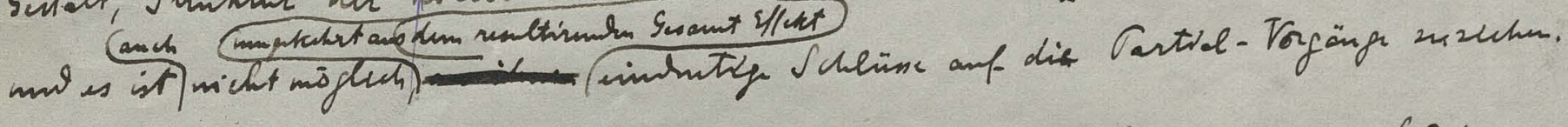

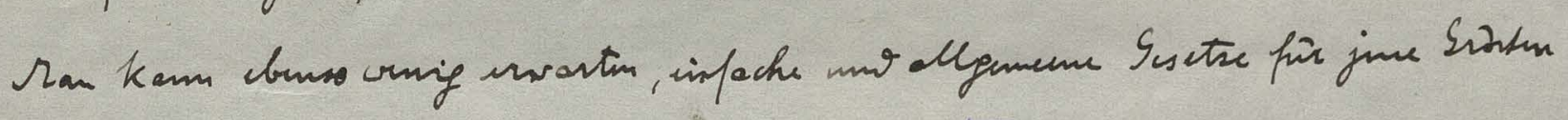

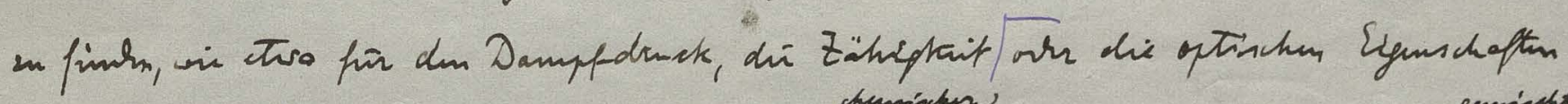

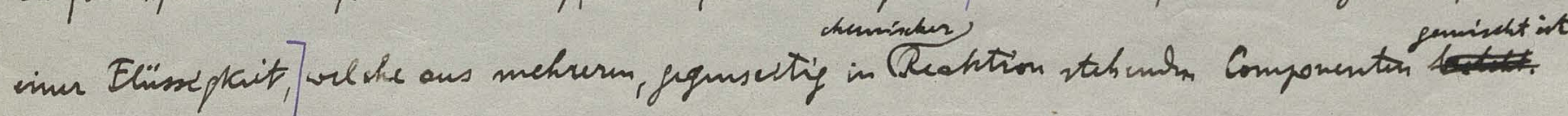

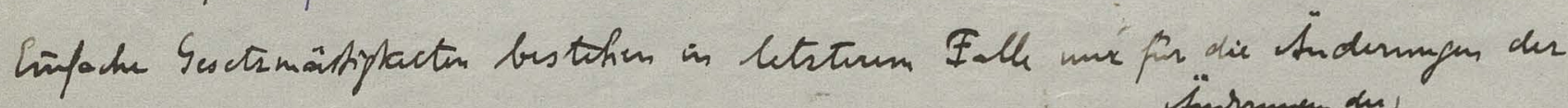

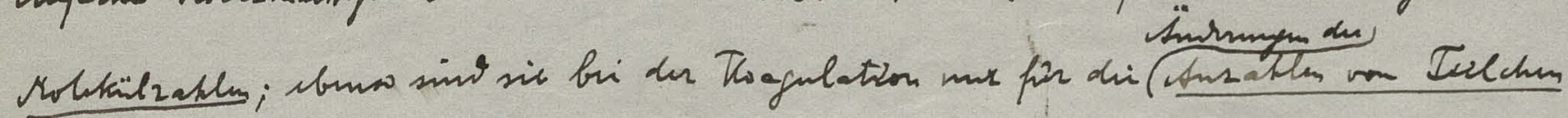

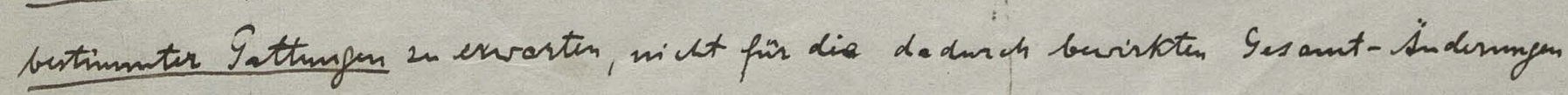

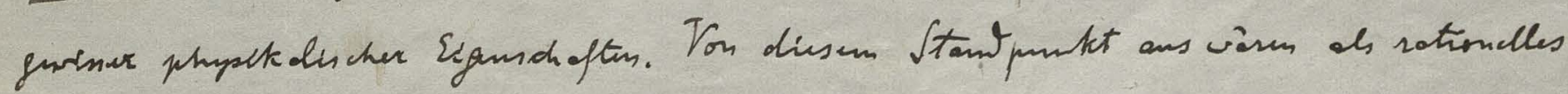

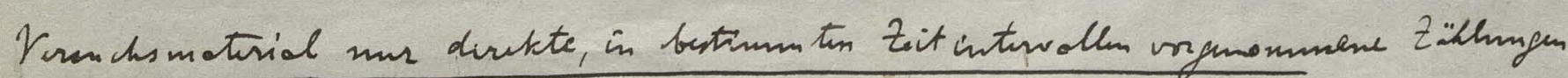




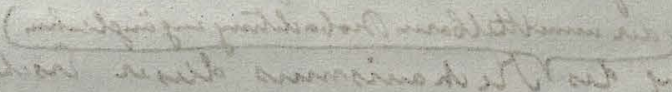

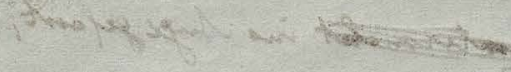

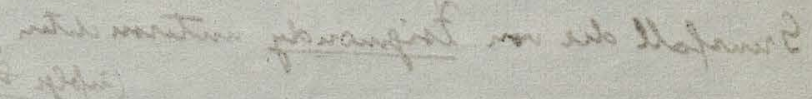

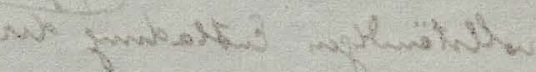

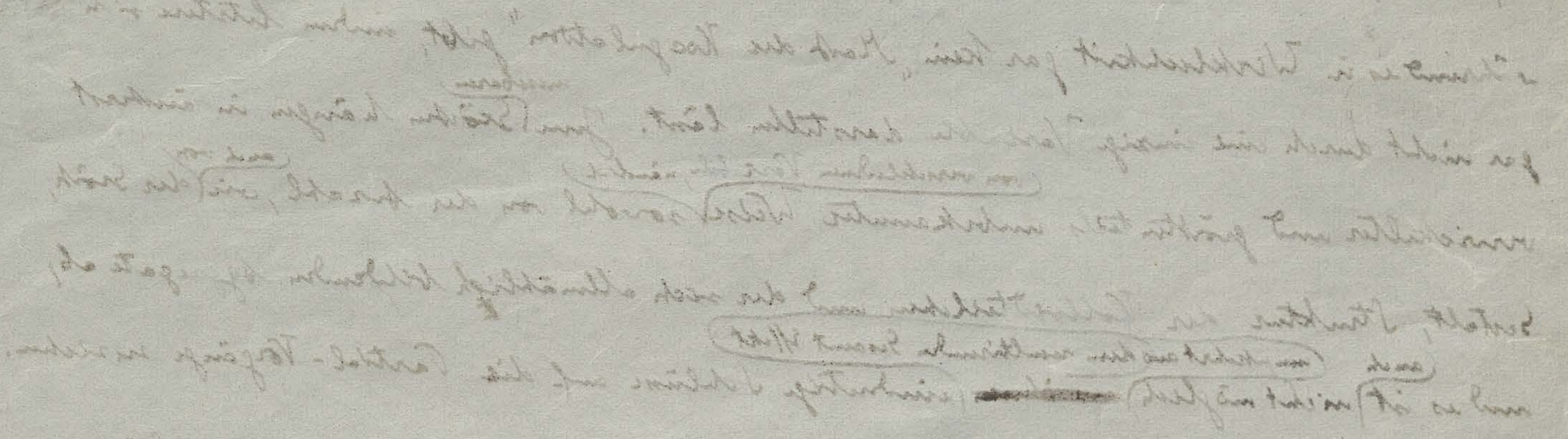
Anow

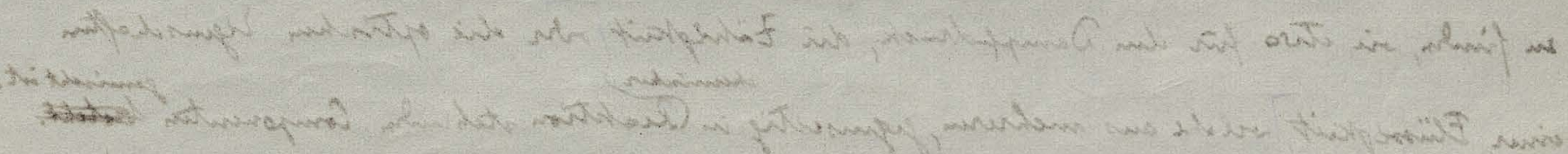

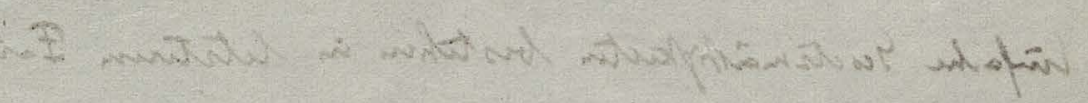$$
\text { chestivinuty }
$$

3it.

$\rightarrow$ wanti:

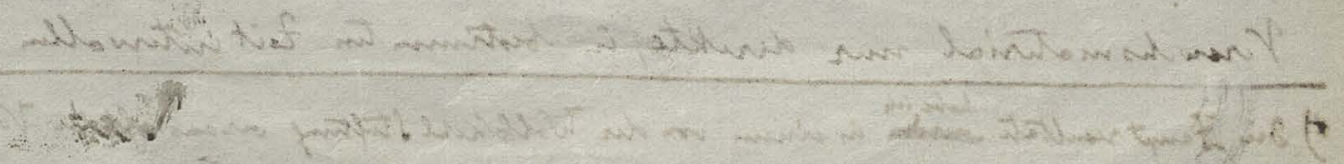




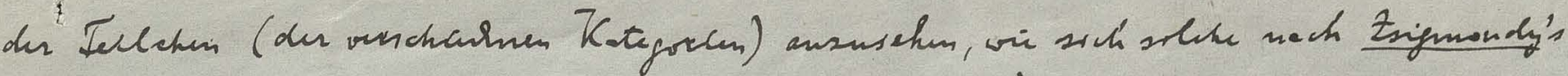

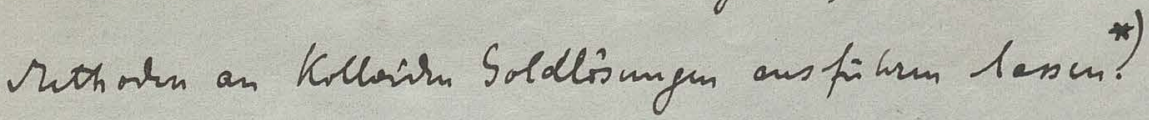

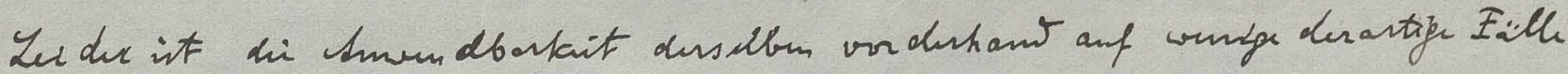

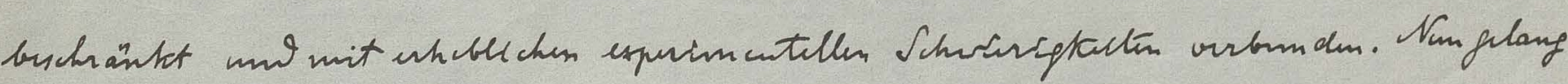

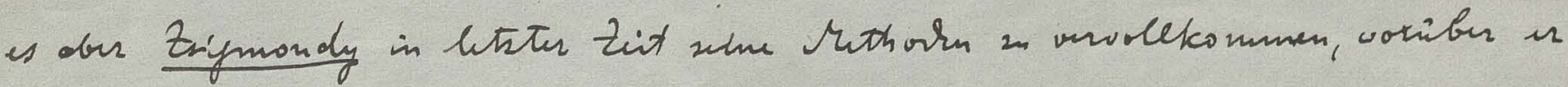
an anbrer Stelle andichlicher berichtm vins, mot it in hoffen, dass in Eutamft

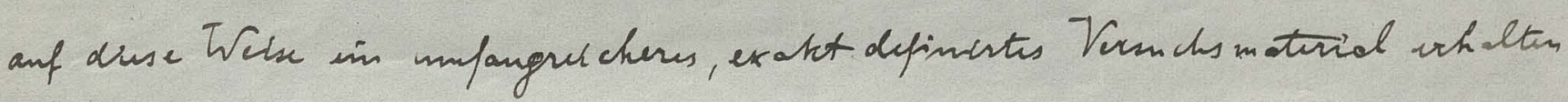

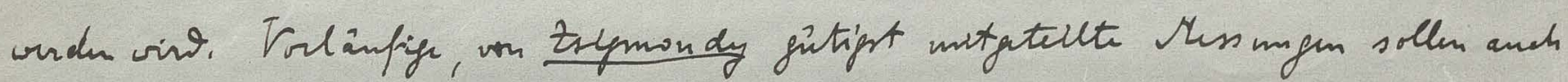

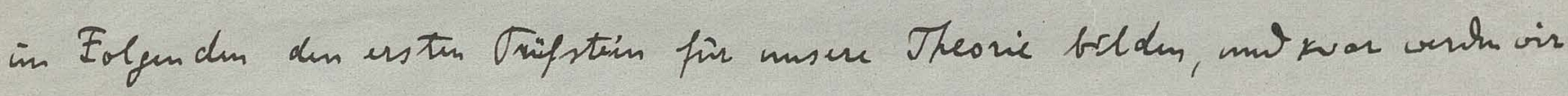

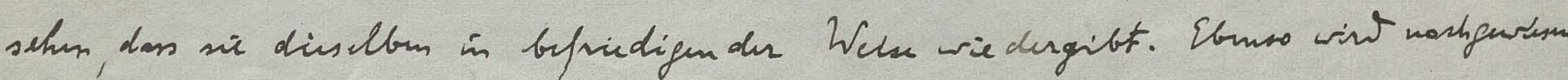

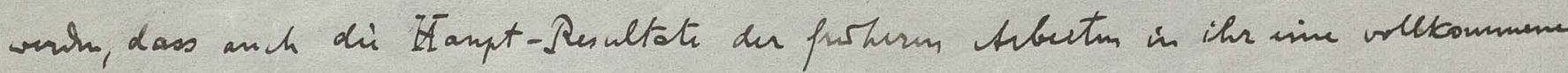

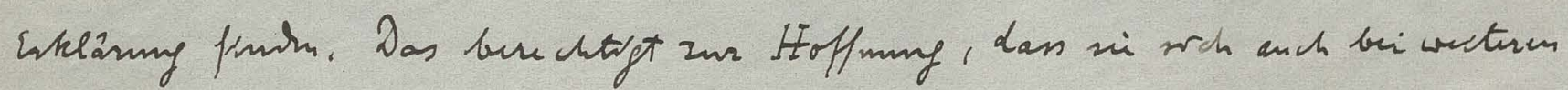

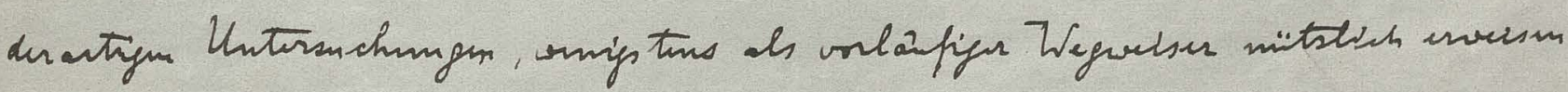
vent

\section{Pryoukduch Srundlagen der Toagulationstherric.}

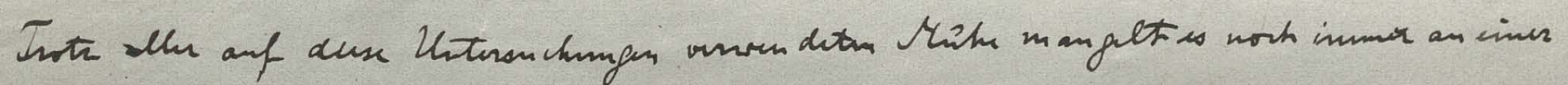

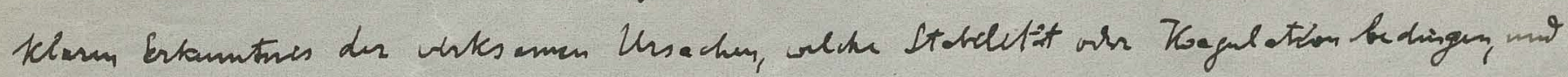

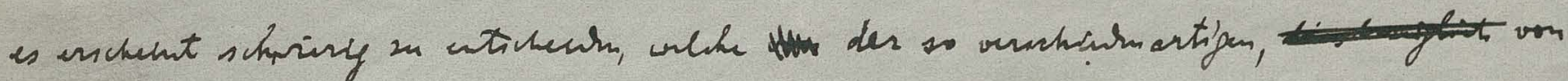

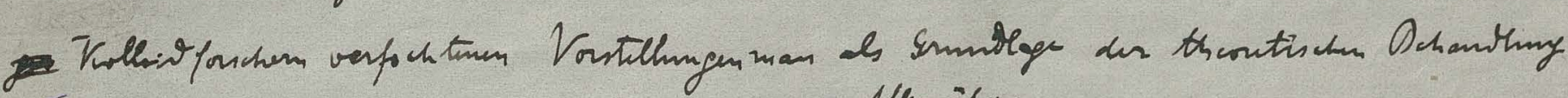

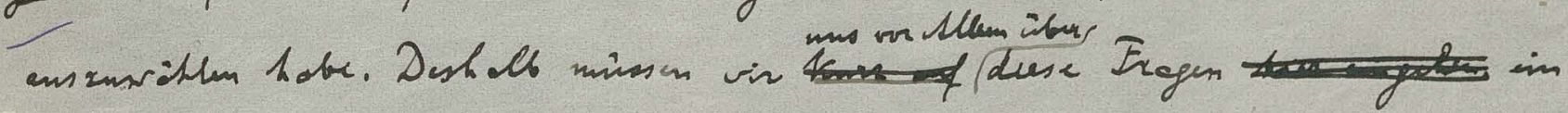
Untile billom.

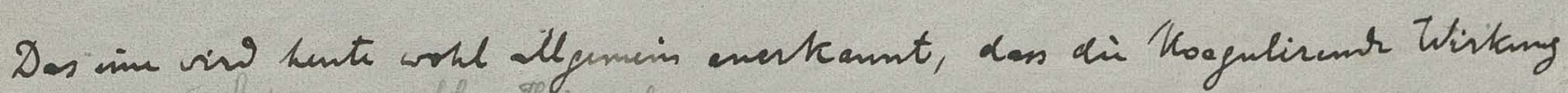

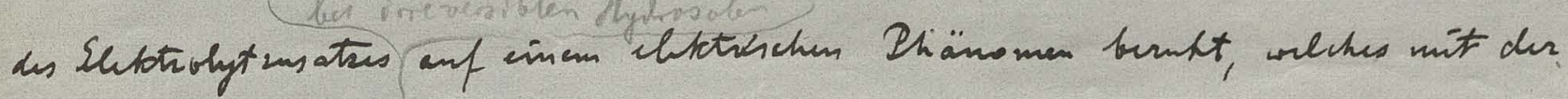

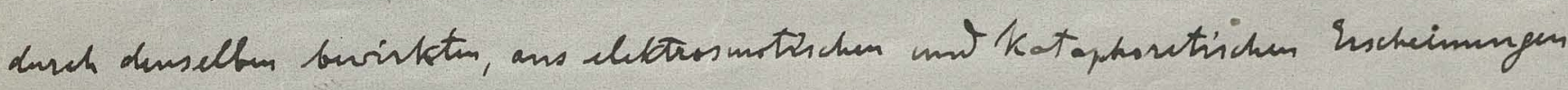

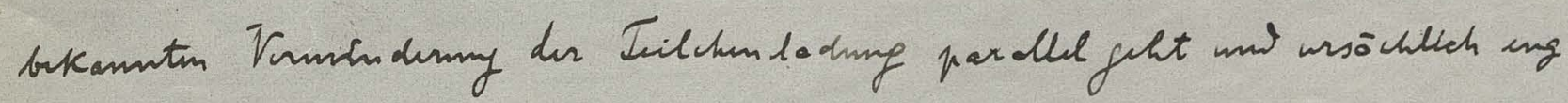
zus amment angt.

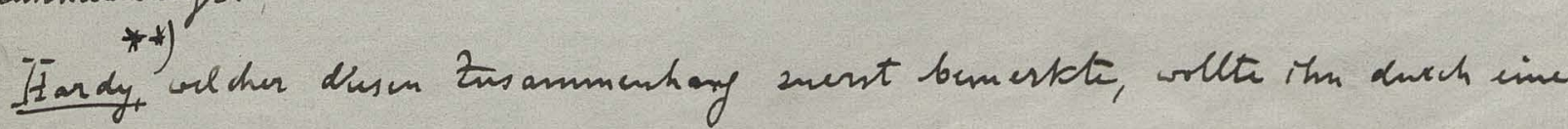

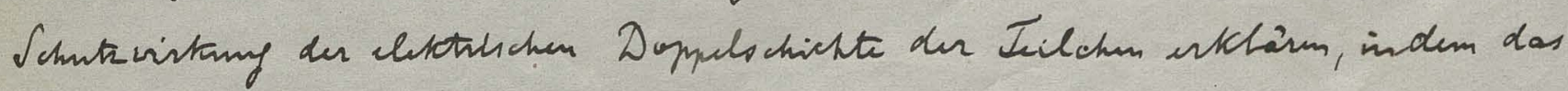

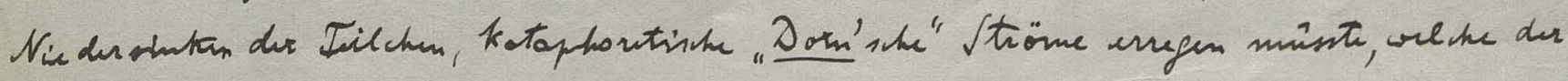

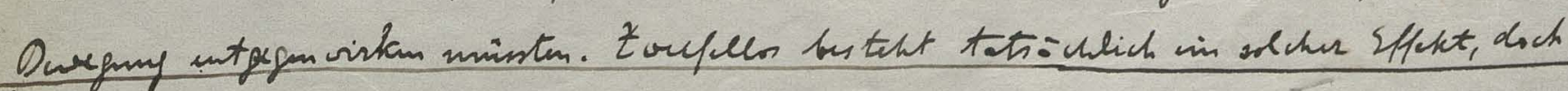




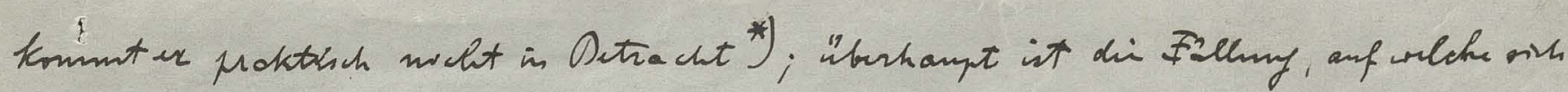

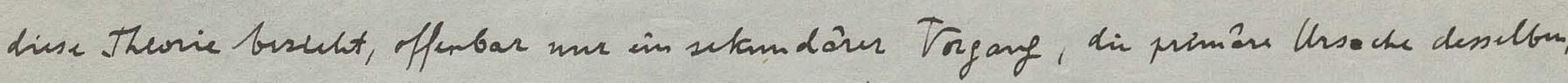

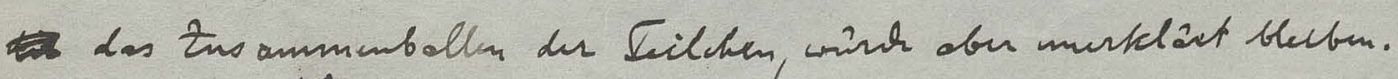

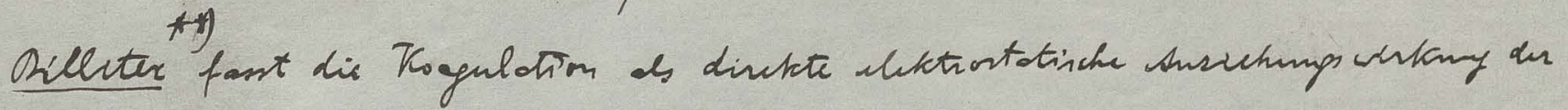
Joven anf di geladenen Filchen anf. So cimpoch Kann doer der Mechanismus zicher

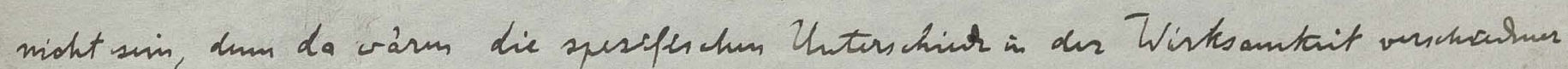

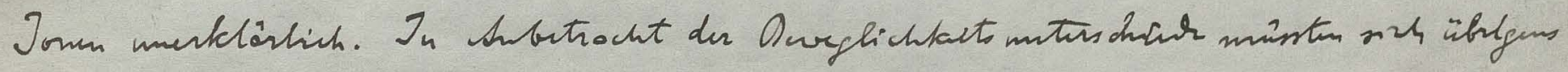

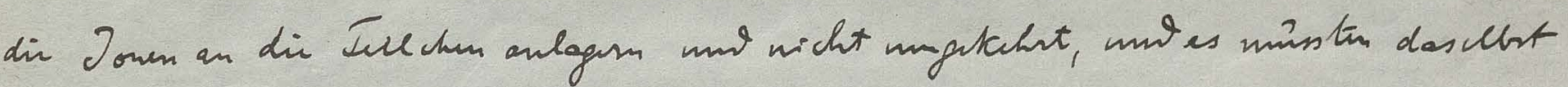

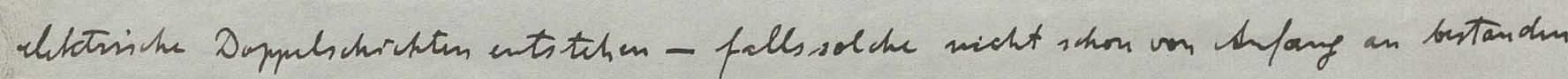
Latten.

Es int ibuhaupt en butorem, dass man jich dir "Teilchm lachmign" nicht, wi des

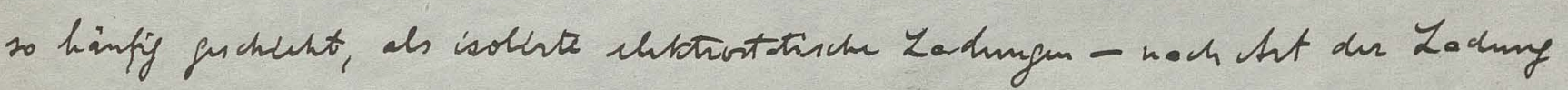

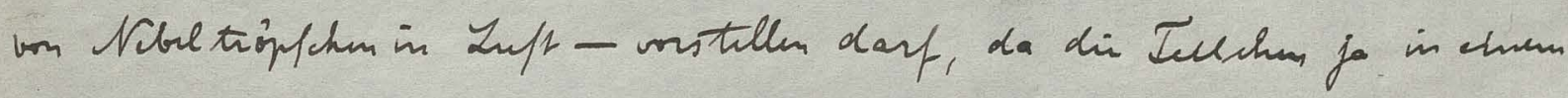

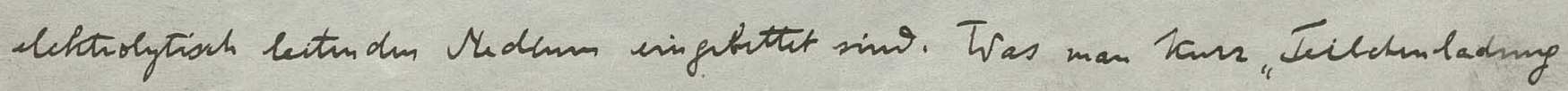

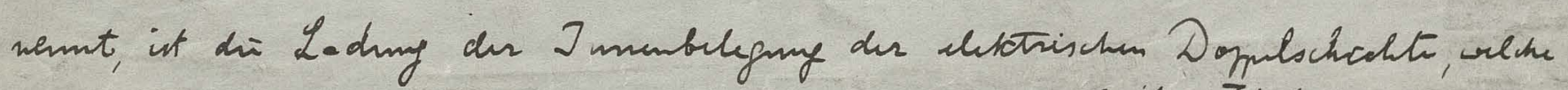

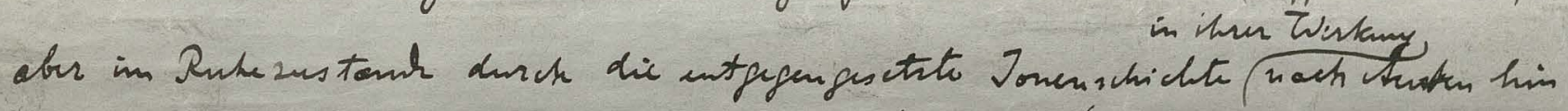

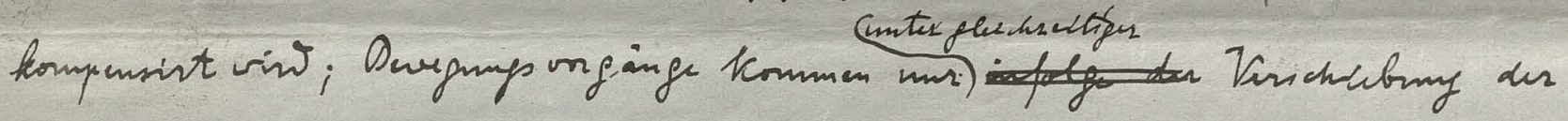

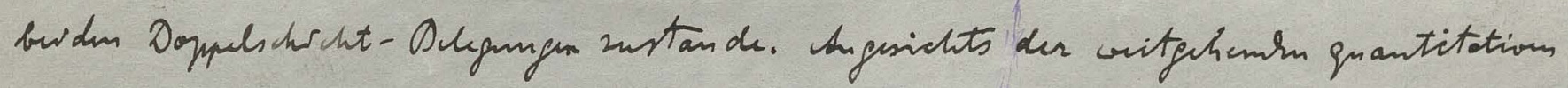

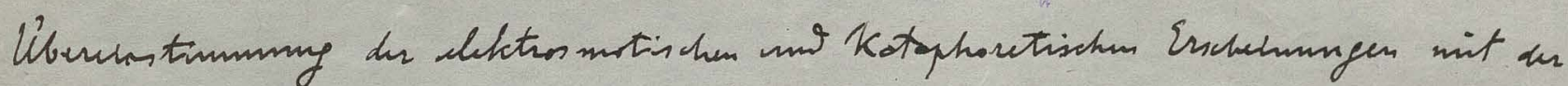

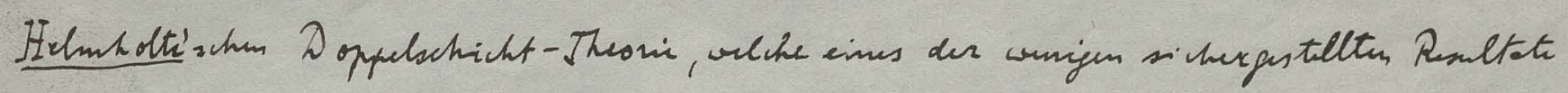

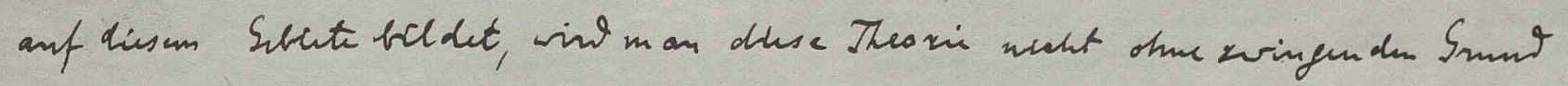
oulessen dirfan.

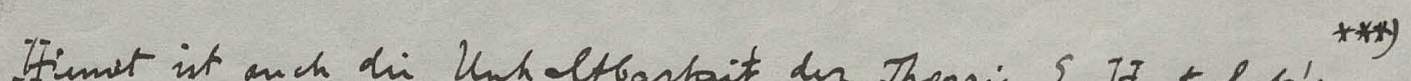

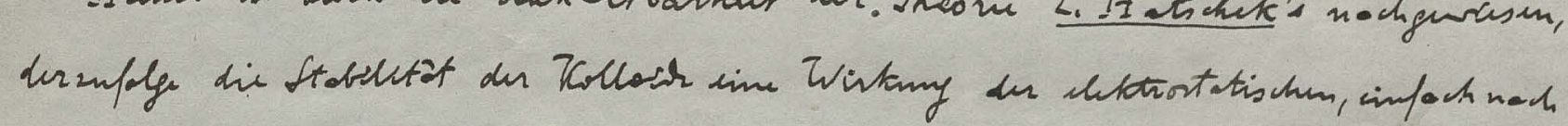

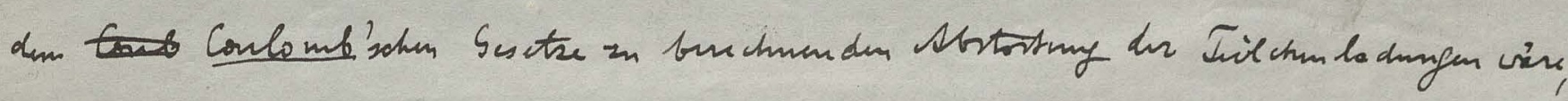

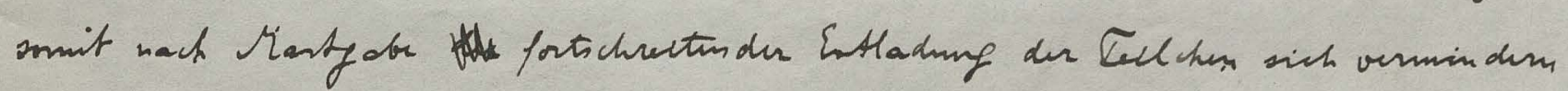

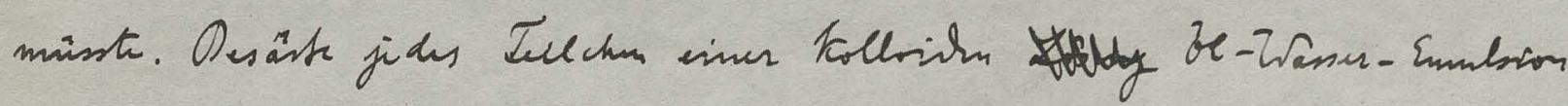

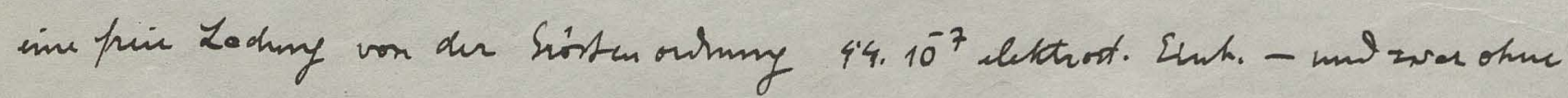

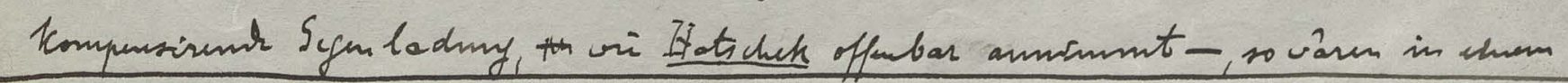

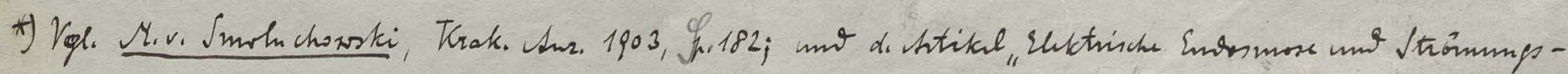

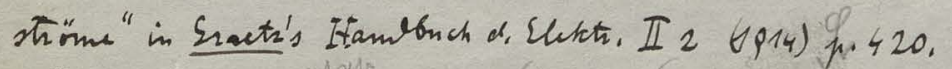

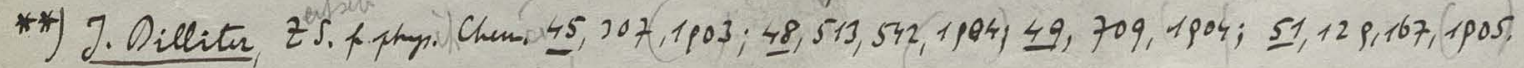

***) E. Ifatichek, Toll. ZS. $9,159,1$ 111. 


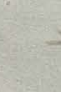

mato nativin
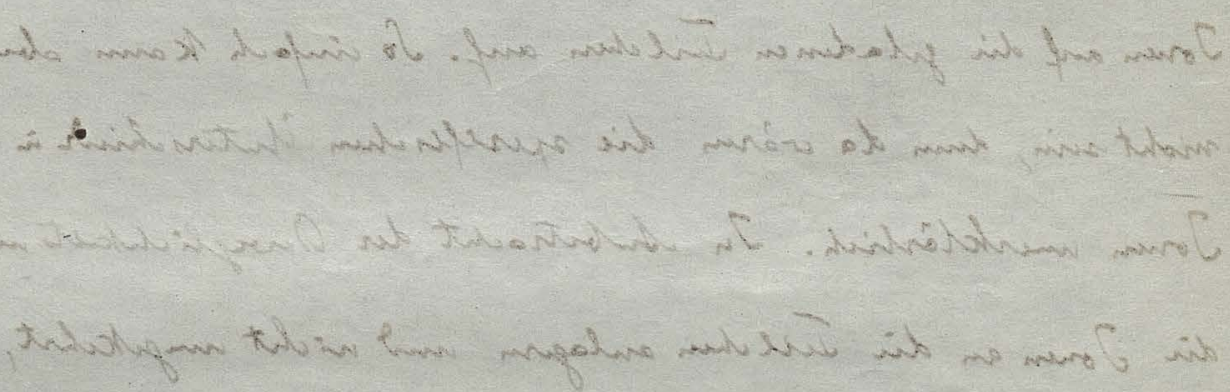

\section{$\rightarrow$}

no wal
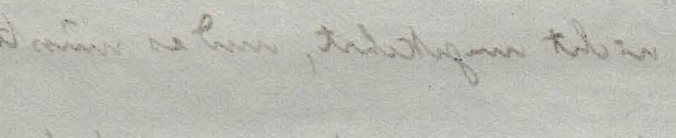

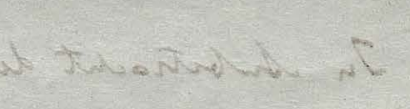

\section{ang $x$}
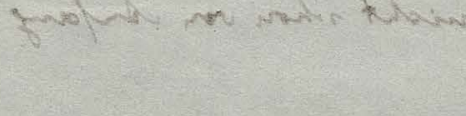

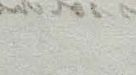

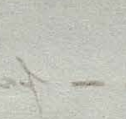

and $\rightarrow \operatorname{man}=5$

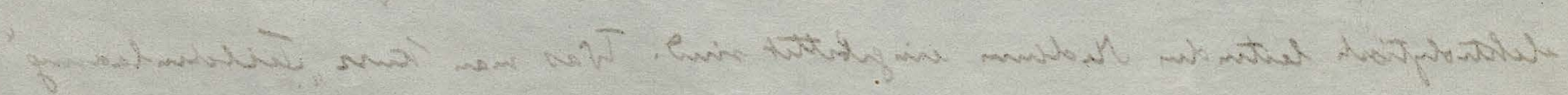

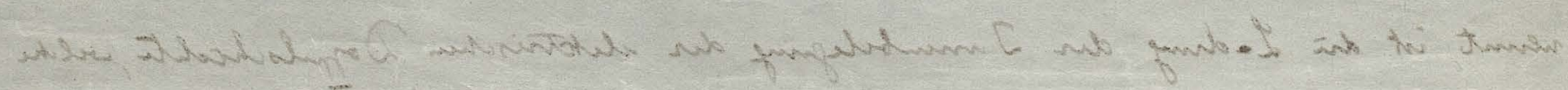

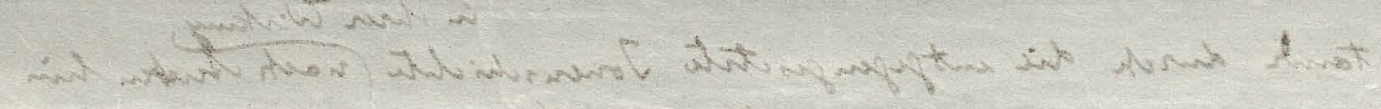

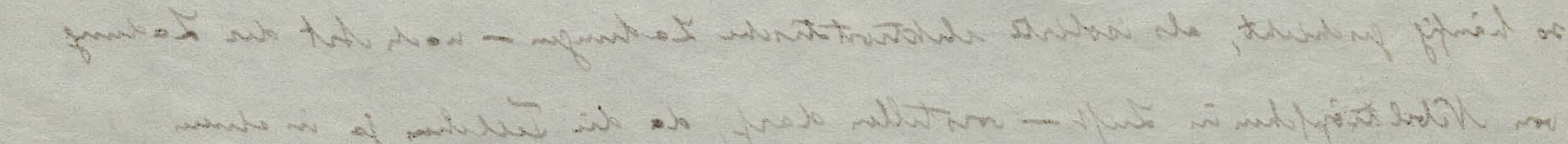

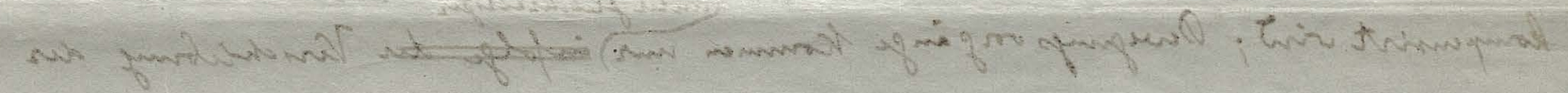

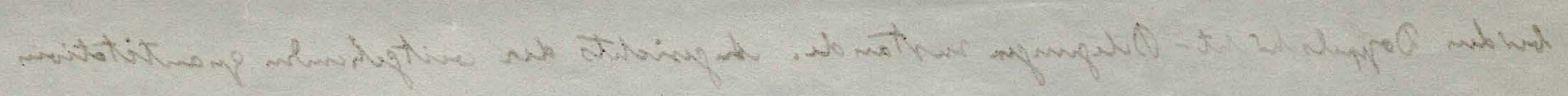

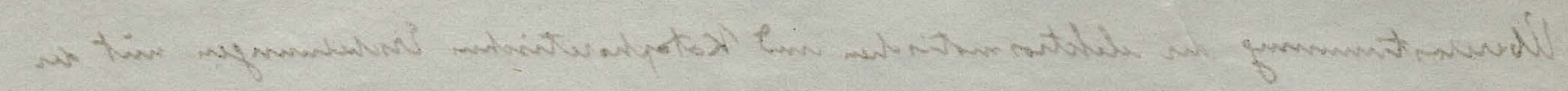

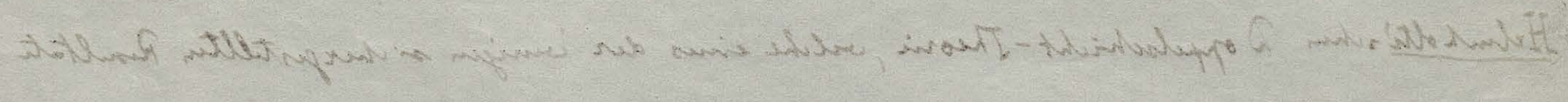

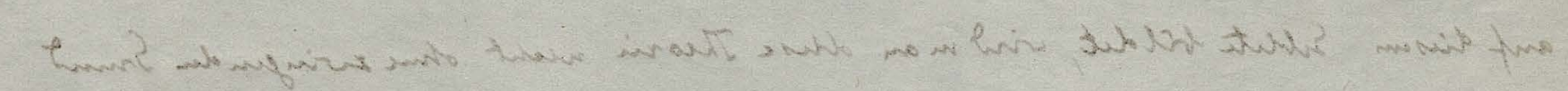

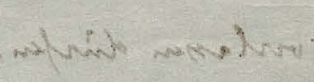

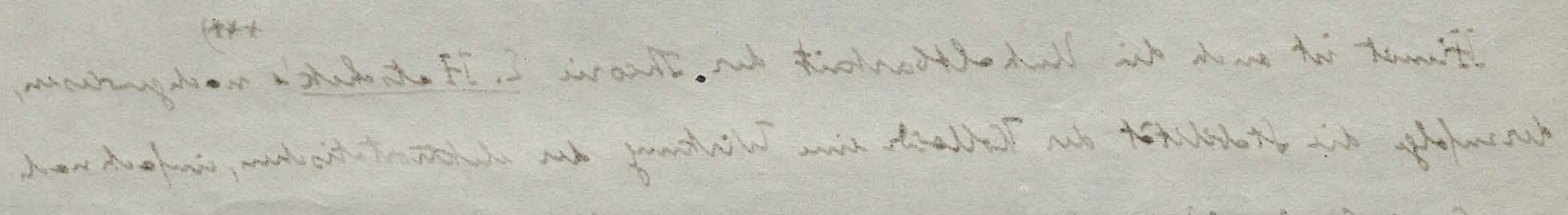

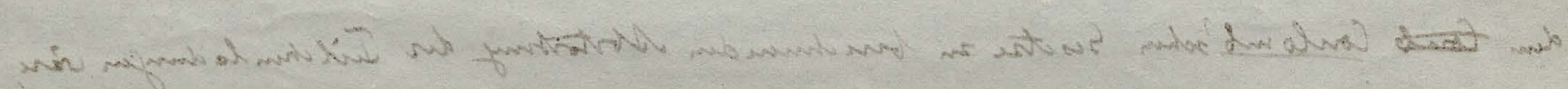

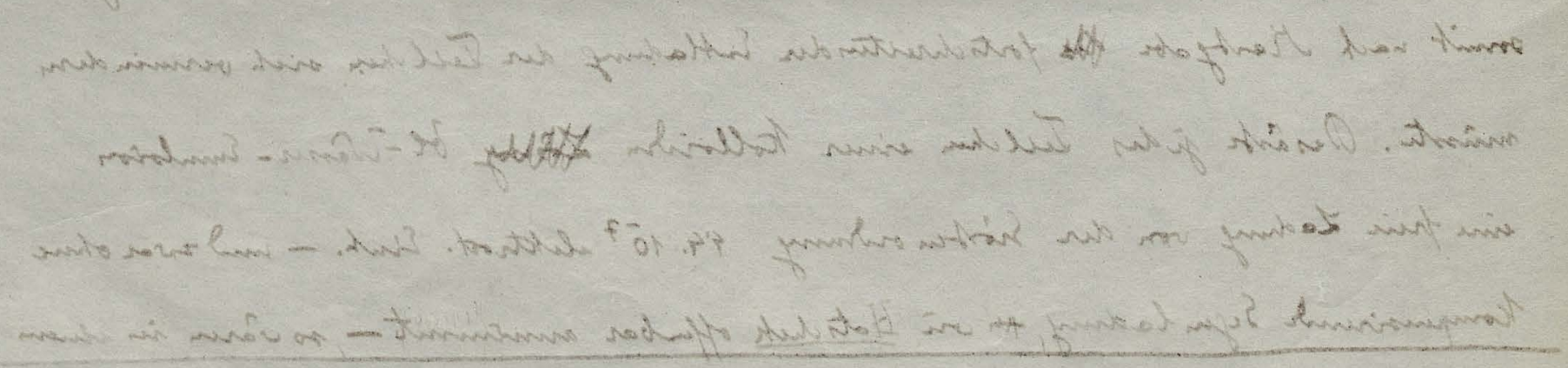




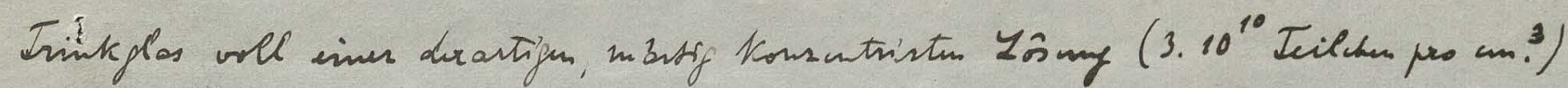

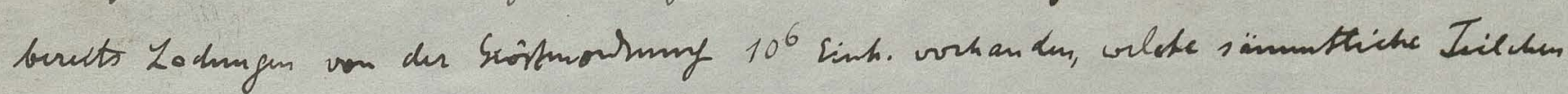

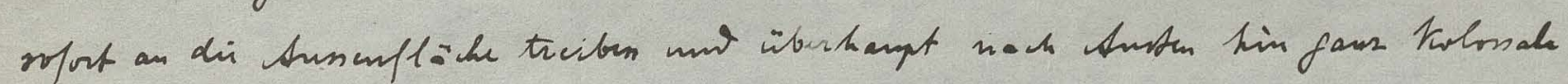

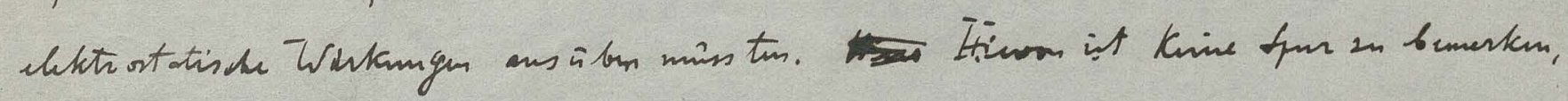

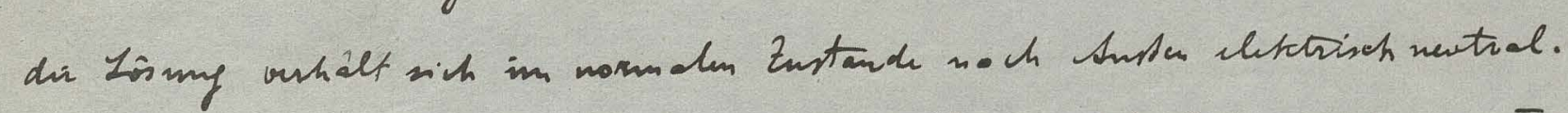

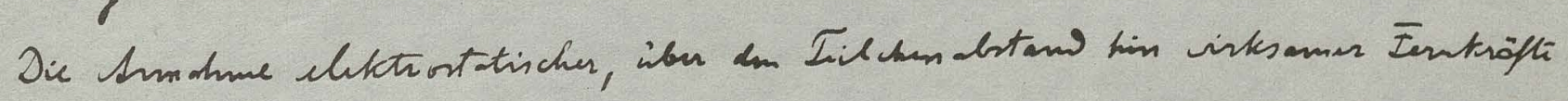

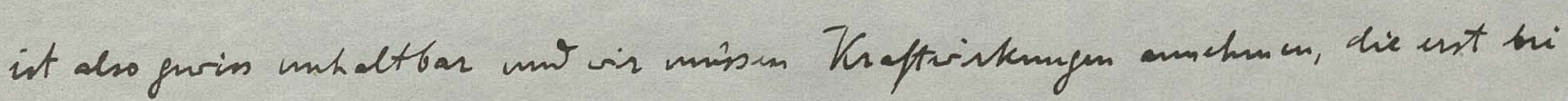

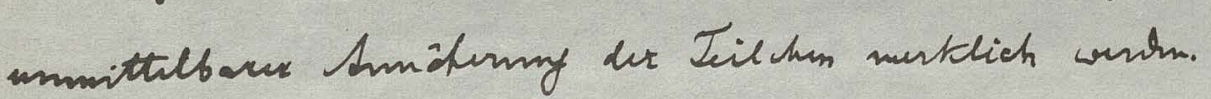

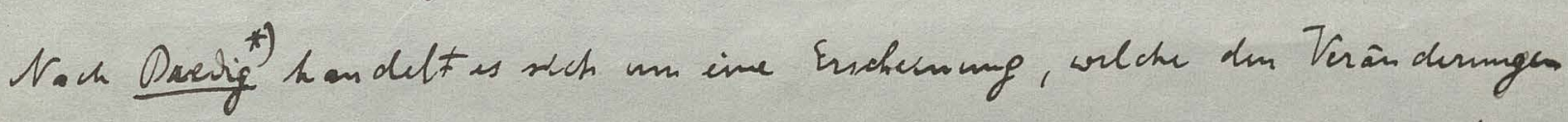

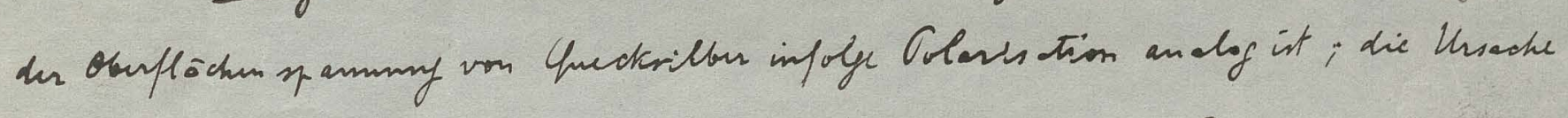

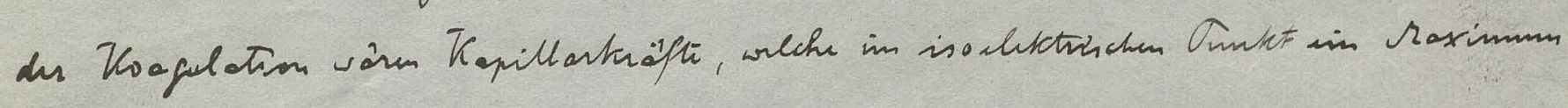

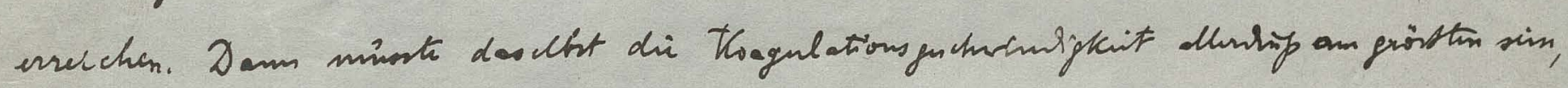

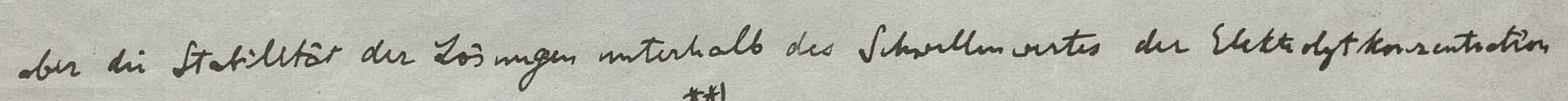

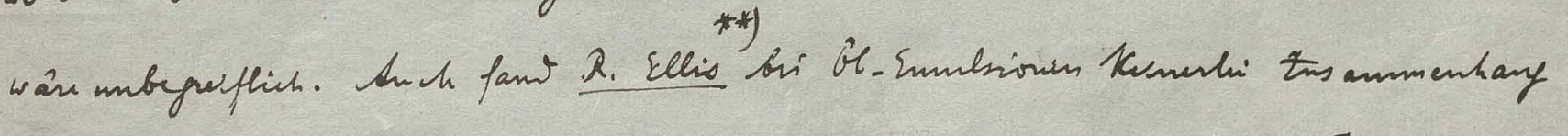

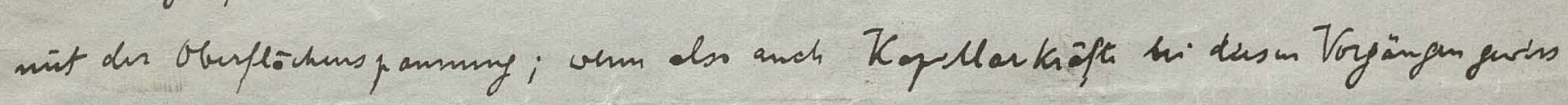

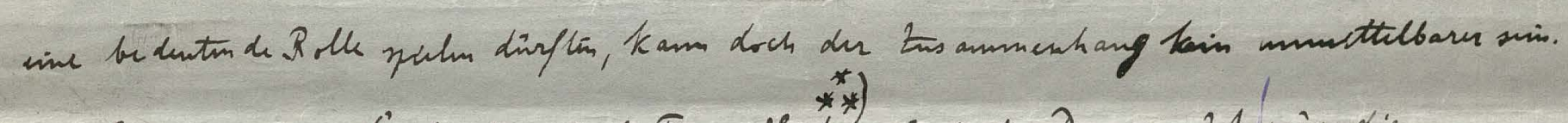

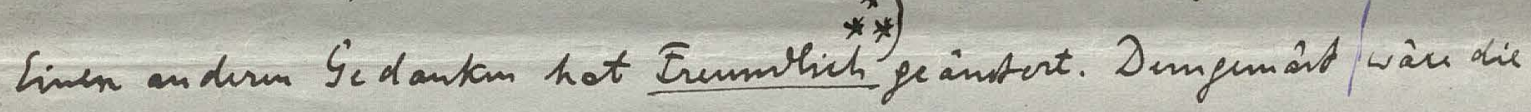

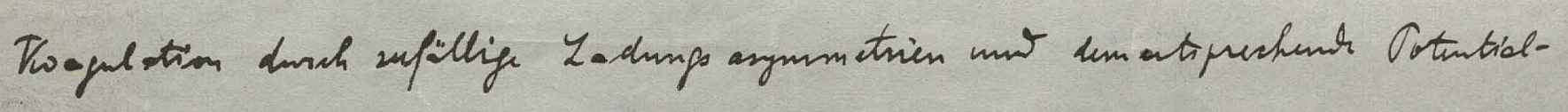

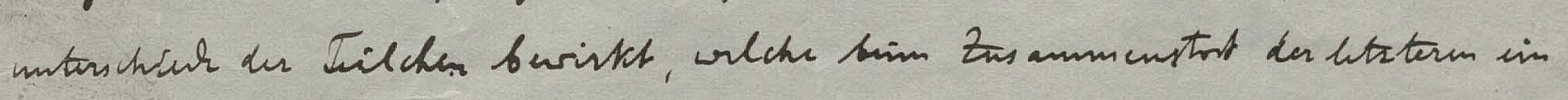

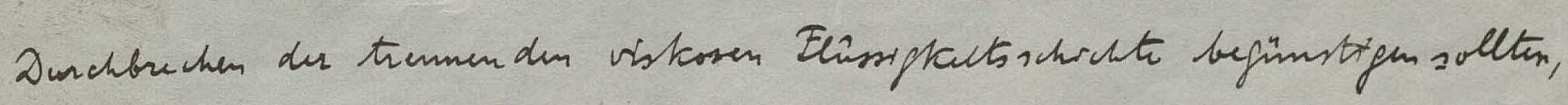

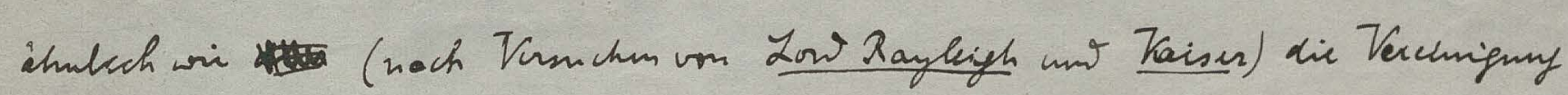

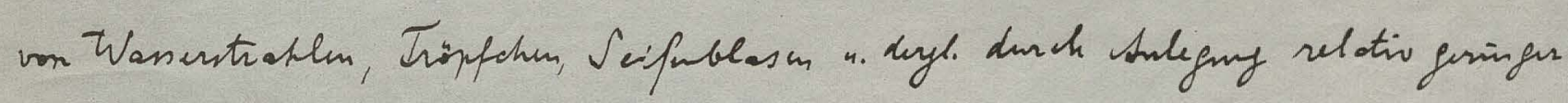
Ootentidunterehies beschlemight wint.

Die Ursache dis letteren Vorganges ist abea Offenber in des Ansamminng

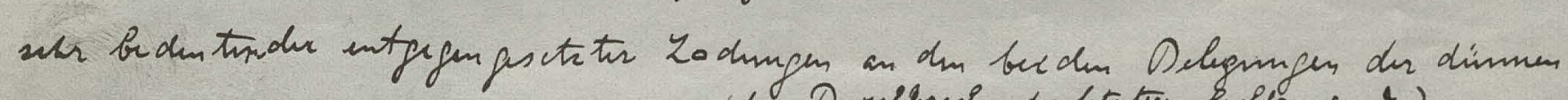

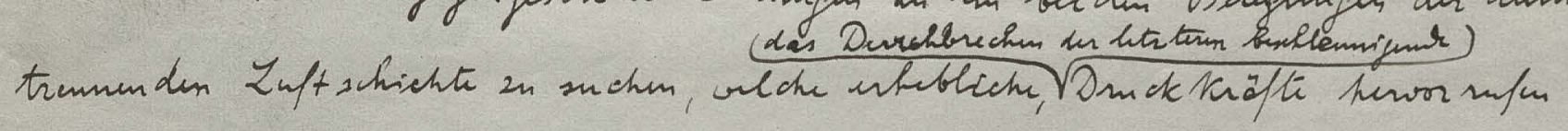

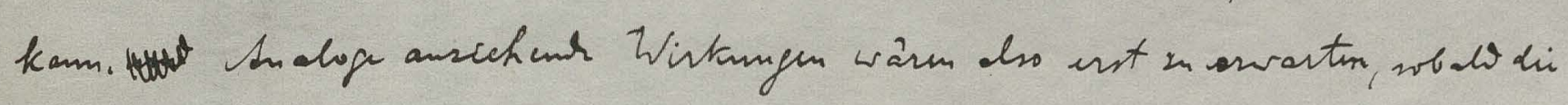

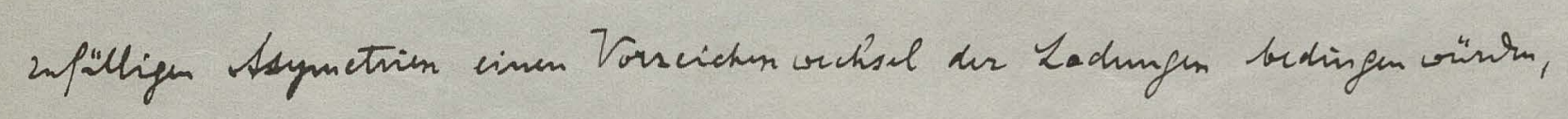

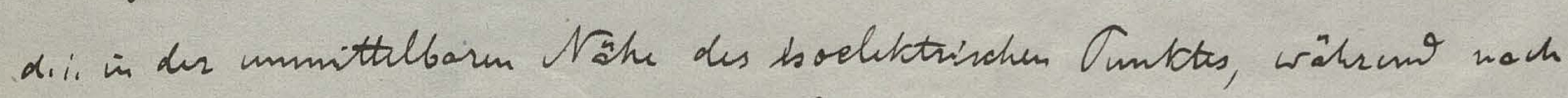

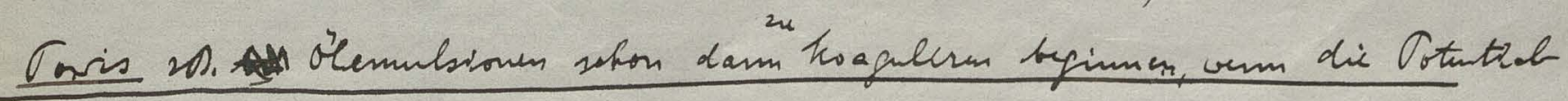

*) S. Orealy, Anorg. Ermente 1po1.

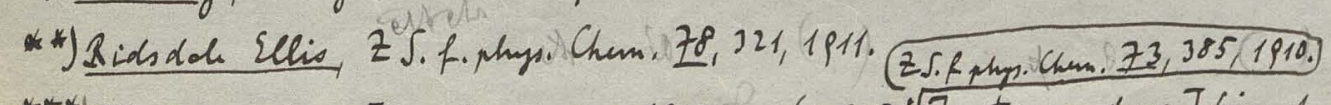

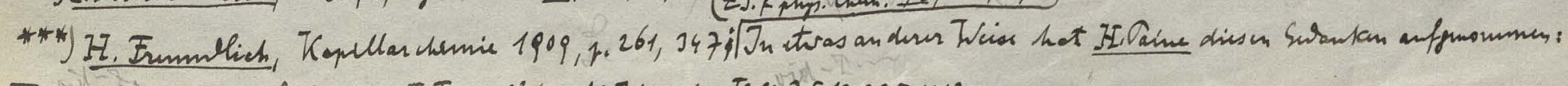

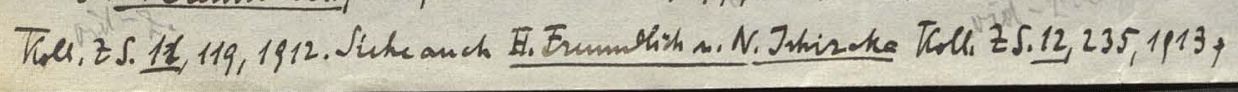




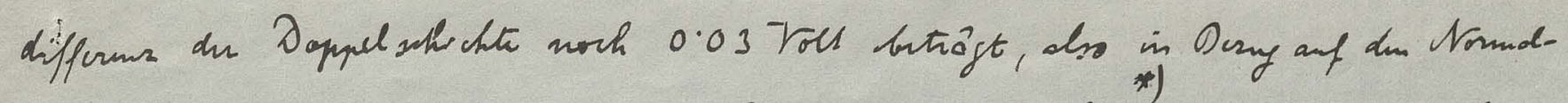

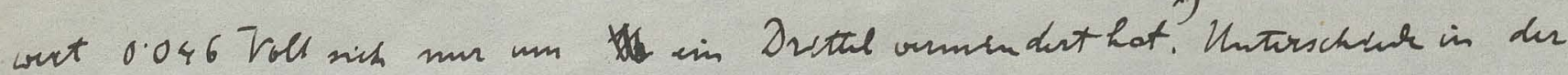

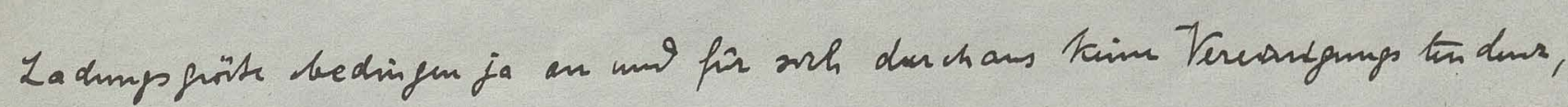

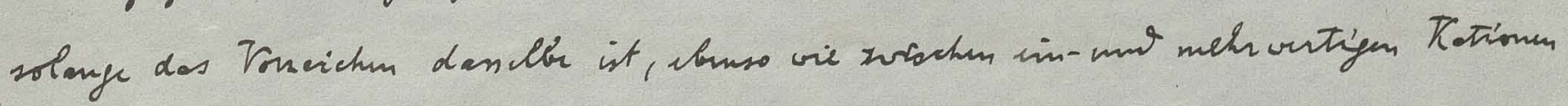
nicht dos unin des te Oestebm einer Vacinigury buteht.

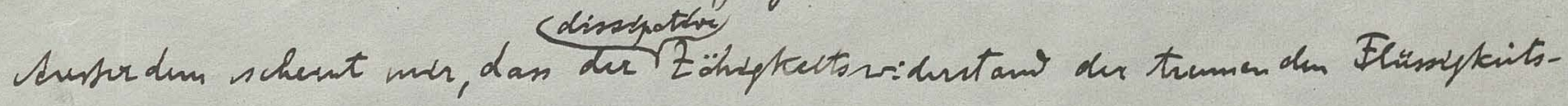

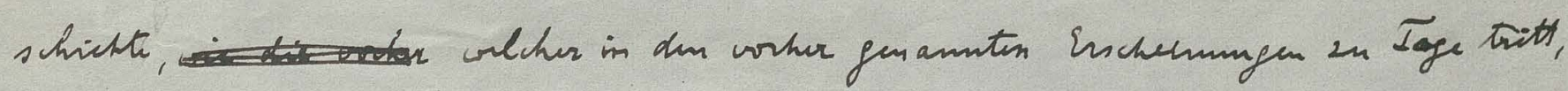

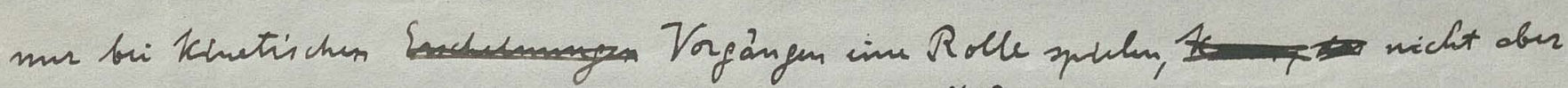

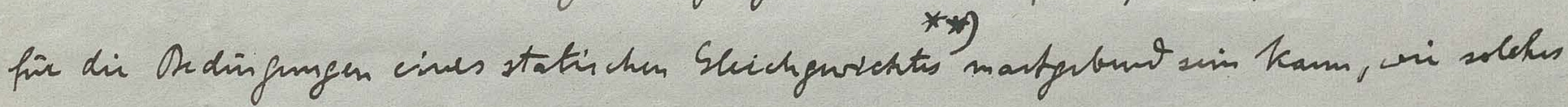

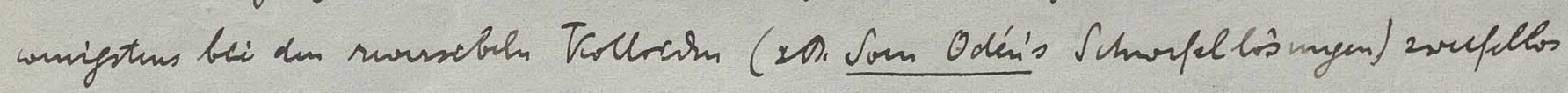

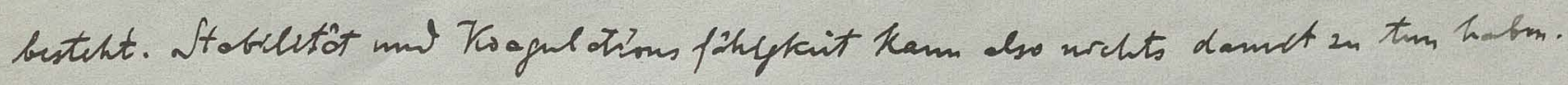

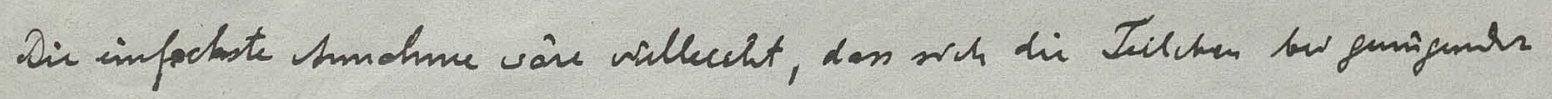

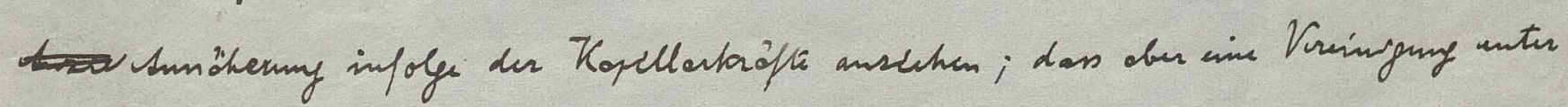

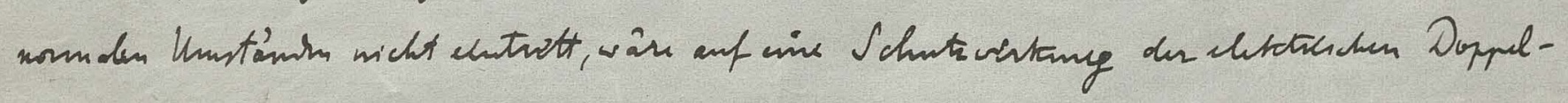

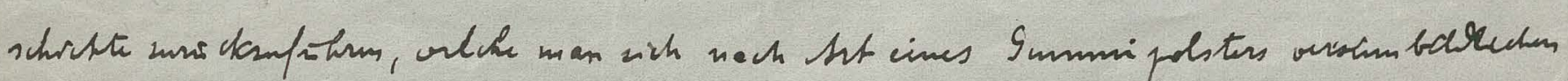

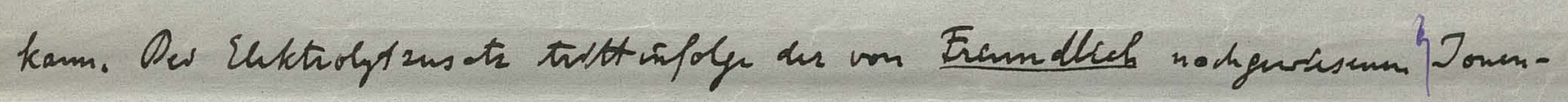

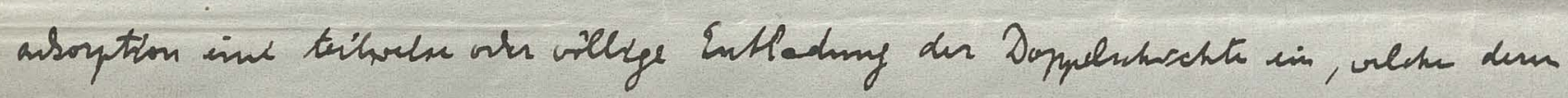

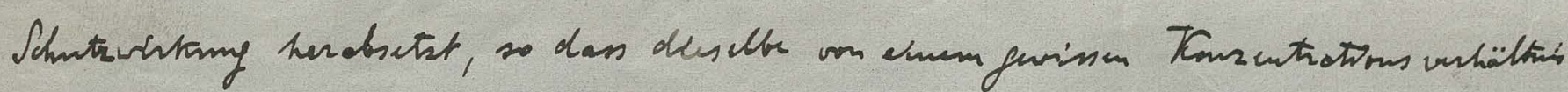

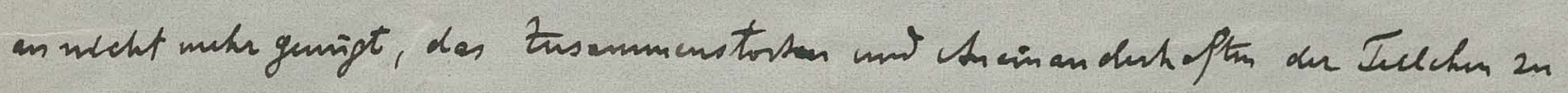
verhinderm.

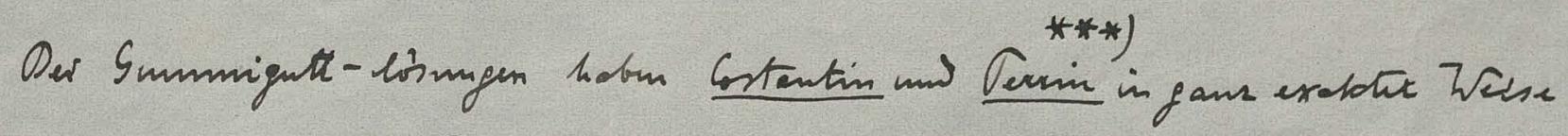

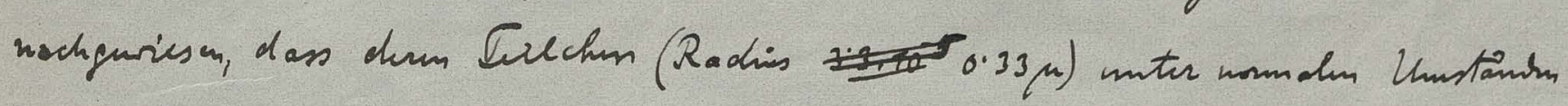

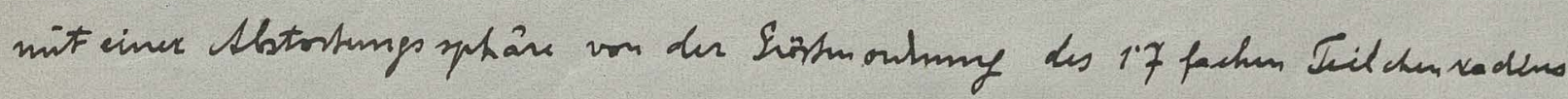

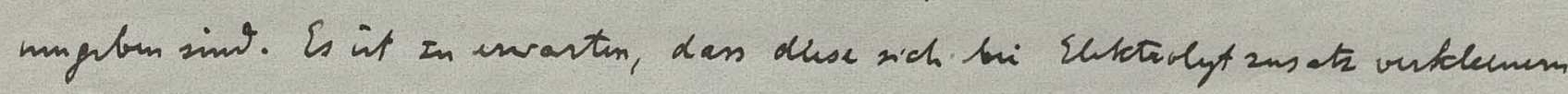

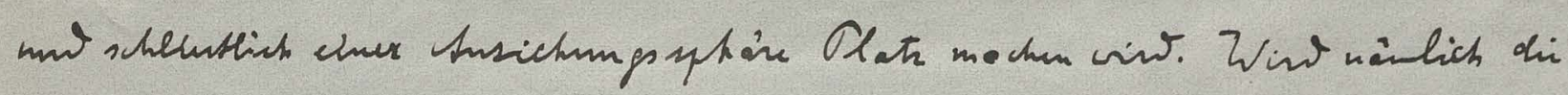

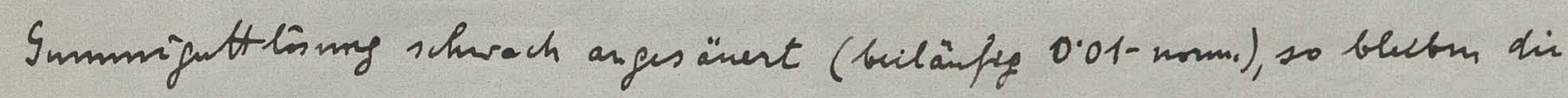

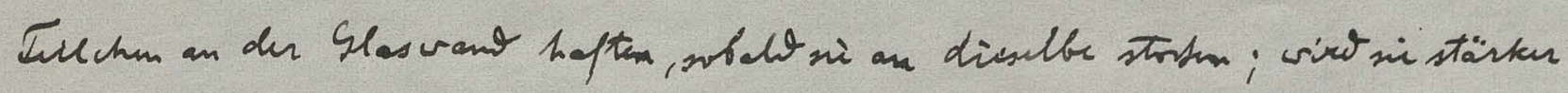
angesinuert, so versinigen sie jich anch nutereinander $2 n$ Aggregatm, "obalo sis

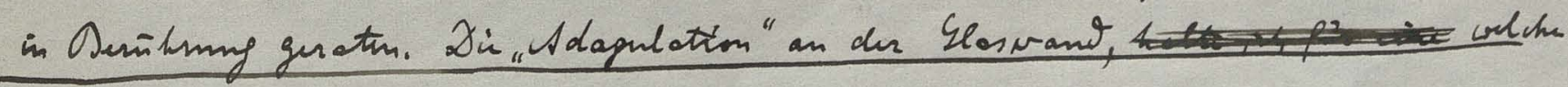

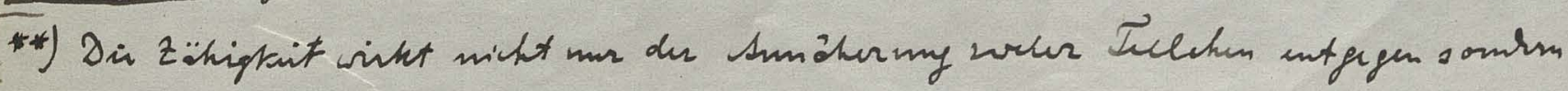
sturso auch derm Entferoming.

***1) J. Ournin, C.R. 158 1168, 1914; R. Contantin, C. R. 158, 1171, 1341, 1914.

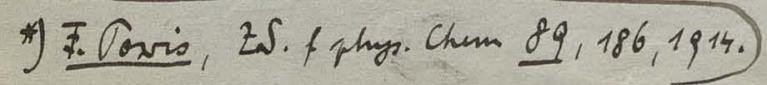


that $\sin$

- Sindwant at

Comatimat:a

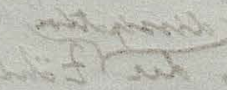

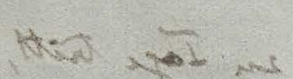

renthos

ator whe sicia

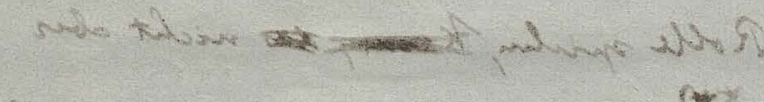

Nato:

$(* x)$

che burans

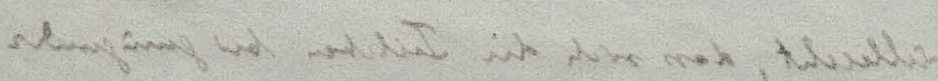

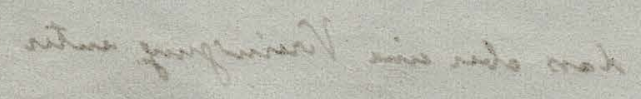

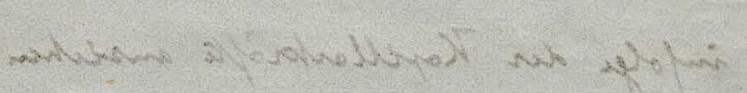

sigento

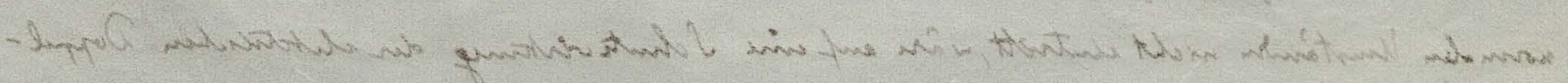

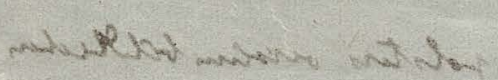

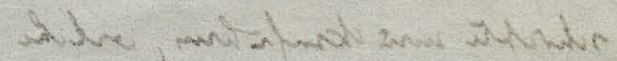

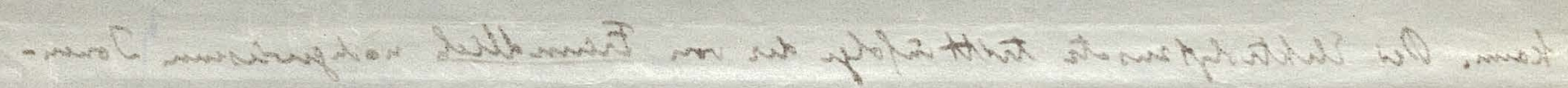

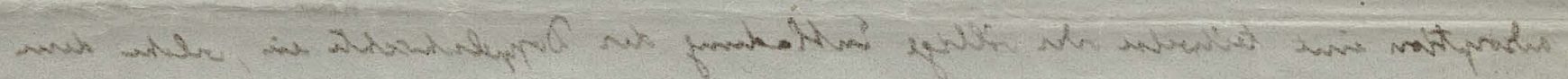

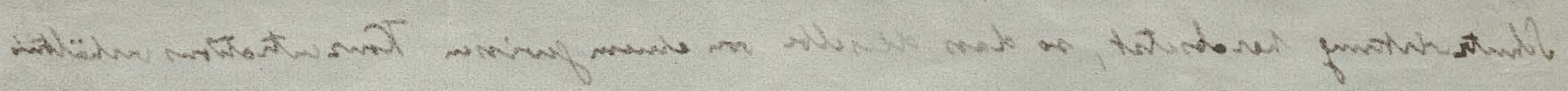

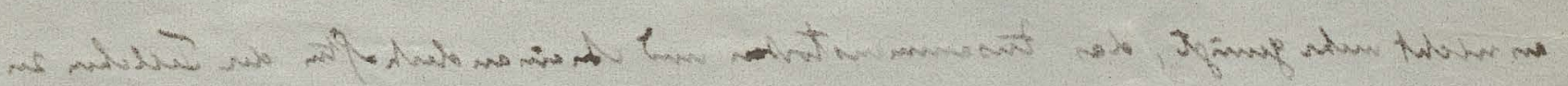

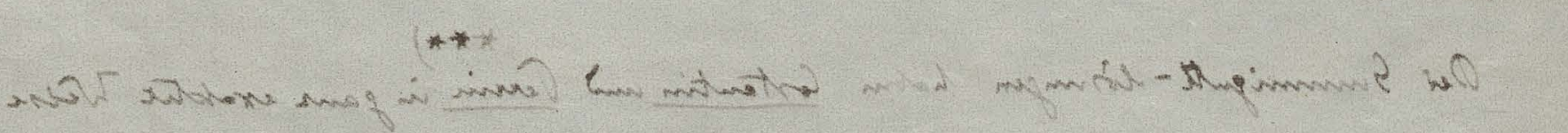

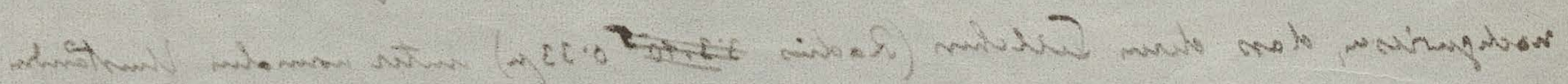

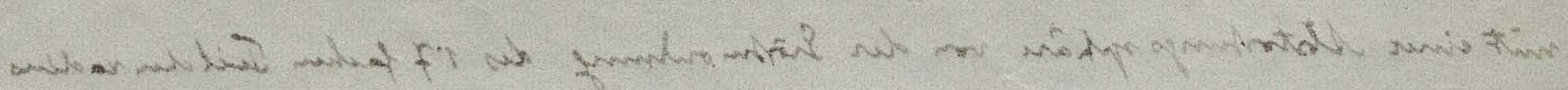

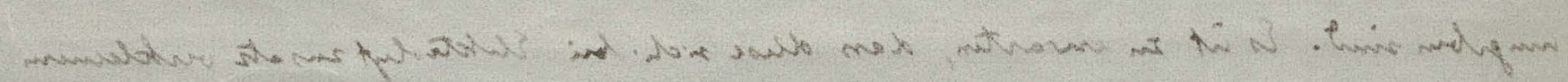

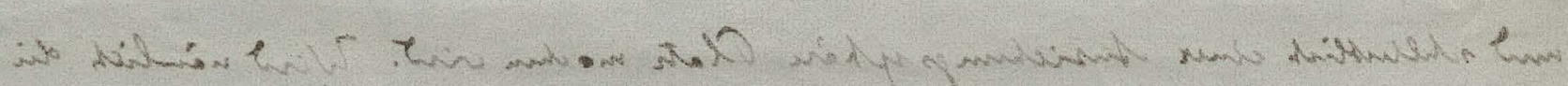

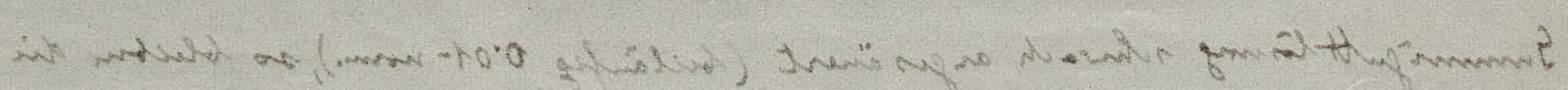

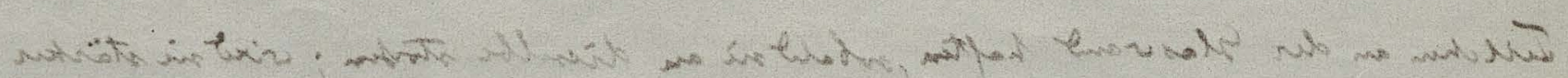

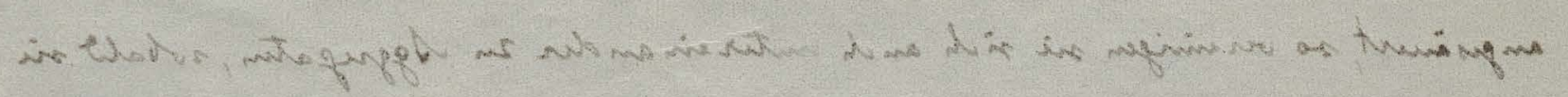

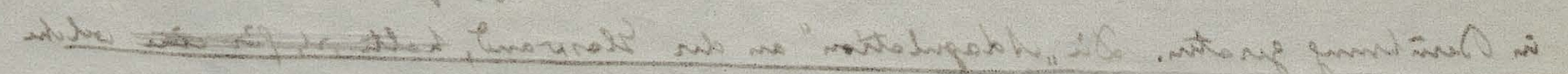

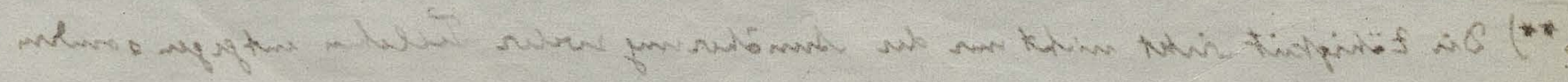

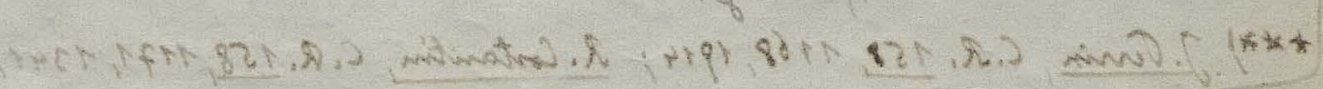

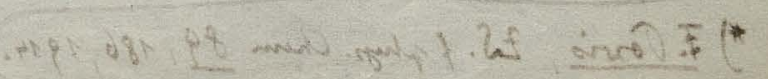




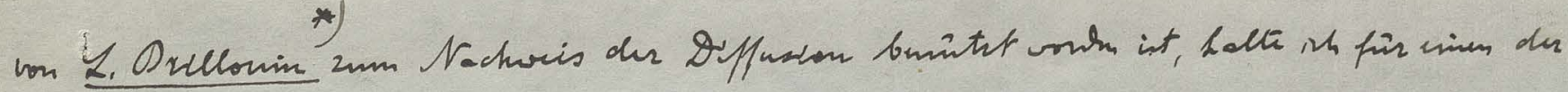

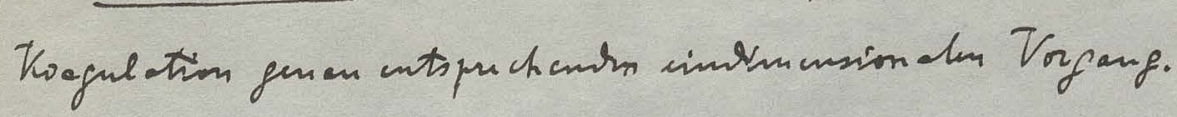

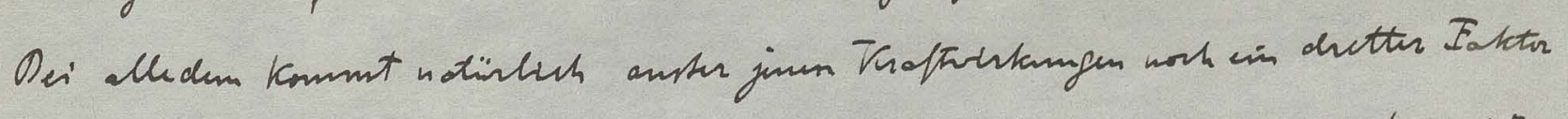

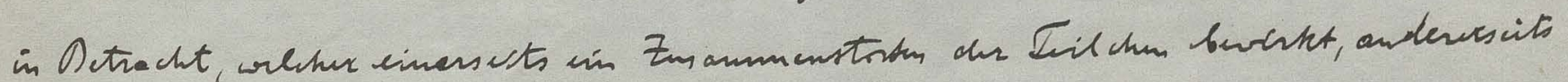

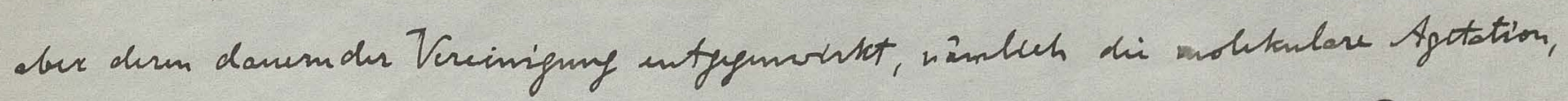

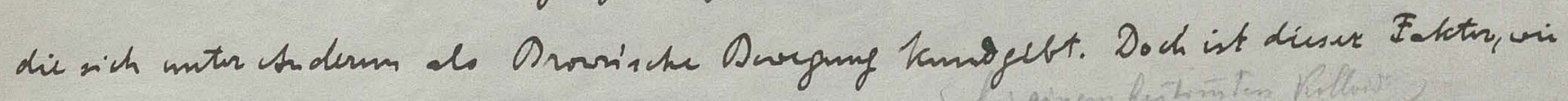

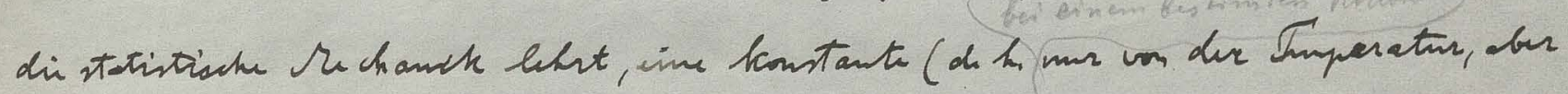

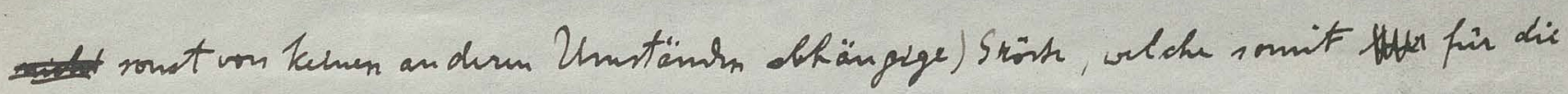

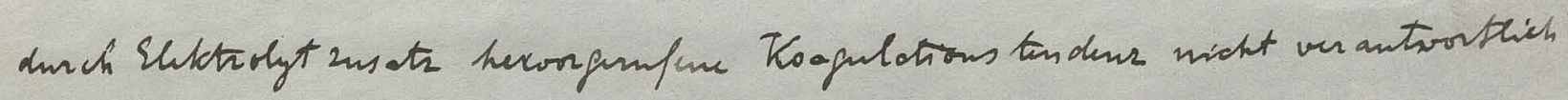
gemacht unden kam,

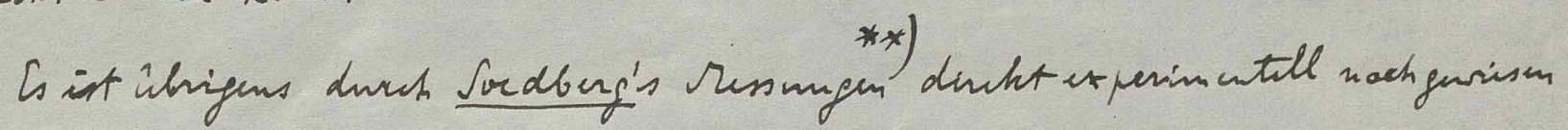

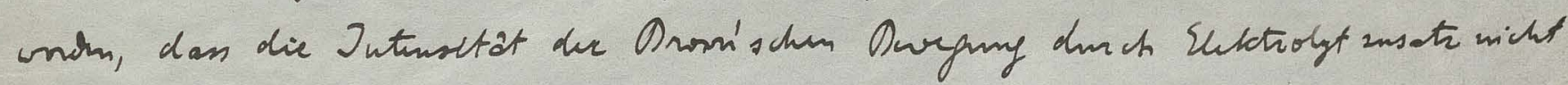

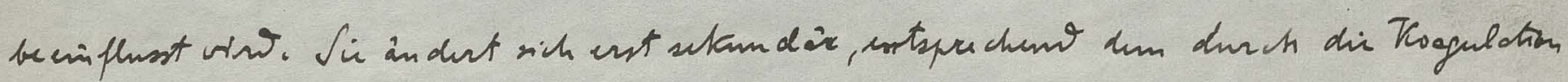

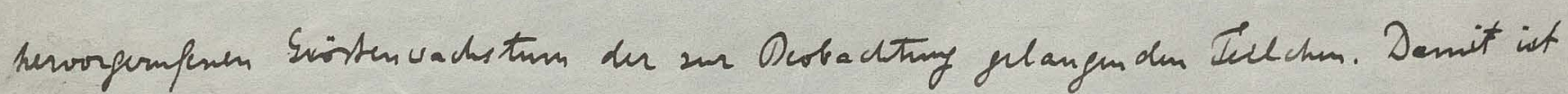
anch die Unhalthartait juner Thersien erviesm, welche die Thogetation anf

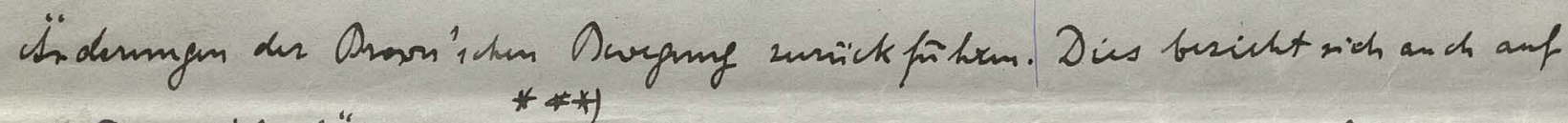

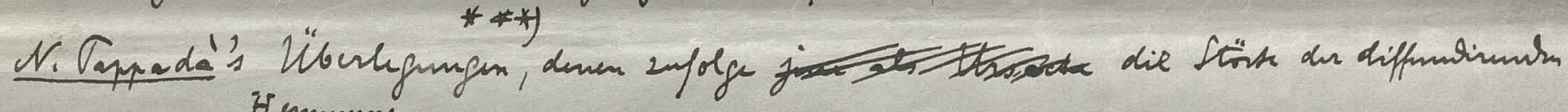

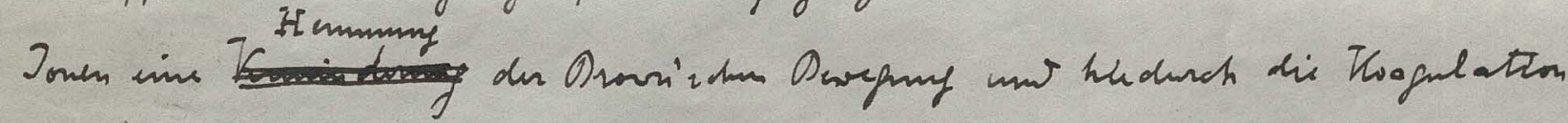

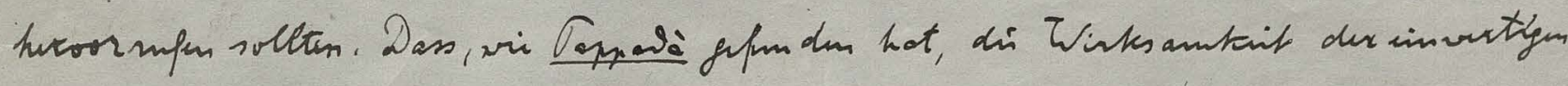

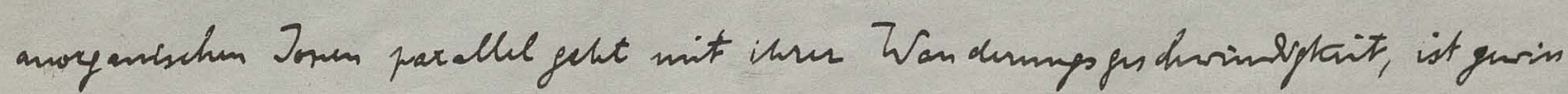

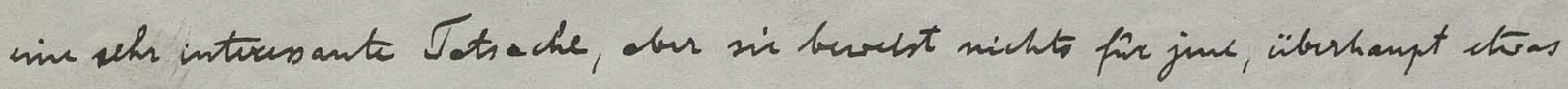

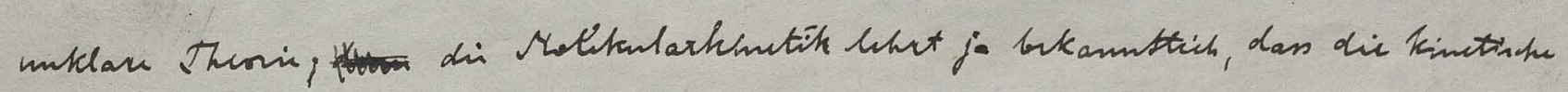

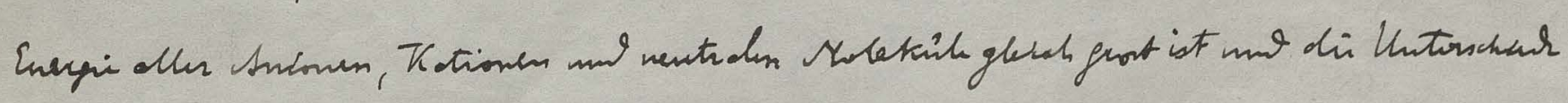

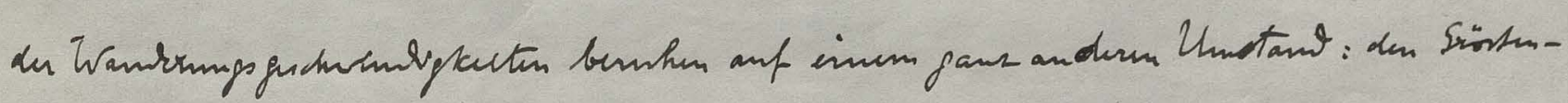

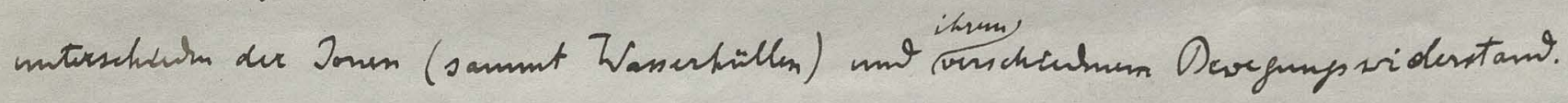

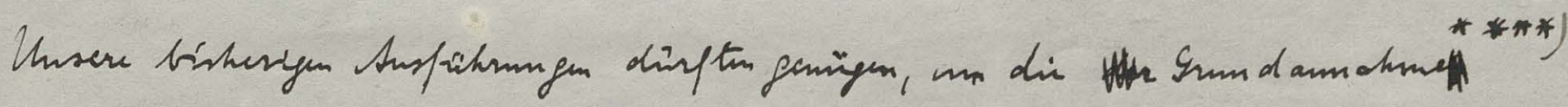

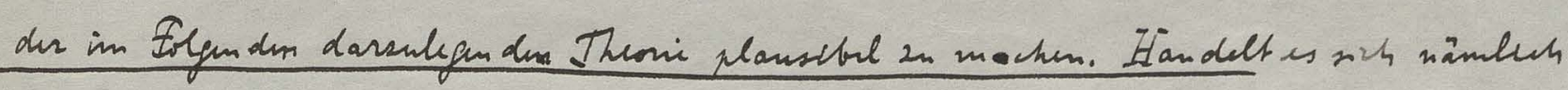

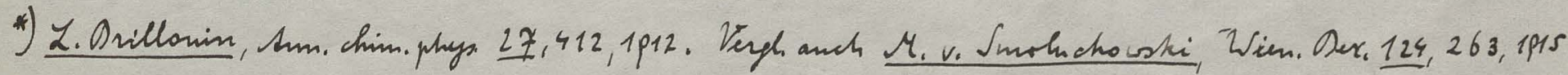

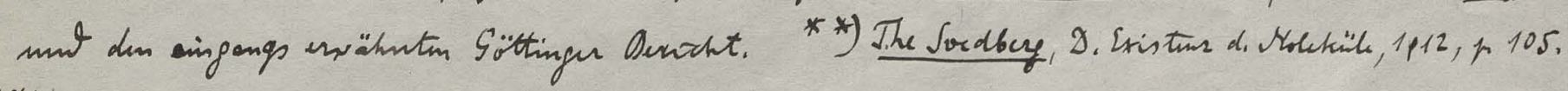

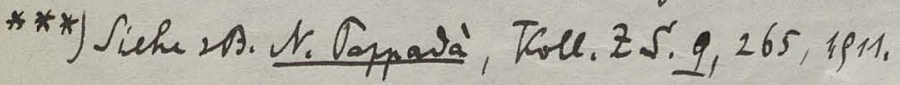

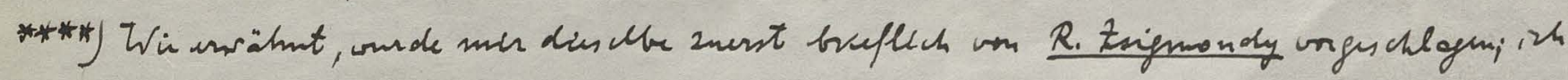

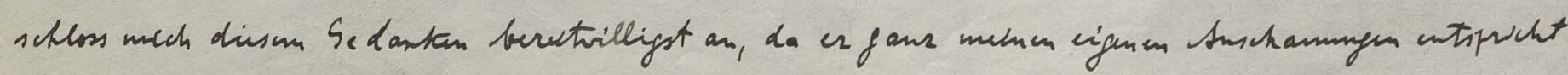

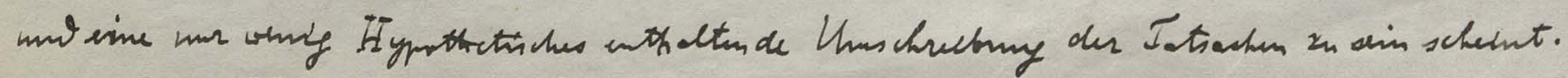


matiant

in atom 7

जoto antors

Lith ait wat से

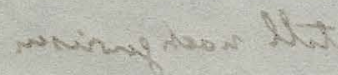

lx*

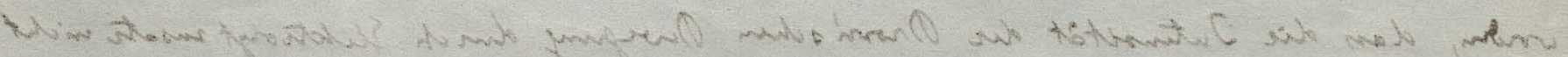
with hanowit indo

$\operatorname{tin} x \min 6$

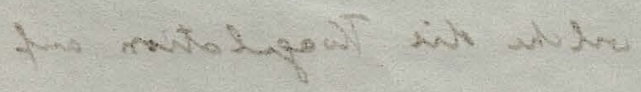

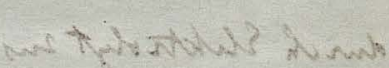

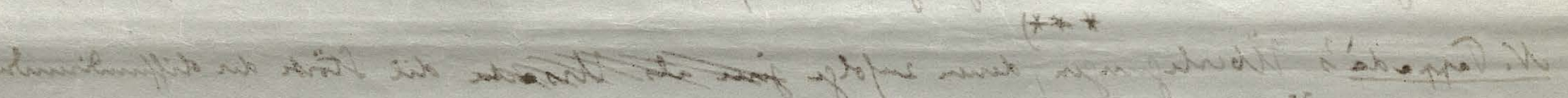

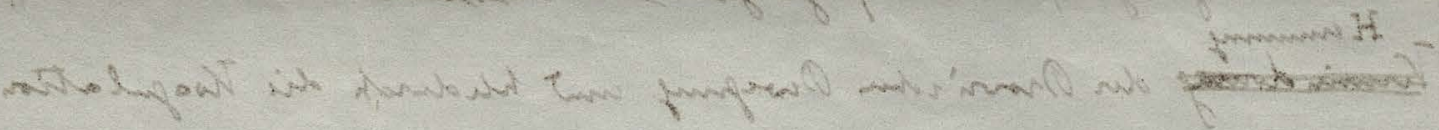

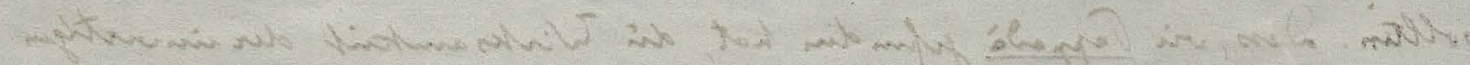

Lis tiothinan

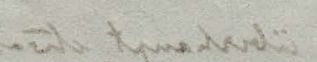

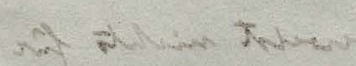

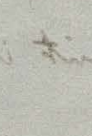

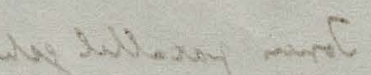

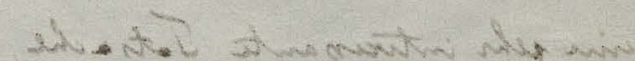

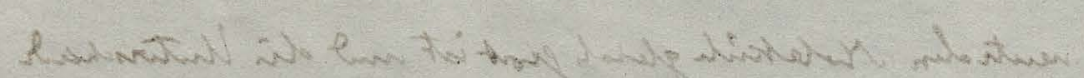

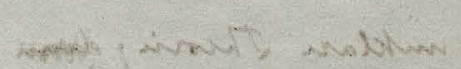

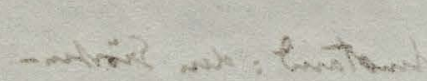

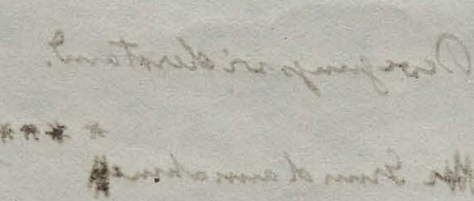

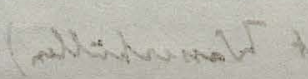

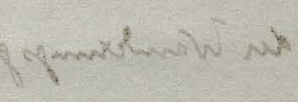

the

\section{Nuenow}

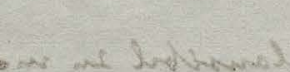




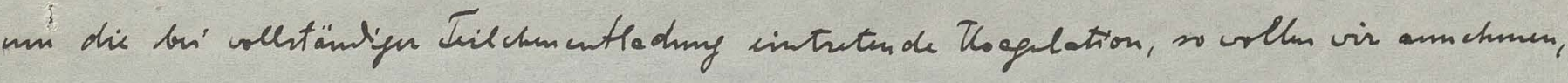

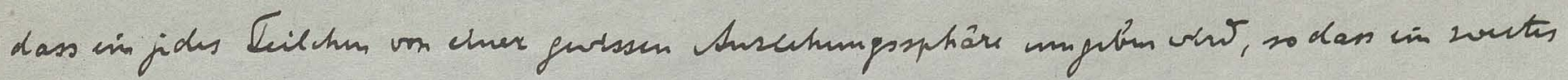

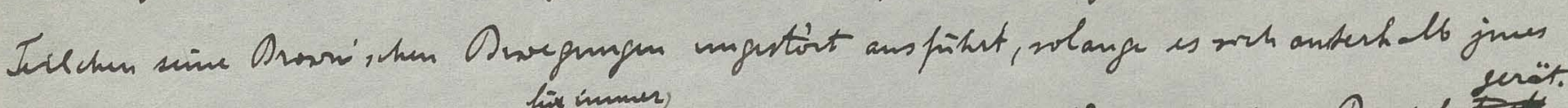

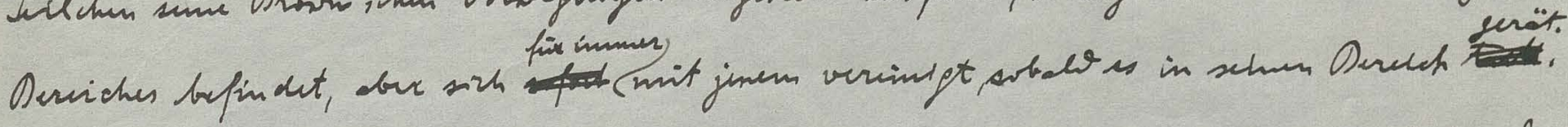

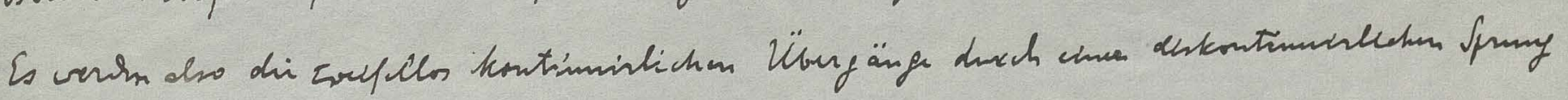

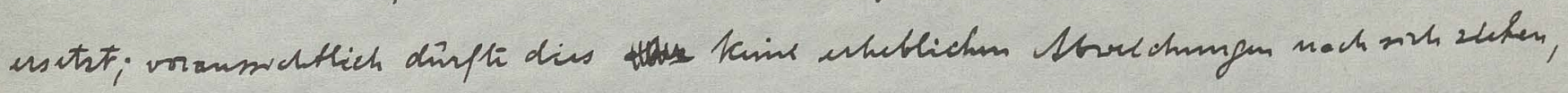

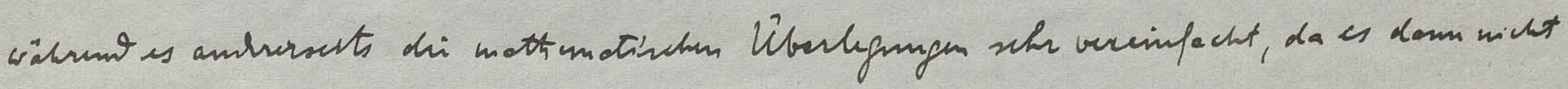

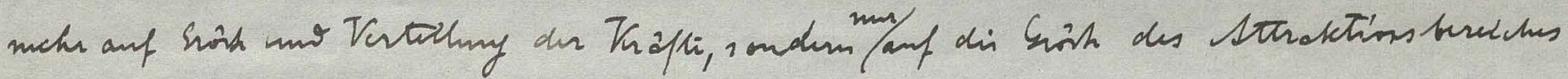
anternunt.

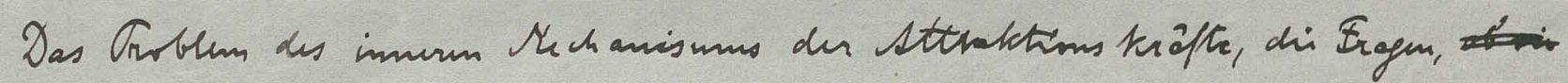

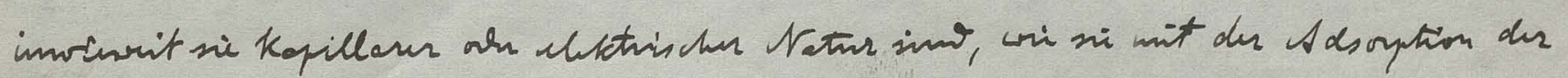

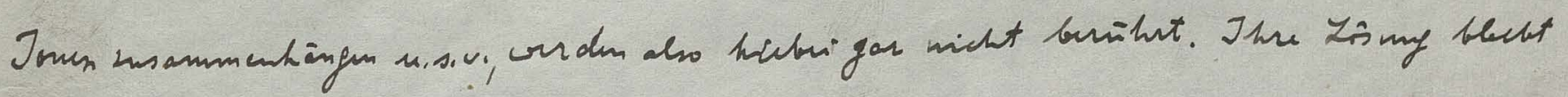

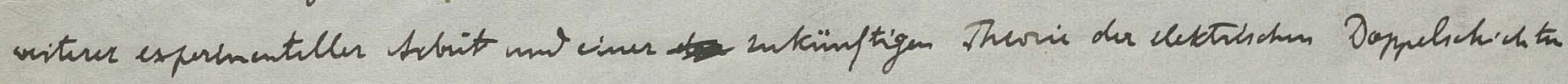

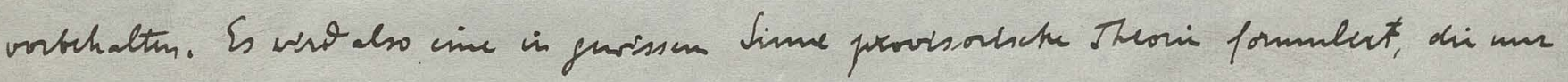

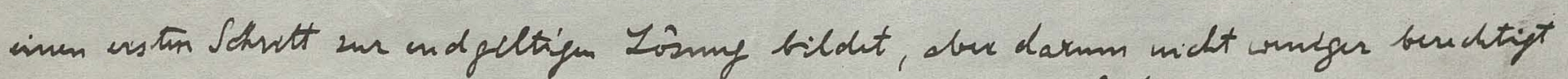

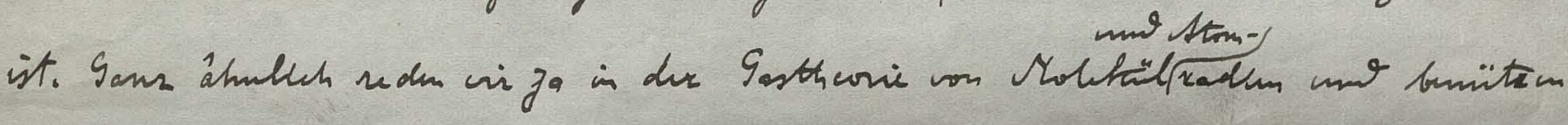

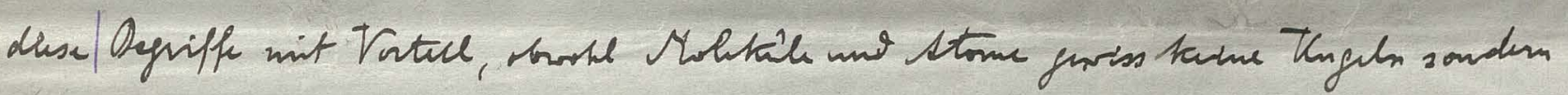

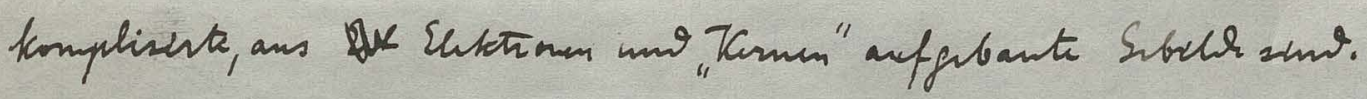

\section{Mathematiscte Theoric der raschen Toegulation.}

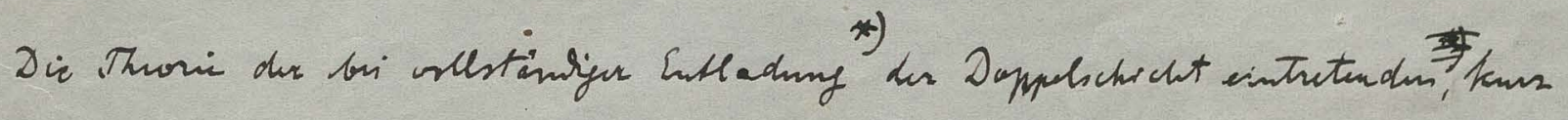
gregt "raschen", Twagelation soll in Folgenden nuter der Anmahme nother ausgebant

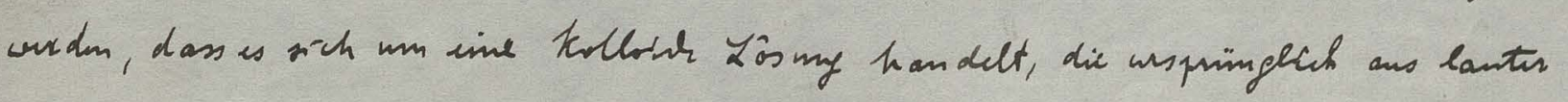

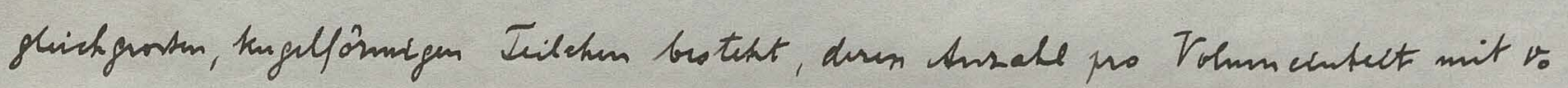

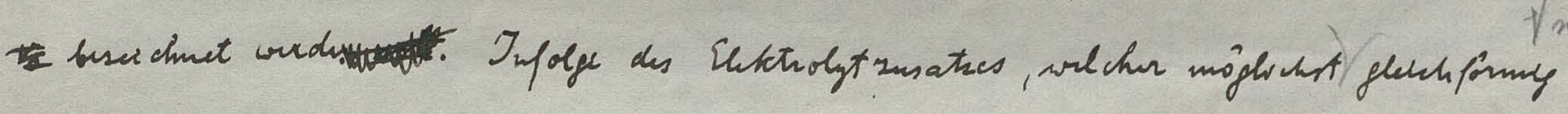

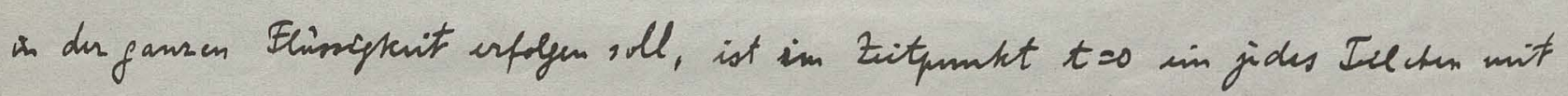

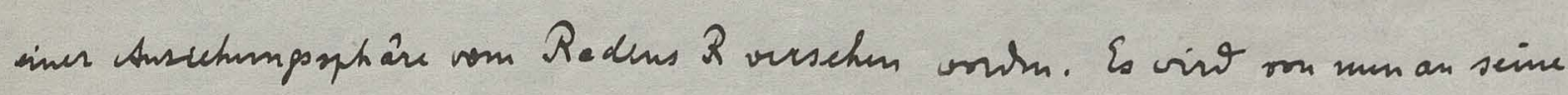

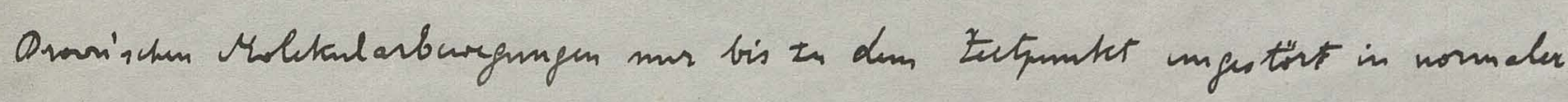

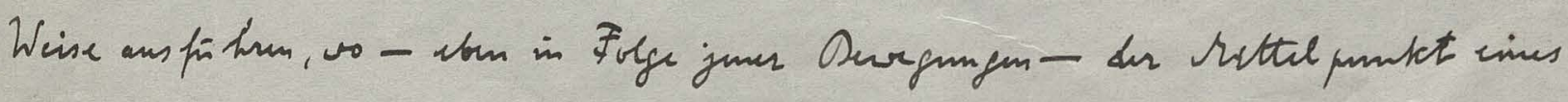

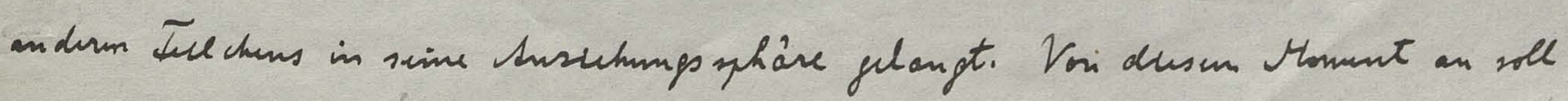

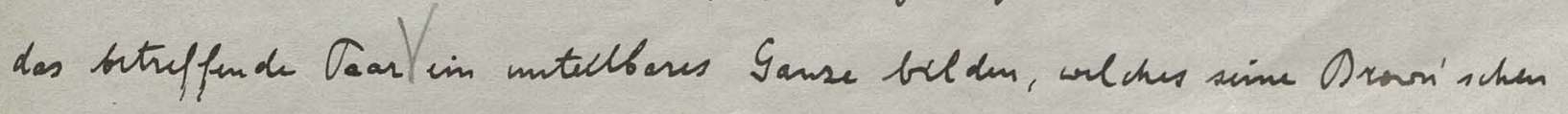

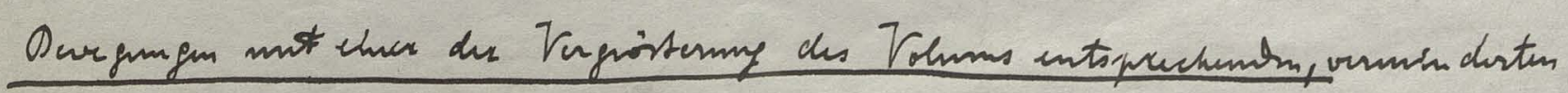

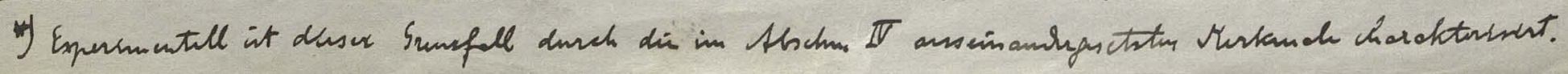


$\rightarrow$

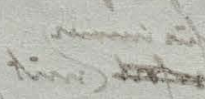

$+4$

tiv

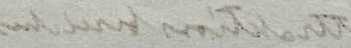

colo histe ino tha

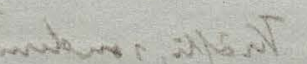

skimoner turs

$-1+x$

Aiton menth ahd

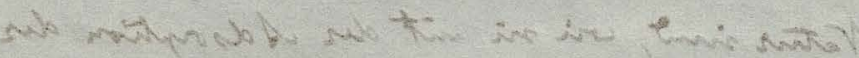

nowit

tank $\cos ^{2}$

unteol

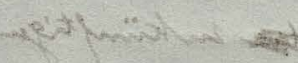

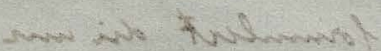

$x+2$

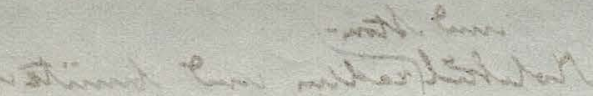

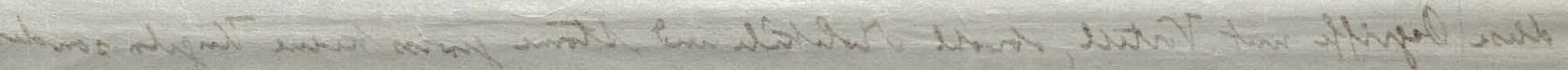

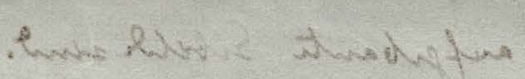

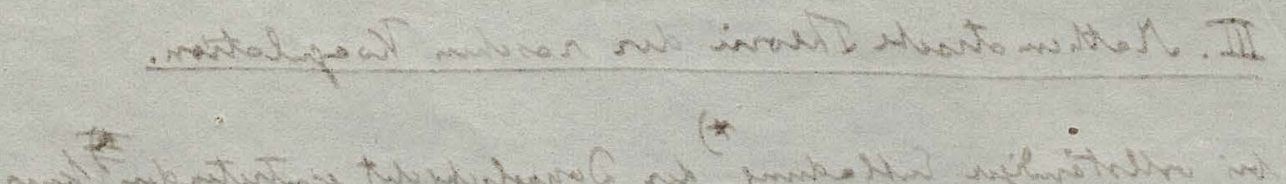

Fimation sion

atinol nas do

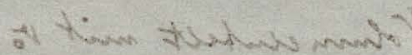

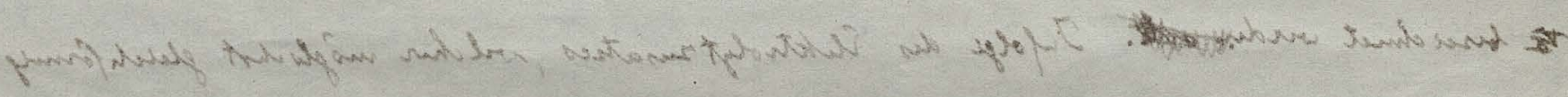

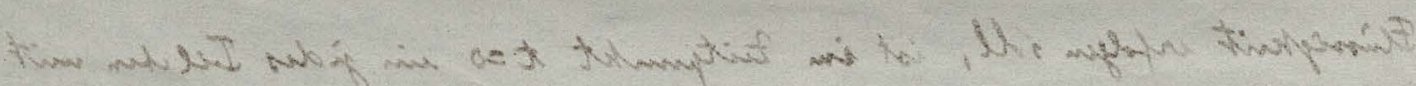

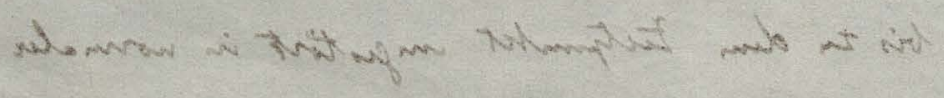
A wave $\hat{x}$ 


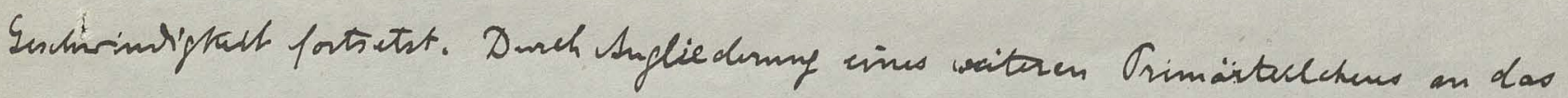

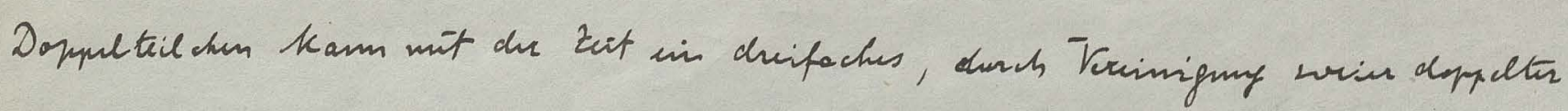

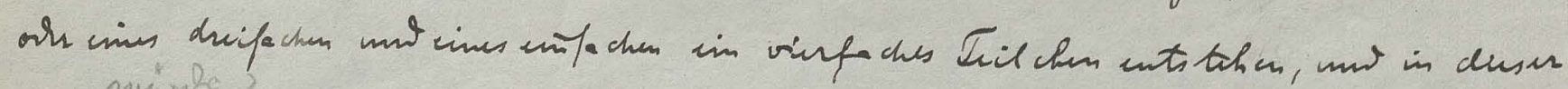

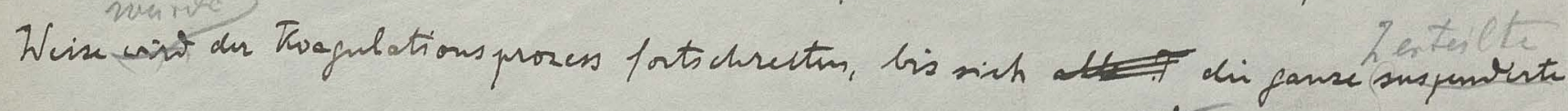

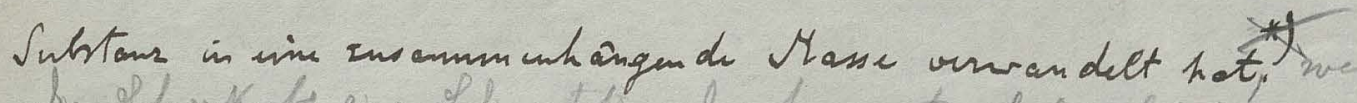

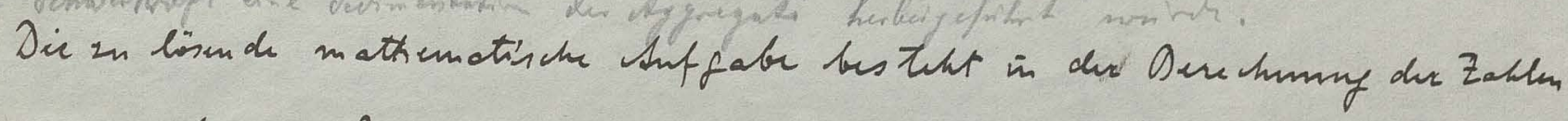

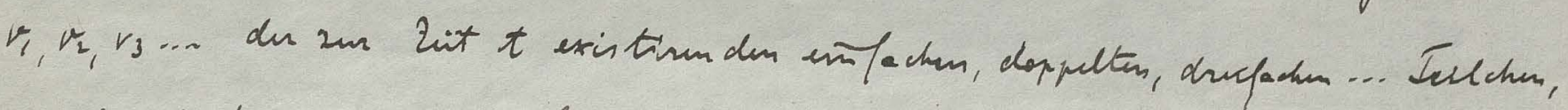

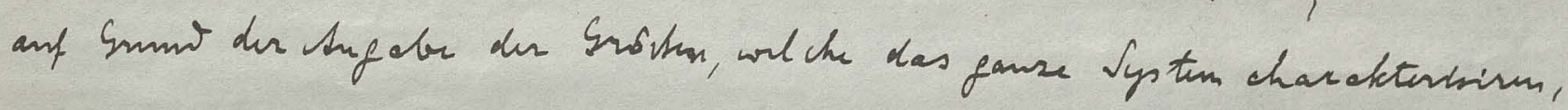

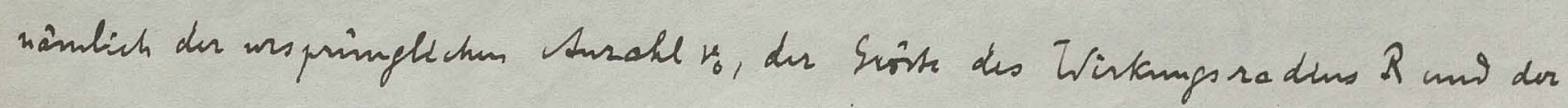
Inchrinteftects toustante $D$ der Dranischen Ourgeng.

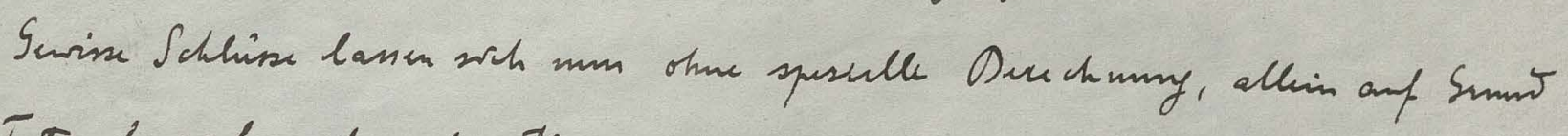

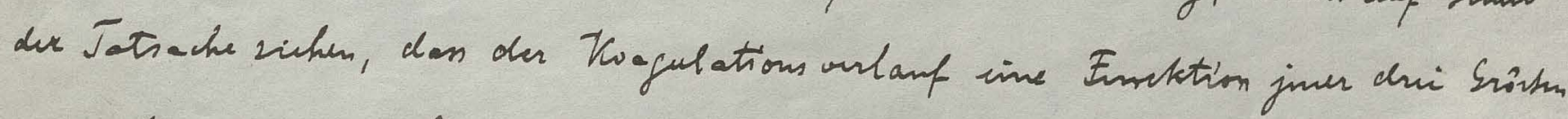
sim soll. Ans dum Scheme der Dimunsionen: $v_{0} \sim l^{-3} ; R \sim l$;

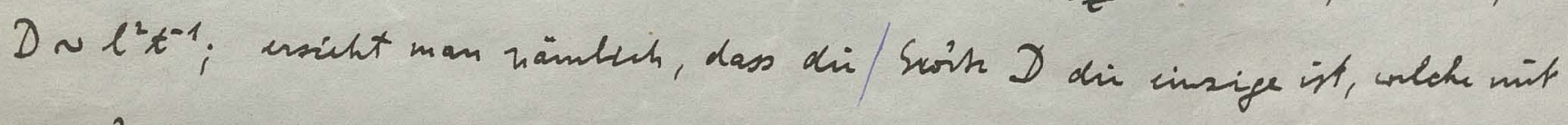

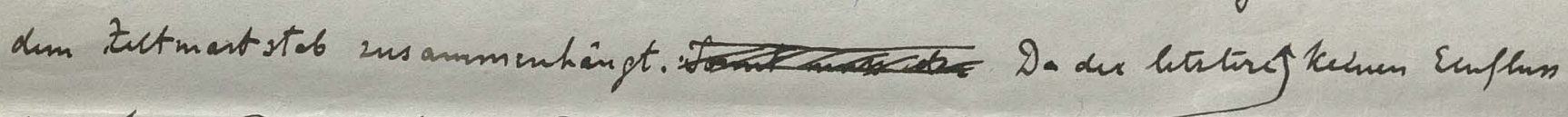

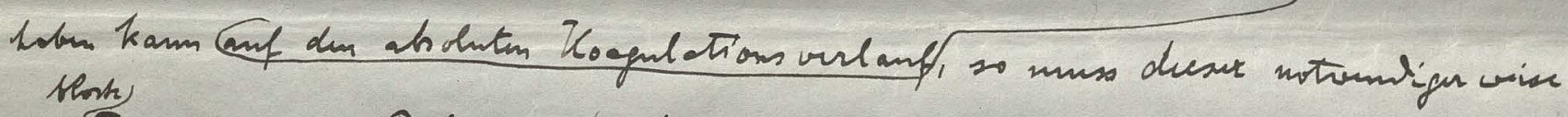

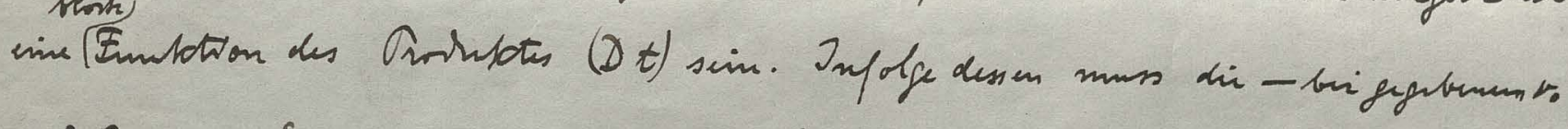

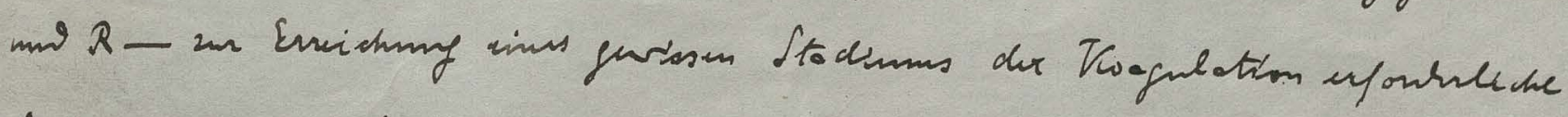

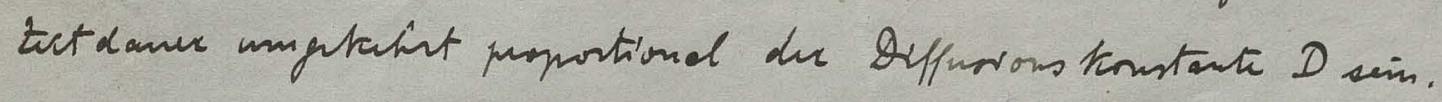

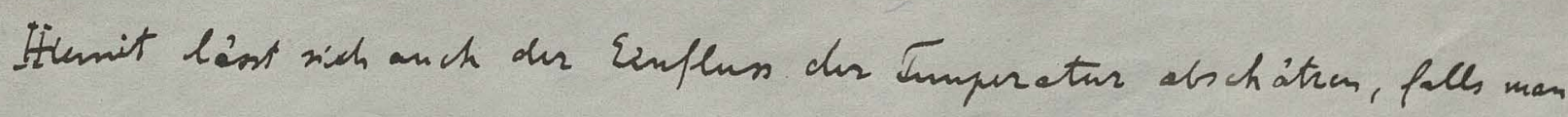

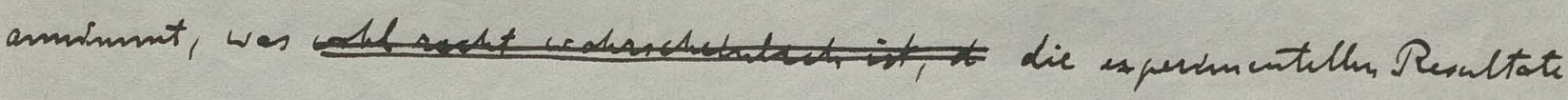

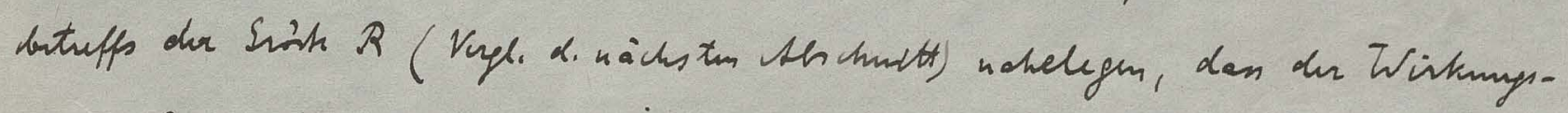

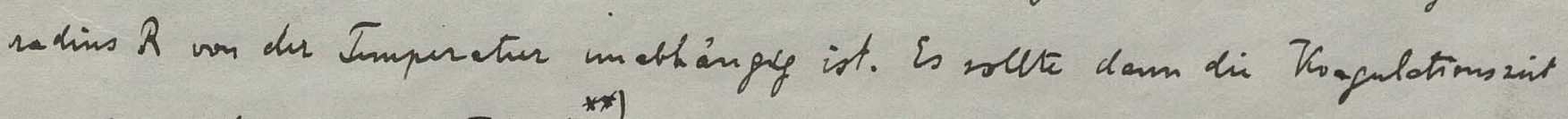
nit Riscresit anf di Formel $\left.{ }^{* *}\right)$

$$
D=\frac{\pi \theta}{\pi} \frac{1}{6 \Omega \mu \theta}
$$

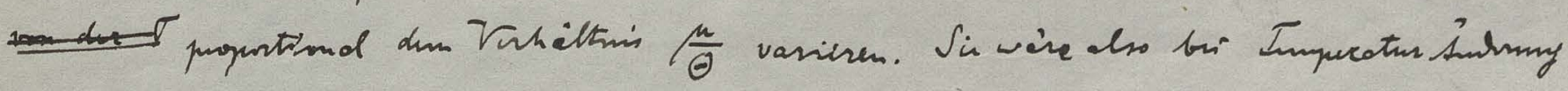

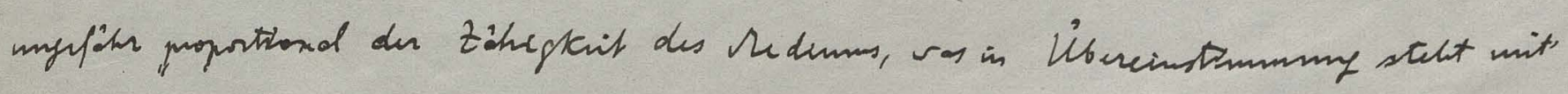

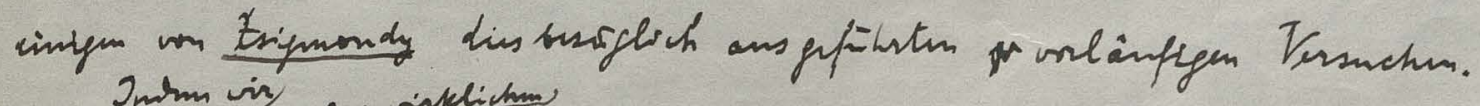

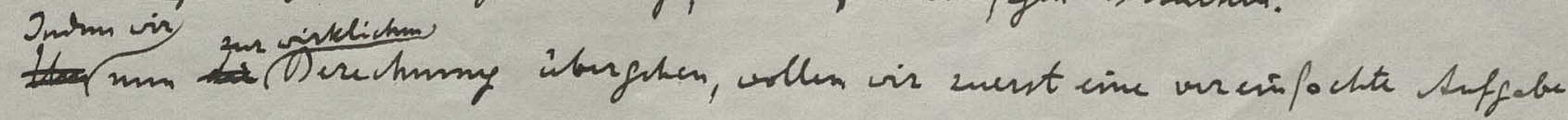

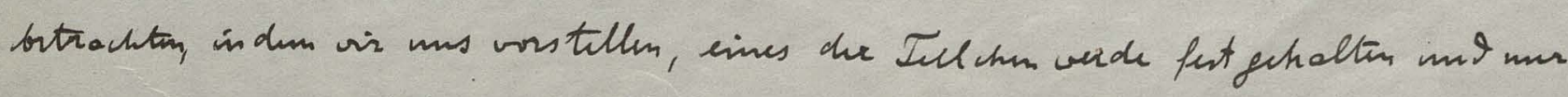

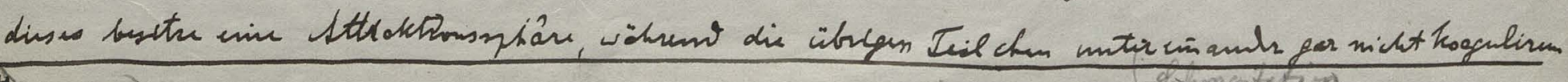

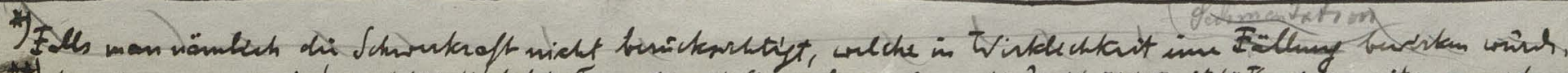

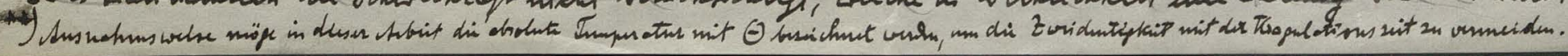




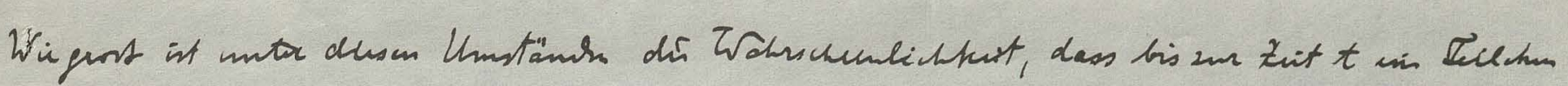
sirh an dos hurovegh obme angelagest haber?

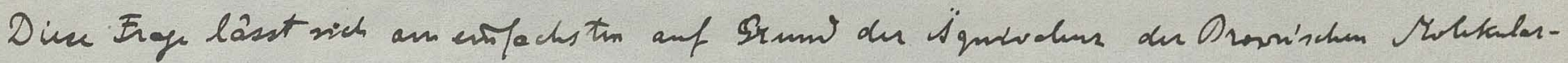

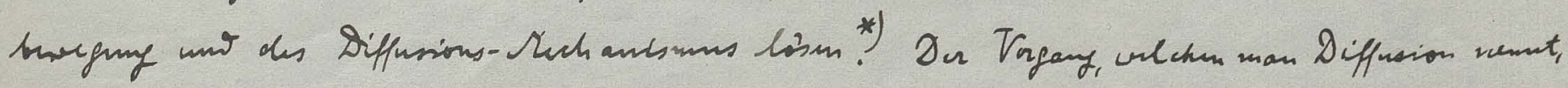

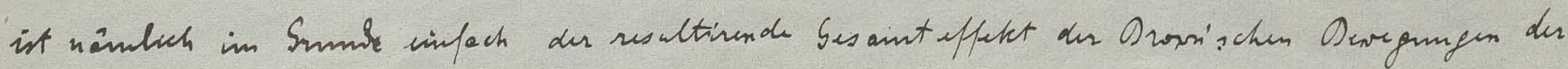

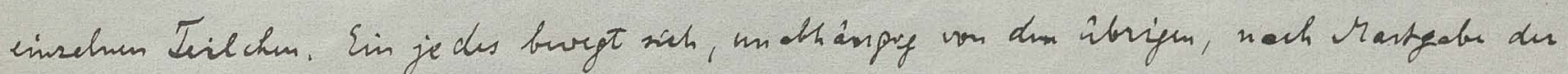
Vutachmps.

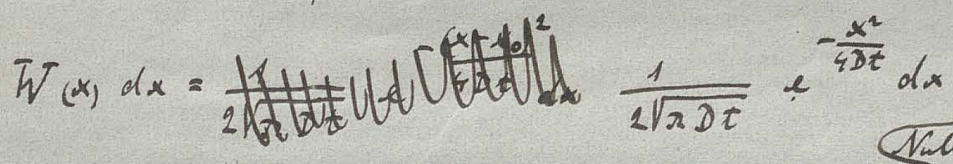

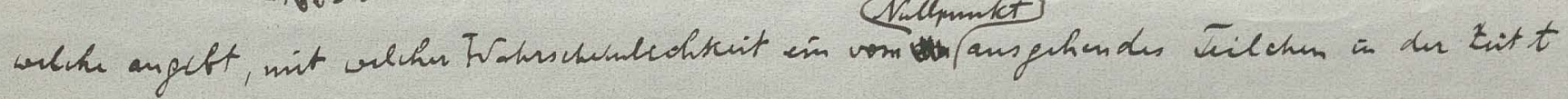

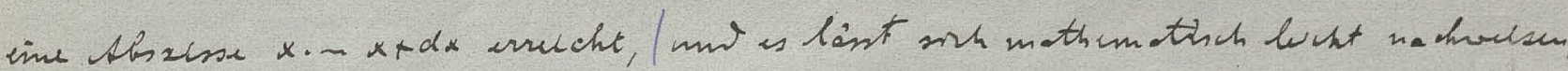

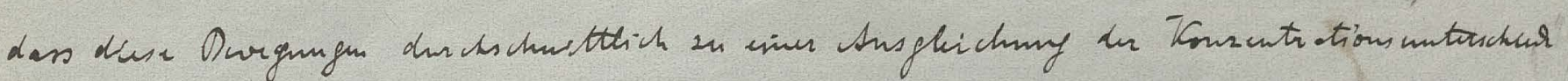

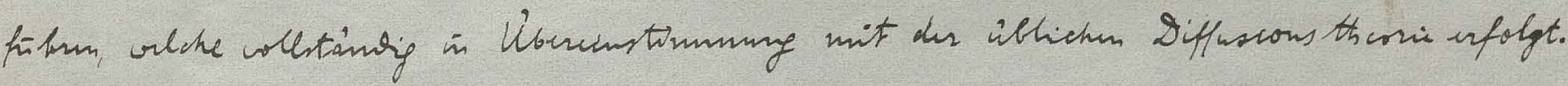

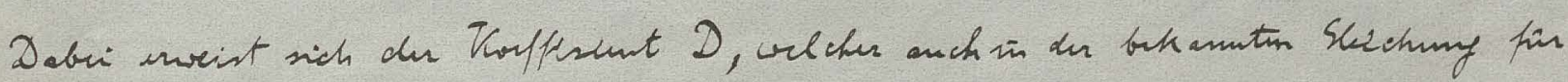

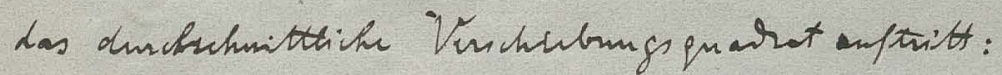

\section{(स)}

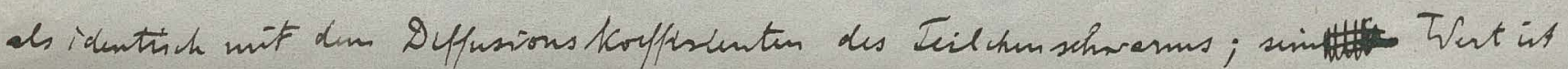

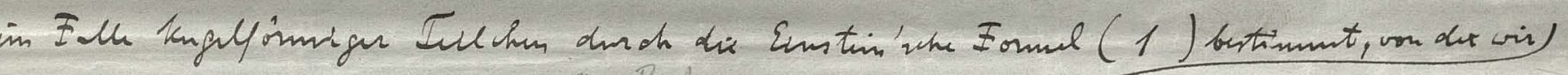

Oie Toransstrany, dans dii Rogelfich $R$

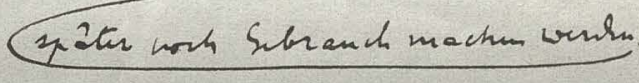

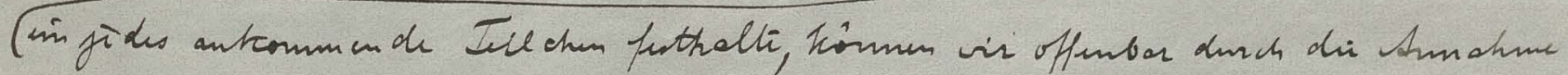

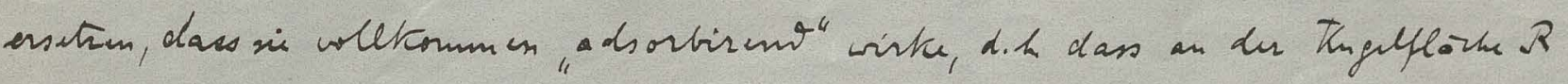

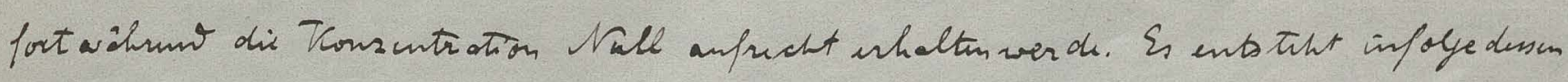

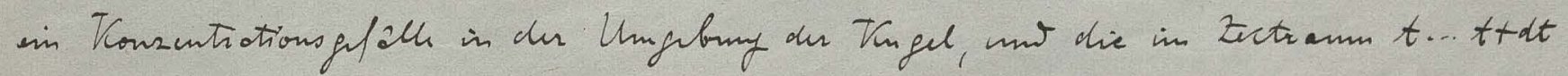

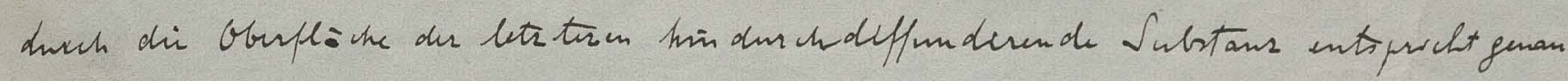

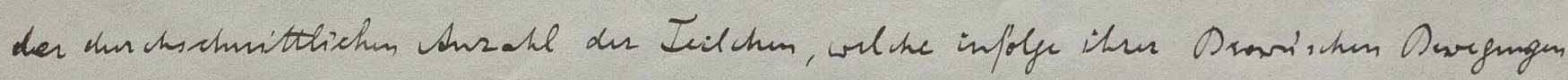

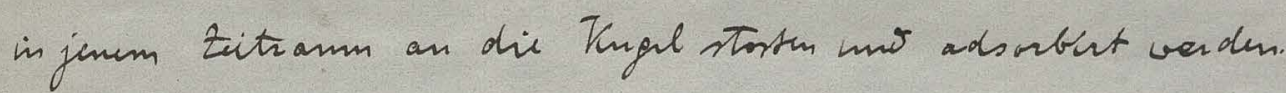

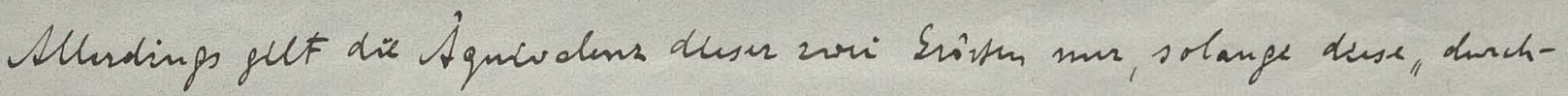

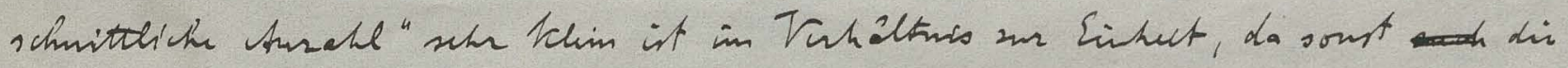

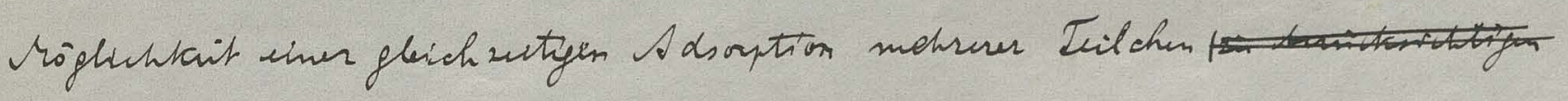

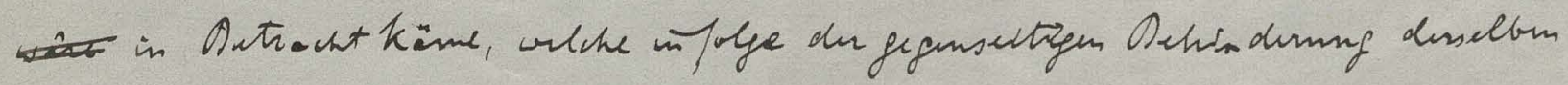
anders verlaufen minste. Sicher it tho die Iquardens geltig, folls dis Ansahe vo

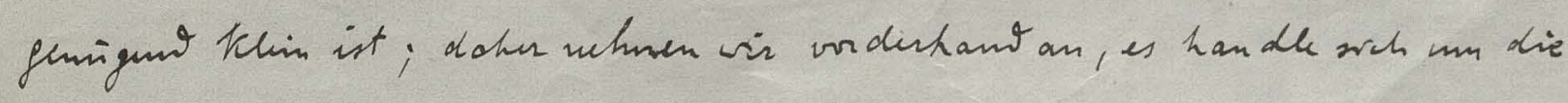

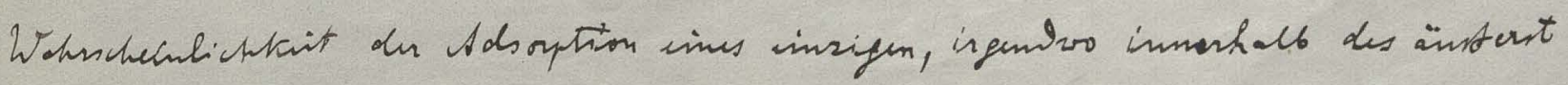
gersten Tolums V woskandenen Filchems. 
of

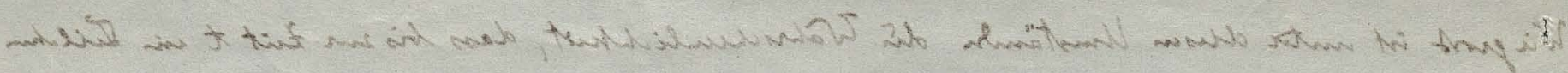

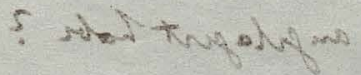

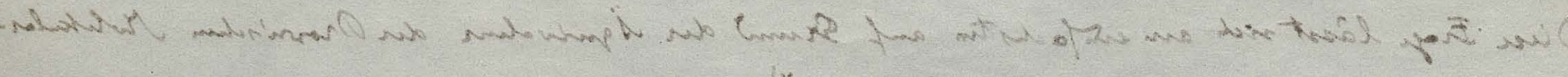

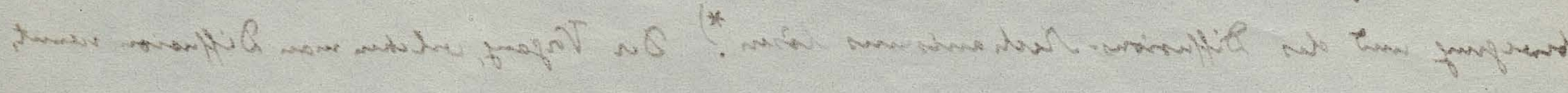

sit

$$
\begin{aligned}
& \text { thatenis the } \\
& \operatorname{lin} \text { - whinges }
\end{aligned}
$$

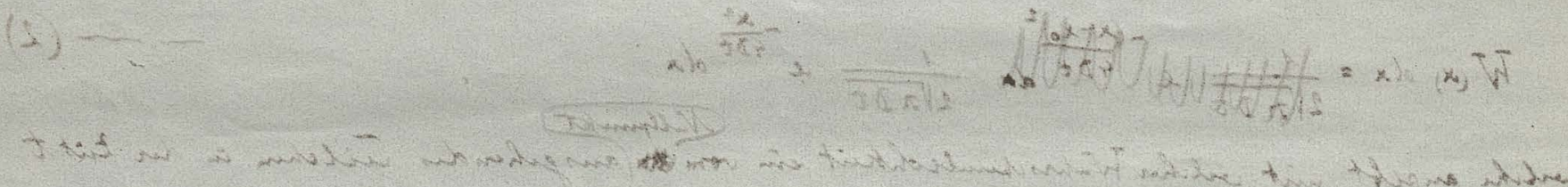

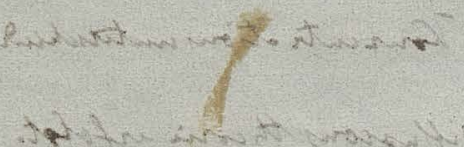

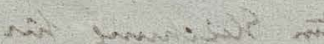

C.

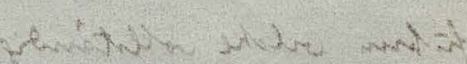

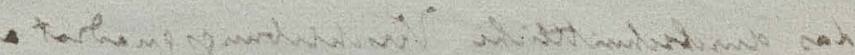

(2)

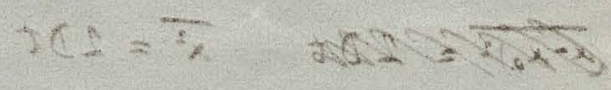

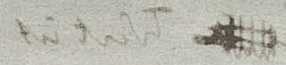

mon the

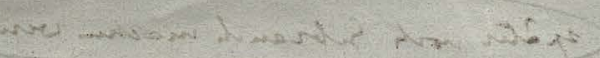

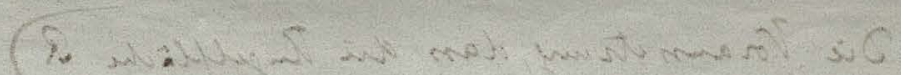
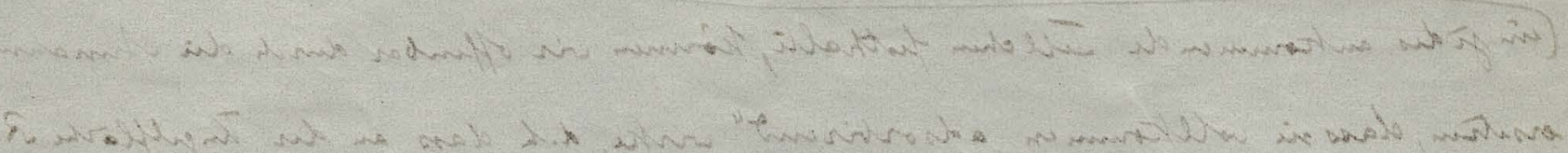

wit.

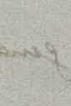

- humita now

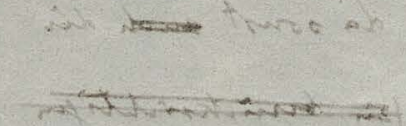




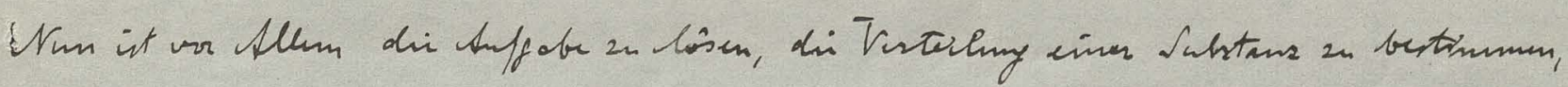

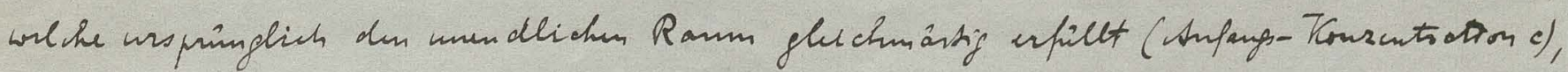

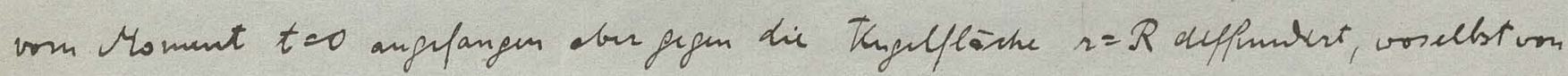

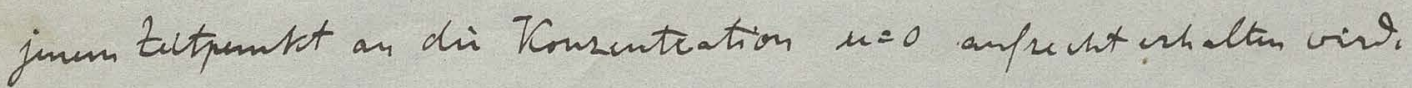

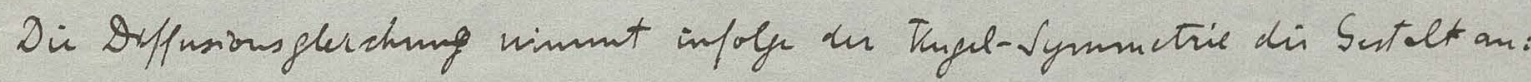

$$
\frac{\partial(2 u)}{\partial t}=D \frac{\delta^{2}(2 u)}{\partial x^{2}}
$$

unit dise Slwhing wint durch di Entition:

$$
n=c\left[1-\frac{R}{n}+\frac{2 R}{n \sqrt{n}} \int_{0}^{\frac{2-R}{2 \sqrt{D t}}} e^{-2^{2}} d x\right]
$$

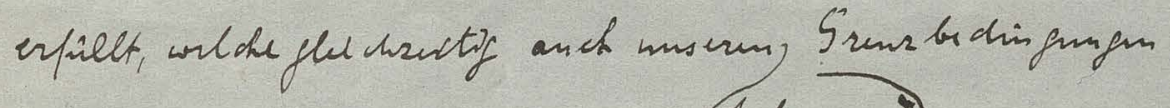

1). $u=c$ fir $t=0,2>R$ Anparp min

2). $n=0$ fin $n=R, t>0$

Seninge lestet.

Serans ezgett zich dir in Zutrann t... As dt an dir Thagl $R$ andiffunderumer Kenge:

$J d t=4 \pi D R^{2} \frac{\partial u}{\partial r} \int_{R} d t=4 \pi D R c\left[1+\frac{R}{\sqrt{n \partial t}}\right] d t$

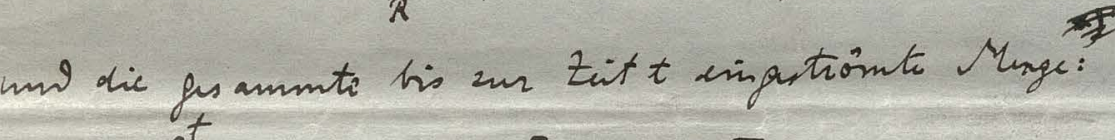

$M=\int_{0}^{t} J d t=4 \Omega D R_{c}\left[t+\frac{2 \pi \sqrt{t}}{\sqrt{2 D}}\right]$

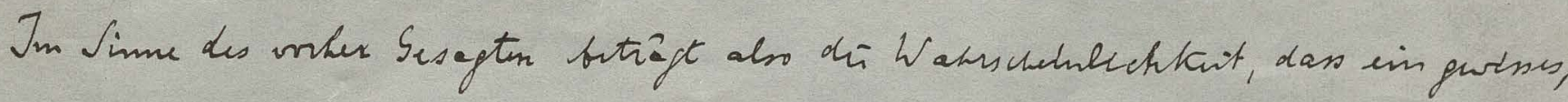

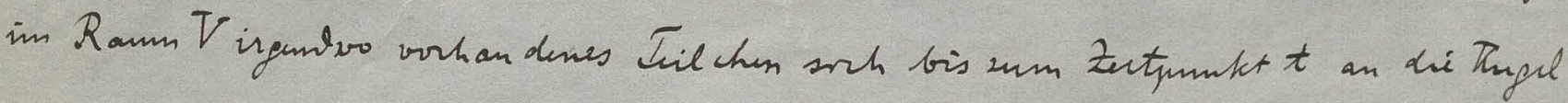
angelagent habe ( $\mathrm{m}$ in $\left.c=\frac{1}{\nabla}\right)$ :

$W_{t}=\frac{4 \pi D R}{V}\left[t+\frac{2 R \sqrt{t}}{\sqrt{\pi D}}\right]$

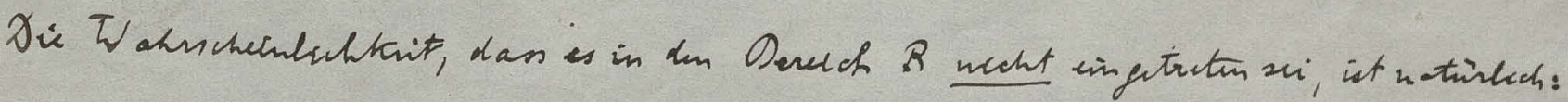

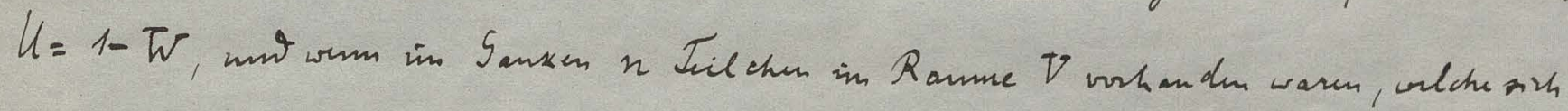

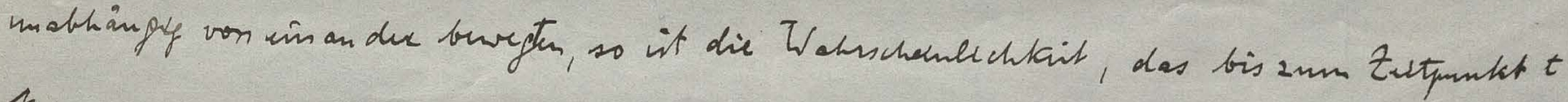

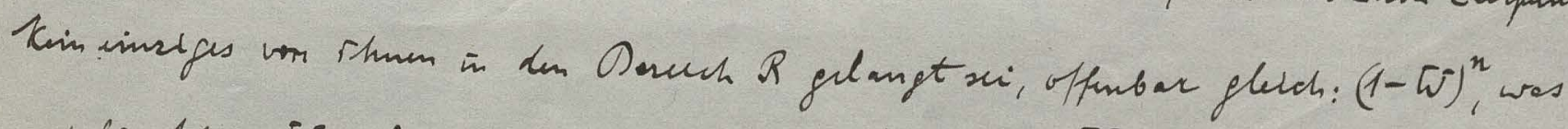

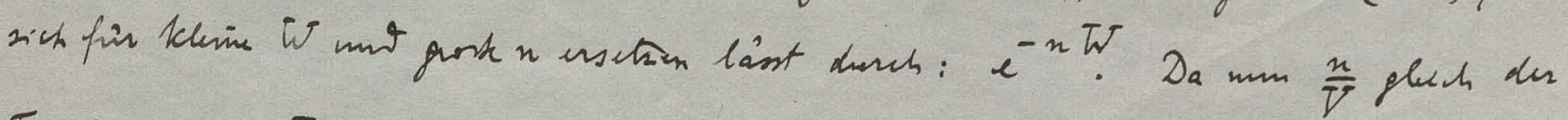

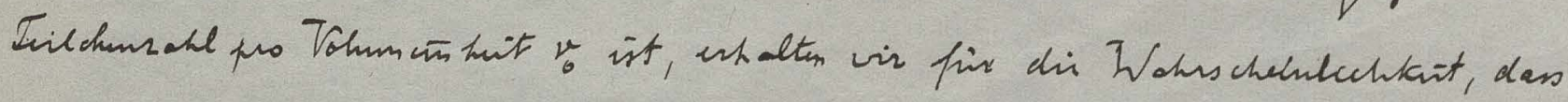
bis in juer Zit kine Snlagerny eingetreter si:

$$
U_{t}=e^{-4 \pi D R t_{0}\left[t+\frac{2 R \sqrt{t}}{\sqrt{\pi D}}\right]}
$$

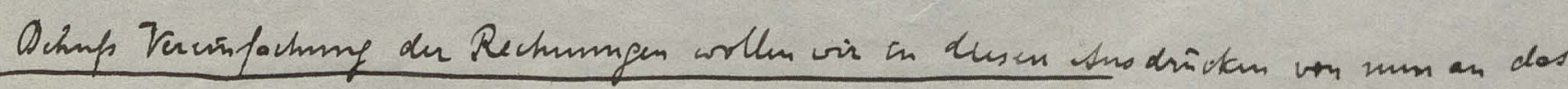

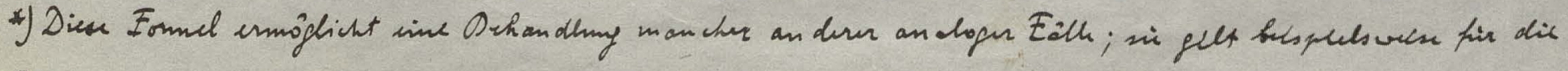

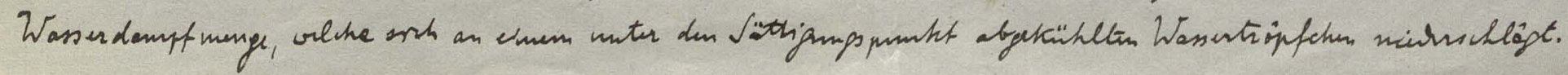




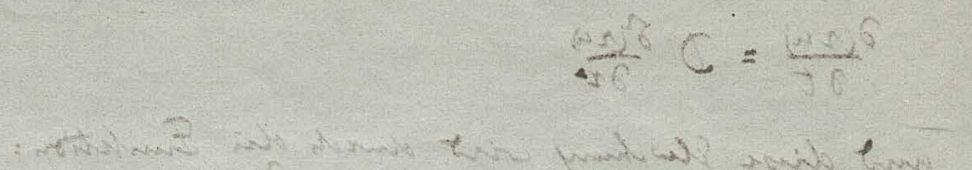

$$
\text { का }
$$

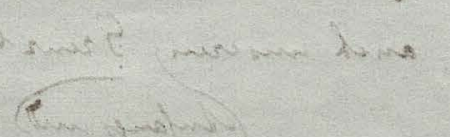

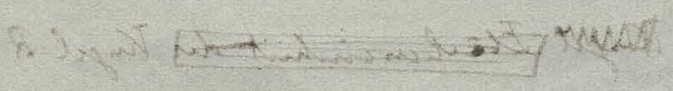

(d)

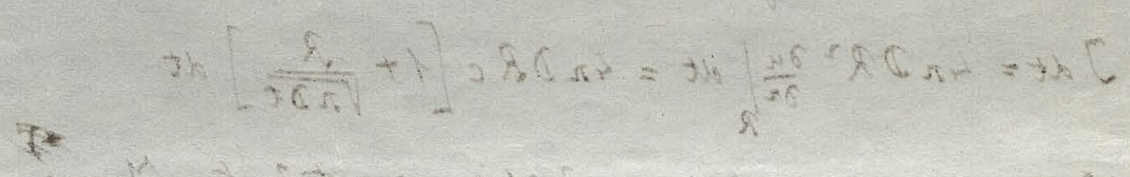

$(f)=$

$$
\left[\frac{7+55}{65}+7\right], \times 6 n+x=
$$

(8)

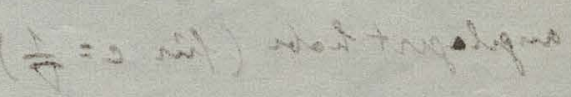

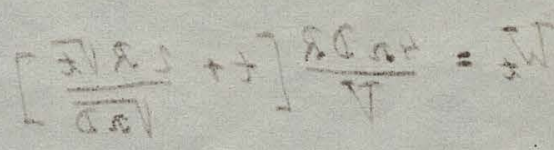

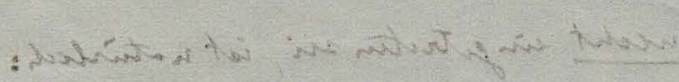

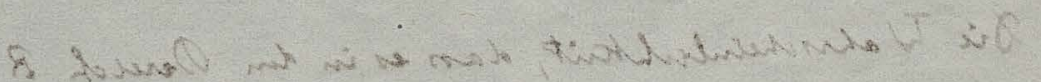

wastater

3 thimation

$$
\text { A } 20.000
$$$$
\text { Giman }-\bar{T}-1=1
$$ 


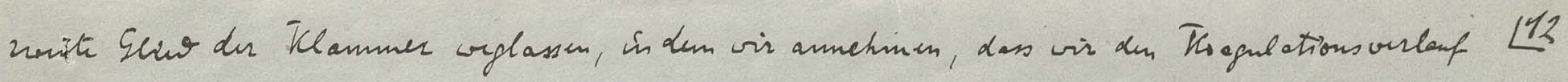

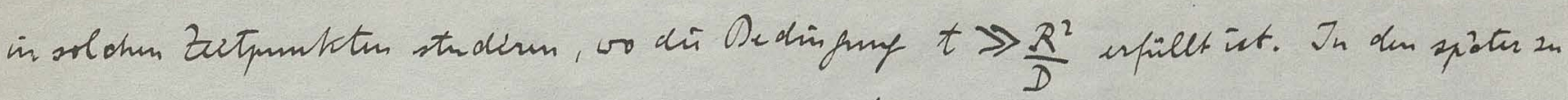

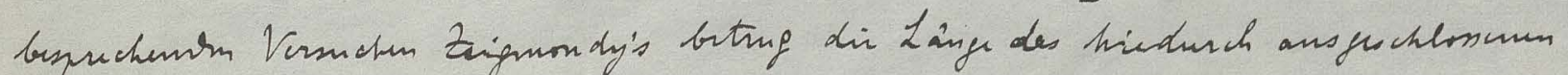

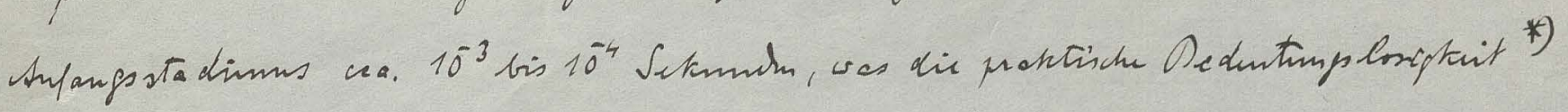

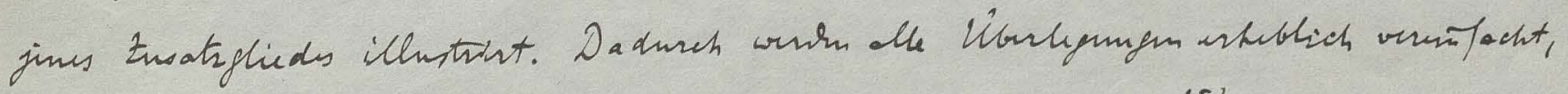

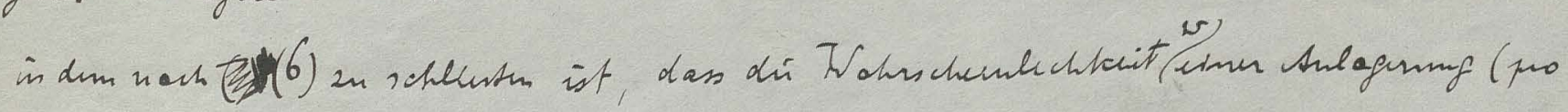

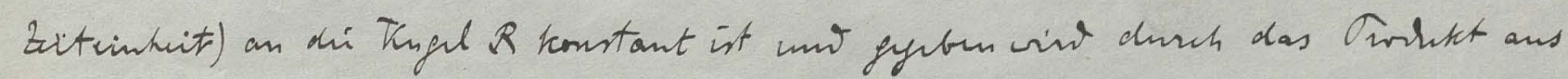

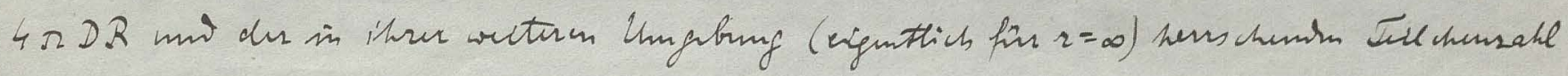
Vo pro Tolum inheit: $\quad w=4 \pi D R v_{0}$

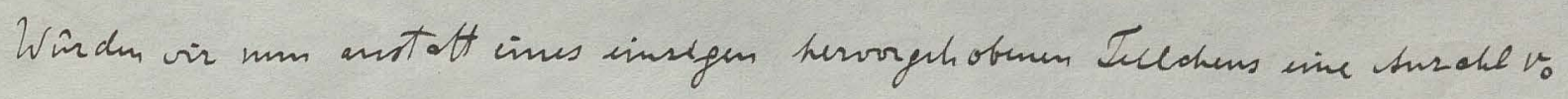

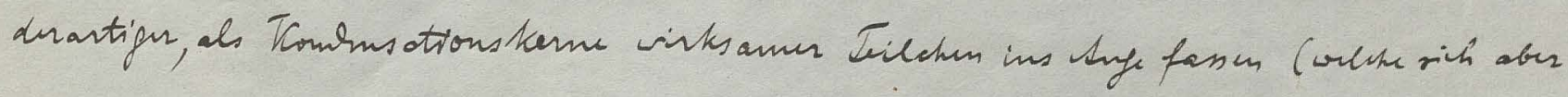

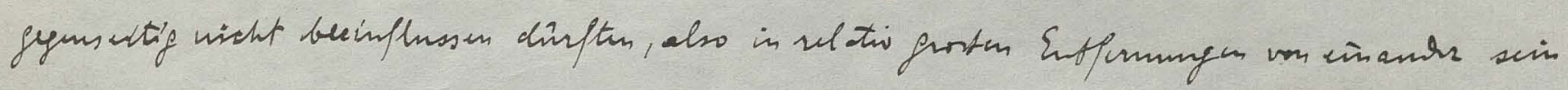

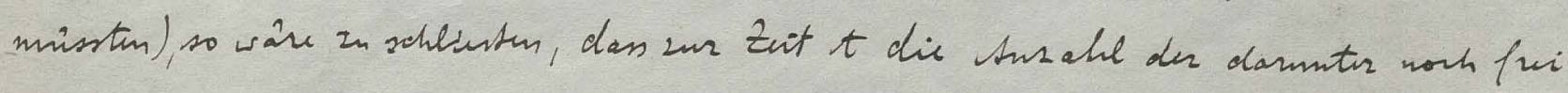

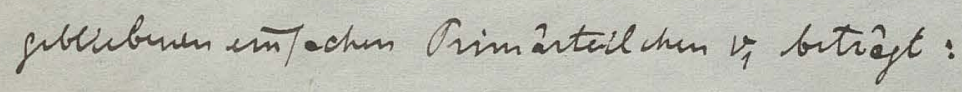

$$
v_{1}=v_{0} e^{-4 \Omega D R v_{0} t}
$$

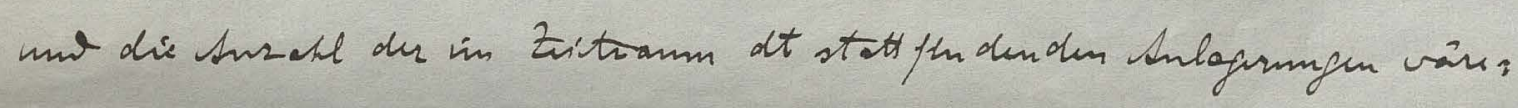
$-d v_{1}=4 \pi D R v_{0} \nu_{1} d t$

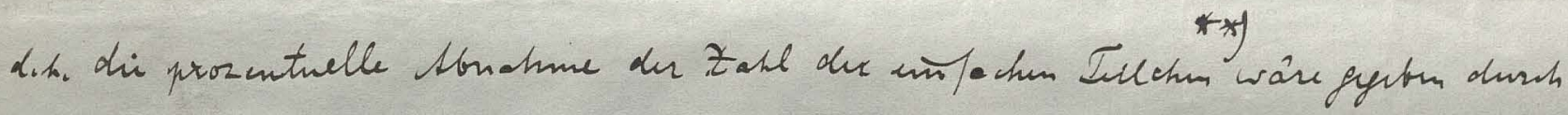

$-\frac{d v_{1}}{v_{1}}=4 \pi D R v_{0} d t$

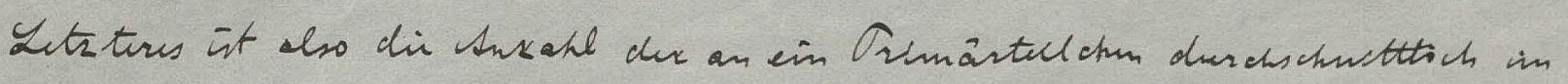

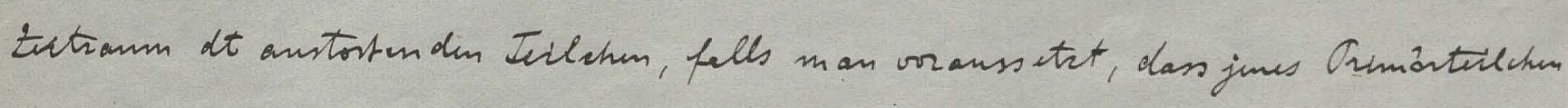

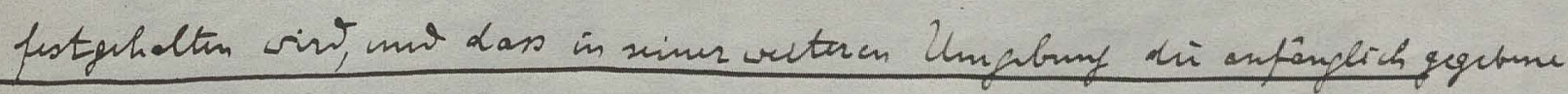

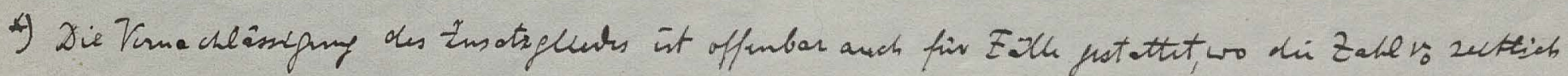

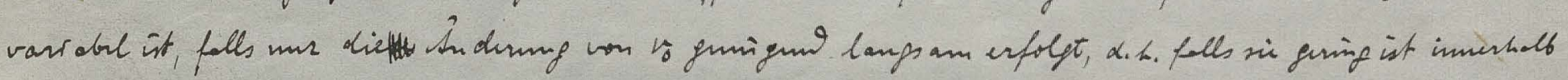

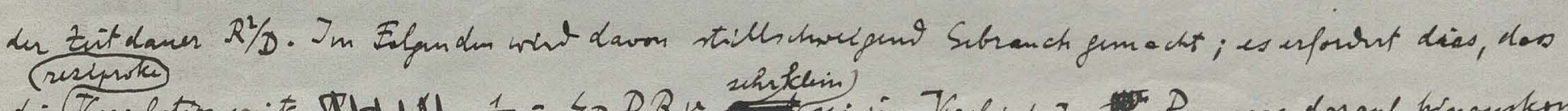

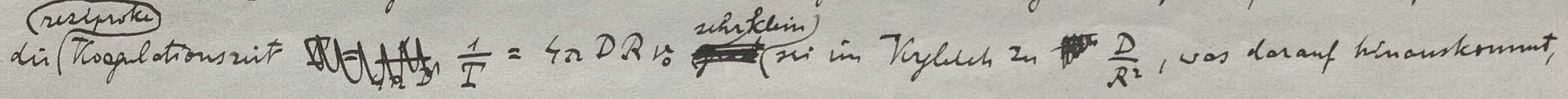

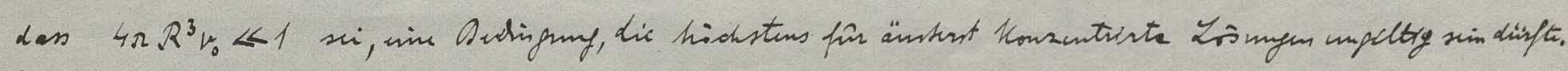

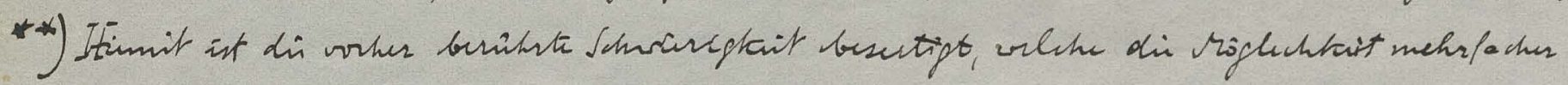

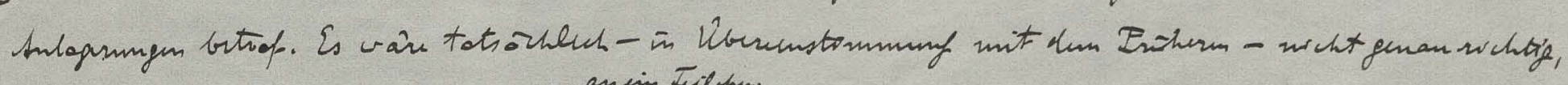

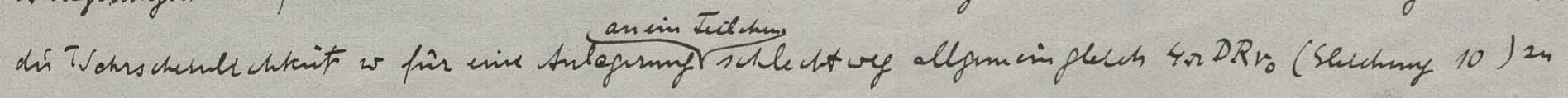

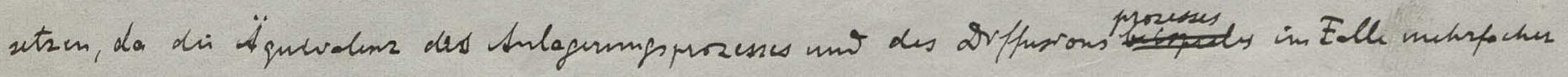

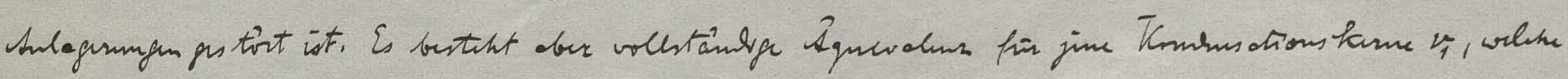

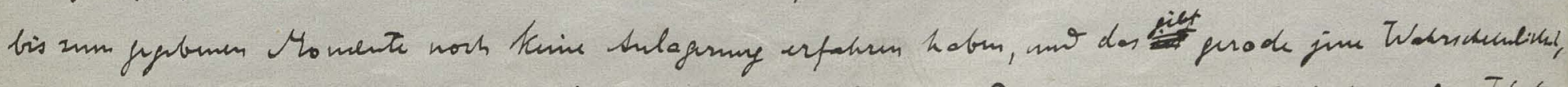

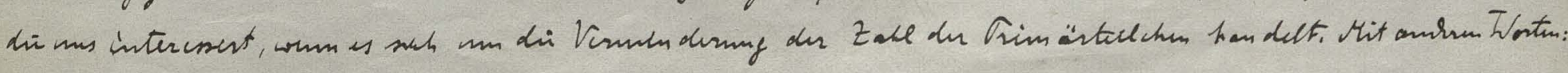

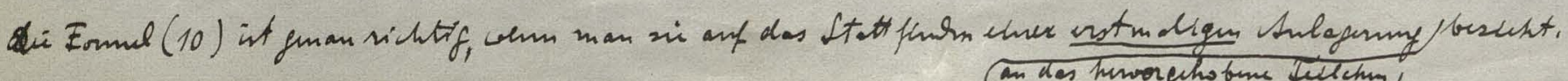


Dis $+x^{2}$

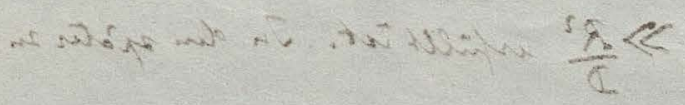

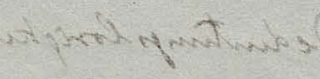

$$
\begin{aligned}
& \text { - ont }
\end{aligned}
$$

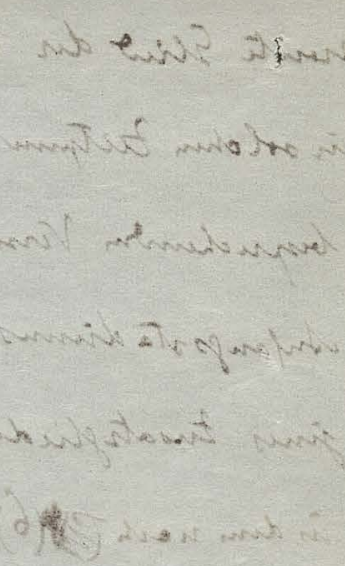

(a)

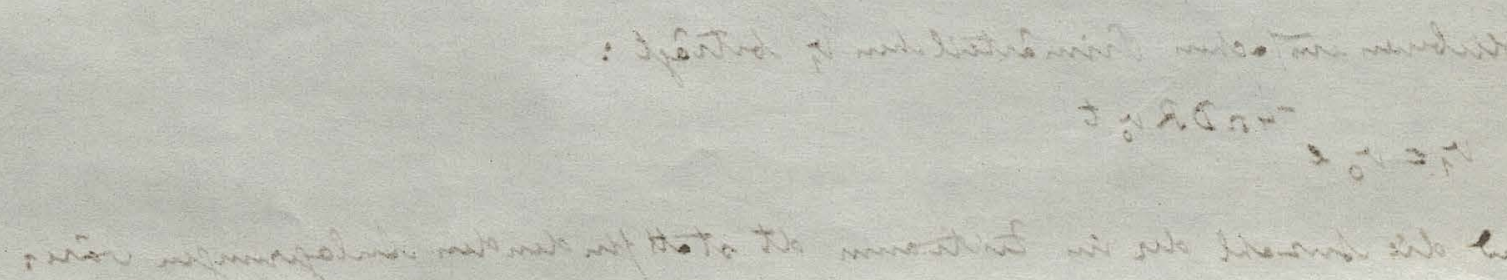

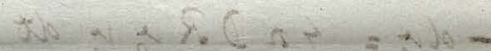

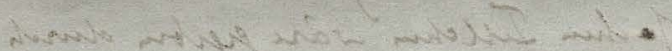

(j)

$$
T_{4}: \pi 6 \pi=\frac{\pi 18}{x}-
$$

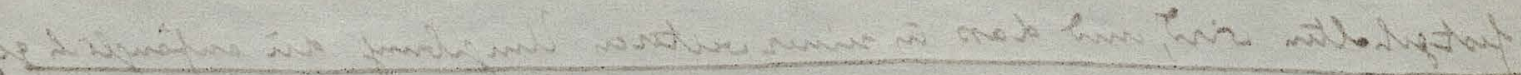

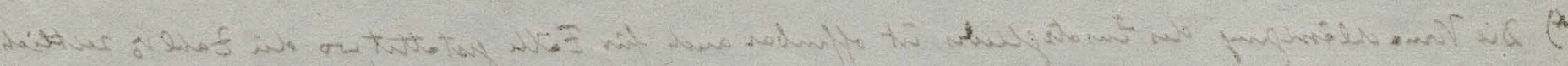

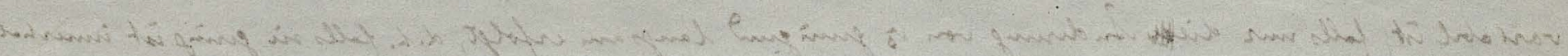

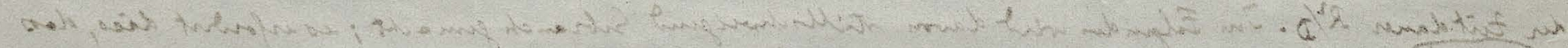

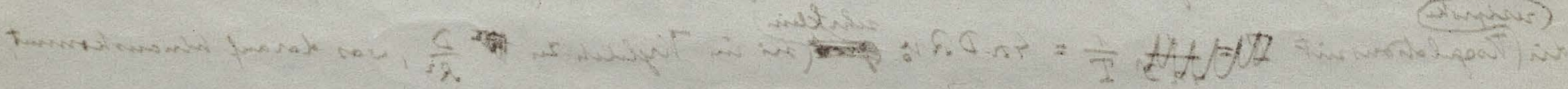

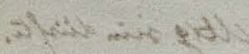

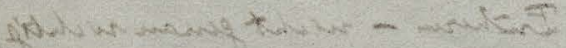

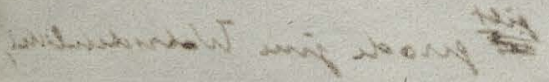

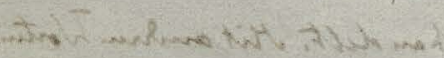

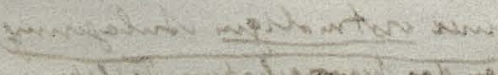




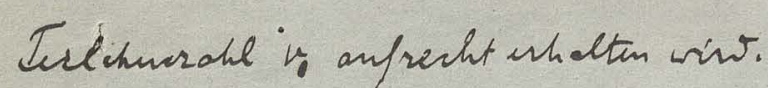

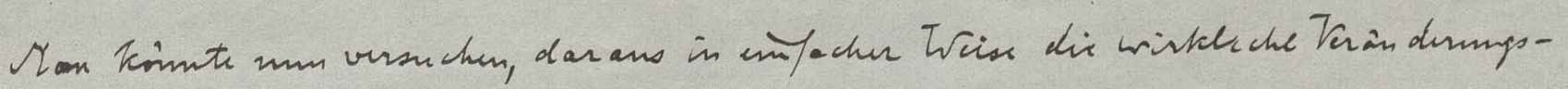

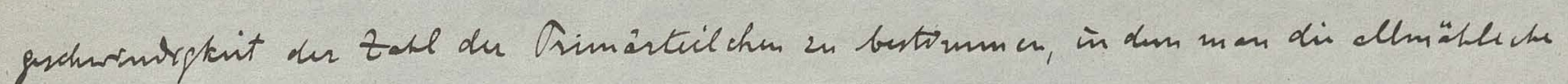

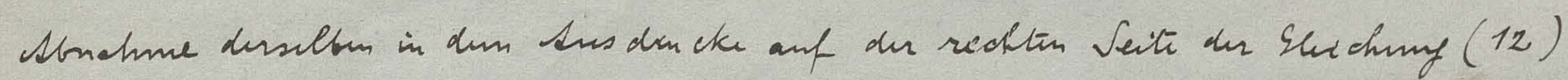

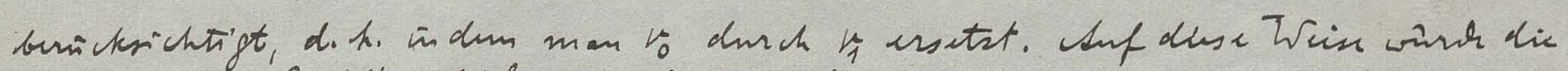

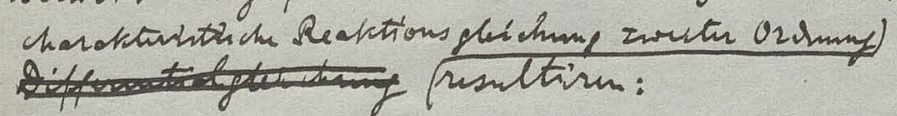

$$
\frac{1}{v_{1}^{2}} \frac{d v^{2}}{d t}=-4 \Omega \partial R
$$

wher das Intigral ergäb:

$$
v_{1}=\frac{v_{0}}{1+4 \pi D R v_{0} t}=\frac{v_{0}}{1+\frac{t}{T}}
$$

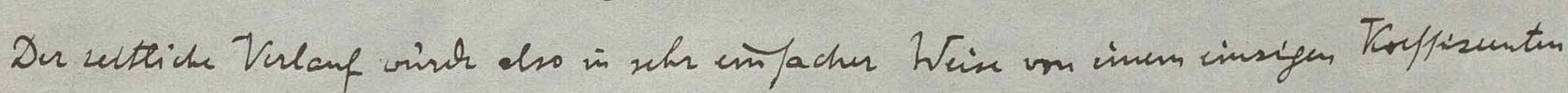

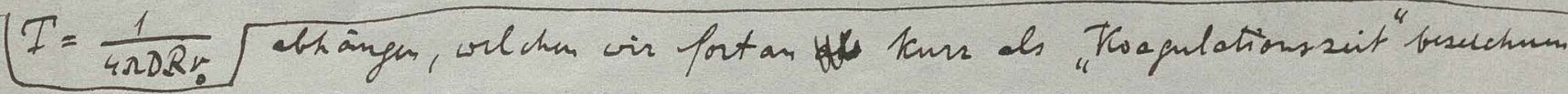
ountur.

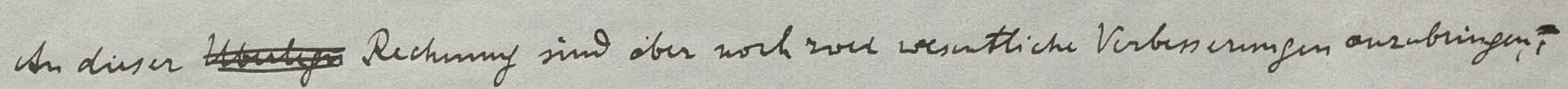

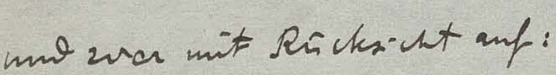

1) dir Elgenburgany des huroorghtobenm Telchers,

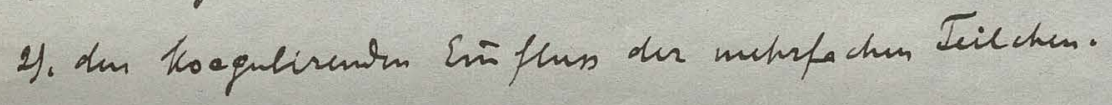

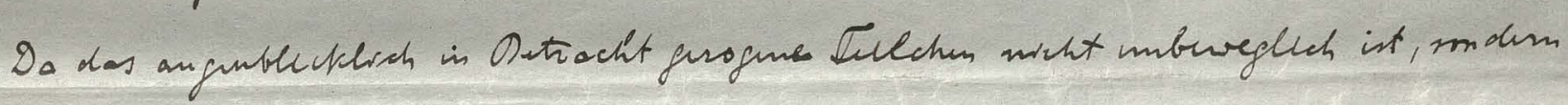

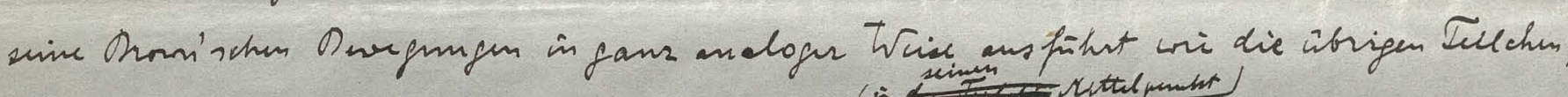

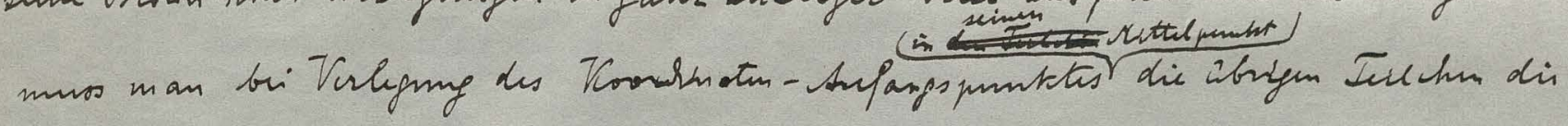
visklichun Relatioburgengen ans fulters lassen.

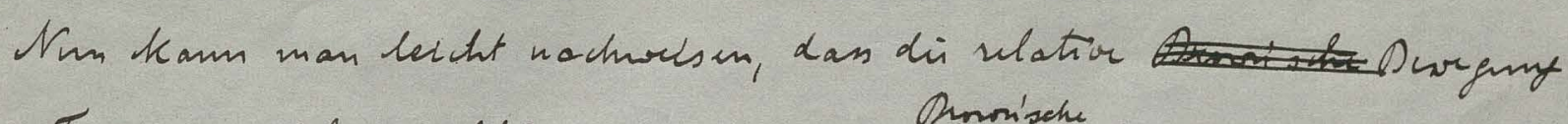

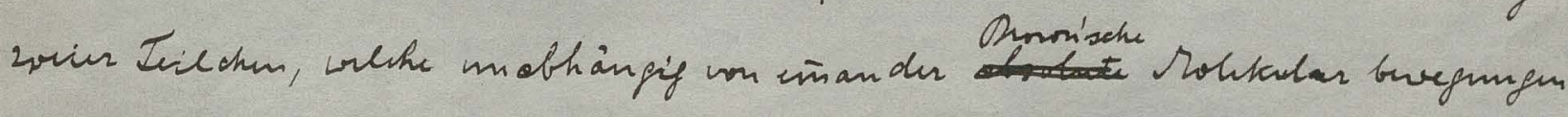

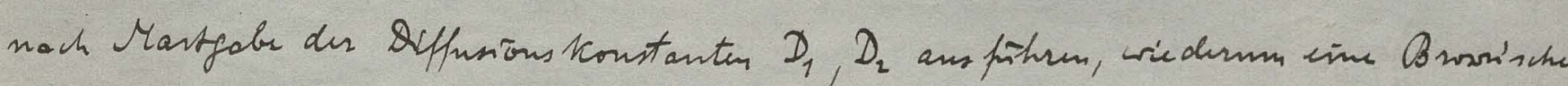

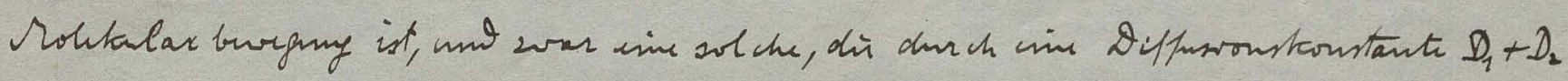

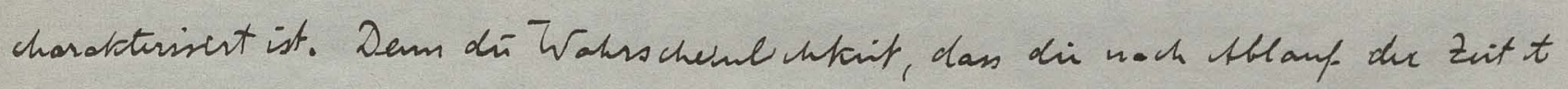

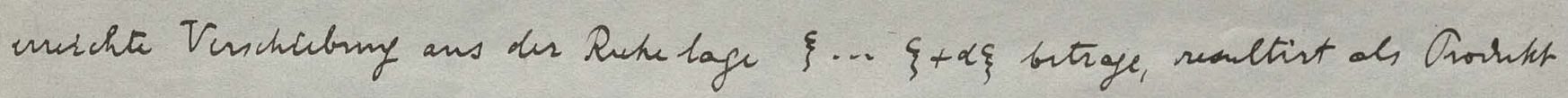

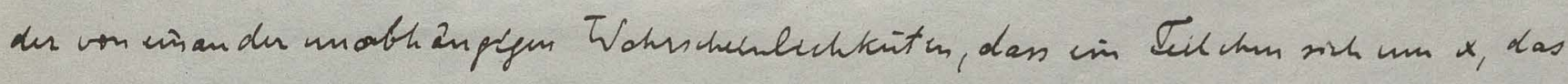

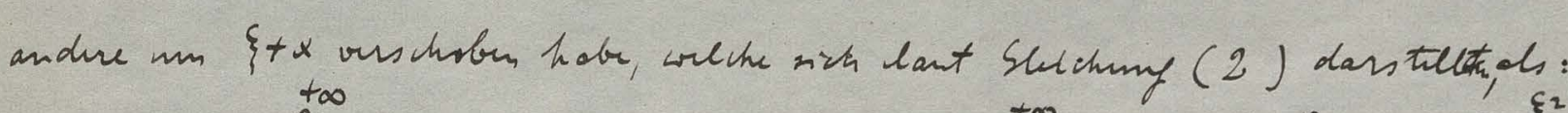

$$
\left.W(\xi) d \xi=\int_{x=-\infty}^{+\infty} T(x) d x T \xi \xi x\right) d \xi=\frac{d \xi}{2 \pi t \sqrt{D_{1} D_{2}}} \int_{-\infty}^{+\infty} e^{-\frac{x^{2}}{4 D_{2} t}}-\frac{(\xi+x)^{2}}{4 D_{1} t} d x=\frac{e^{-\frac{\xi_{2}}{4\left(D_{1}+D_{2}\right) t}}}{2 \sqrt{2\left(D_{1}+D_{2}\right)}} d \xi
$$

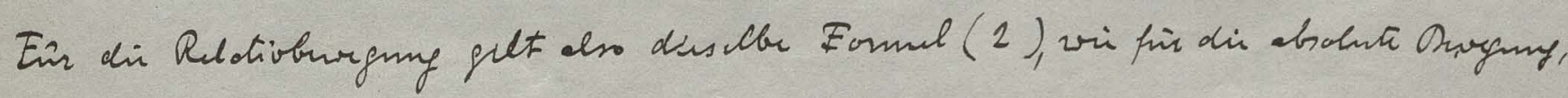

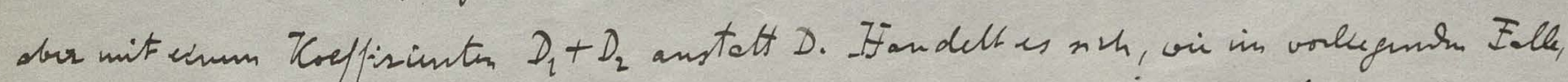

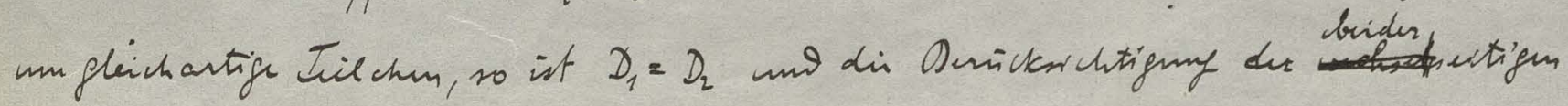

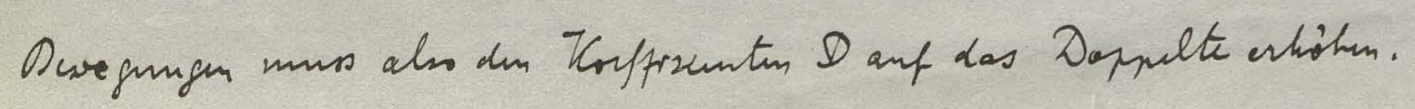





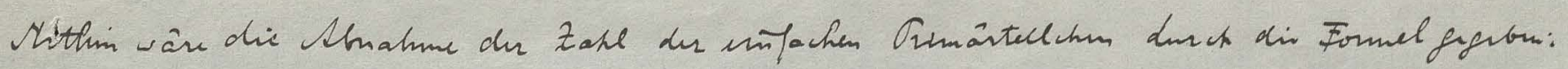

$v_{1}=\frac{v_{0}}{1+8 n D R \nu_{0} t}=\frac{\nu_{0}}{1+\frac{2 t}{T}}$

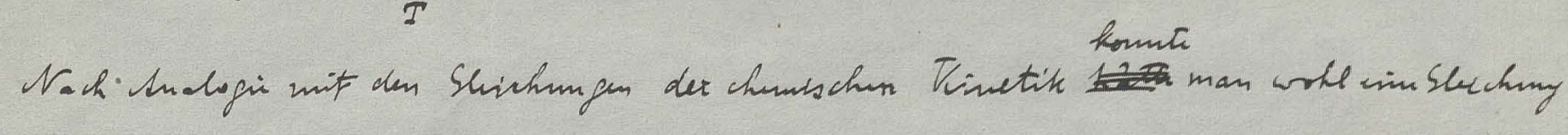

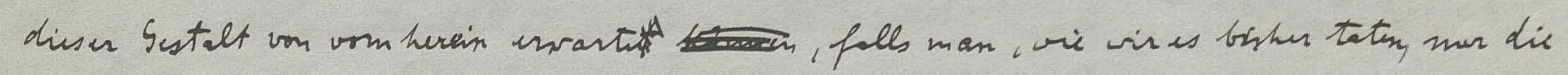

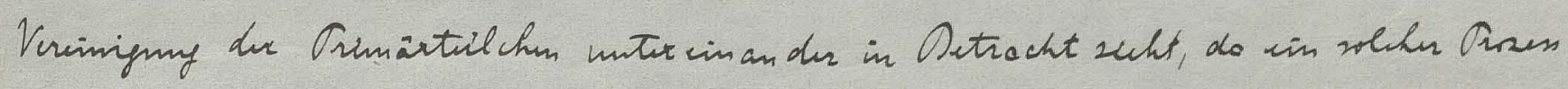

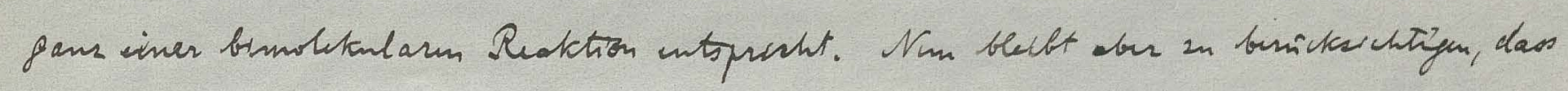

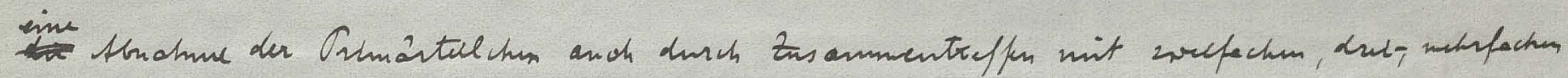

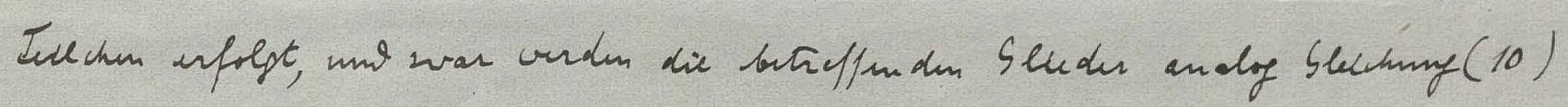

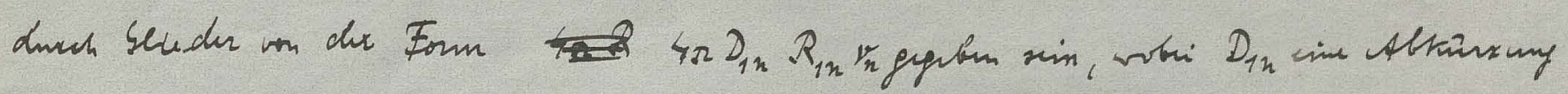
ist fir $D_{1 n}=D_{1}+D_{n}$ mot $R_{1 n}$ den Radins der Wirkmpophair bedentet, wolche dem

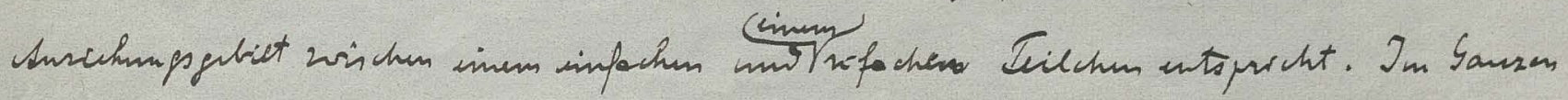

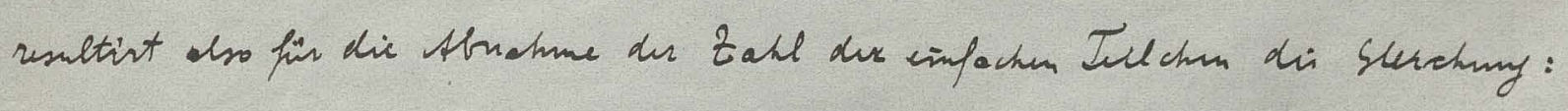

$\frac{1}{4 \Omega} \frac{d v_{1}}{d t}=-D_{11} R_{11} v_{1}^{2}-D_{12} R_{12} r_{1} v_{2}-D_{13} R_{13} v_{1} r_{3}-\ldots$

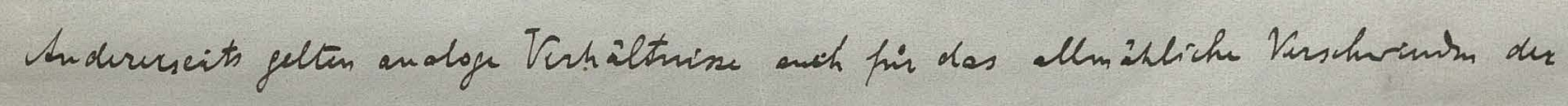

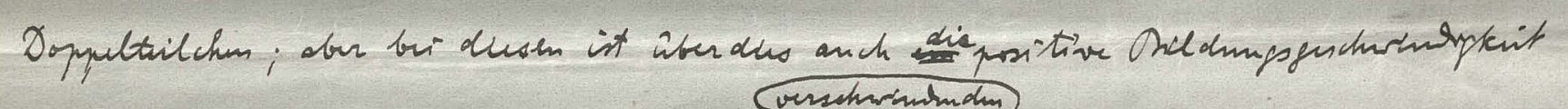

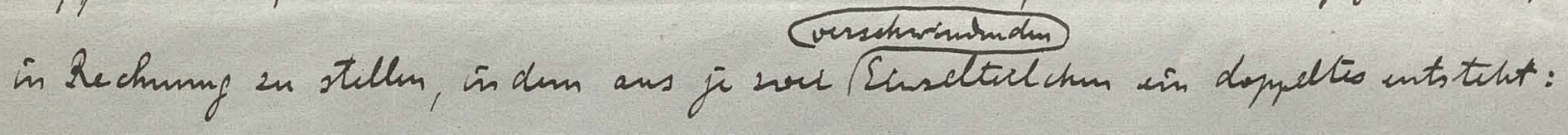

$\frac{1}{4 \Omega} \frac{d v_{3}}{d t}=\frac{1}{2} D_{11} R_{11} v_{1}^{2}-D_{12} R_{12} v_{1} v_{2}-D_{22} R_{22} v_{2}^{2}-D_{23} R_{23} v_{2} v_{3}$

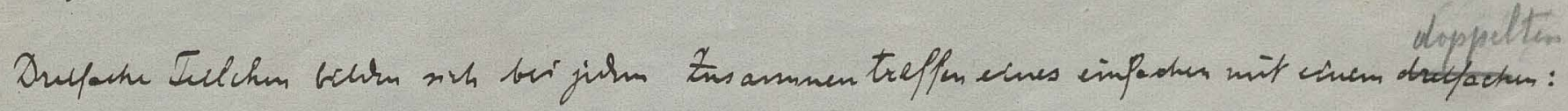

$\frac{1}{4 \Omega} \frac{d v_{3}}{d t}=D_{12} R_{13} r_{1} r_{2}-D_{13} R_{13} v_{1} v_{3}-D_{23} R_{23} r_{2} r_{3}-\cdots$

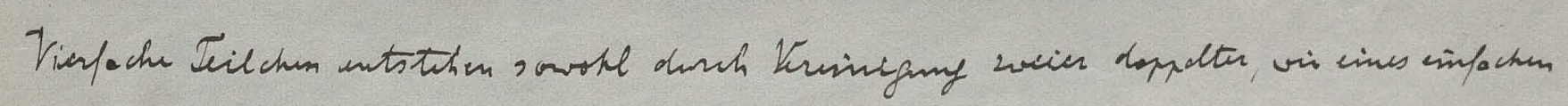
unt cines drufechen:

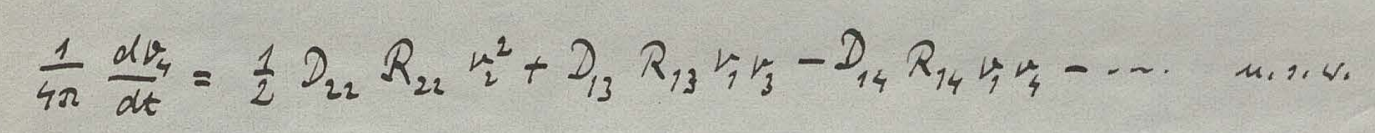

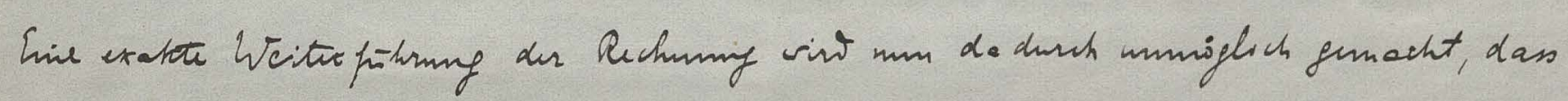

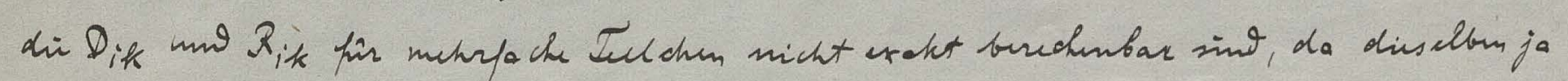

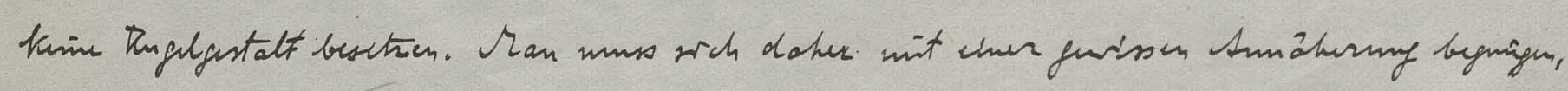

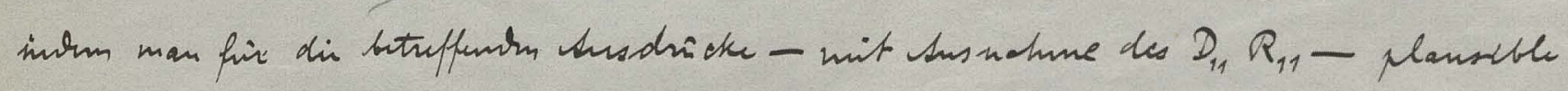

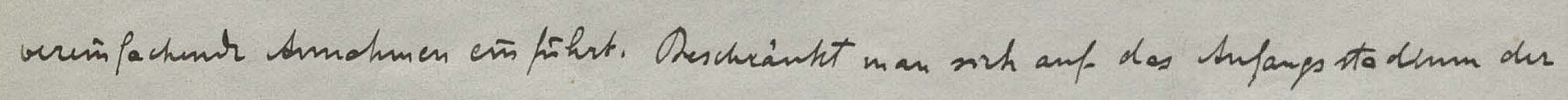

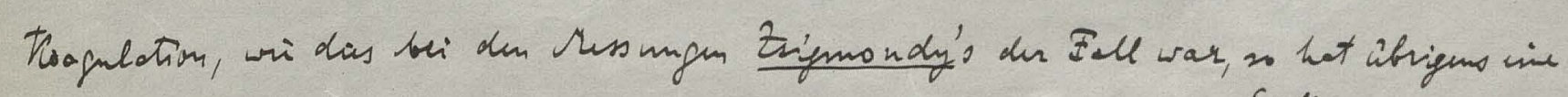

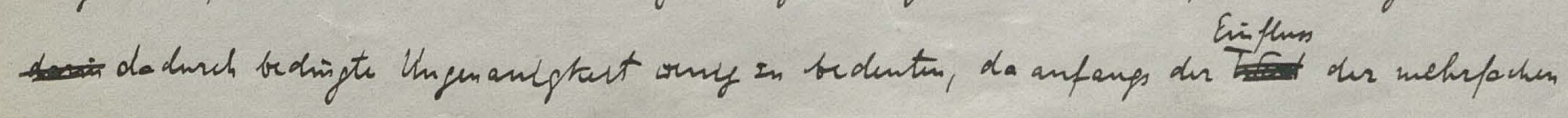
Tilchen whishampt cony ansmocht. 



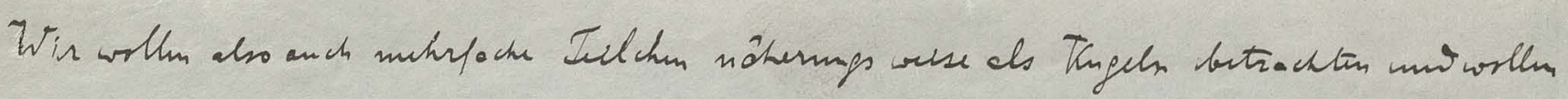

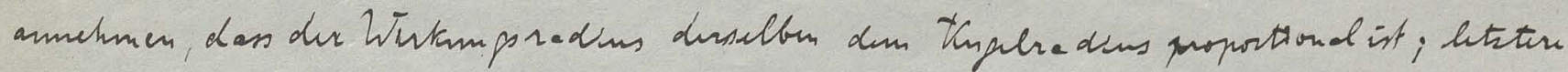

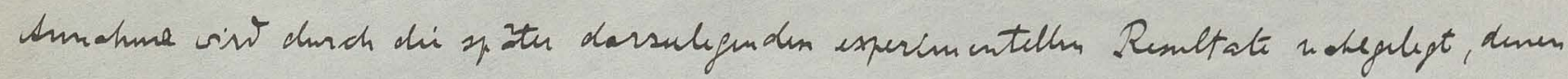

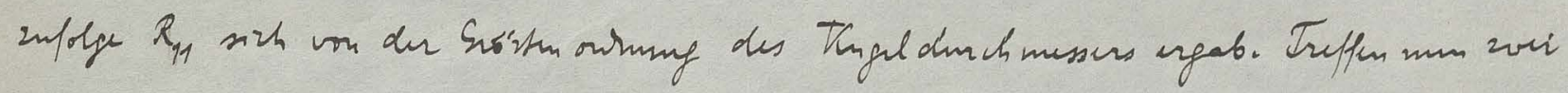

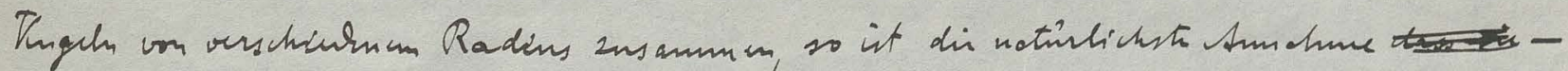

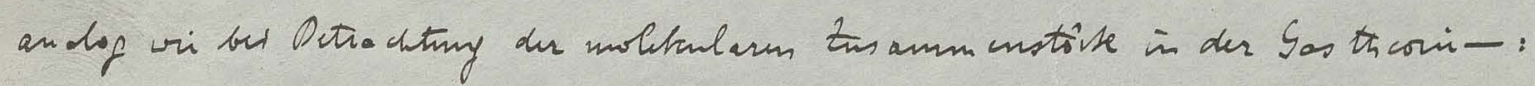

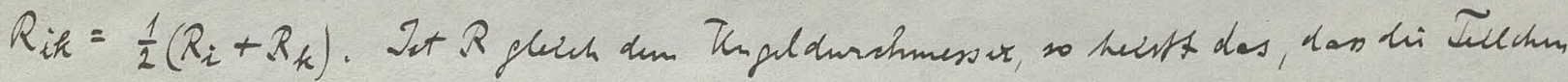
with bu Ocsitinny answhen.

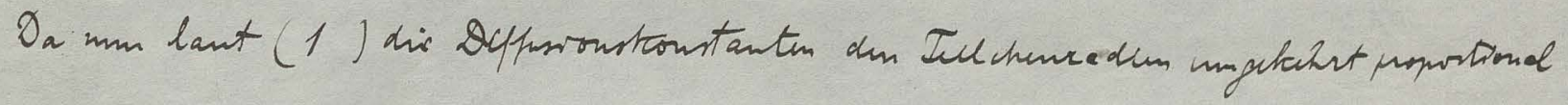
sinit, gilts

$D_{i k} R_{i k}=\frac{1}{2}\left(D_{i}+D_{f}\right)\left(R_{i}+R_{k}\right)=\frac{\partial R}{2} \frac{\left(R_{i}+R_{k}\right)^{2}}{R_{L} R_{k}}$

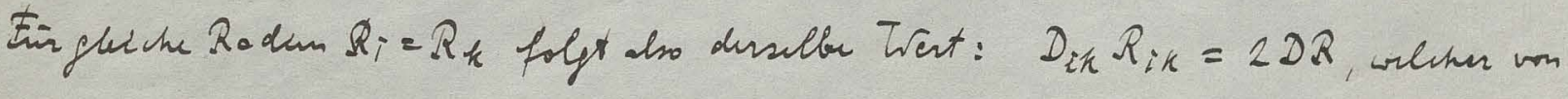

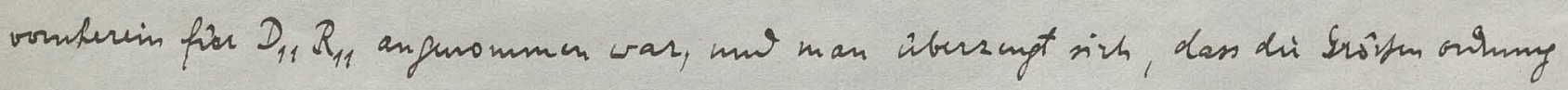

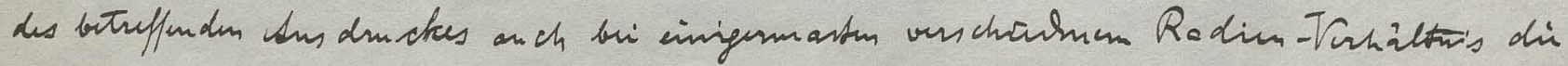

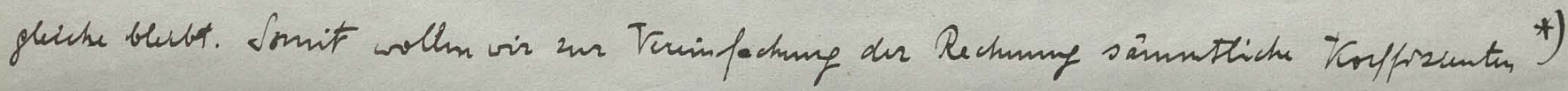
$4 \Omega D_{i k} R_{i \pi}=8 \Omega D R=2 \alpha$ utrm min whalten derart das Sle chimgsypten:

$$
\begin{aligned}
& \frac{1}{2 \alpha} \frac{d v_{1}}{d t}=-v_{1}^{2}-v_{1} v_{2}-v_{1} v_{3}-v_{2} v_{4}-\ldots \\
& \frac{1}{2 \alpha} \frac{d v_{2}}{d t}=\frac{v_{1}^{2}}{2}-v_{1} v_{2}-v_{2}^{2}-v_{2} v_{3}-\cdots \\
& \frac{1}{2 \alpha} \frac{d v_{3}}{d t}=v_{1} v_{2}-v_{1} v_{3}-v_{2} r_{3}-v_{3}^{2}-\cdots \\
& \frac{1}{2 \alpha} \frac{d v_{4}}{d t}=\frac{v_{2}^{2}}{2}+v_{1} v_{3}-v_{1} v_{4}-v_{2} v_{4}-\cdots
\end{aligned}
$$

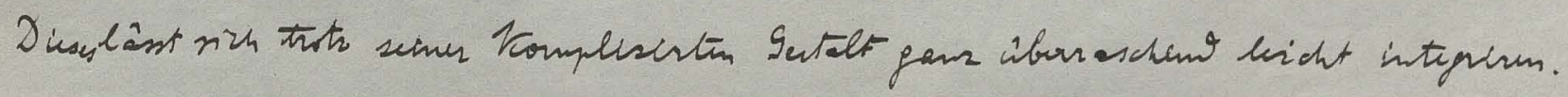

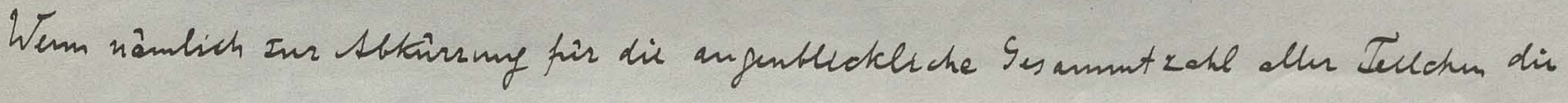
Descichnony

$$
r_{1}+v_{2}+r_{3}+v_{4}+\cdots=\sum r
$$

ingefintert writ, so minint jumes System die Sutalf an:

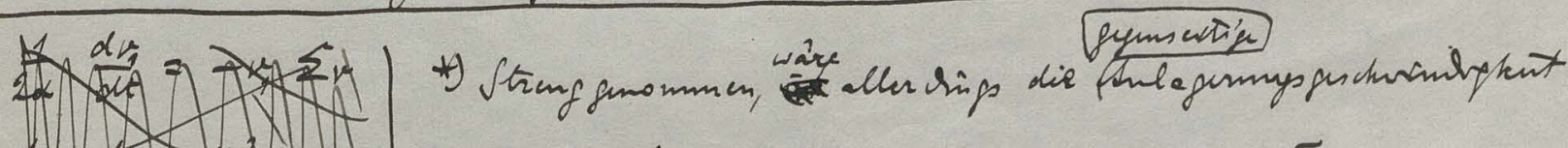

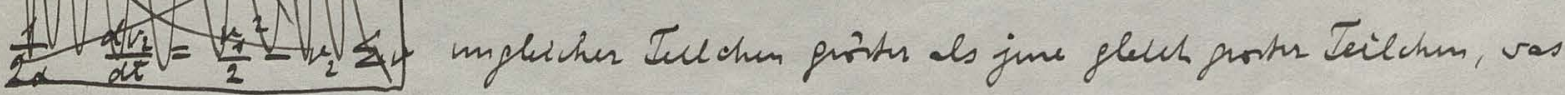

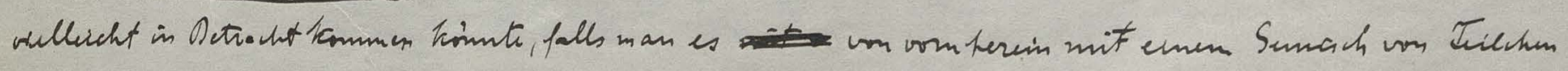

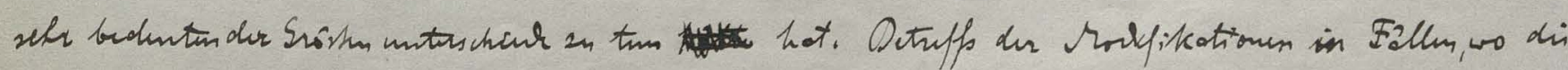

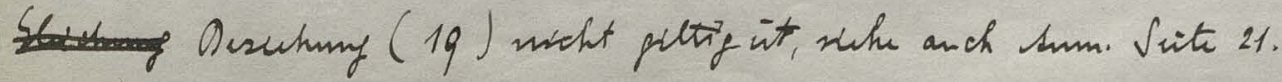


(8)

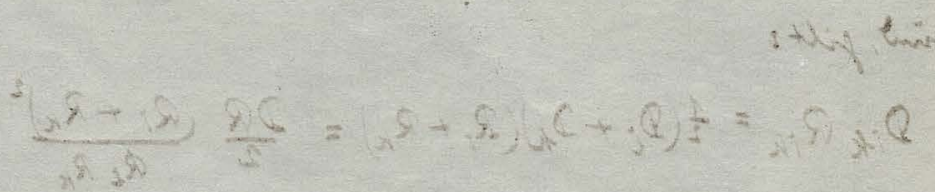

(+

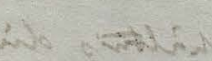

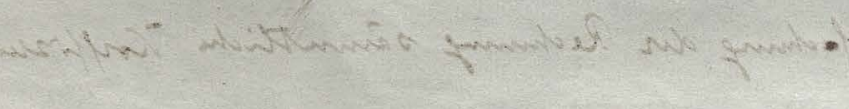

- whे

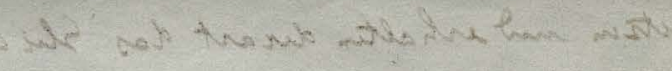

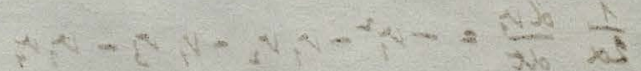

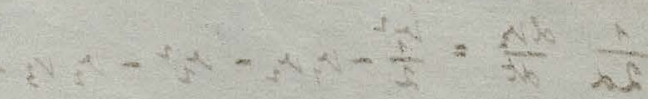

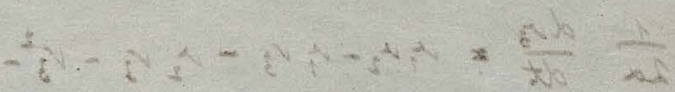

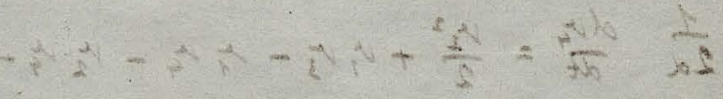

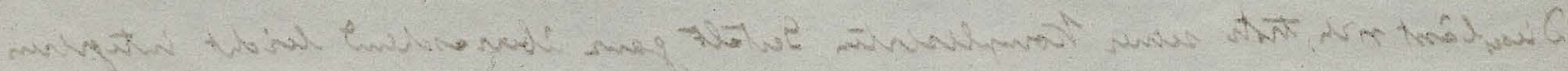

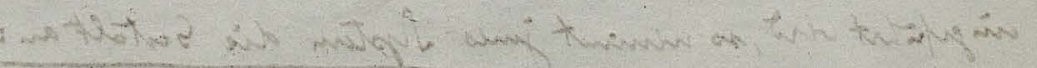

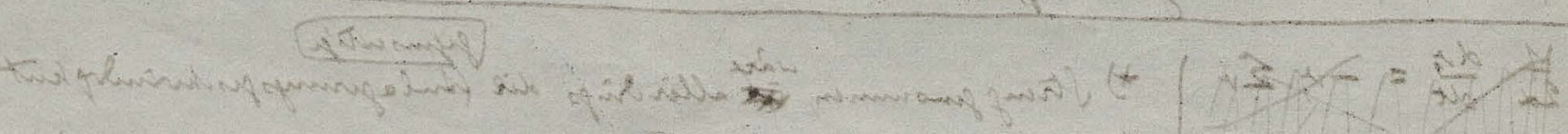

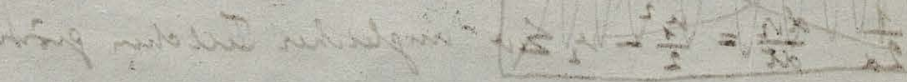

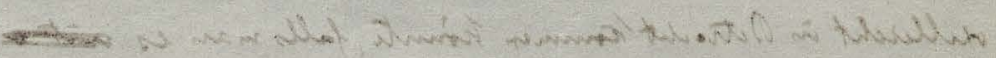

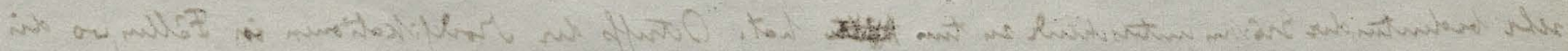

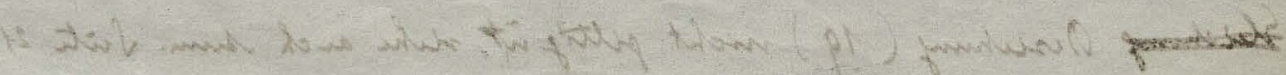




$$
\begin{aligned}
& \frac{1}{2 \alpha} \frac{d r_{1}}{d t}=-r_{1} \sum r_{\mu} \\
& \frac{1}{2 \alpha} \frac{d r_{2}}{d t}=\frac{r_{r}^{2}}{2}-r_{2} \sum r \\
& \frac{1}{2 \alpha} \frac{d r_{3}}{d t}=r_{1} r_{2}-r_{3} \sum r \\
& \frac{1}{2 \alpha} \frac{d r_{k}}{d t}=\frac{1}{2}\left[r_{1} r_{k-1}+r_{2} r_{k-2}+r_{3} r_{k-1}+\cdots r_{k-1} r_{1}\right]-r_{k} \sum v
\end{aligned}
$$

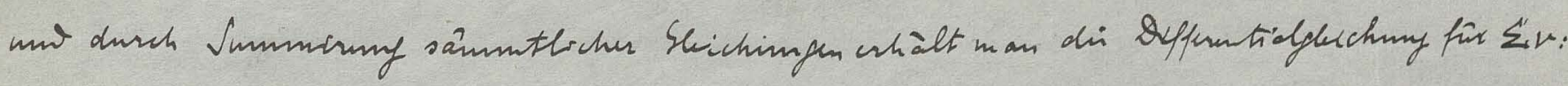

$$
\frac{1}{\alpha} \frac{d \sum v}{d t}=-\left(\sum v\right)^{2}
$$

ans colcher numecter folft:

$$
\sum \mu_{2} \frac{v_{0}}{1+\alpha \nu_{0} t}=\frac{r_{0}}{1+4 \Omega D R \mu_{0} t}=\frac{v_{0}}{1+\frac{t}{T}}
$$

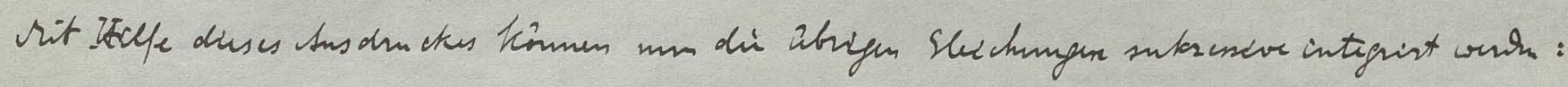

$$
\begin{aligned}
& v_{1}=\frac{v_{0}}{\left[1+\alpha v_{0} t\right]^{2}}=\frac{v_{0}}{\left[1+4 \pi D R v_{0} t\right]^{2}}=\frac{v_{0}}{\left[1+\frac{t}{T}\right]^{2}} \\
& v_{2}=v_{0} \frac{\alpha v_{0} t}{\left[1+\alpha v_{0} t\right]^{3}} \\
& v_{3}=v_{0} \frac{\left[\alpha v_{0} t\right]^{2}}{\left[1+\alpha v_{0} t\right]^{4}} \\
& v_{k}=\frac{v_{0}\left[\alpha v_{0} t\right]^{k-1}}{\left[1+\alpha v_{0} t\right]^{k+1}}
\end{aligned}
$$

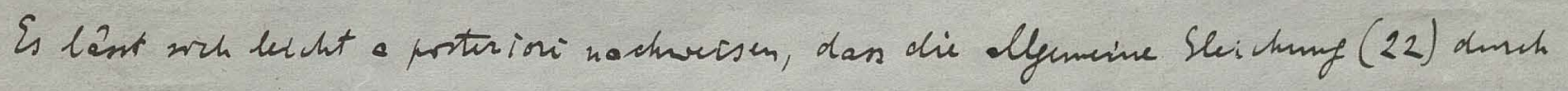

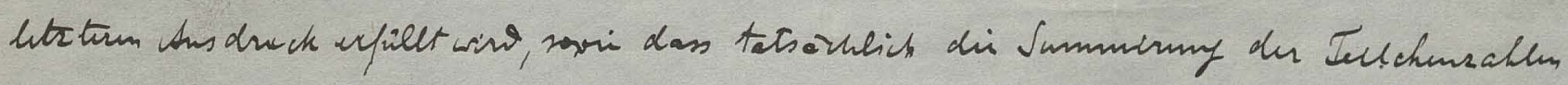
den Susdrick (23) upits.

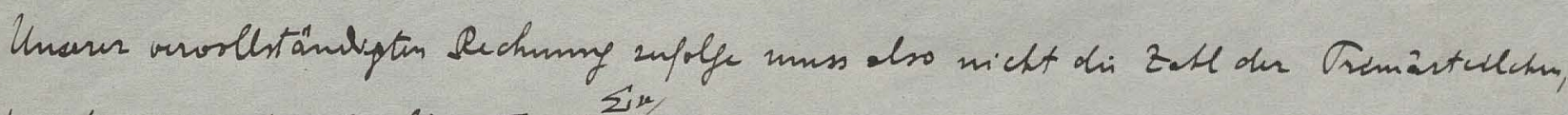

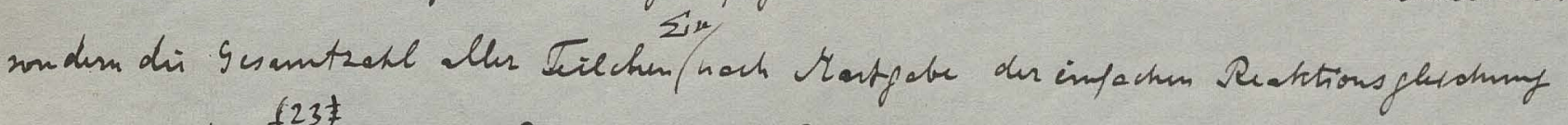

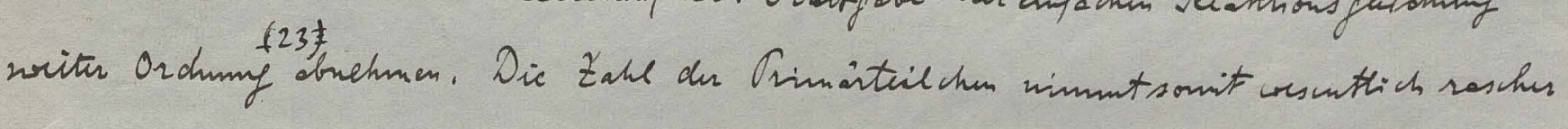

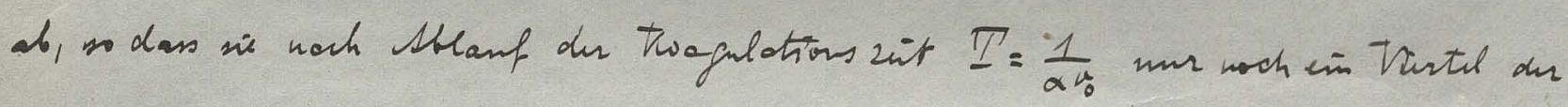

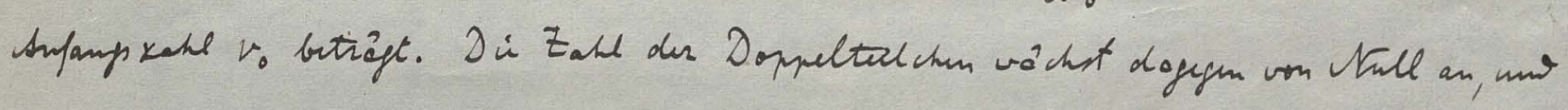

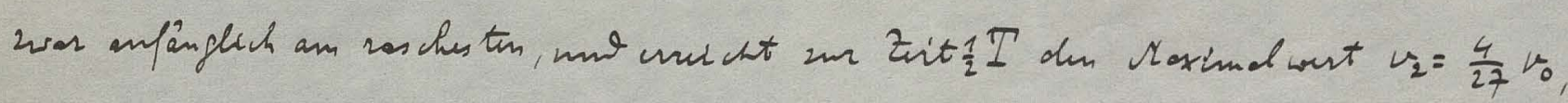

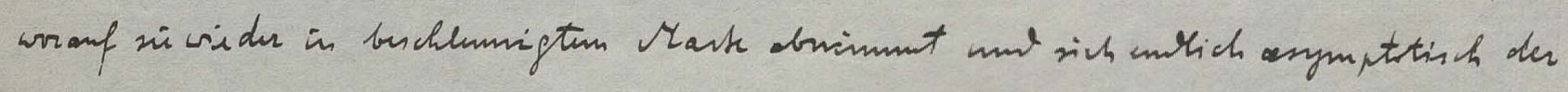

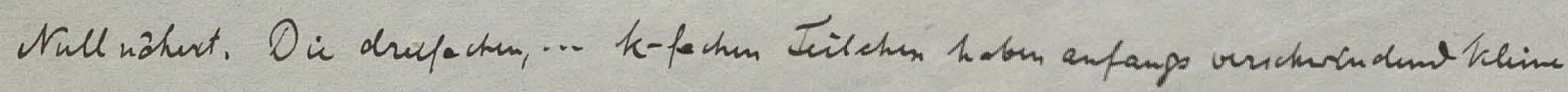

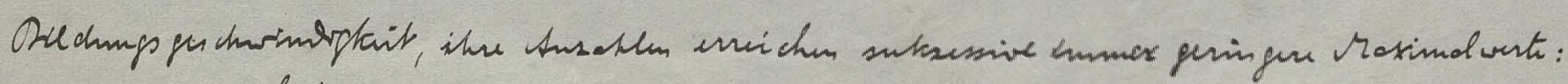

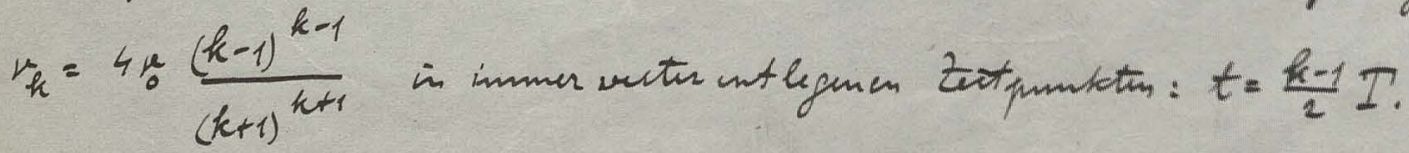

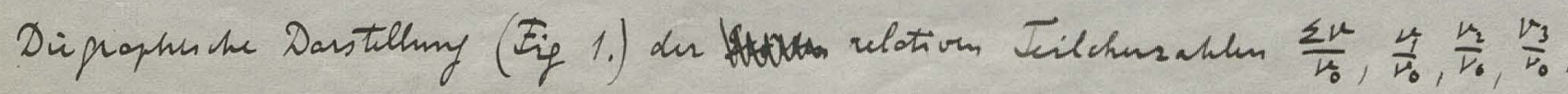




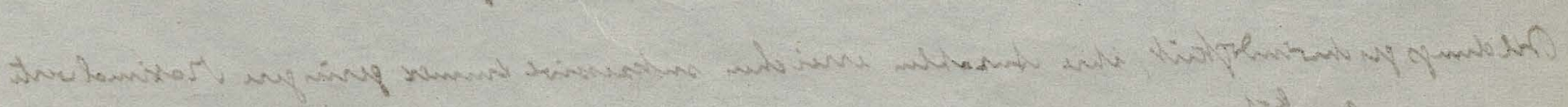

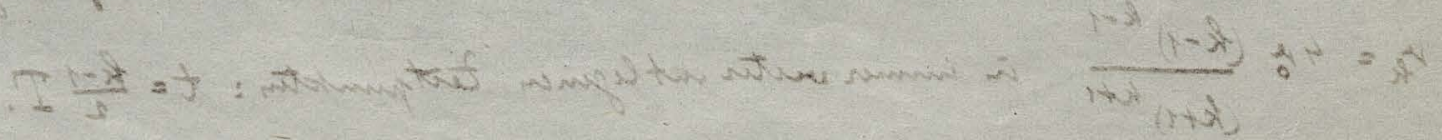

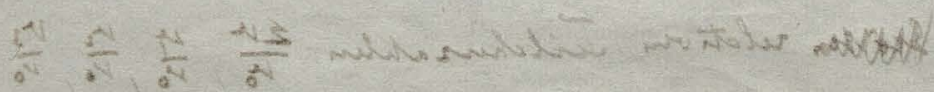

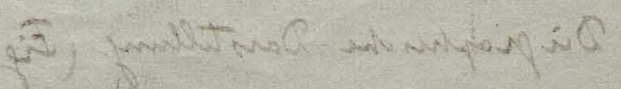




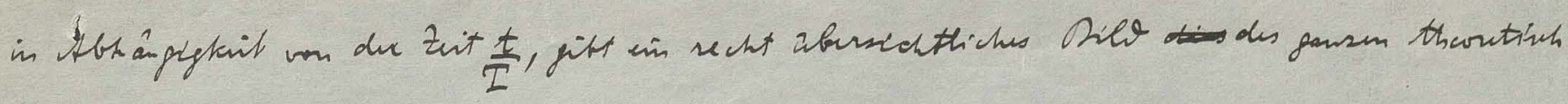
mansensechenden tiogulations unganges.

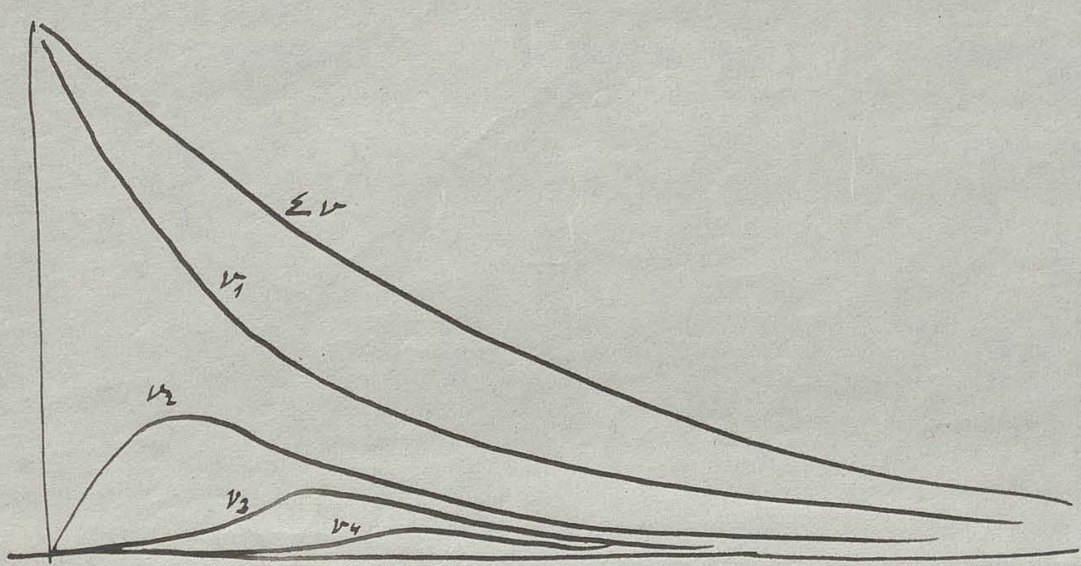

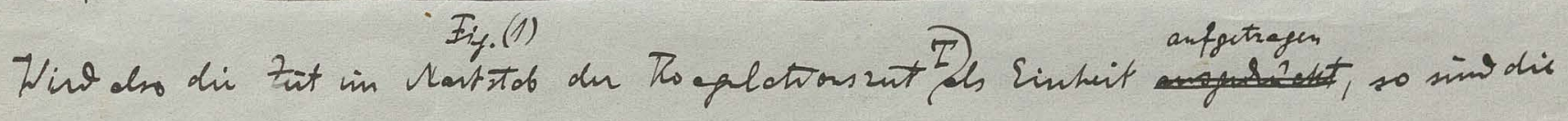

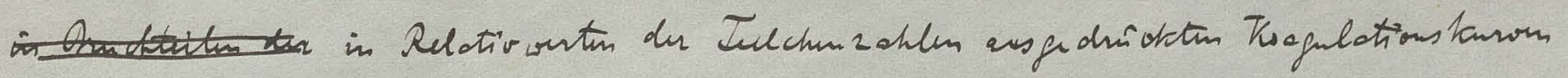

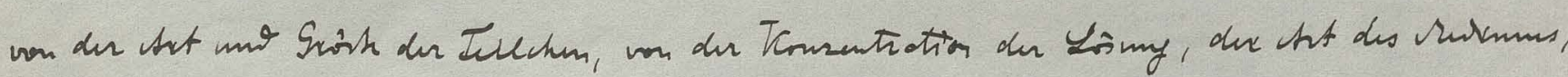

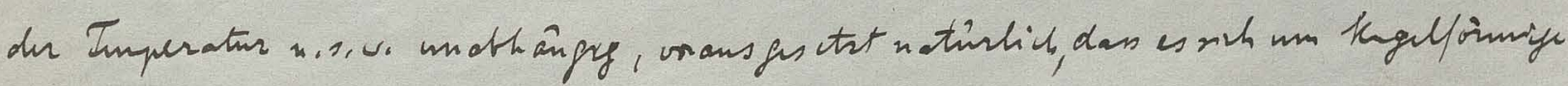

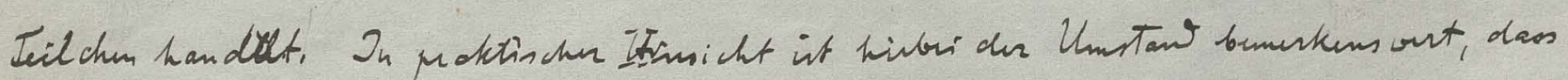

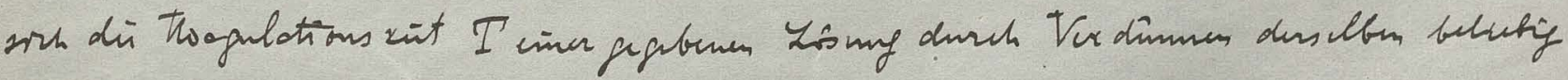

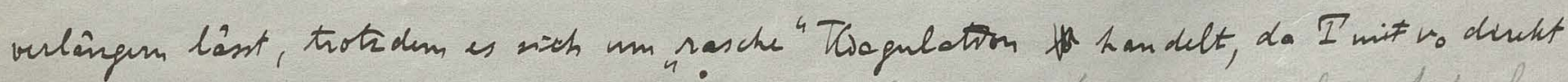
popistiond oin nuss,

\section{Vugleetch nit Zrigmondy's Messumen.}

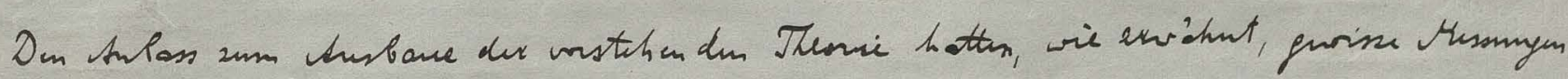

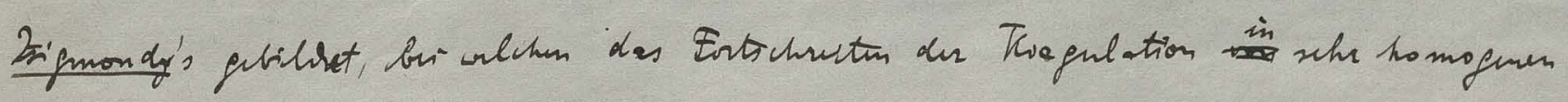

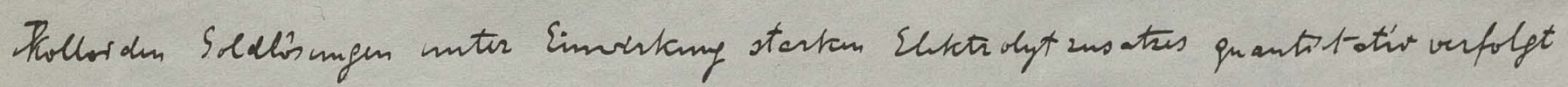

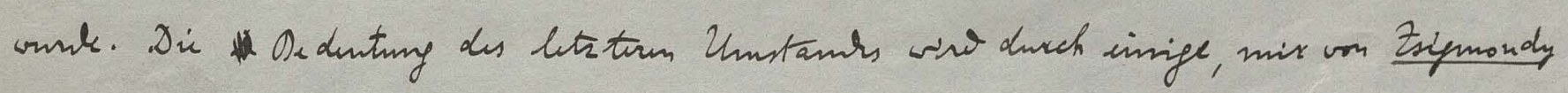

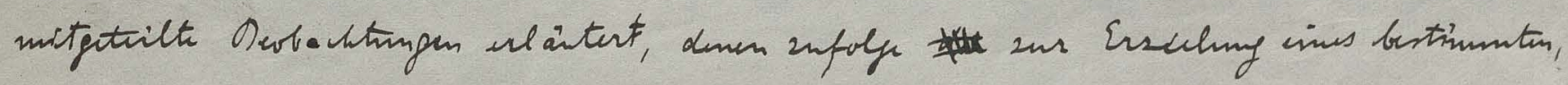

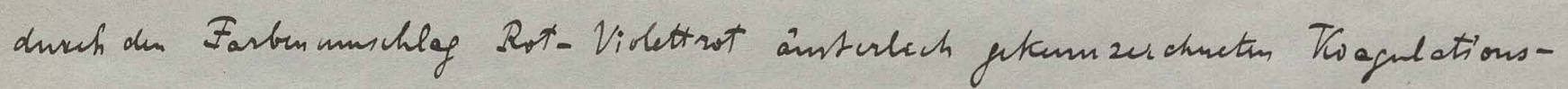

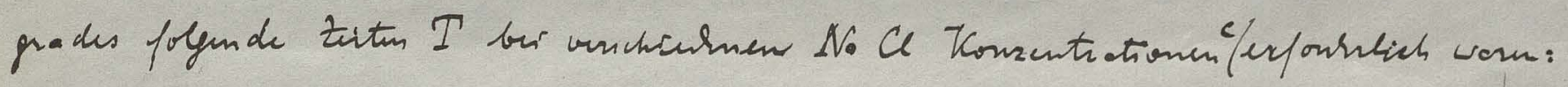

\begin{tabular}{|c|c|c|c|c|c|c|c|c|c|c|}
\hline c & $\mathcal{S}$ & 10 & 20 & 50 & 100 & 150 & 200 & 300 & Millinnd i.zitu & 500 \\
\hline$I$ & $>150$ & 12 & 7,2 & 7 & 7 & 6 & 6,5 & 7,5 & Stemmen & 7 \\
\hline
\end{tabular}

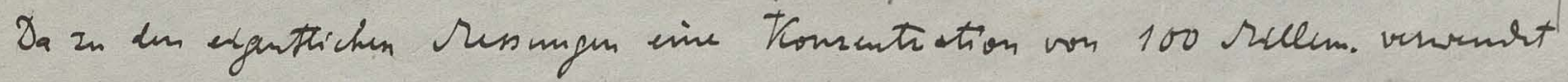

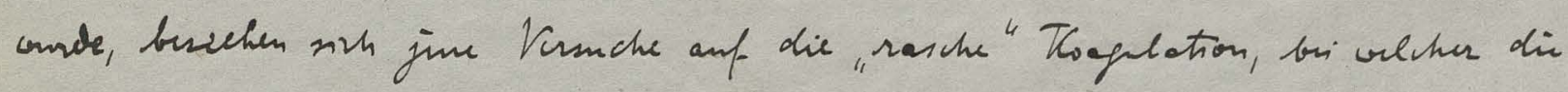

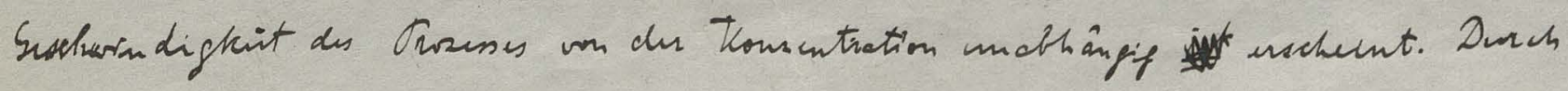

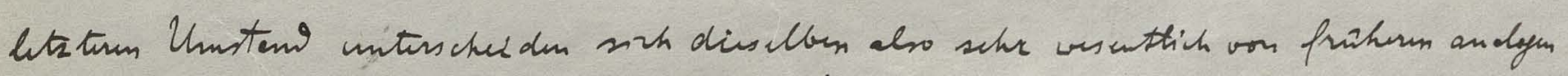

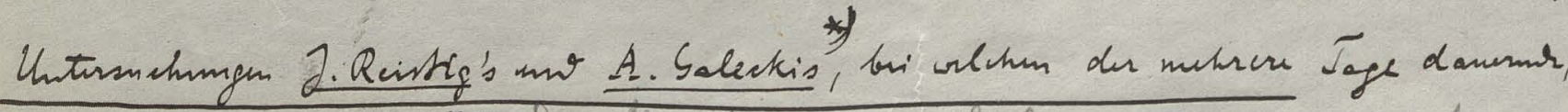

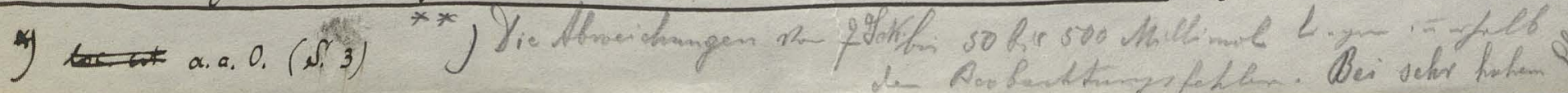




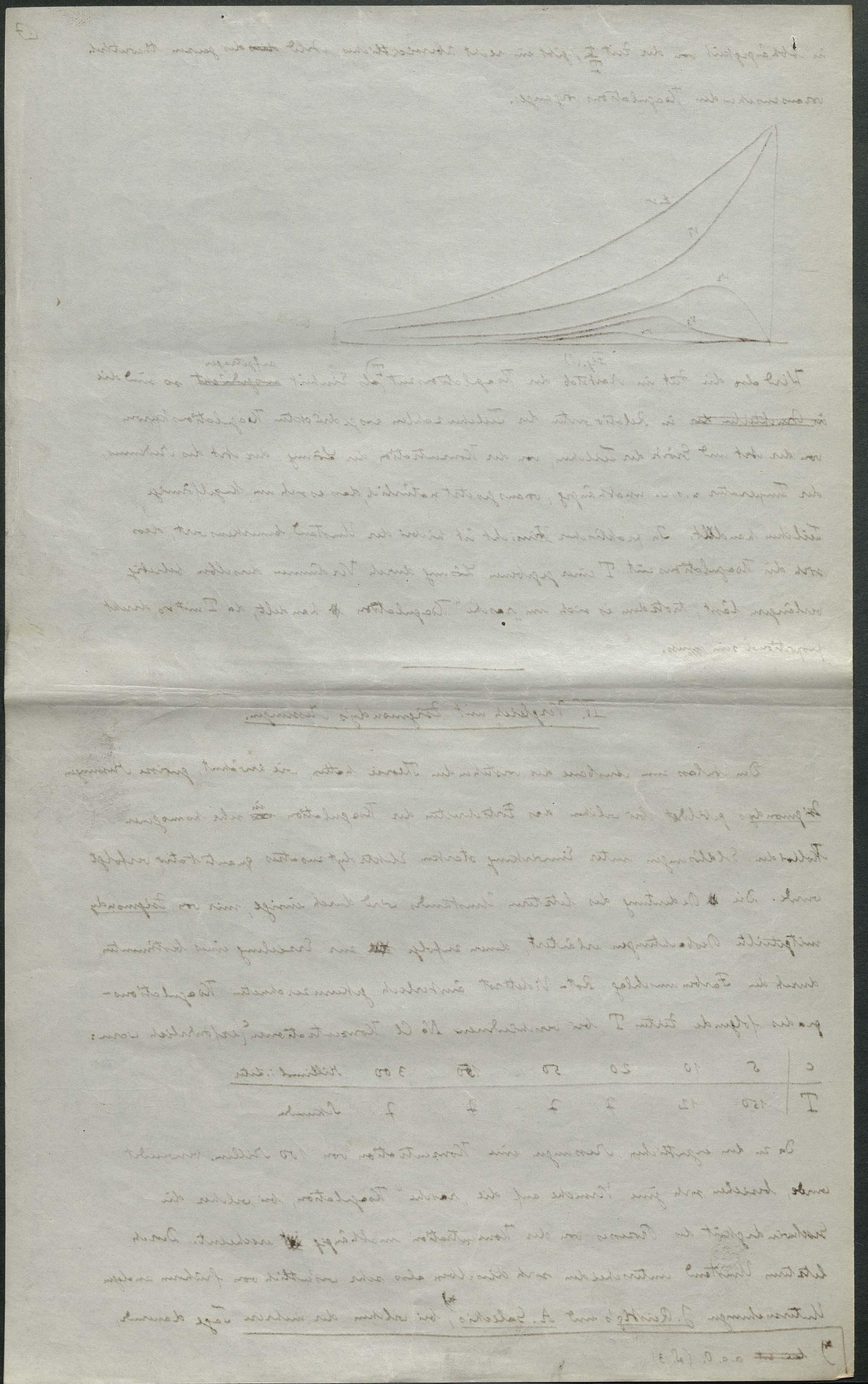




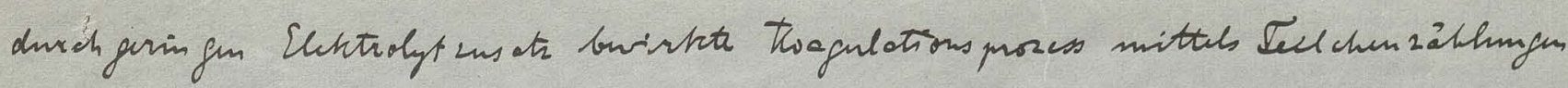
ouplet ouns.

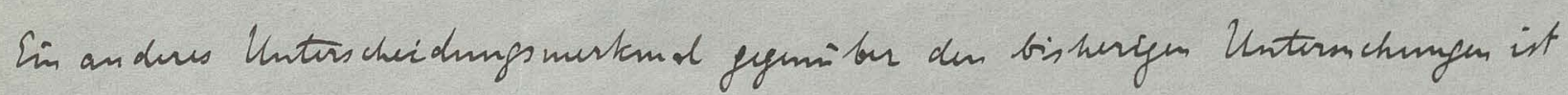

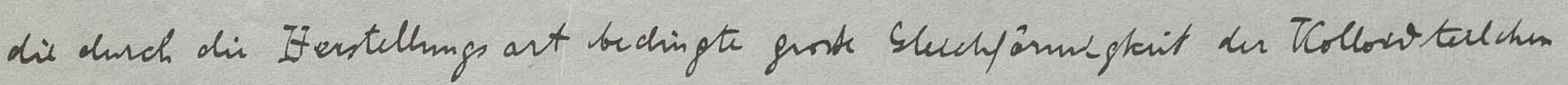

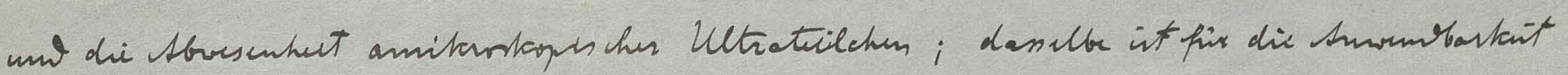

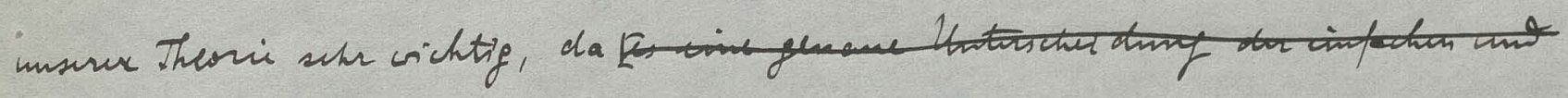

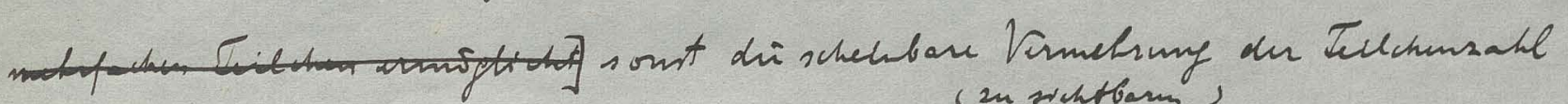

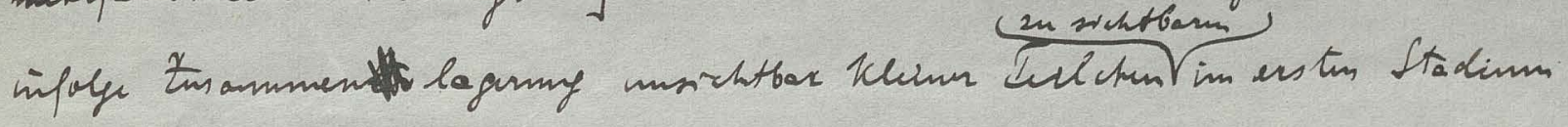

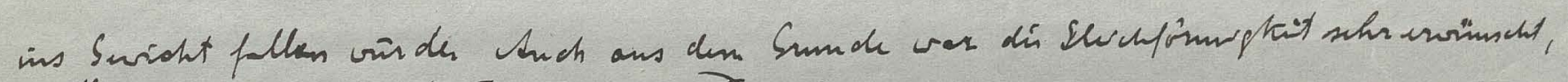

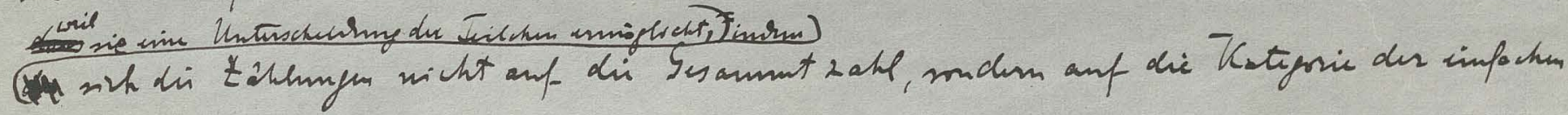

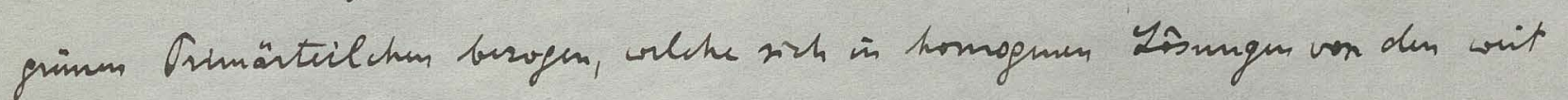

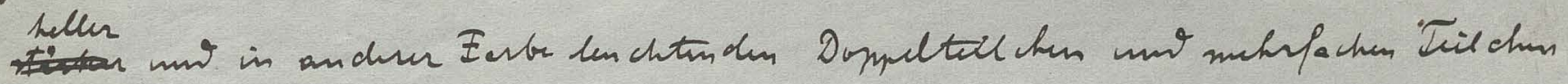
lecolt unterschecden lassen.

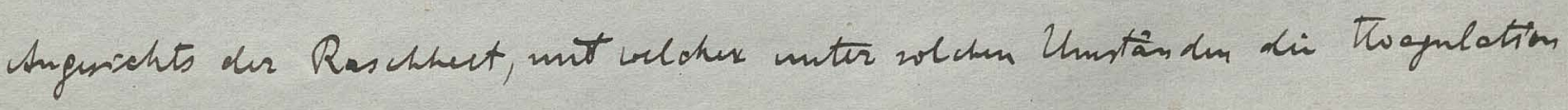

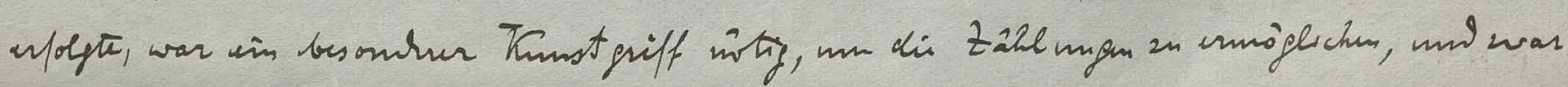

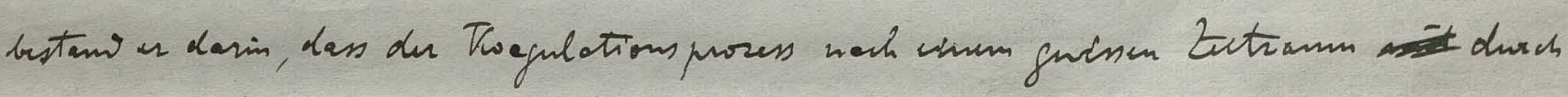

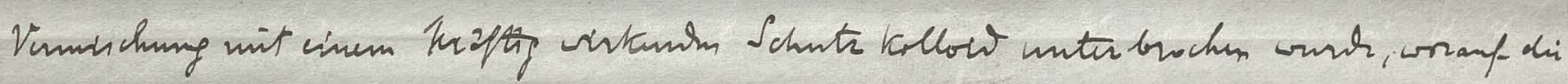

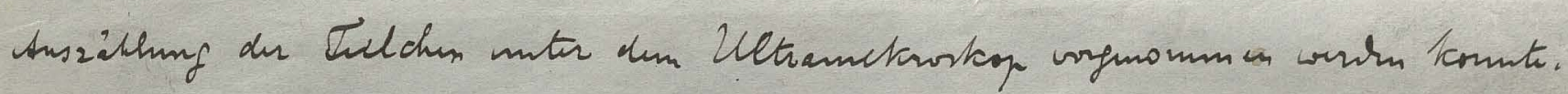

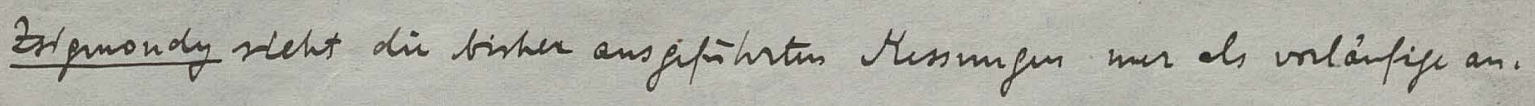

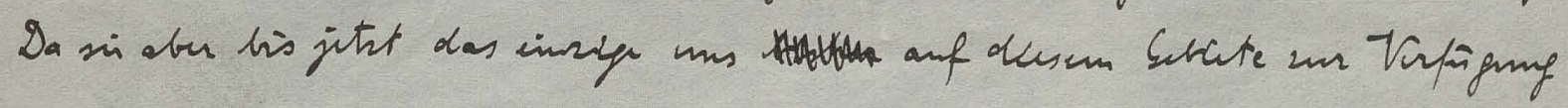

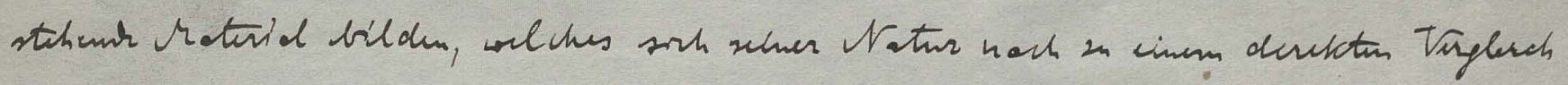

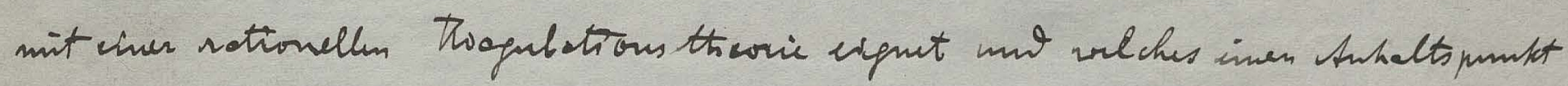

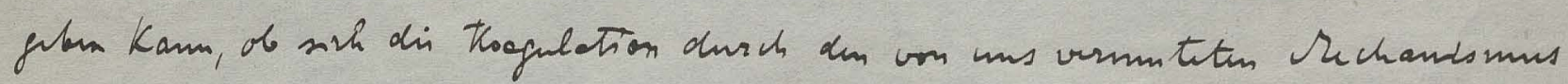

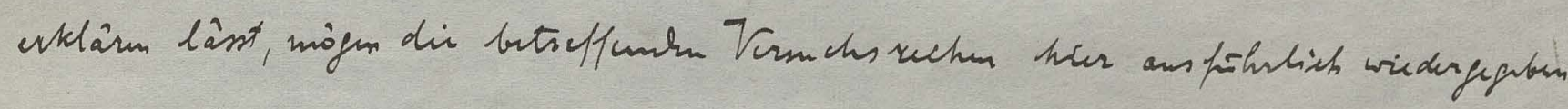

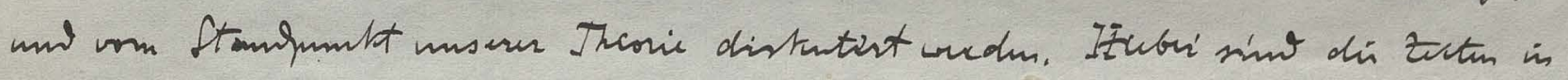

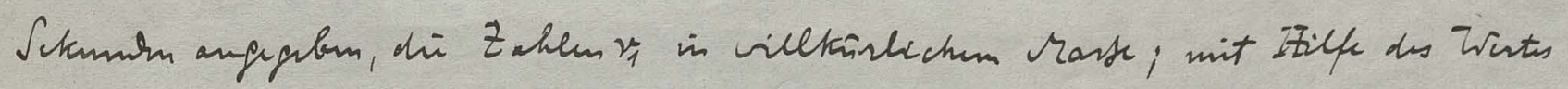

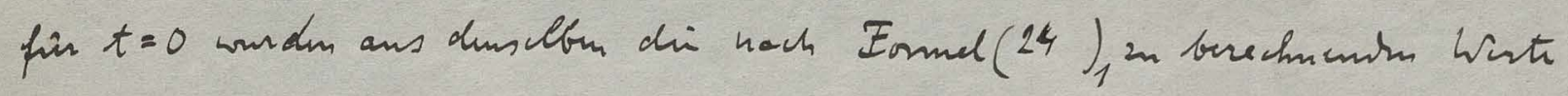
der eseypotem Troagulations sit

$\frac{1}{T}=4 \pi \partial R v_{0}=\frac{1}{t}\left[\sqrt{\frac{r_{10}}{r_{1}}}-1\right]$

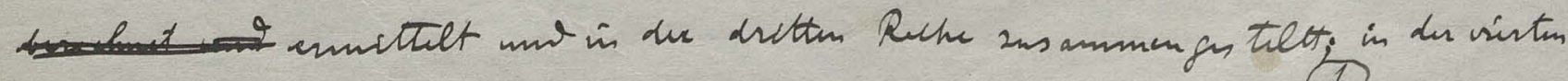

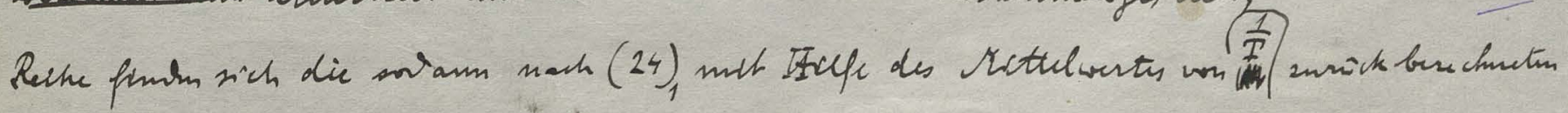
$v_{1}-$ Werta. 
whit dus emath

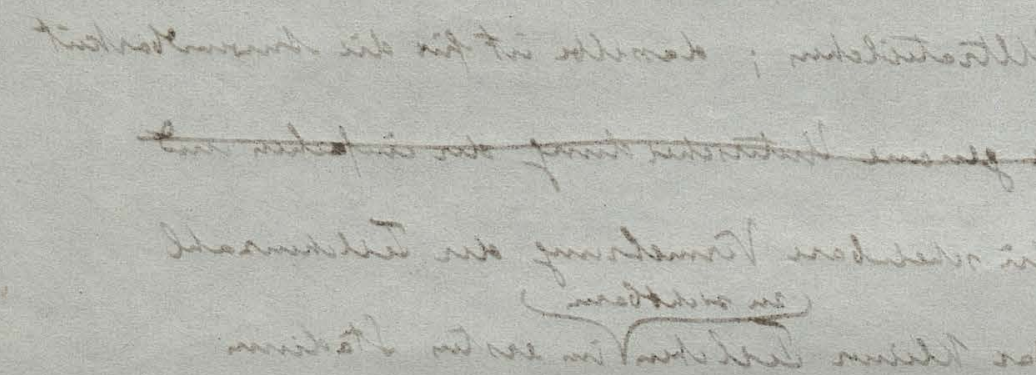

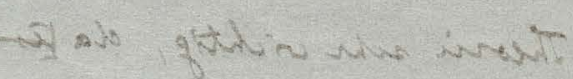

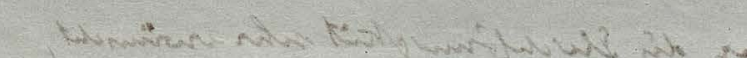

whathis and imata

$$
\text { 项 }
$$

$\left(x+\frac{20}{20}\right.$

\section{mintolutew}

now

sundo tiese

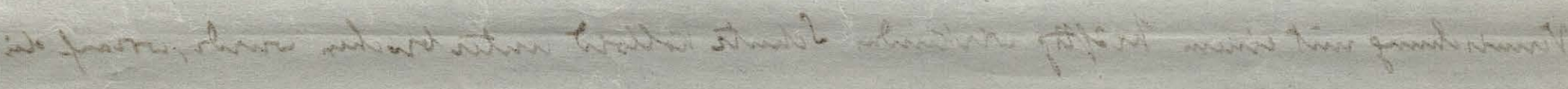

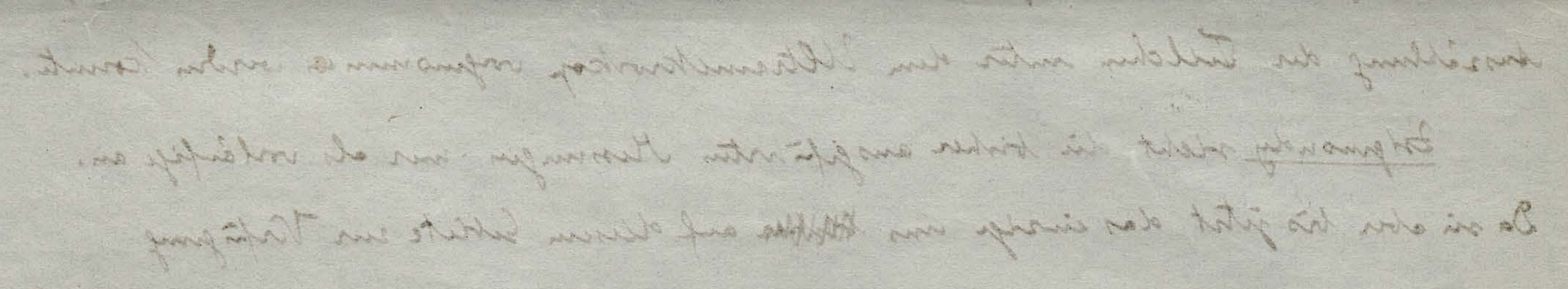

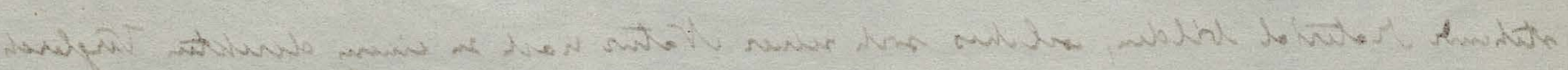

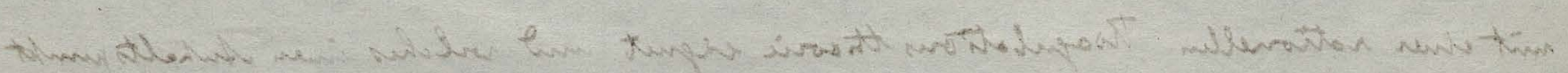
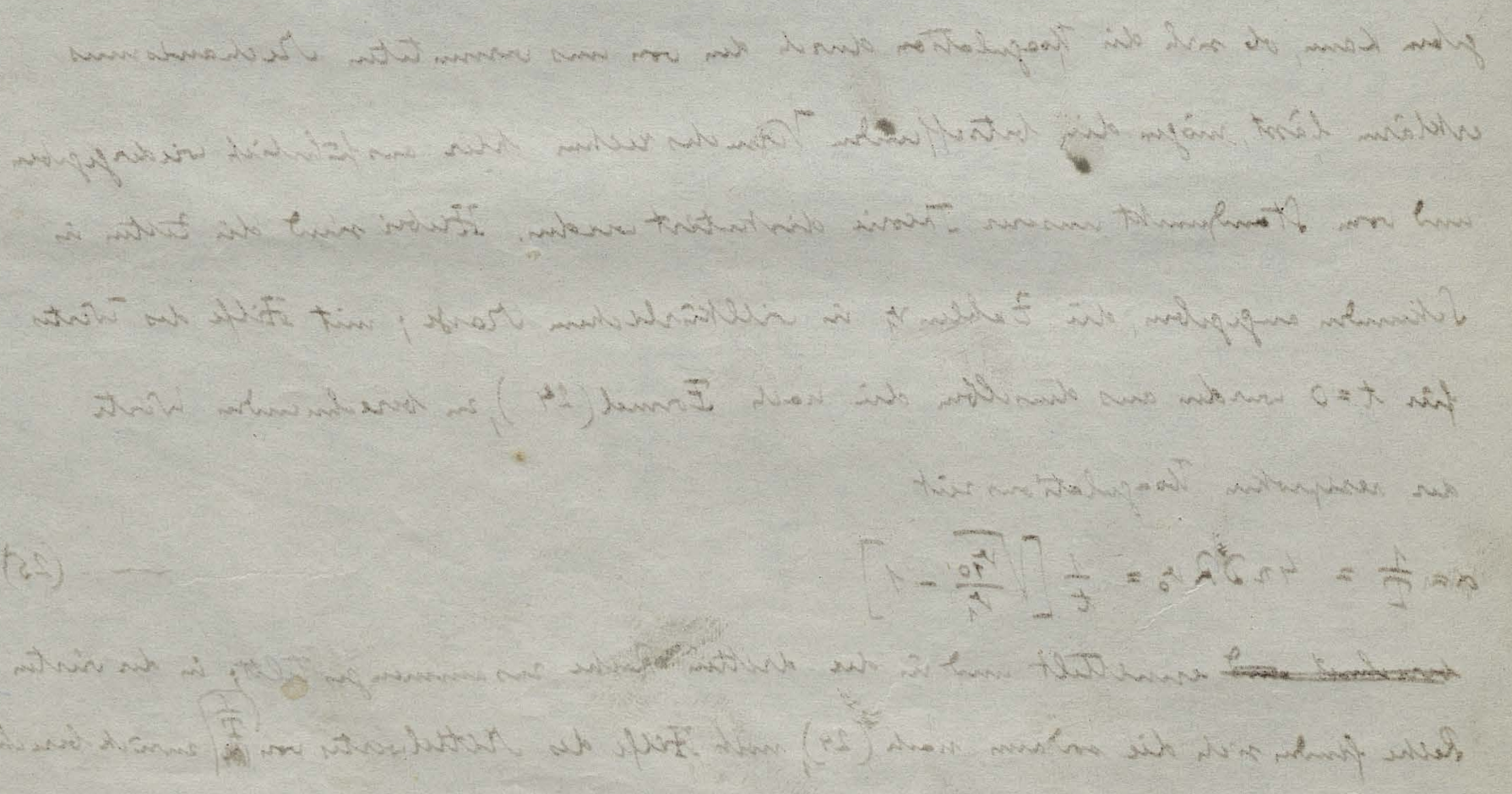
Fersuch D: $r_{0}=0.80 \cdot 10^{10} ; x=134 \cdot 10^{-7} \mathrm{~cm}$.

\begin{tabular}{c|c|c|c}
\hline$t$ & $v_{1}$ & $\frac{1}{T}$ & $r_{1} \mathrm{bur}$ \\
\hline 0 & 1.93 & & 1.93 \\
2 & 1.42 & $0.083)$ & 1.71 \\
10 & 1.17 & 0.0286 & 1.14 \\
20 & 0.75 & 0.0202 & 0.76 \\
30 & 0.52 & 0.0309 & 0.53
\end{tabular}

Mattel $\frac{1}{T}=0.0299 ; \quad \frac{R}{r}=1.40$

Vursuch $F: v_{0}=0 \cdot 27 \cdot 10^{10} ; s=24 \cdot 2 \cdot 10^{-7} \mathrm{~m}$

\begin{tabular}{|c|c|c|c|}
\hline$t$ & $\nu_{1}$ & $\| \frac{1}{T}$ & $v_{1}$ ber. \\
\hline 0 & 1.97 & & 197 \\
\hline 3 & 156 & $(0.040)$ & $1 \cdot 36$ \\
\hline 20 & $1 \cdot 02$ & 0.0195 & 1.04 \\
\hline 40 & 0.66 & 0.0183 & 0.64 \\
\hline$"$ & $0.76(2)$ & $(0.0153)$ & 4 \\
\hline 60 & 0.44 & 0.0187 & 0.44 \\
\hline 80 & $0.49(.2)$ & $(0.0126)$ & 0.31 \\
\hline
\end{tabular}

Vusuch $E: v_{0}=0.552,10^{10} ; 2=24 \cdot 2,10^{-7} \mathrm{~cm}$

\begin{tabular}{c|c|c|c}
\hline$t$ & $v_{1}$ & $\frac{1}{T}$ & $4_{1}$ ber \\
\hline 0 & 1.97 & & 1.97 \\
2 & 1.35 & $(0.105)$ & 1.65 \\
5 & 1.19 & $(0.058)$ & 1.31 \\
10 & 0.89 & 0.0490 & 093 \\
20 & 0.52 & 0.0475 & 0.54 \\
40 & 0.29 & 0.0403 & 0.25 \\
& 17 & 0.04 &
\end{tabular}

rettel: $4^{\frac{1}{2}}=0.0456 ; \frac{R}{2}=3.12$

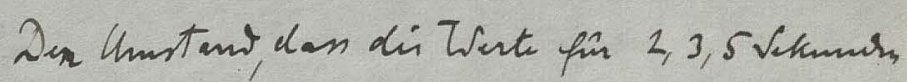
so bedentenit ans der Rethe heram fallen, firunte man daduech an extelarm versucht sim, dan wis in meserer Thoxi sing geverse Noterning

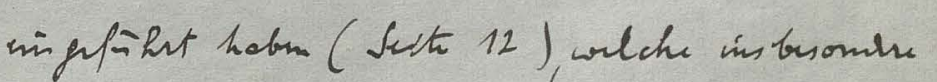
das errte Anfangs stodem be einflust. Doch

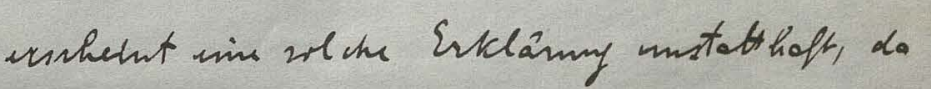
ine Toutsolle nut IEClf der a.c. O. angefishotem Oedingung Whit, dass des Eller hiser gans

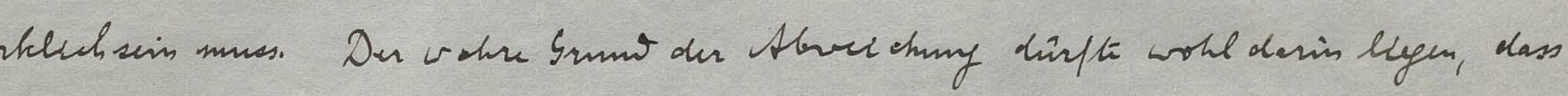

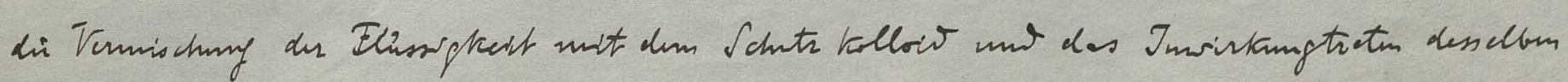

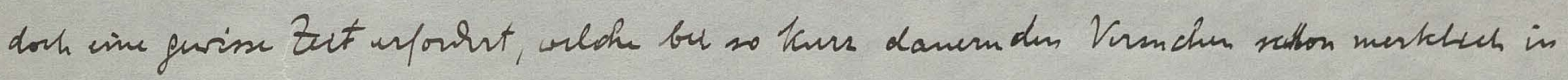

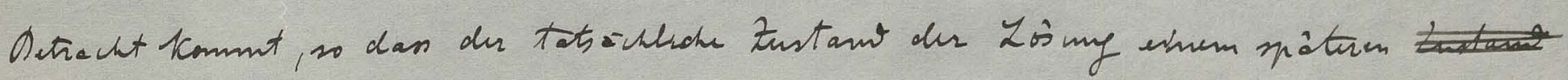
roment entsprach els die angegeberm Zitpuntet.

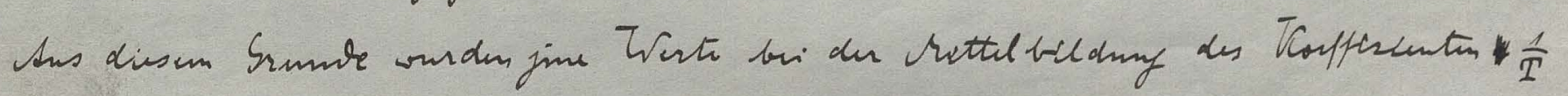

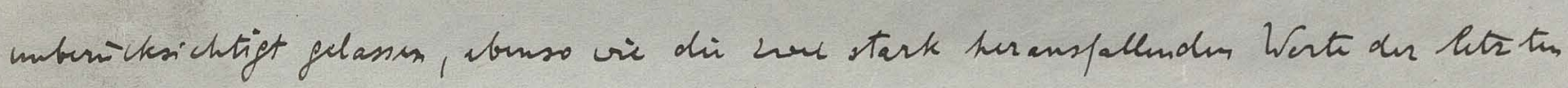

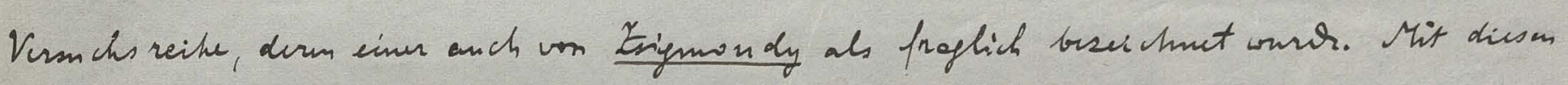

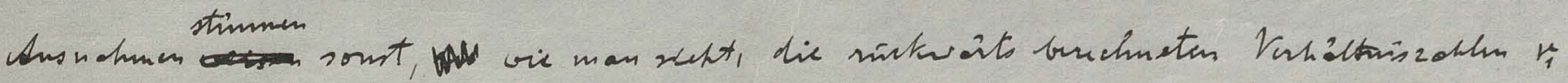

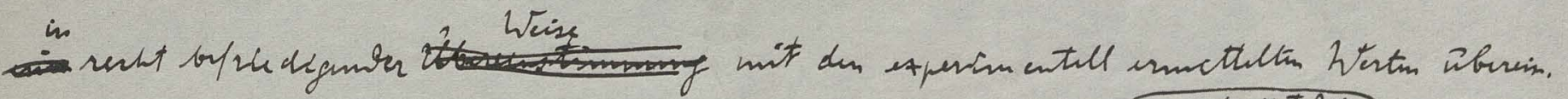

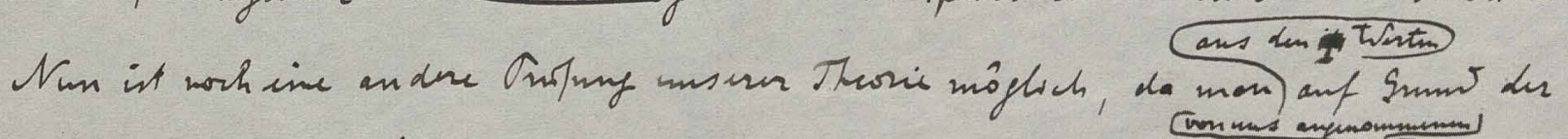

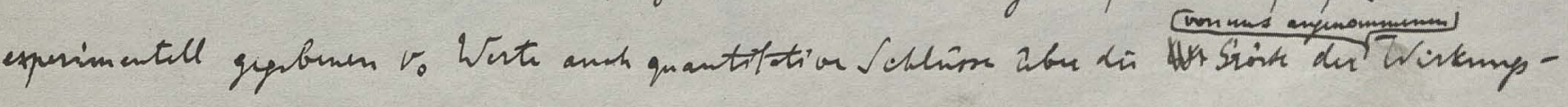

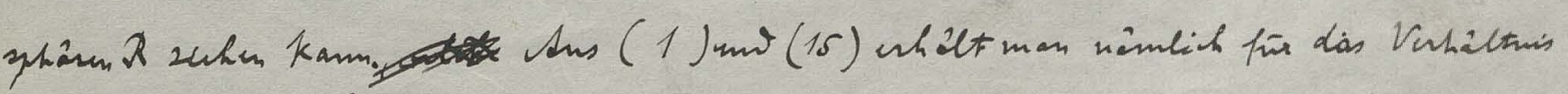
des Wivitamprodinstemn Feshuncedurs $n$ :

$$
\frac{R}{2}=\frac{N}{I 2} \frac{3 \mu}{2 v_{0}} \frac{1}{T}
$$



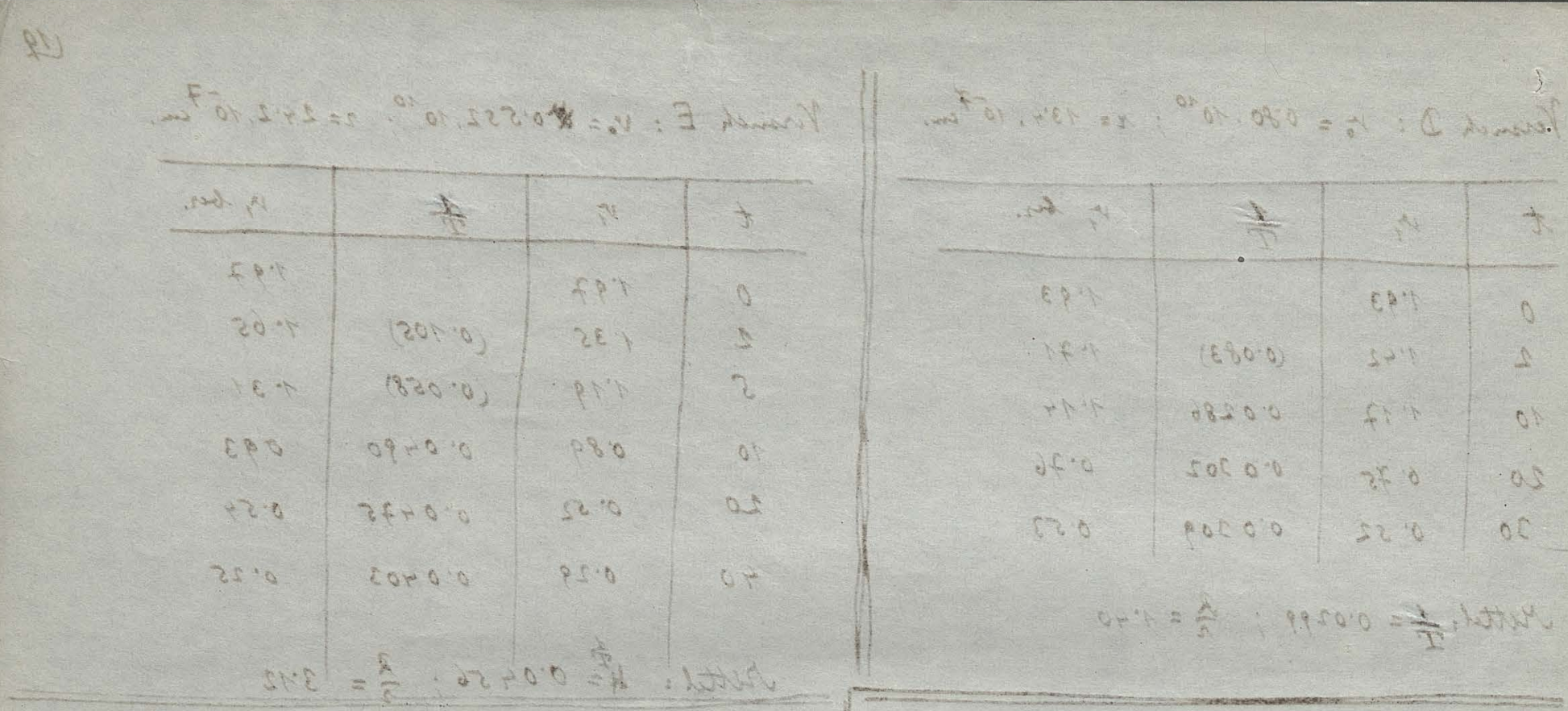

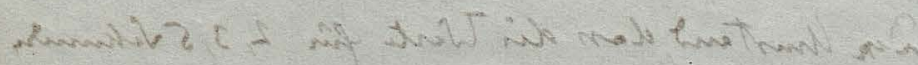

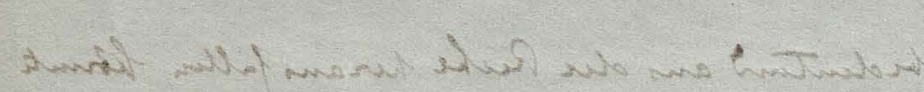

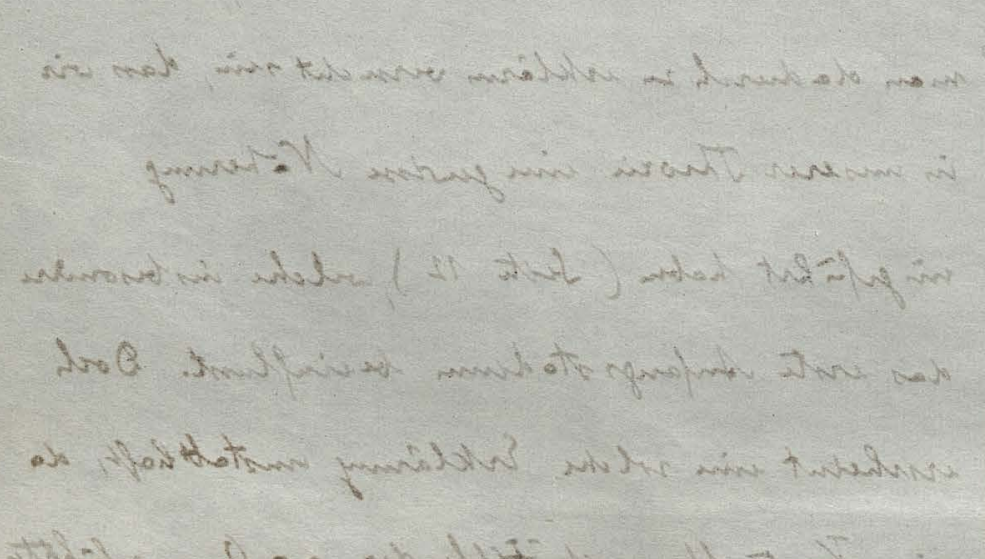

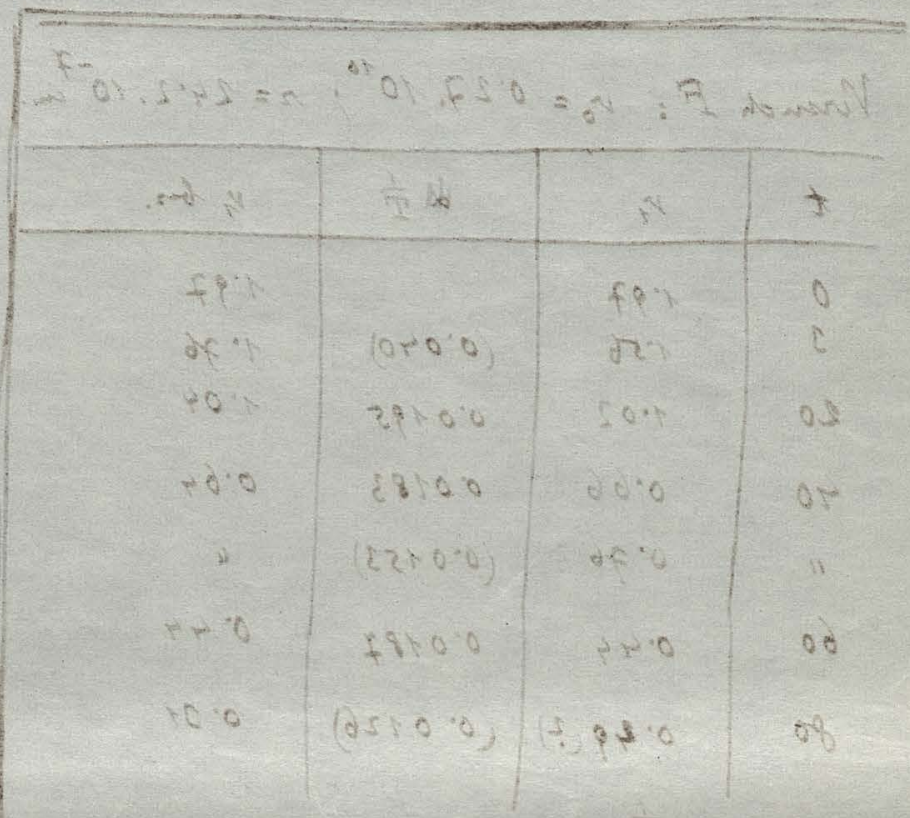

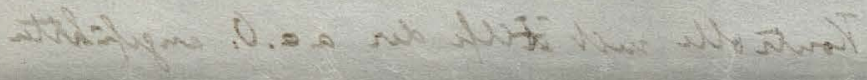

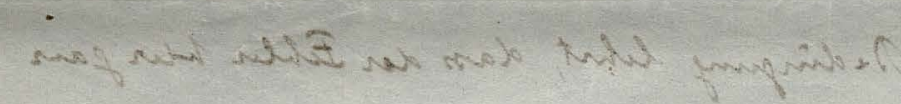

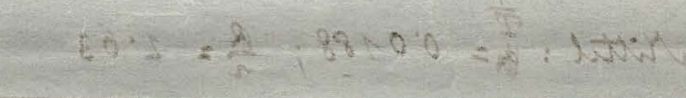

exalo

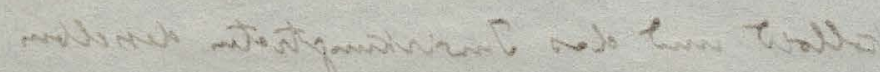

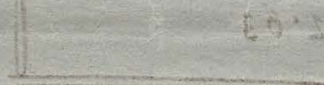

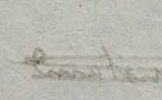

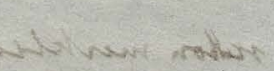

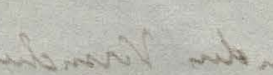

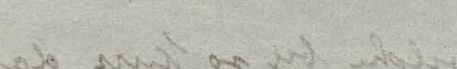

Awationits at

ins.

Whits? 


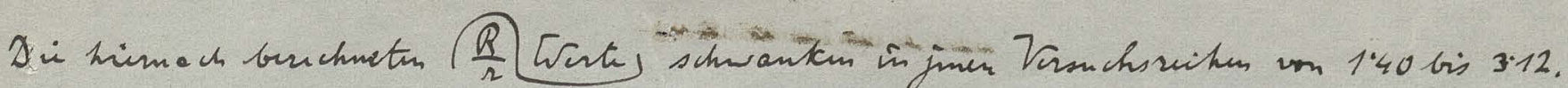

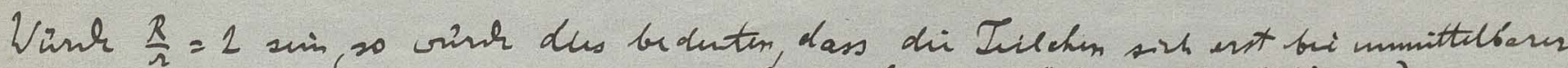

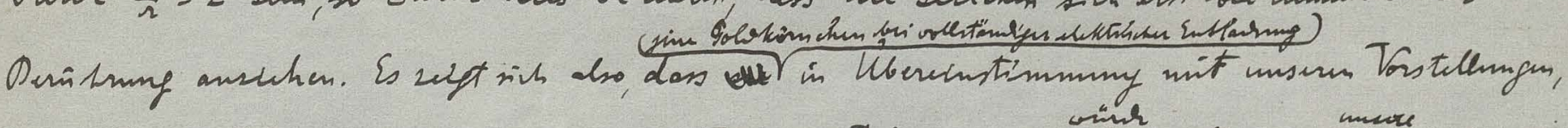

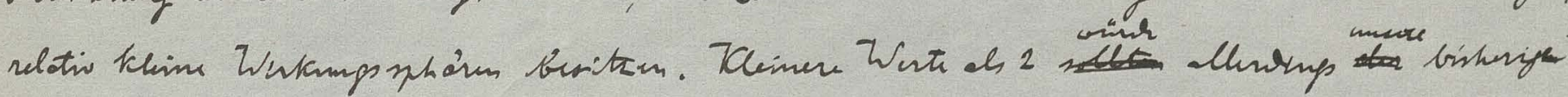

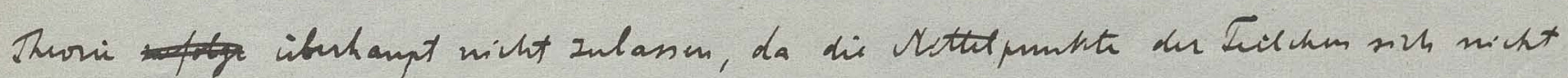

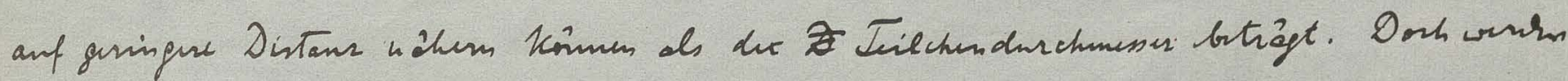

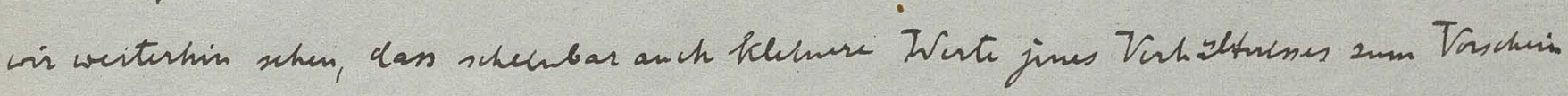

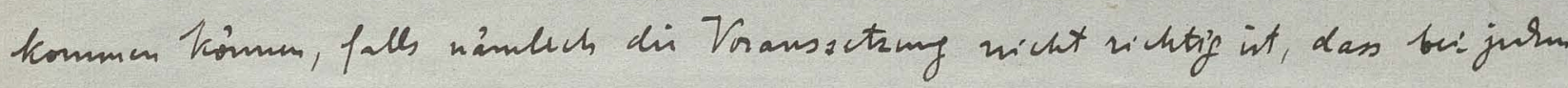

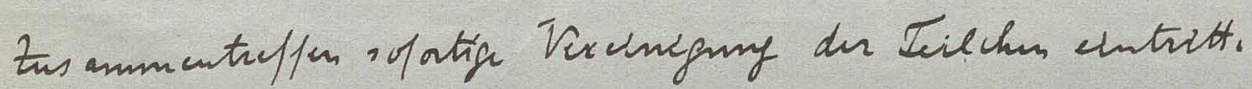

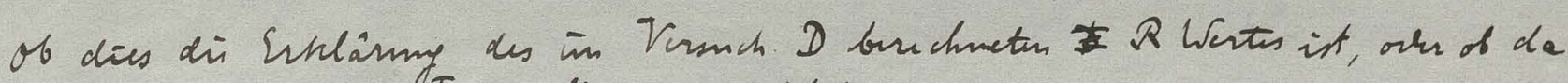
Enlugudlem mitgenult haben

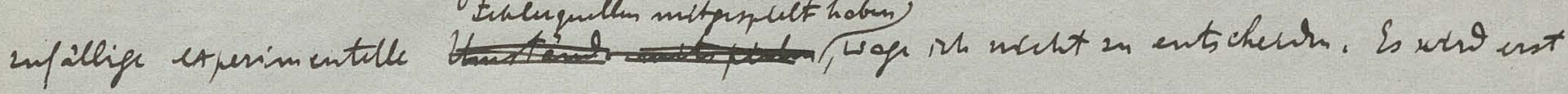

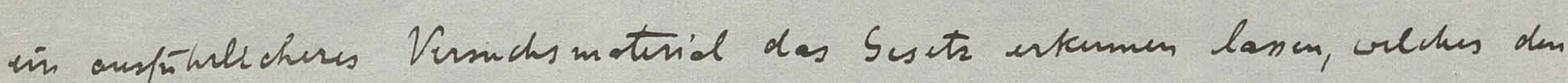

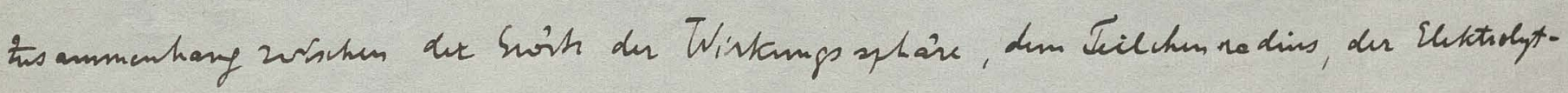

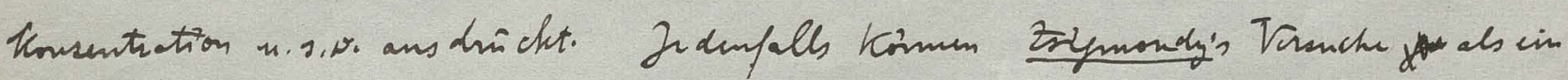

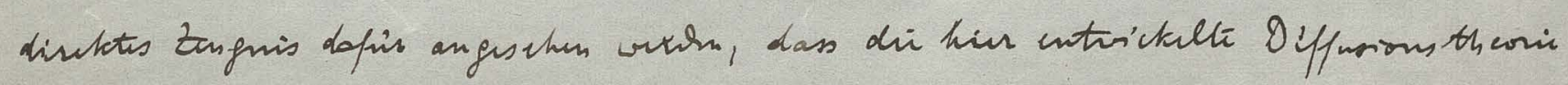

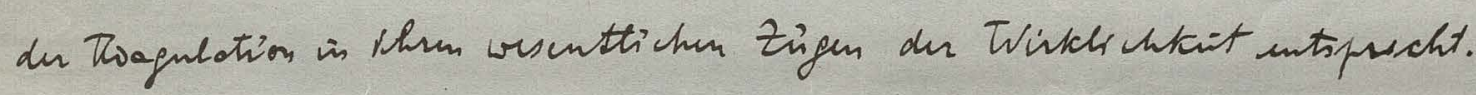

\section{T. Theosic der langsanen thogenlation.}

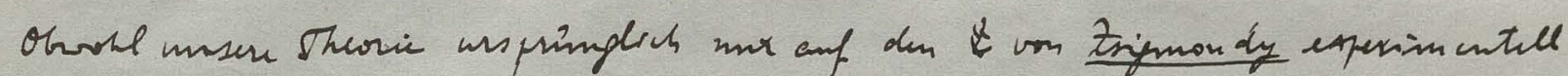

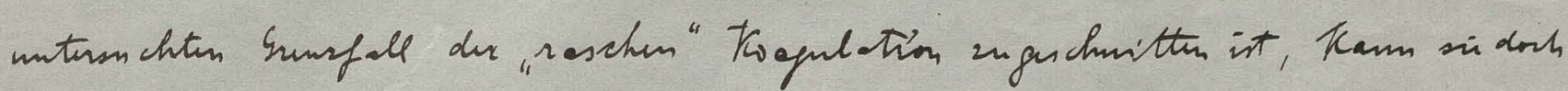

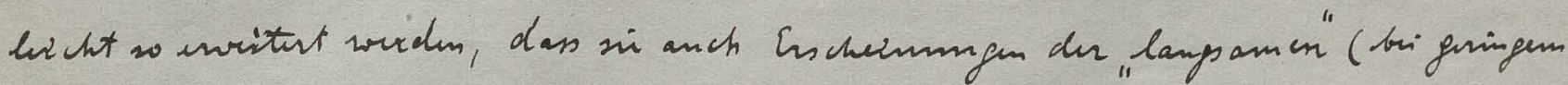

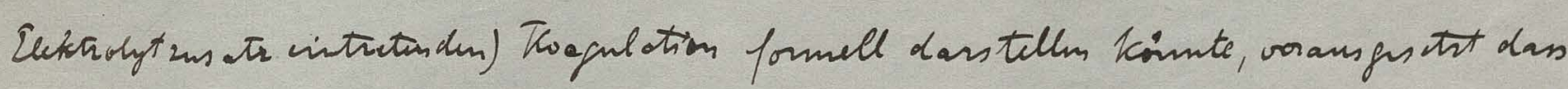

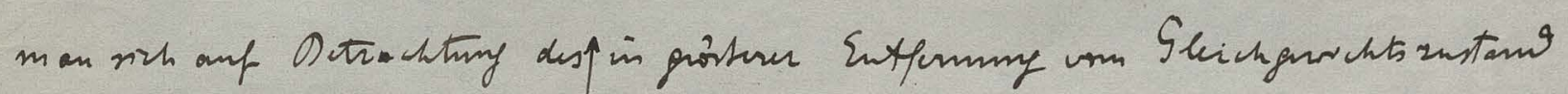

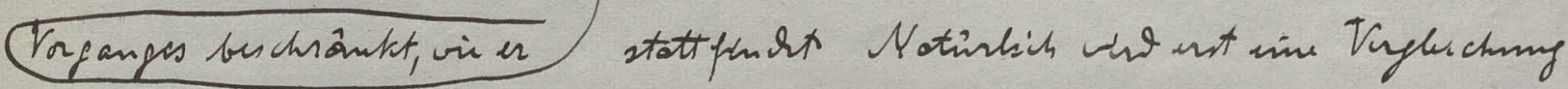

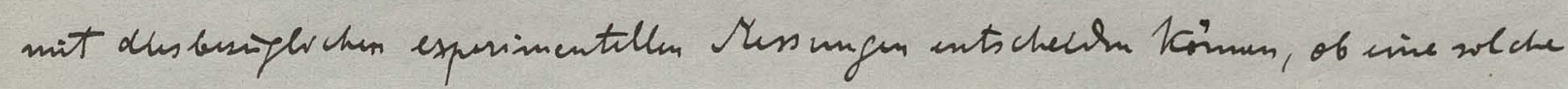

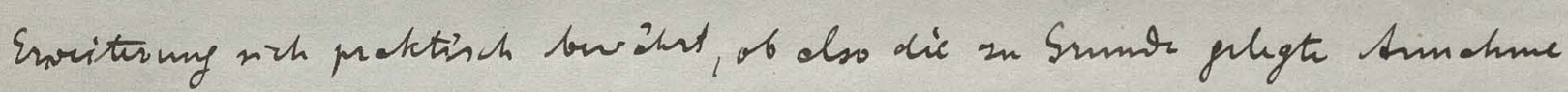

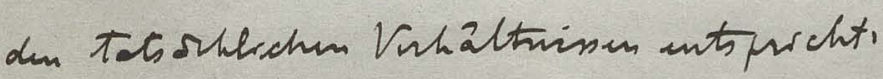

Diese infoche Enocterny unseres Thoui buttht dasin, dass wir uns wos tellen,

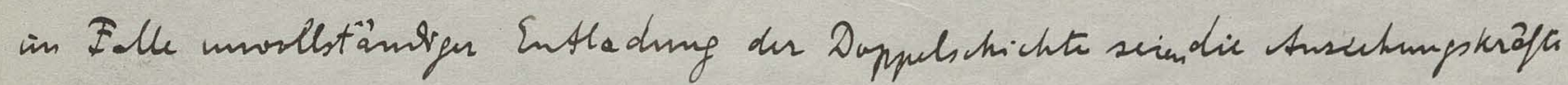

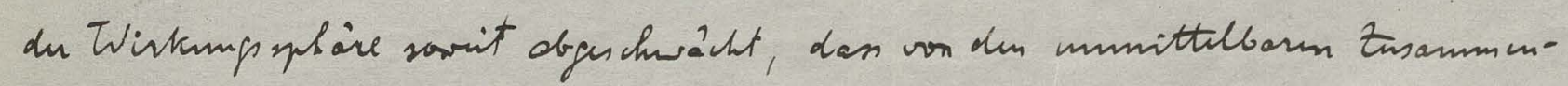

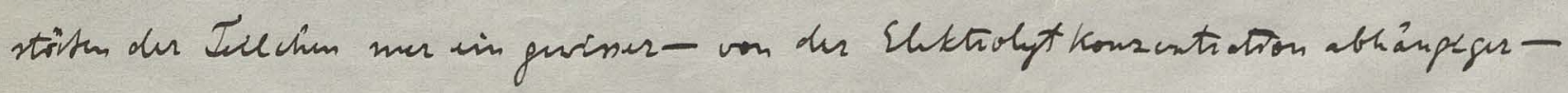

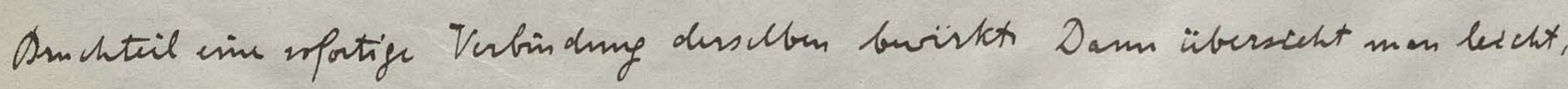
dan Mr Formch (24) anch fin di langsame Troogulation giltig blectur, folls 
Q

$-m^{2}+2+x ;$

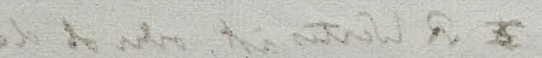

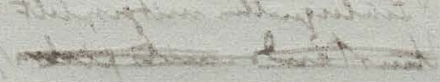

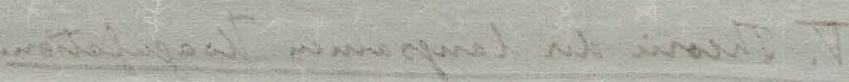

14.

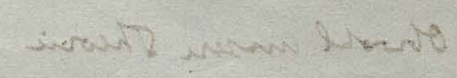

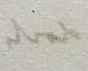


main dasin imfoch $\alpha=4 \Omega R D$ crsitst durch

$\alpha=\sin \Omega D_{\varepsilon}=\frac{4}{3} \frac{T E}{N} \frac{\varepsilon}{\mu}$

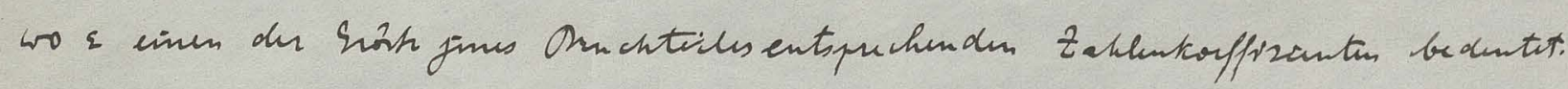

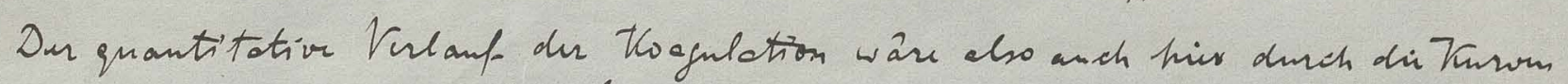
der Eig (1) butinnt; sir sairenalso fir elle Tousuntrationen des Twollois uno des Shktiolyts Affin, d. h. zi listmmindedurch

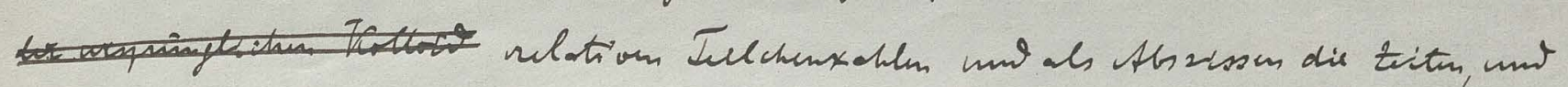

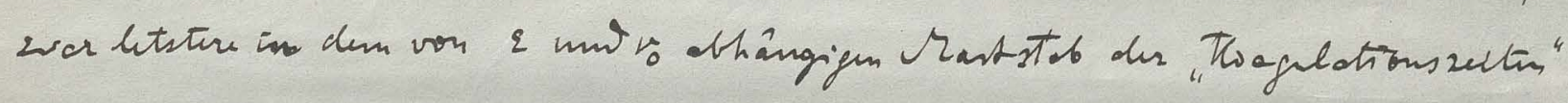

$I=\frac{3}{4} \frac{N}{H} \mu \frac{1}{\varepsilon \nu_{0}}$

amptrat.

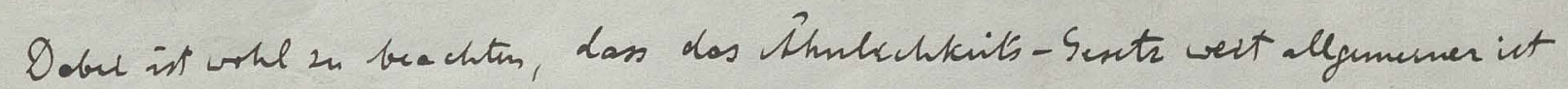

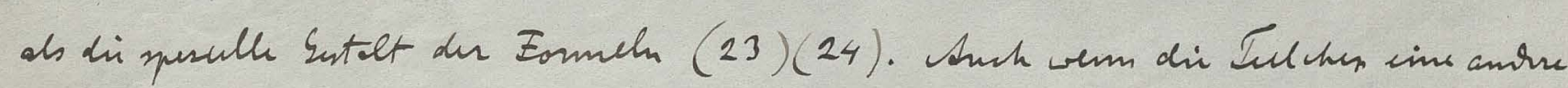

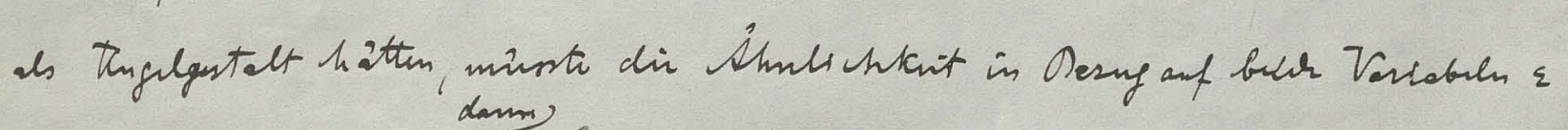

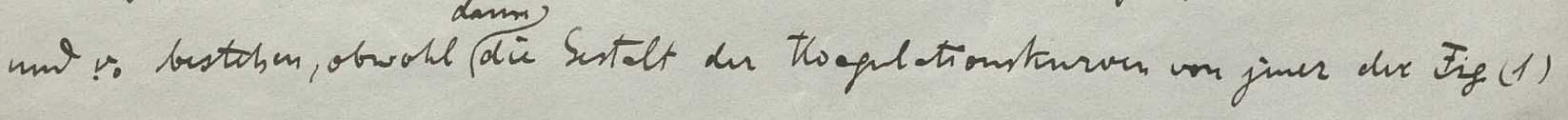

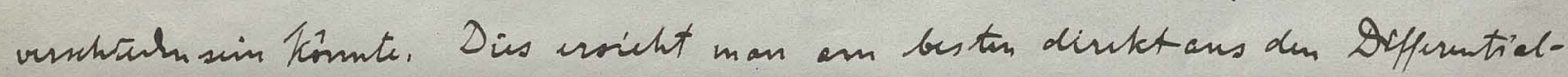

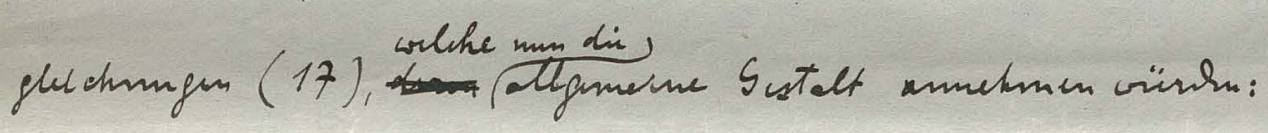

$$
\frac{d v_{k}}{d t}=\varepsilon \sum_{1} A_{1 h} v_{i} v_{k}-\varepsilon \sum_{2}^{1} B_{i k} v_{i} v_{k}
$$

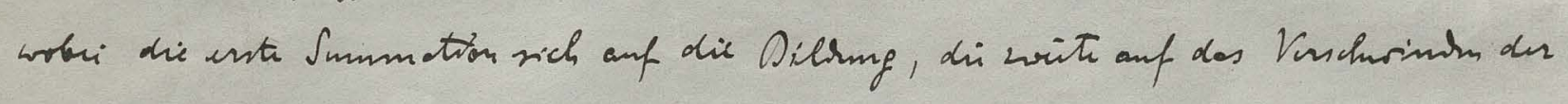

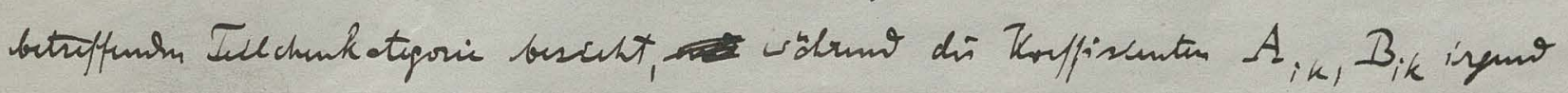

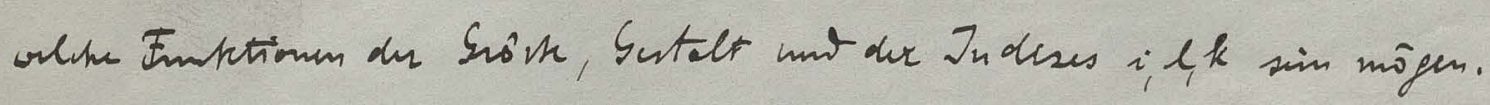

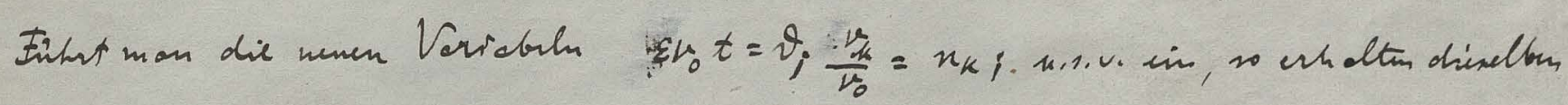
di sutalt:

$$
\frac{d x_{l}}{d v}=\sum_{1}^{1} A_{i k} x_{i} x_{k}-\sum_{2}^{1} B_{i k} x_{i} x_{k}
$$

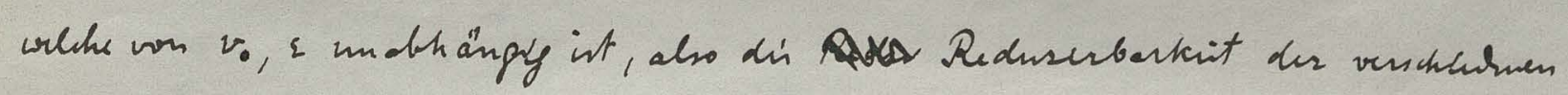

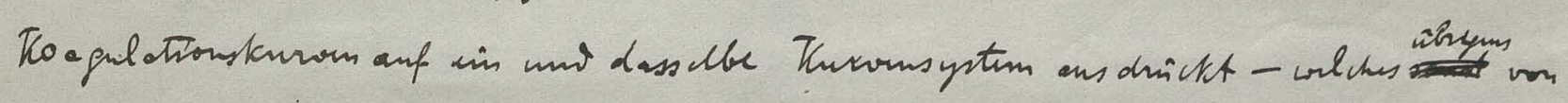
(24) musklich abouction tïnuts.

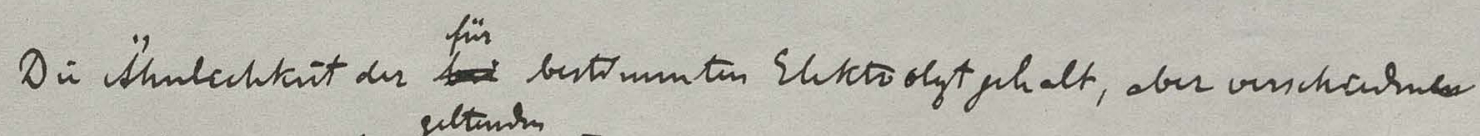

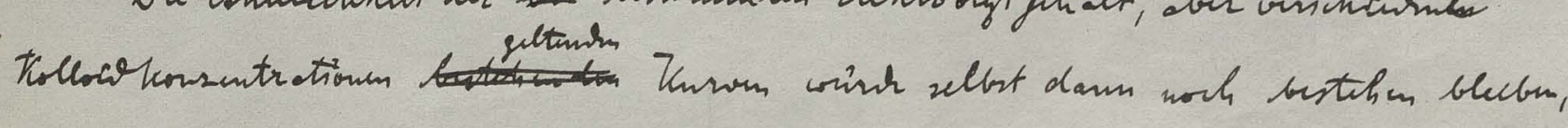

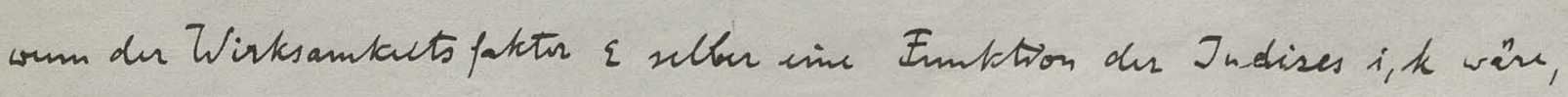

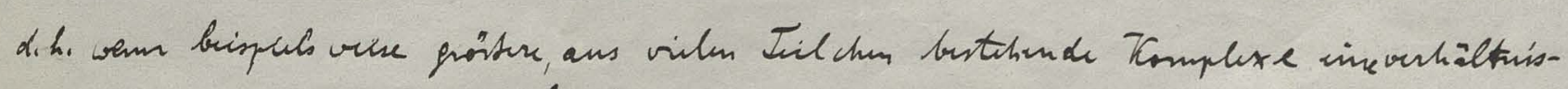

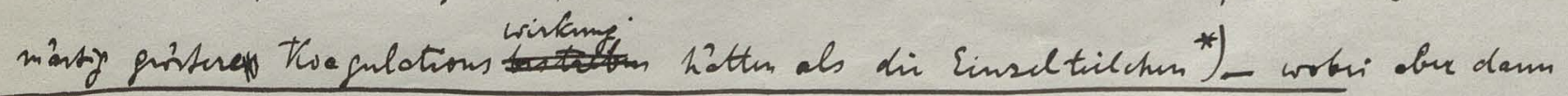

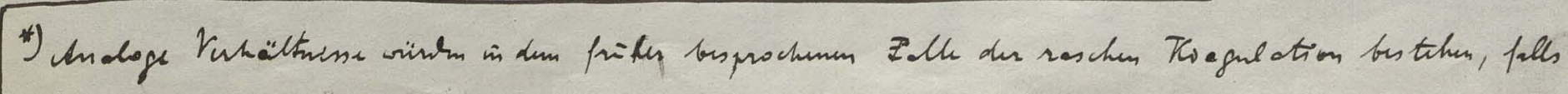

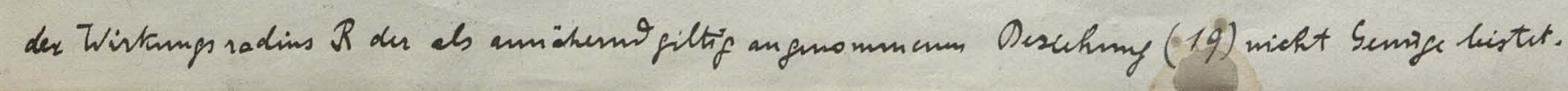





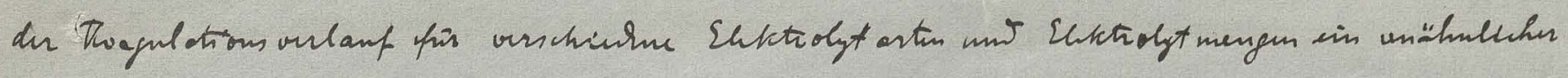

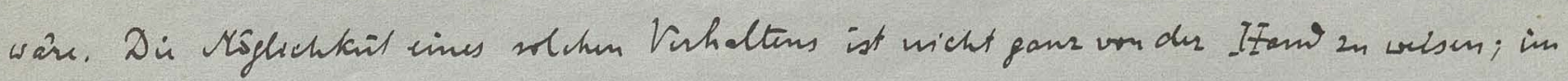

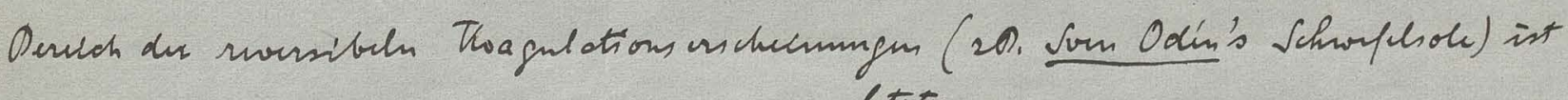

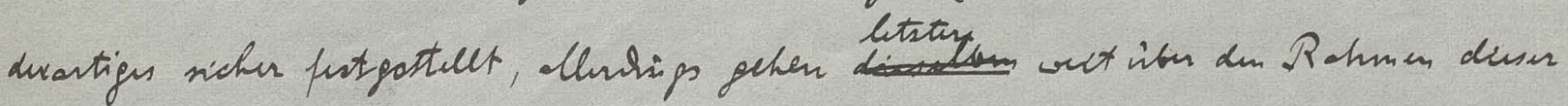

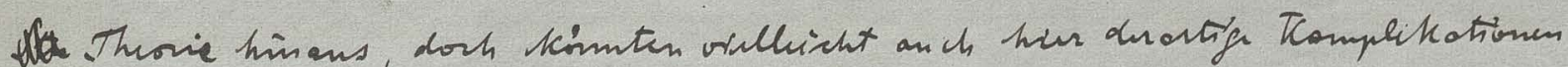

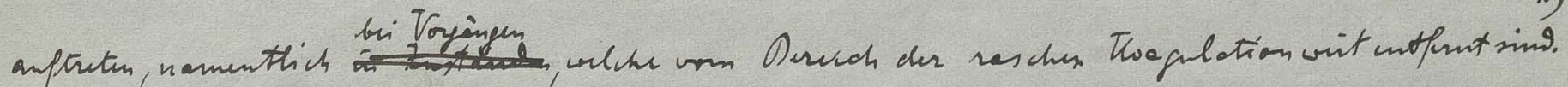

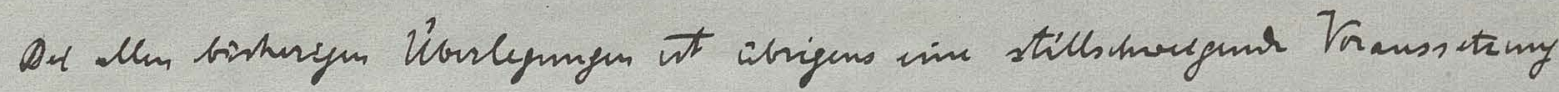

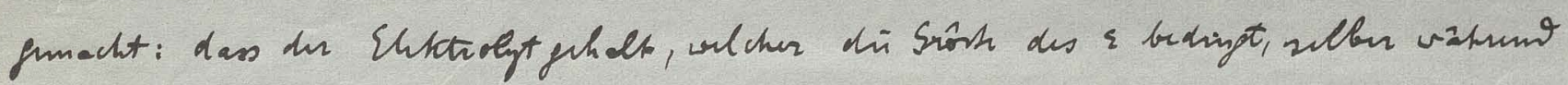

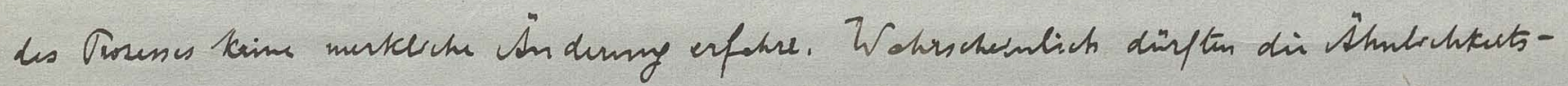

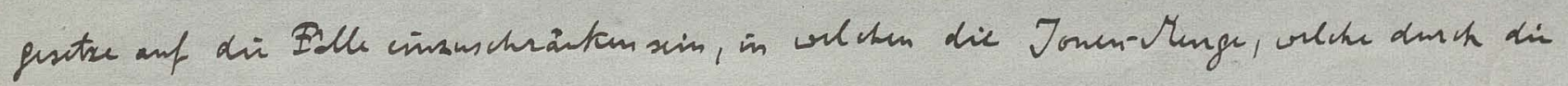

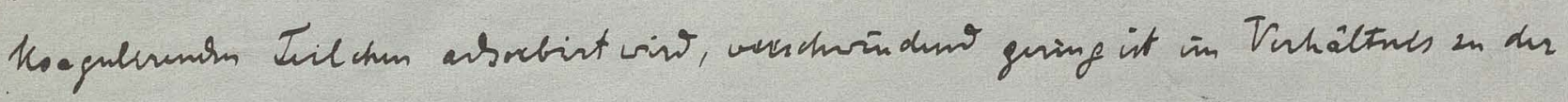

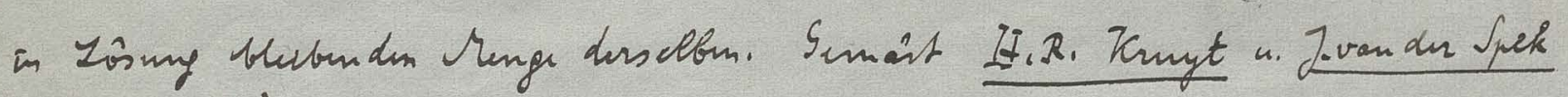

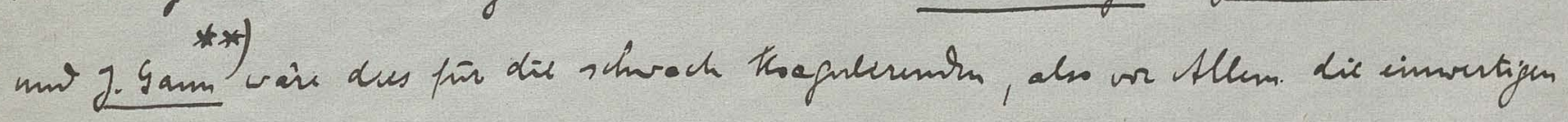

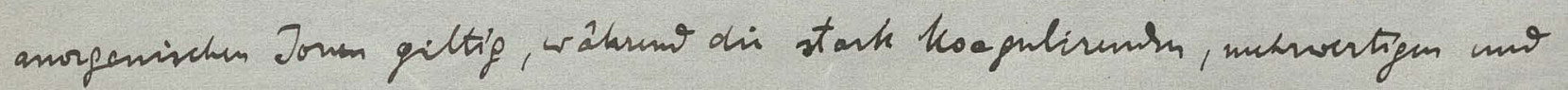

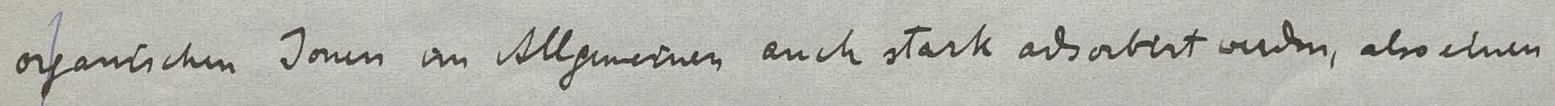

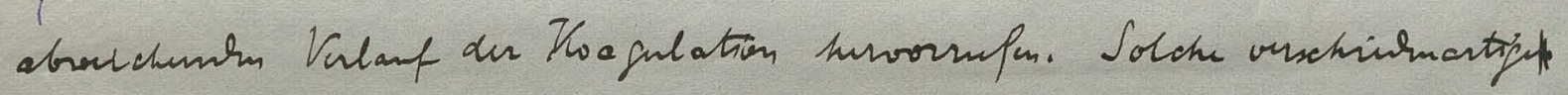

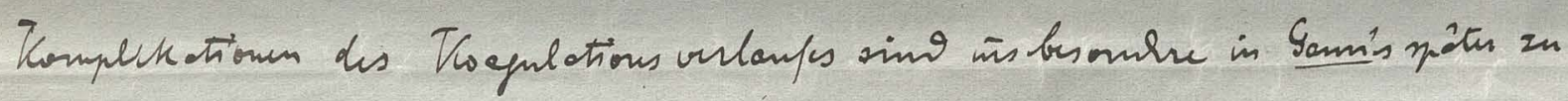
beprechuder subert 2n Toge getrictur.

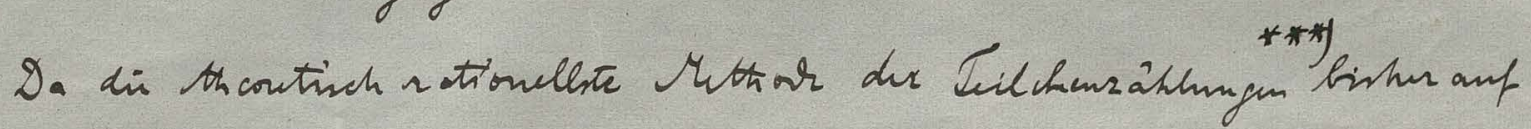

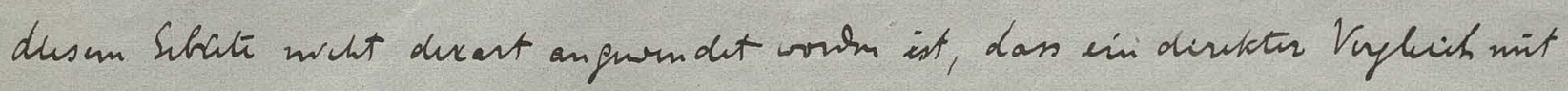

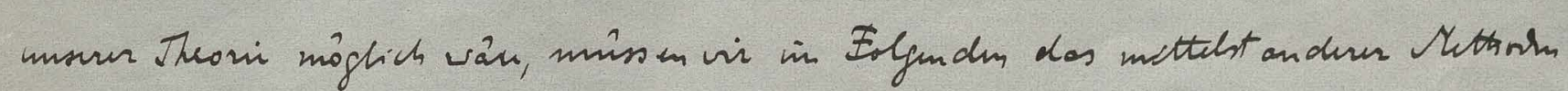

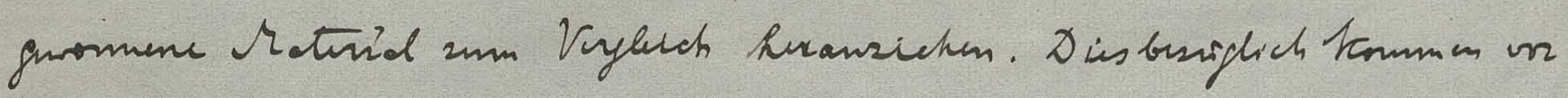
Mlem wori Methosh in Oitracht.

\section{Vughech mit IF. Taine's Messmagn.}

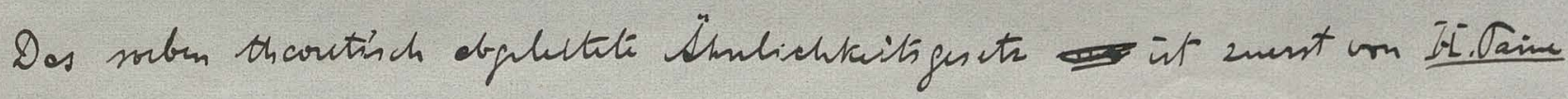

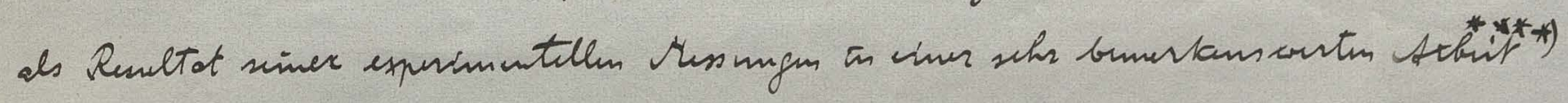

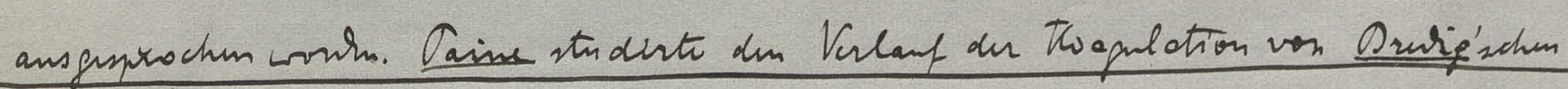

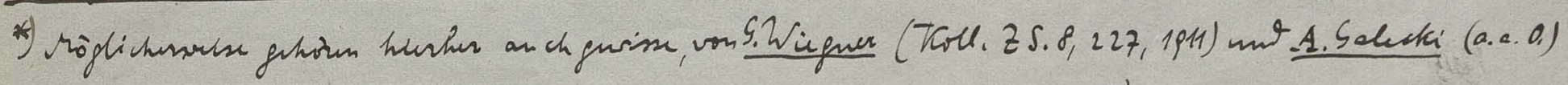

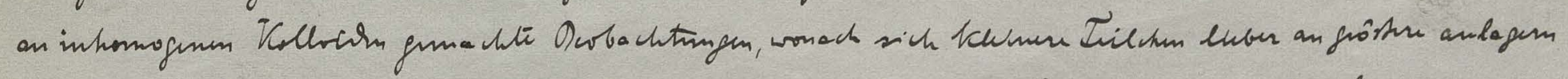

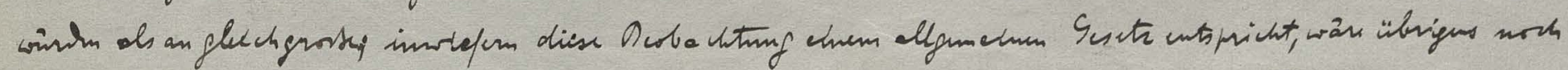

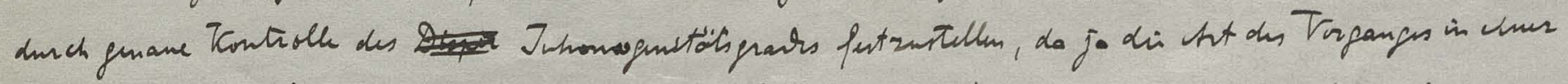

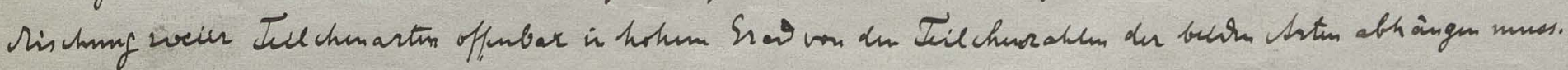

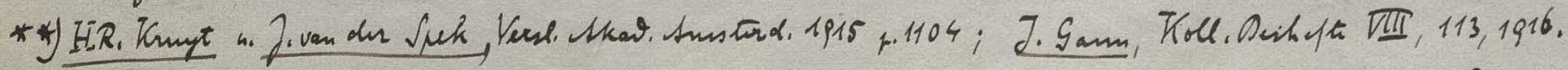

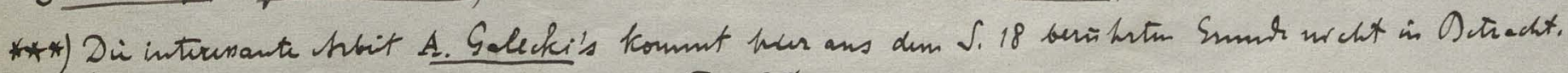

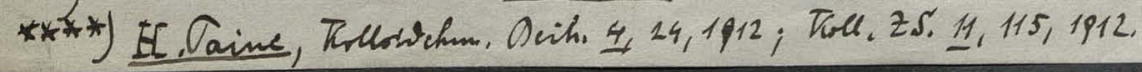




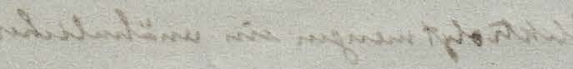
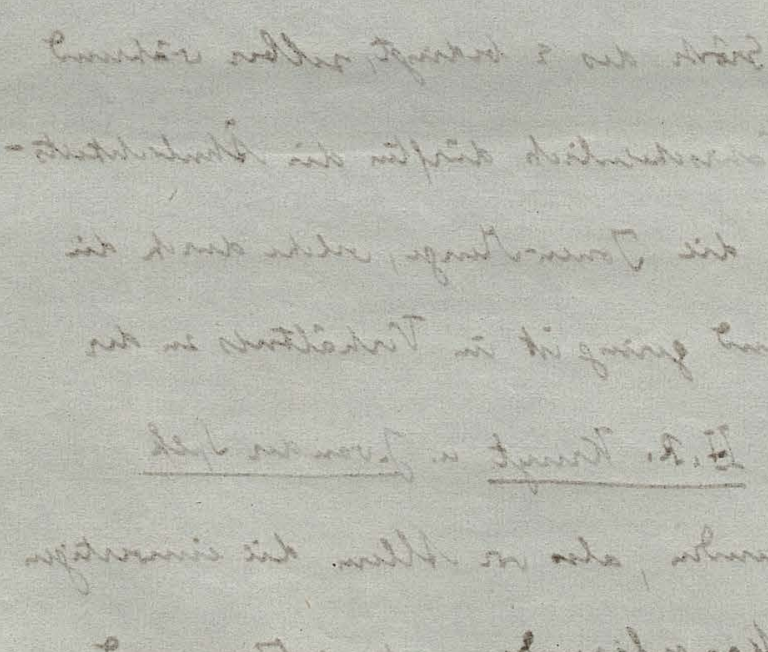

ande

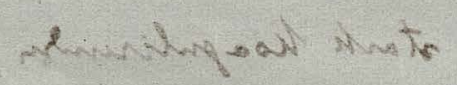

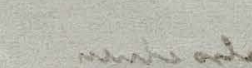

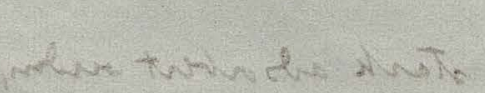

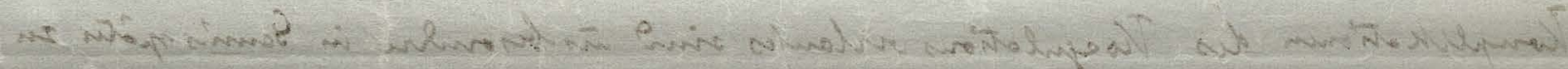
$t \rightarrow *$

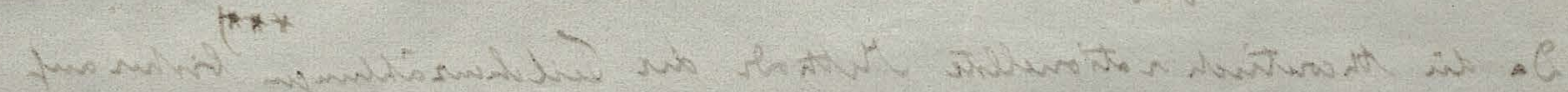

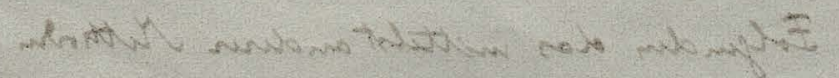

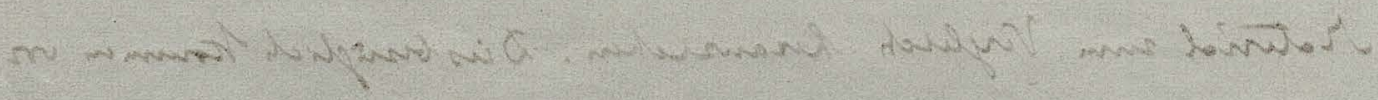

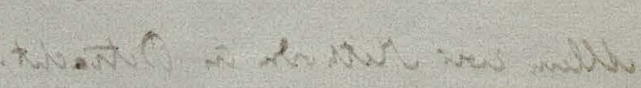

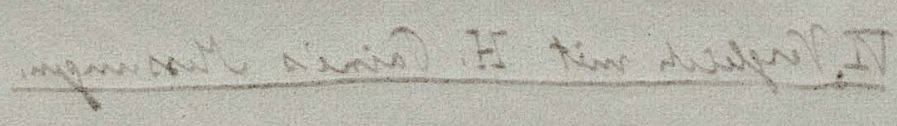

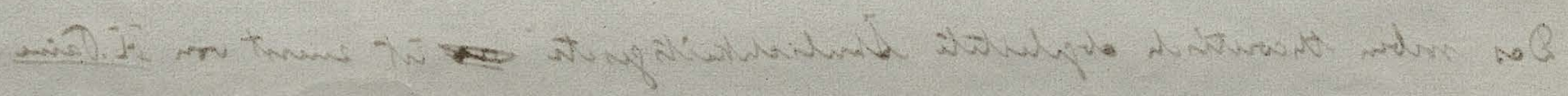

$$
\begin{aligned}
& +* x+
\end{aligned}
$$

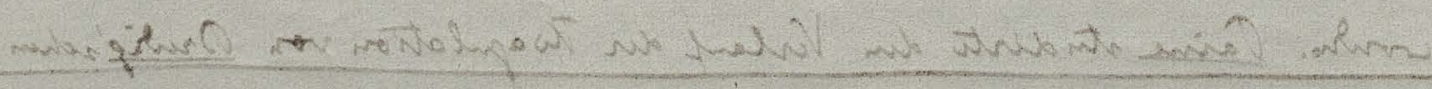

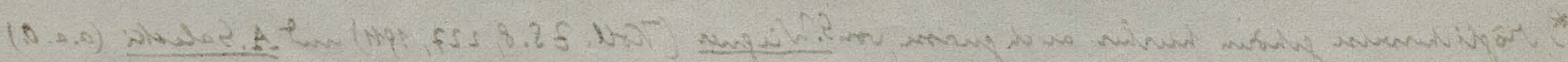

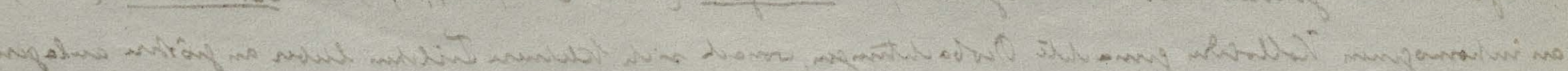

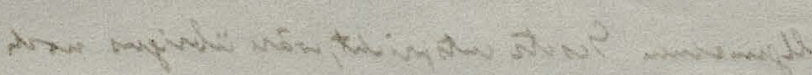

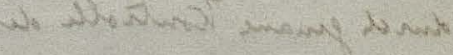

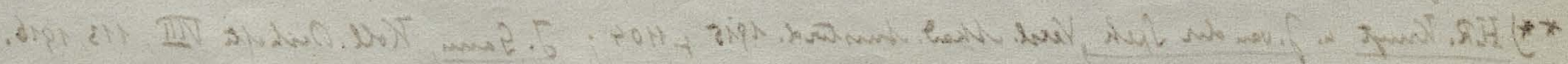

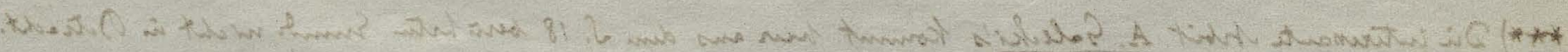




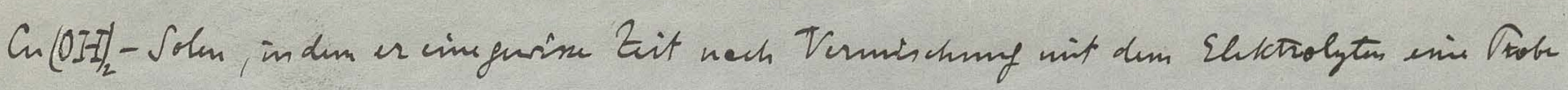

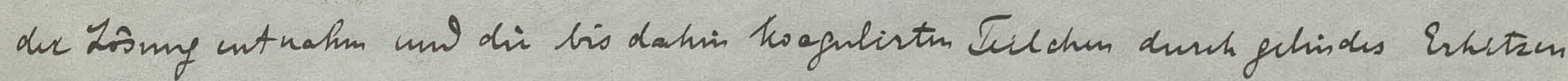

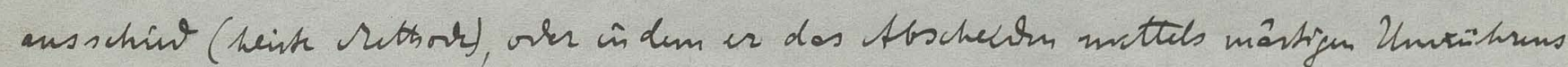

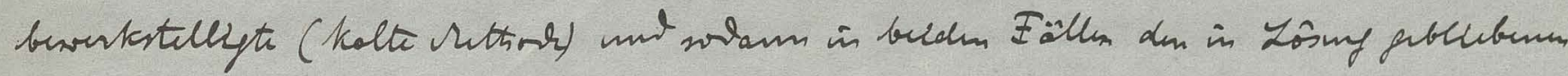
Thperghalt andytasch butimunte.

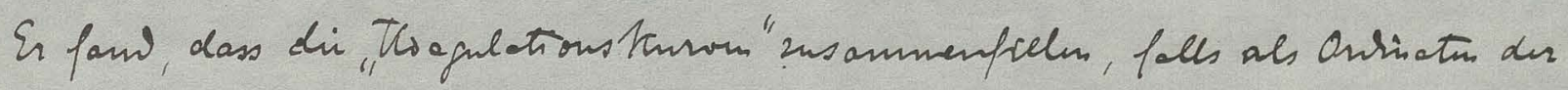

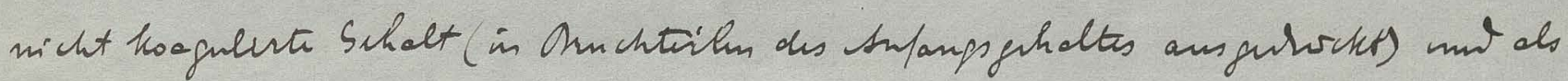

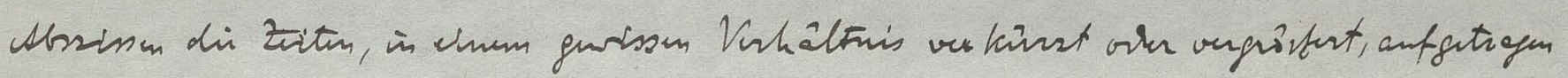

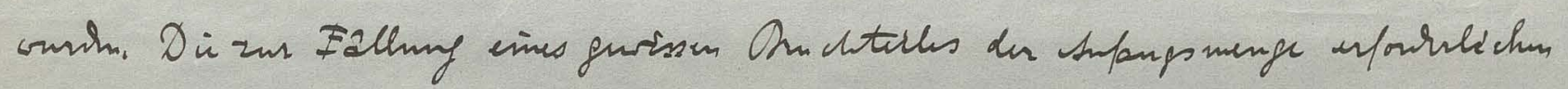

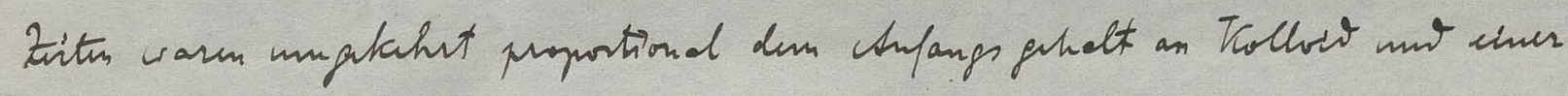

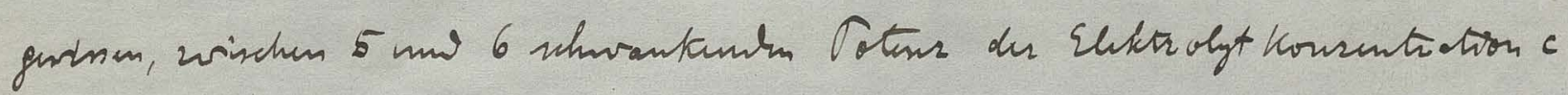
in der toing.

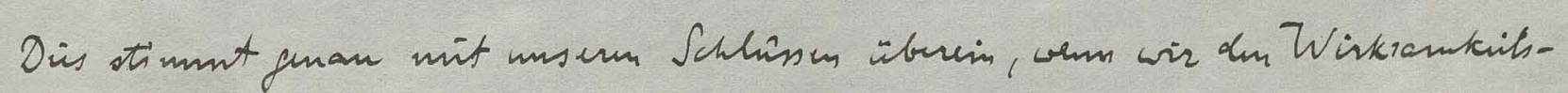

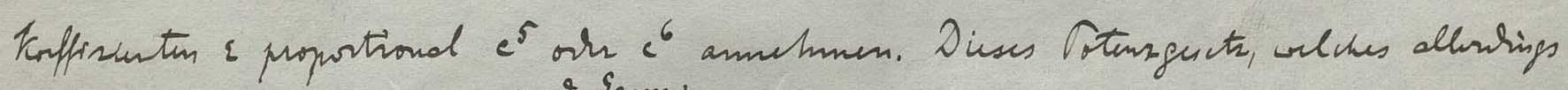

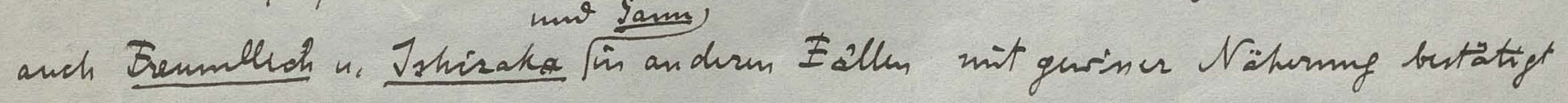

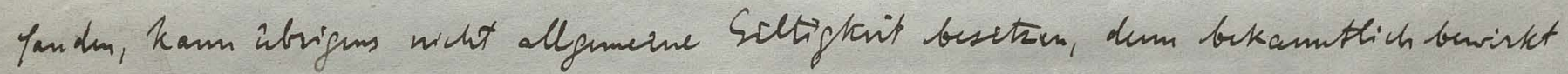

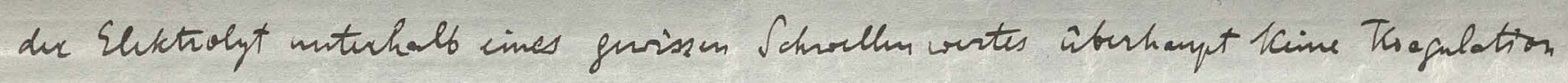

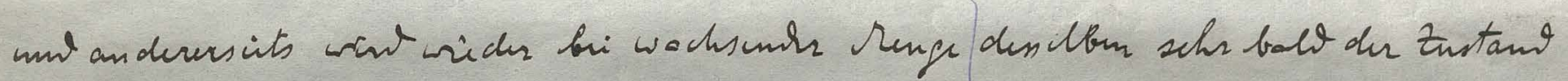

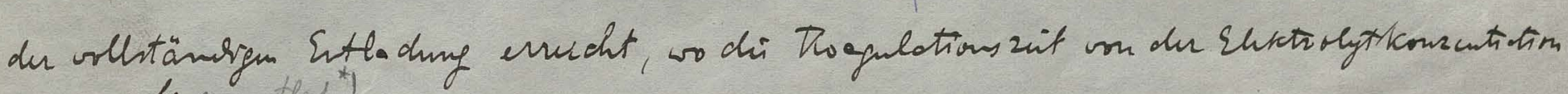

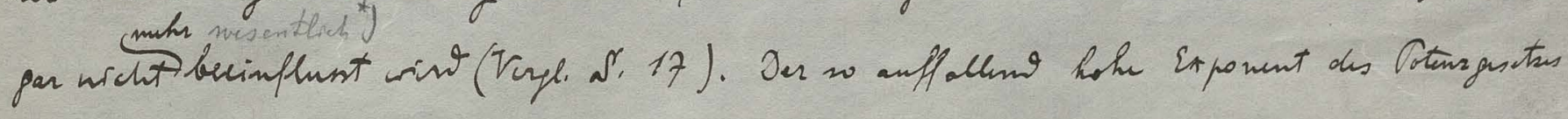

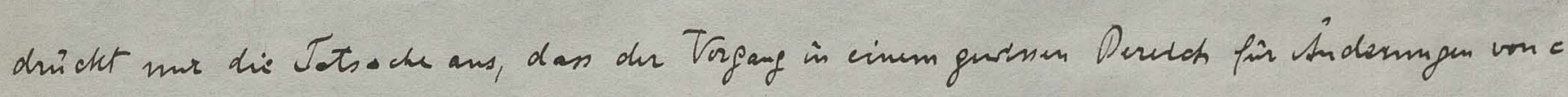
so ansterst unfimblech int.

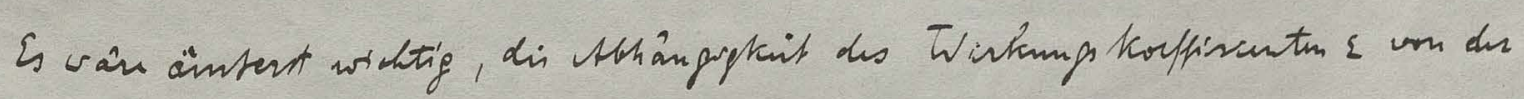

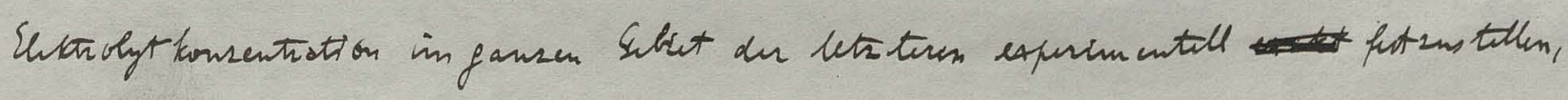

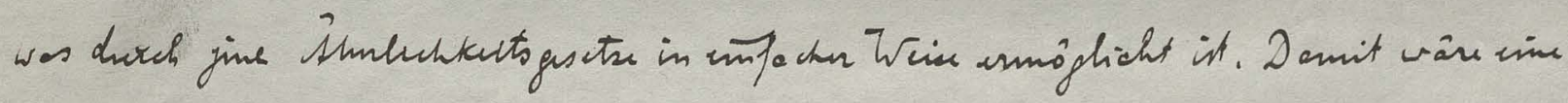

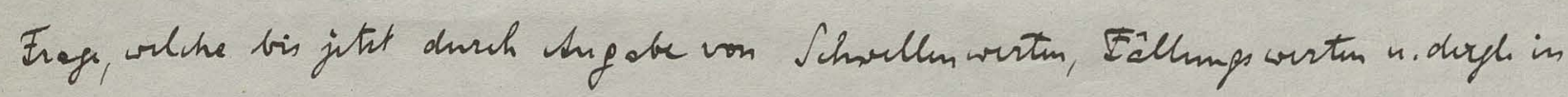
recht wher Triss be antwortet wint, exakt gelost.

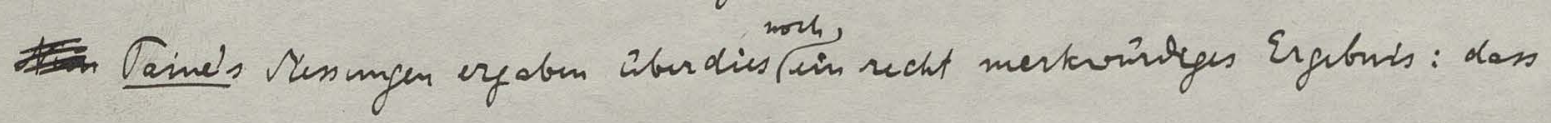
Harmi eimititing Uambhng witake las

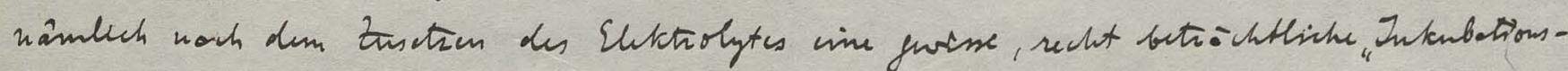

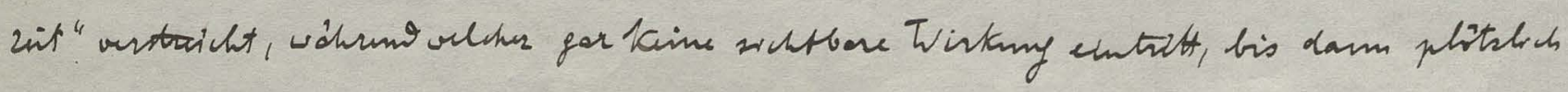

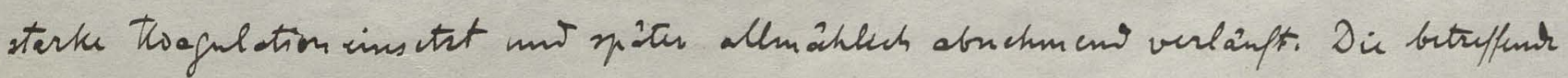

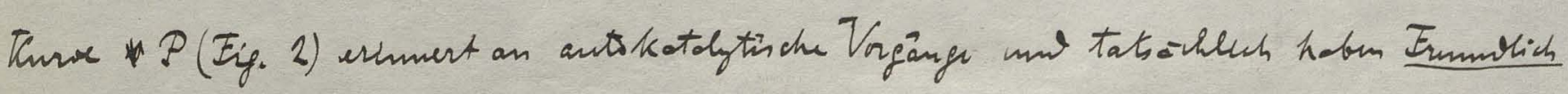

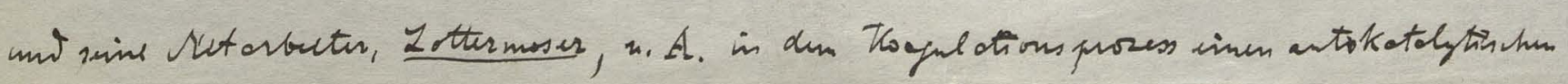


wasting

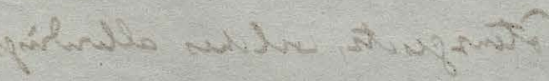

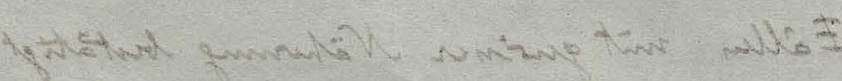

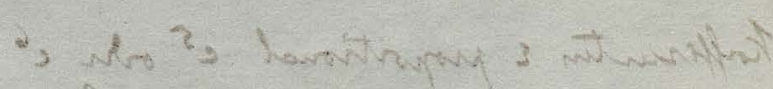

\section{anses? entorat} a. क्ष

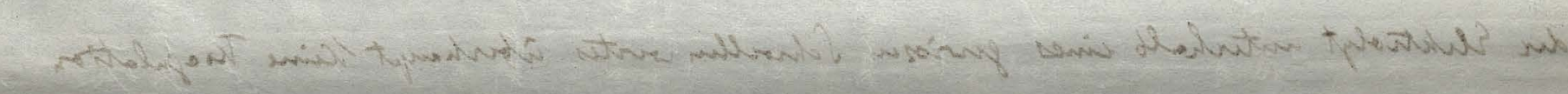

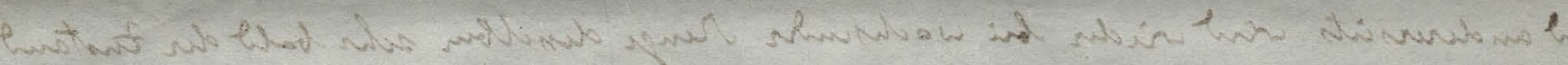

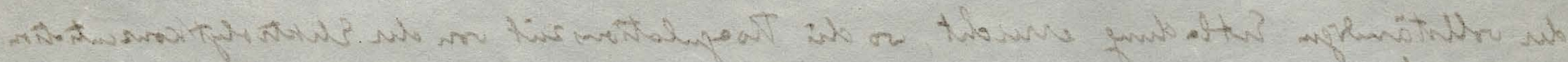

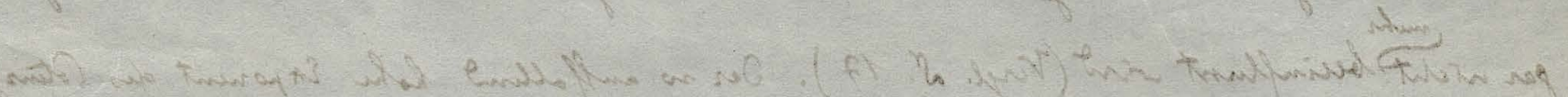

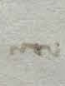

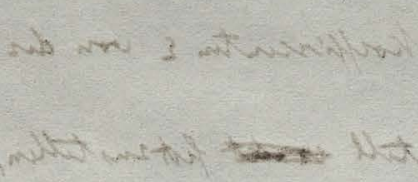




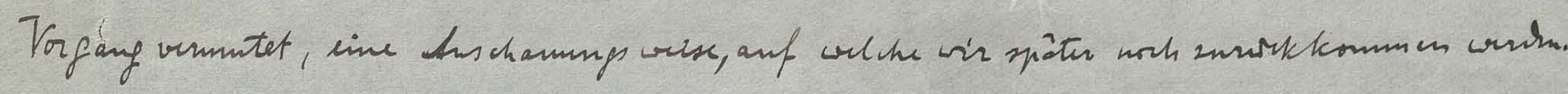

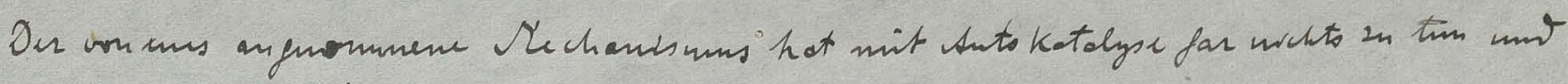

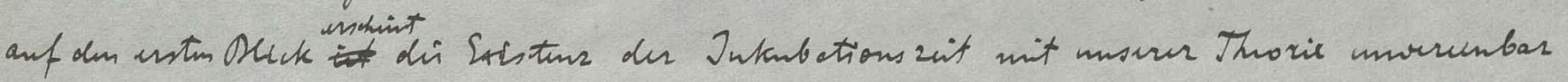

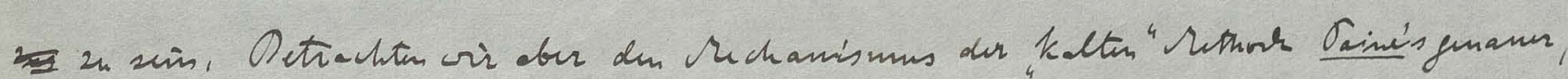

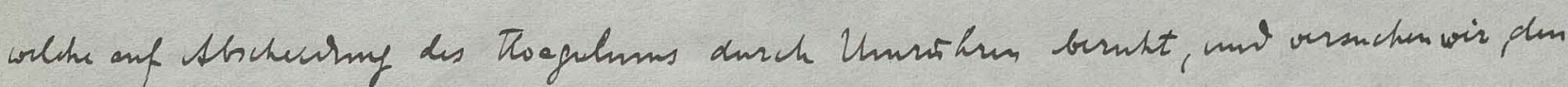

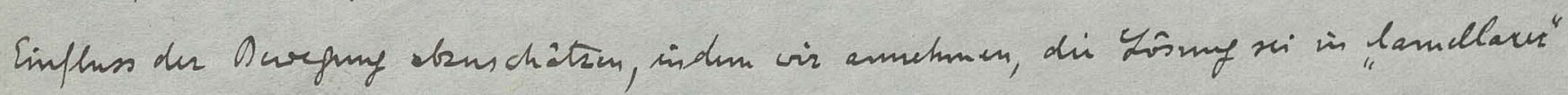

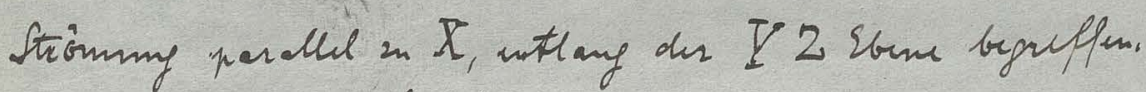

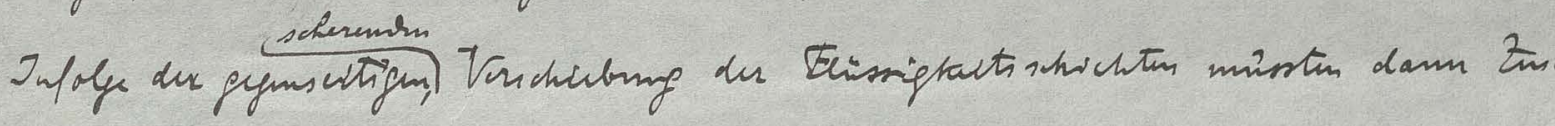

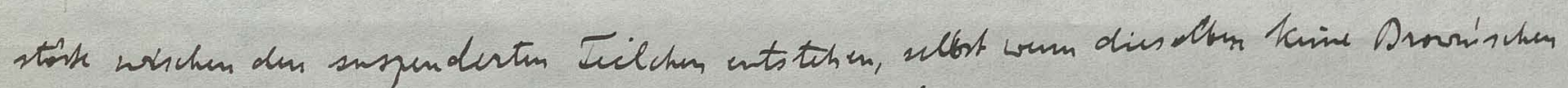

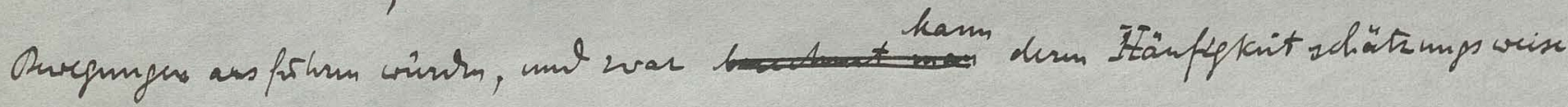

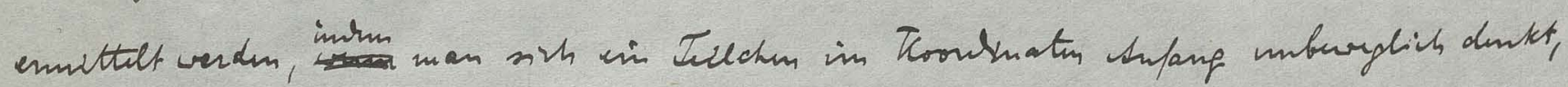

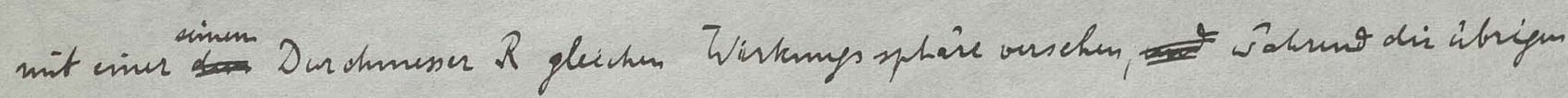

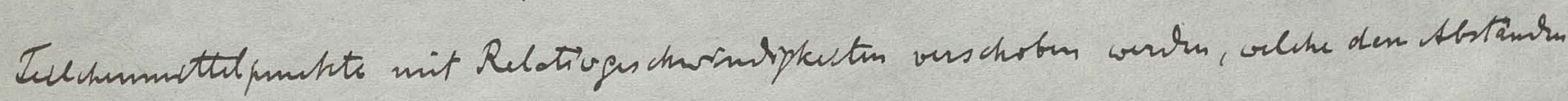

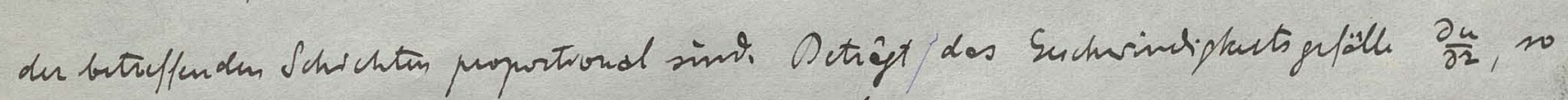

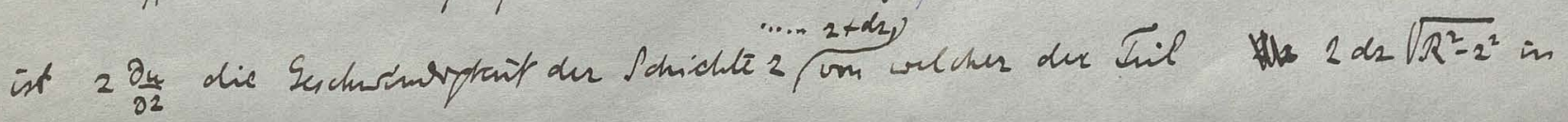

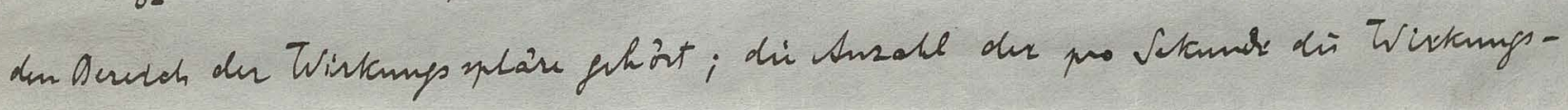
Yhâre durchstostenden Tulchen vine dro gegebm dusch:

$$
x=4 \frac{\partial u}{\partial z} \mu \int_{0}^{R} 2 \sqrt{R^{2}-z^{2}} d x=4 \frac{\partial u}{\partial x} R^{3} \int_{0}^{\frac{R}{2}} \cos ^{2} y \sin \varphi d y=\frac{4}{3} R^{3} \nu \frac{\partial u}{\partial x}
$$

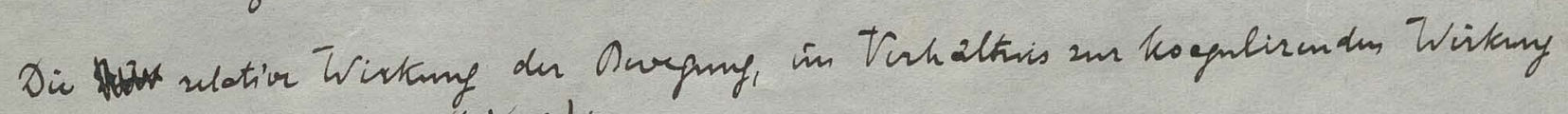

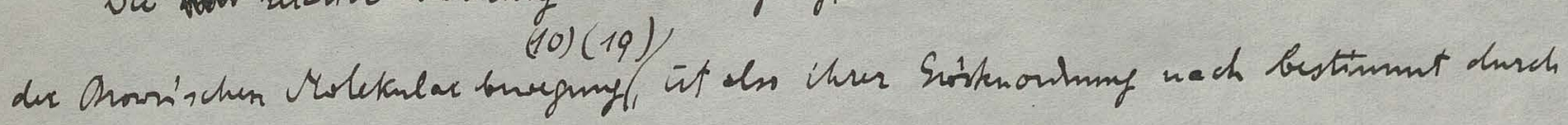
dun Sus druck:

$$
\beta=\frac{1}{6 \lambda} \frac{\partial u}{\partial z} \frac{R^{2}}{D N}=\frac{4 r^{2} \mu}{\frac{H u}{\partial 2}}
$$

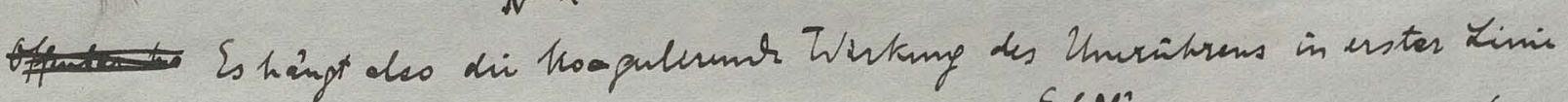

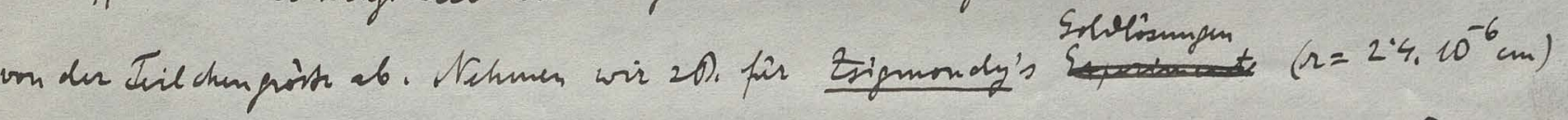

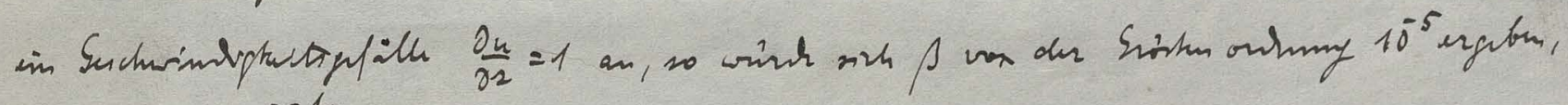

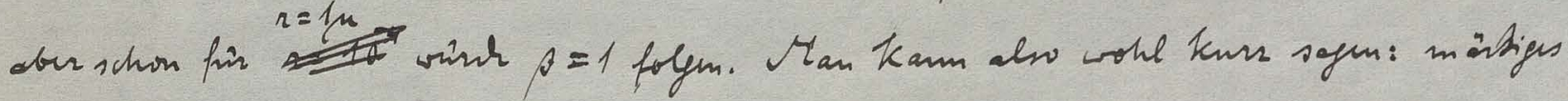

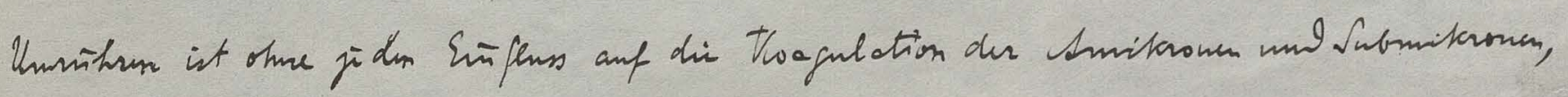

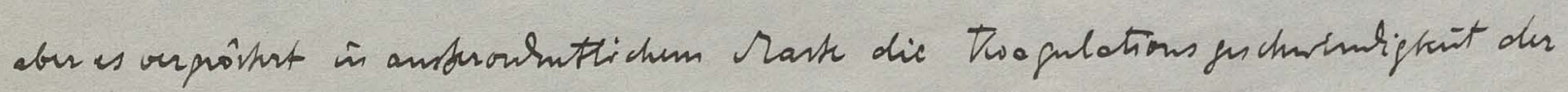

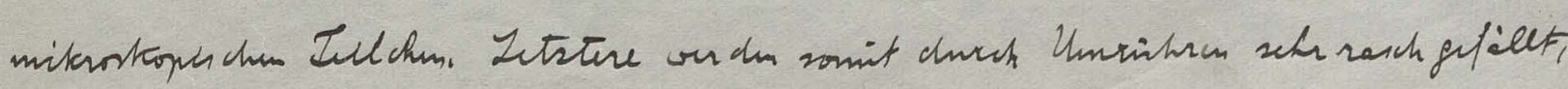

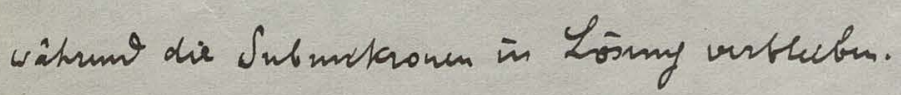

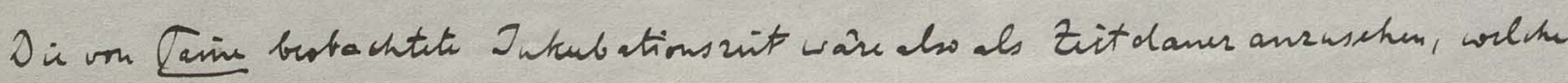

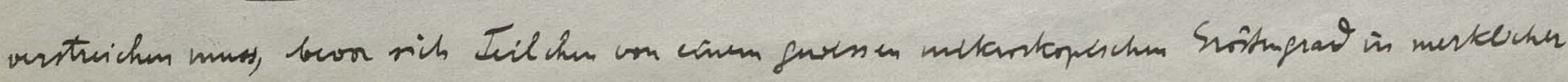




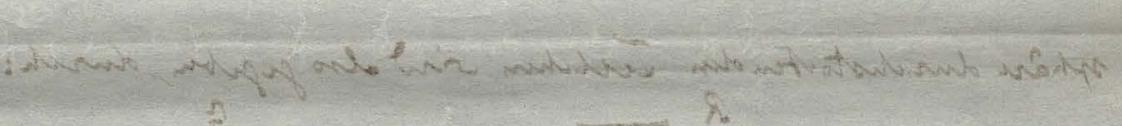

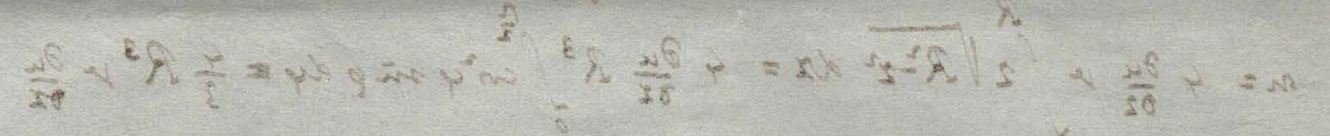

(81)

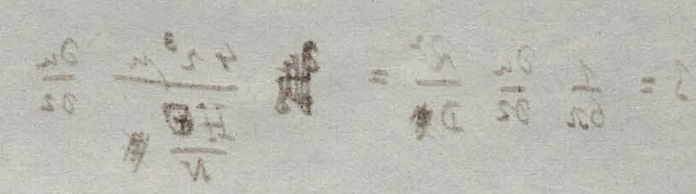

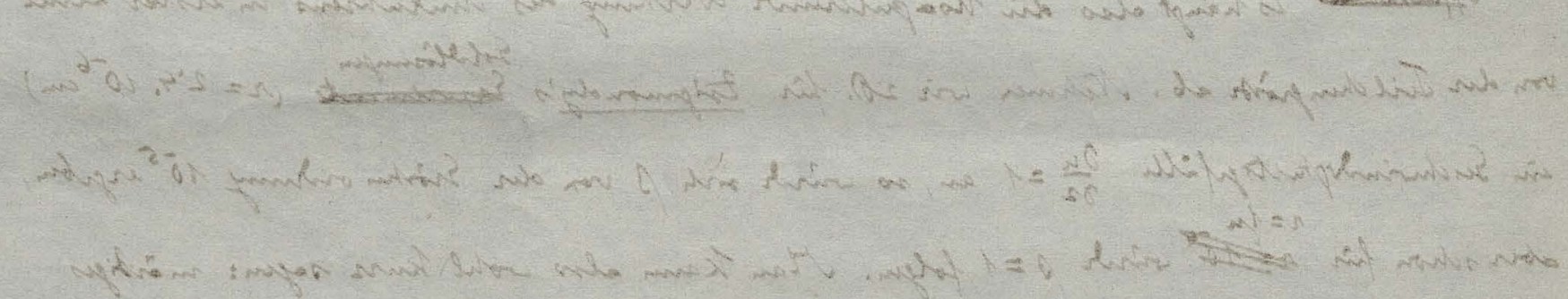

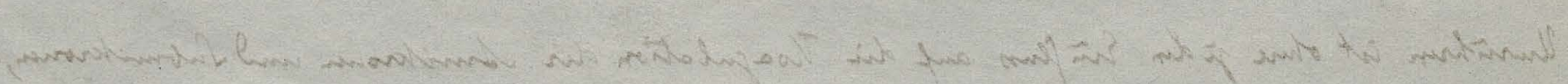

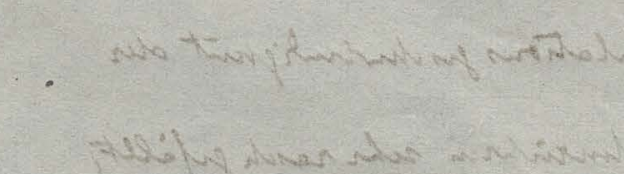




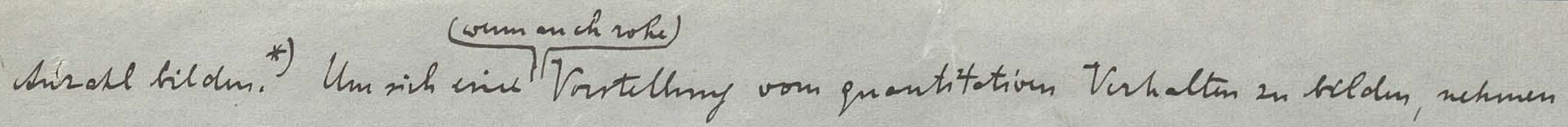

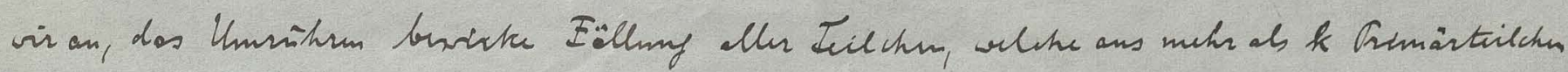

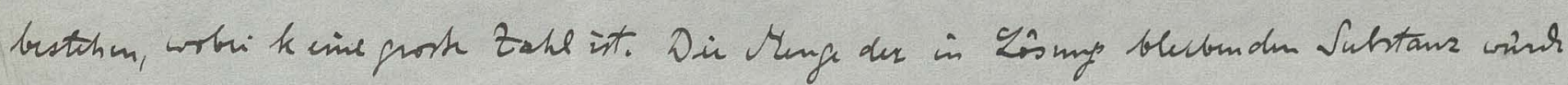

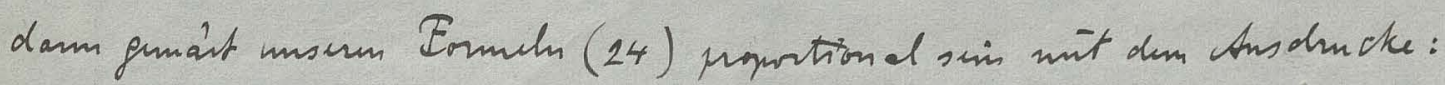

$$
y=v_{1}+2 v_{2}+3 v_{3}+\cdots k v_{k}=1-\left(\frac{t}{t+T}\right)^{k}\left[1+\frac{k t}{t+T}\right]
$$

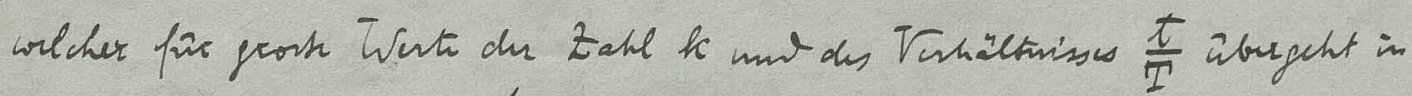

$$
\lim y=1-\left(1+\frac{1}{x}\right) e^{-\frac{1}{x}}
$$

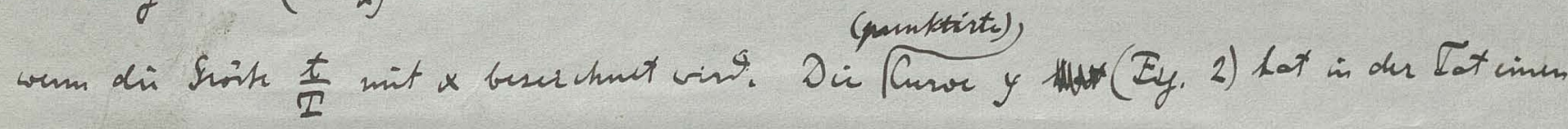

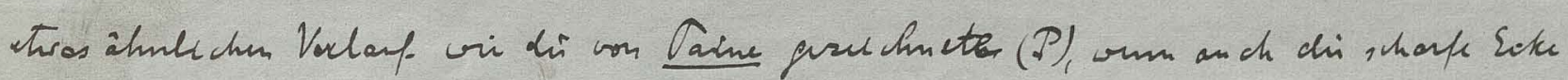

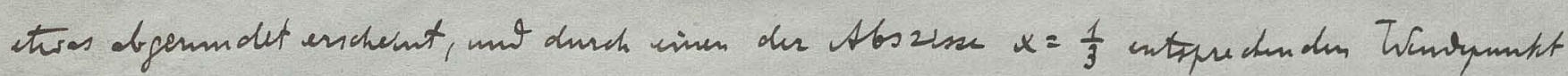
esstet int.

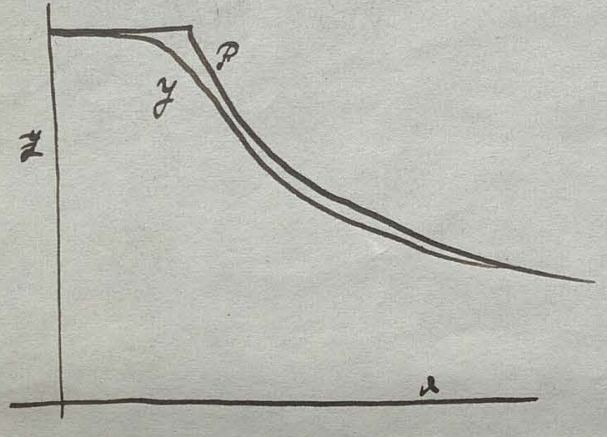

Ey. 2.

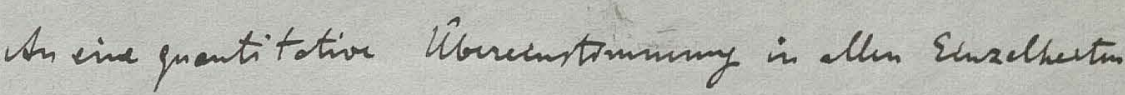
it bei cines derartigen Shchatany notislich nicht on dentem, aber wovl karm man worl igen, dass sich di

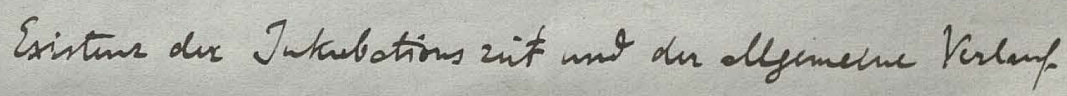

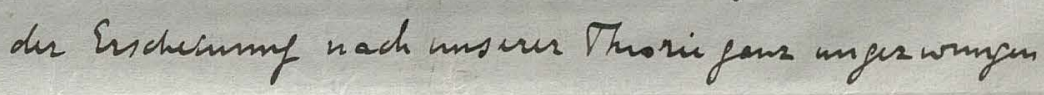
utulân.

\section{Veaglerch mit Vistosetiots mesungen.}

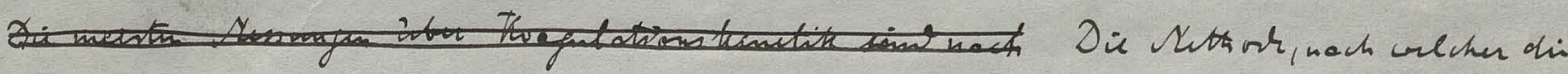

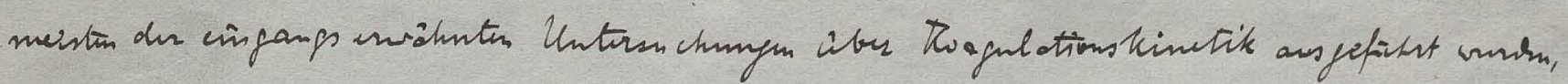

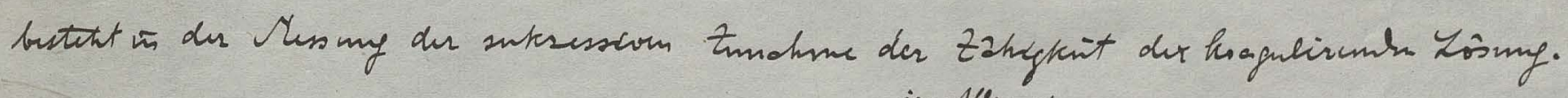

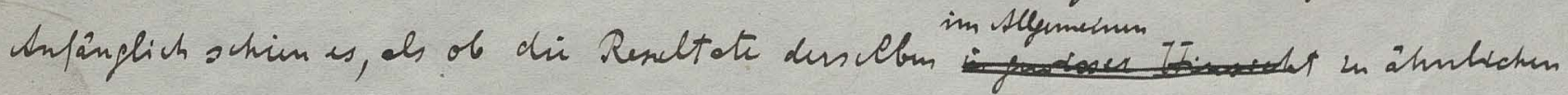

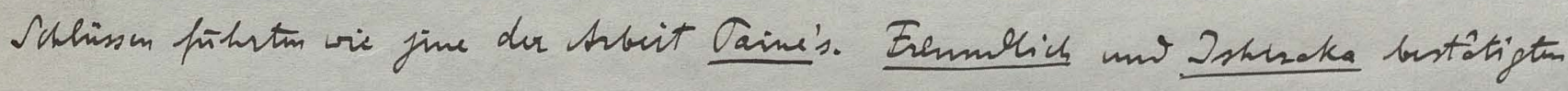

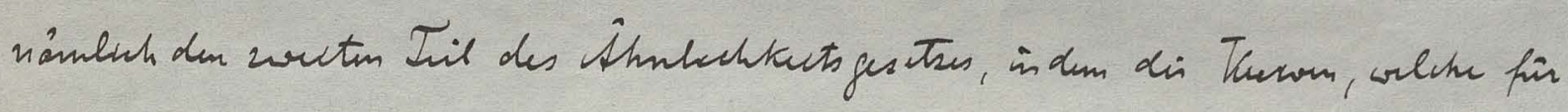

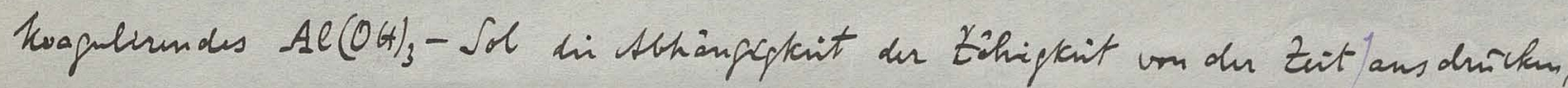

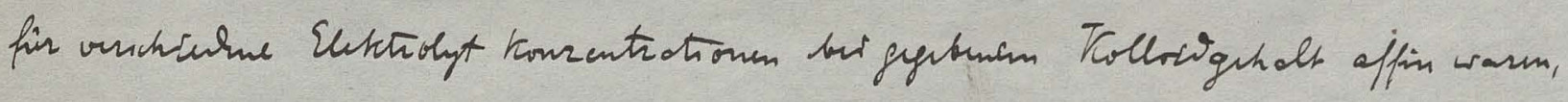

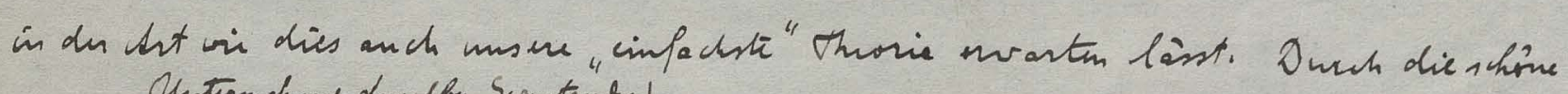

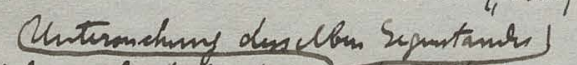

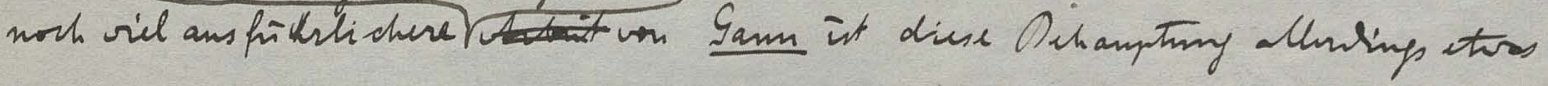

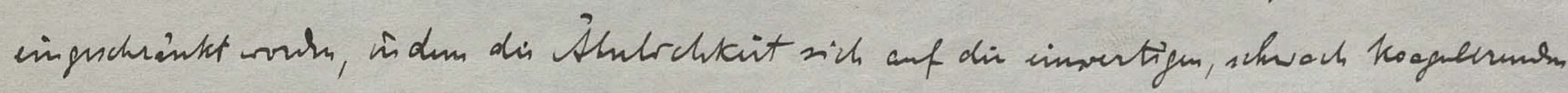

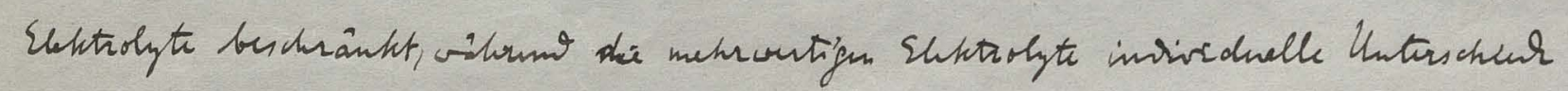

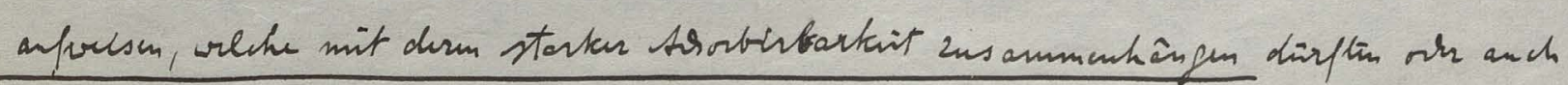

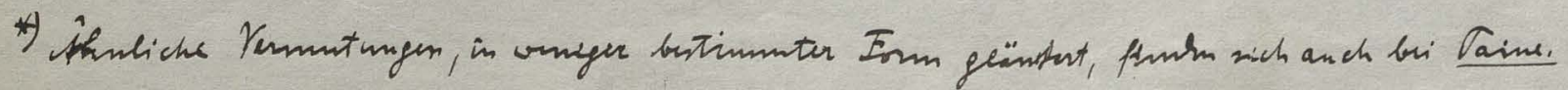


25

$(9+1)$

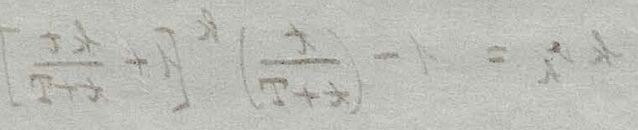

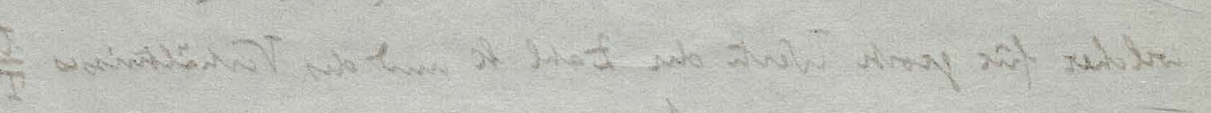

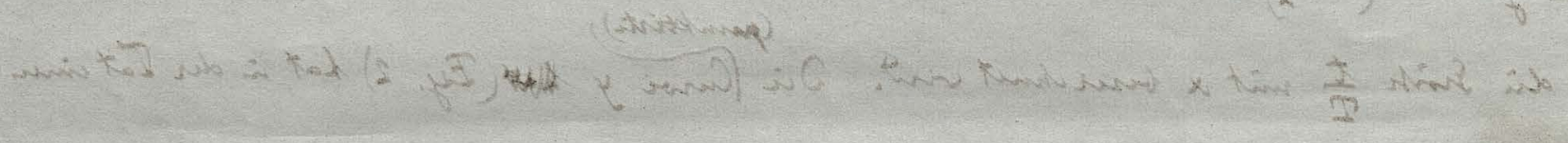

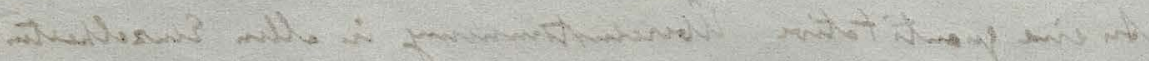

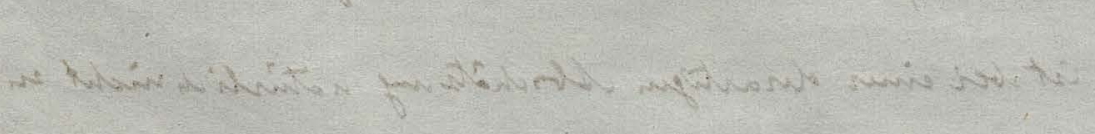

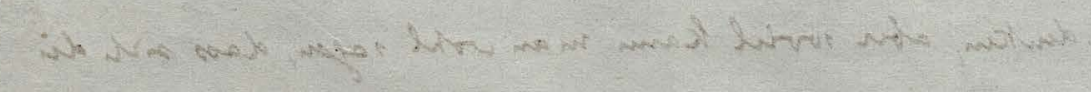

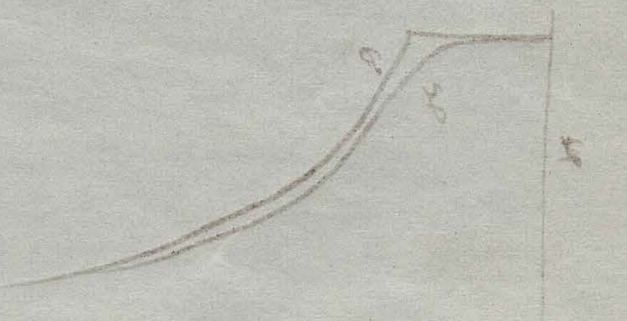

tat

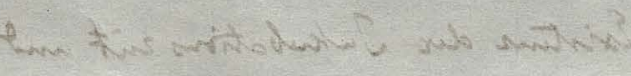

inculation

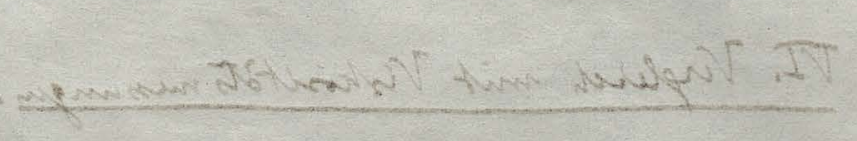

$5 \cdot y^{3}$

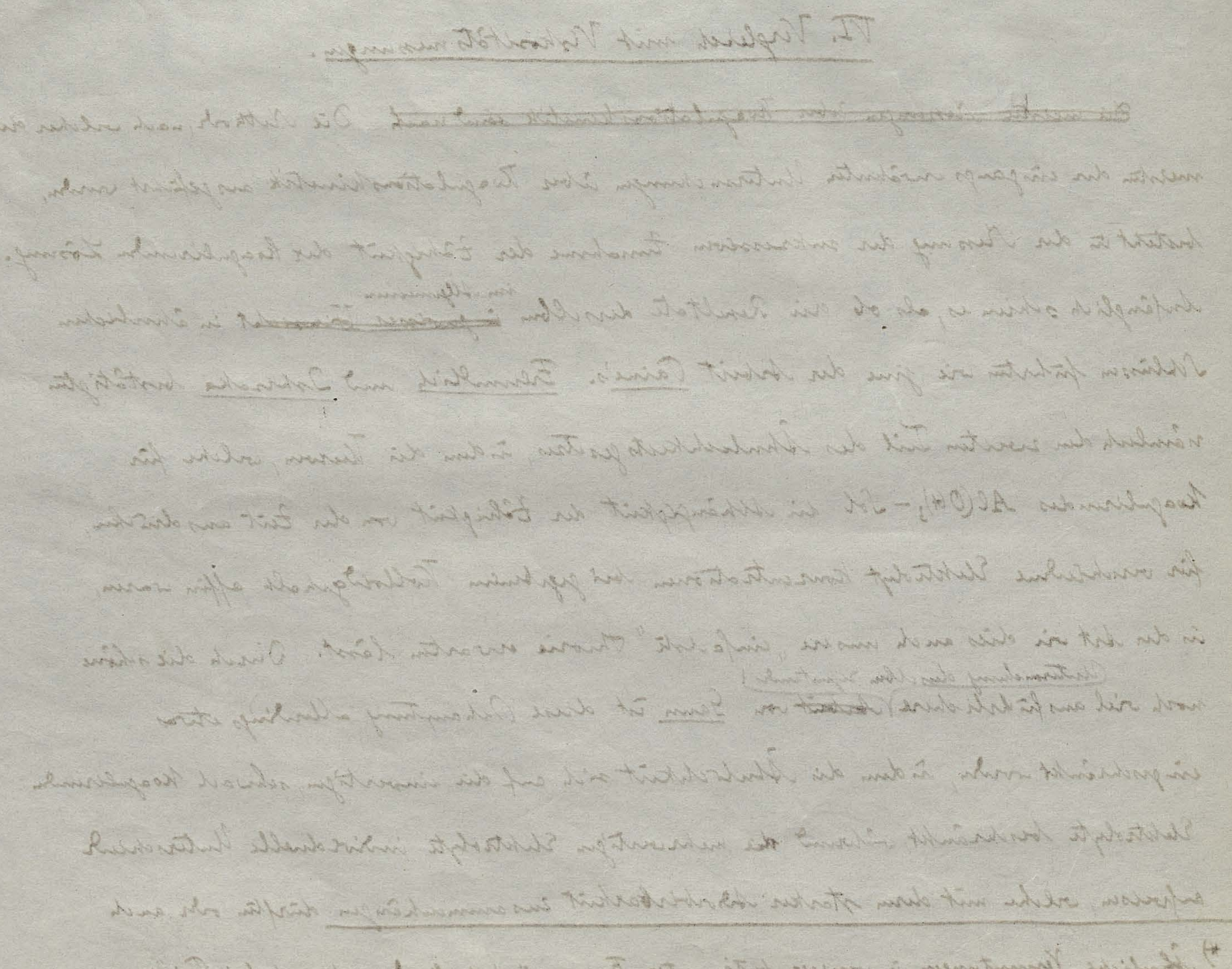




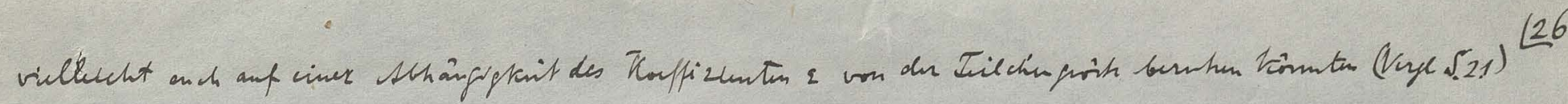

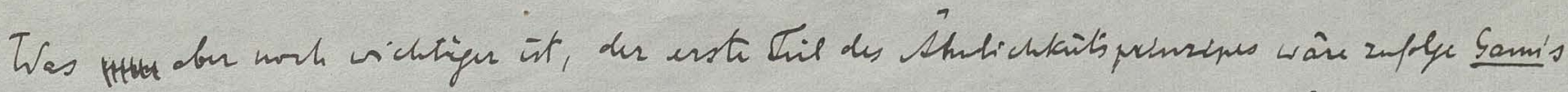

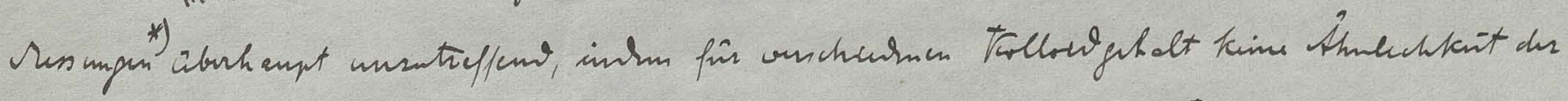

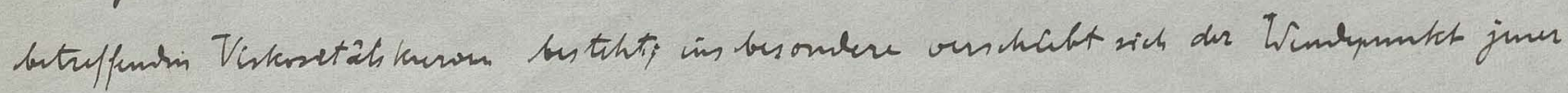

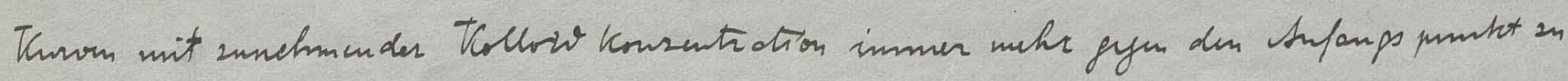

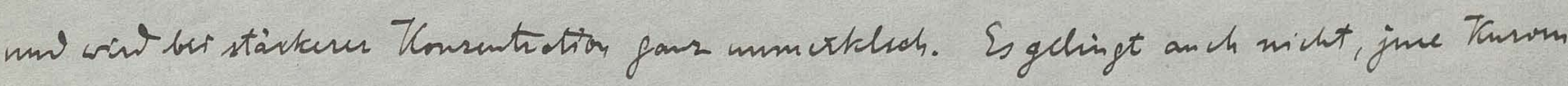

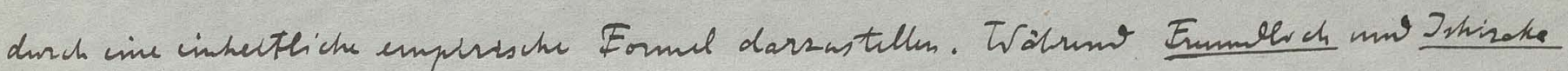
Slei Aningen der Gutals

$$
\frac{d x}{d t}=k t(1+6 x)(1-x)^{2} \quad \text { orr } \frac{d x}{d t}=k^{\prime} t\left(1+b^{\prime} x\right)(1-x)
$$

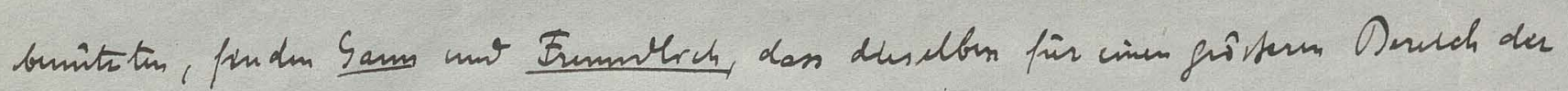

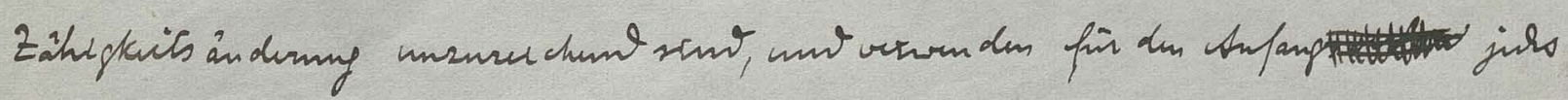

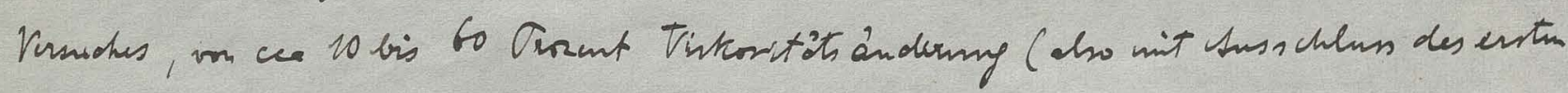

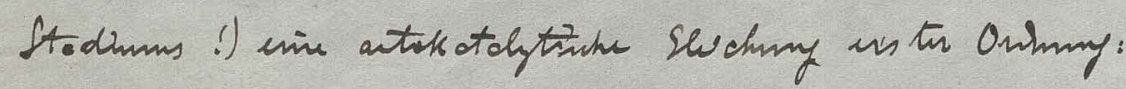

$$
\frac{d x}{d t}=k_{1}\left(1+b_{1} x\right)(1-x)
$$

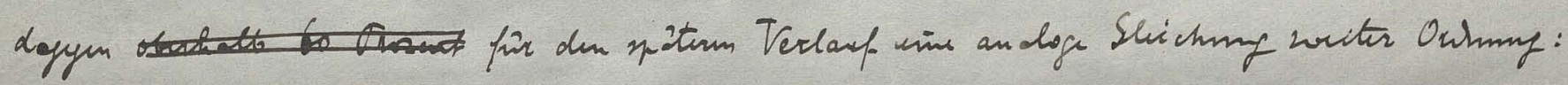

$$
\frac{d x}{d t}=h_{2}\left(1+b_{2} x\right)(1-x)^{2}
$$

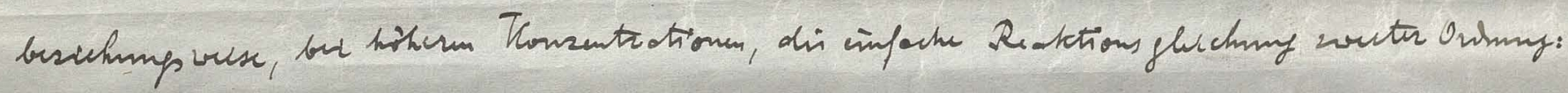

$$
\frac{d x}{d t}=k^{\prime \prime}(1-x)^{2}
$$

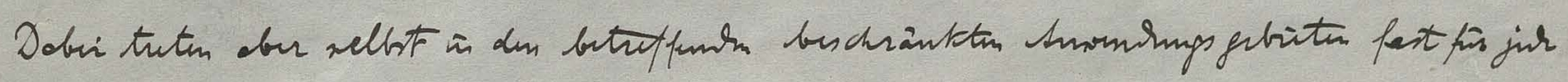

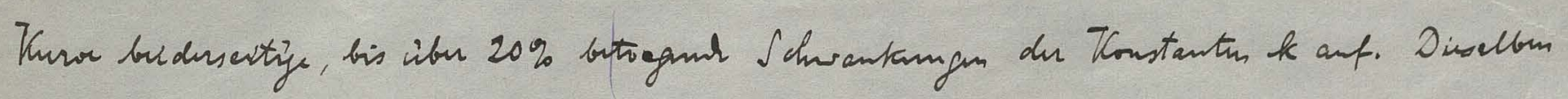

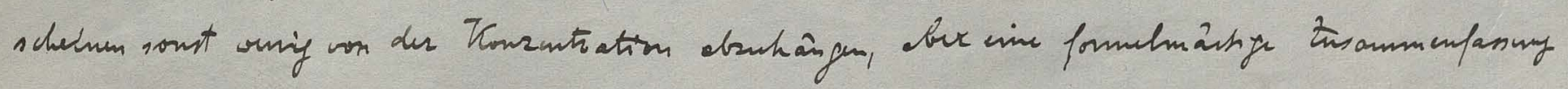

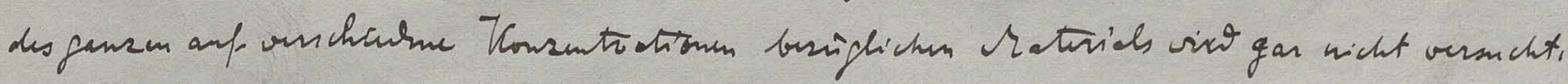

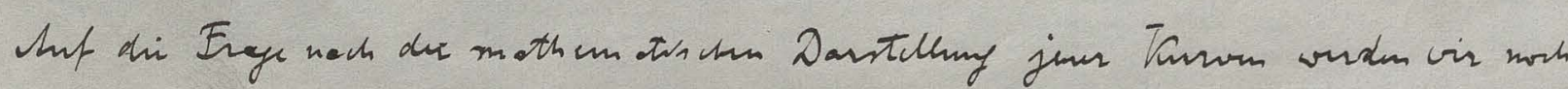

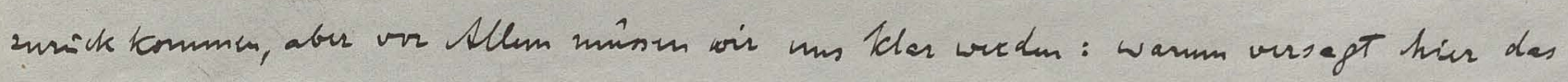

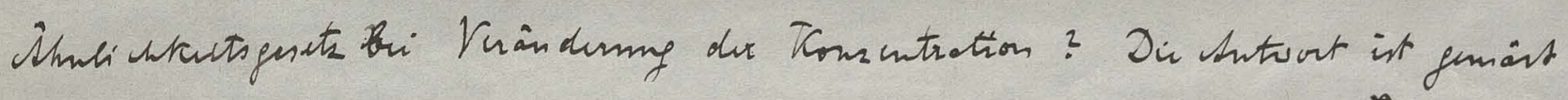

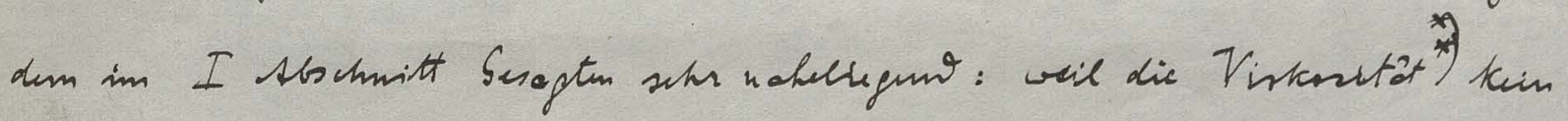
dirukter rastatob fir den Toagulations prosess ist.

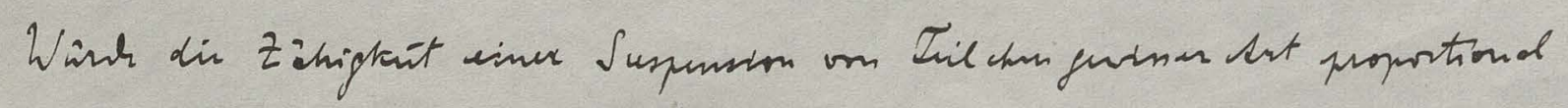

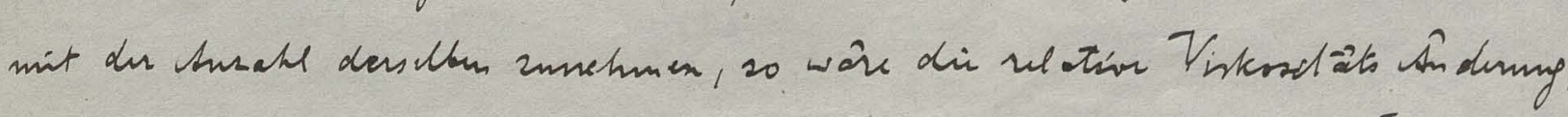

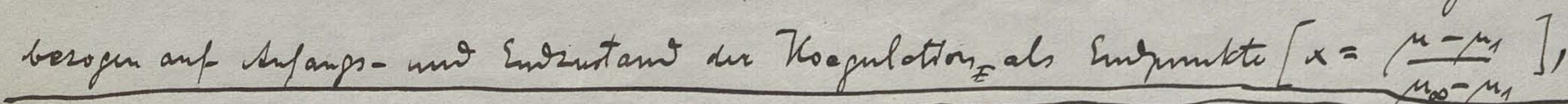

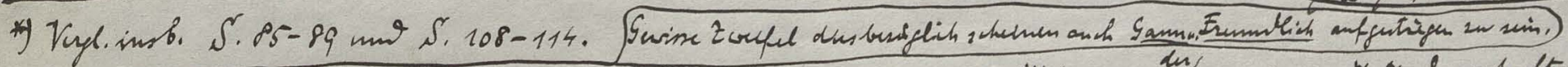

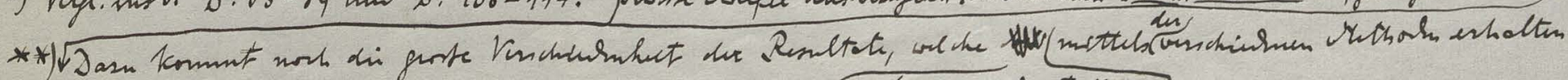
cuin (Vuge Sarn's Atbut).

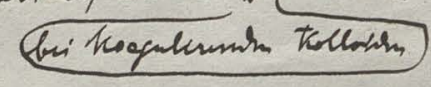




$$
\begin{aligned}
& (x-1),(x+x+1)+x)=\frac{x y}{x+6}
\end{aligned}
$$

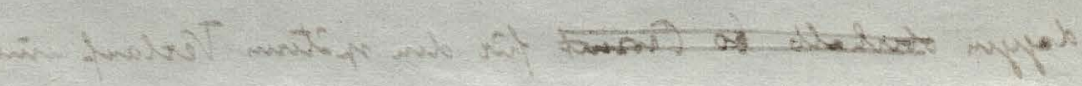

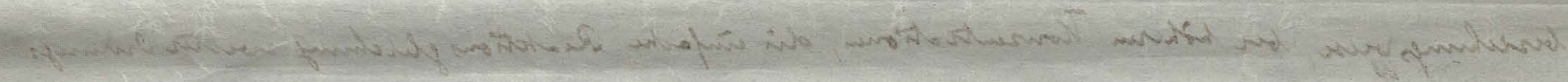
(1) $\varepsilon_{\text {) }}$

$$
\left.(x-1)^{3}\right)=\frac{4 \lambda}{x-10}
$$




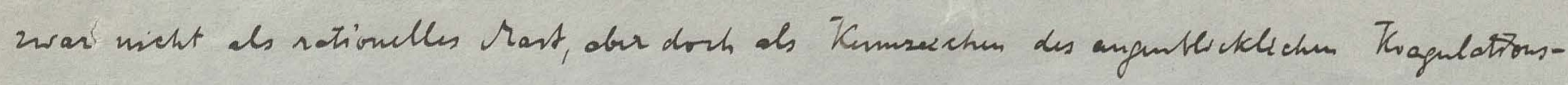

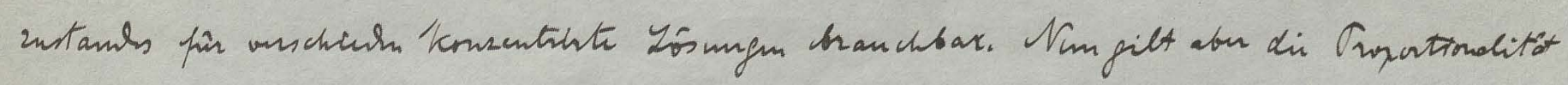

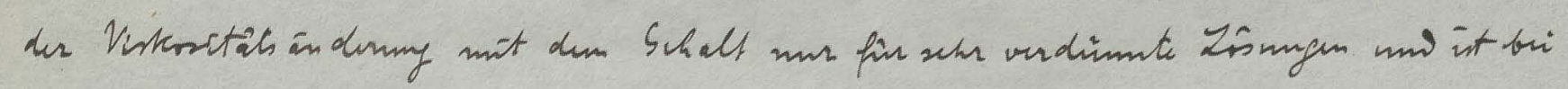

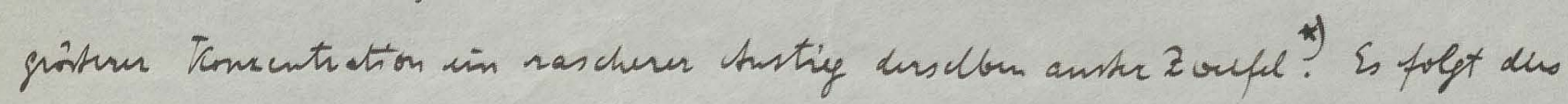

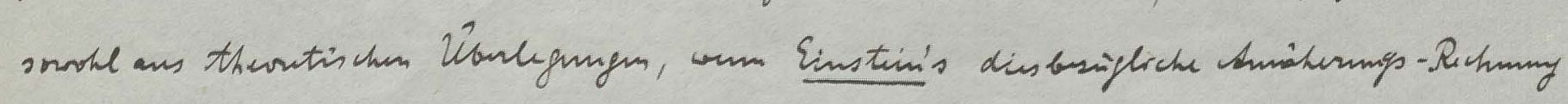

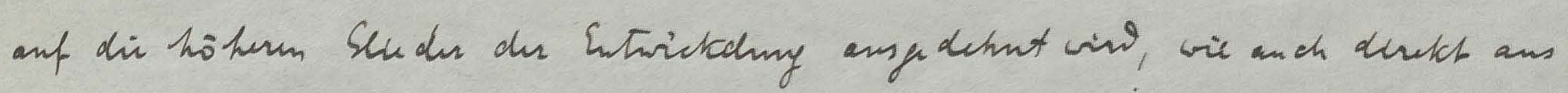

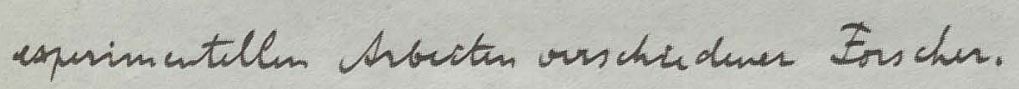

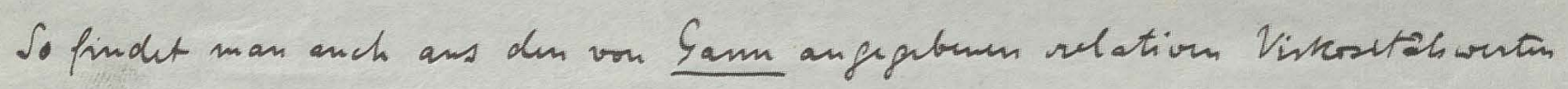

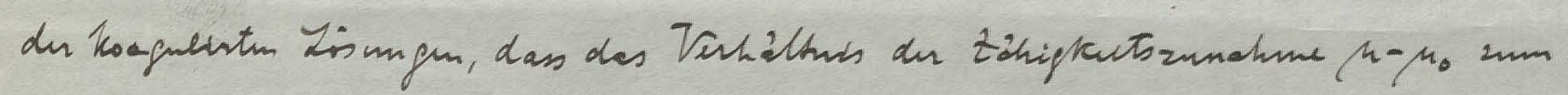

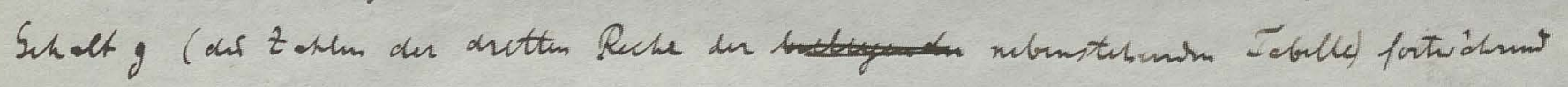
strift:

\begin{tabular}{l|cc|c|c|c|c|c}
$g$ & 0 & 11 & 1.5 & 2.0 & 2.5 & 3.0 & 4.0 \\
\hline$\frac{\mu}{\mu_{0}}$ & 1.000 & 1.260 & 1.429 & 1.578 & 1.837 & 2.175 & 2.897 \\
\hline$\frac{\mu_{0}-\mu_{0}}{\mu_{0} g}$ & & 0.236 & 0.286 & 0.287 & 0.335 & 0.392 & 0.474
\end{tabular}

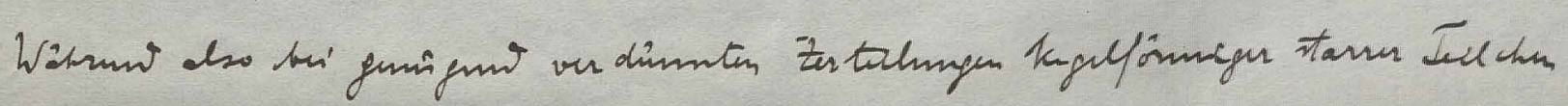
di Einstim 'rue Eonnul: $\mu=\mu_{0}\left[1+\frac{5}{2} \varphi\right]$

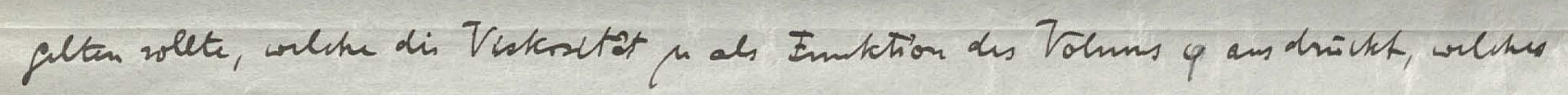

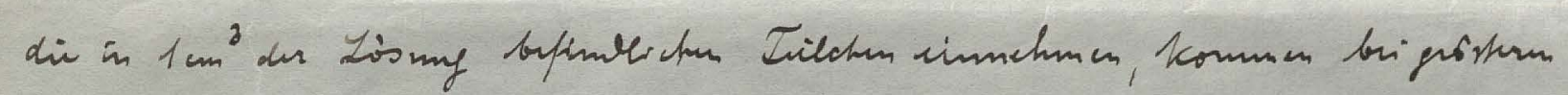

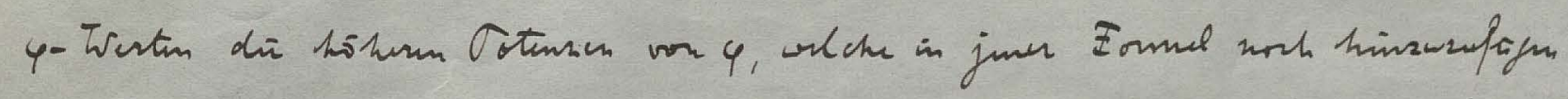

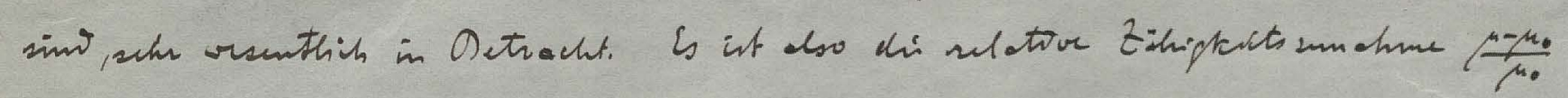

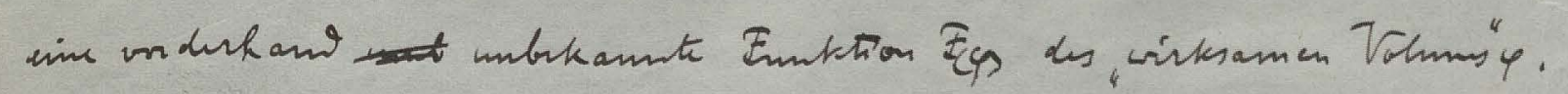

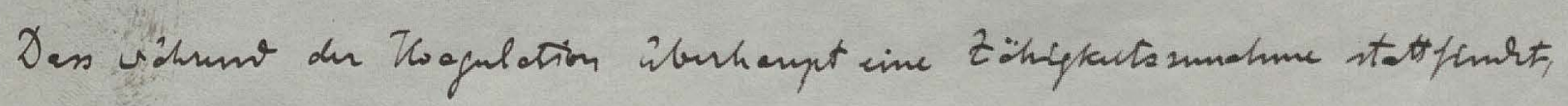

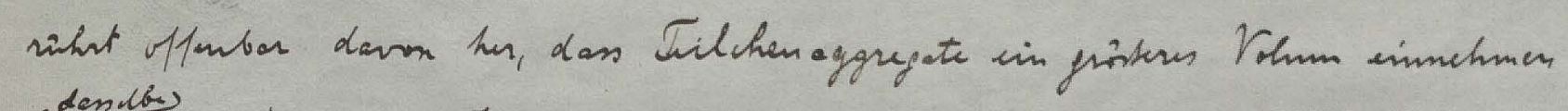

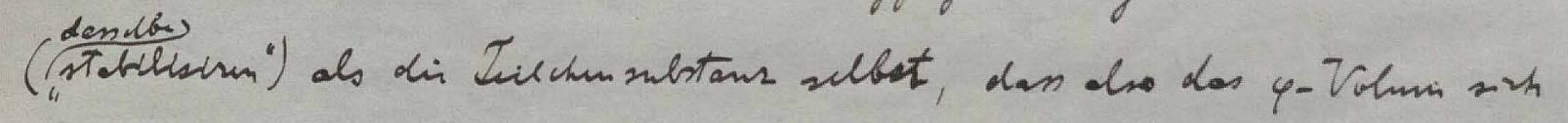

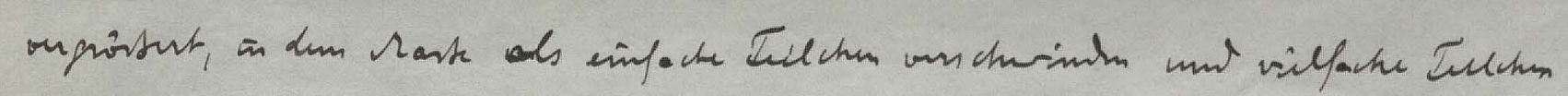

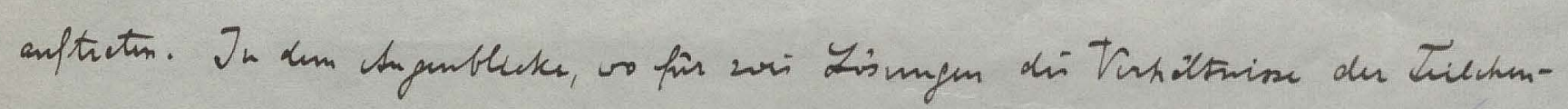

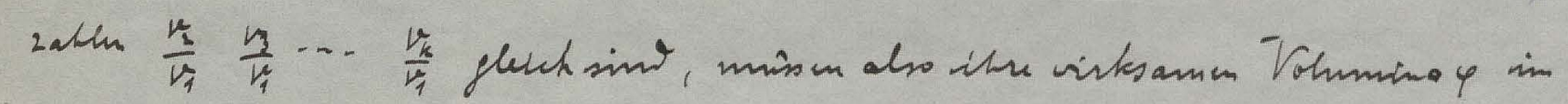

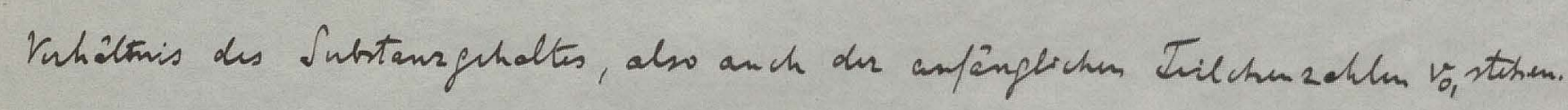

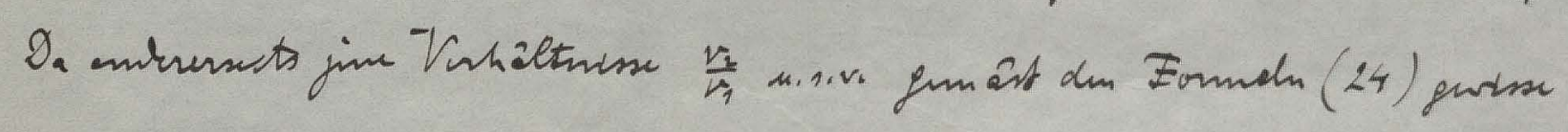

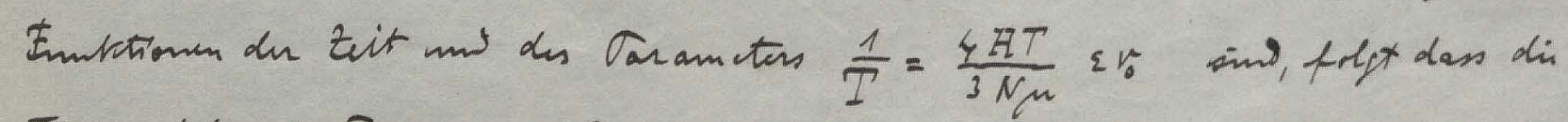

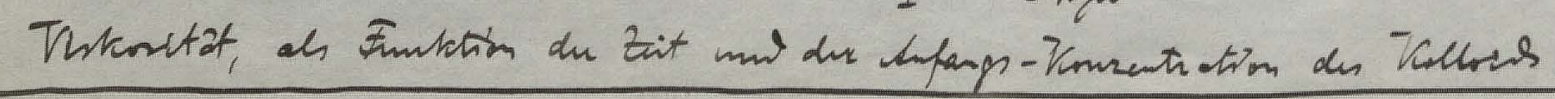

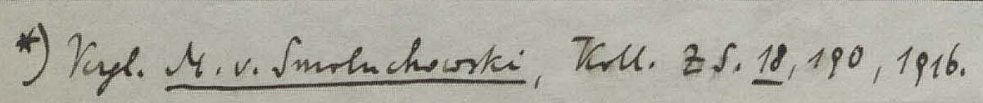




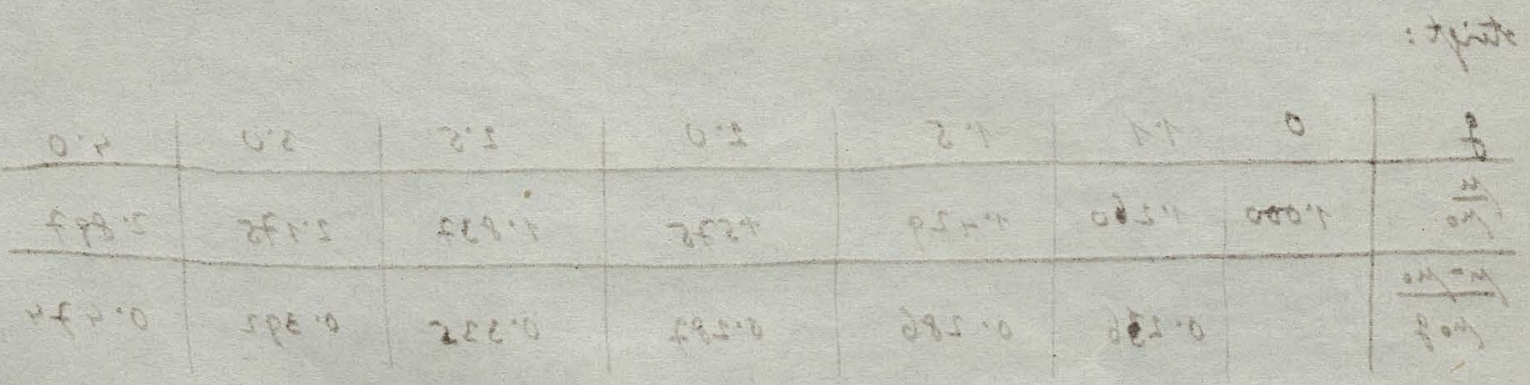

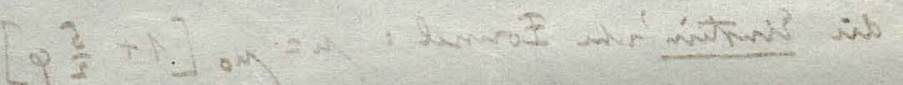

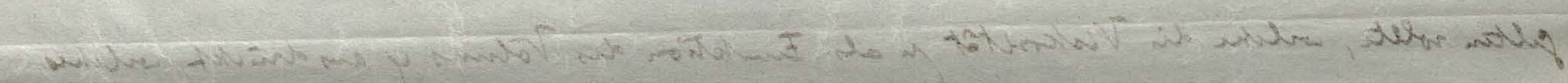

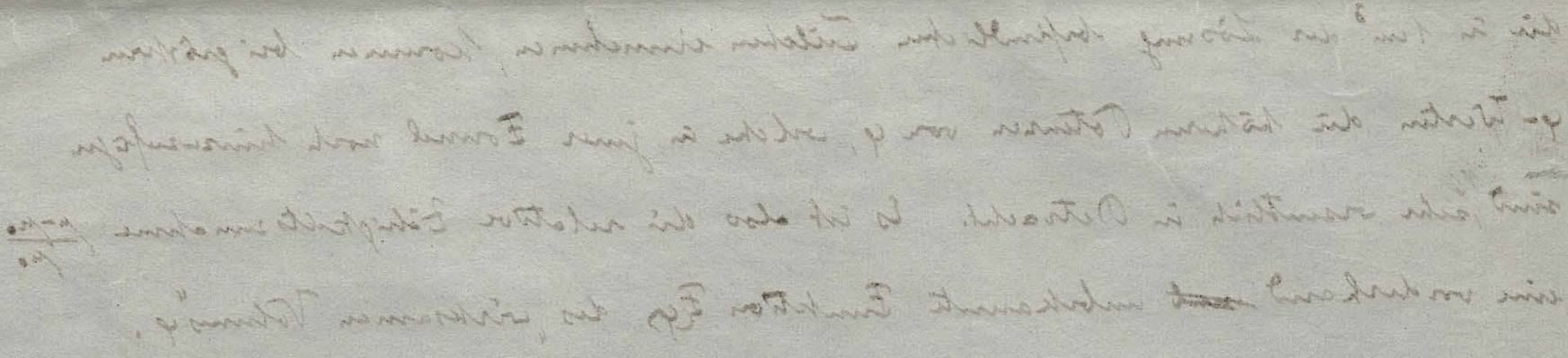
thenation

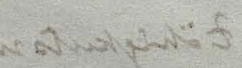

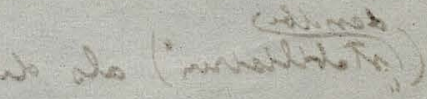

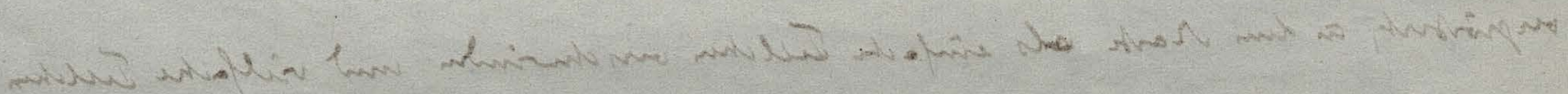

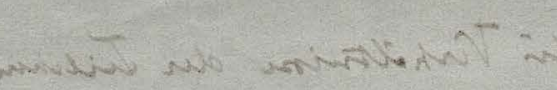

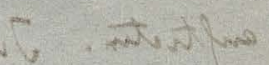

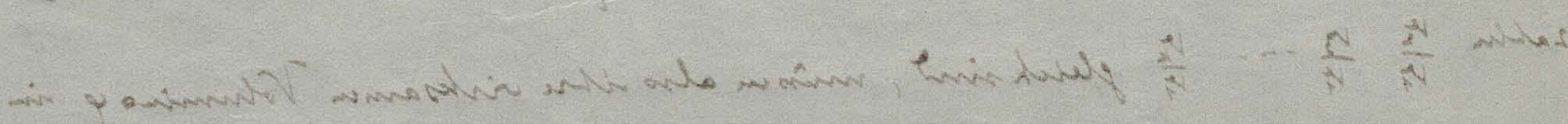

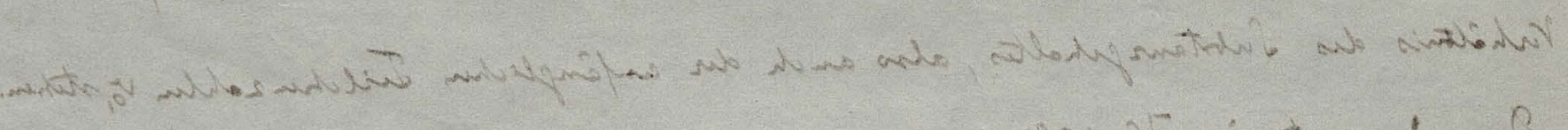

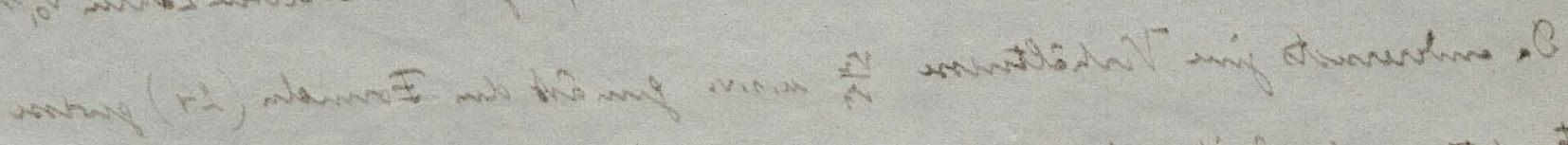

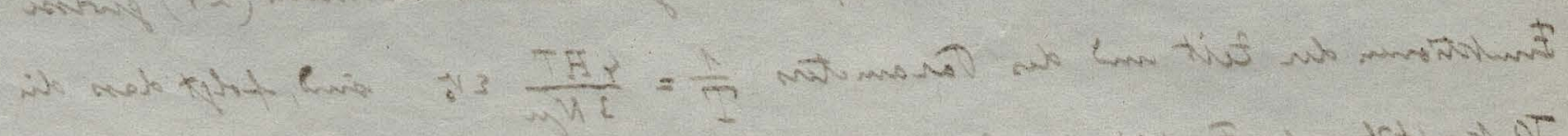

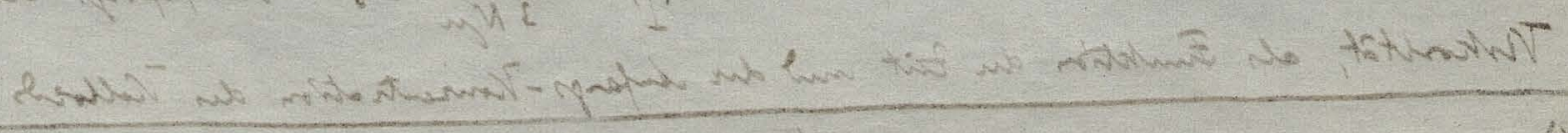




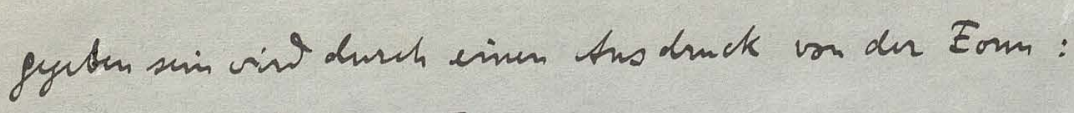

$$
\mu=\mathcal{F}\left[\nu_{0} \Phi\left(v_{0} t\right)\right]
$$

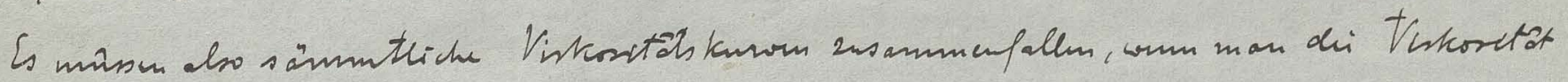

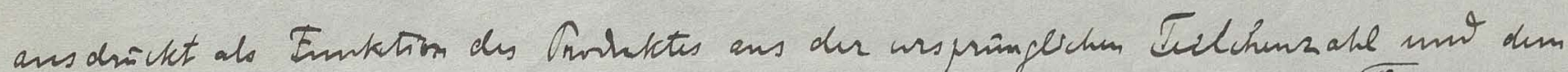

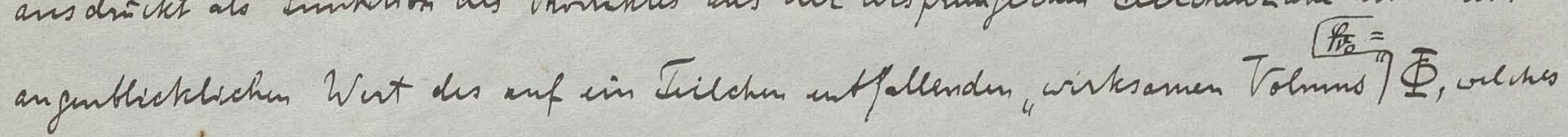

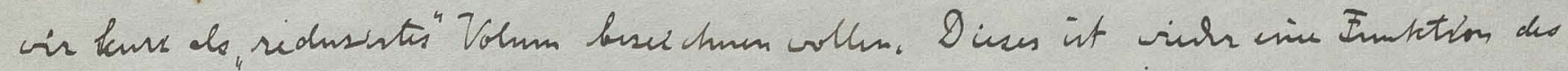

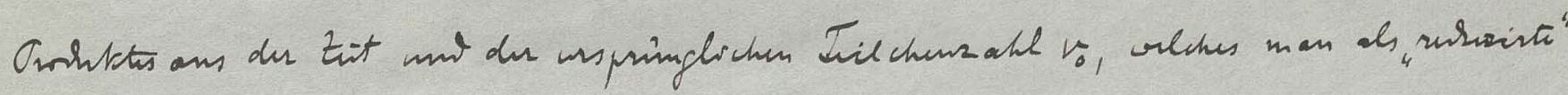
tit $\vartheta$ besuchnen k onnte.

Ein sytum atisches Vusuchsmaterial solte di emperesche Ernittilung jiner

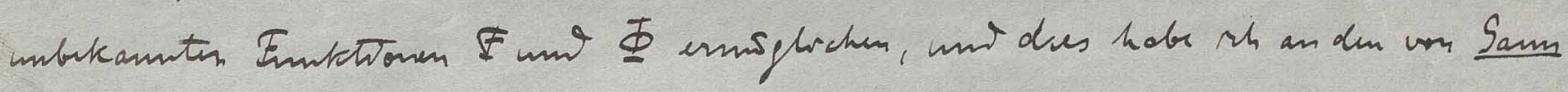

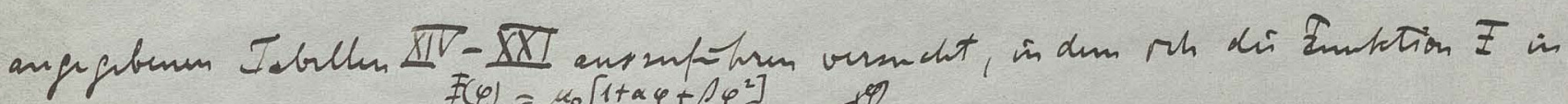

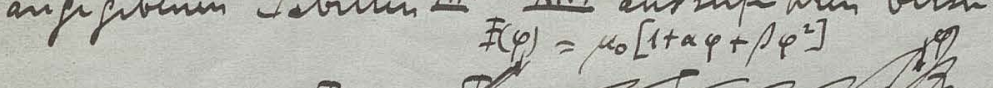

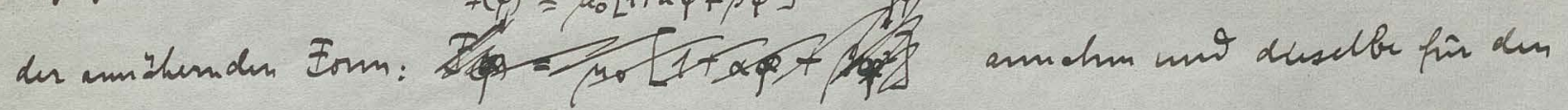

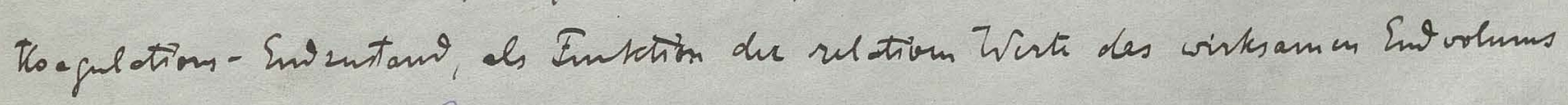

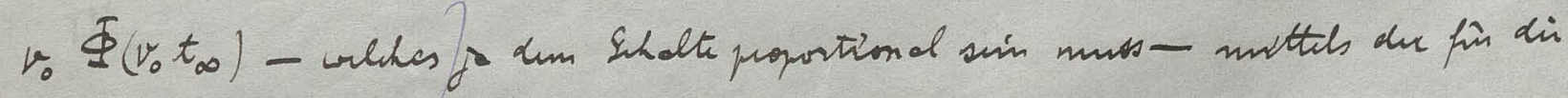

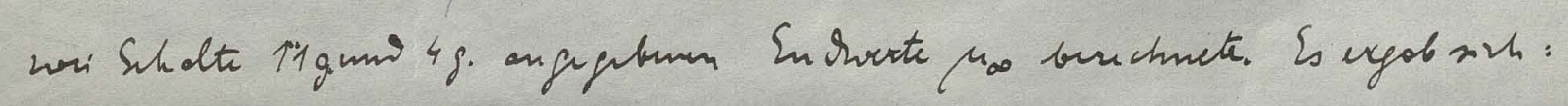
$\alpha=0.146 ; \beta=0.082$.

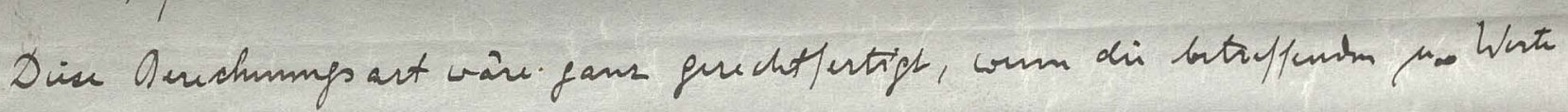

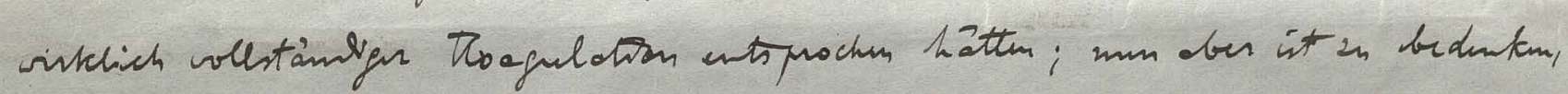

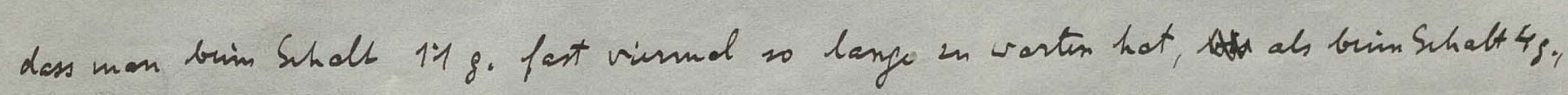

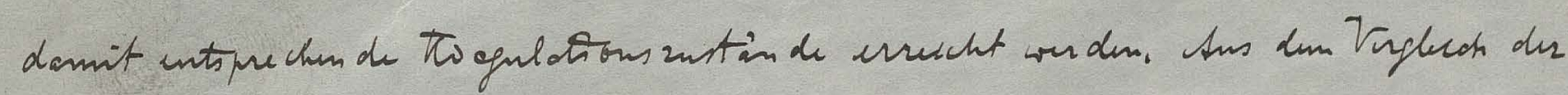

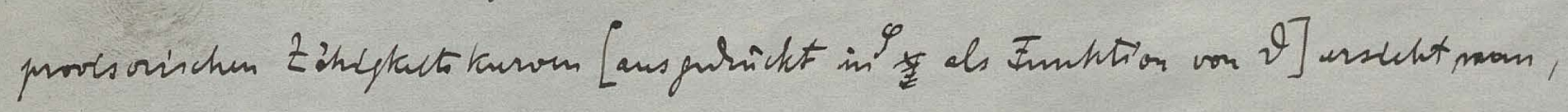

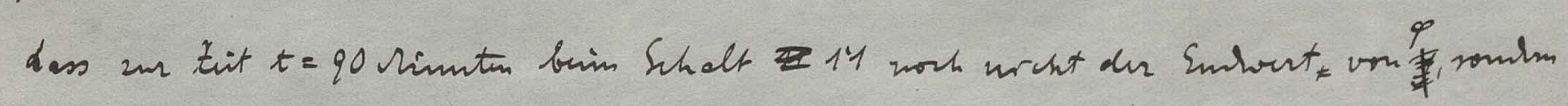

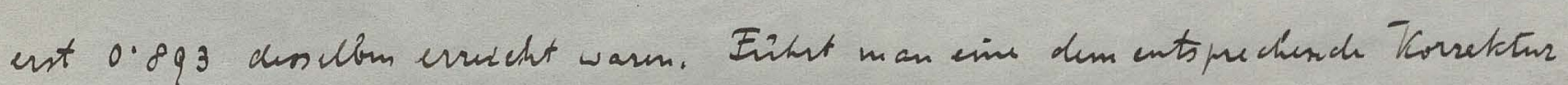

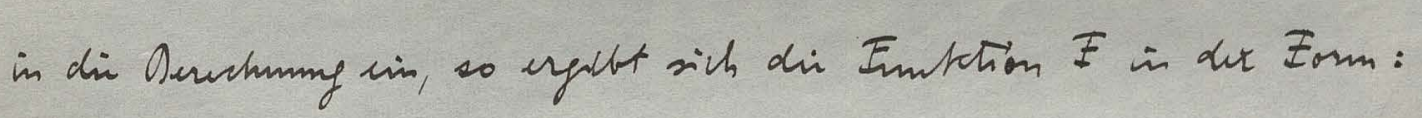

$$
F=\mu=\mu_{0}\left[1+0.1989+0.0699^{2}\right]
$$

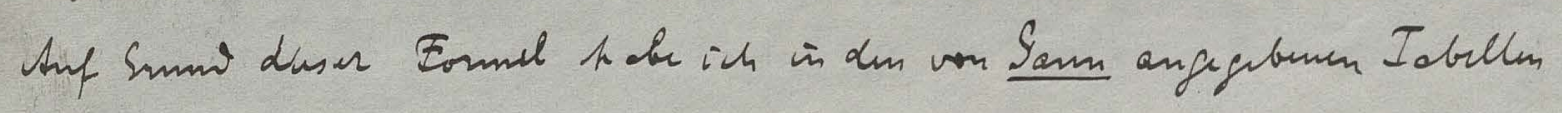

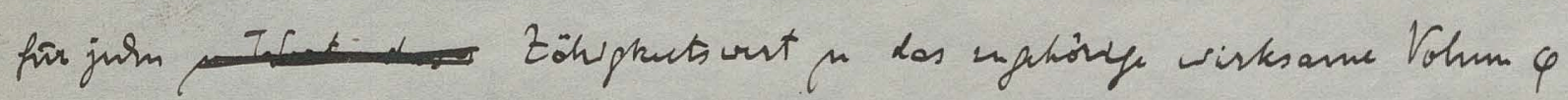

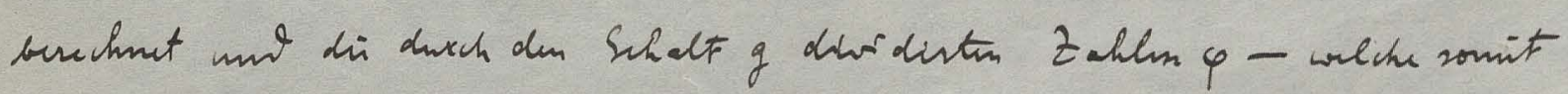

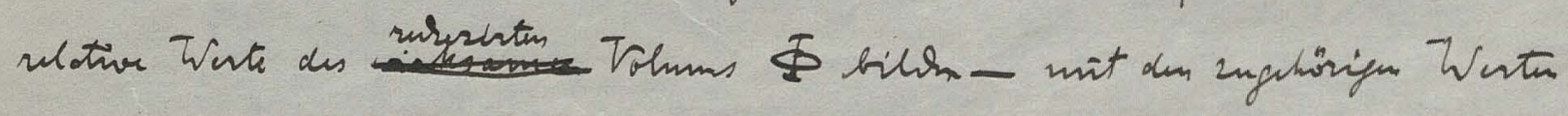

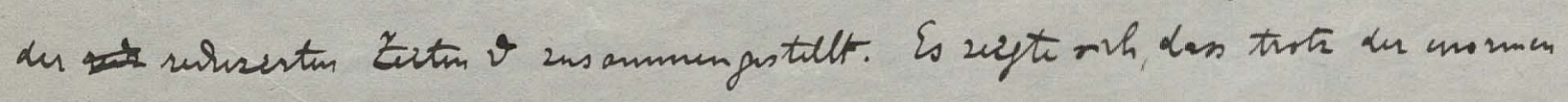

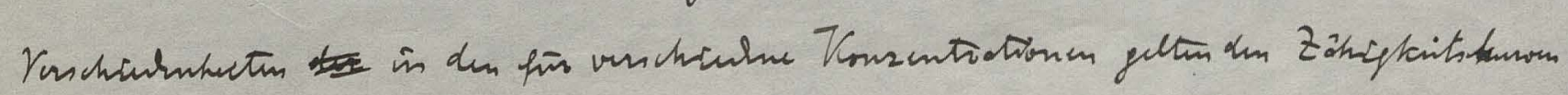

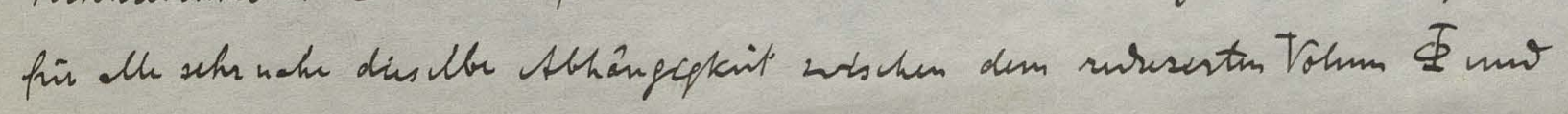


Ad tores

\$

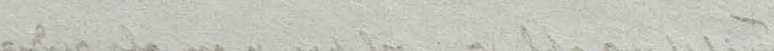

und जotwhing

$\sin x$ id

$+\frac{1}{2}$

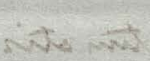

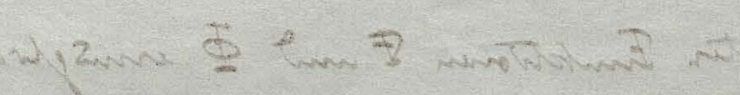

ENantons

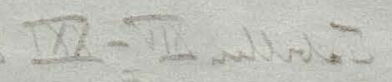

$x+2 x+2, x^{2}=x^{5}$

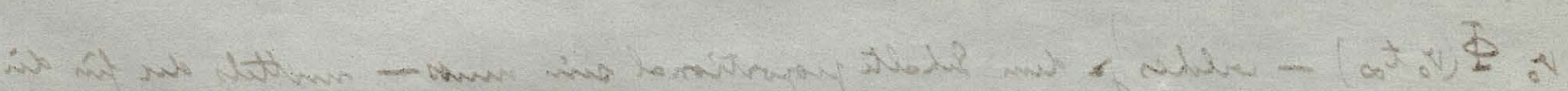

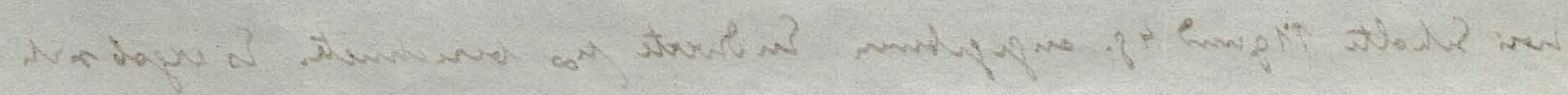

$=x$

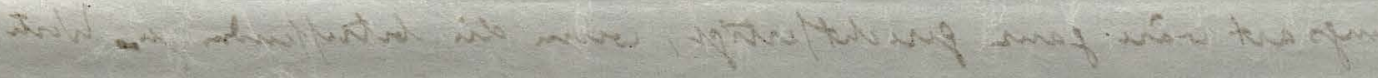

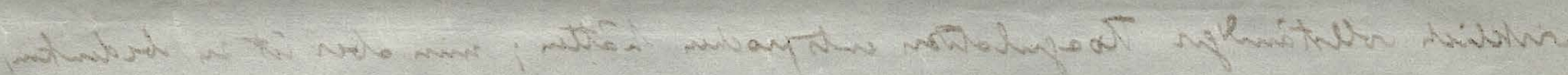

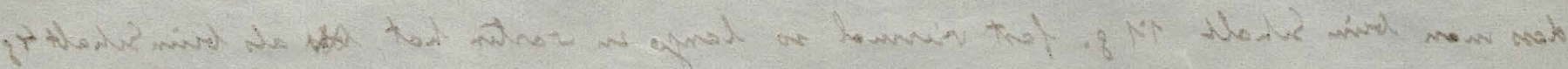

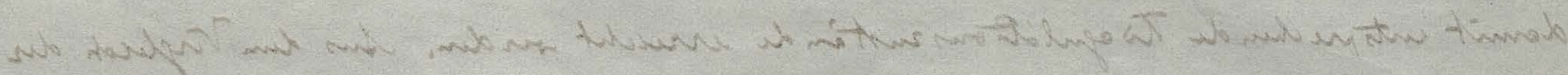

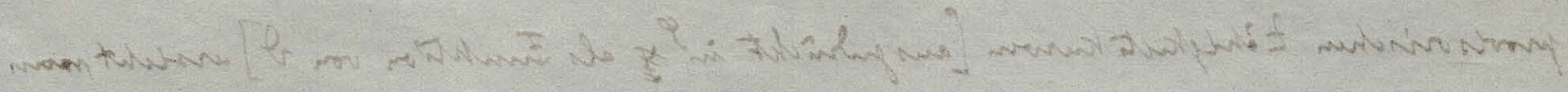

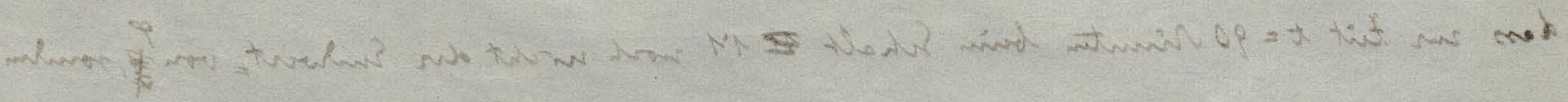

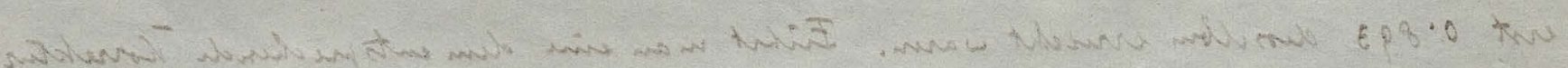

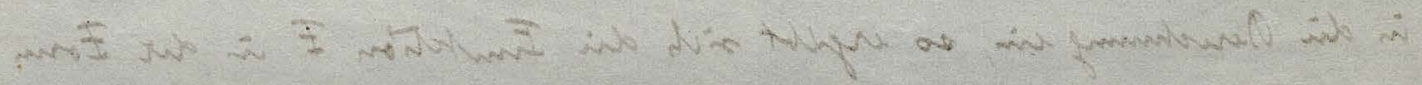
$(\pi \ell)$

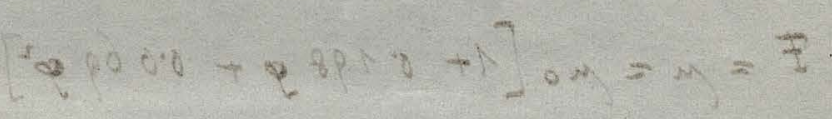

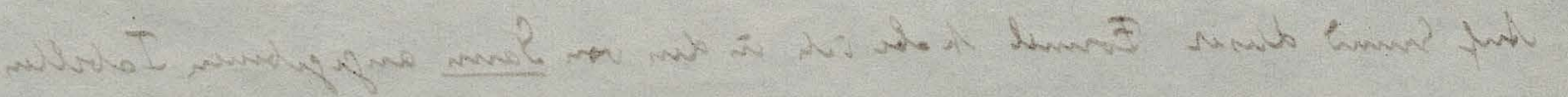

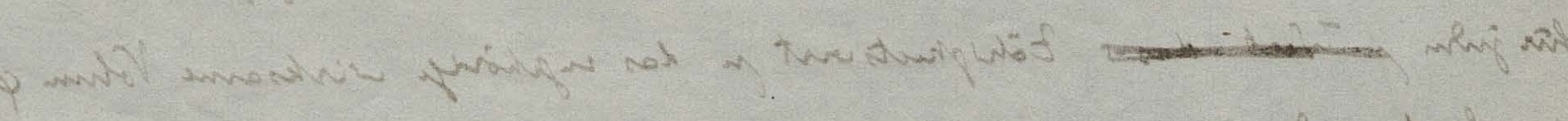

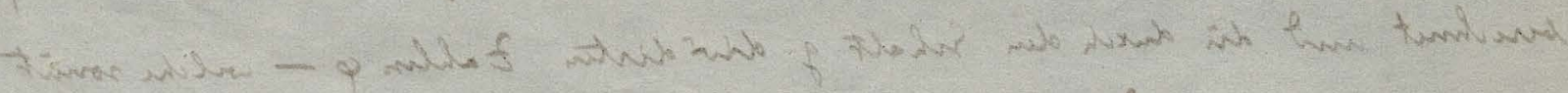

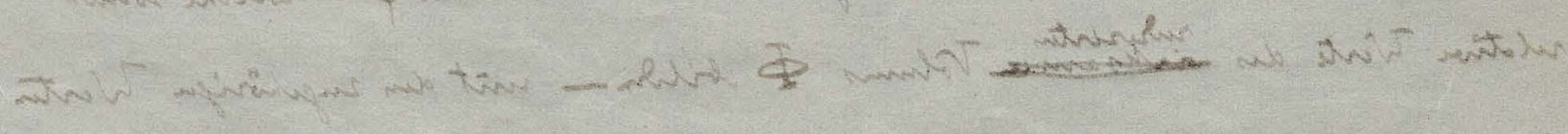

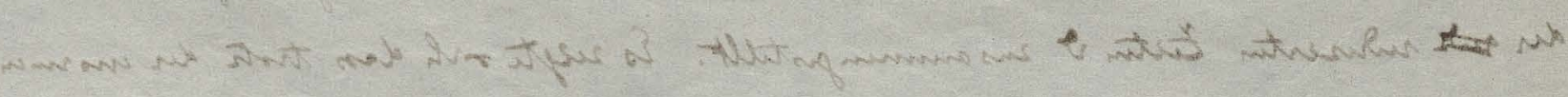

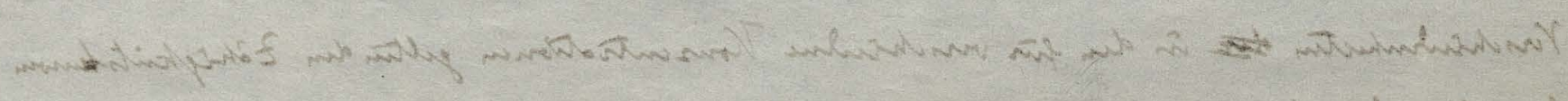

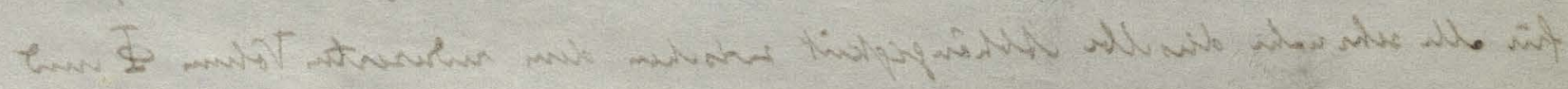




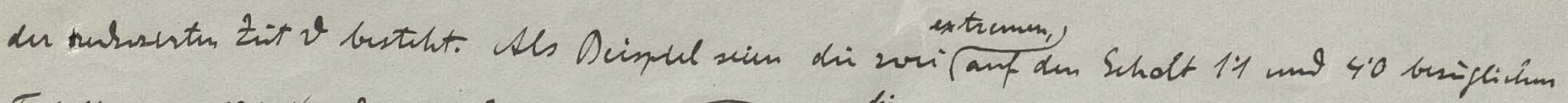

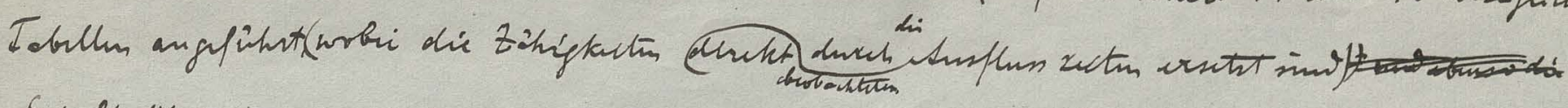

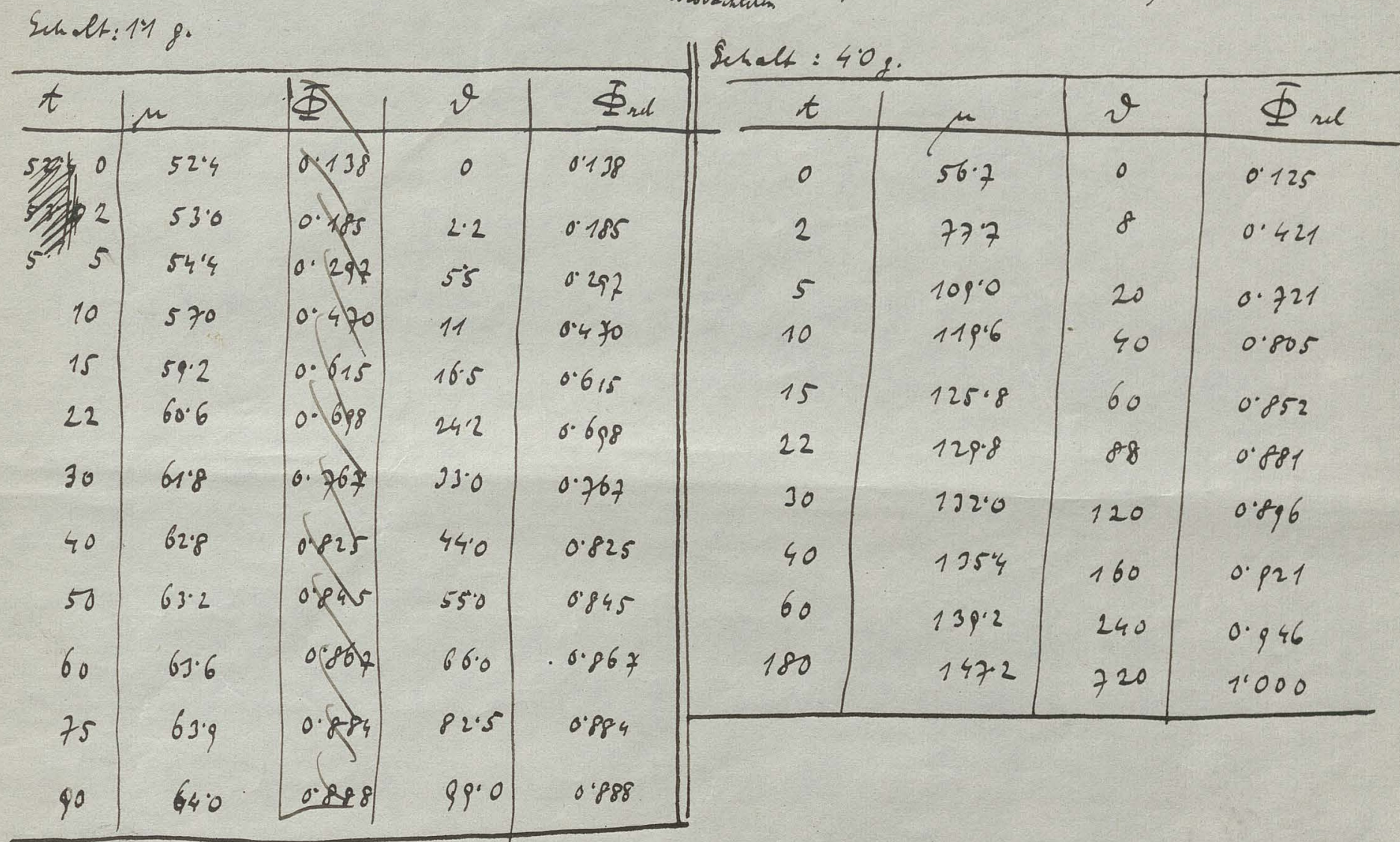

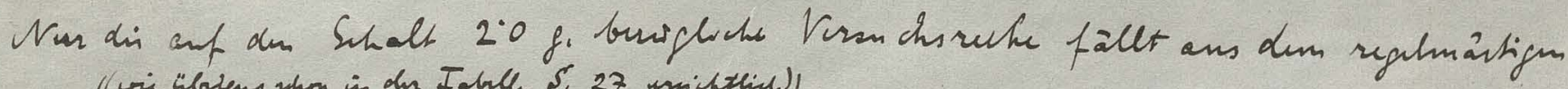

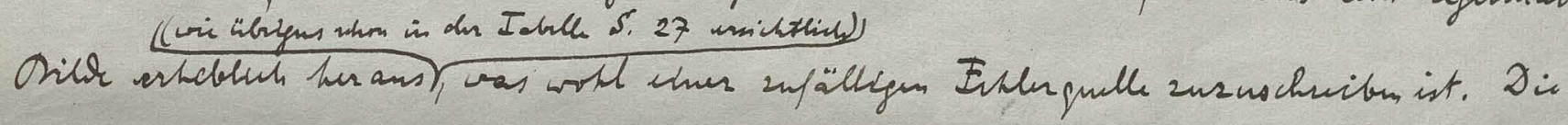

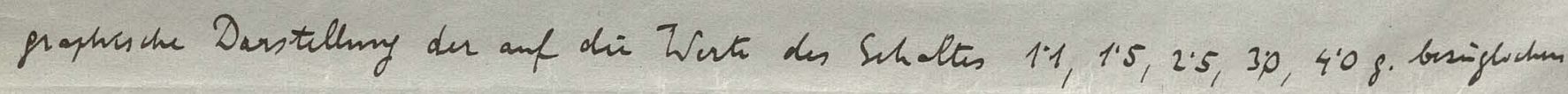

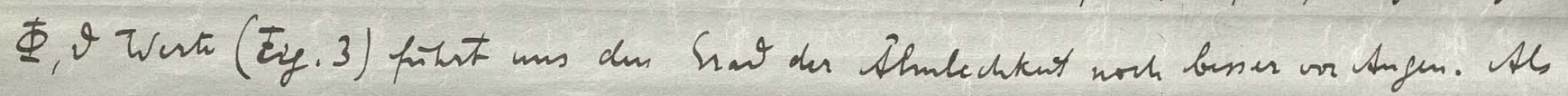

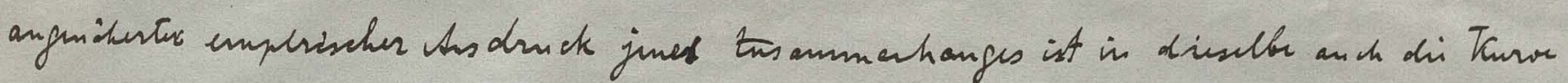
$\Phi=0.131+0.869\left[\frac{\vartheta}{\vartheta+3.94}\right]^{3}$

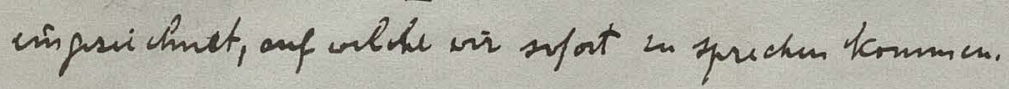

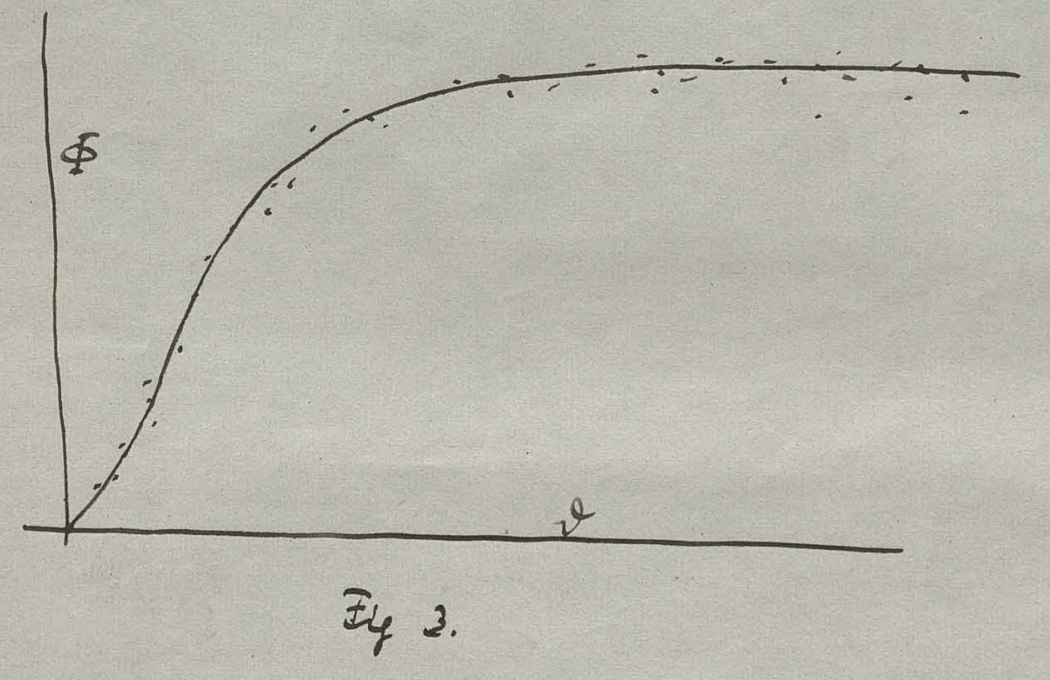

Sit Anprant gristure Rechmasbit linters nach dis Touffisunter in dm bet dm cuperischen Eosmeln gures worch pasumber best numen, so das di woch bestchemin Abouchingen.

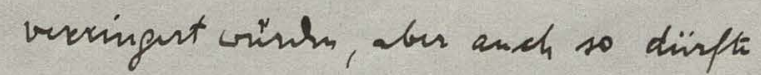
dese tom numentelleng genigen, un dis Eristurs des theoritischen

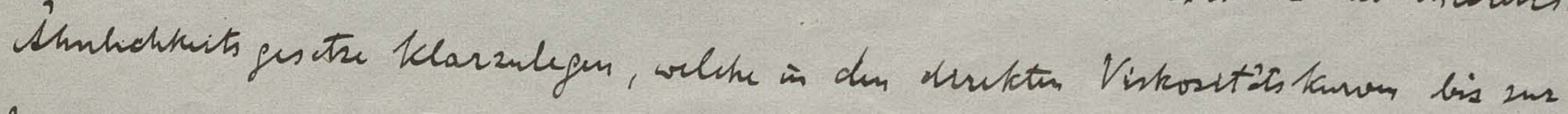
Untanntlichkit unhillt was.

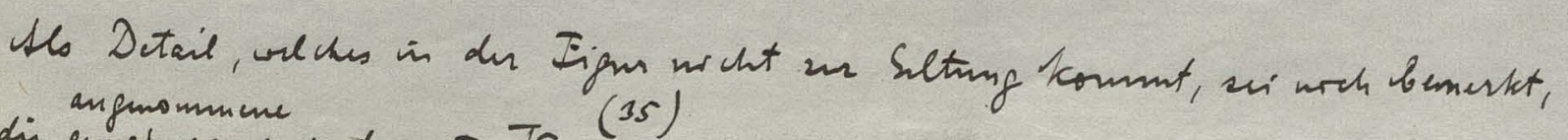
dass dir angenomoume duserte TC (15) Angubleck hovisoutel oulanft, dam 


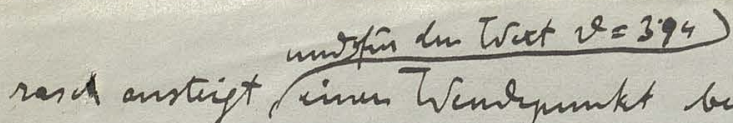

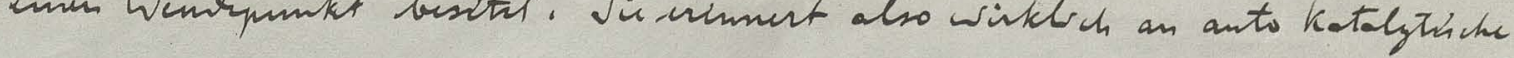

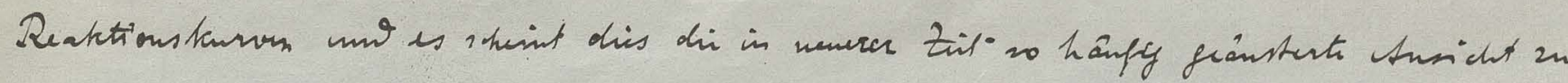

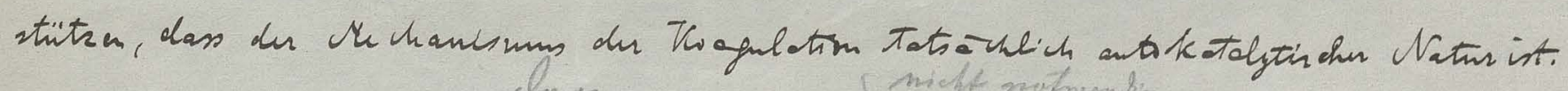

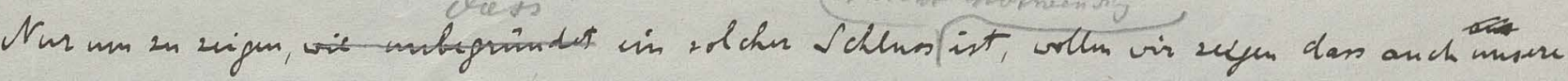

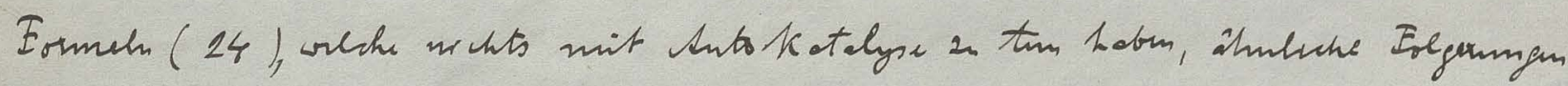

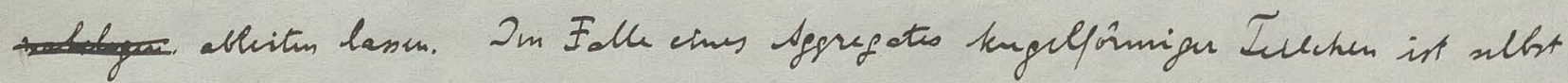

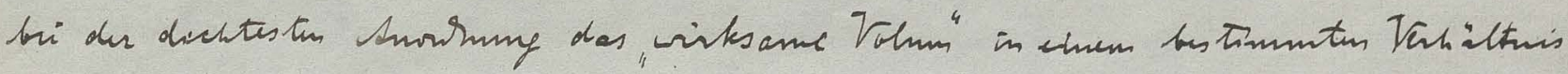

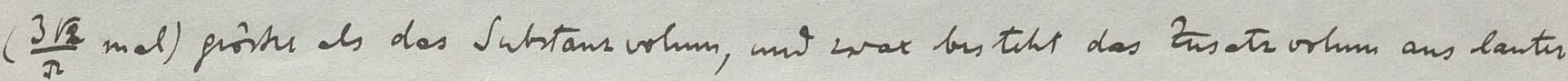

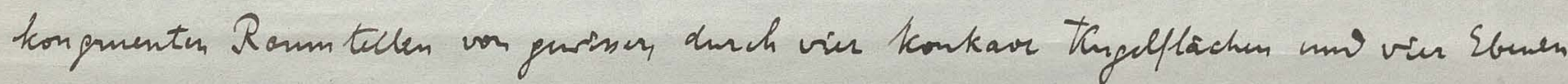

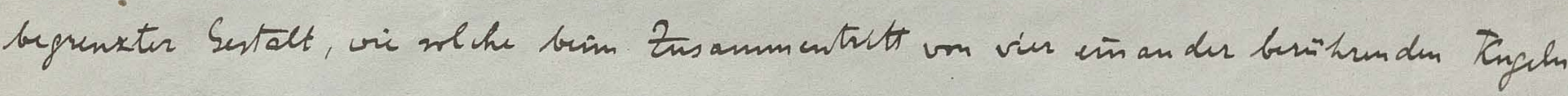

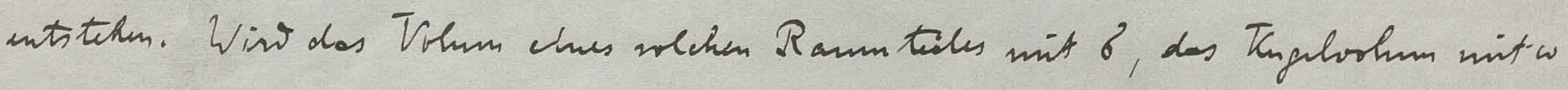

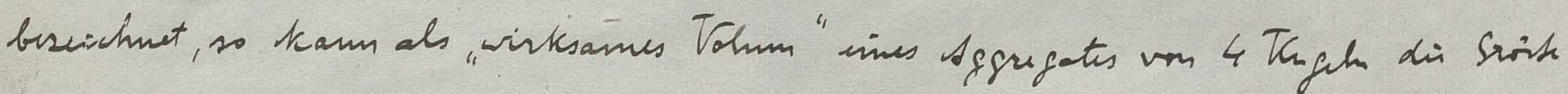

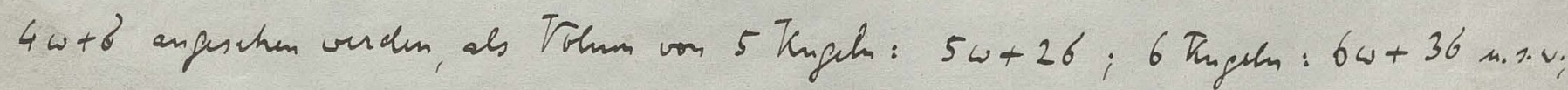

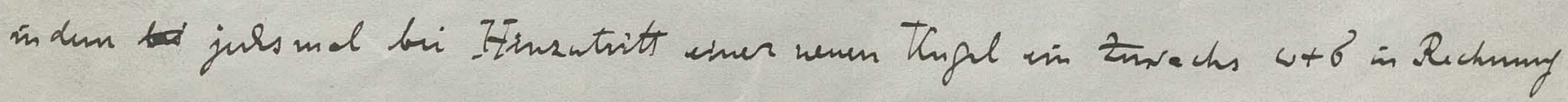
2n vithen ist.

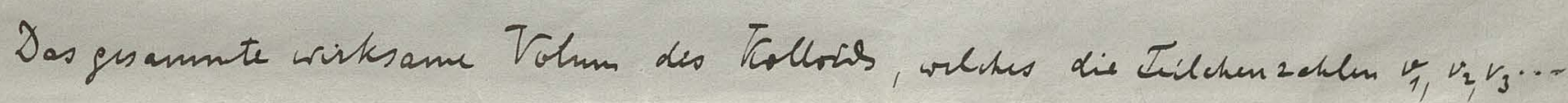

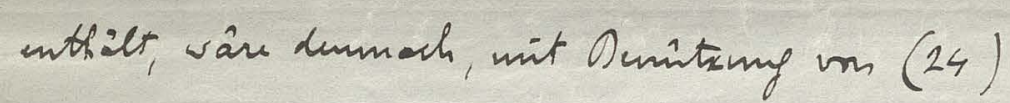
$\varphi=\left(v_{1}+2 v_{2}+3 v_{3}\right) \omega+v_{4}(4 \omega+6)+v_{5}(5 \omega+6)+v_{6}(6 \omega+36)+\cdots$

$=\omega \sum k v_{k}+\sigma \sum k v_{k+3}=\omega v_{0}+\sigma v_{0}\left(\frac{t}{t+T}\right)^{3}$ mithos seduscera Tolum

$\Phi=\omega+6\left[\frac{t}{t+T}\right]^{3}$

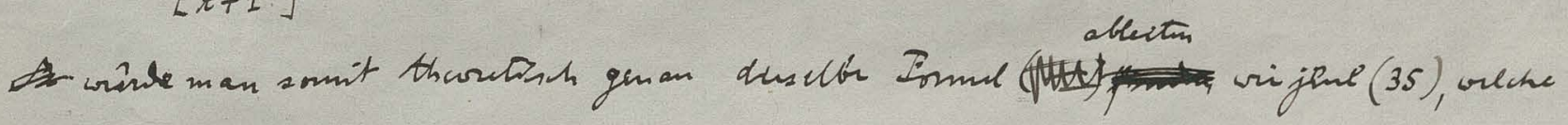

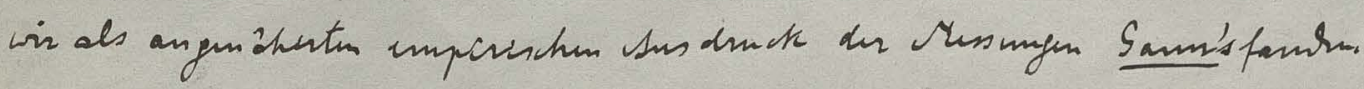

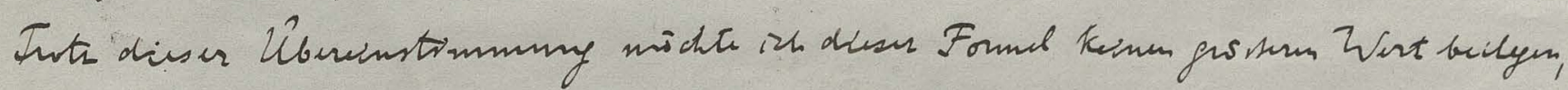

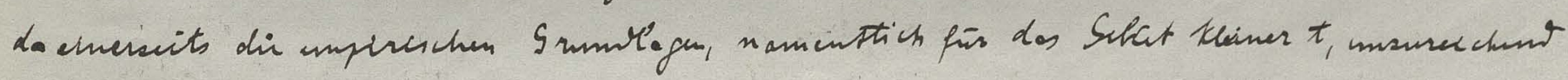

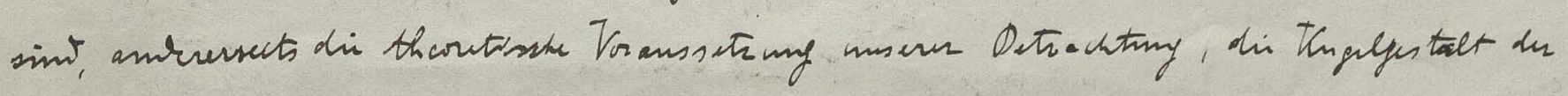

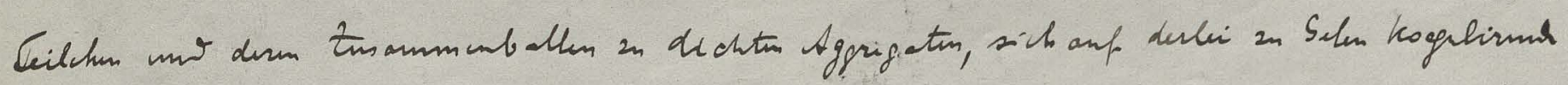

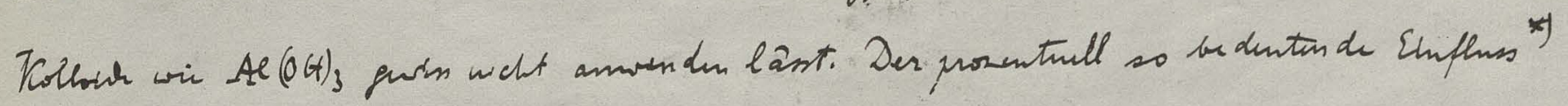

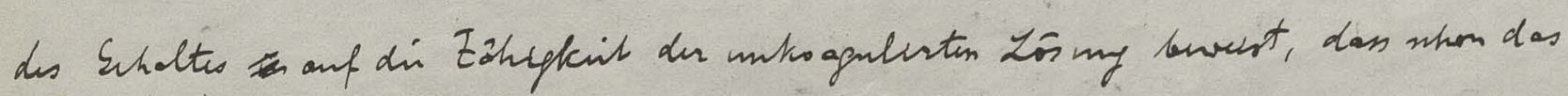

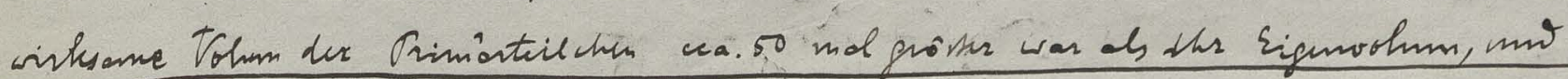

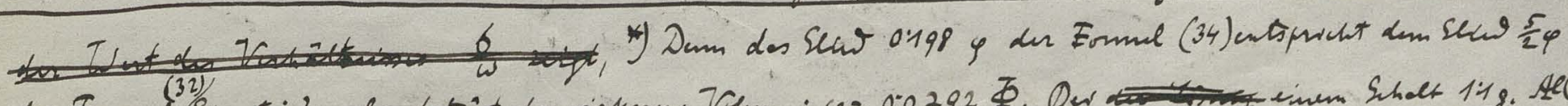

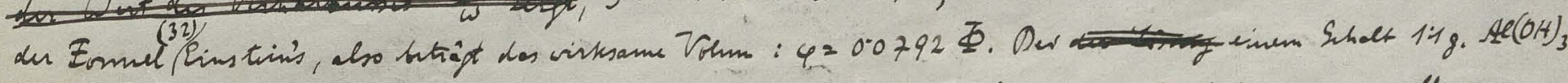

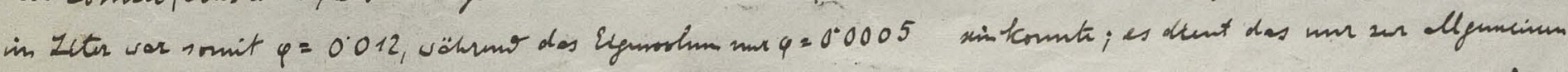

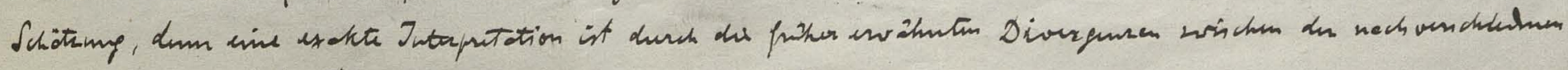

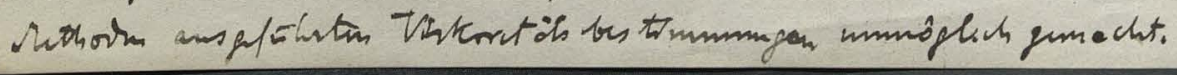




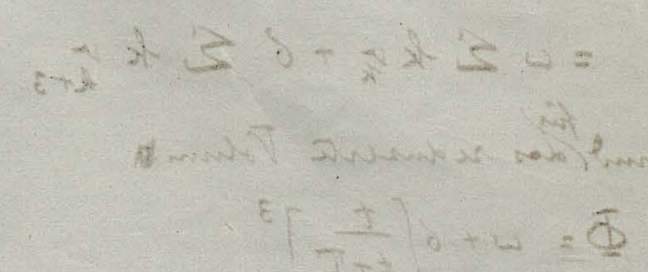

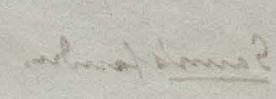




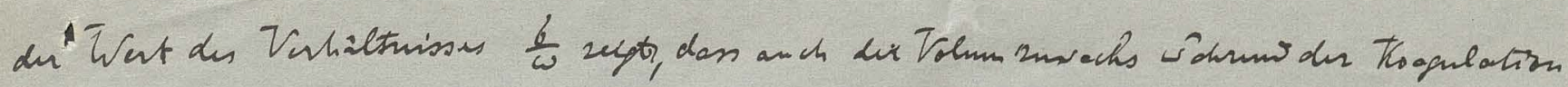

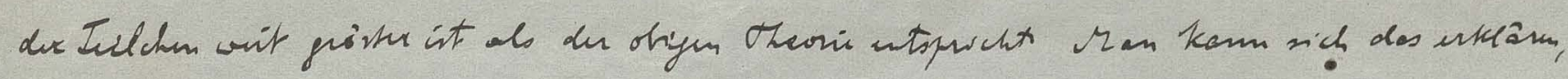

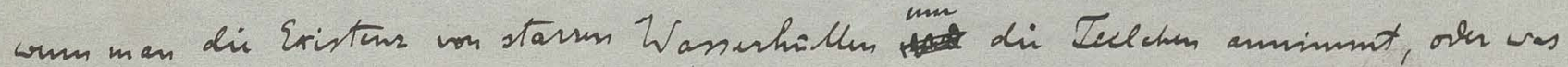

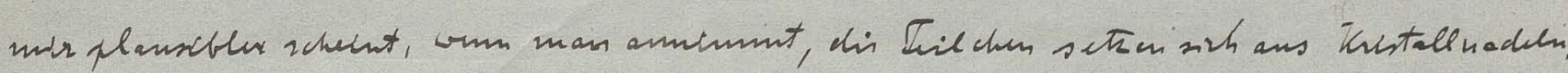

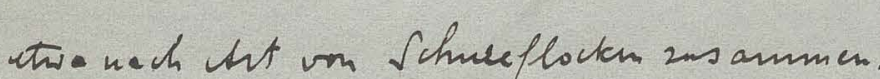

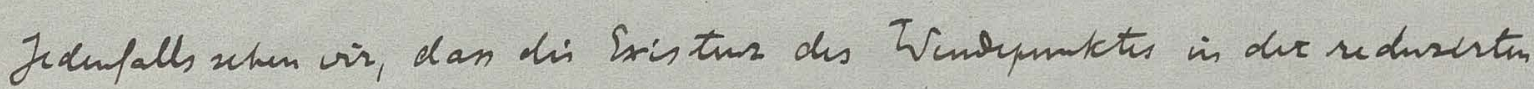

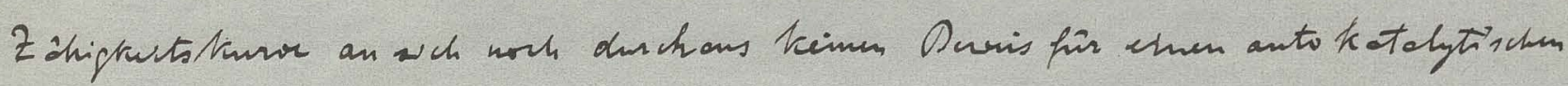

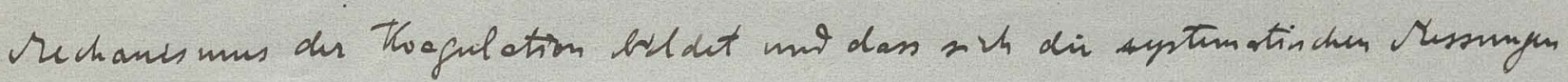

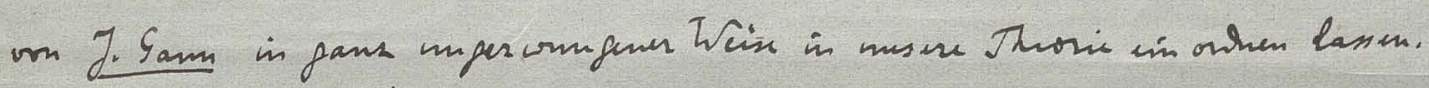

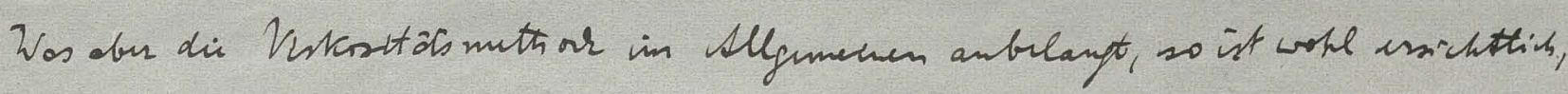

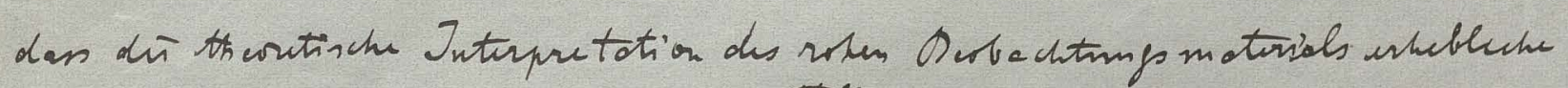

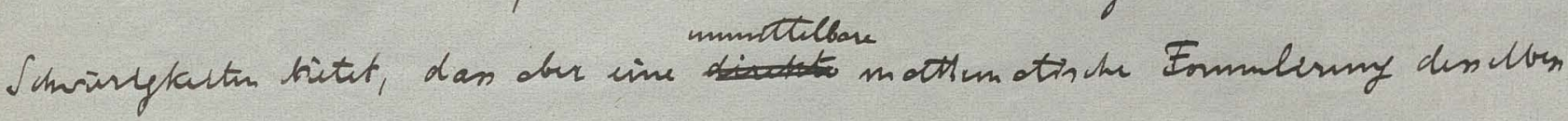

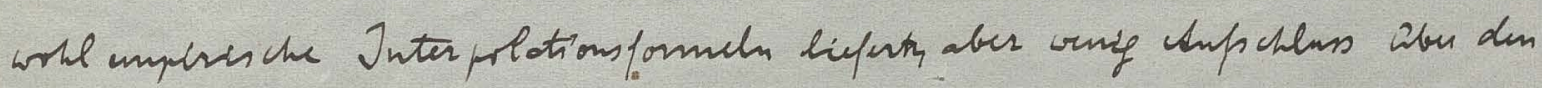

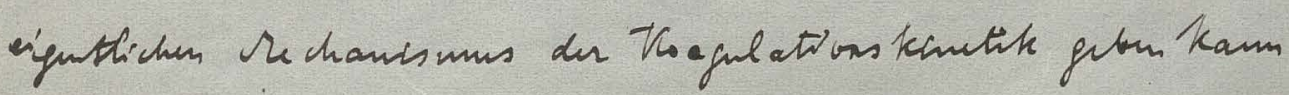

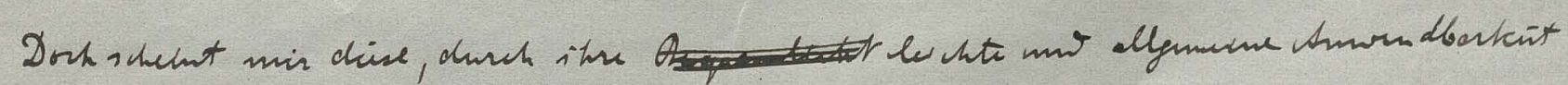

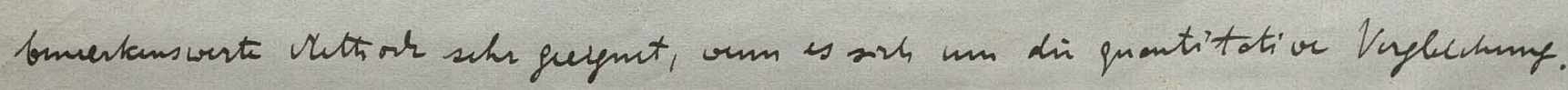

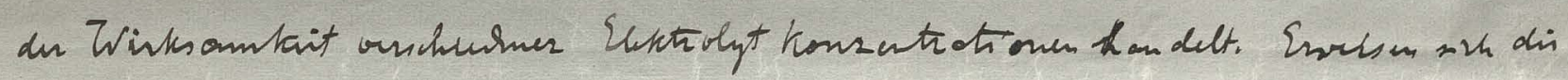

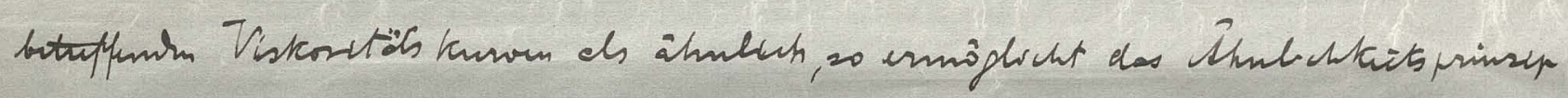

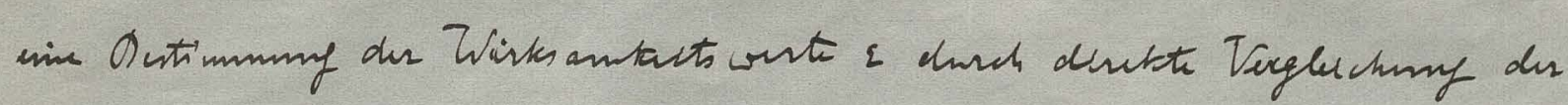

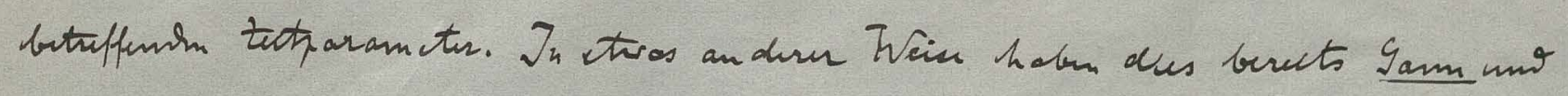

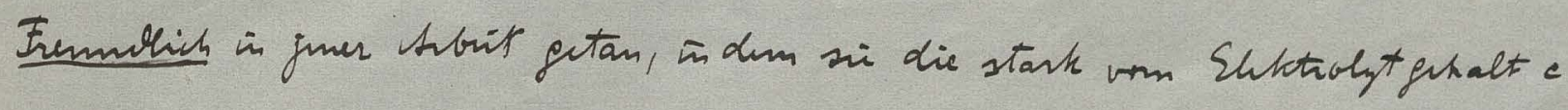

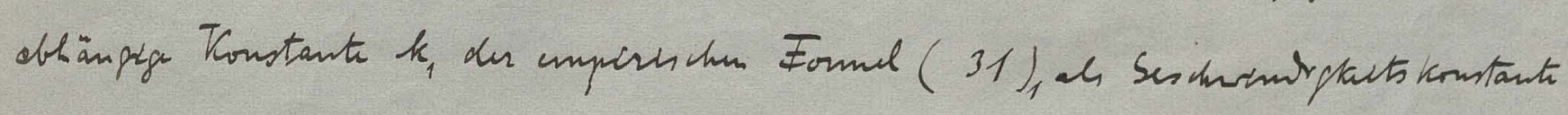

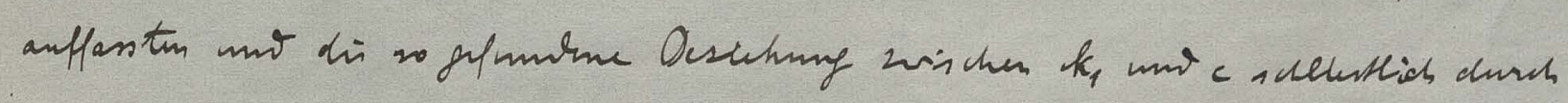

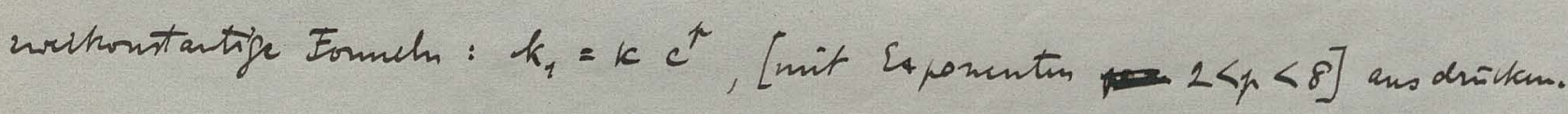

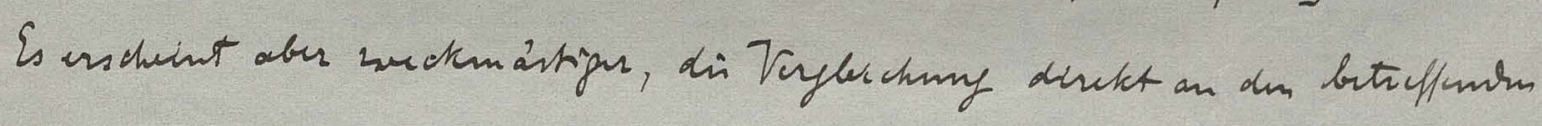

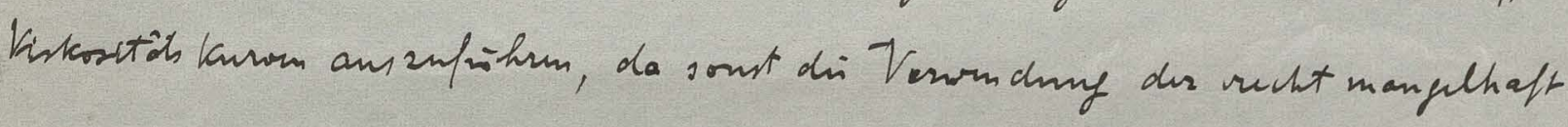

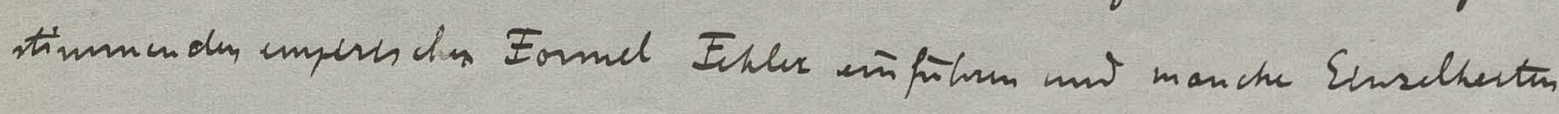

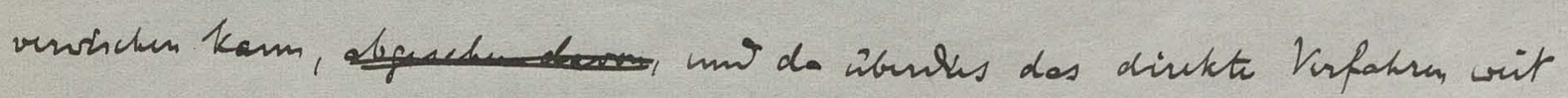
infocher itt.

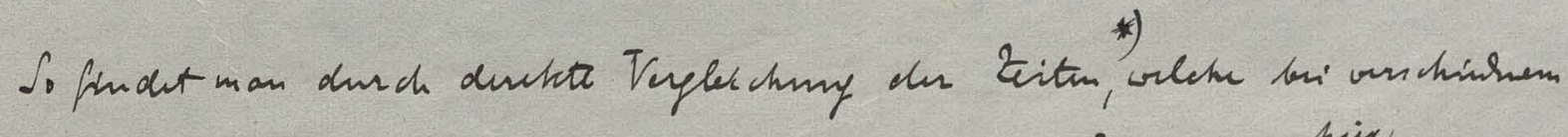

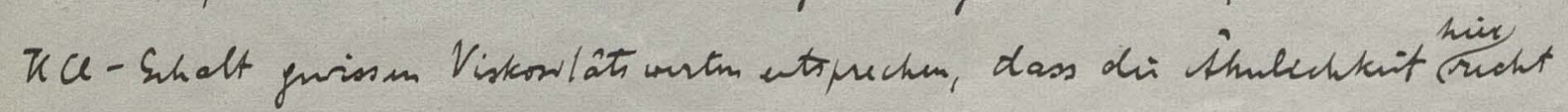

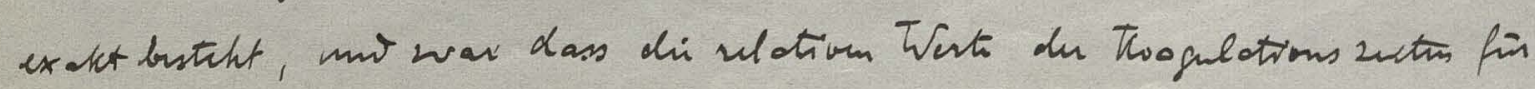

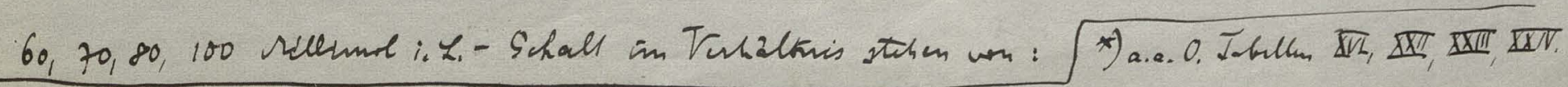




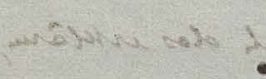

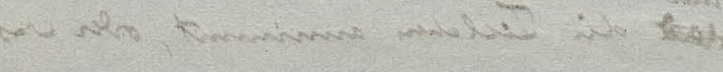

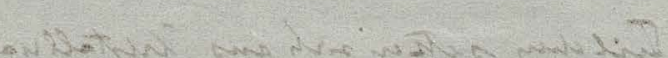

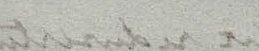

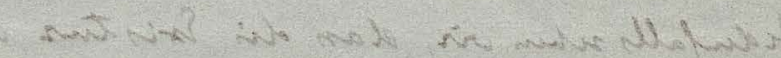

$+6$

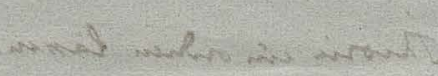

(A)

eri

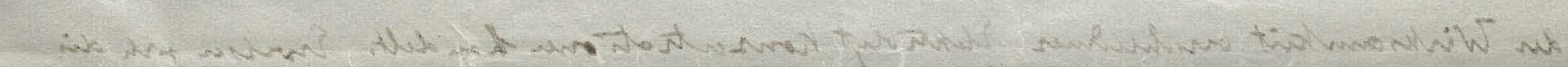

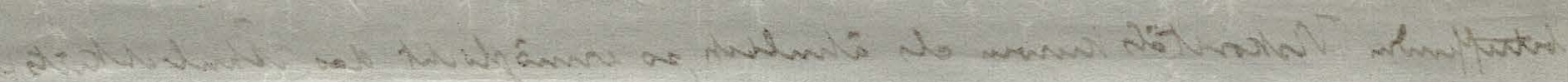
No

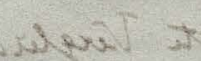

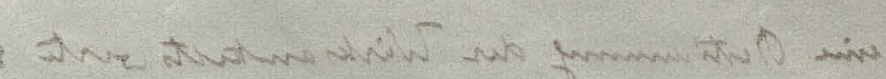

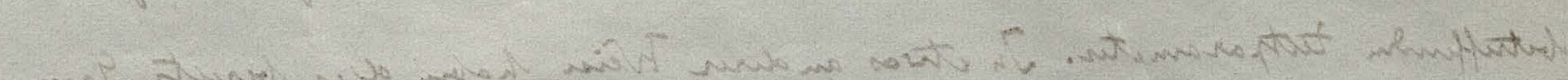

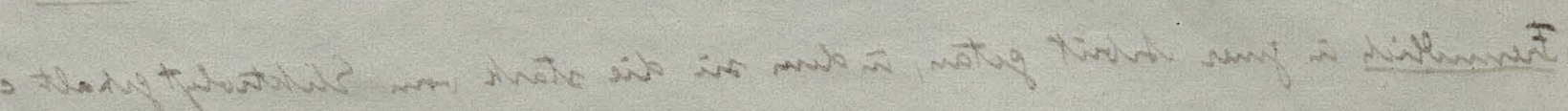

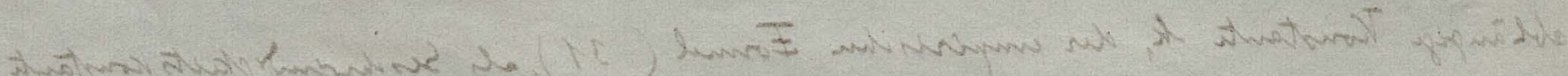

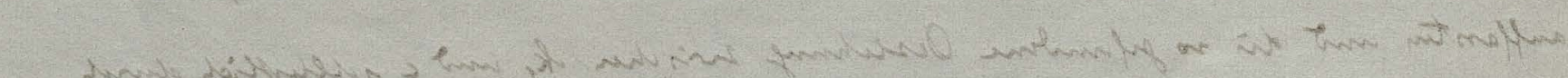

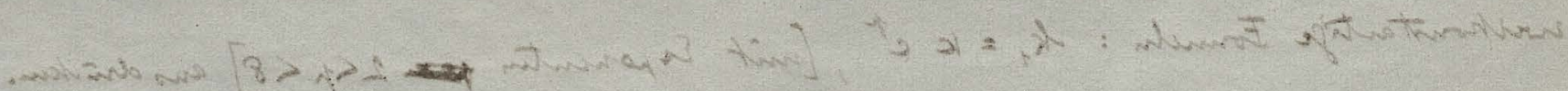

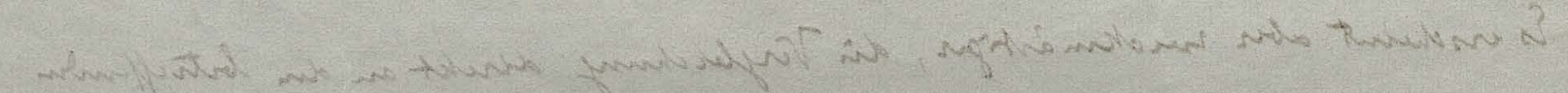

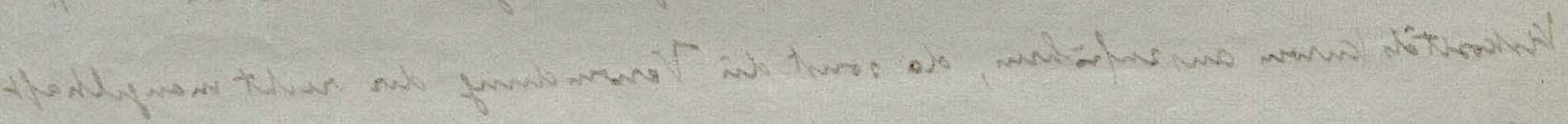

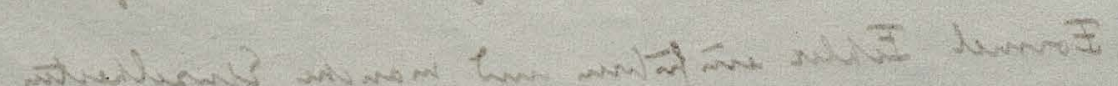

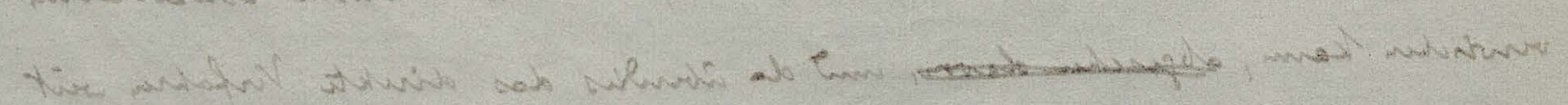

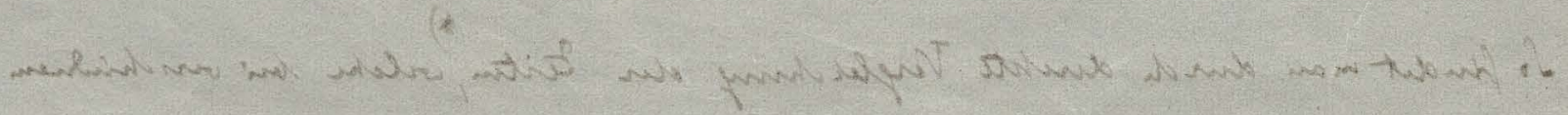

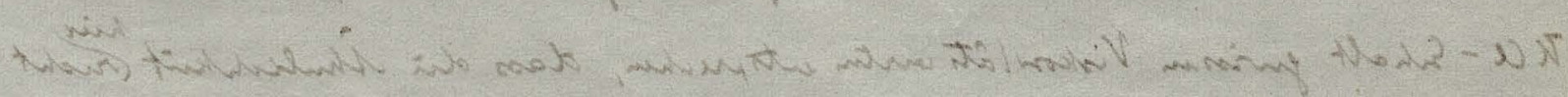

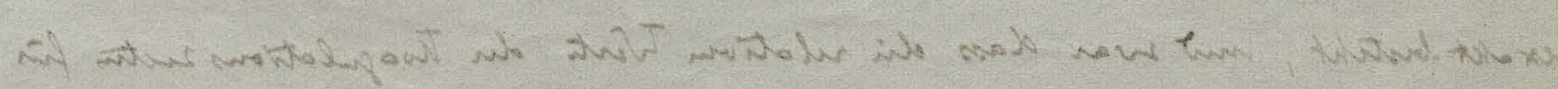

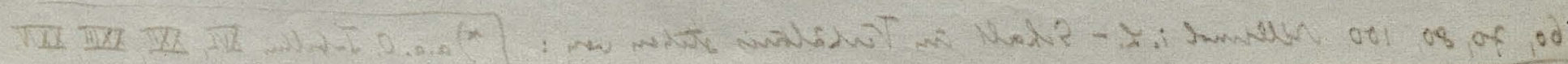


$T_{100}: I_{80}: I_{70}: T_{60}=1: 2 \cdot 75: 5 \cdot 92: 12 \cdot 7$

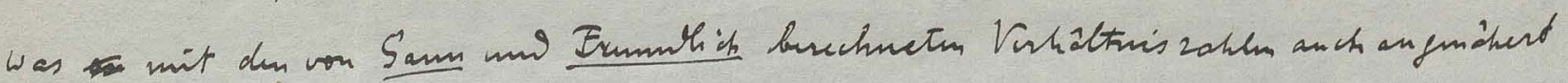

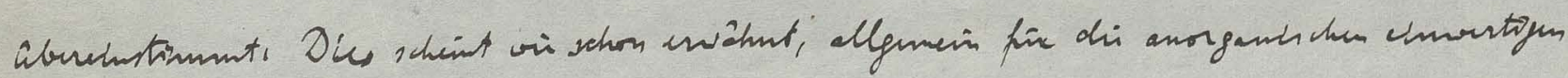
Swionen en gltm.

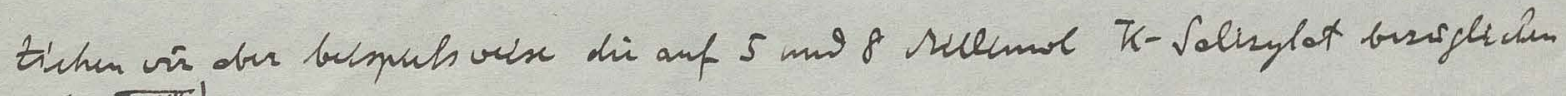

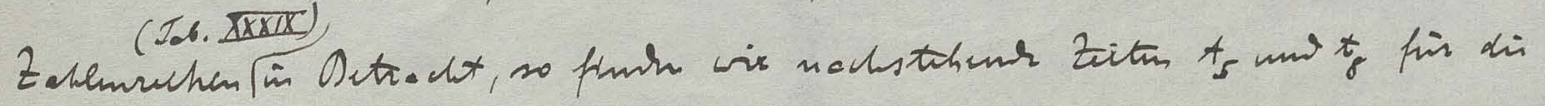
enguhorifum Vistaritits outs $\mu$ :

\begin{tabular}{|c||c|c|c|c|c|c|c|c|c|c|c|}
\hline$\mu$ & 52.4 & 52.5 & 52.9 & 53.8 & 550 & 57.7 & 62.0 & 6507 & 67.4 & 687 & 70.1 \\
\hline$t_{5}$ & 0 & 12.5 & 30 & 56 & 78 & 107 & 135 & 168 & 186 & 206 & 240 \\
\hline$t_{8}$ & 0 & 2 & 5 & 10 & 15 & 22 & 30 & 40 & 50 & 60 & 75 \\
\hline$t_{5 / t_{8}}$ & & 6.3 & 6.0 & 5.6 & 5.2 & 4.9 & 4.5 & 4.2 & 303 & 3.5 & 300 \\
\hline
\end{tabular}

Di unjune Sutorm angusendete Oeschumpmethor da dir

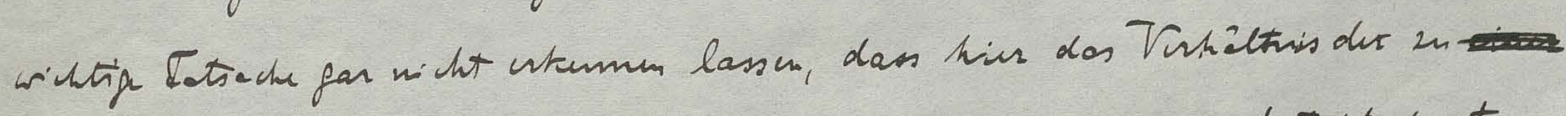

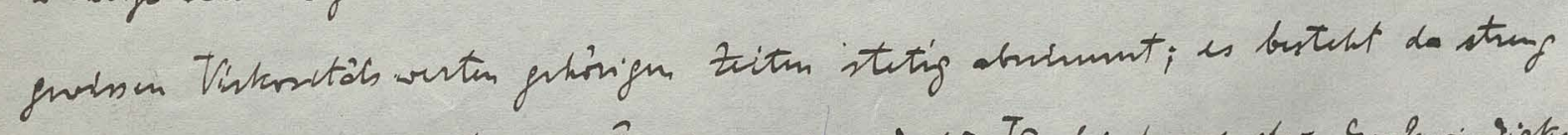

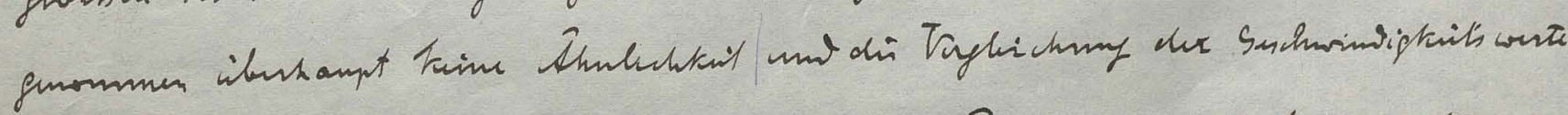

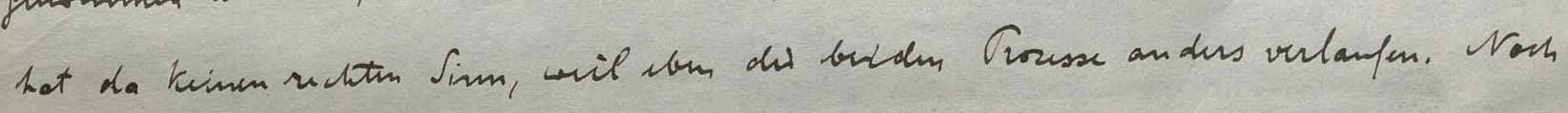

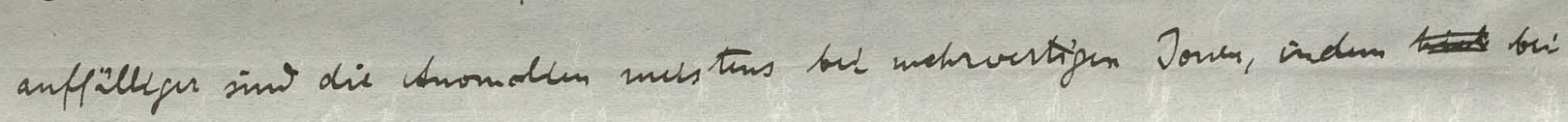

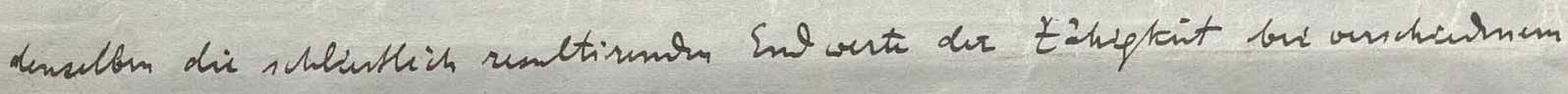

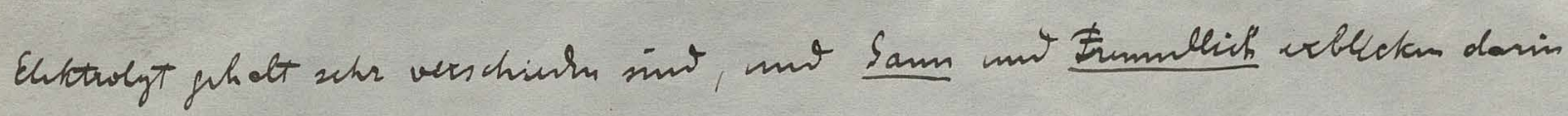

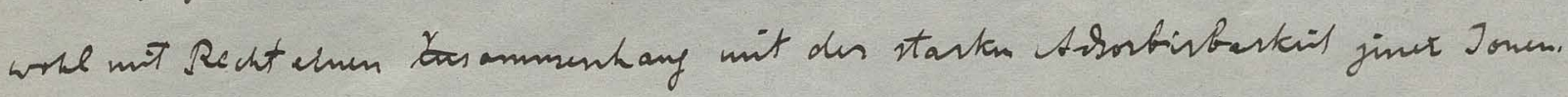

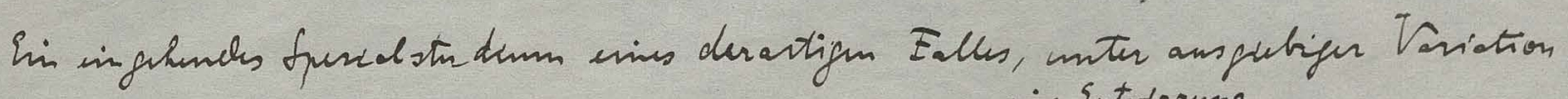

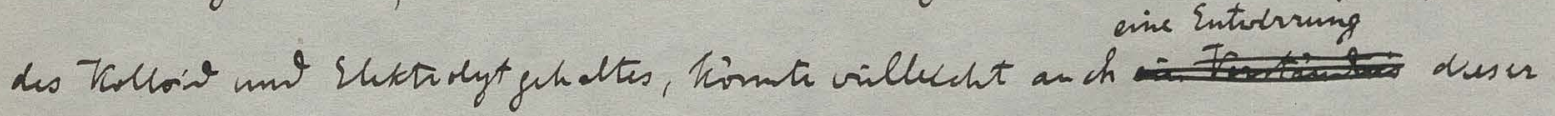

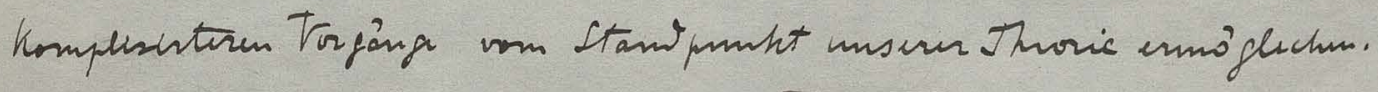

\section{Vugleich mat chumescher Tinutits.}

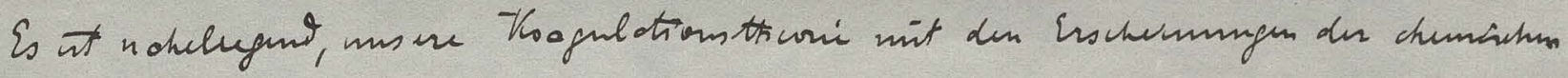

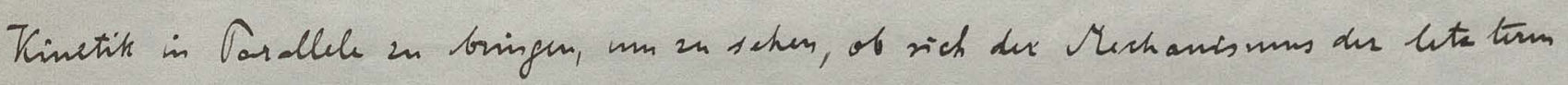

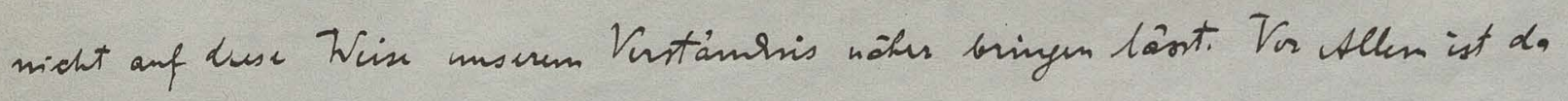

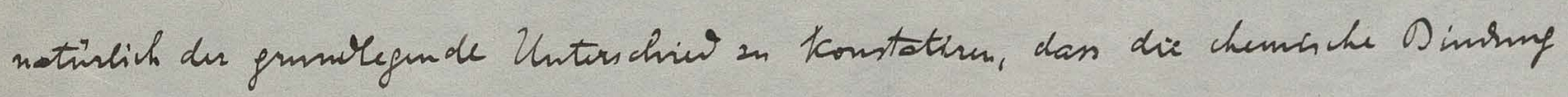

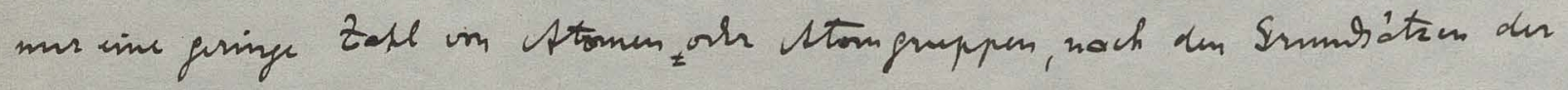

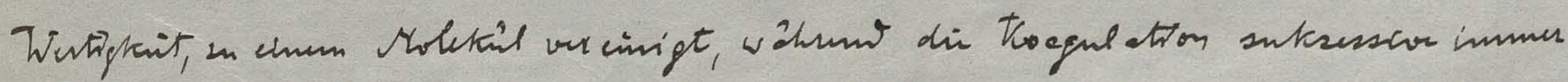

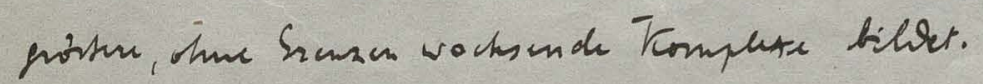

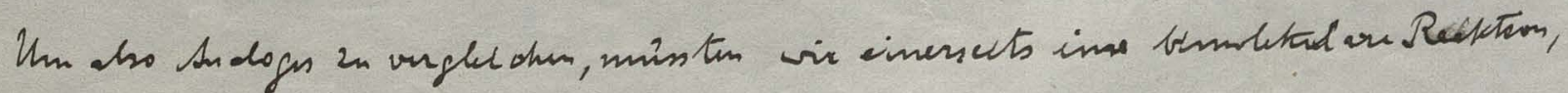

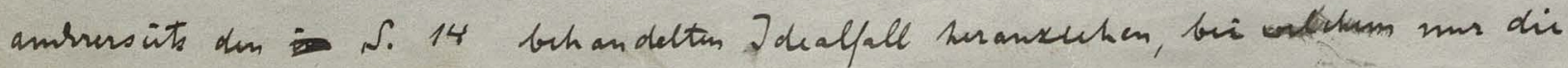




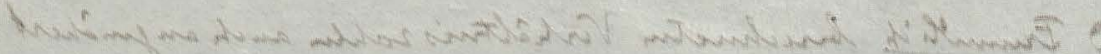

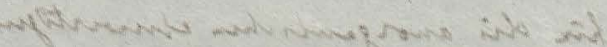

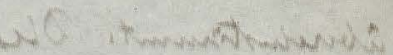

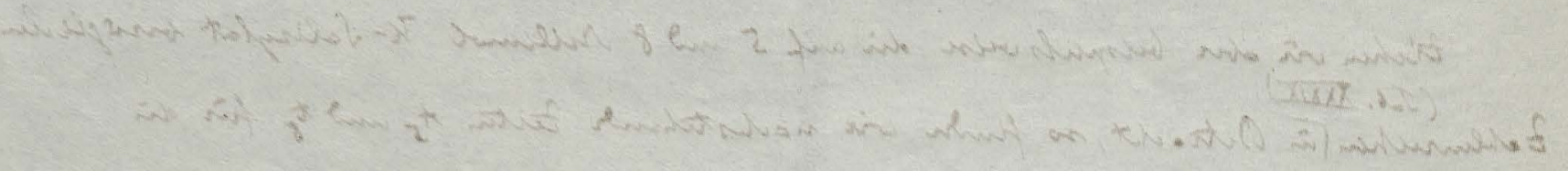

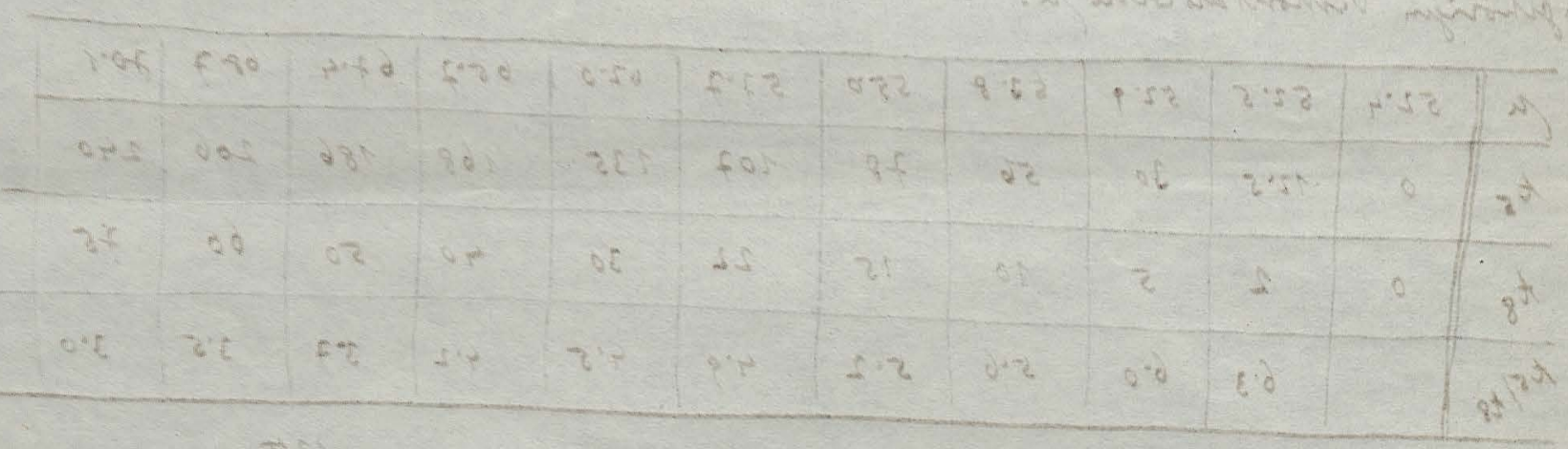

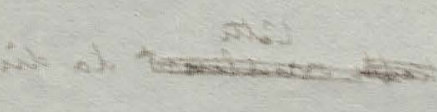

sickese

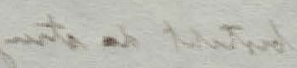

wh the

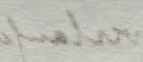

$$
\text { ats }
$$

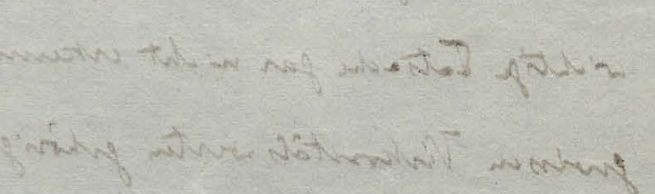

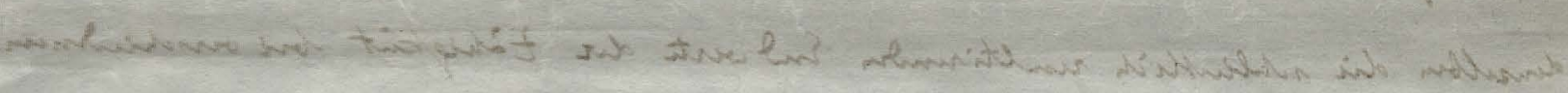

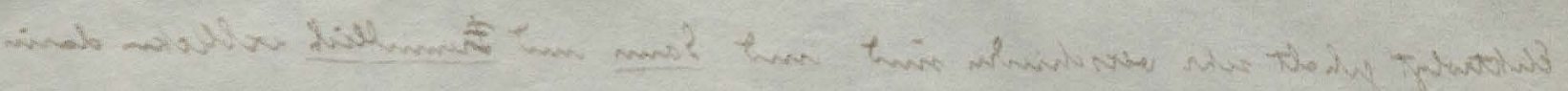

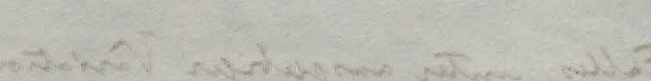

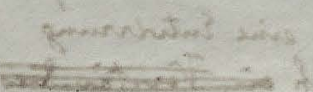

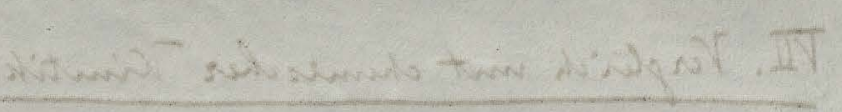

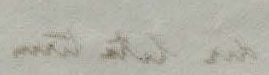

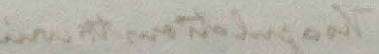
enmentivion to 3

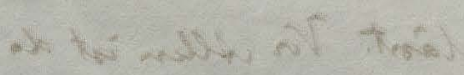
Kandenc $\mathrm{O}$

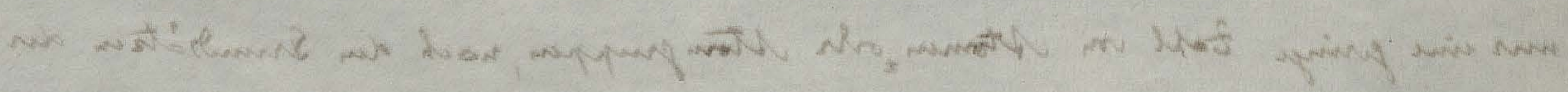

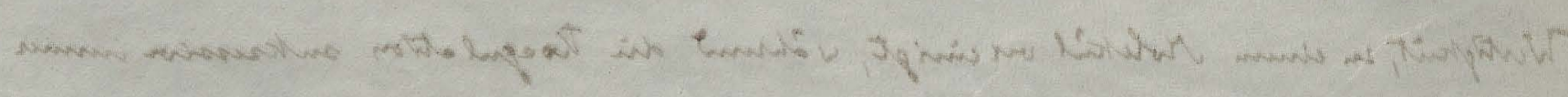

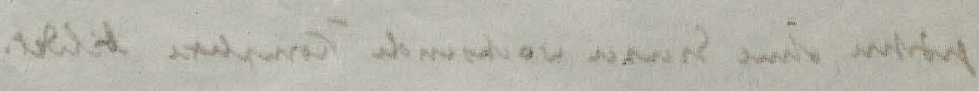

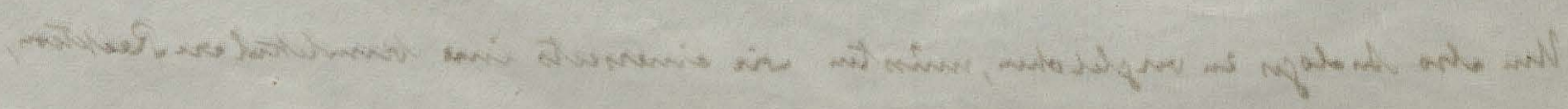

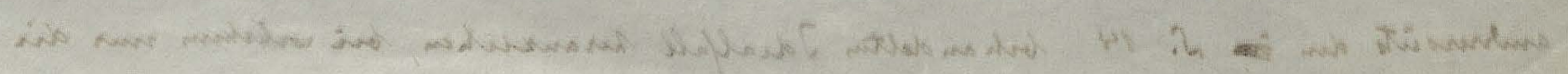




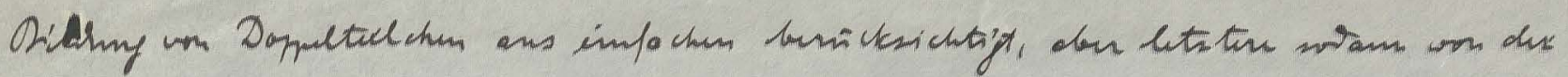

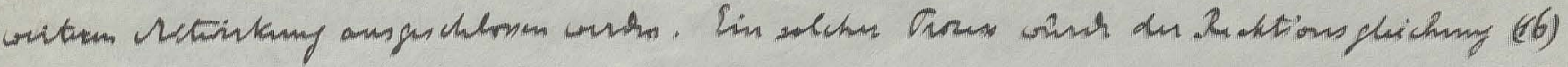
funingm *

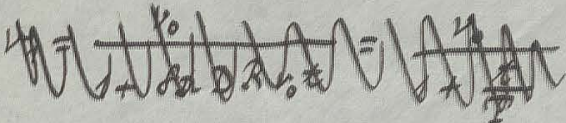

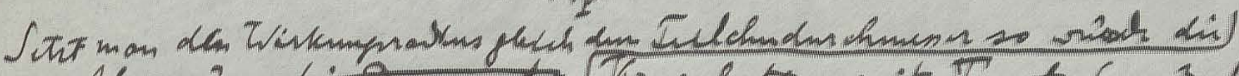

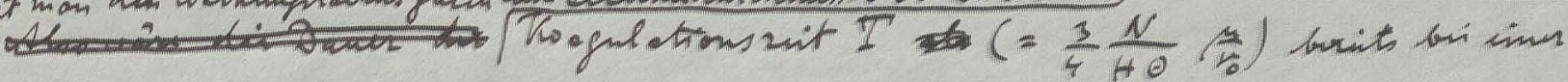

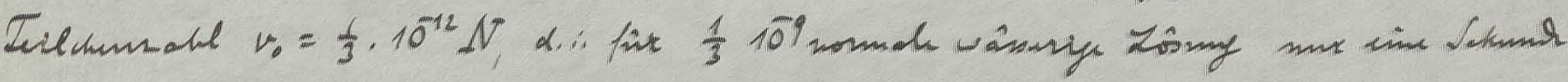

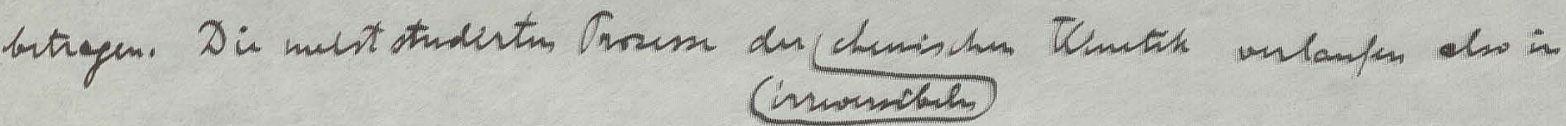

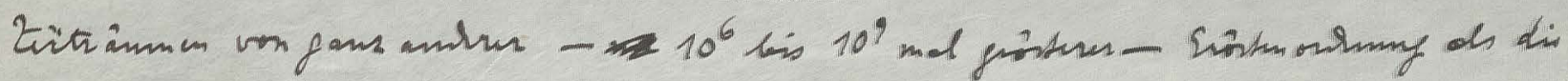

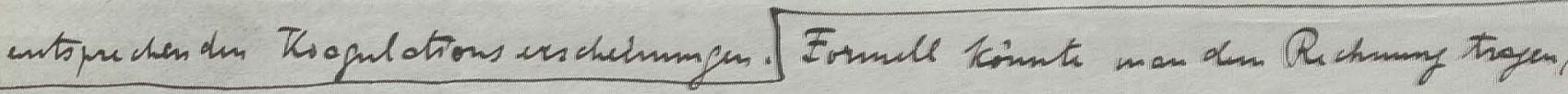

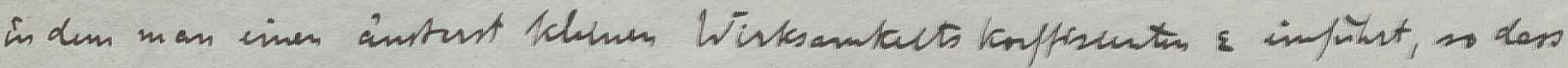

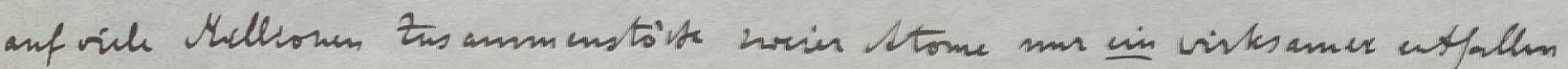

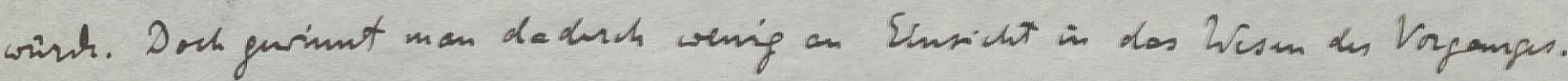

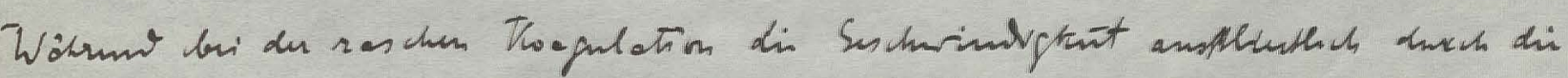

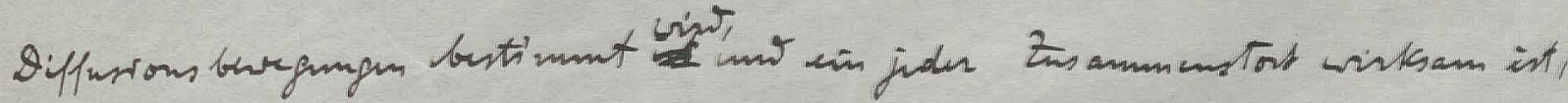

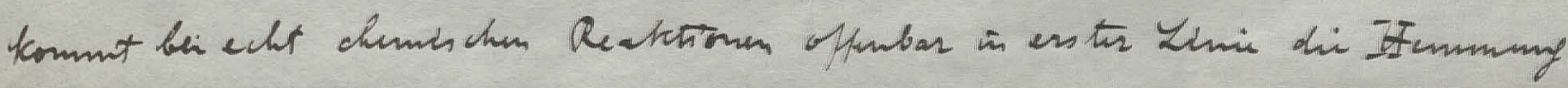

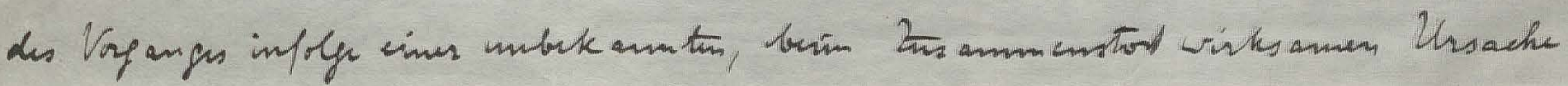

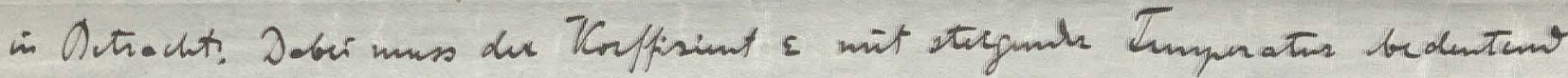

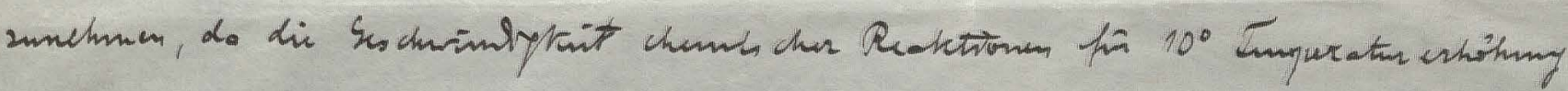

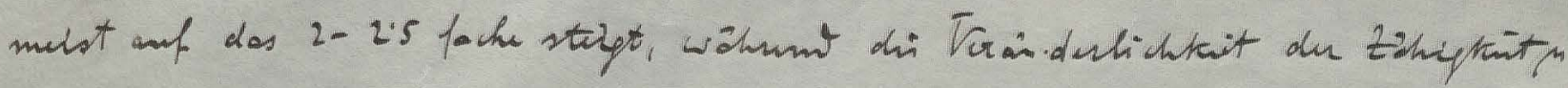

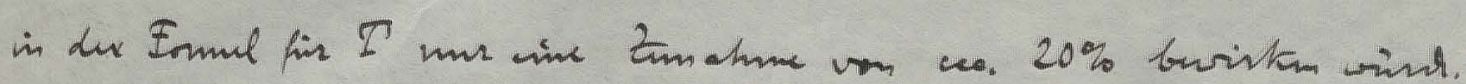

Orkanntlich hat wh Doltrmann in simm Dissiations thasio dunch

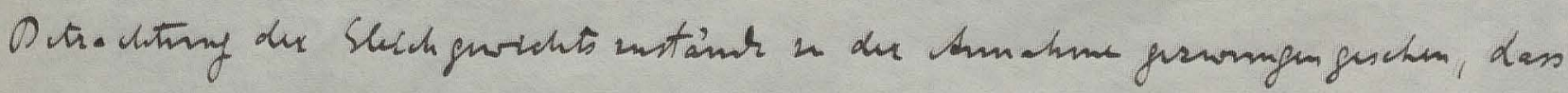

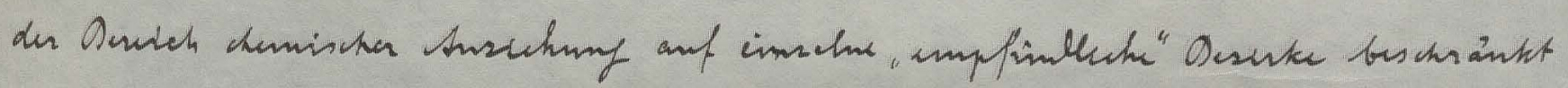

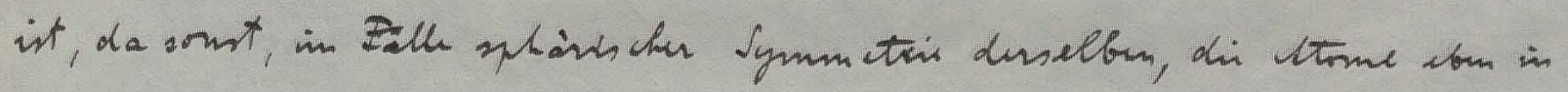

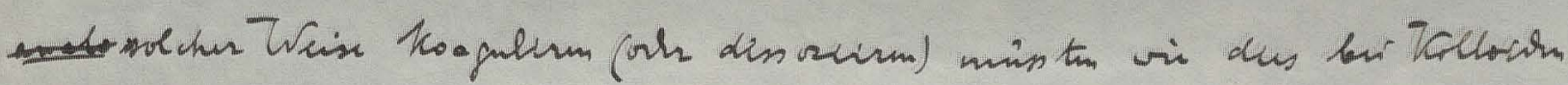

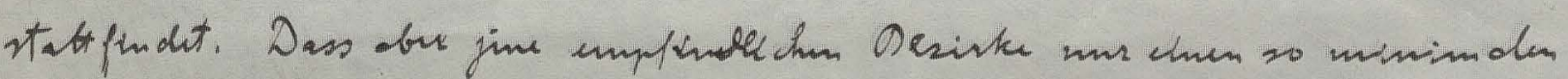

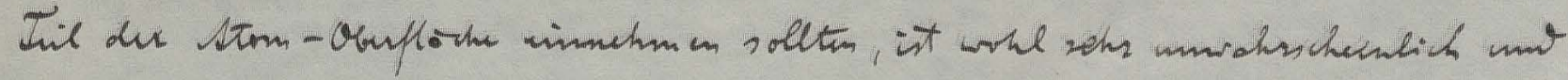

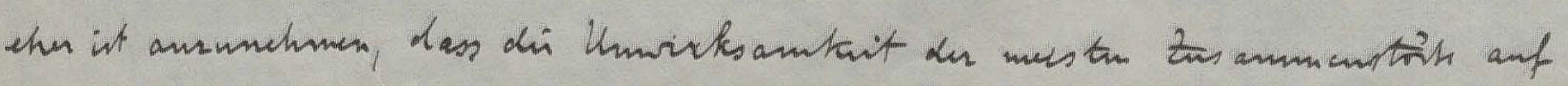

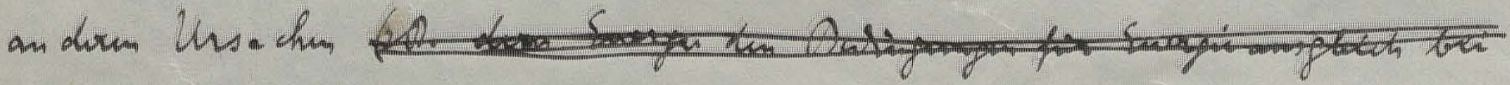

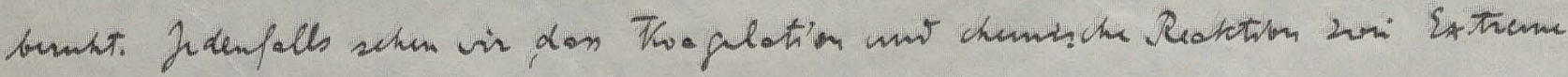

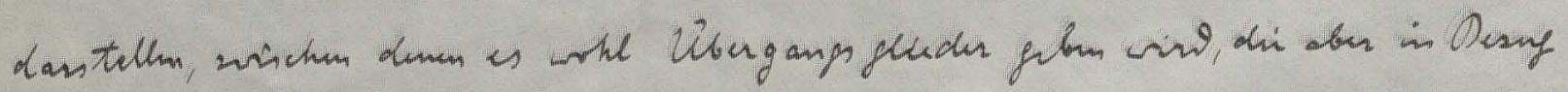

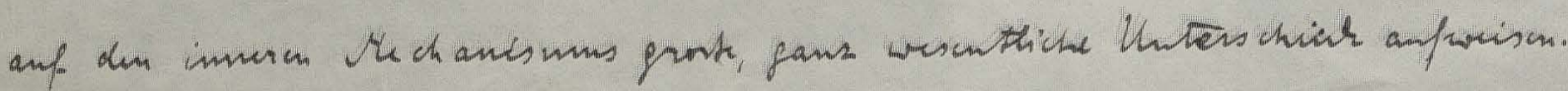


(d)

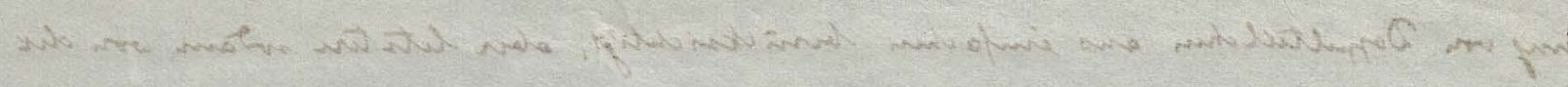

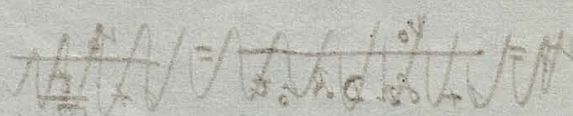

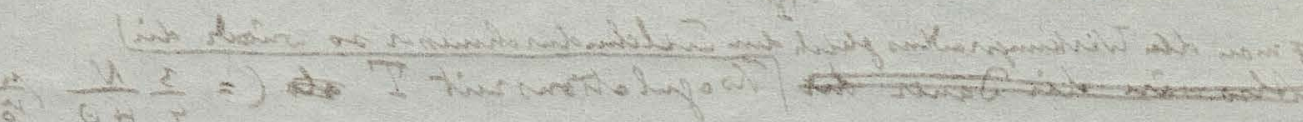

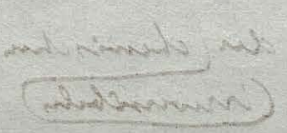

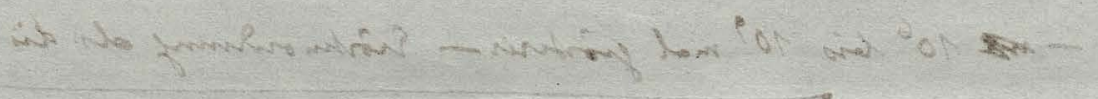

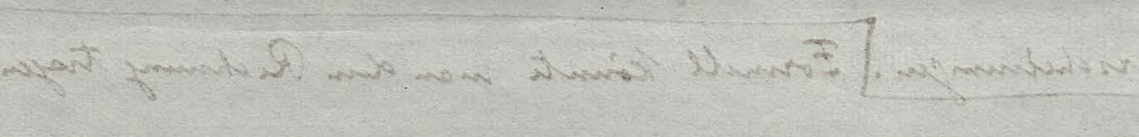

$$
\begin{aligned}
& -4
\end{aligned}
$$

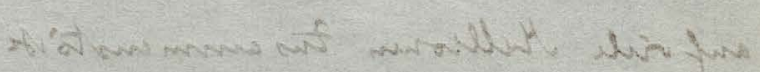

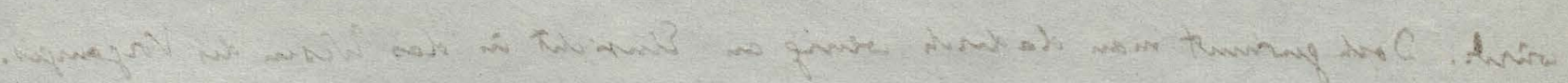

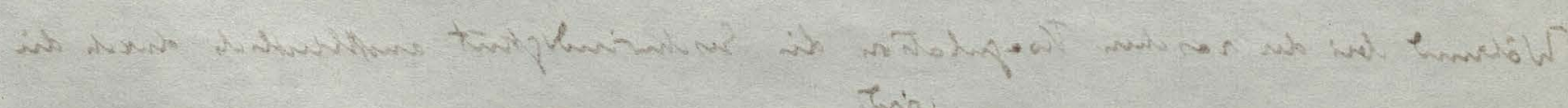

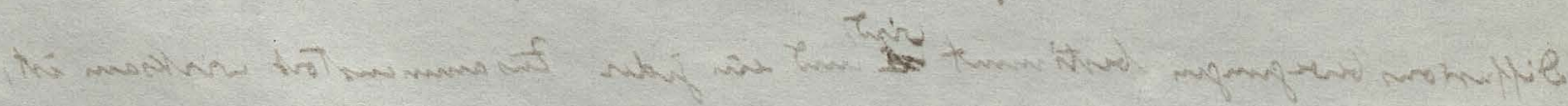

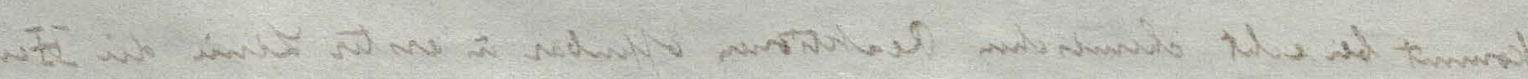

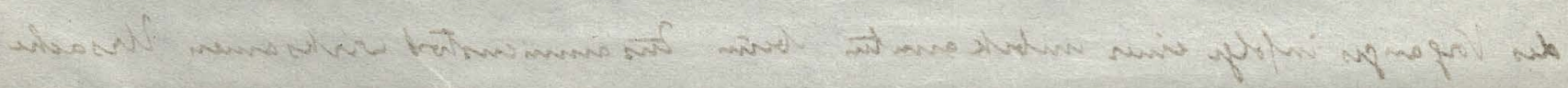

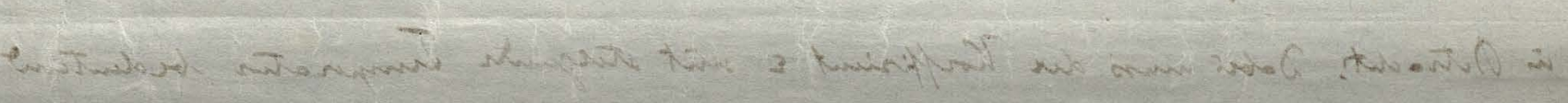

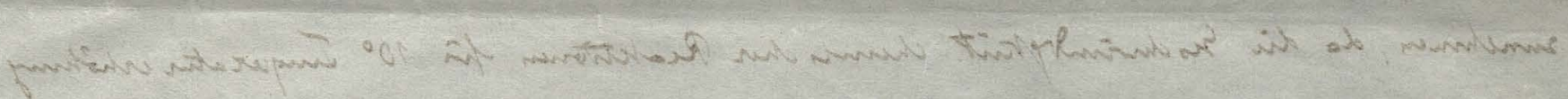

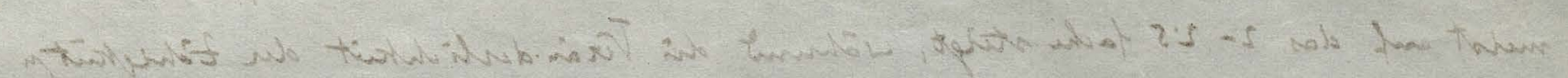

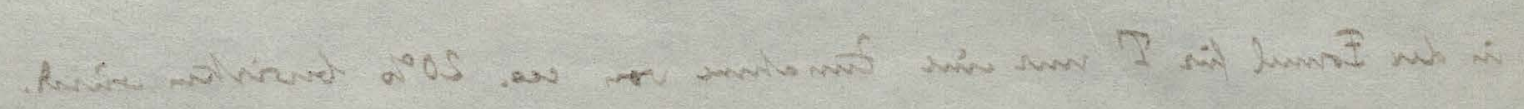

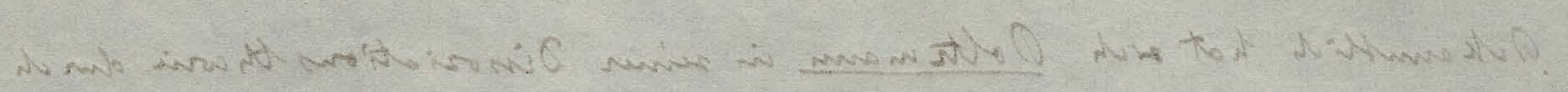

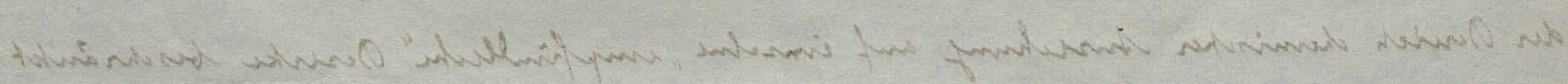

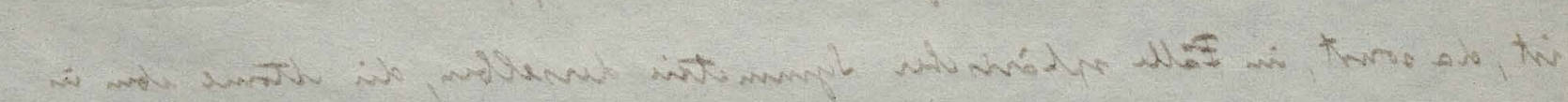

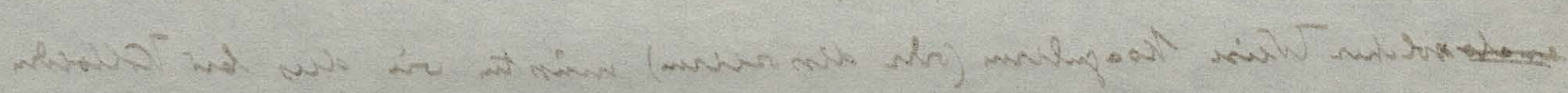

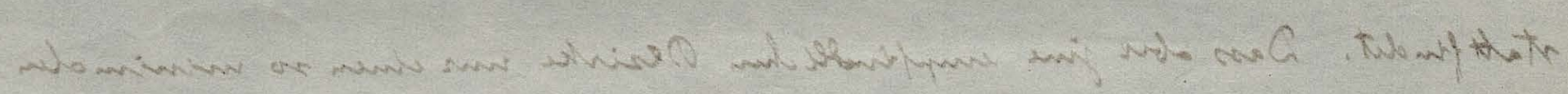

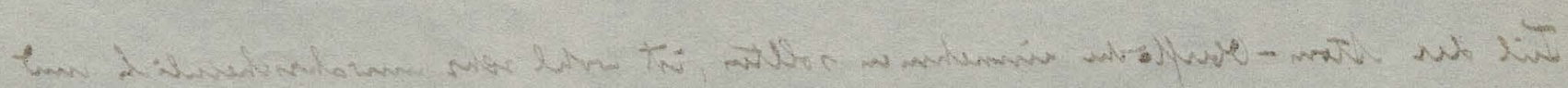

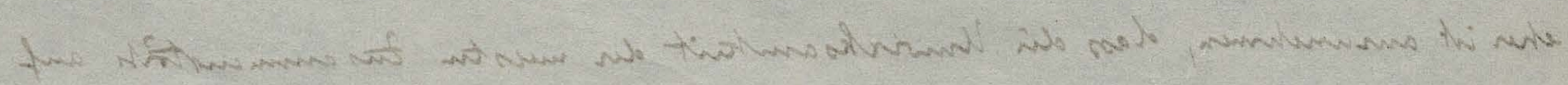

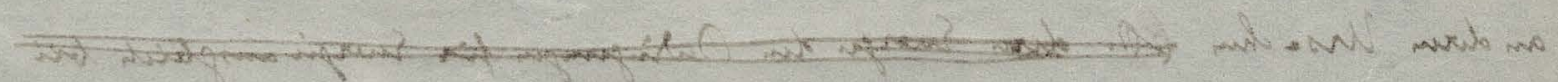

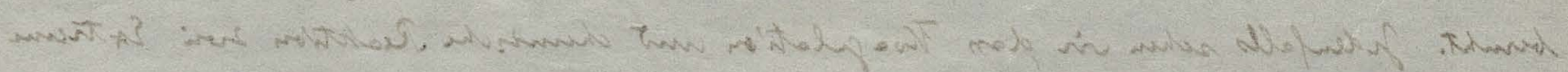

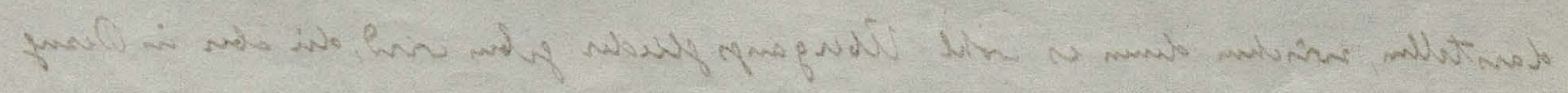

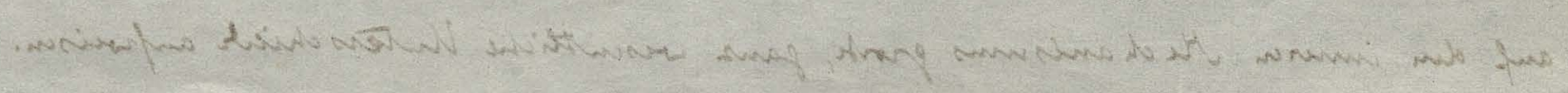




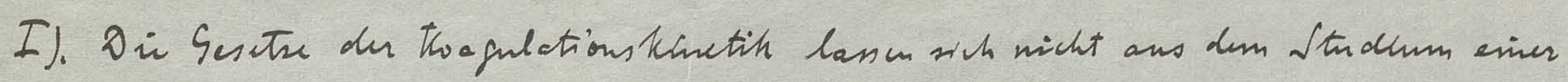

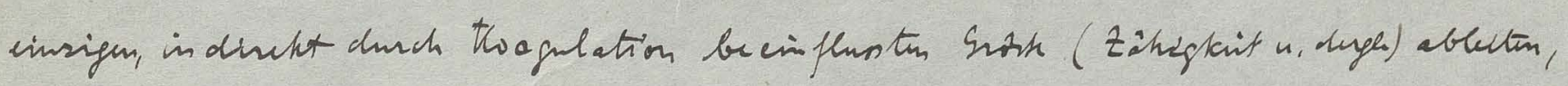

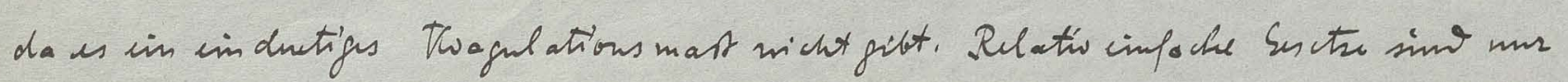

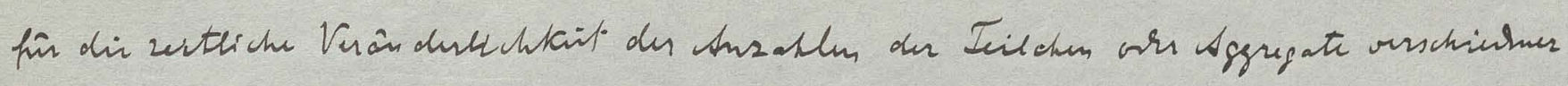
Totegorm in exrartem.

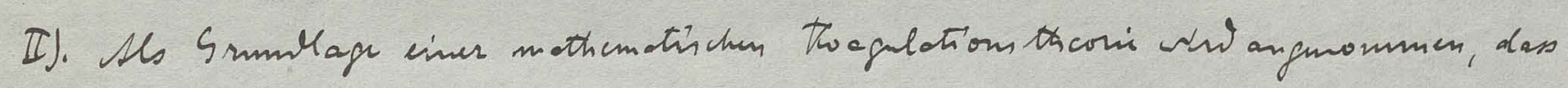

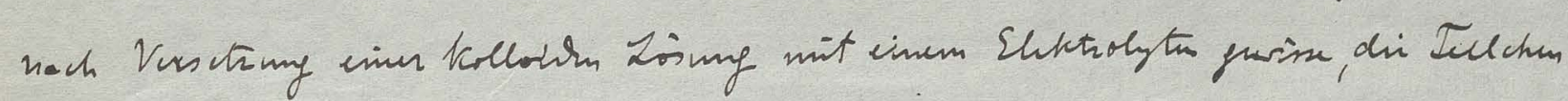

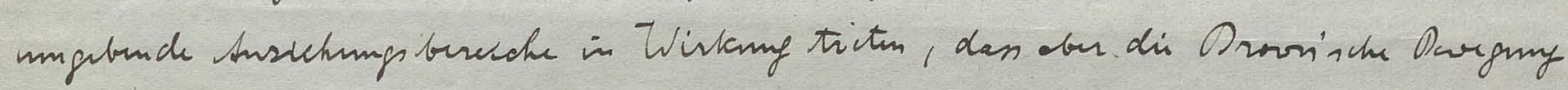

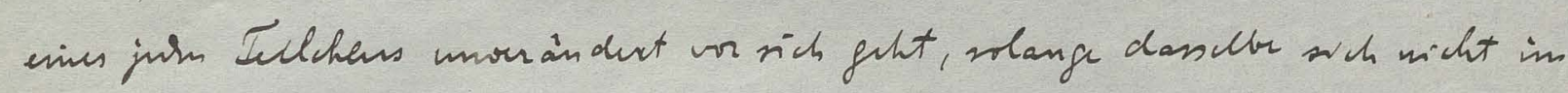

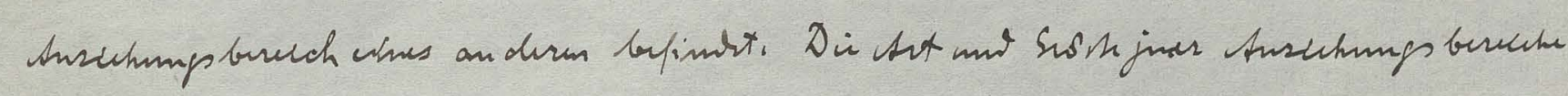

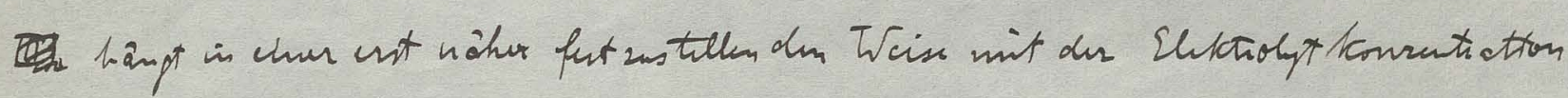

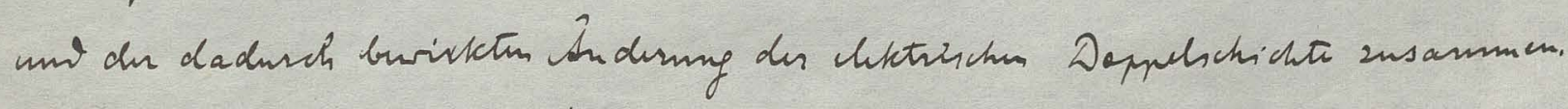

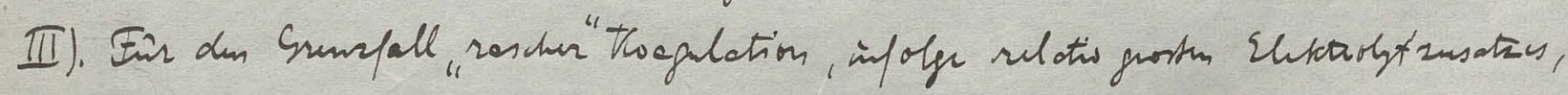

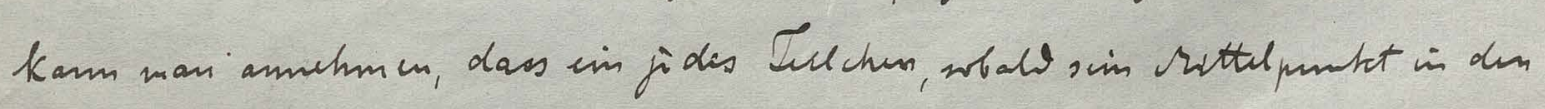

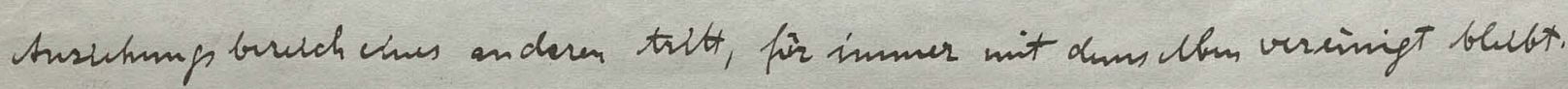

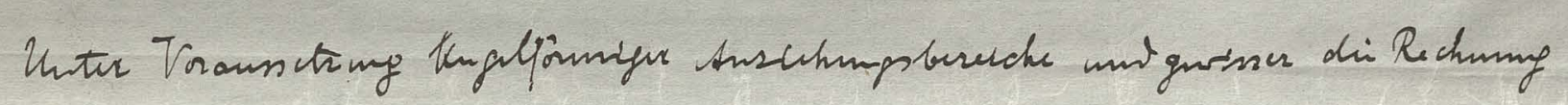

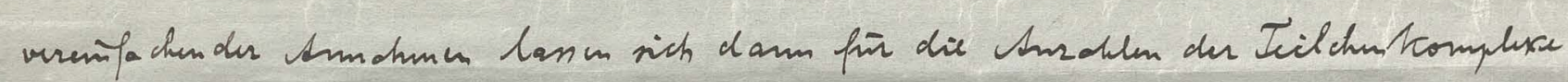

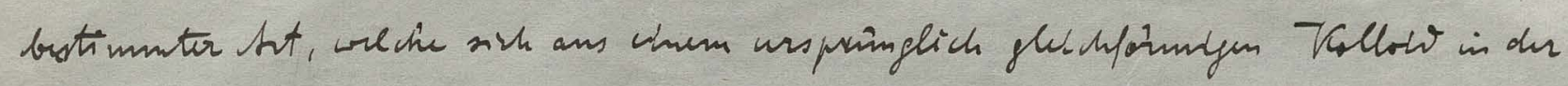

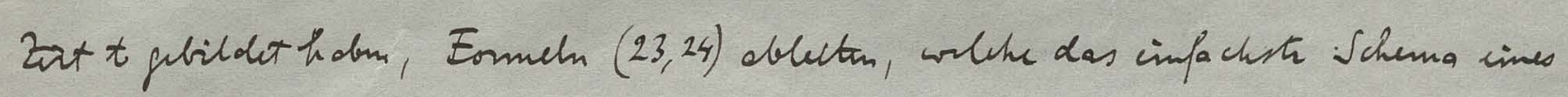

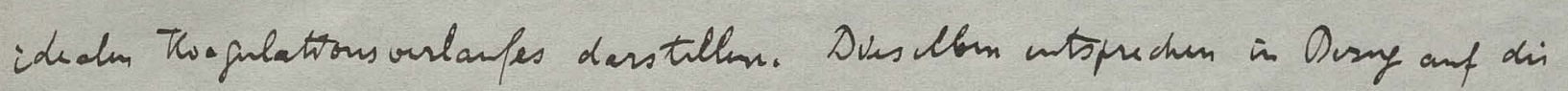

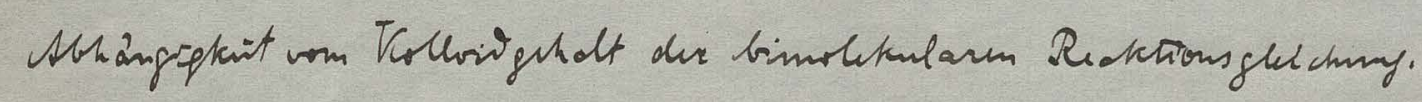

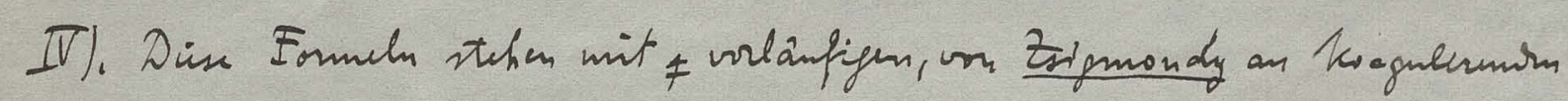

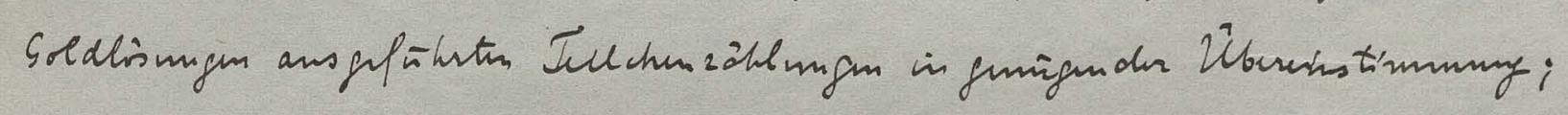

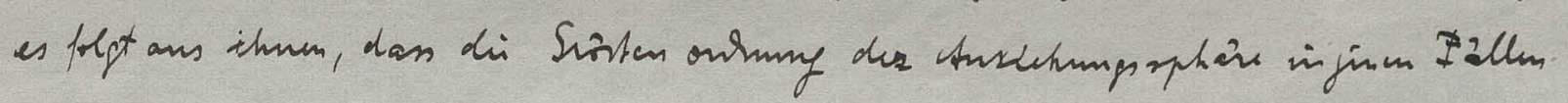

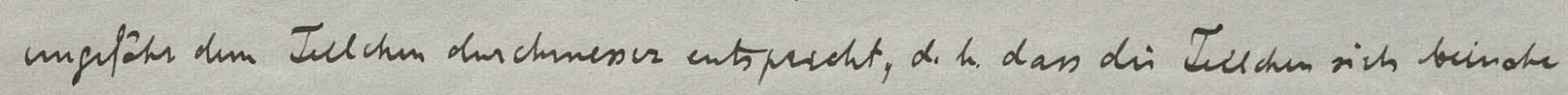

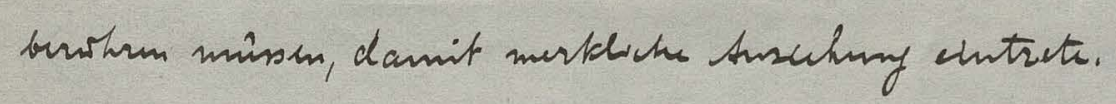

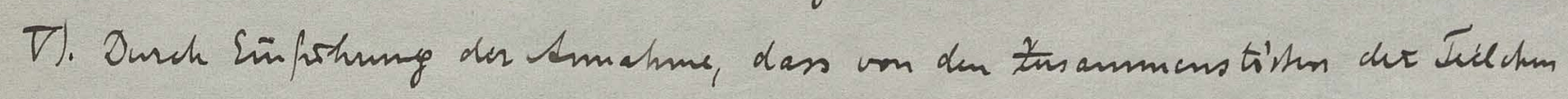

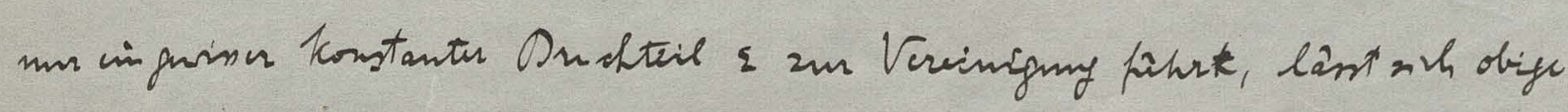

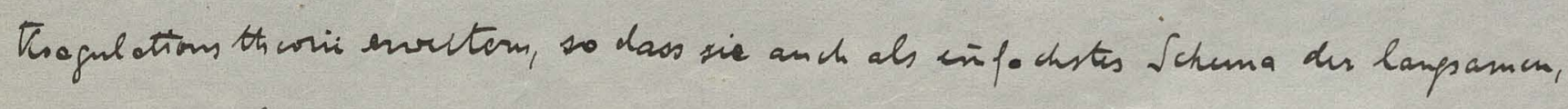

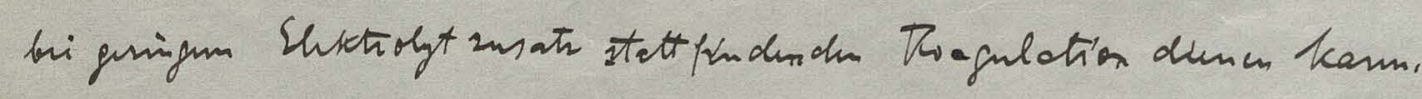

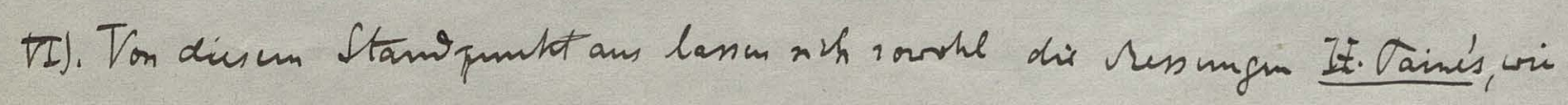

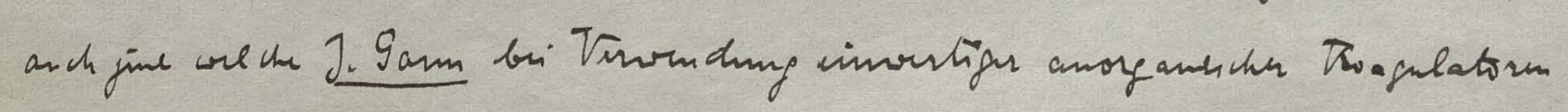


$n+k$

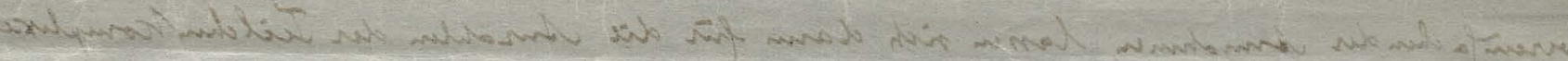

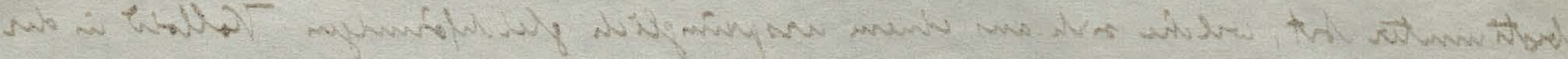

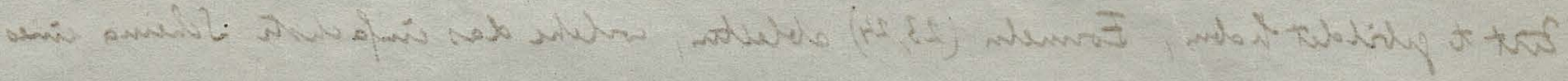

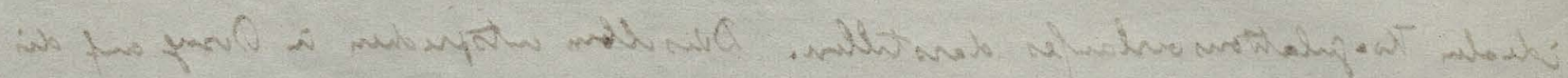

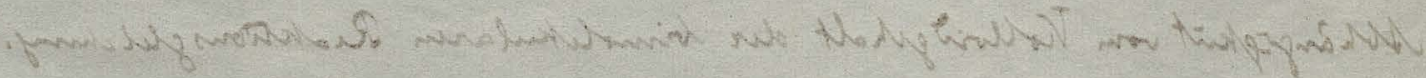

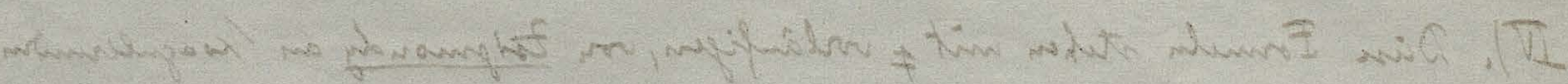

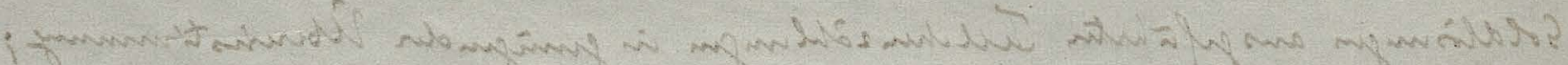

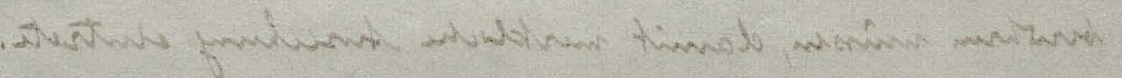

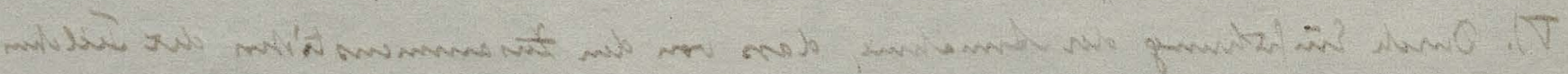

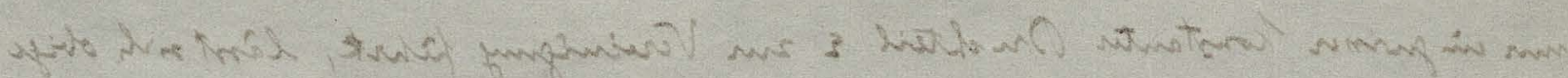

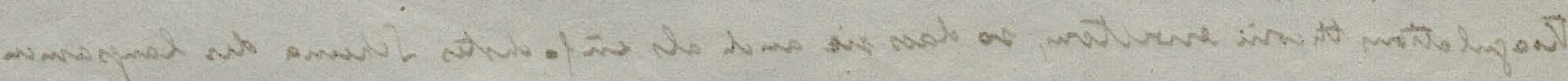

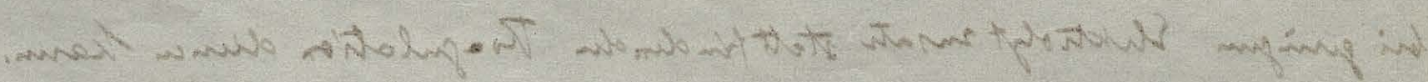

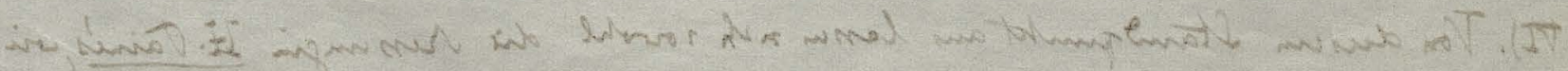

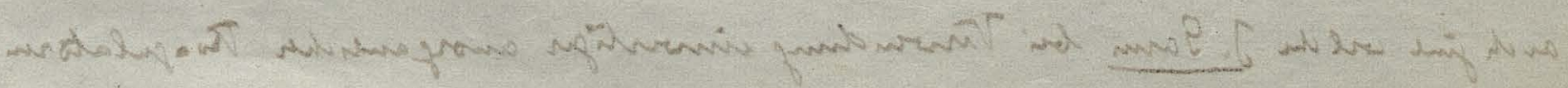




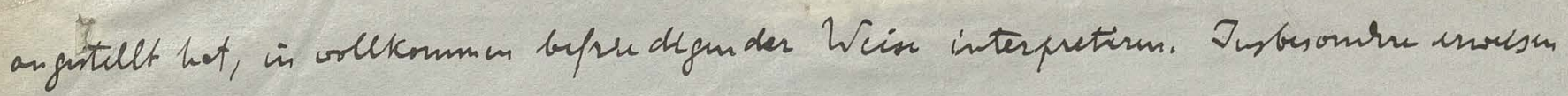

35

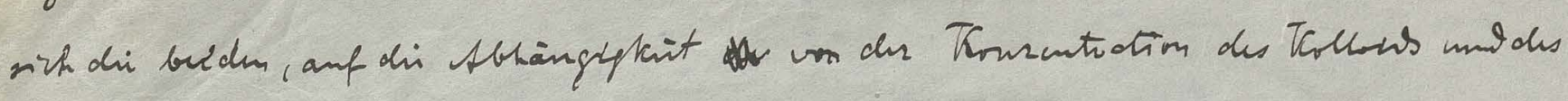

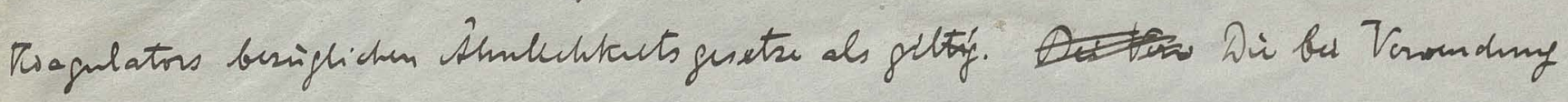

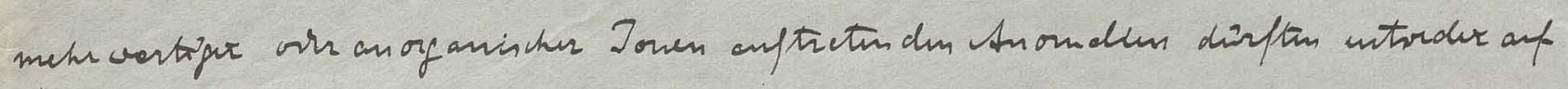

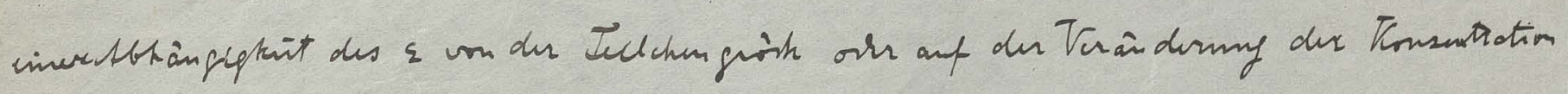

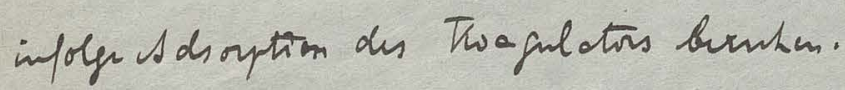

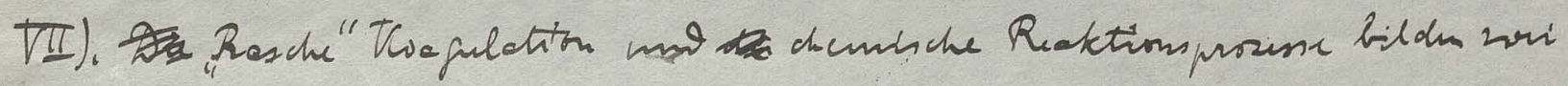

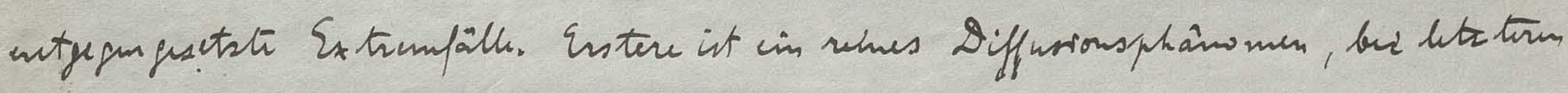

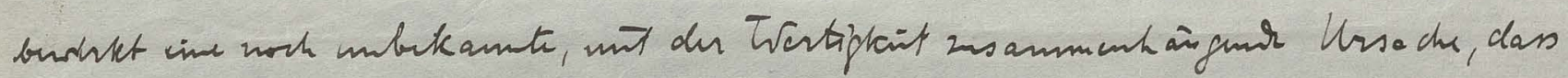

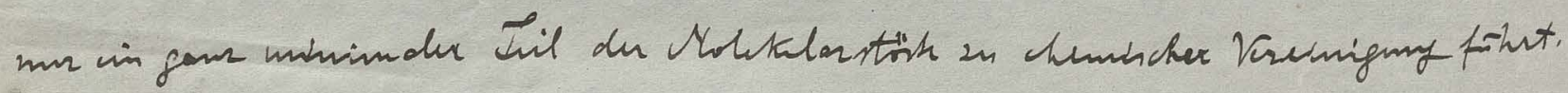

Krokan, Juti 1916.

Sdruse d. Tupaness: Mof. A. M.v. Smohechowste:

Trakan, Studencka 27 


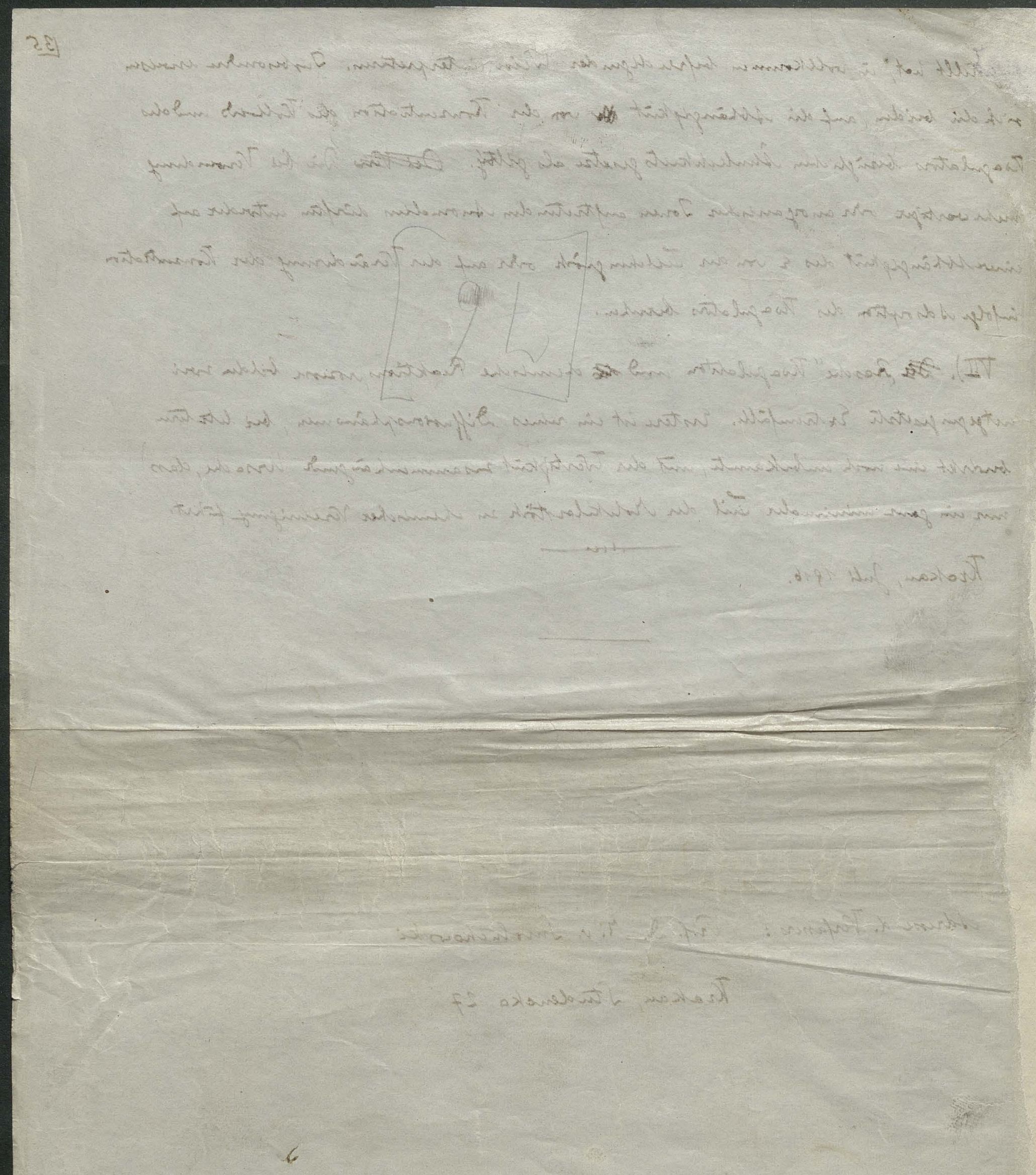


Inie tuts hilf of phyokdence Chemie

Algesmert an tripmonily 11/8 1966

$$
\begin{aligned}
& {[76]} \\
& \text { Wh. 7et t. Jux the } 9 \text { ? }
\end{aligned}
$$




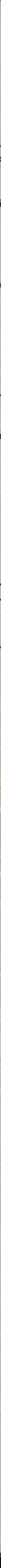


Vusush inum mattsucteren

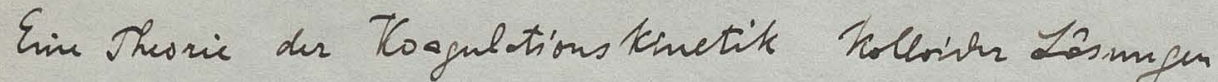

M. v. Sunrushowker

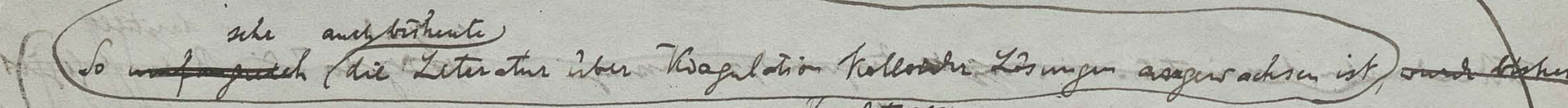

\section{Einleitang.}

Som

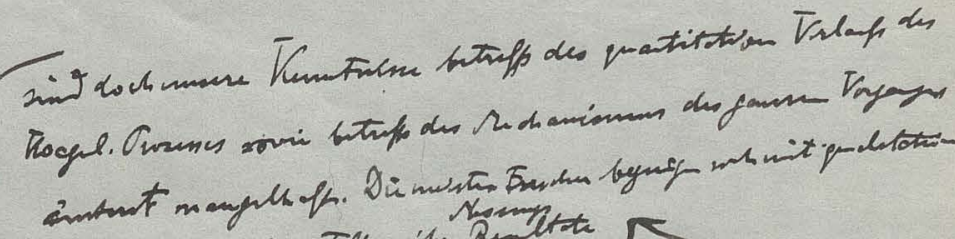

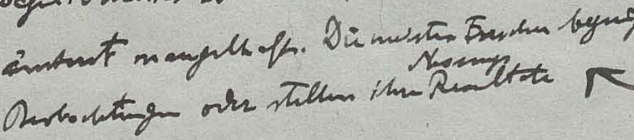

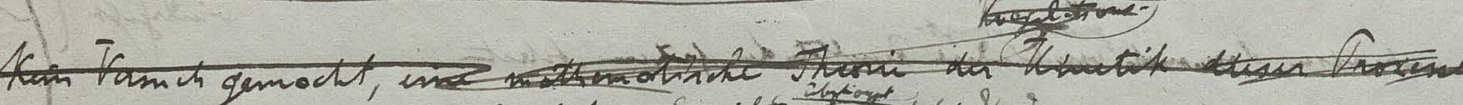

की

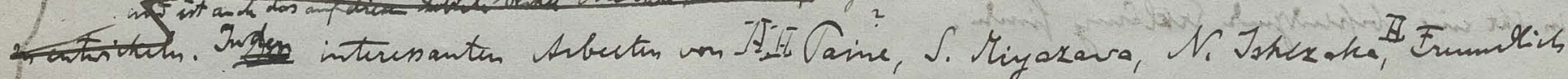
Sam, vinallenting.

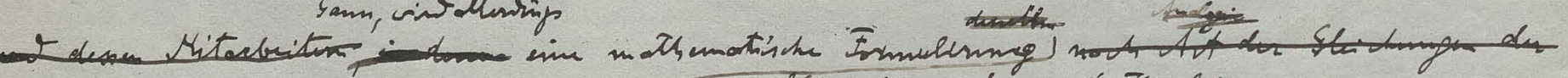

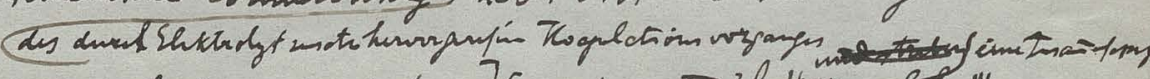

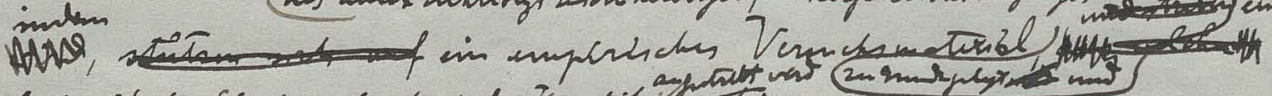

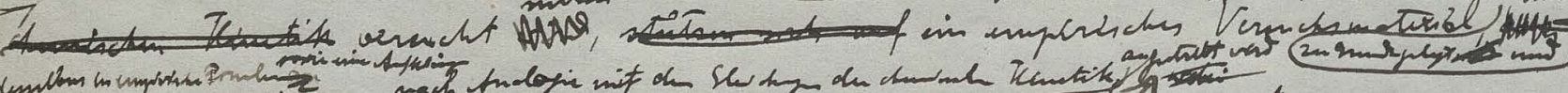

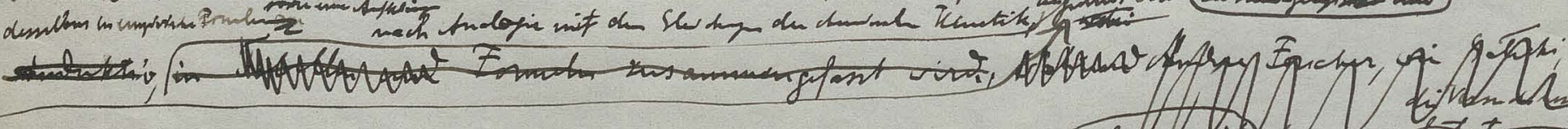

Iothenger

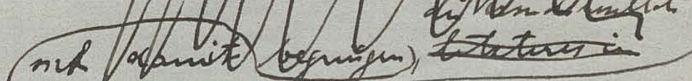

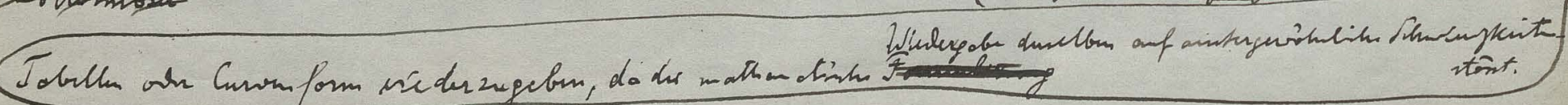

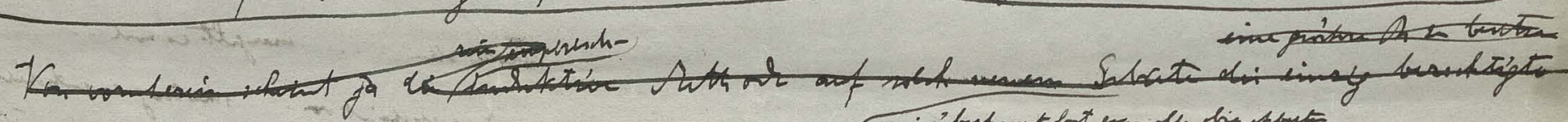

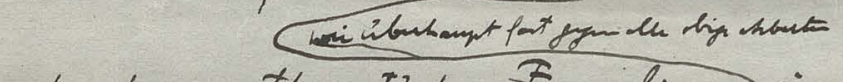

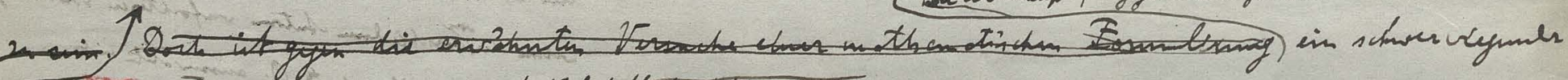

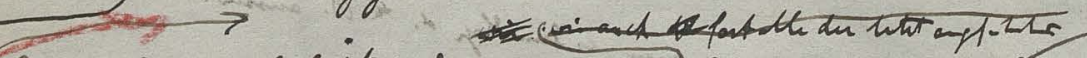

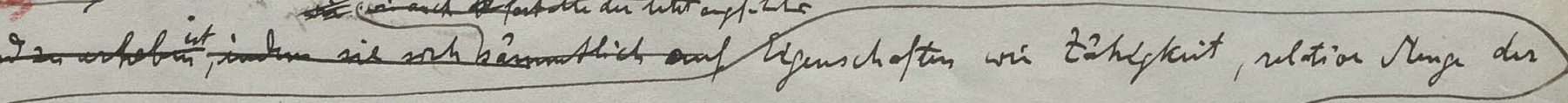

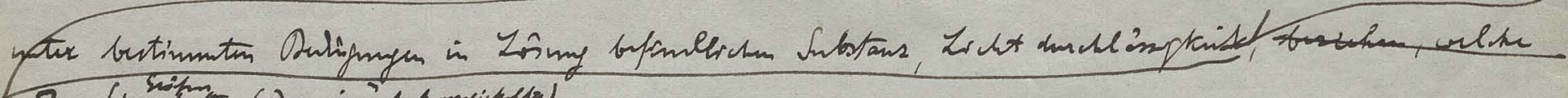

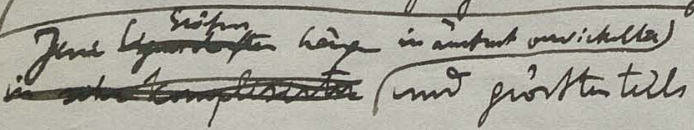

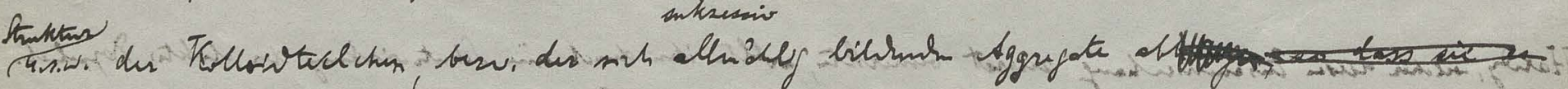

bikanter tris refmeris

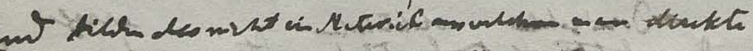

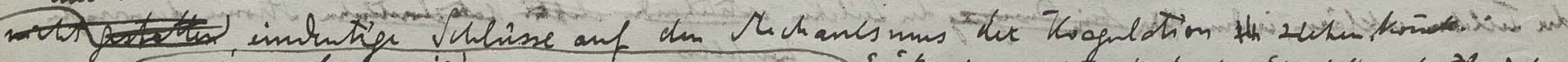

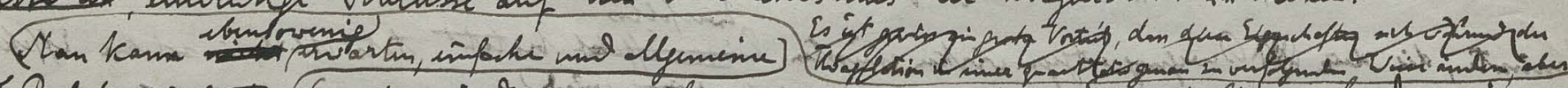

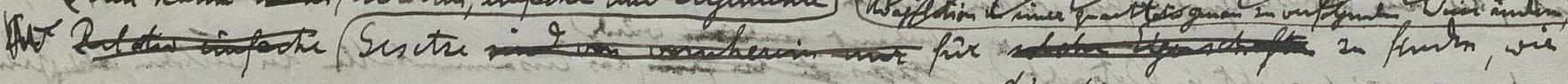

wher tivetom

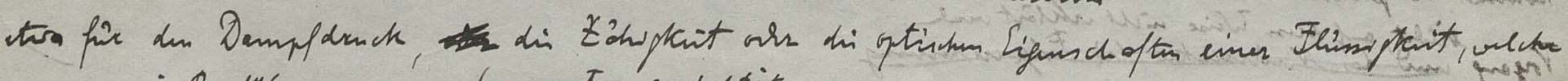

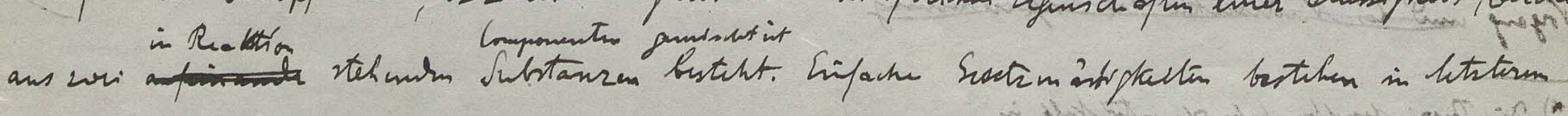

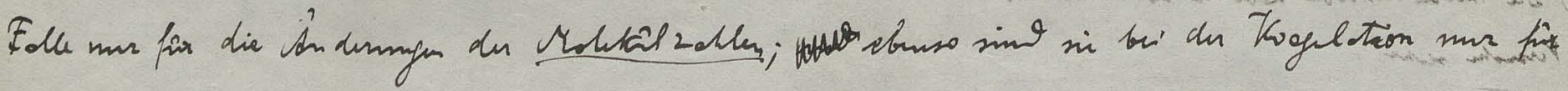

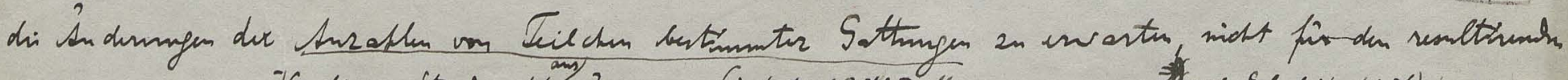

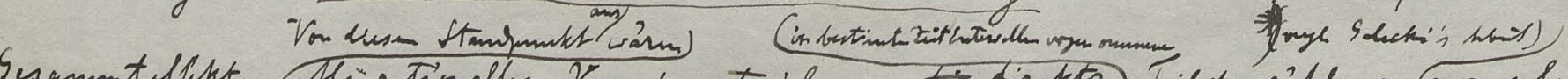

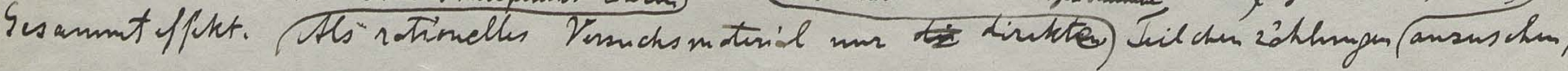
in moth rolche tellewh

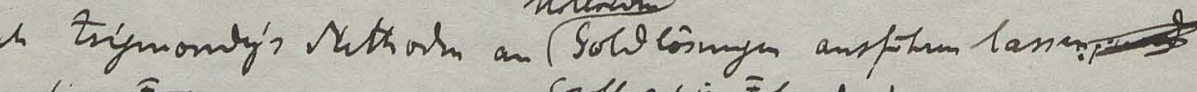

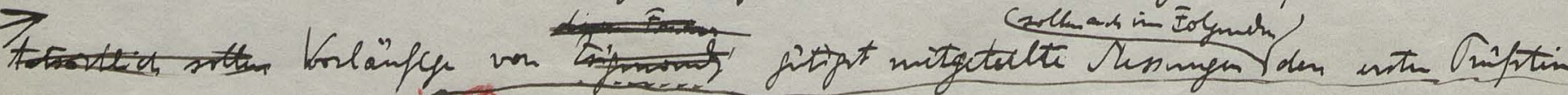

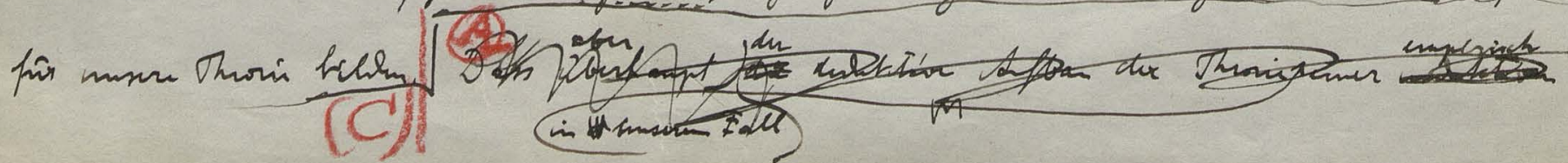




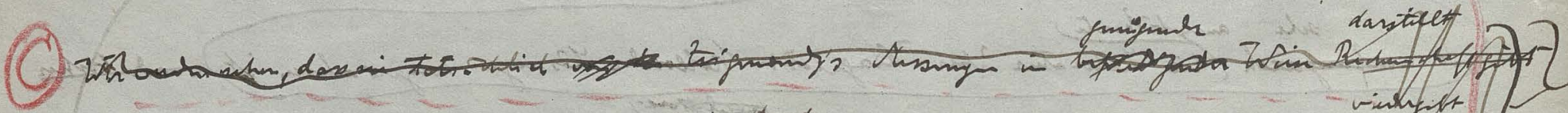

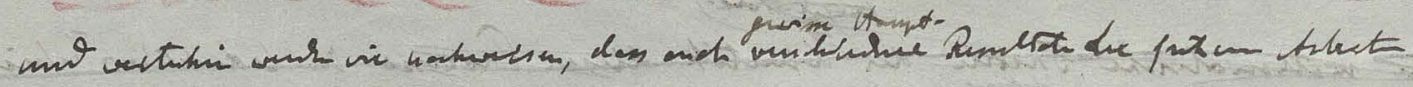
in the eine bopentymer velelormy finch.

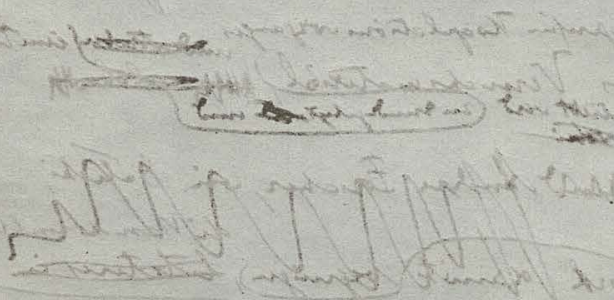

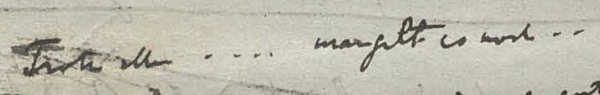

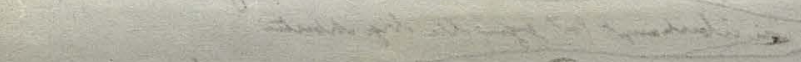

A.

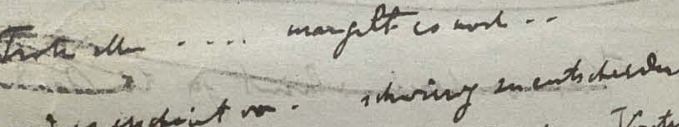

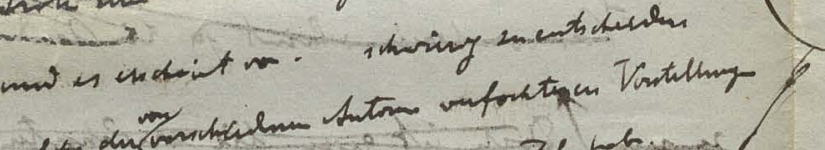

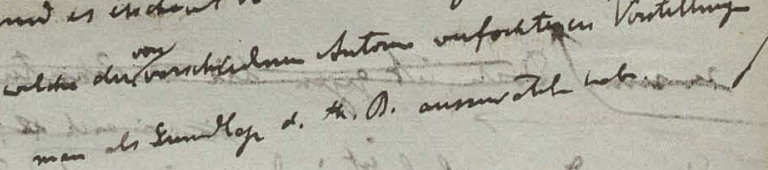

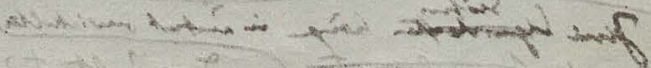

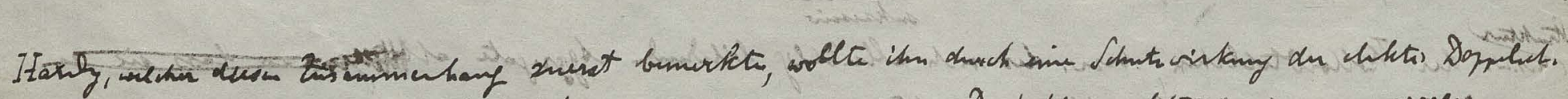

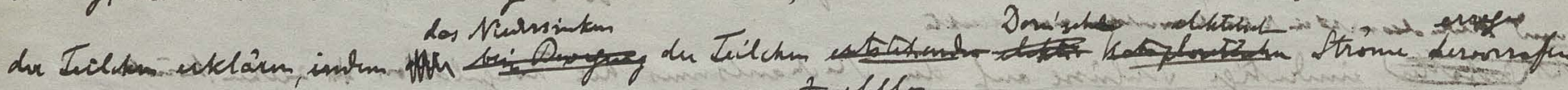

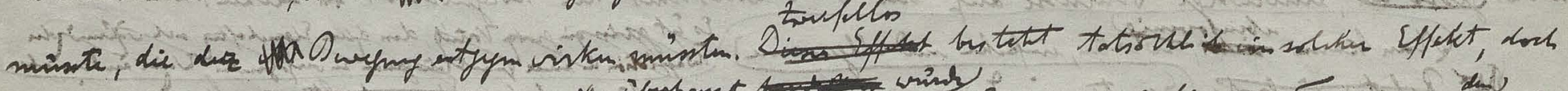

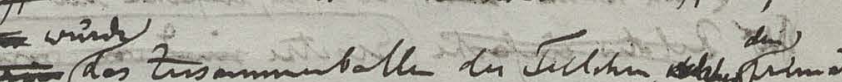

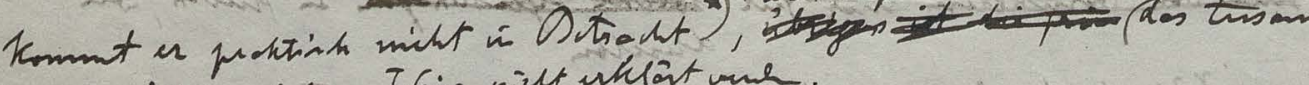

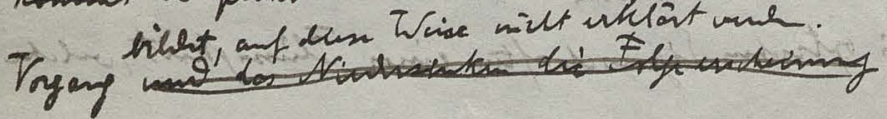

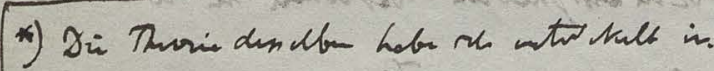

and $\rightarrow+n$ 


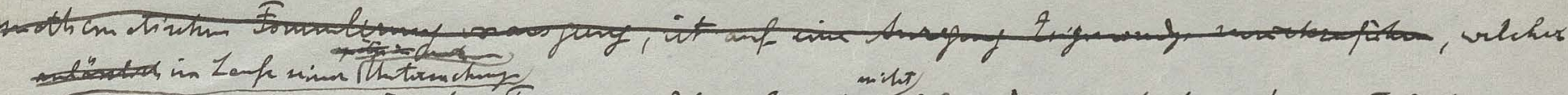

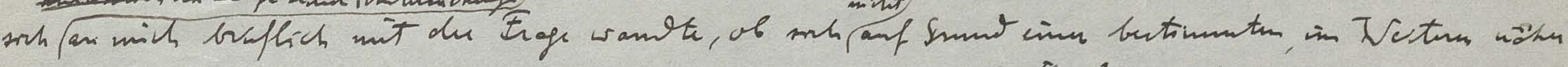

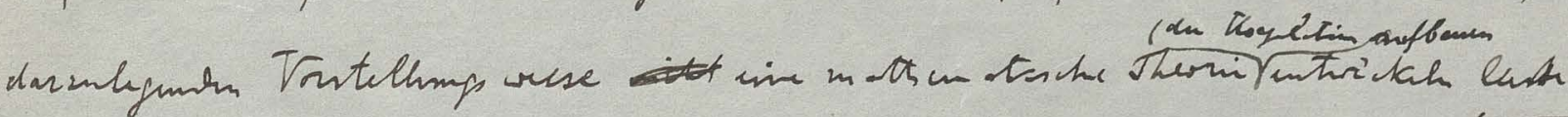

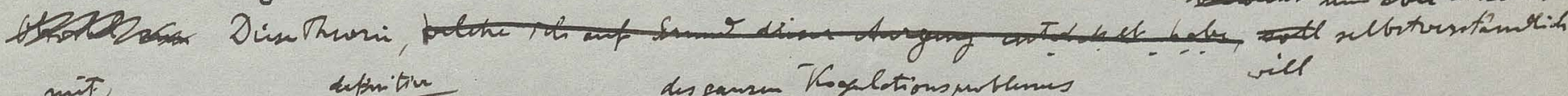

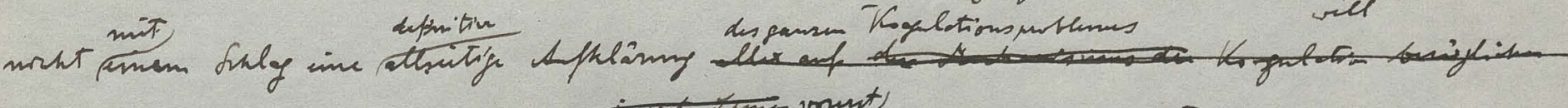

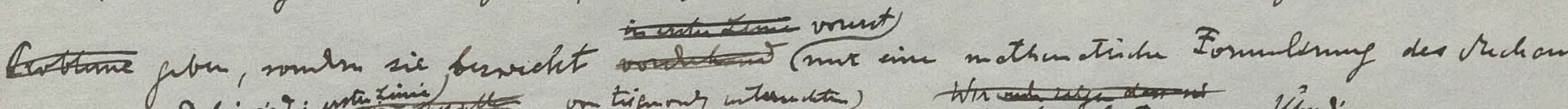

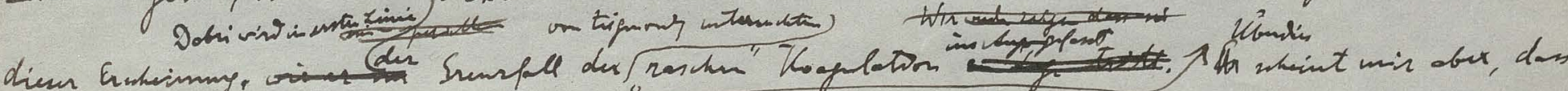

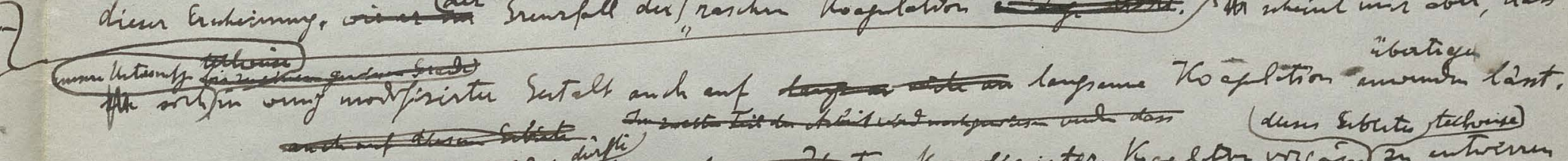

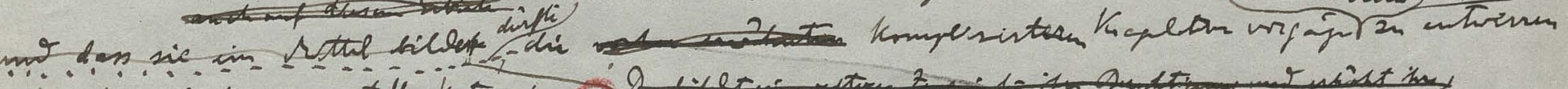

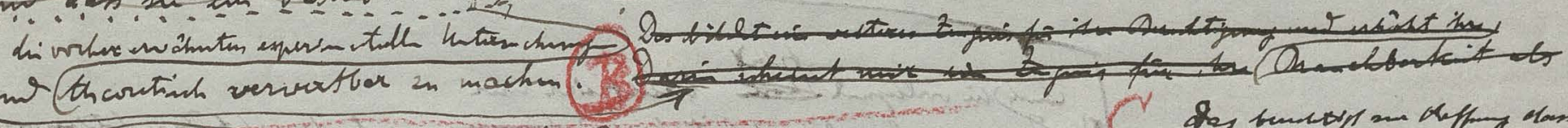

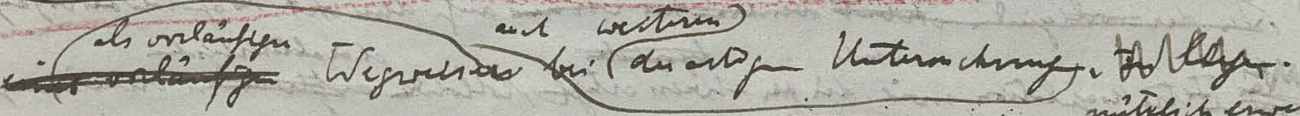

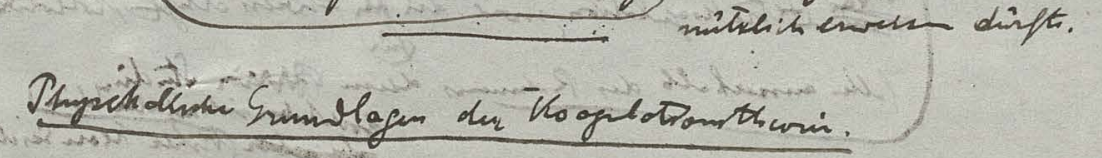

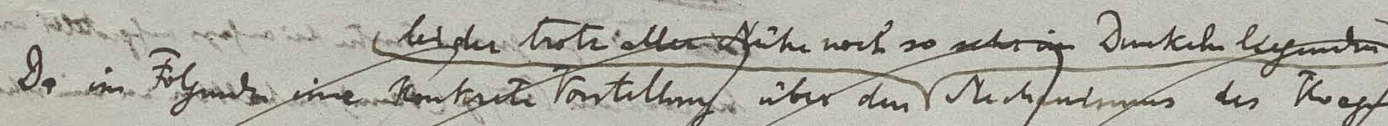

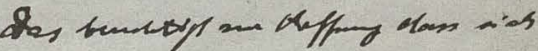

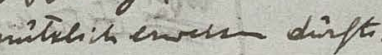

作

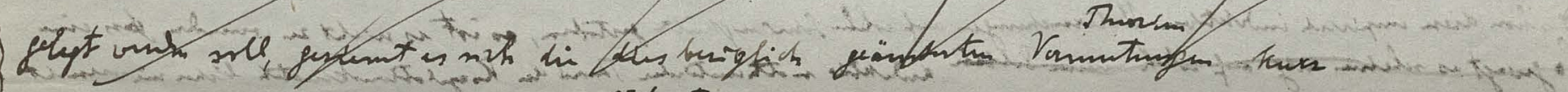

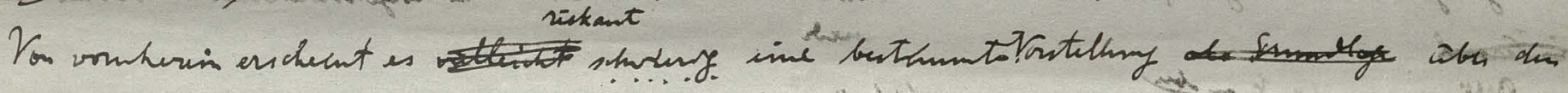

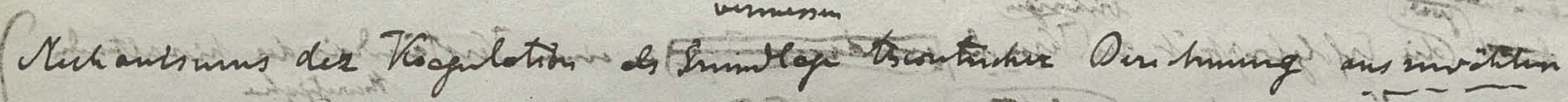

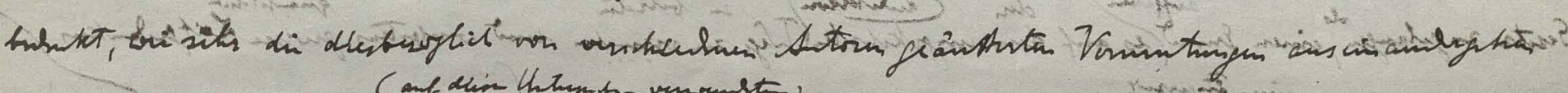

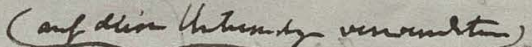

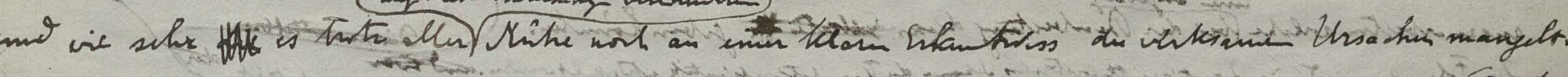

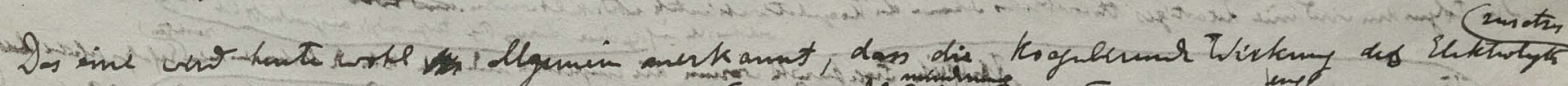

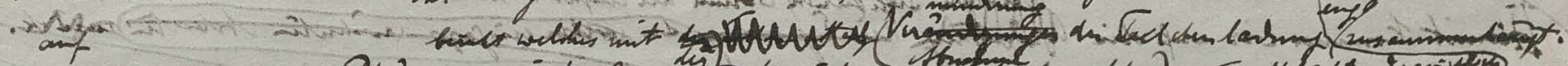

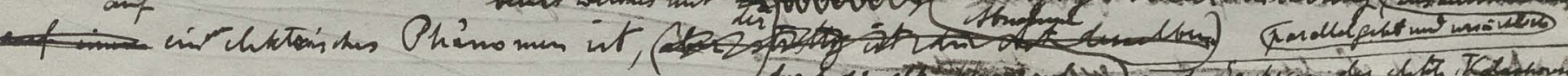

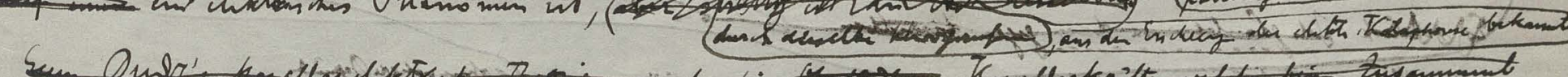

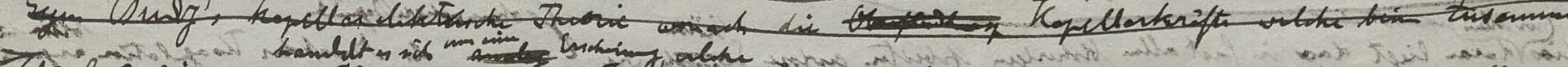

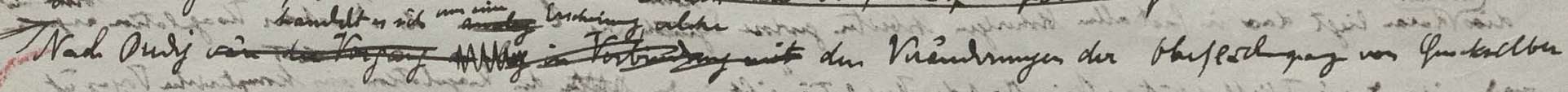

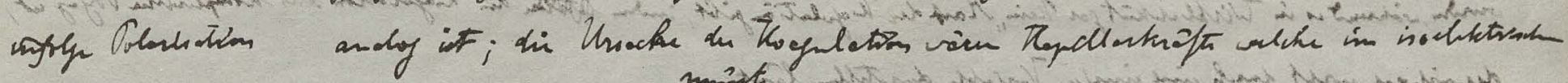

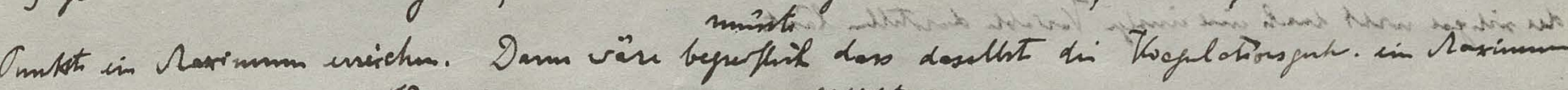

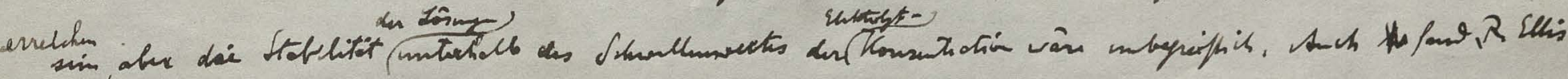

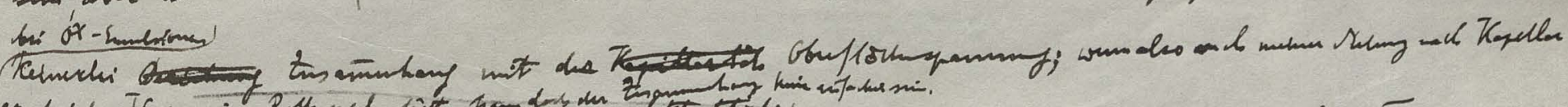

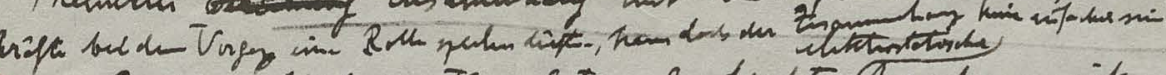

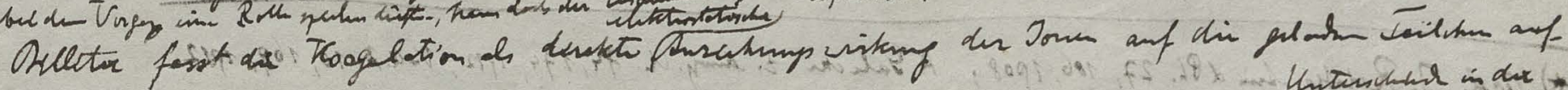

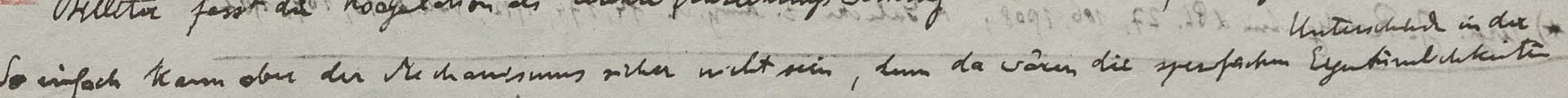
Whtmennex)

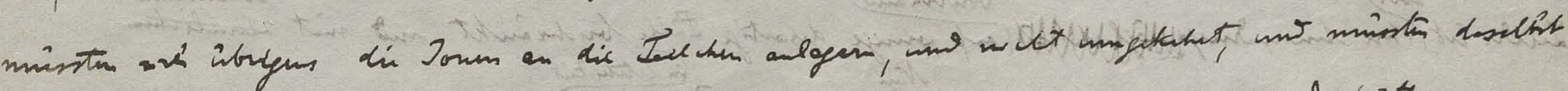

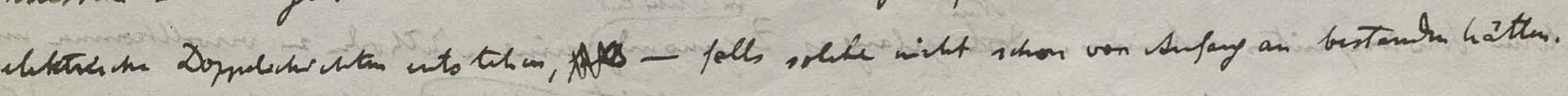




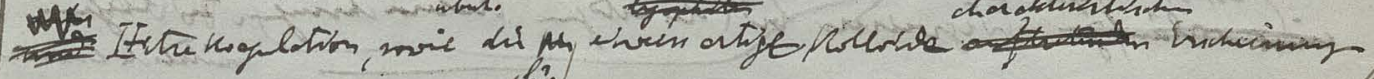

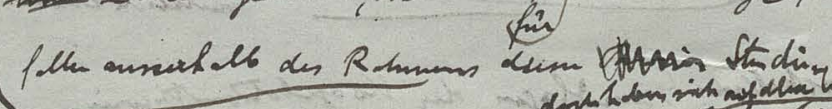

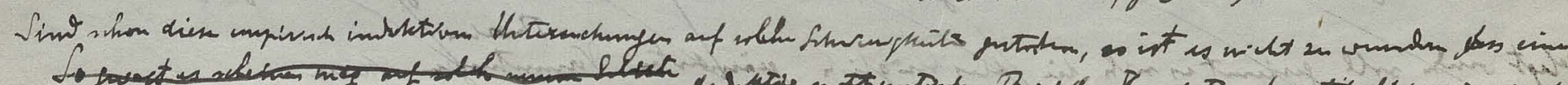

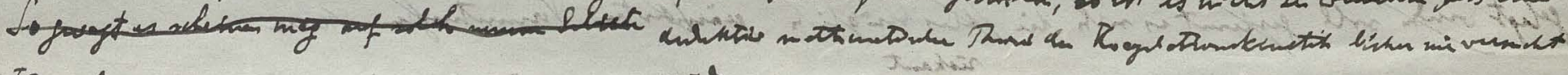
coner

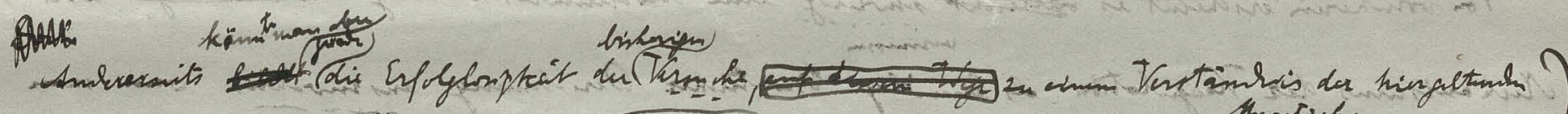

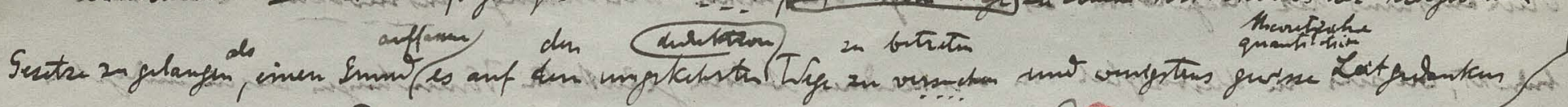

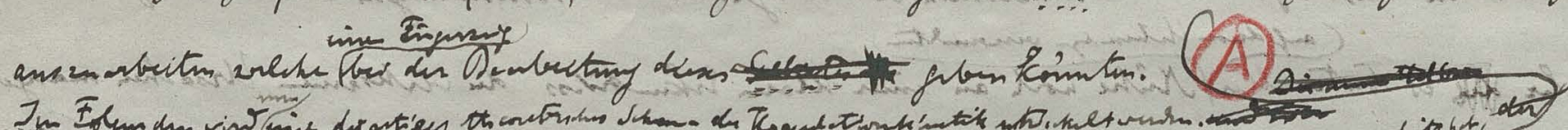

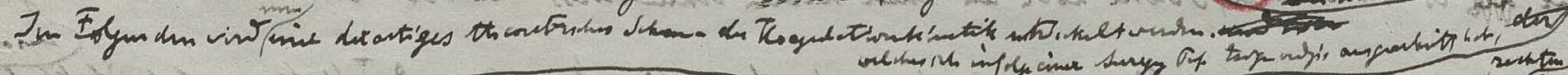

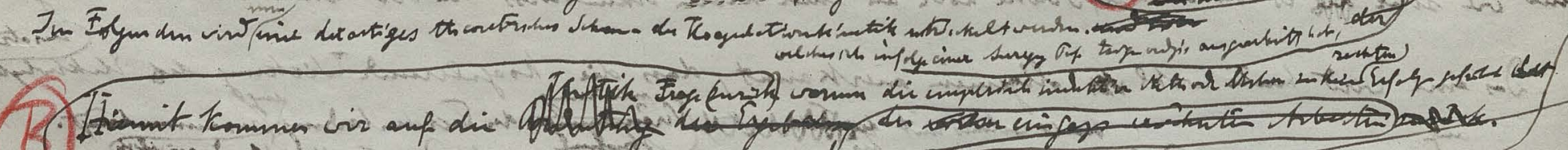

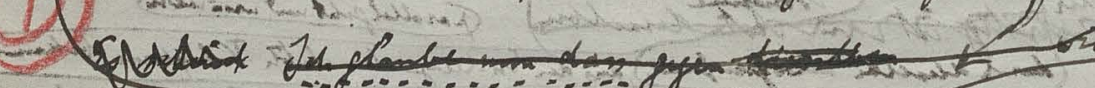

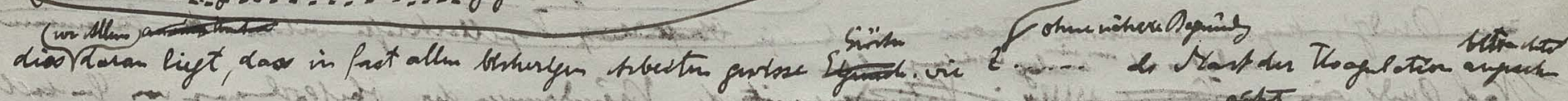

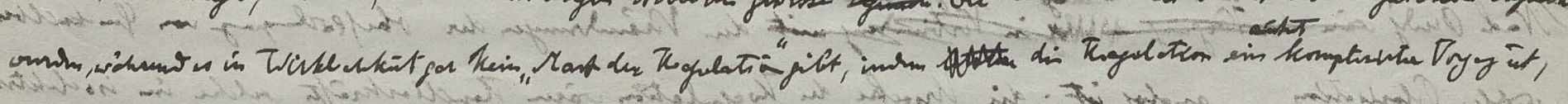

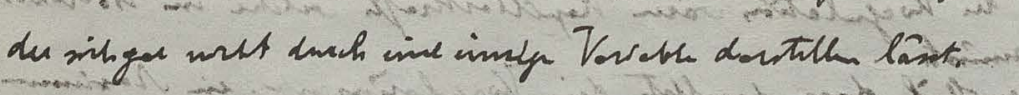
ind 5 lem $x+\infty$
Sto 2 then

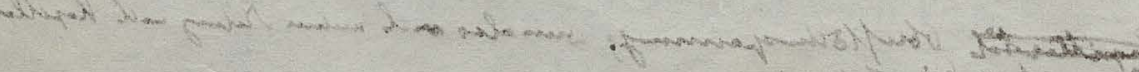

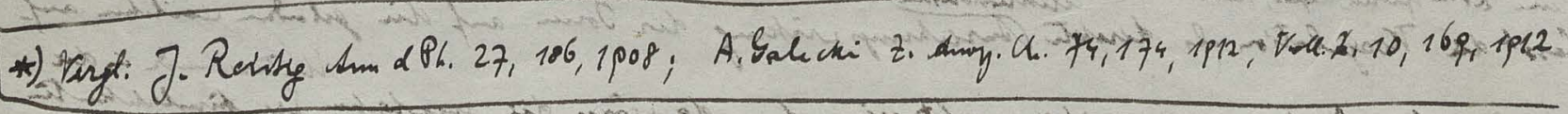

$10 x+2,10,160+1012$

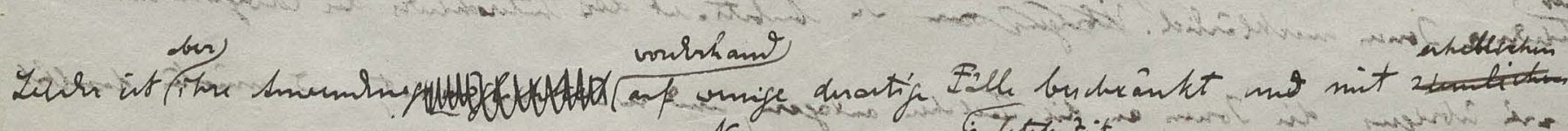
Sivinanthis

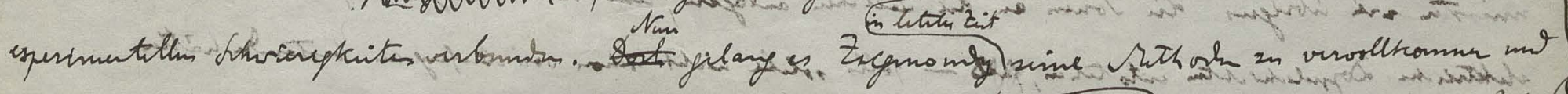

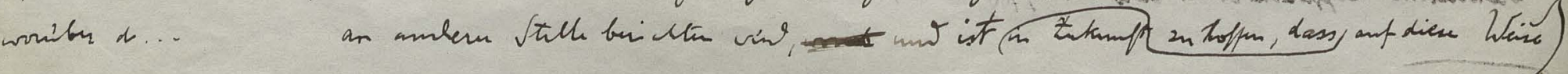

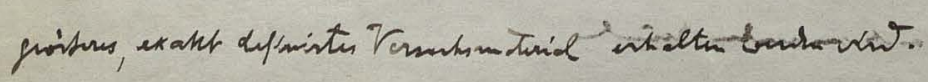




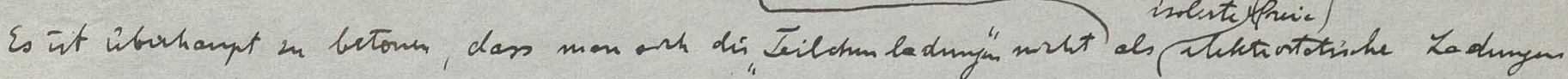

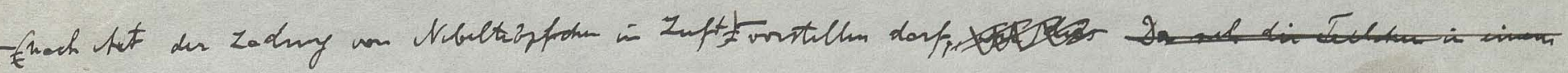

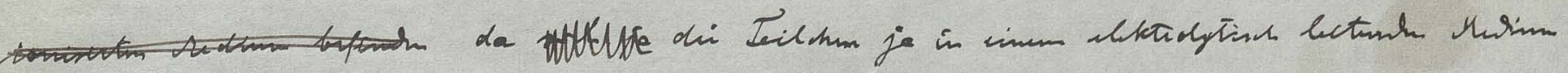

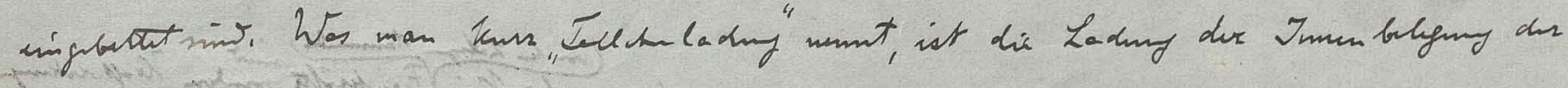

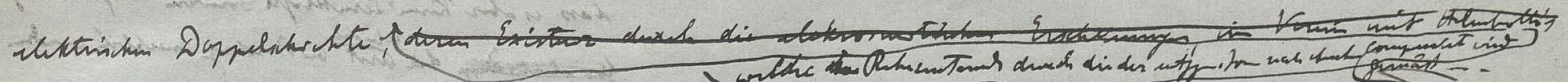

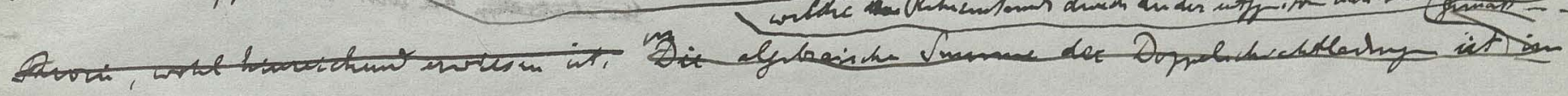
(7) Vuyl. Suml howh

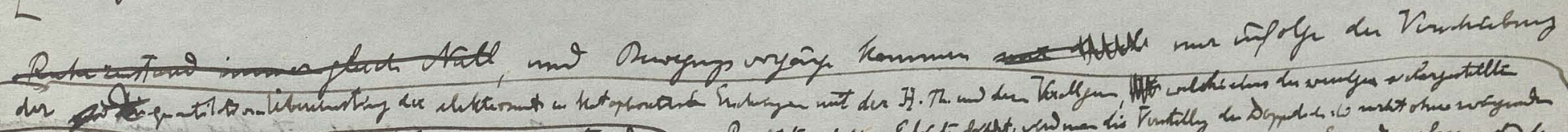

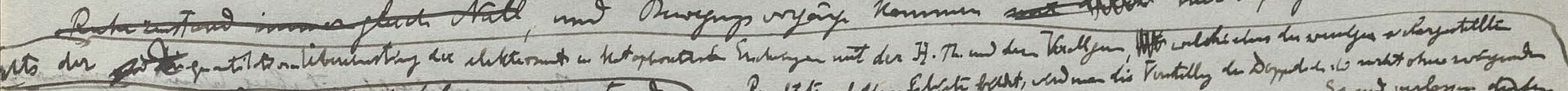

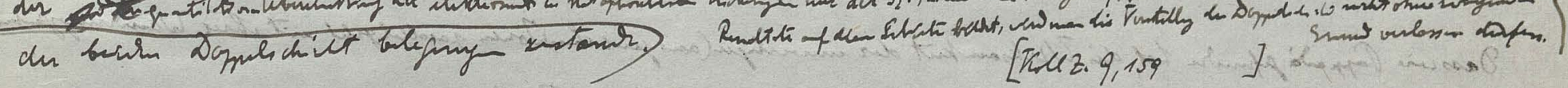

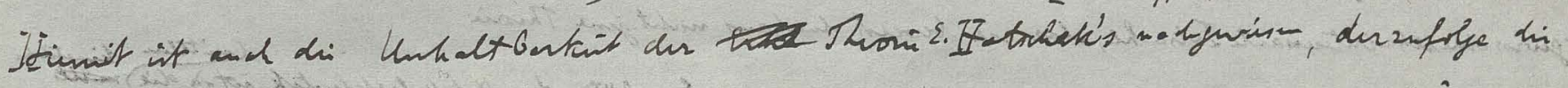

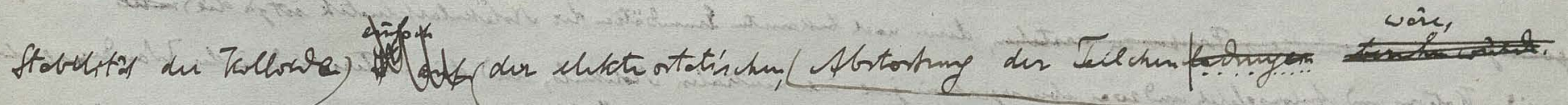

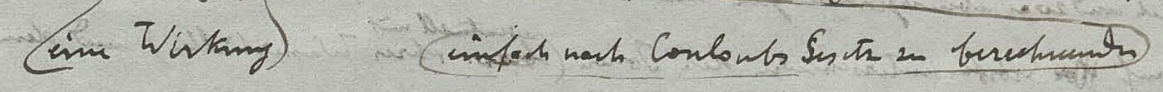

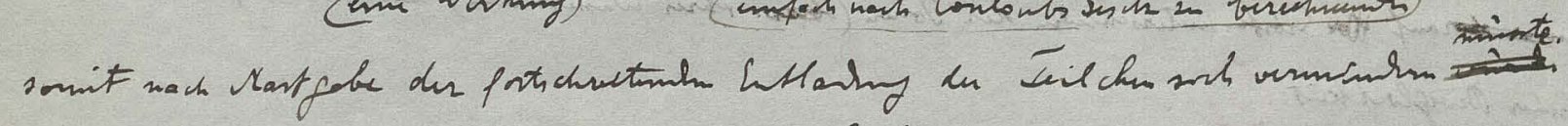

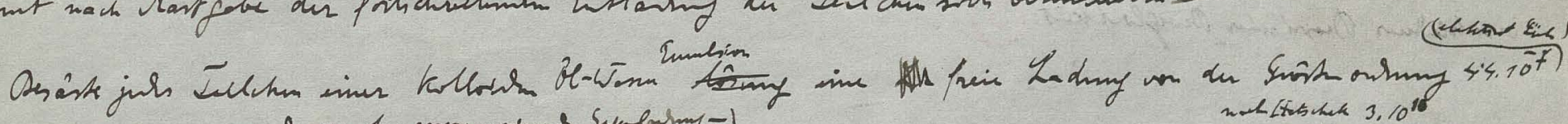

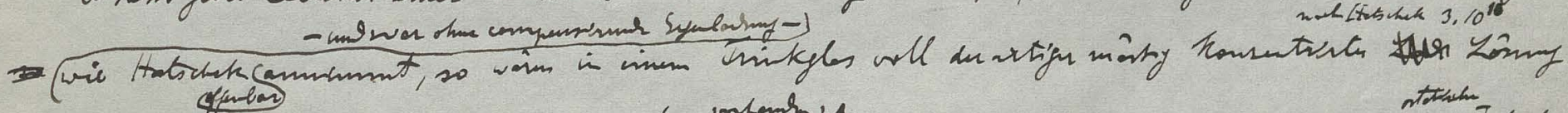

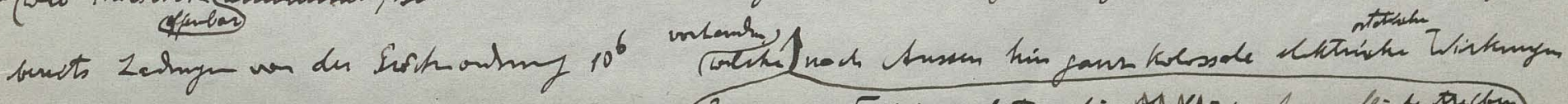
ansabu minton

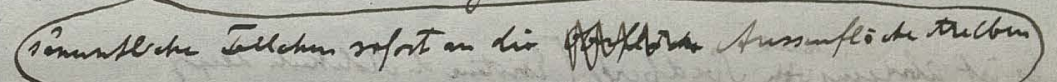

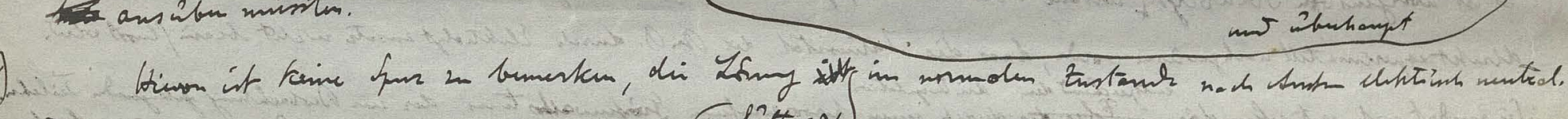

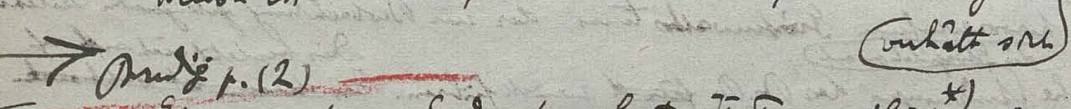

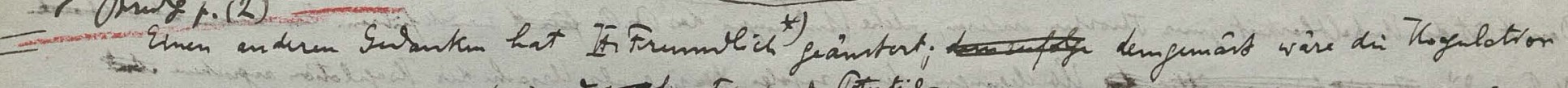

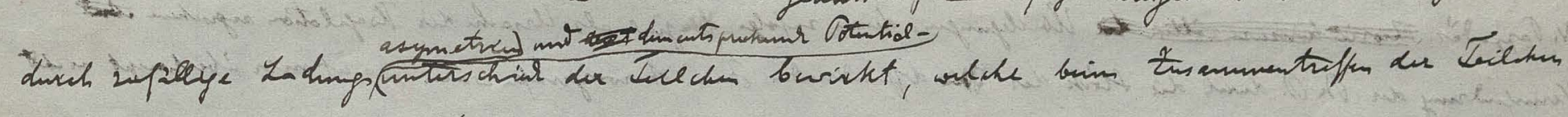

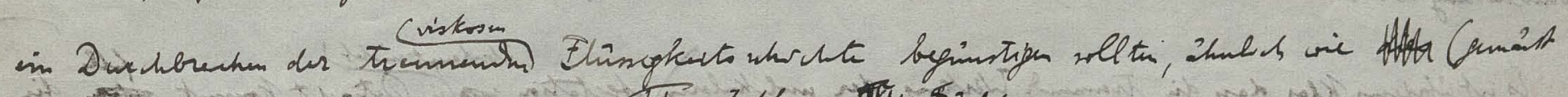

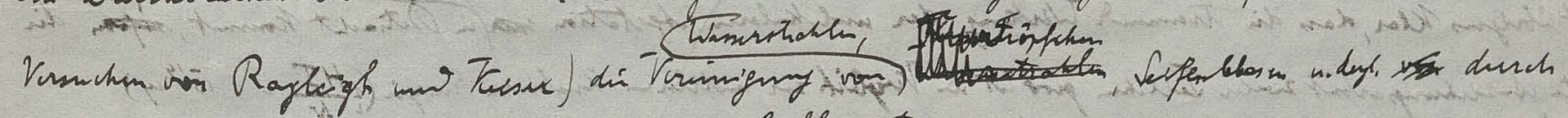

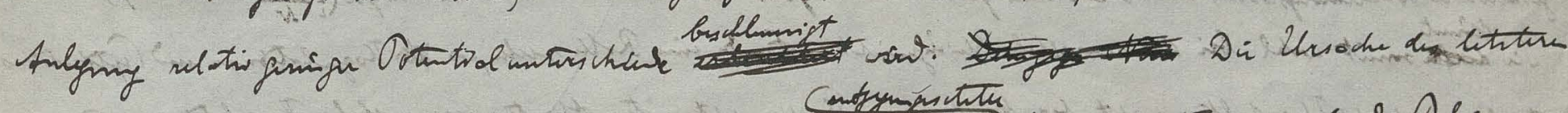

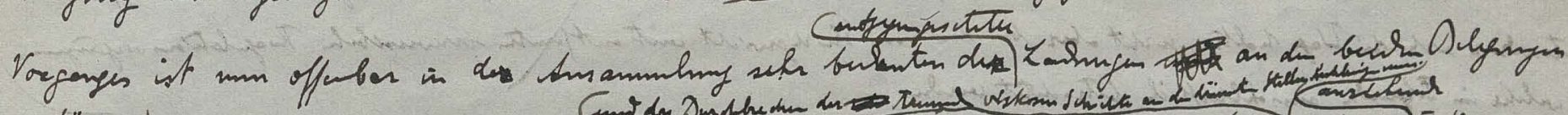

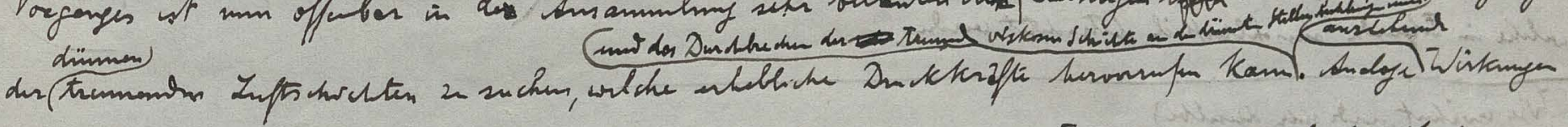

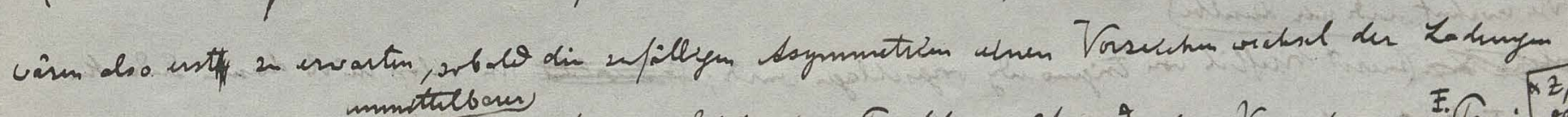

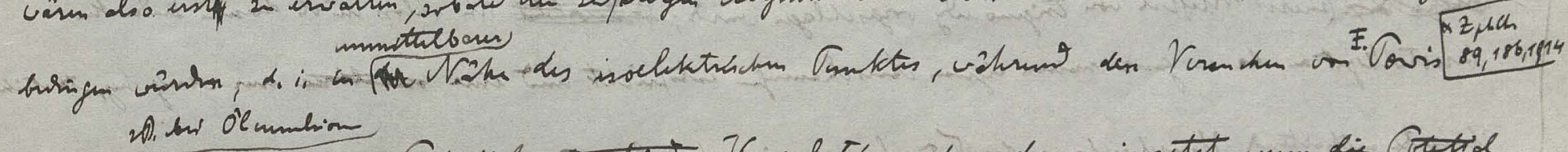

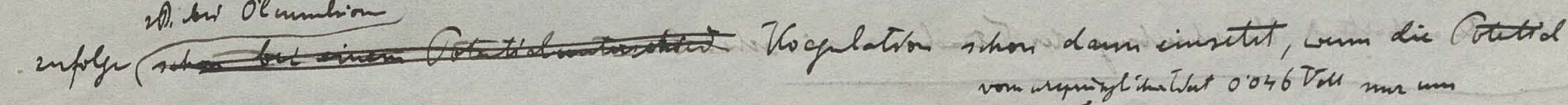

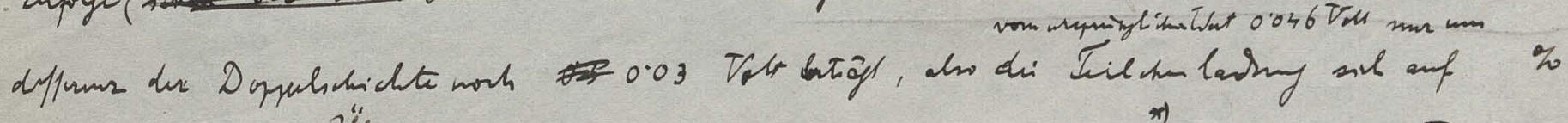

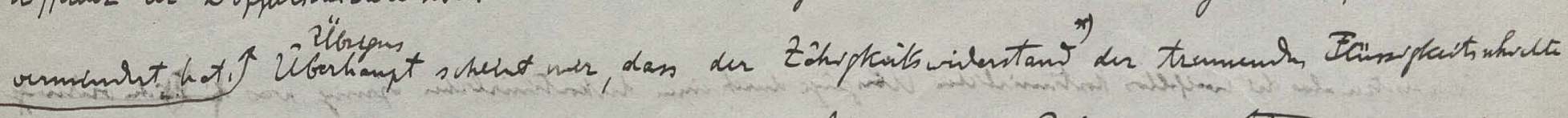

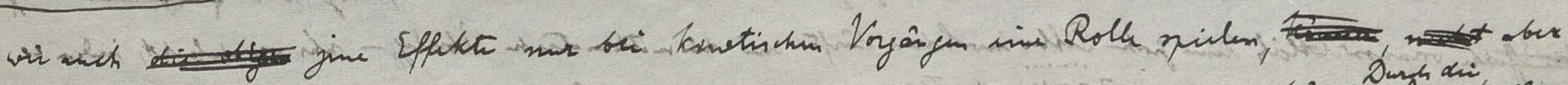

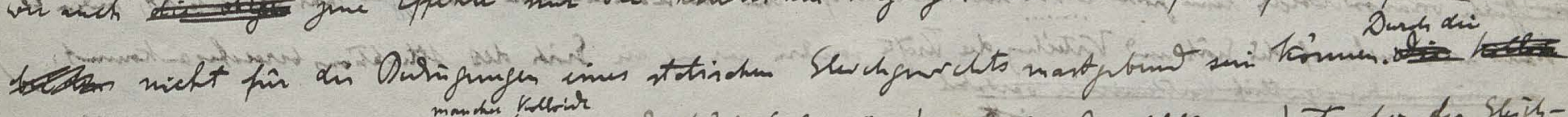

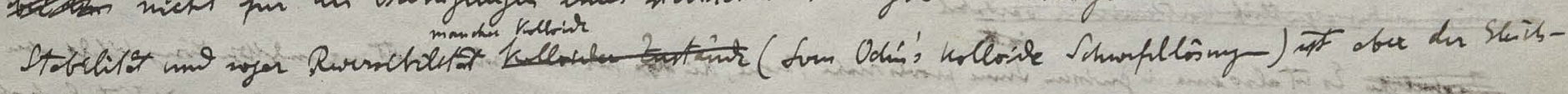

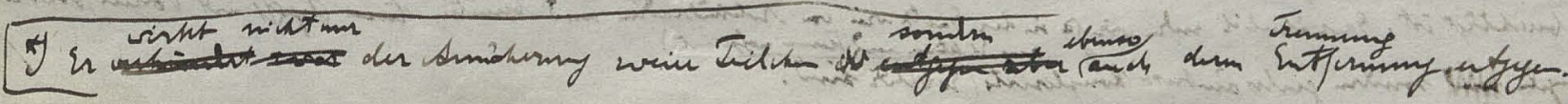




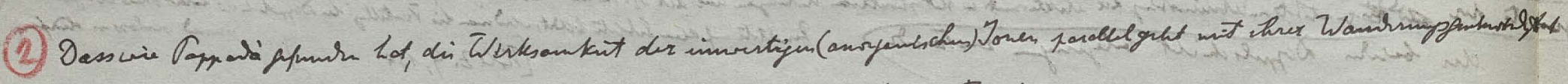

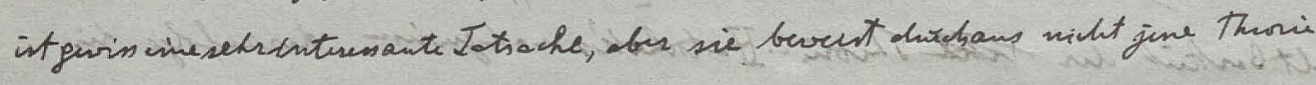

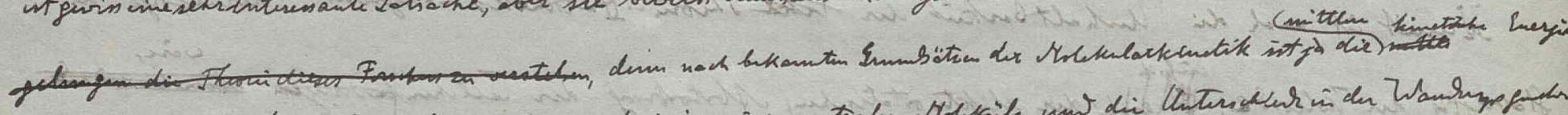

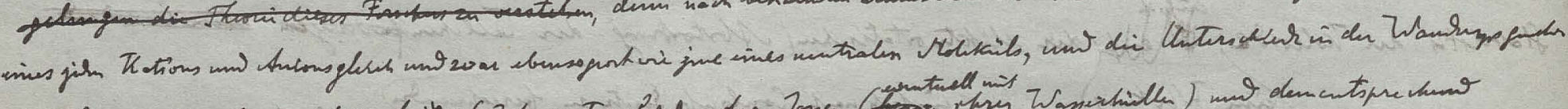

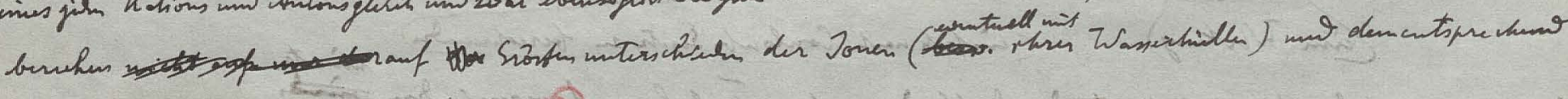

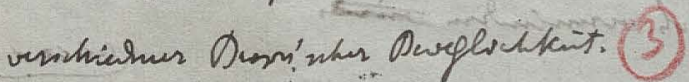

ar

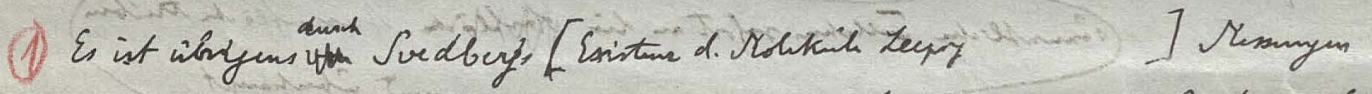

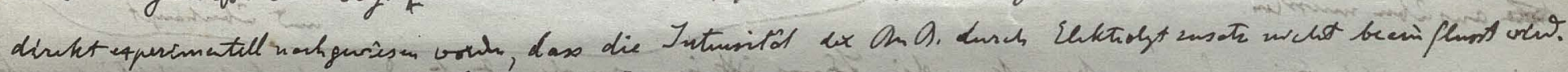

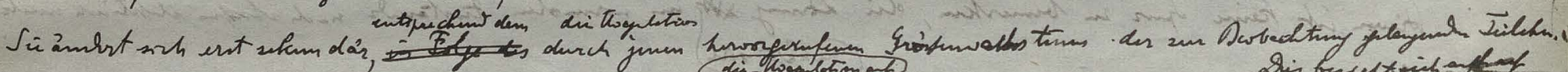

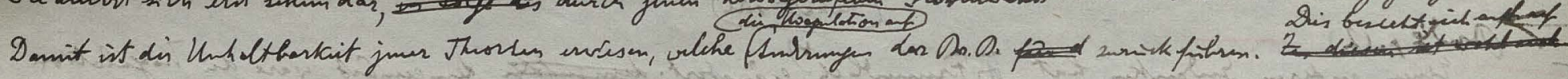

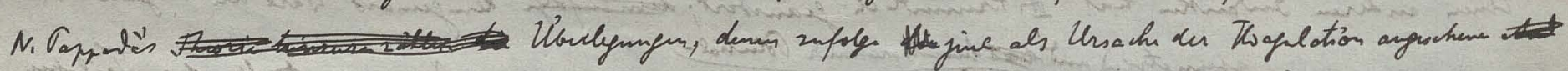

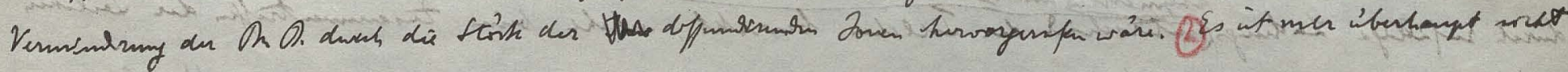

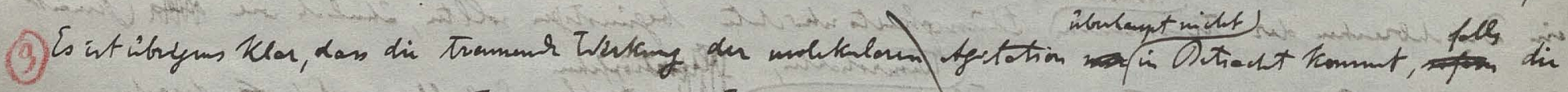

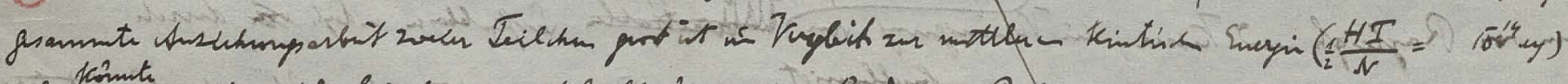

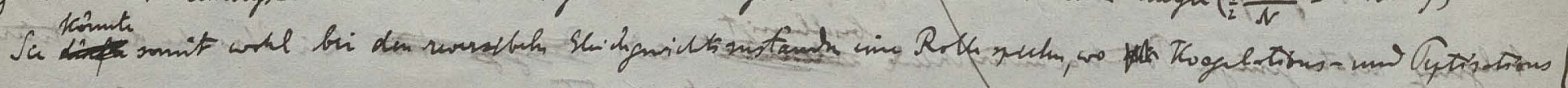

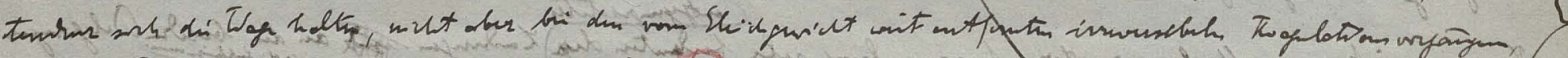

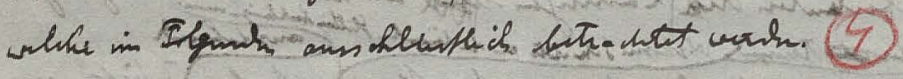

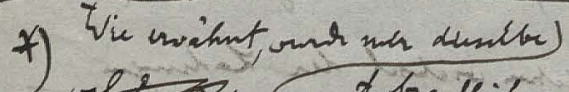

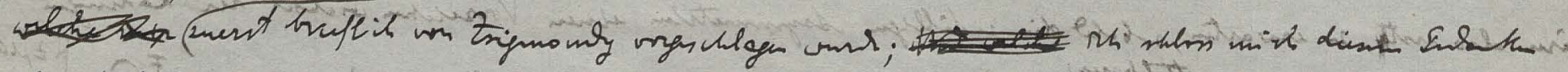

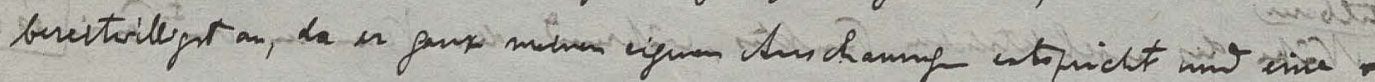

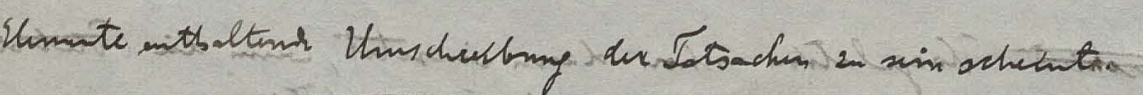

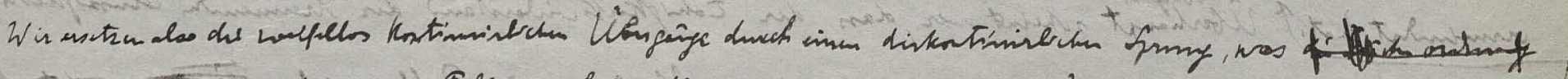

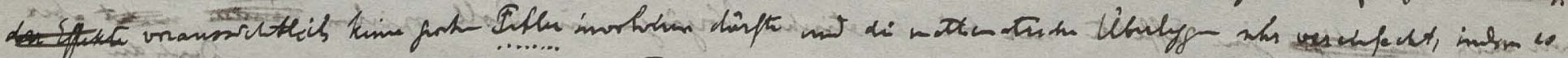

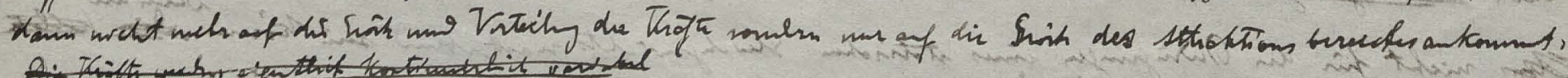

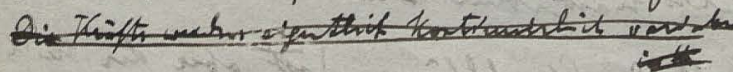

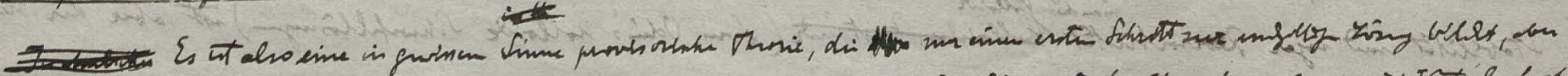

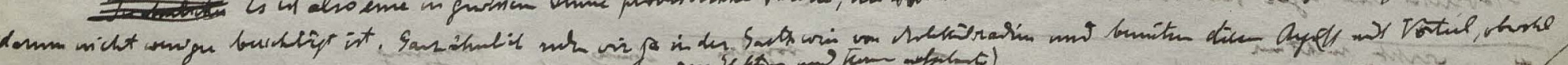

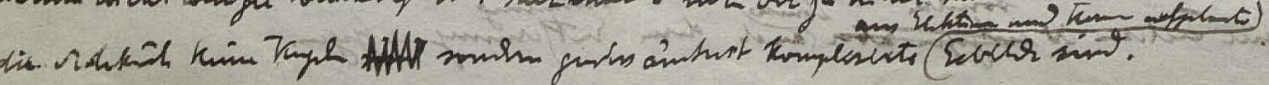




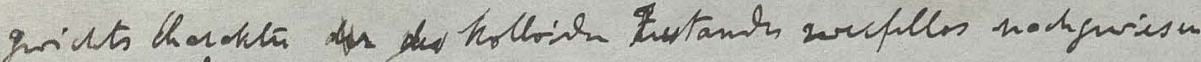

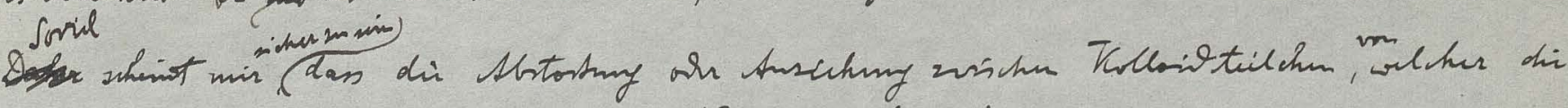

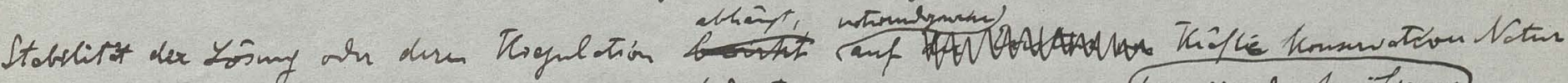

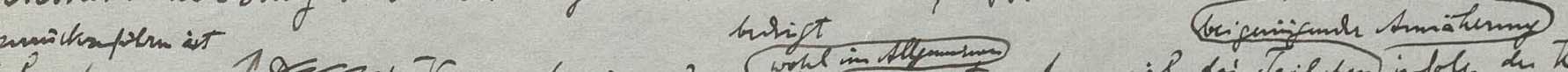

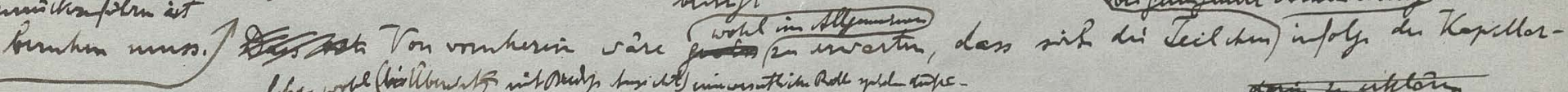

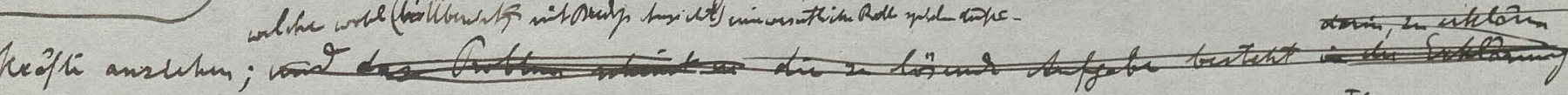

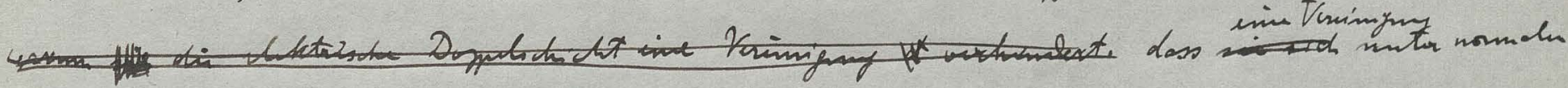

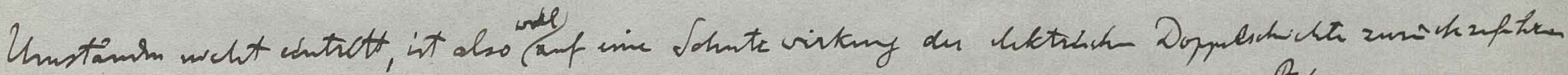

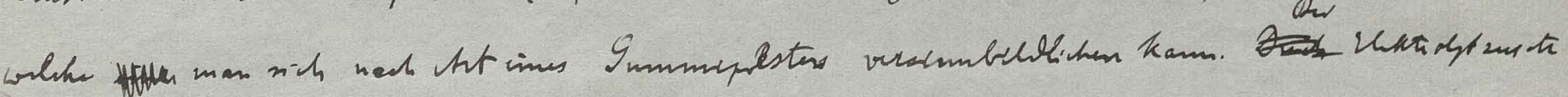

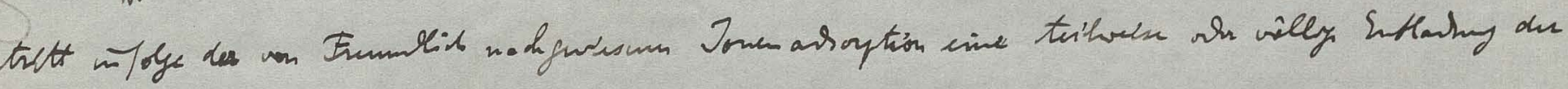

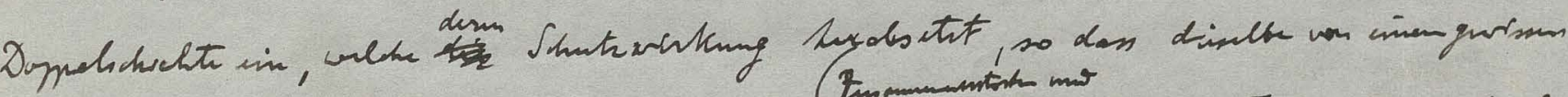

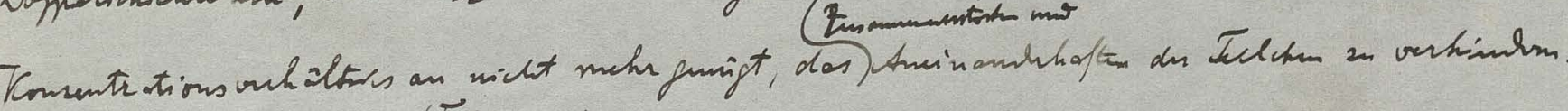

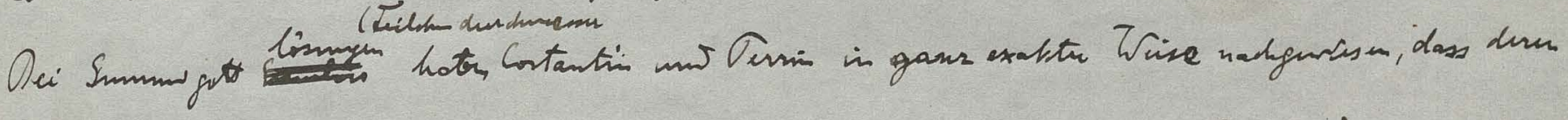

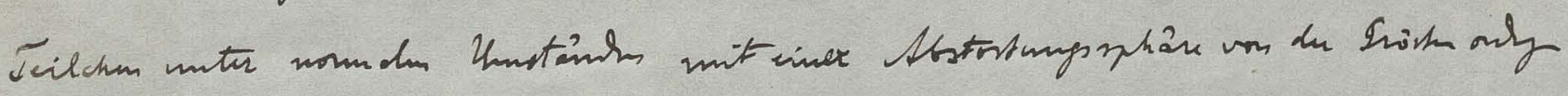

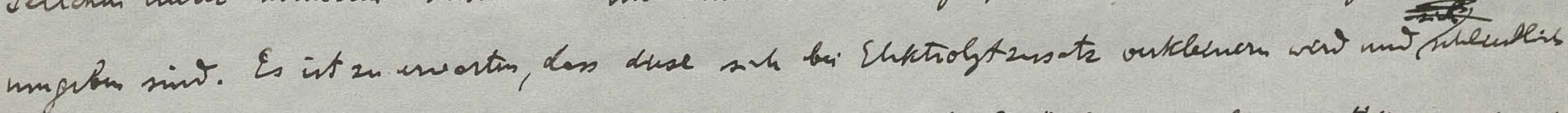

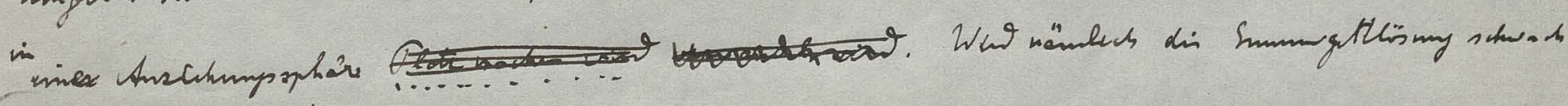
(helset) $\frac{1}{100}$ un

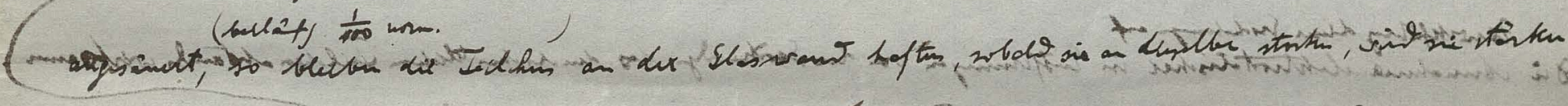

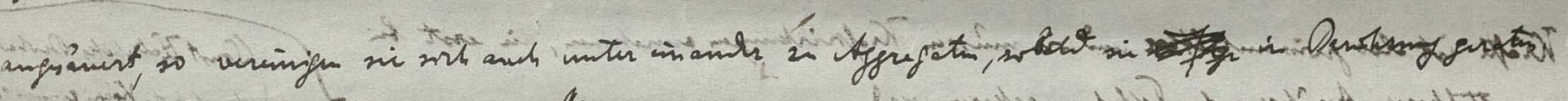

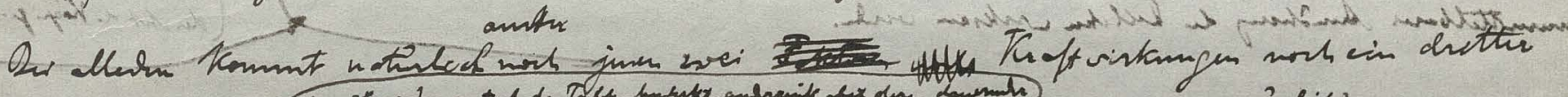

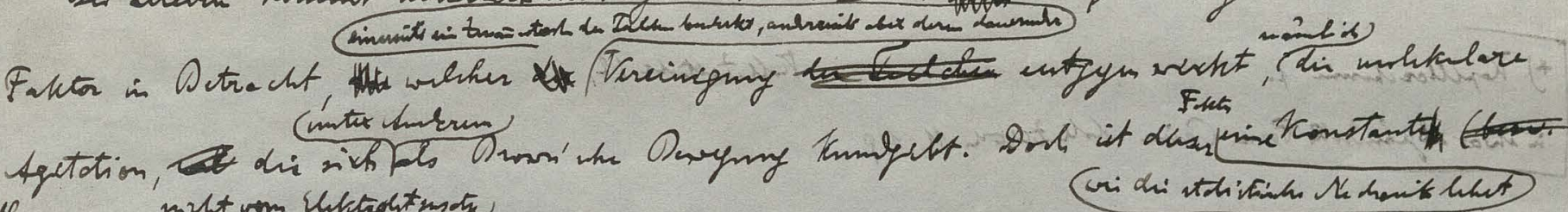
(oll.

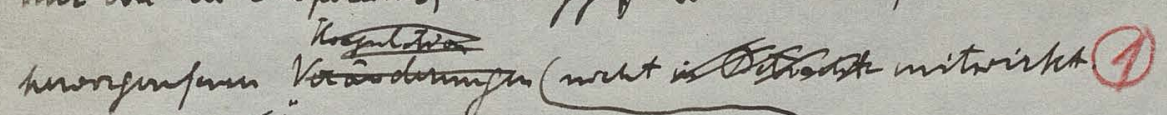

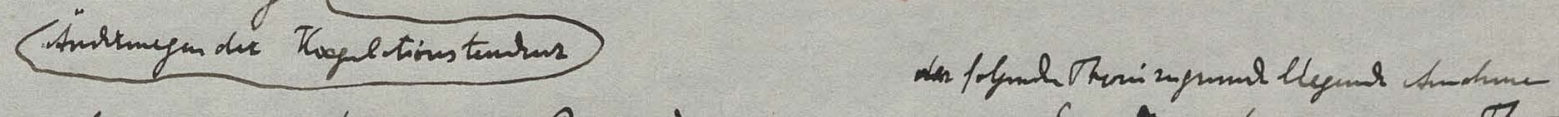

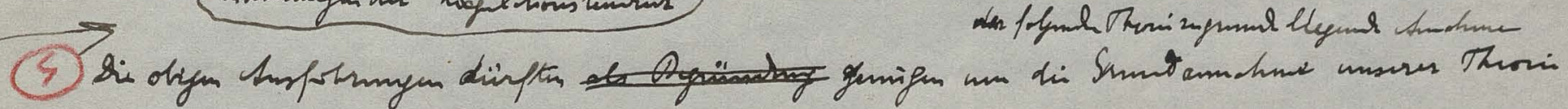

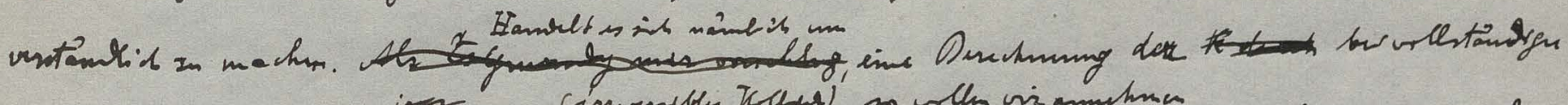

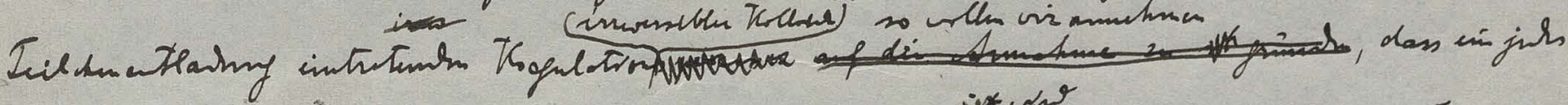

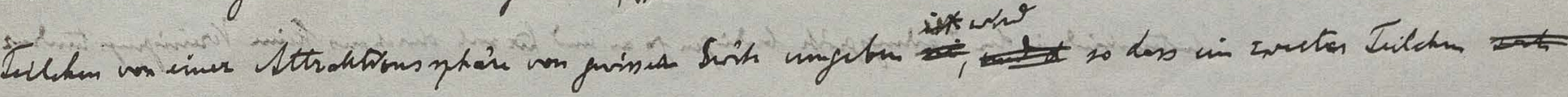

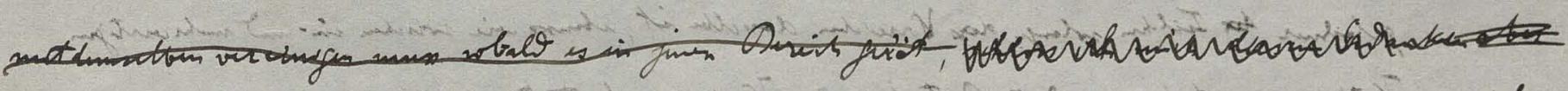

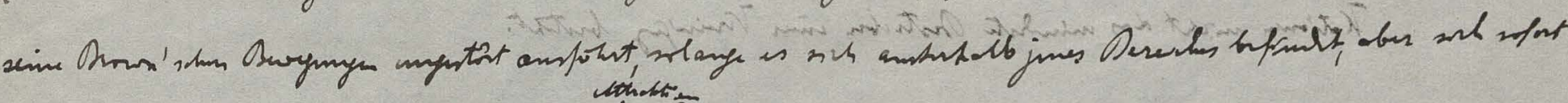

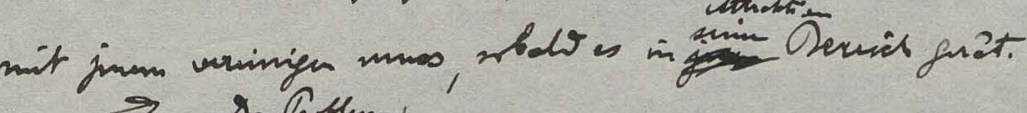

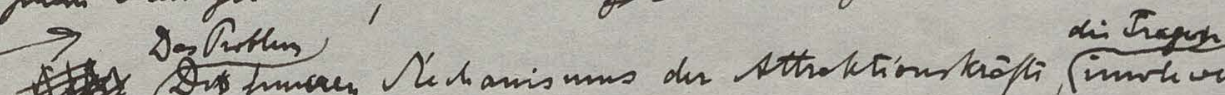

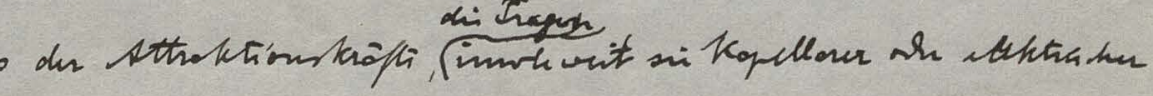

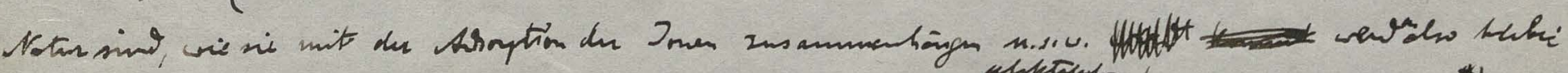

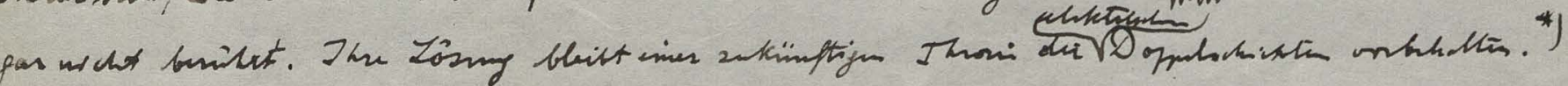

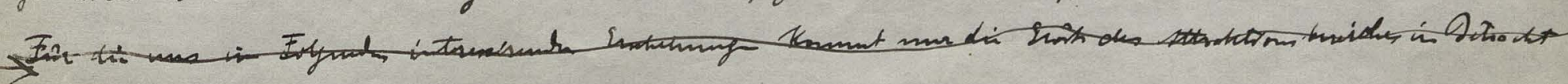




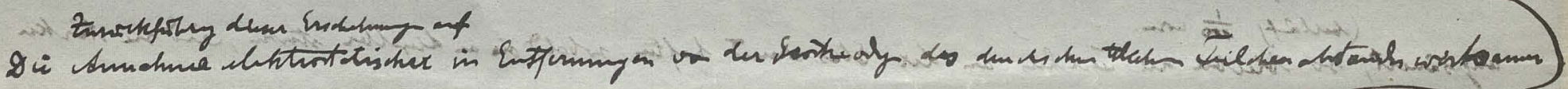

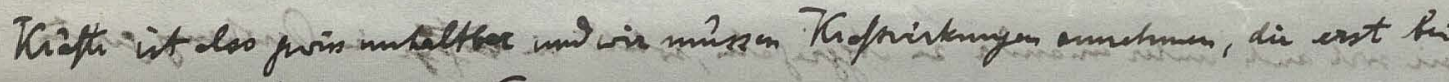

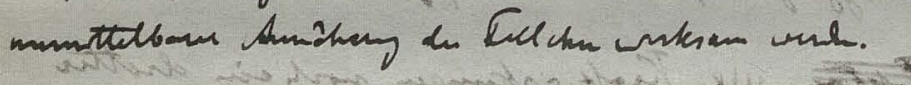

*) Topelloritimin if. then.zentin 12

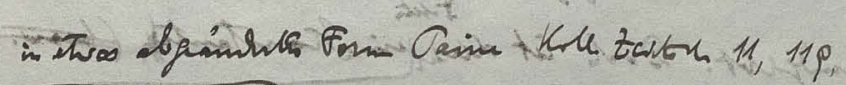

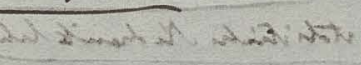

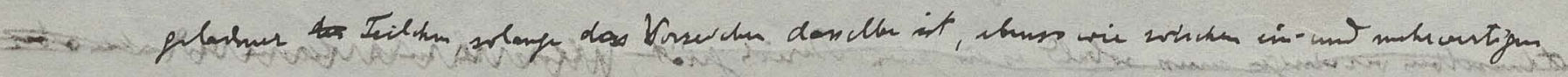

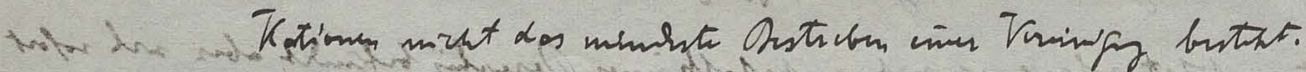




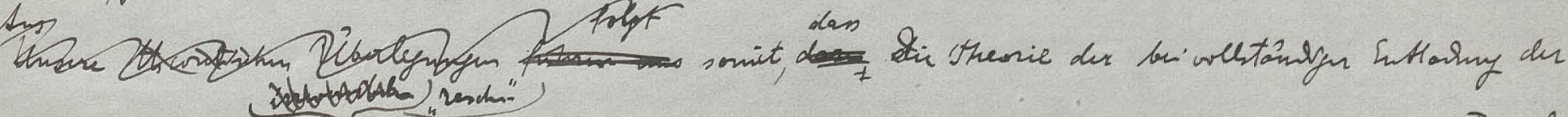

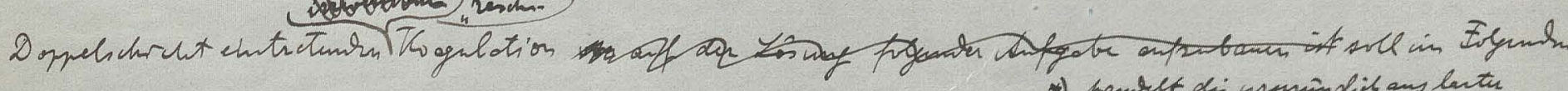

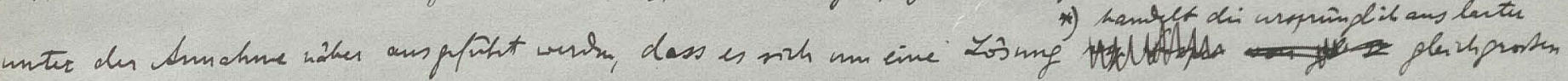

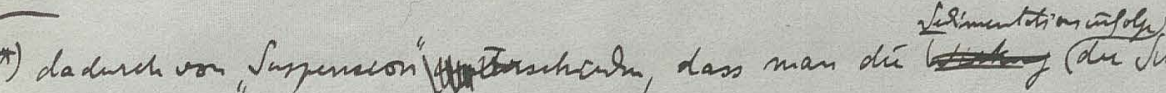

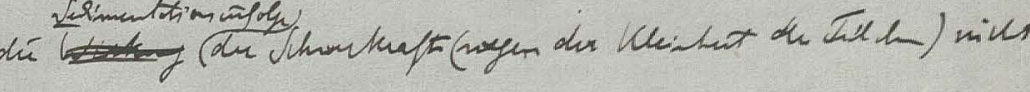

in Retunny in whem bancht

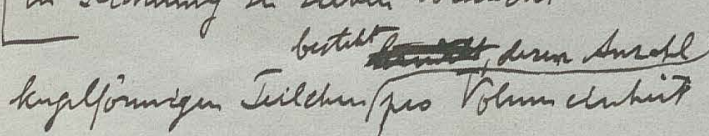

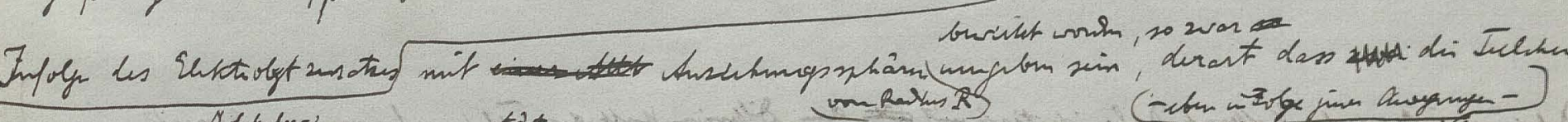

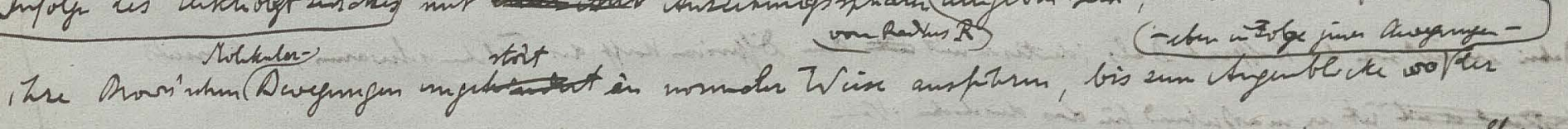

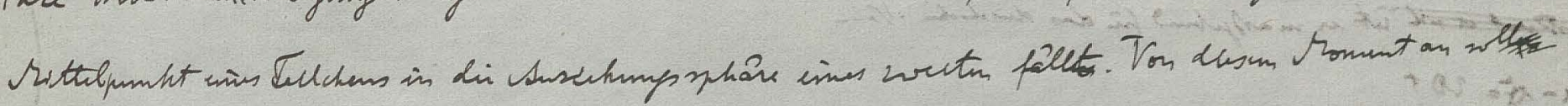

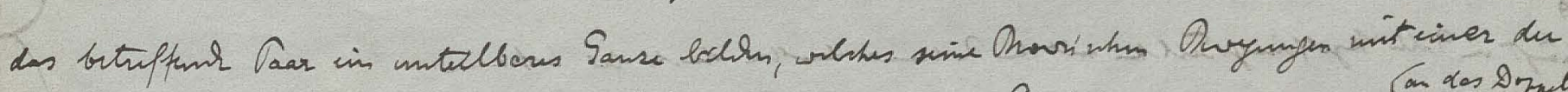

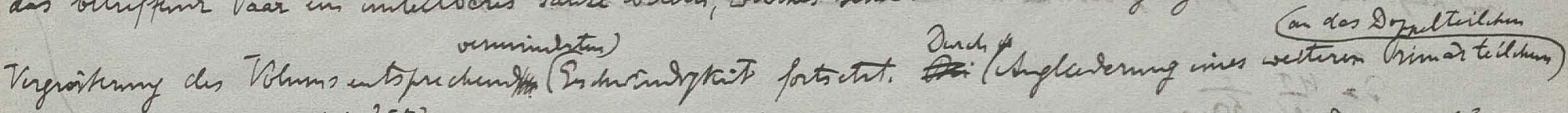

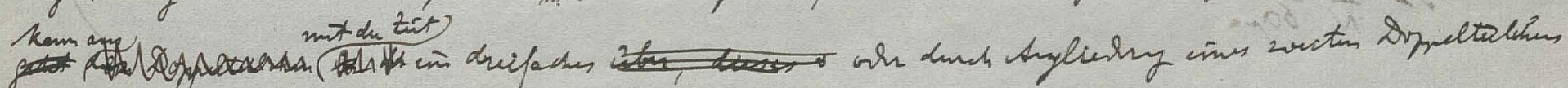

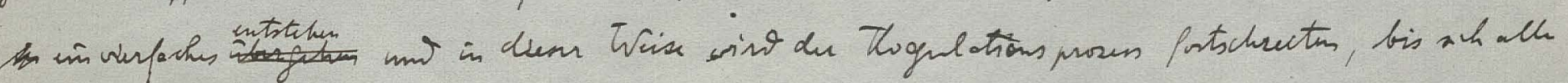

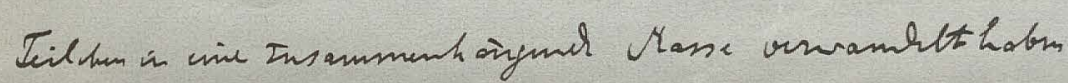

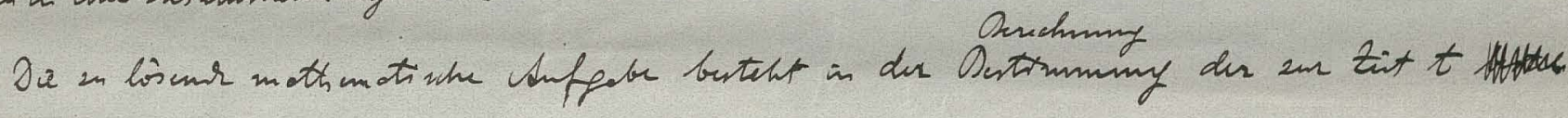

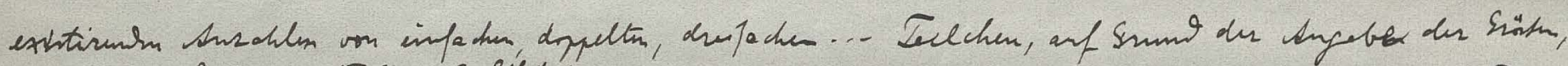

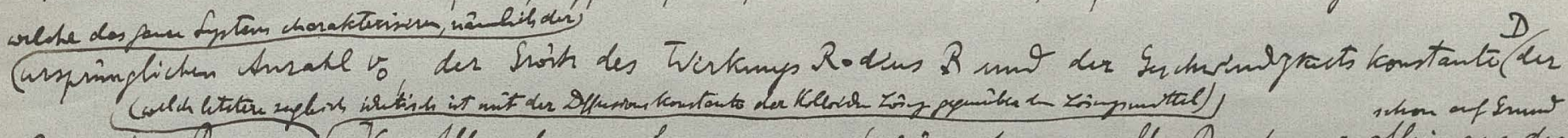

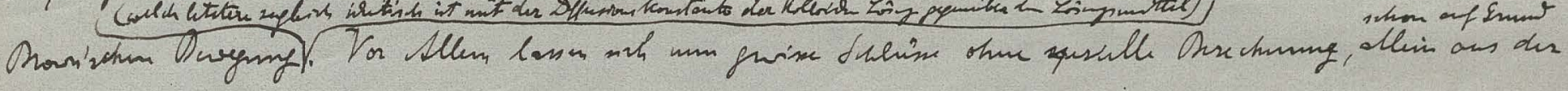

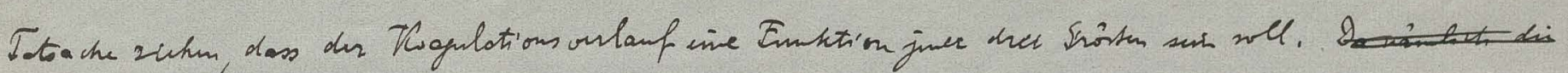

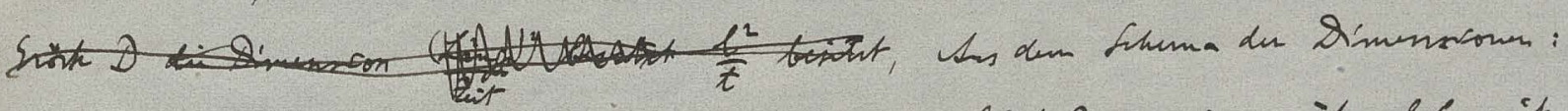

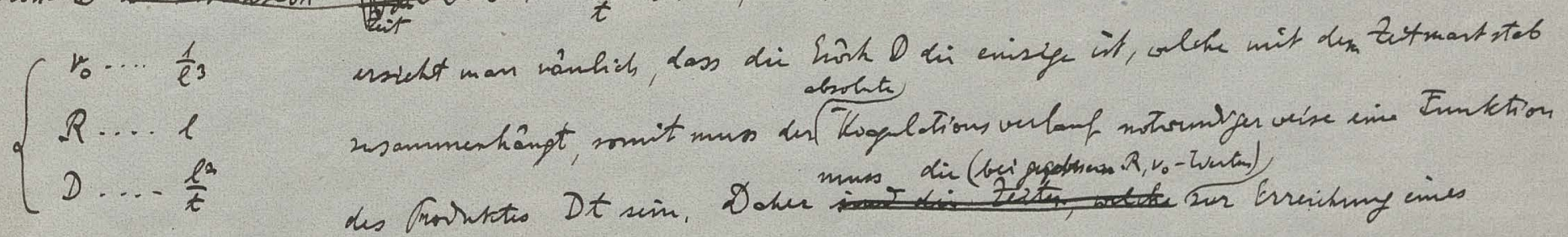

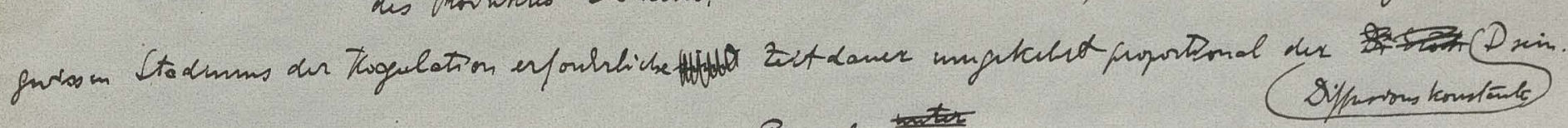

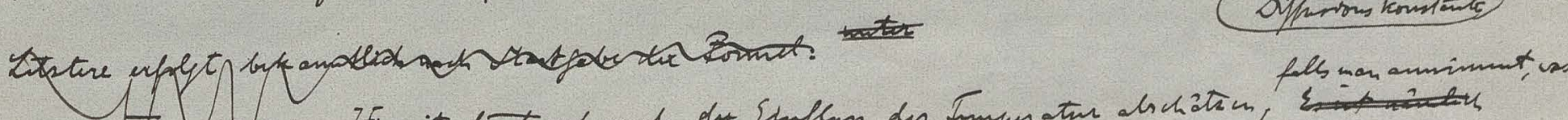

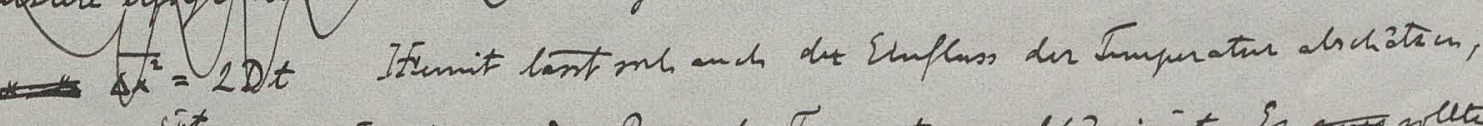
th orenume

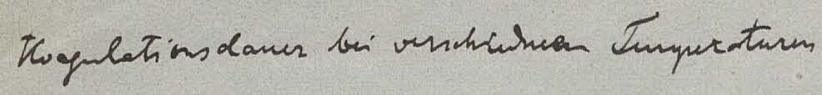
afly den End $D=\frac{H T}{N} \frac{1}{6 n \mu a}$

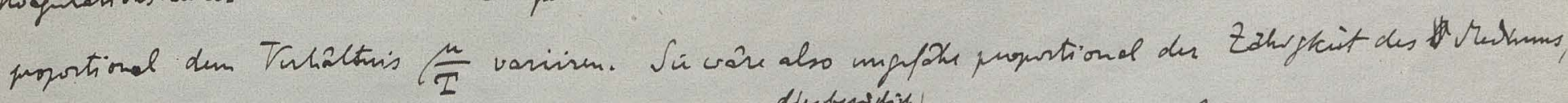

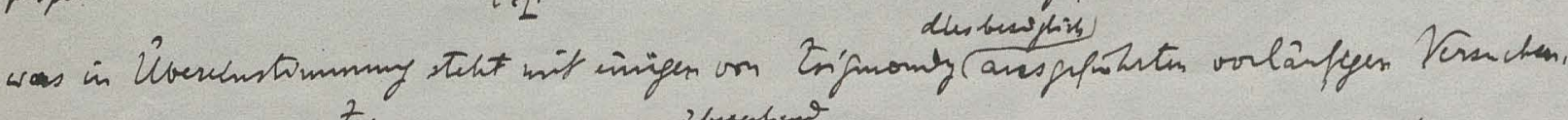

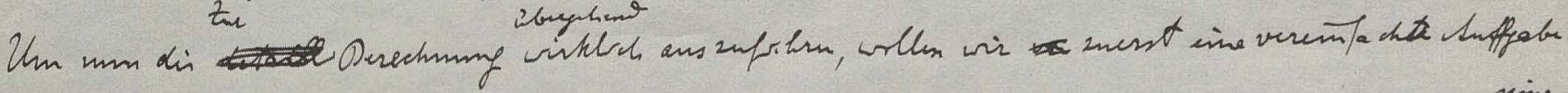

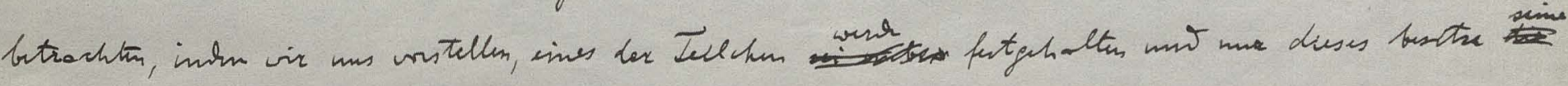

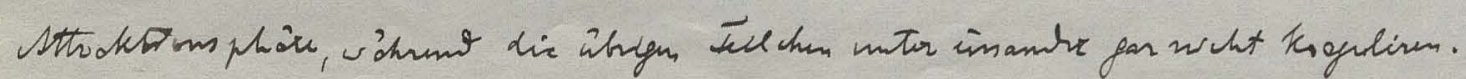




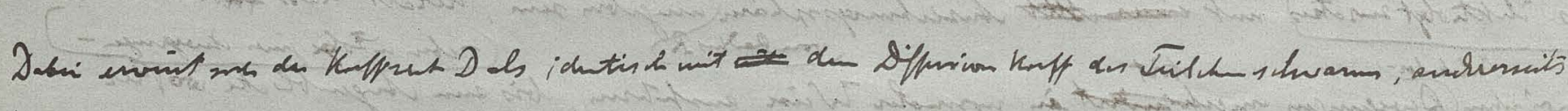

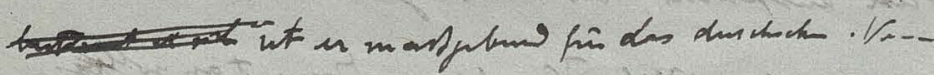

$(x-y)^{2}=20+$

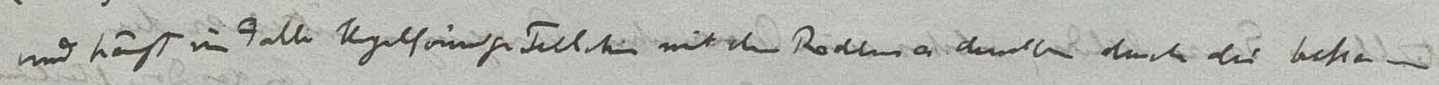
$\partial=\frac{H \pi}{N} \frac{1}{60 \% a}$

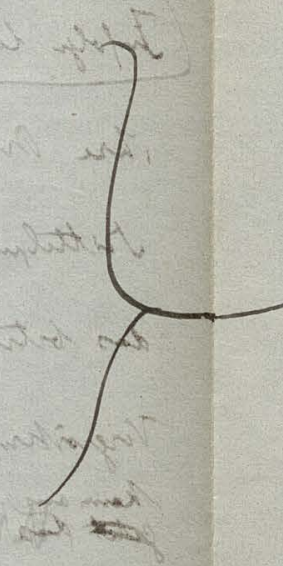

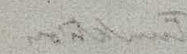

$\sin x+3 x$

$(3+x)+a n d t$

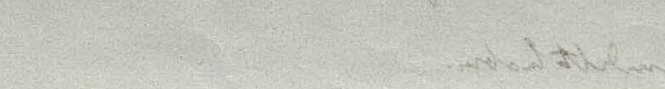

and 
426

(enar.')

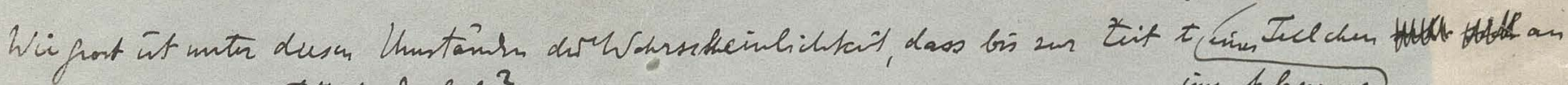
dum heroryshobum Totoptumber hate? inn Anleysurny)

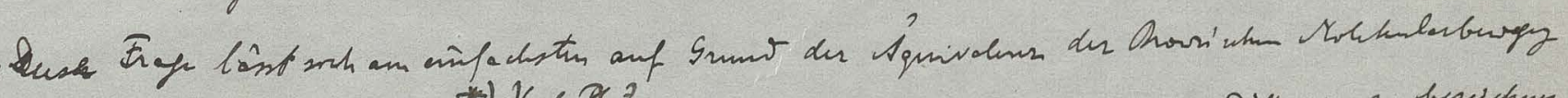

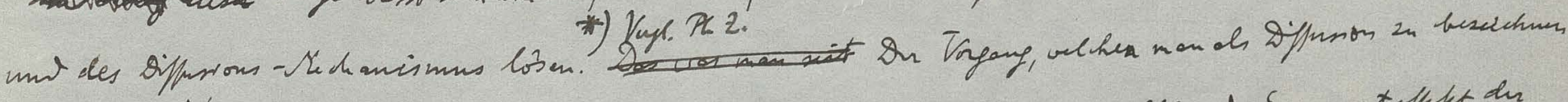

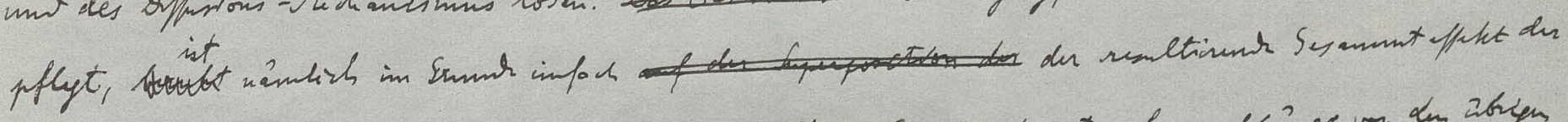

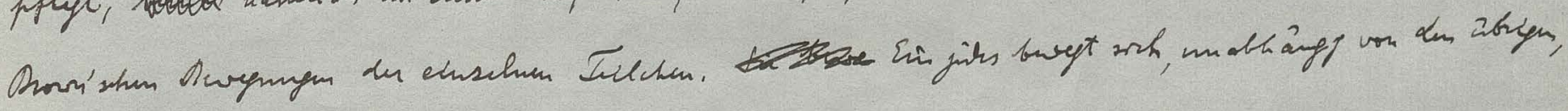
wach Mantgabe der Wolndenticinteits forme

$$
W_{(x)} d x=\frac{1}{2 \sqrt{206}} e^{-\frac{(x-t)^{2}}{40 t}} d x
$$

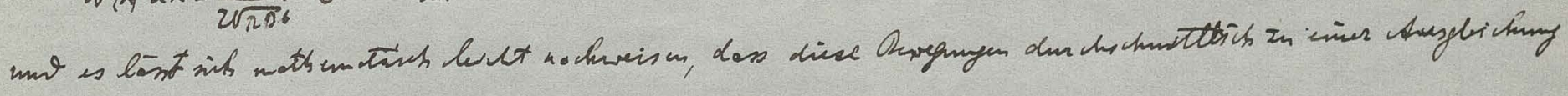

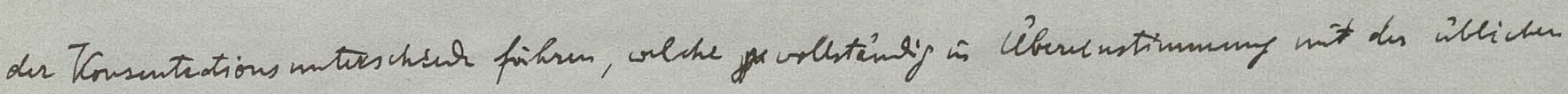

Dipuriourtacioni esflyt.

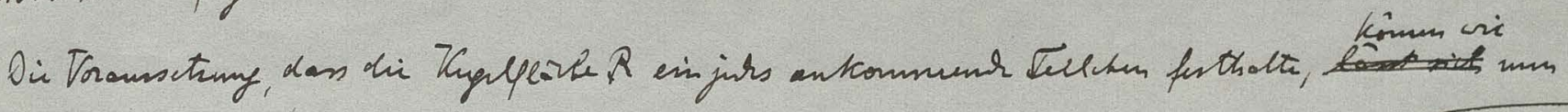

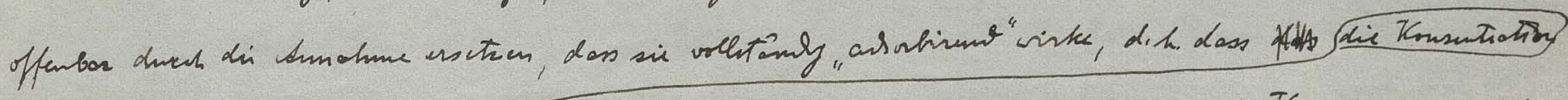

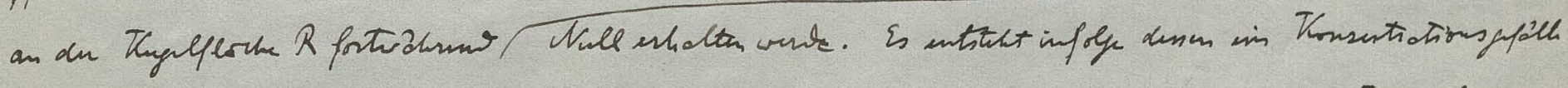

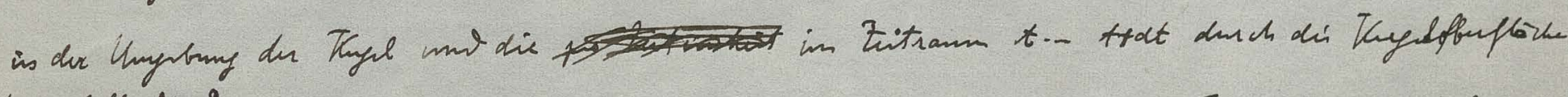

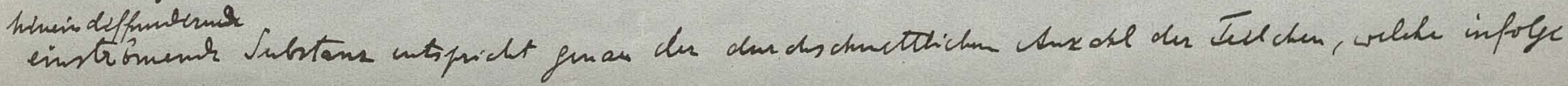

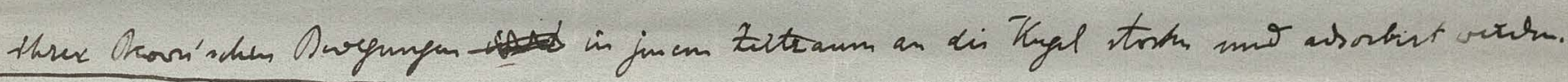

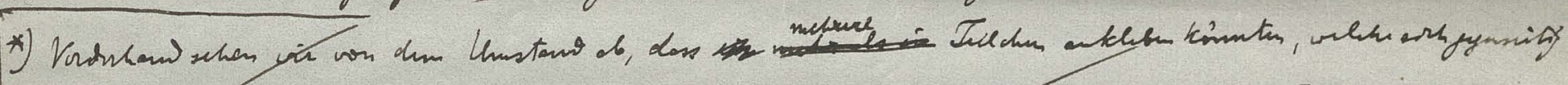

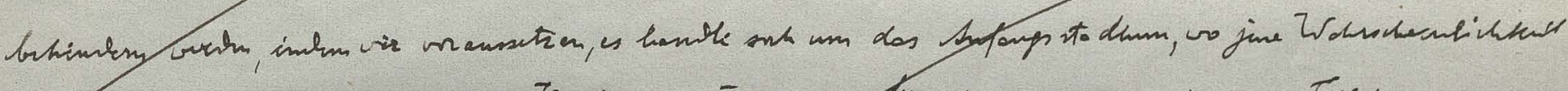

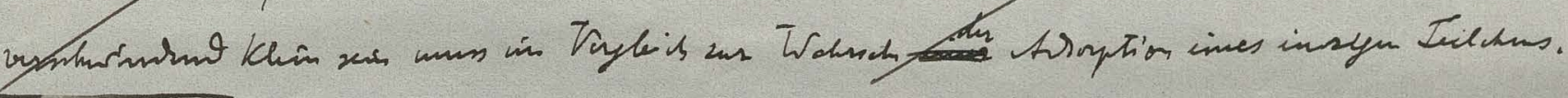

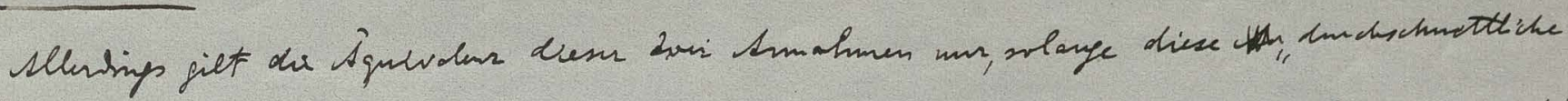

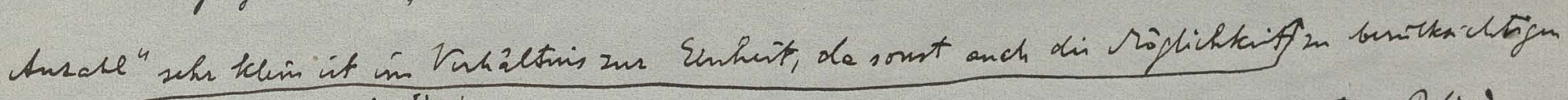

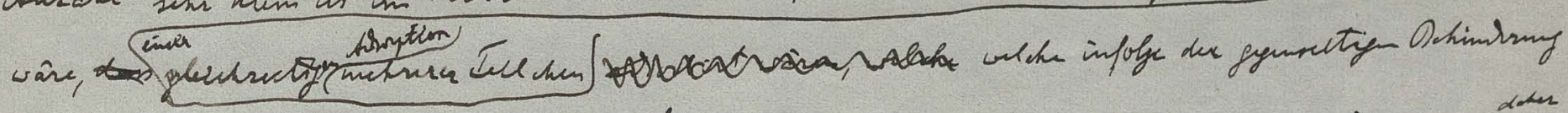

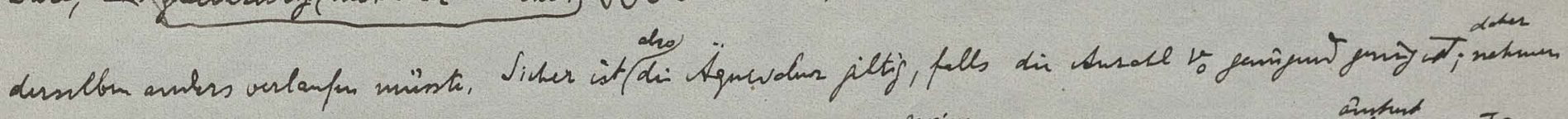

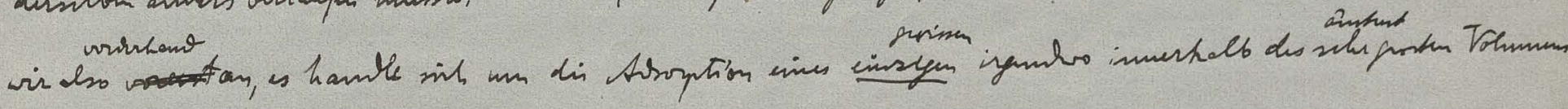
Vorchaniman Fellchers.

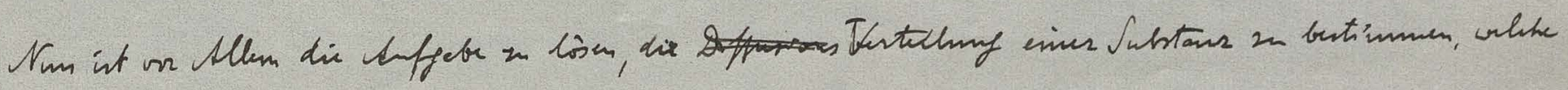

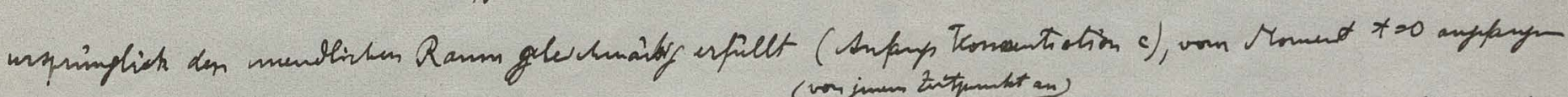

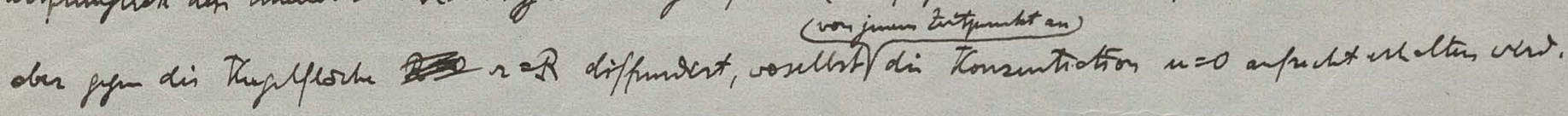

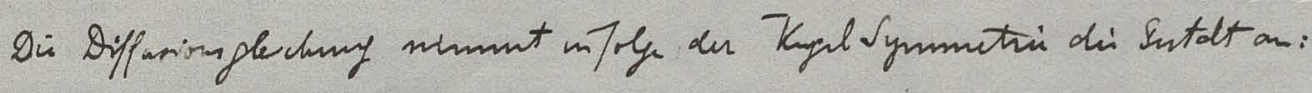

$$
\frac{\partial(2 u)}{\partial t}=D \frac{\partial^{2}(u 2)}{\partial z^{2}}
$$

not dese vers dunch dic Envation

$$
n=c\left[1-\frac{R}{n}+\frac{2 R}{n \sqrt{n}} \int_{0}^{\frac{1-R}{2 \sqrt{2}}} e^{-2^{2} d r}\right]
$$

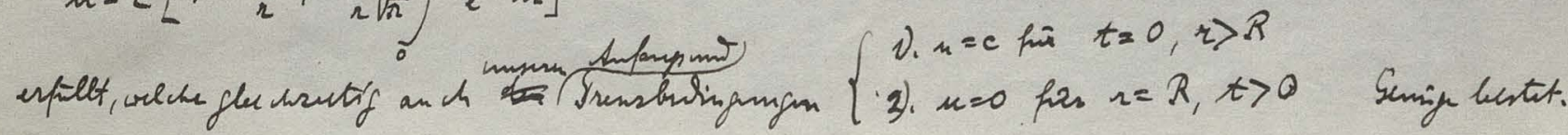


जing

a.

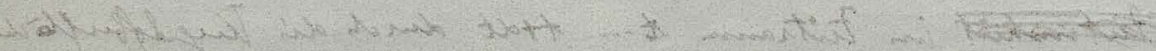

e

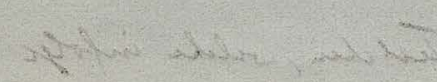

$\therefore$. 


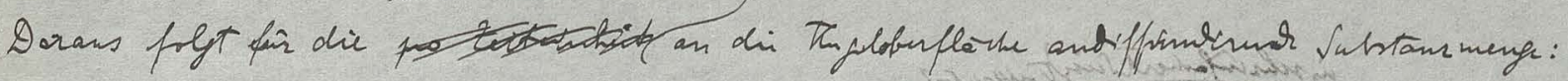

$J d t=\left.4 \pi D R^{2} \frac{\partial u}{\partial r}\right|_{2=R} ^{N} d t=4 \pi D R c\left[1+\frac{R}{\sqrt{n D t}}\right] d t$

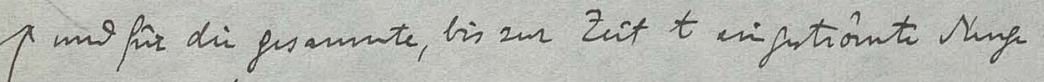

*) $\quad M=\int_{0}^{t} J d t=4 \pi 0 \Omega c\left[t+\frac{2 R \sqrt{t}}{\sqrt{x D}}\right]$

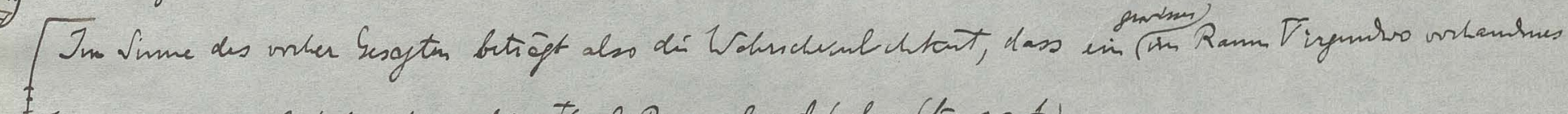

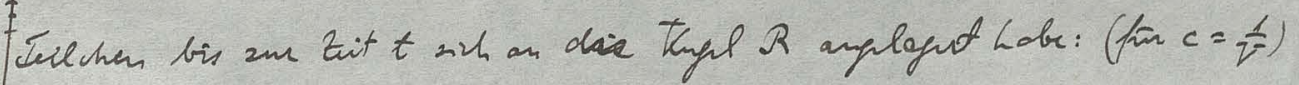

$W_{t}=\frac{4 \Omega D R}{V}\left[t+\frac{2 R \sqrt{t}}{\sqrt{\Omega D}}\right]$

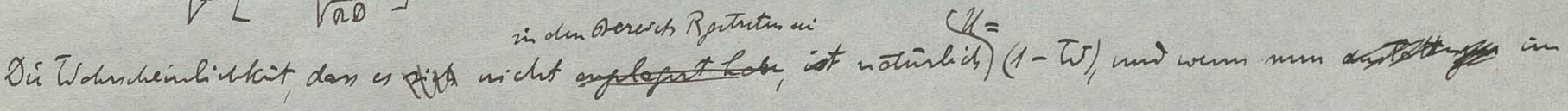

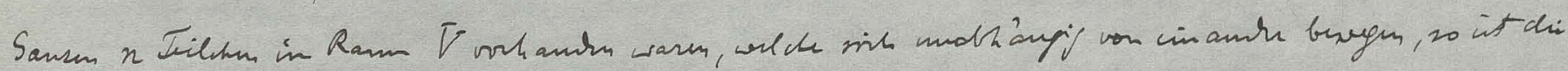

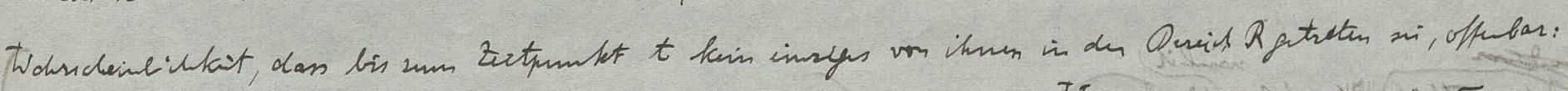

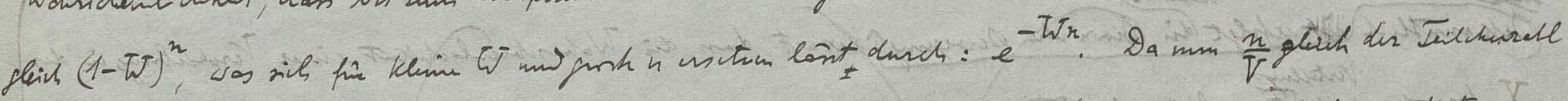

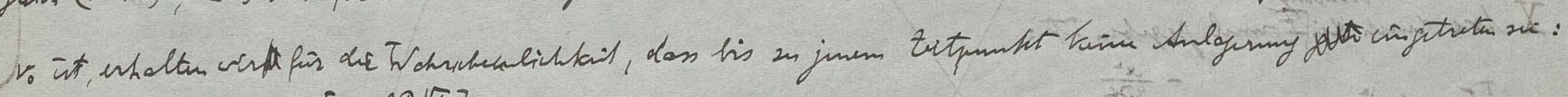

$$
U_{t}=e^{-4 \Omega \partial R_{0}\left[t+\frac{2 R \sqrt{t}}{\sqrt{n D}}\right]}
$$

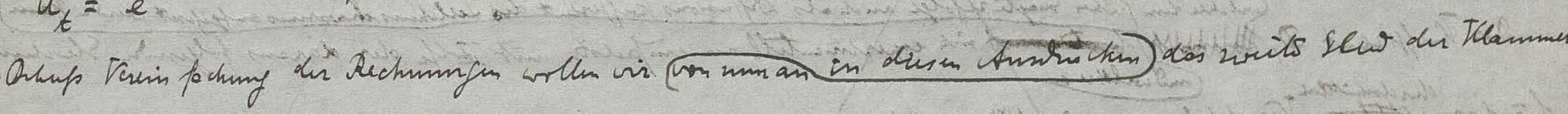

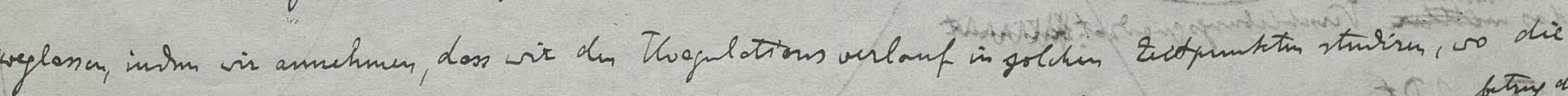

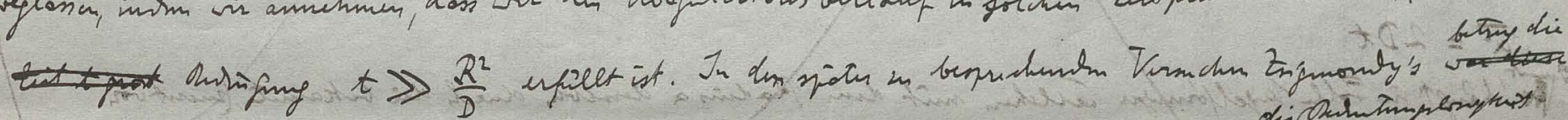

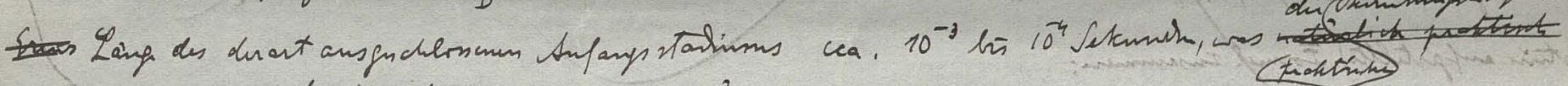

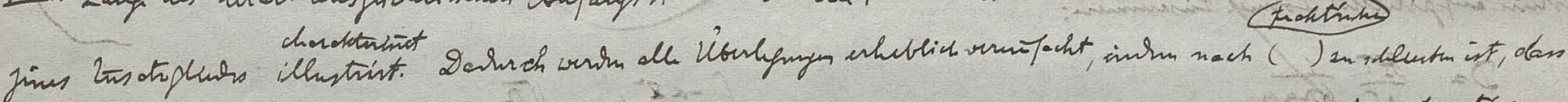

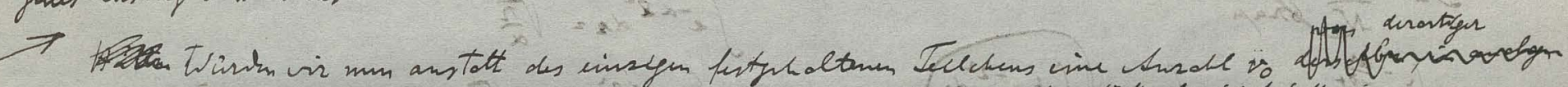

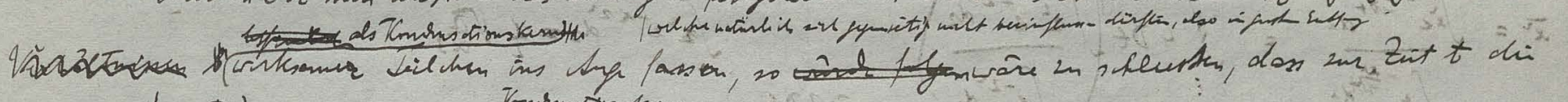

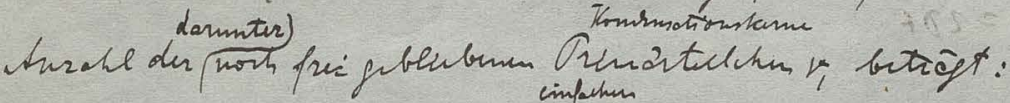

$$
\mu_{1}=v_{0} e^{-\sin \partial R v_{0} t}
$$

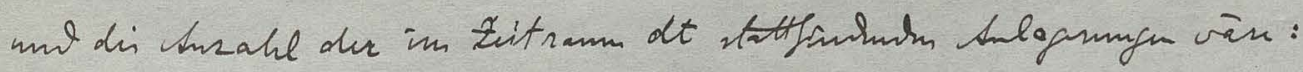

$-d v_{1}=4 \pi D R r_{0} v_{1} d t$

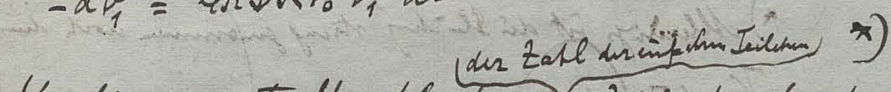

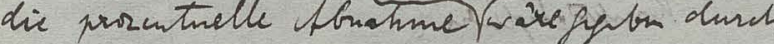

$-\frac{d v_{1}}{v_{1}}=4 \pi D R v_{0} d t$

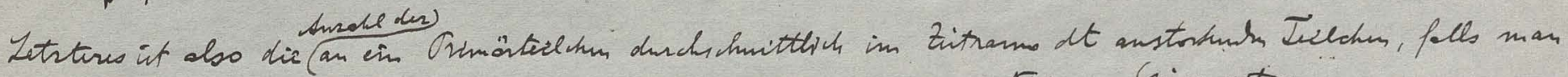

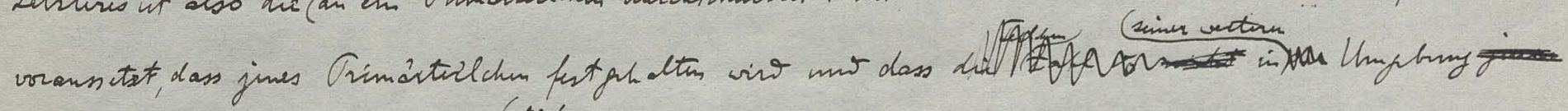

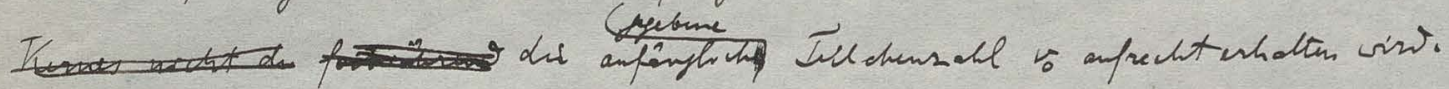

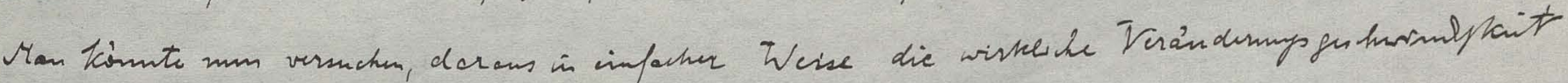

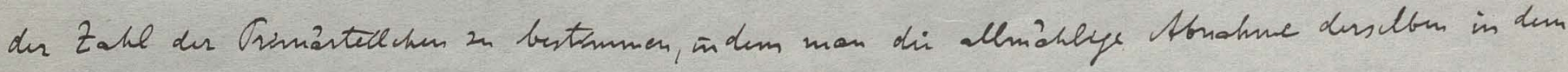

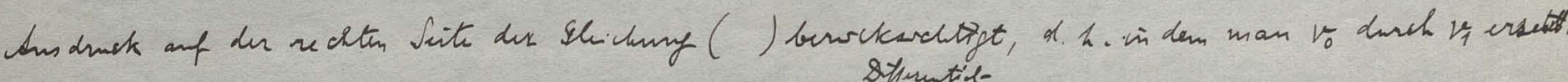

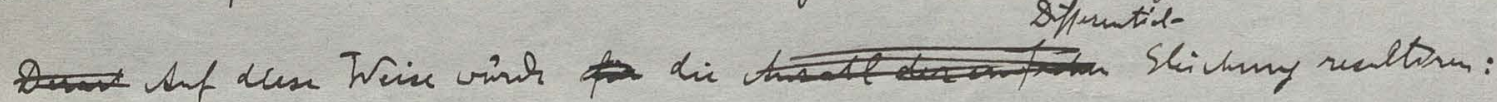

$$
\frac{11}{v_{1}^{2}} \frac{d v_{7}}{d t}=-4 \pi \partial R
$$




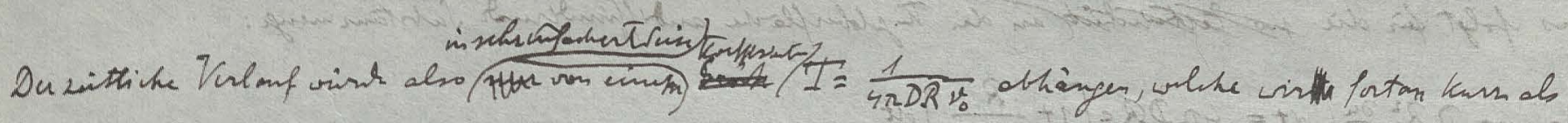

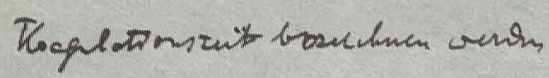

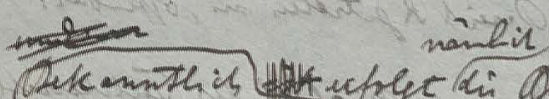

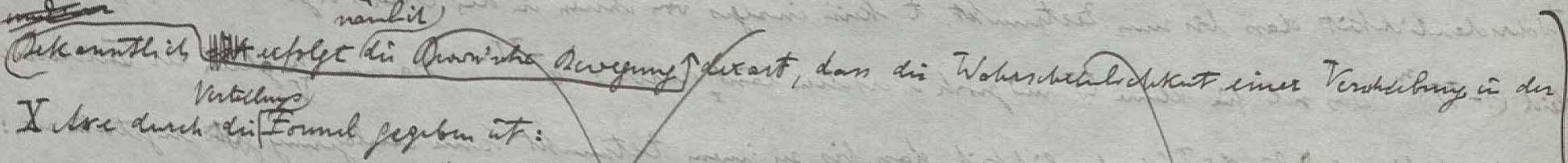

$T(x) d x=\frac{1}{2 \sqrt{n+5 x}} e^{-\frac{x^{2}}{2 D x}} d x$

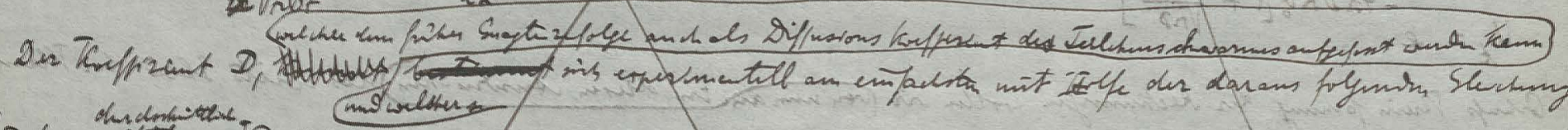

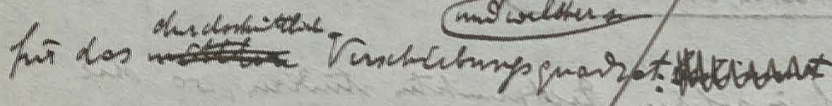

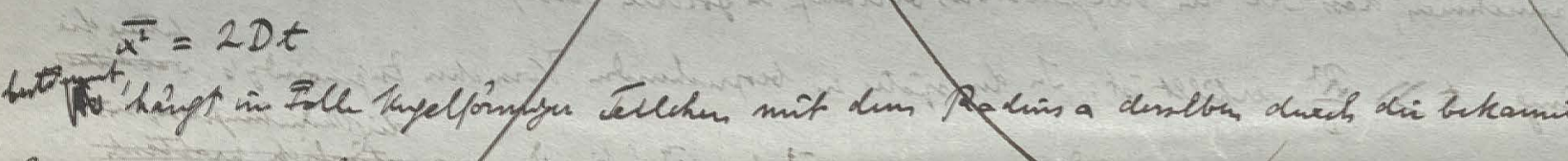

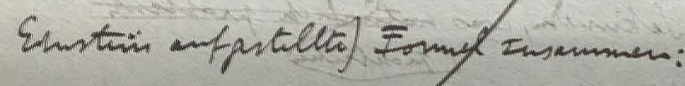

$$
\begin{aligned}
& D=\frac{\text { IIT }}{N} \frac{1}{\mathrm{Bra} / \mu}
\end{aligned}
$$

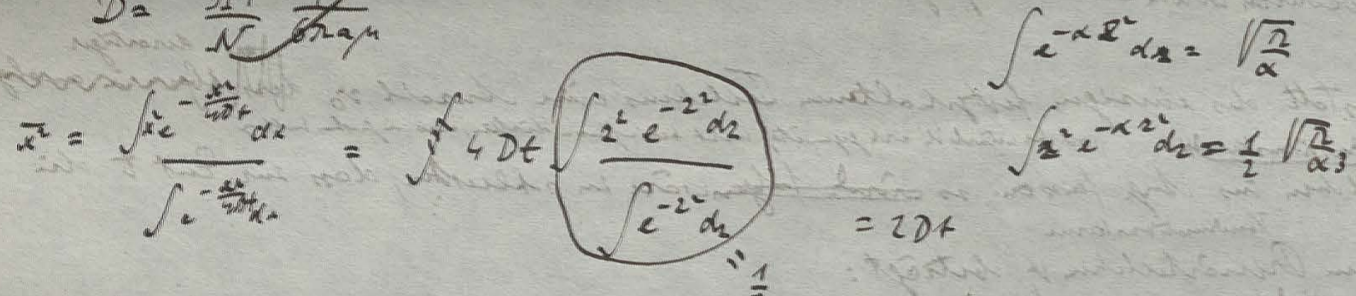

$$
\begin{aligned}
& \int e^{-\alpha z^{2} d z}=\sqrt{\frac{2}{\alpha}}
\end{aligned}
$$

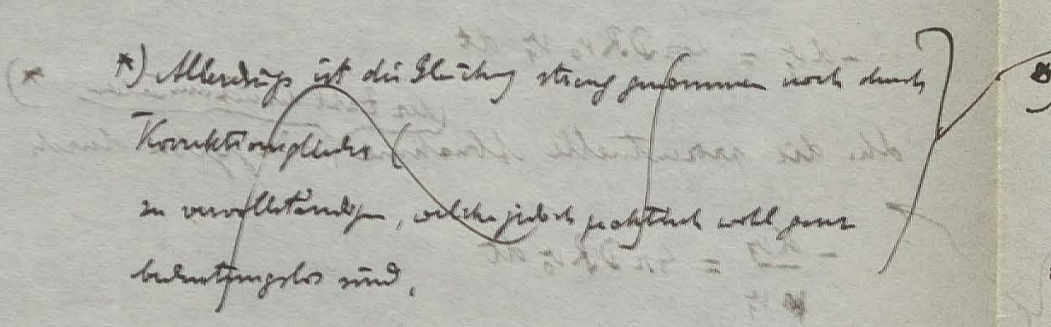




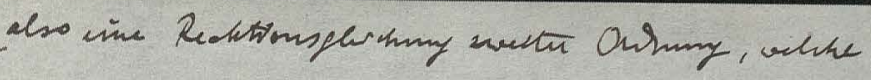

448 Antegnt tegih:

$$
v_{1}=\frac{v_{0}}{1+\varepsilon n \partial R_{0} t}=\frac{\nu_{0}}{1+\frac{t}{T}}
$$

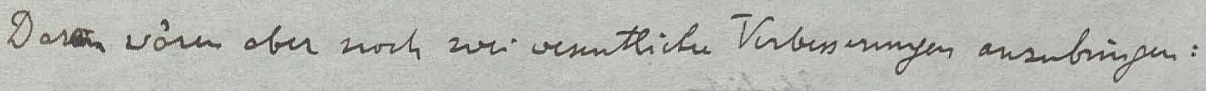

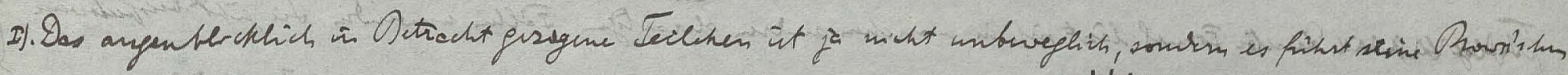

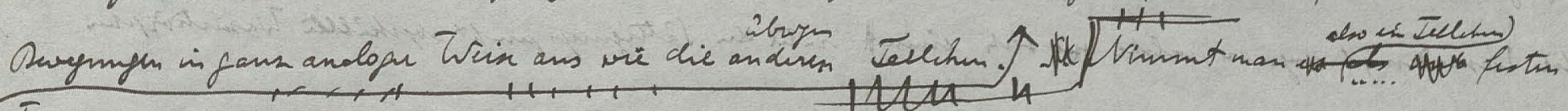

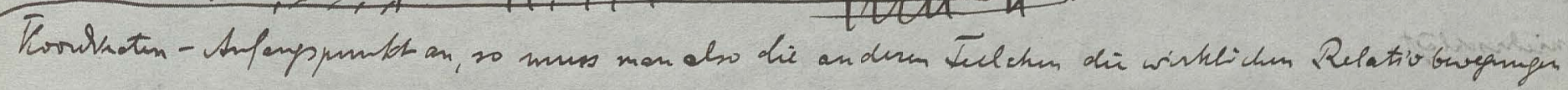
ensfincu lassm,

8

$$
\begin{aligned}
& \text { 14 lent a k } \\
& \text { Cun ohoingy uncinanls }
\end{aligned}
$$

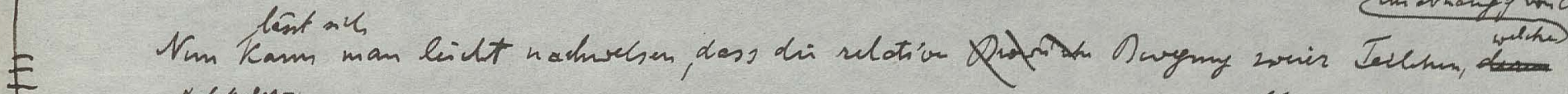

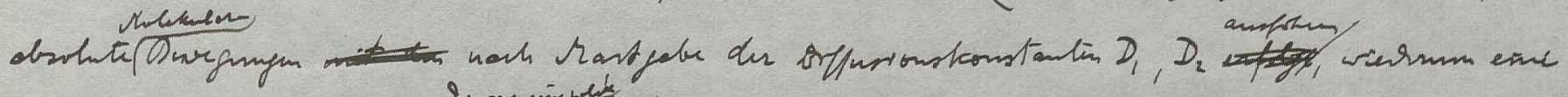

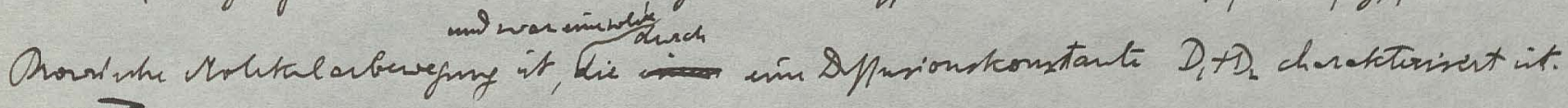

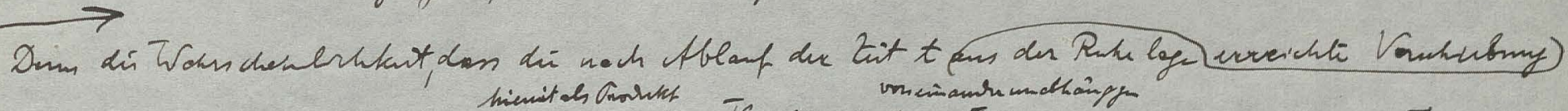

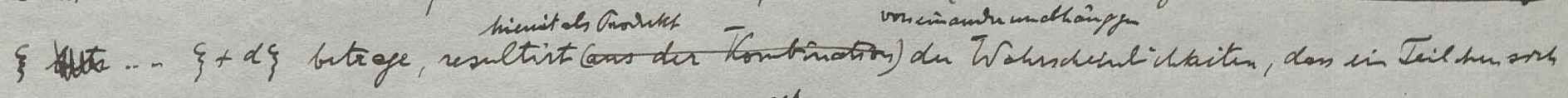

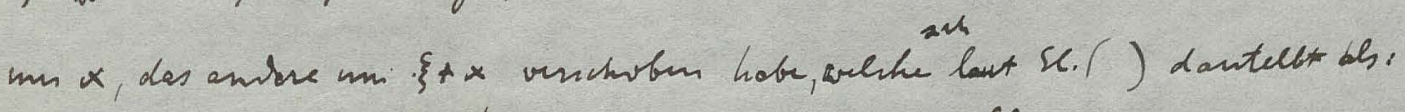

$$
\begin{aligned}
& W(\xi) d \xi=\frac{d \xi}{2 n+\sqrt{D, D_{2}}} \int^{+\infty}-\frac{(\xi+x)^{2}}{4 D_{1} t}-\frac{x^{2}}{4 \lambda_{2} t} d x=\frac{e^{-\frac{\xi^{2}}{\left.4 D_{1}+D_{2}\right) t}}}{2 \sqrt{\left(\pi_{n}\left(D_{1}+\lambda_{2}\right) t\right.}} d \xi \\
& \int_{x=-\infty}^{+\infty} T(x) d x \bar{L}(\xi+x) d \xi=
\end{aligned}
$$

$$
\text { ? }
$$

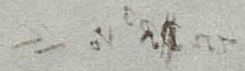

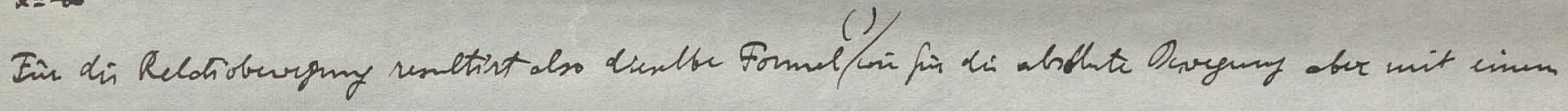
Toppisunter $D_{1}+D_{2}$ anstoto $D$.

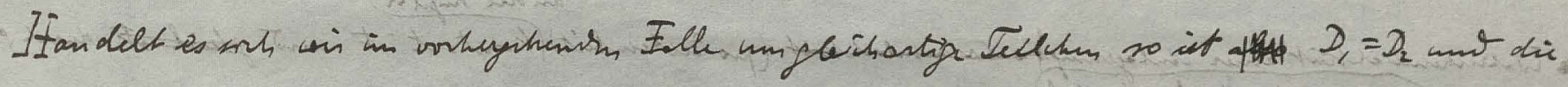

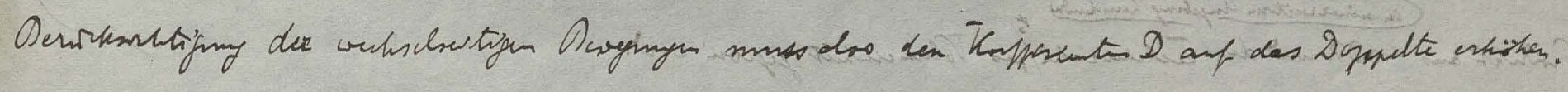

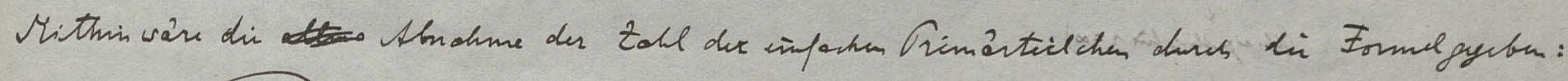

$$
v_{1}=\frac{v_{0}}{1+\delta_{n} D R v_{0} t}=\frac{v_{0}}{1+\frac{2 t}{T}}
$$

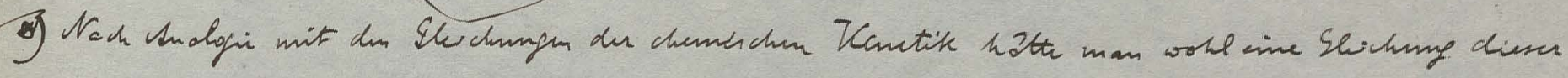

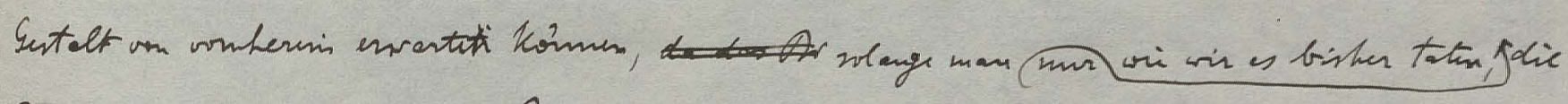

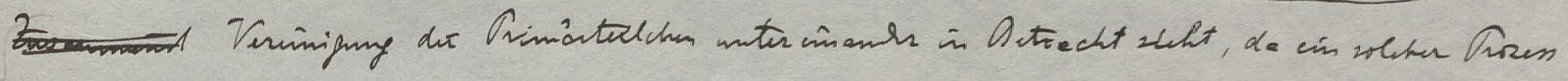

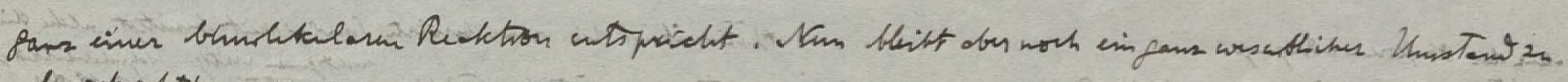
buiskerstigen:

D).

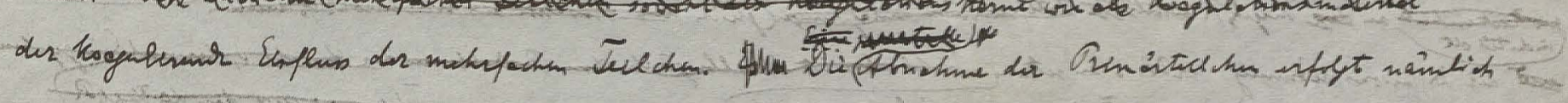

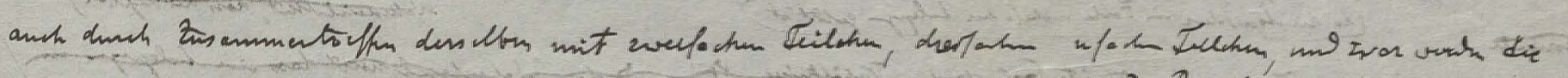

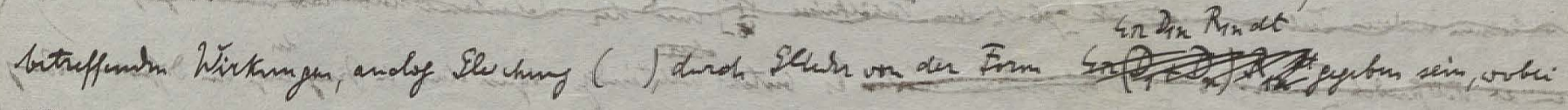

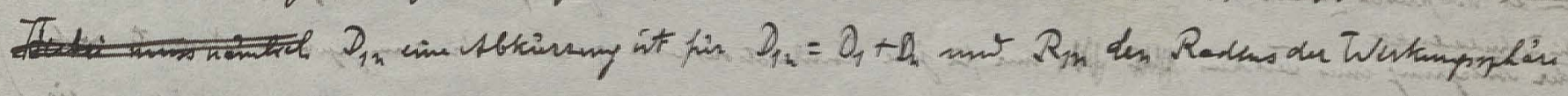

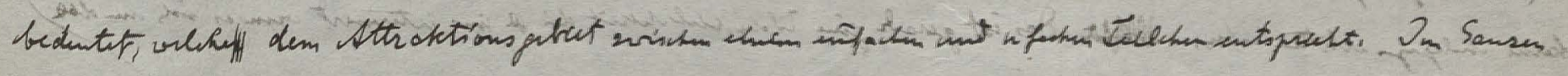

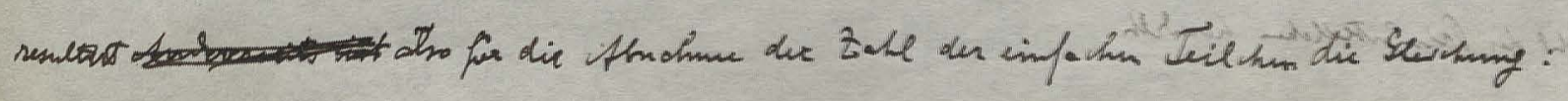




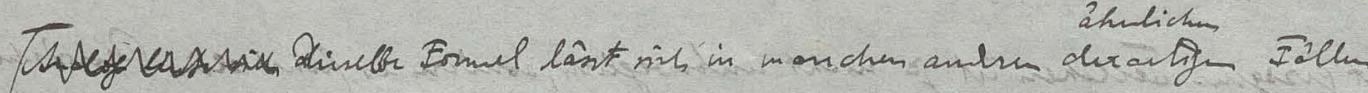

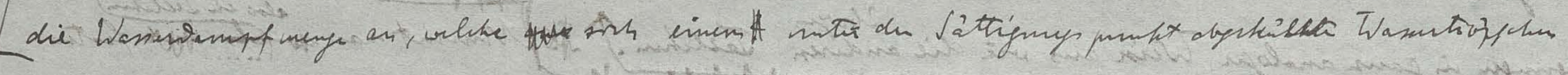
nimuregt.

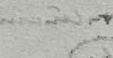
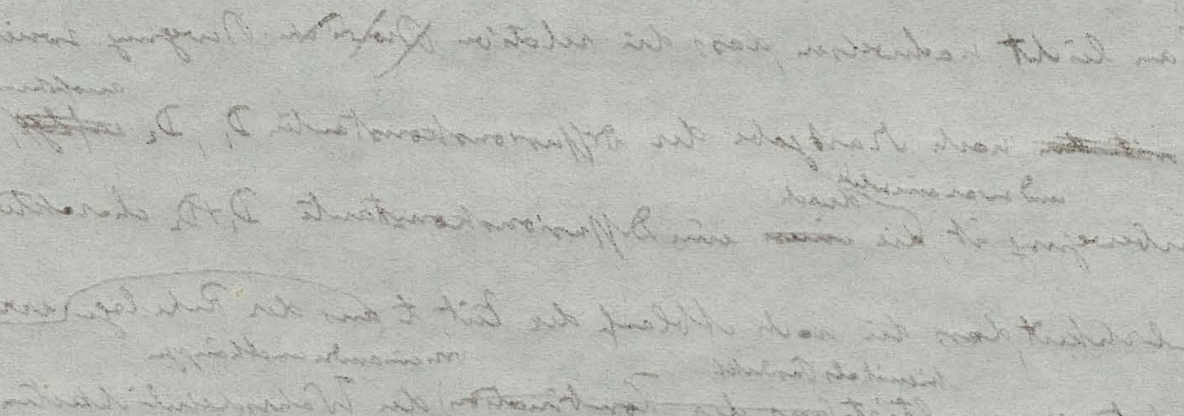

$4 \Omega \not R^{3} v_{0} \ll 1$

$2+3$

$x+4$

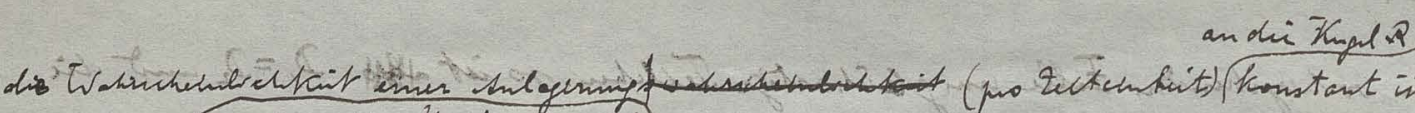
in sinerveitom Unyedmy romismas

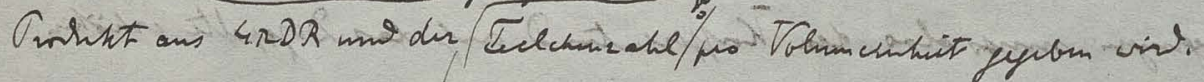

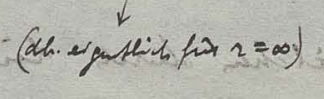

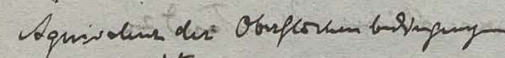

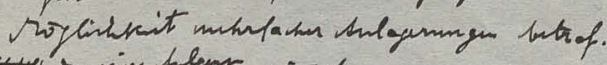

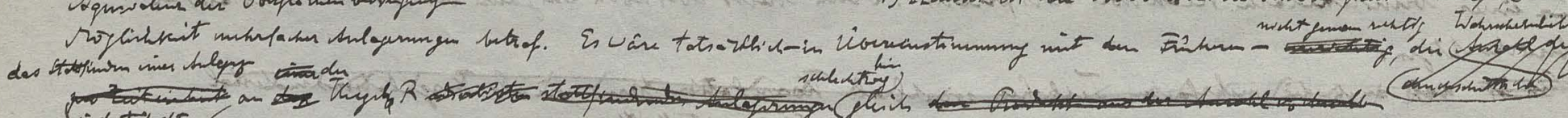

Tromente (da dir dis $^{3}$

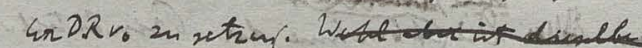

egenih de

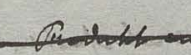

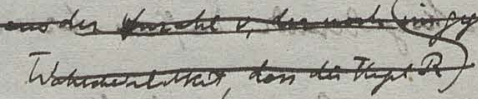

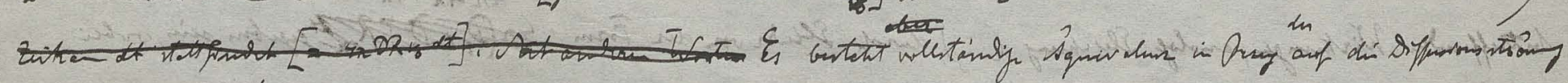

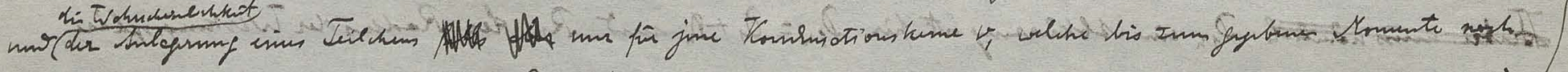

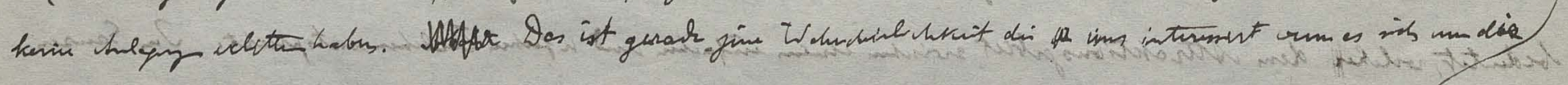

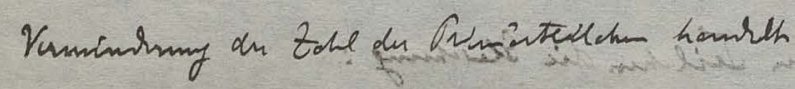


154

$$
+\frac{1}{4 \Omega} \frac{d v_{1}^{2}}{d t}=-D_{11} R_{11} v_{1}^{2}-D_{12} R_{12} r_{1} v_{2}-D_{13} R_{13} r_{3}-\ldots
$$

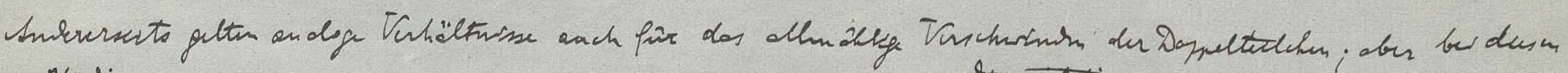

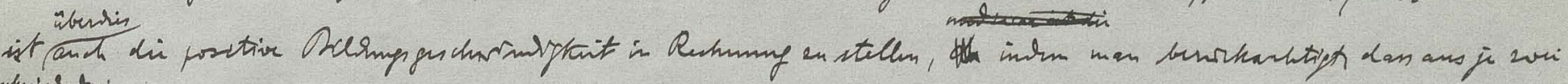

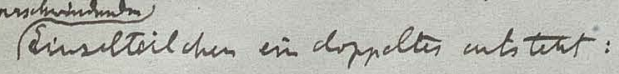

$$
\frac{1}{42} \frac{d v_{1}}{d t}=\frac{1}{2} D_{11} R_{11} v_{11}^{2}-D_{12} R_{12} v_{1} v_{2}-D_{22} R_{22} v_{2}^{2}-\partial_{23} R_{25} v_{3}-\cdots
$$

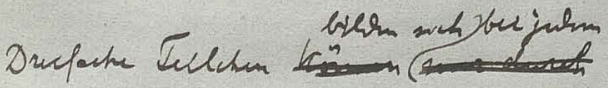

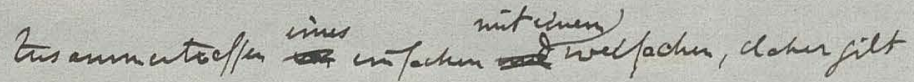

$$
\frac{1}{42} \frac{d v_{3}}{d t}=D_{12} R_{12} v_{1} v_{2}-D_{13} R_{13} v_{i} v_{3}-D_{23} \cdot R_{23} v_{2} v_{3} \ldots
$$

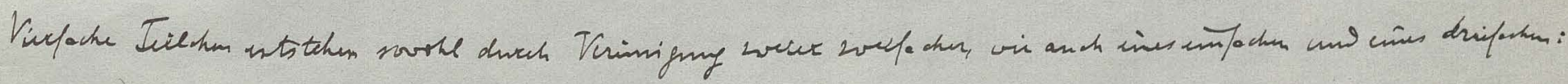

$$
\frac{1}{4 \Omega} \frac{d v_{4}}{d t}=\frac{1}{2} D_{22} R_{22} v_{2}^{2}+D_{13} R_{13} v_{1} v_{3}-D_{14} R_{14} v_{1} v_{4}-\ldots u_{1} 1_{1} N_{1}
$$

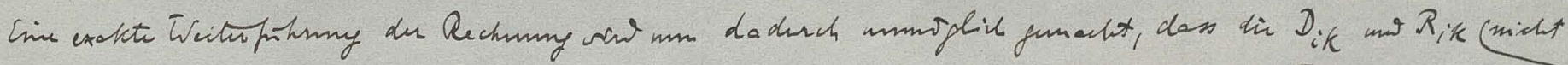

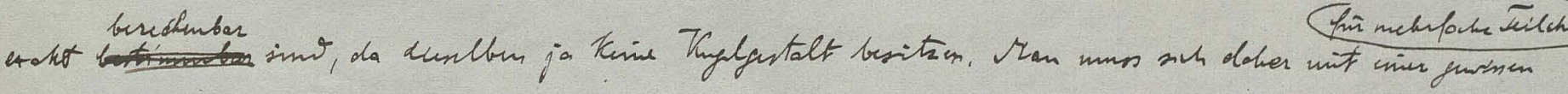

Fin mebrote Filchm

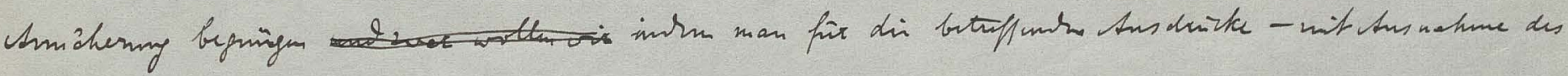

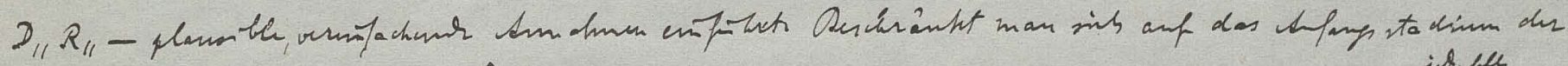

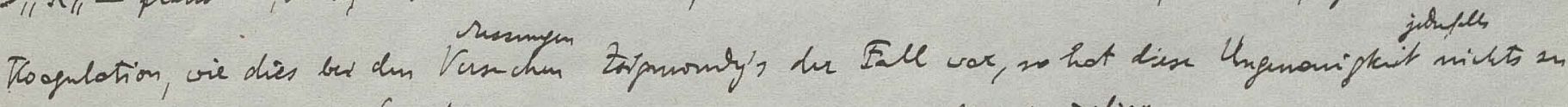

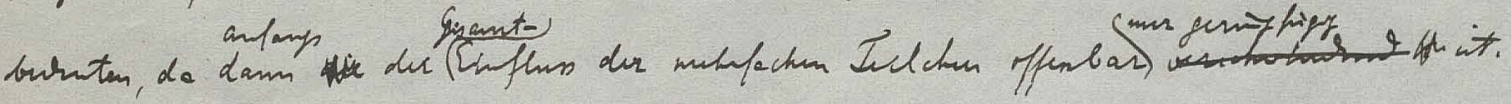

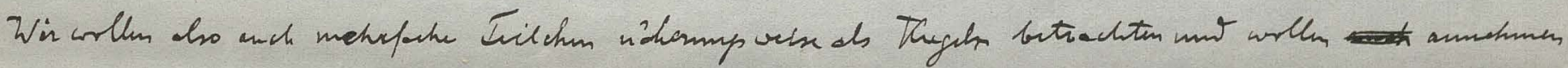

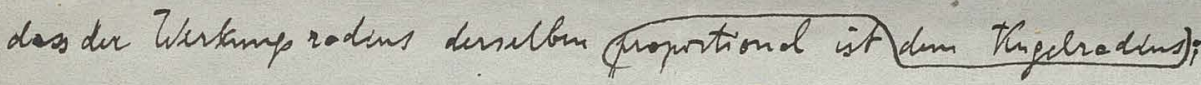

betare

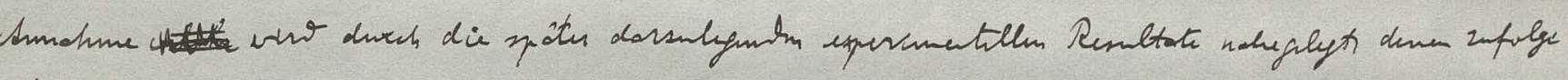

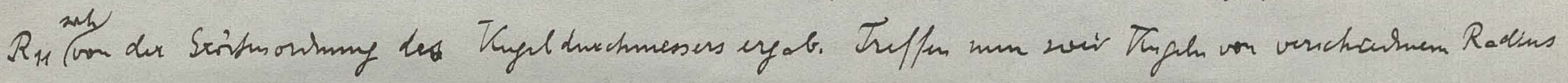

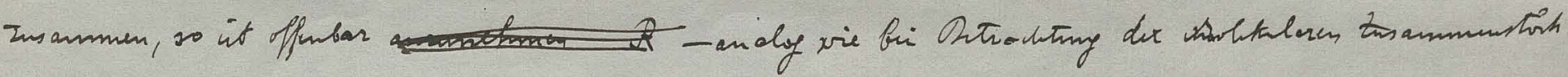

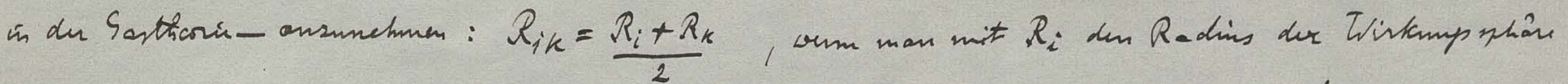

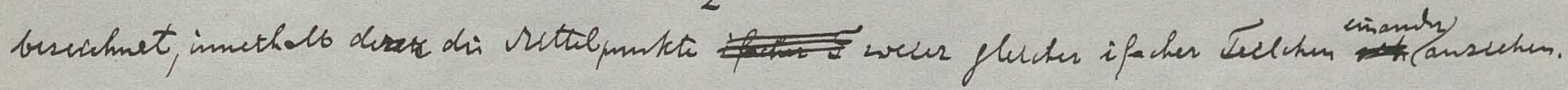

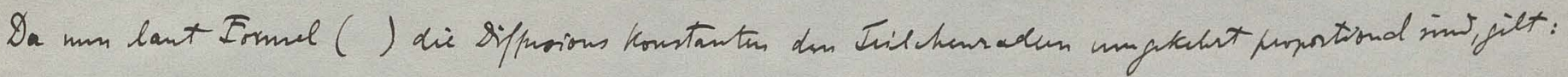

$$
D_{i k} R_{i k}=\frac{1}{2}\left(D_{i}+D_{k}\right)\left(R_{i}+R_{k}\right)=\frac{\partial R}{2}\left(R_{i}+R_{k}\right)\left(\frac{1}{R_{i}}+\frac{1}{R_{k}}\right)=\frac{\partial R}{2} \frac{\left(R_{i}+R_{k}\right)^{2}}{R_{i} R_{k}}
$$

En gleiche $R_{a}$ dlem $Q_{i}=R_{k}$ folgt also is Dik $R_{i k}=2 D R$

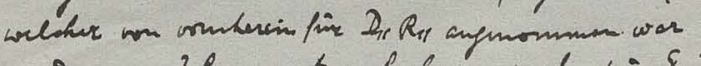

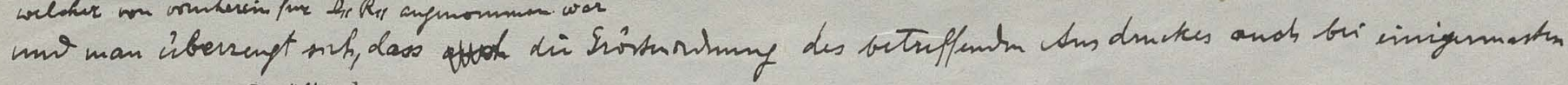

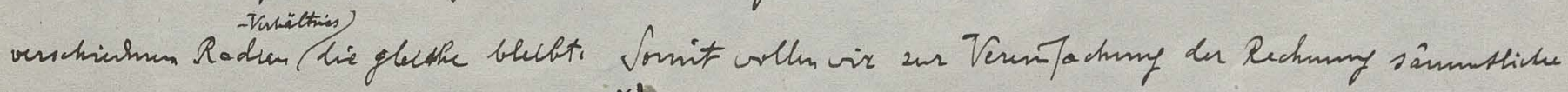

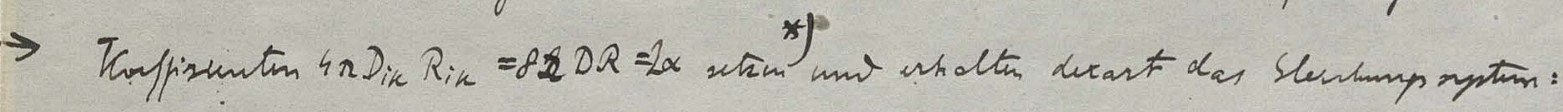

$$
\begin{aligned}
& \int \frac{d v_{1}}{d t}=-v_{1}^{2}-r_{1} r_{2}-r_{1} r_{3}-r_{1} r_{4} \\
& \frac{1}{d x} \frac{d v_{2}}{d t}=\frac{v_{1}^{2}}{2}-v_{1} r_{2}-r_{2}^{2}-v_{2} r_{3}-\cdots \\
& \frac{1}{2 \alpha} \frac{d r_{4}}{d t}=\frac{d r_{3}}{d t}=r_{1} r_{2}-v_{1} r_{3}-r_{2} r_{3}-r_{3}^{2}-\cdots
\end{aligned}
$$




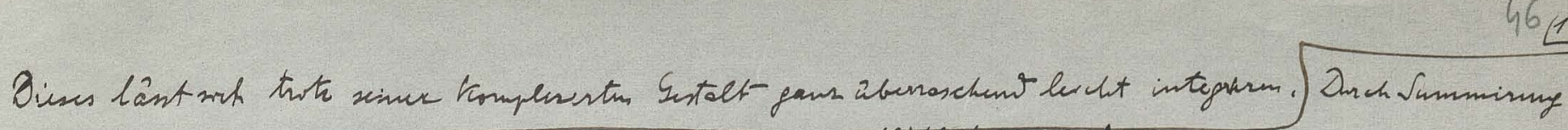

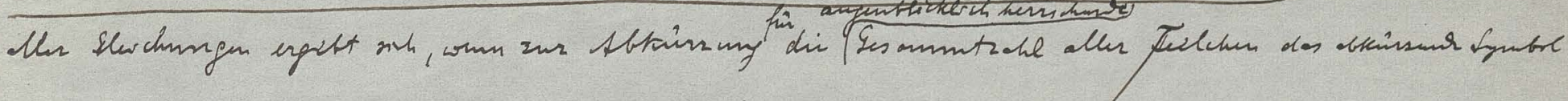

$$
\begin{aligned}
& r_{1}+k_{2}+r_{3}+r_{4} \ldots=\sum_{r} \quad \text { ingphent wint: } \\
& \frac{1}{2 \alpha} \frac{d \sum_{r}}{d t}=\frac{\left(\sum v\right)^{2}}{2}
\end{aligned}
$$

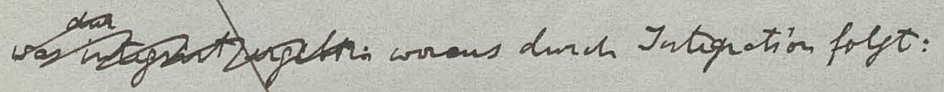

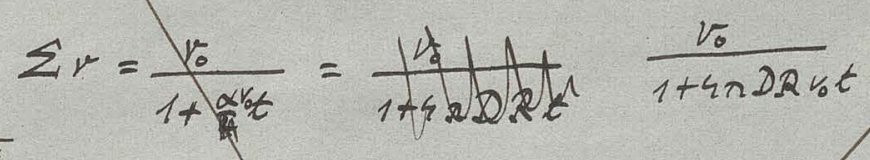

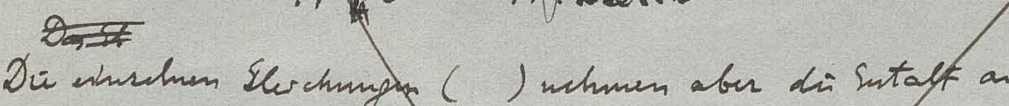

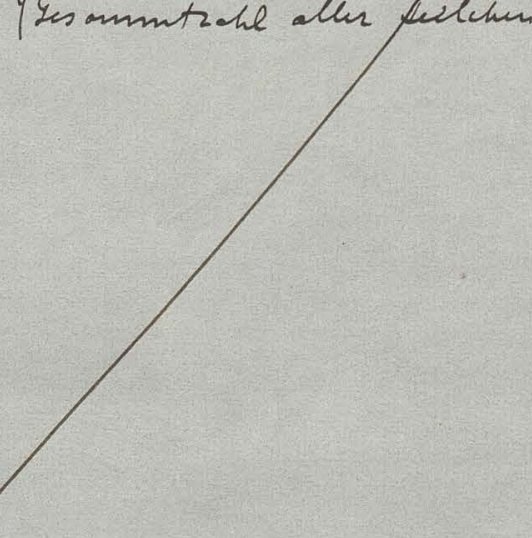

$$
\begin{aligned}
& 2 \frac{1}{\alpha} \frac{d v_{1}}{d t}=-v_{1} \sum r \\
& 2 \frac{1}{\alpha} \frac{d v_{2}}{d t}=\frac{v_{1}^{2}}{2}-v_{2} \sum_{r} \\
& -\frac{1}{2 \alpha} \frac{d v_{3}}{d t}=v_{1} v_{2}-v_{3} \sum r \\
& 2 \frac{1}{2 \alpha} \frac{d v_{4}}{d t}=\frac{v_{2}^{2}}{2}+v_{1} v_{3}
\end{aligned}
$$

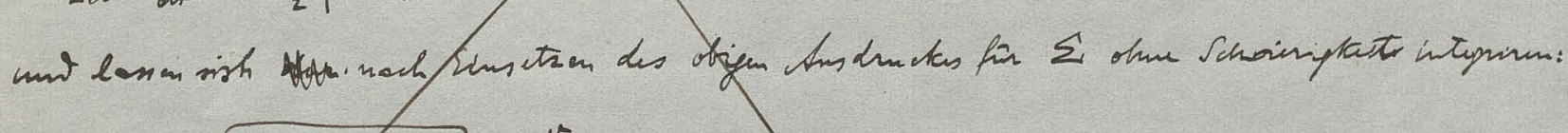

$v_{1}=\frac{v_{0}}{\left(1+4 y 0 R_{0} t\right)^{2}}=\frac{v_{0}}{\left(1+\frac{\alpha}{t} t\right)^{2}}$

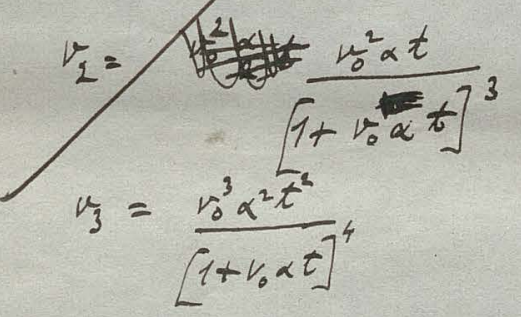

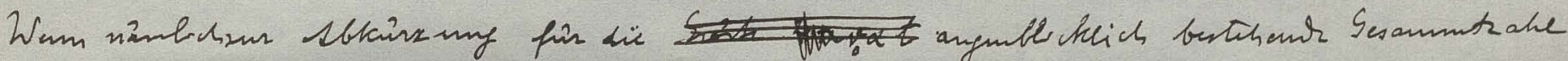

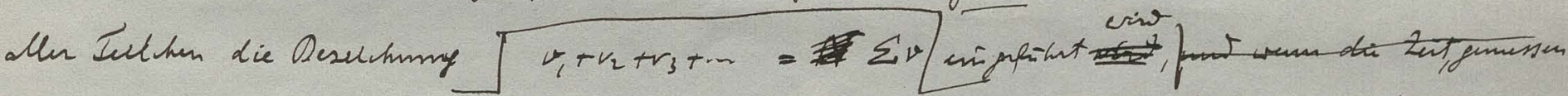

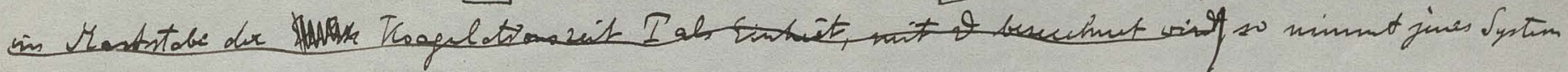
dis Extolt an:

$$
\begin{aligned}
& \frac{1}{2} \frac{d x}{d x}=-v_{1} \sum \pi v \quad \frac{1}{2 \alpha} \frac{d v_{1}}{d t}=-\nu_{1} \sum_{i v} \\
& \frac{1}{2} \frac{d v_{2}}{d t}=\frac{x_{1}^{2}}{2}-v_{2} \quad \frac{1}{2 \alpha} \frac{d v_{2}}{d t}=\frac{r_{1}^{2}}{2}-v_{2} \sum \\
& \frac{1}{2 \alpha} \frac{d r_{3}}{d t}=r_{1} r_{2}-r_{3} \sum \\
& \frac{1}{2 \alpha} \frac{d v_{k}}{d t}=\frac{1}{2}\left[v_{1} v_{k-1}+r_{2} v_{k-2}+v_{3} v_{k-3}+\cdots v_{k-1} v_{1}\right]-v_{k} \sum v
\end{aligned}
$$

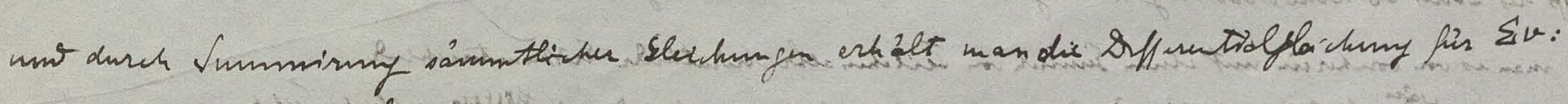

$$
\begin{aligned}
& \frac{1}{1 \alpha} \frac{d \sum_{v}}{d t}=-\left(\sum, y\right)^{2} \\
& \text { ans wicher nummetr folgt: }
\end{aligned}
$$

$$
\Sigma x=\frac{r_{0}}{1+\alpha r_{0} t}=\frac{r_{0}}{1+i_{n} D R v_{0} t}
$$

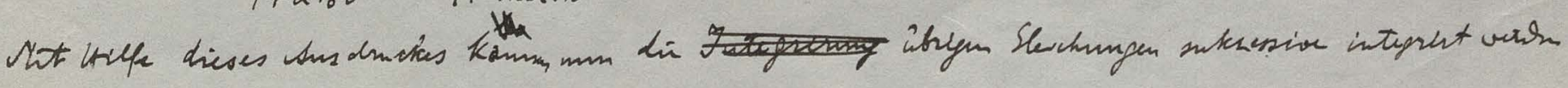

$$
\begin{aligned}
& v_{1}=\frac{v_{0}}{\left[1+\alpha v_{0} t\right]^{2}}=\frac{v_{0}}{\left[1+4 n \partial R v_{0} t\right]^{2}} \\
& v_{2}=\frac{\alpha v_{0} t}{v_{0}} \frac{\left.v_{0} v_{0} t\right]^{3}}{\left[1+\quad v_{3}\right.}=v_{0} \frac{\left[\alpha v_{0} t\right]^{2}}{\left[1+\alpha v_{0} t\right]^{4}}
\end{aligned}
$$




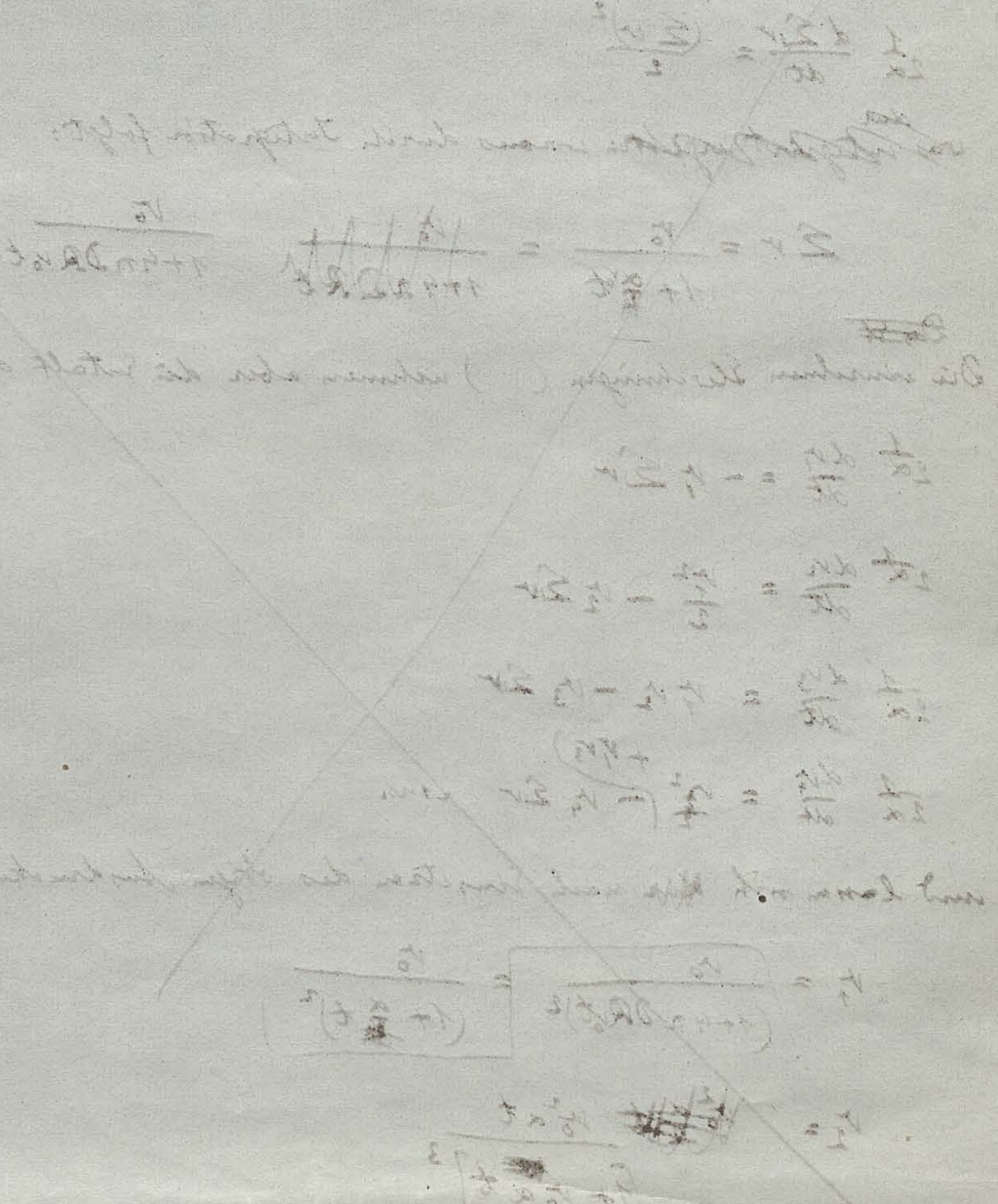

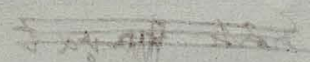

is 7 is: $=$

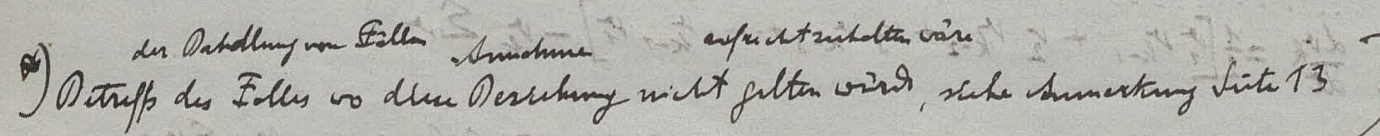

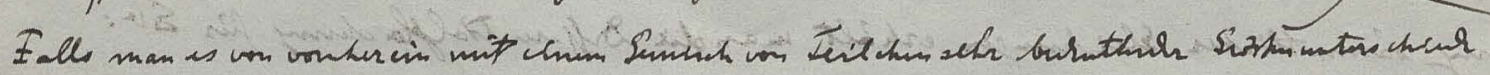

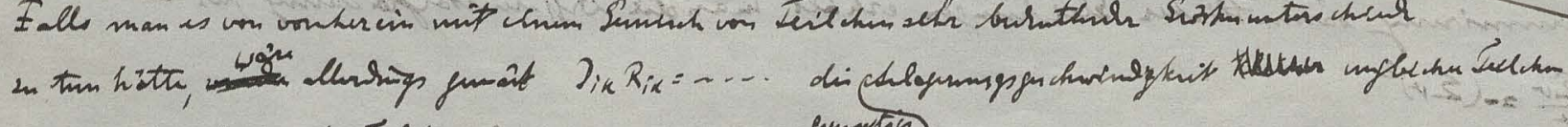

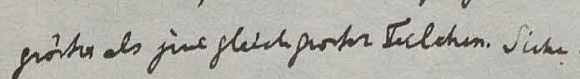

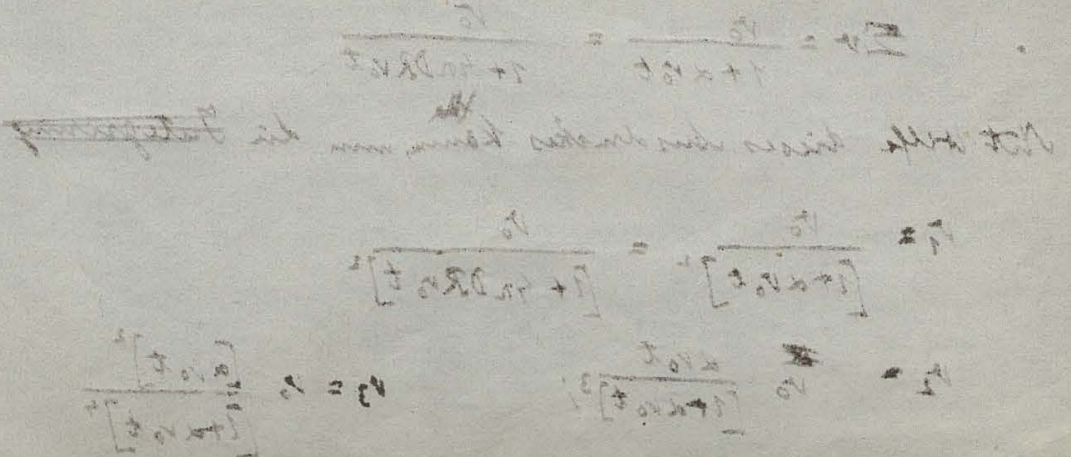




$$
r_{k}=r_{0} \frac{\left[\alpha v_{0} t\right]^{k-1}}{\left[1+\alpha r_{0} t\right]^{k+1}}
$$

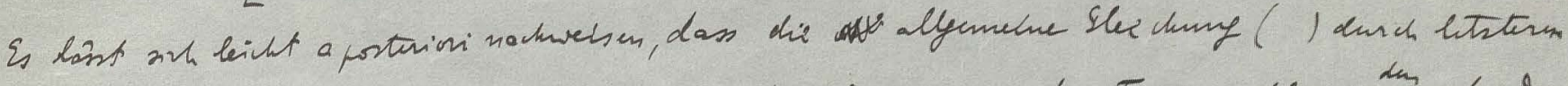

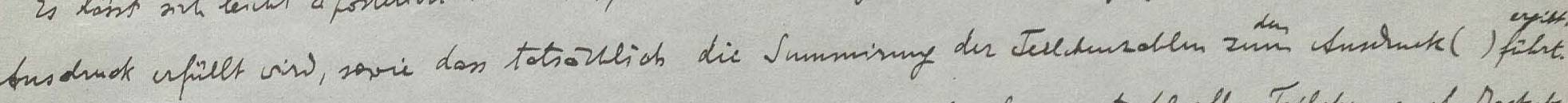

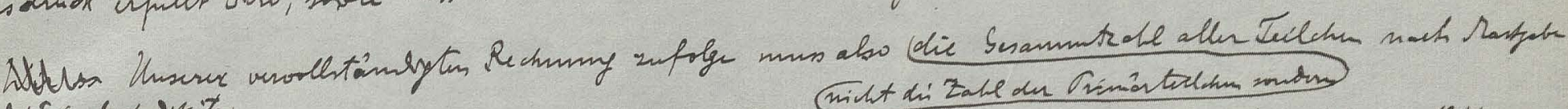

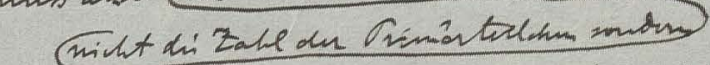

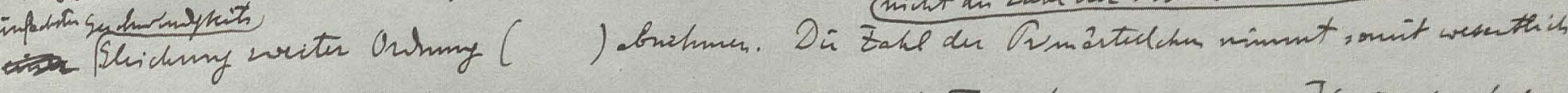

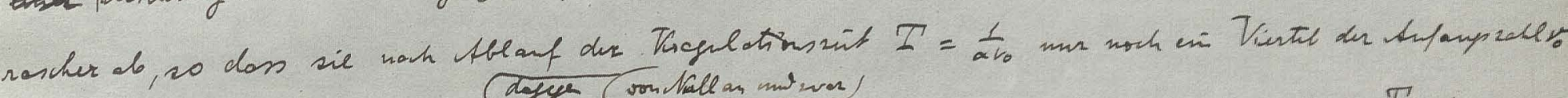

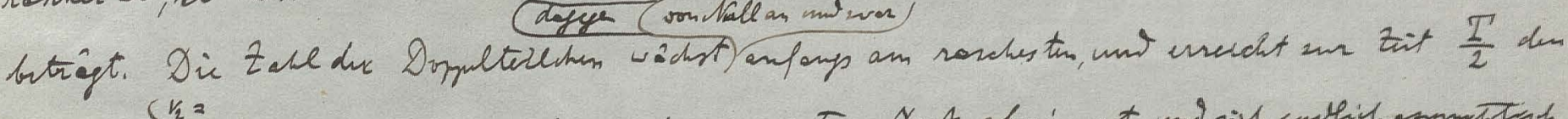

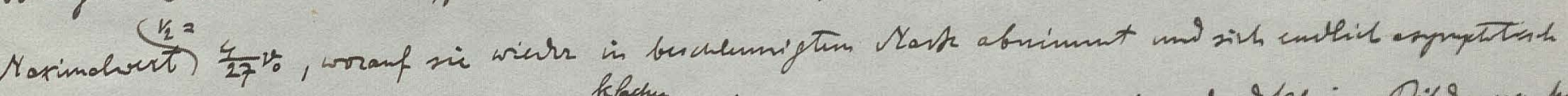

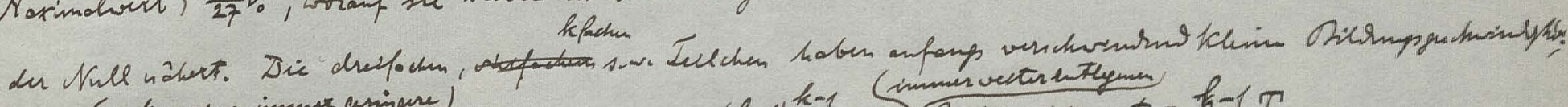

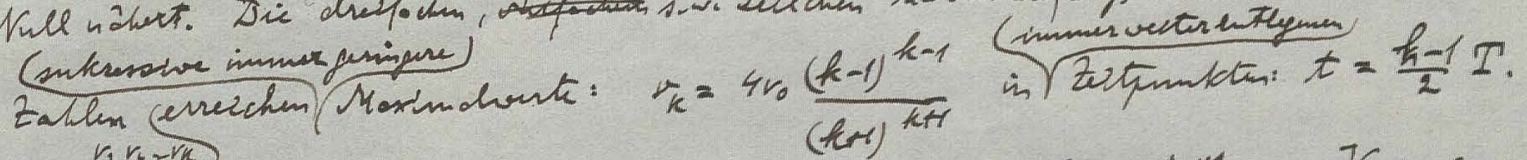

ihe tallon (krt) ktt

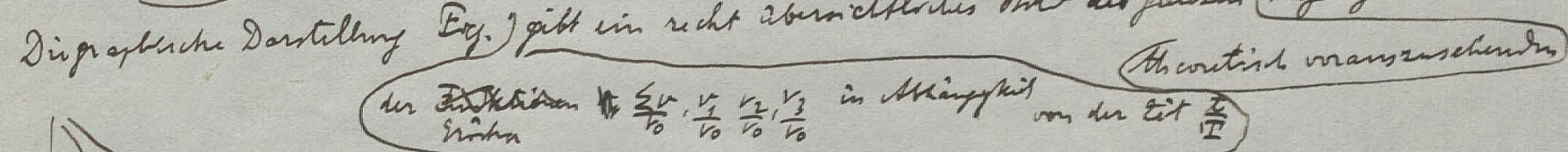

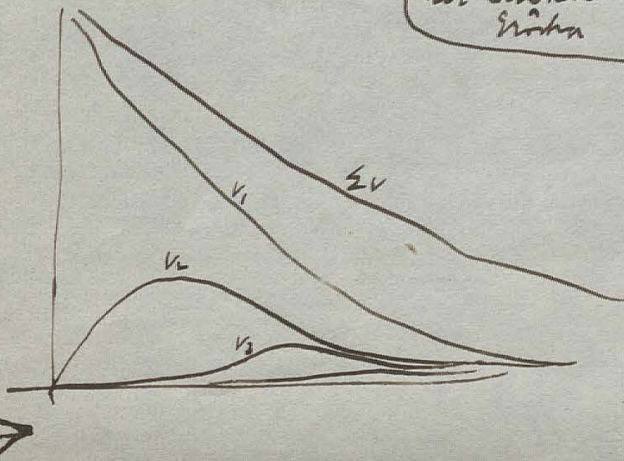

\section{Veylevidh int Frigmondy's Mesungen.}

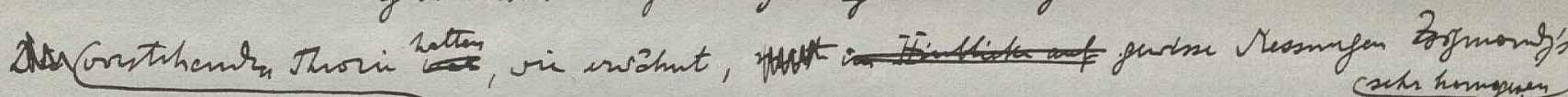

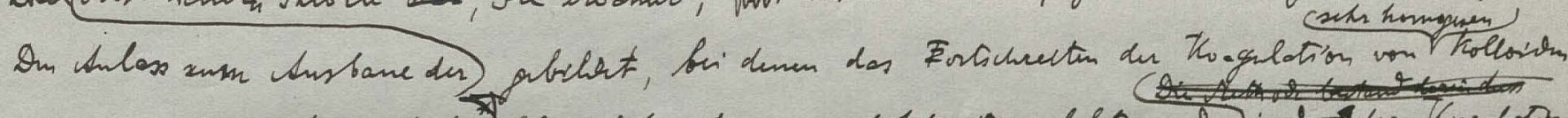

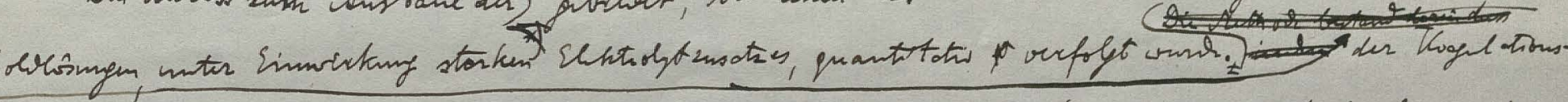

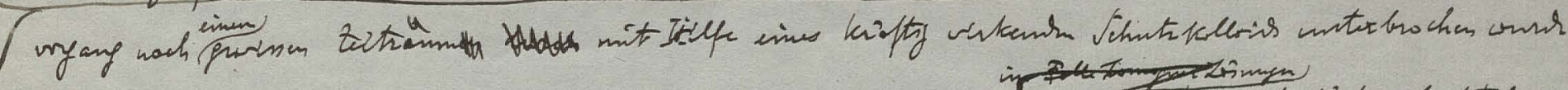

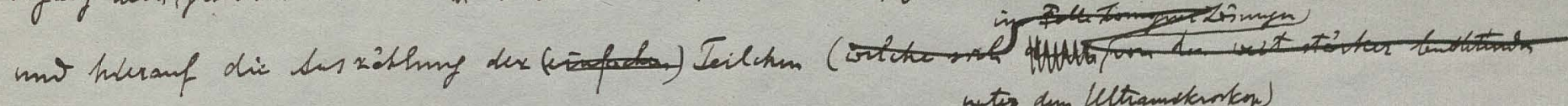

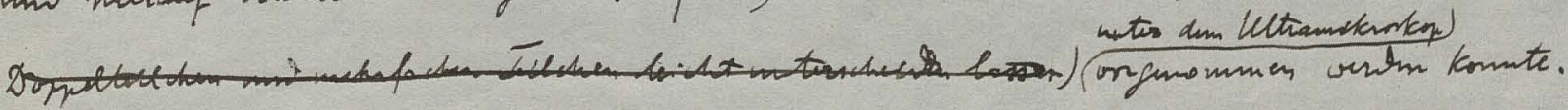

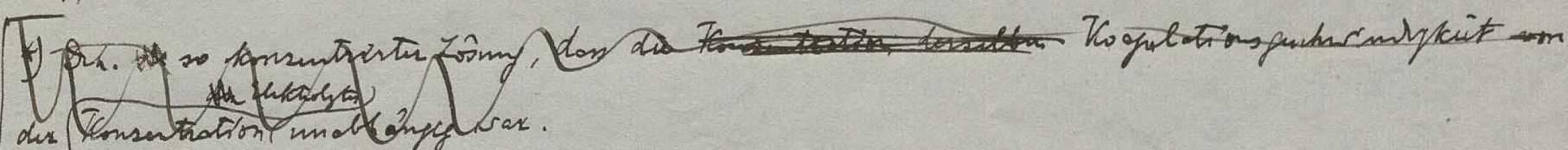

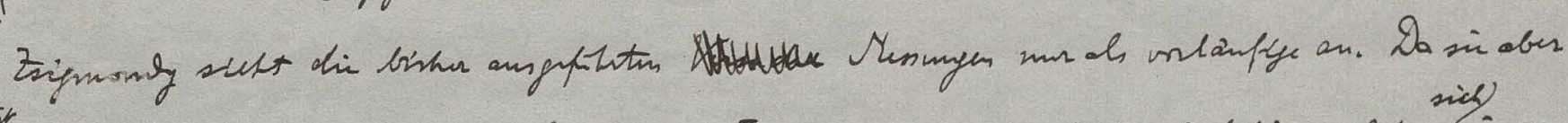
bisjtit

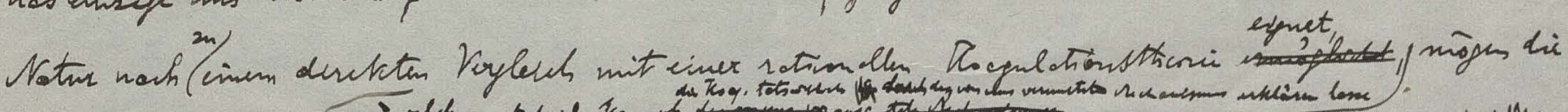

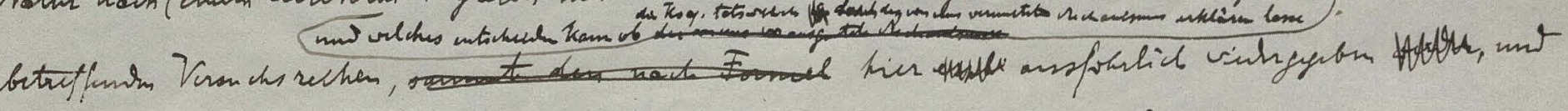

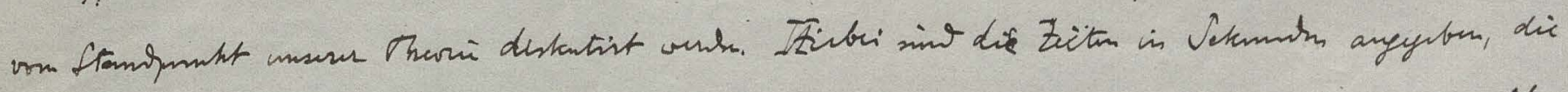

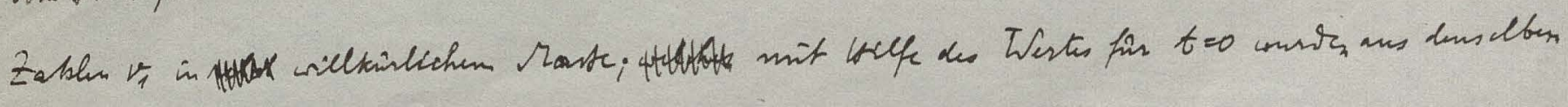

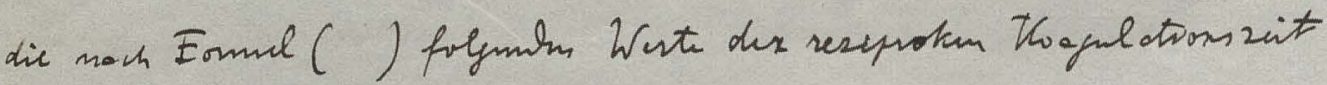

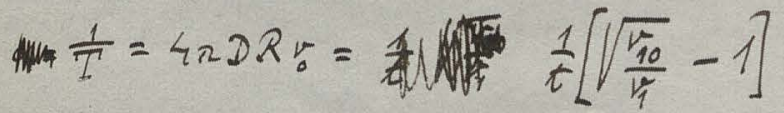

berechnet min is der 3 Reche insommengut telt 
(2)
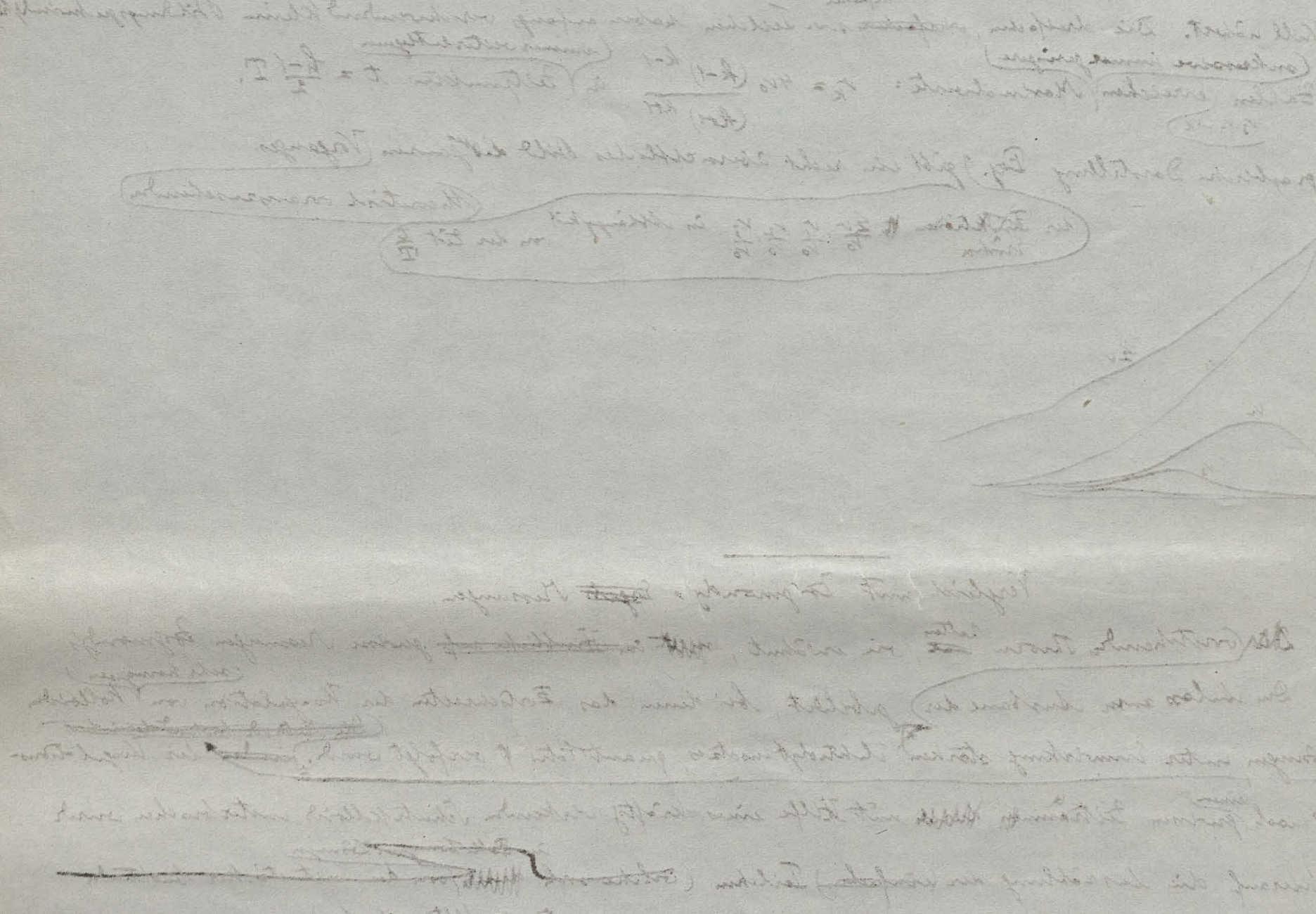
Visunh $D: w_{0}=0.80 \cdot 10^{10} ; r=13.4 \cdot 10^{-7}$

\begin{tabular}{c|c|c|c}
$t$ & $v_{1}$ & $\frac{1}{T}$ & $r_{1}$ bex. \\
\hline 0 & 1.93 & & 1.93 \\
2 & 1.42 & $(0.083)$ & $t .717$ \\
10 & 1.17 & $0.0 \underline{286}$ & 1.14 \\
20 & 0.75 & $0.0 \frac{302}{309}$ & 0.76 \\
30 & 0.52 & $0.0 \underline{30}$ &
\end{tabular}

situl $\frac{1}{T}=0.0299 ; \frac{R}{2}=140$

Vusand E: $v_{0}=0.552 \cdot 10^{10} ; 2=242 \cdot 10^{-7}$

\begin{tabular}{c|c|c|c}
\hline$t$ & $v_{1}$ & \multicolumn{1}{|c|}{$\frac{1}{T}$} & $v_{1}$ bu. \\
\hline 0 & 1.97 & & 1.97 \\
2 & 1.35 & $(0.105)$ & $t_{1.657}$ \\
5 & 1.19 & $(0.058)$ & $f 1.317$ \\
10 & 0.89 & 0.0490 & 0.93 \\
20 & 0.52 & 0.0475 & 0.54 \\
40 & 0.29 & 0.0403 & 0.25 \\
\hline
\end{tabular}

Nitul $\frac{1}{T}=0.0456, \frac{R}{2}=3 \% 12$

\begin{tabular}{|c|c|c|c|}
\hline$t$ & $v_{1}$ & $\frac{1}{2}$ & $v_{1}$ bus \\
\hline 0 & 1.92 & & 197 \\
\hline 3 & 1.56 & $(0.0 \leqslant 0)$ & $7(1 \cdot 76)$ \\
\hline 20 & 1202 & 0.0195 & 104 \\
\hline 40 & 0.66 & 0.0183 & 0.64 \\
\hline " & 0.76 & $(0.0153)$ & " \\
\hline 60 & 044 & 0.0187 & $-0.44 \quad 5 \quad$ \\
\hline 80 & $0.49(2)$ & $(0.0126)$ & $t^{0.317}$ \\
\hline
\end{tabular}

sital $\frac{1}{T}=0.0188 ; \frac{R}{2}=2.63$

\section{Whan Dass}

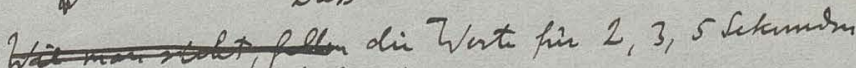

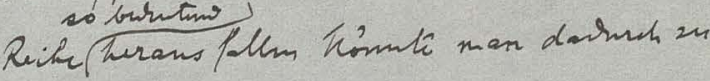

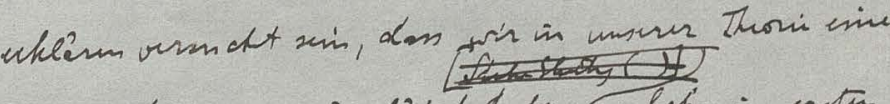

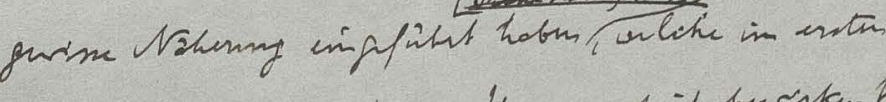

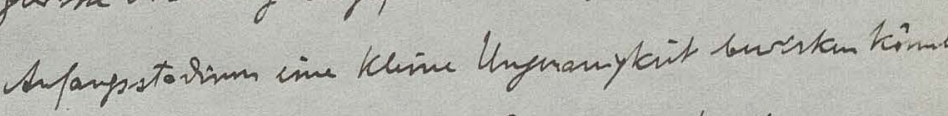

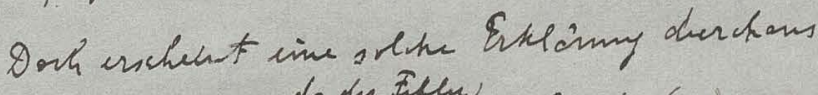

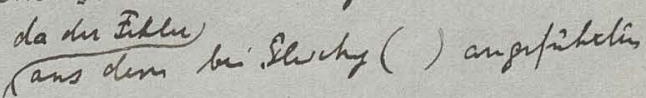
sunor heer gans nomertades sim nuts. Ththe In voles Enmit du thed hung dirfte wore dasin

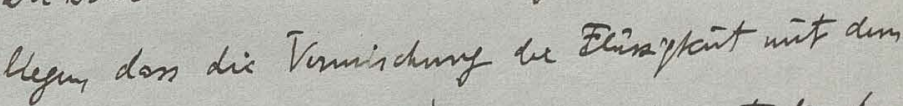

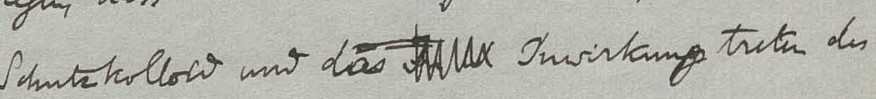
letstom doch ine gurime test erforiht, wiche bei

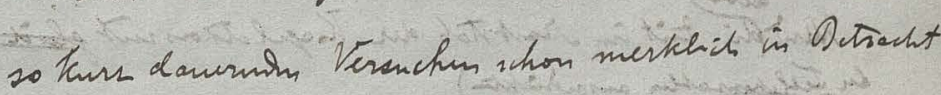

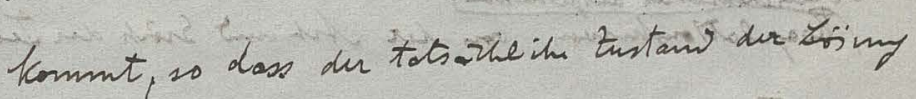

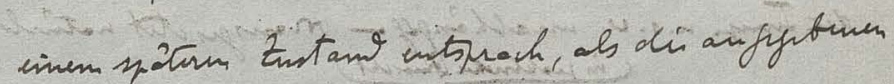
Tersuch sectm.

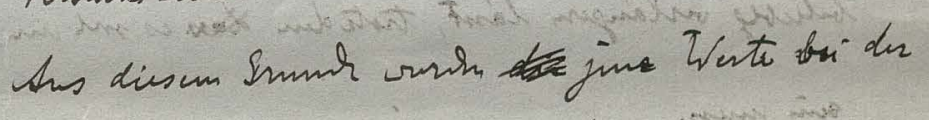

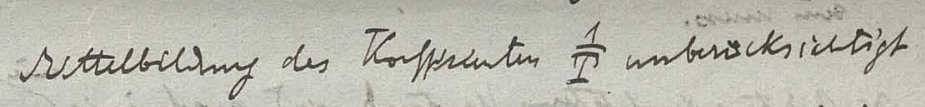
glanem, eburso wi die woi stork herarisfollumin

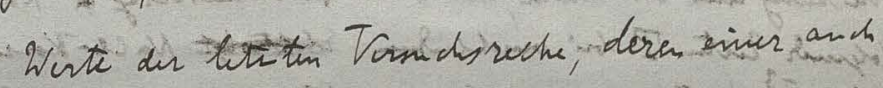

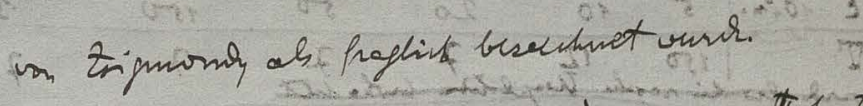

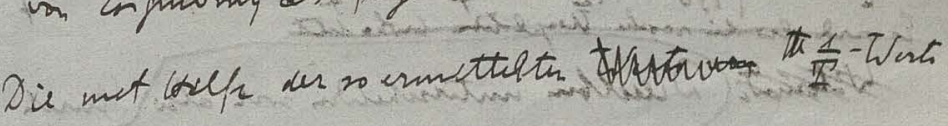

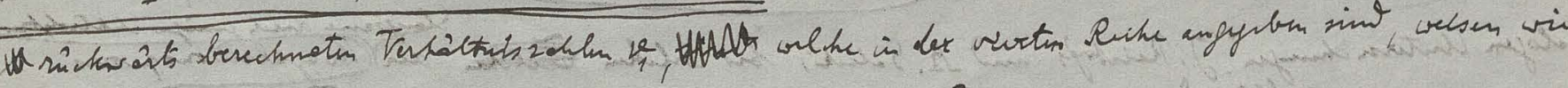

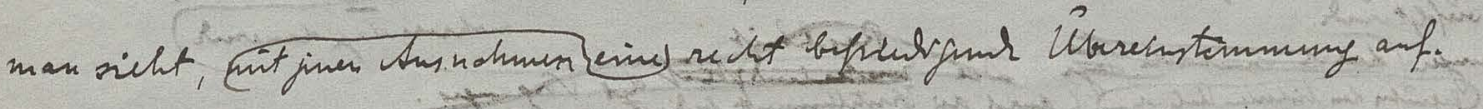

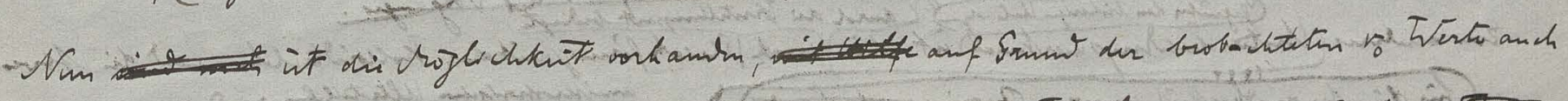

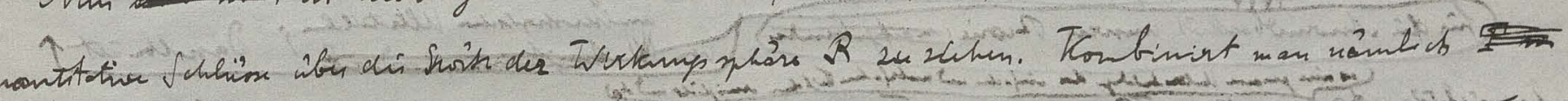

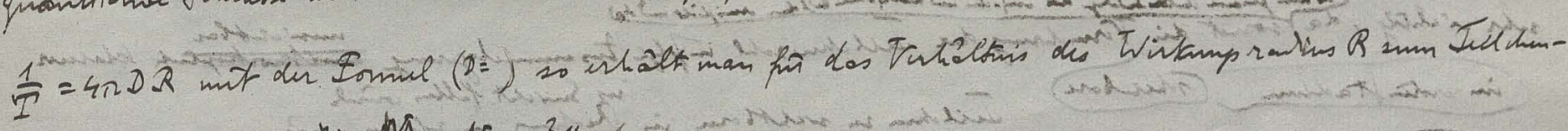
rodus r: $\frac{R}{2}=\#$ Her $\frac{N}{H \theta} \frac{3 \mu}{2 V_{0}} \frac{1}{T}$

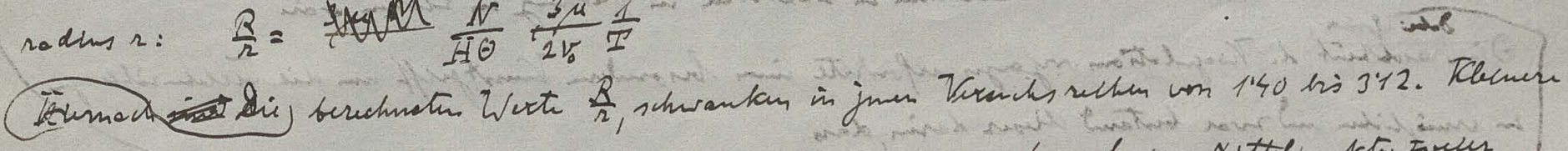

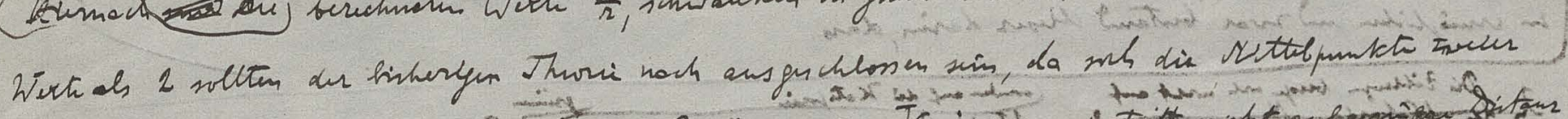

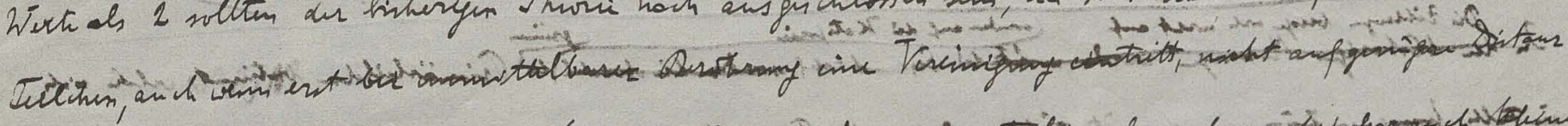

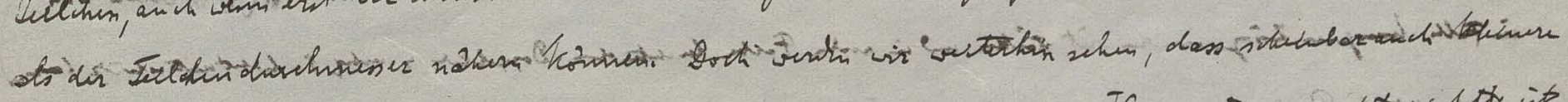

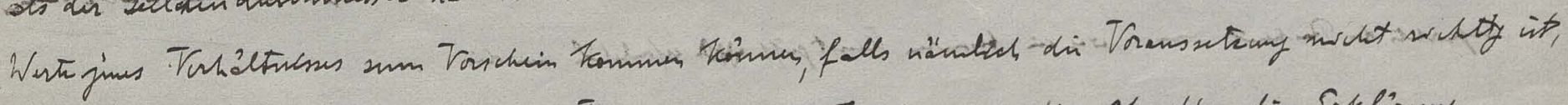

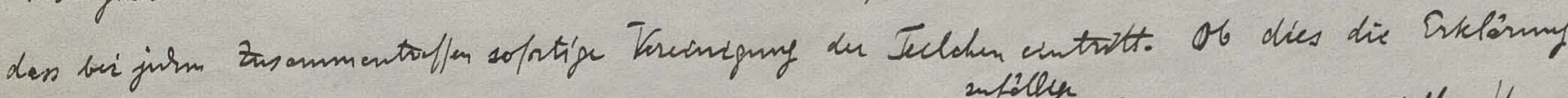

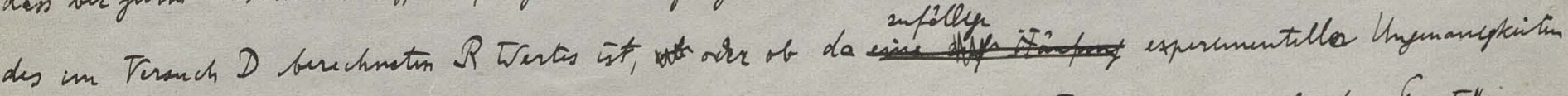

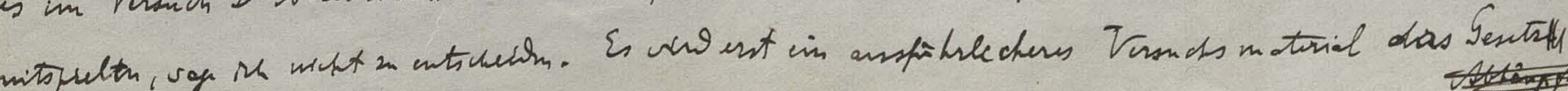


The alos

en Eerennerem aspulisto)

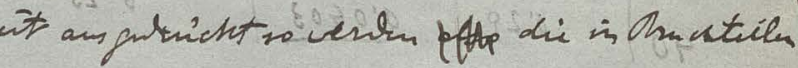

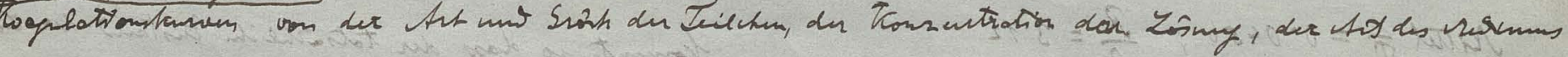

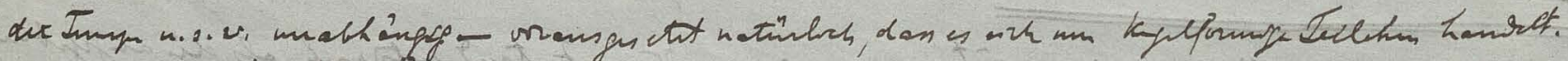

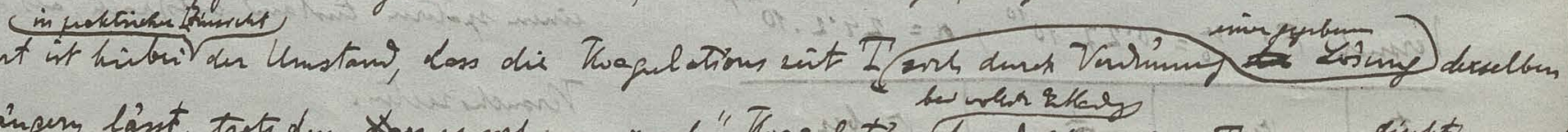

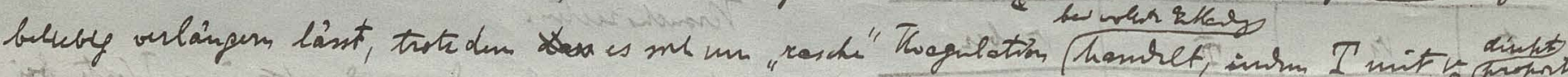
sin unis.

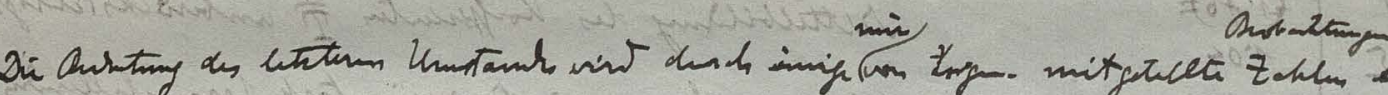

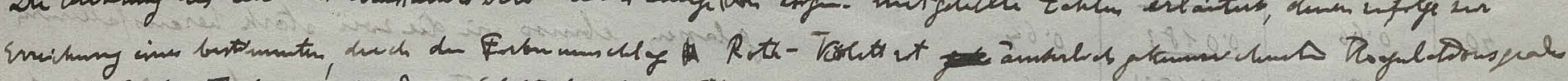

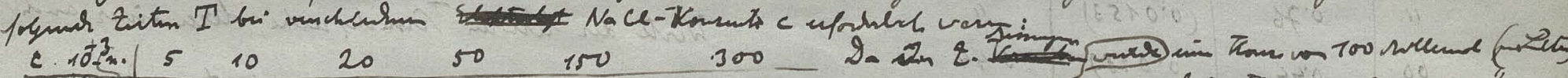

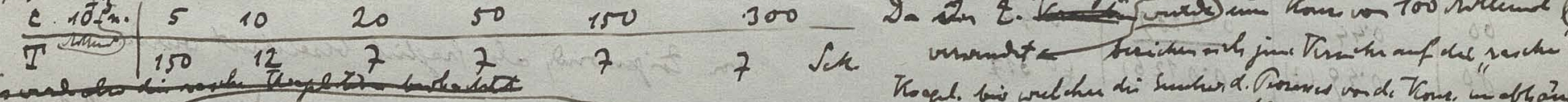

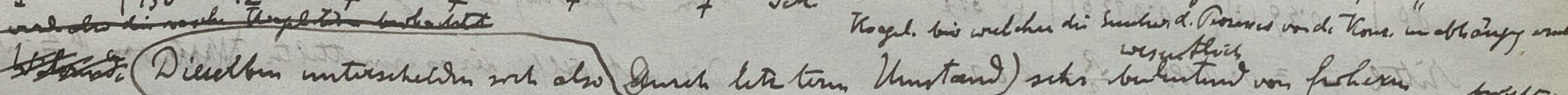

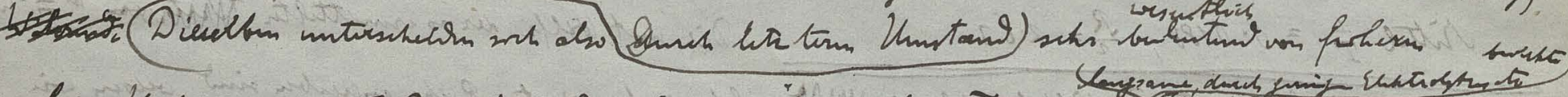

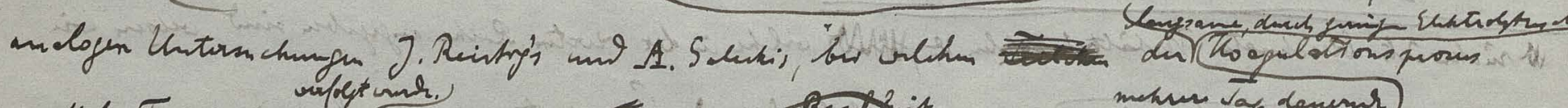

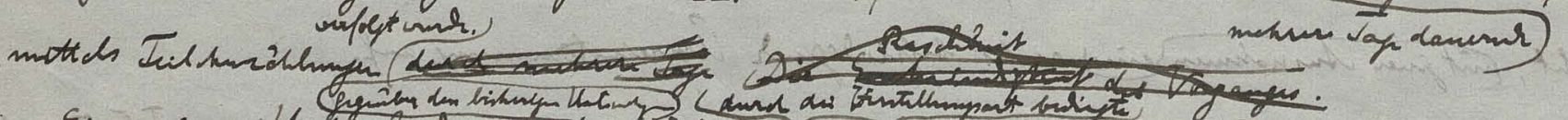

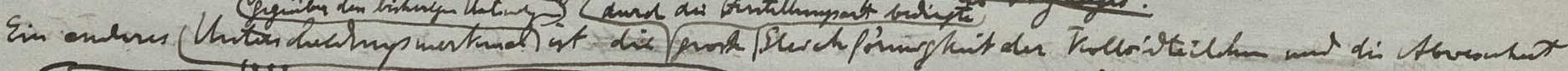

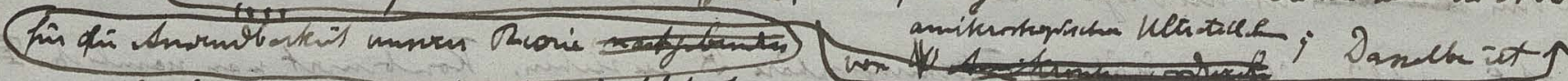

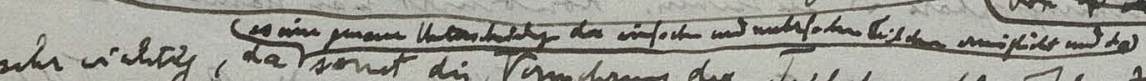

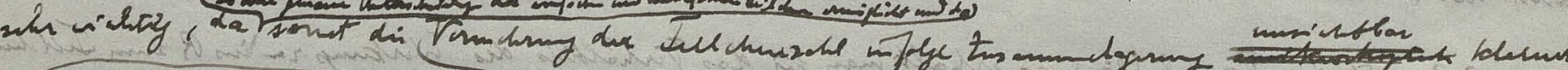

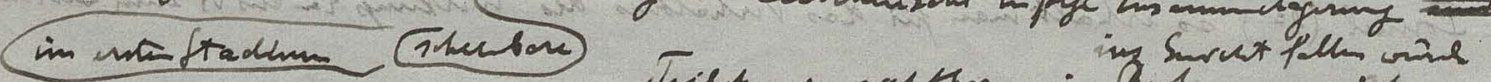
o. Sheigher,

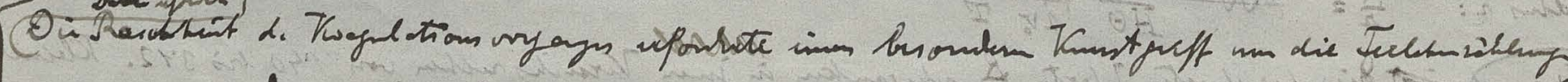

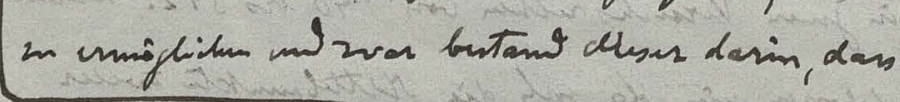

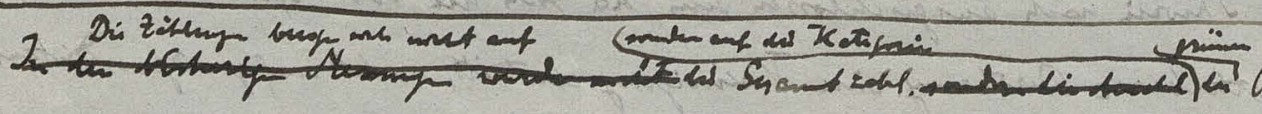

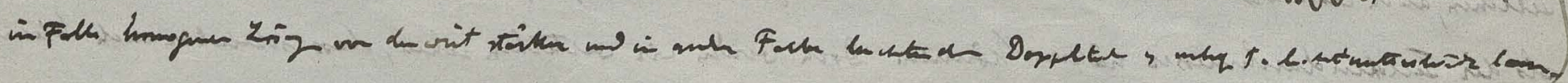

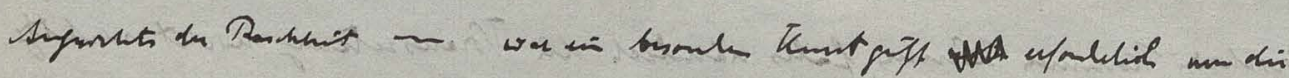




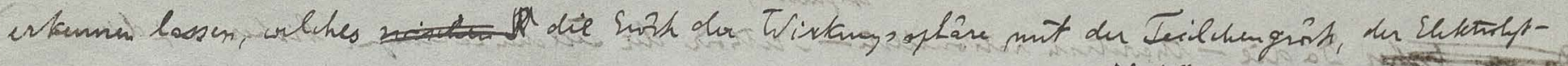

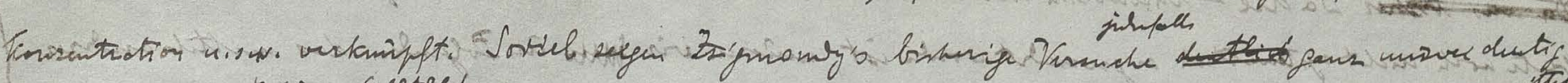
A jime Sorolley

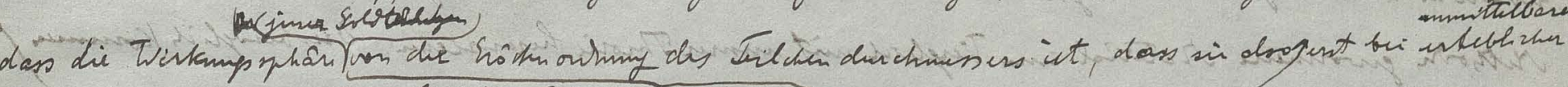

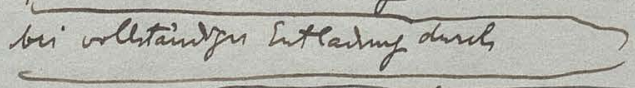

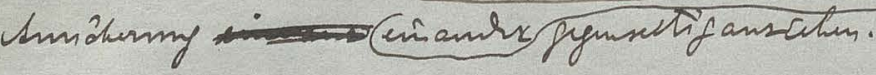

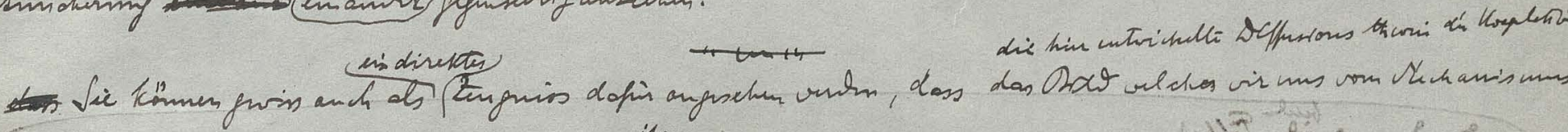

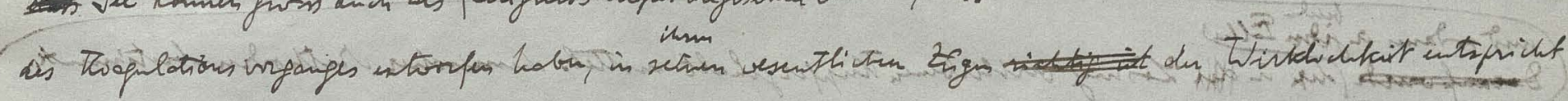

$$
\text { V (Turvi der Zargamen Kogelation) }
$$

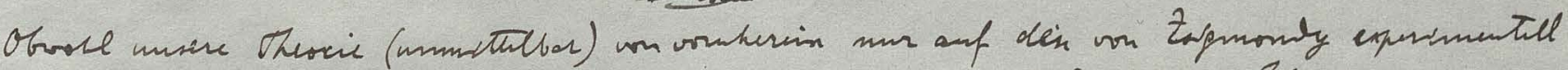

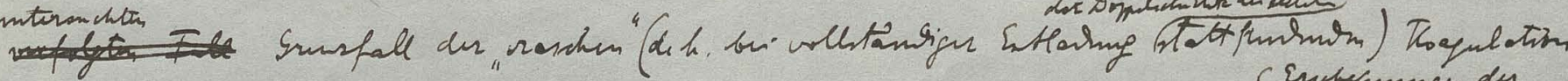

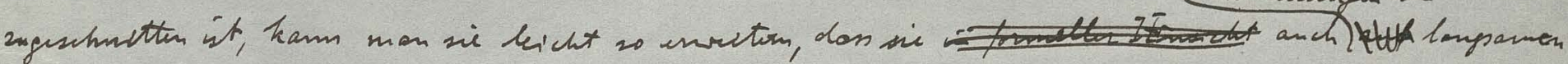

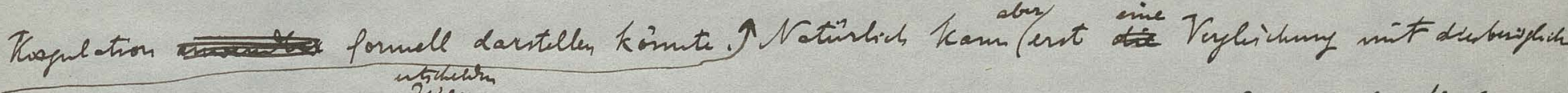

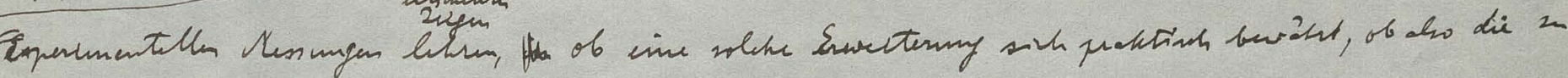

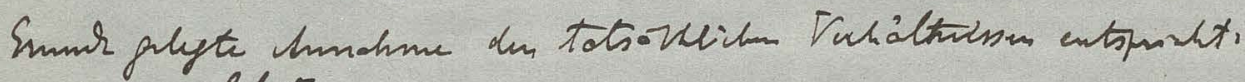

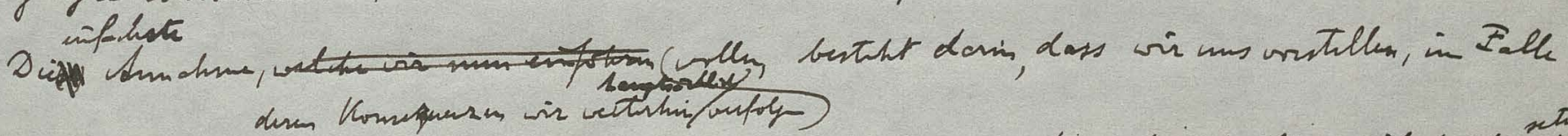

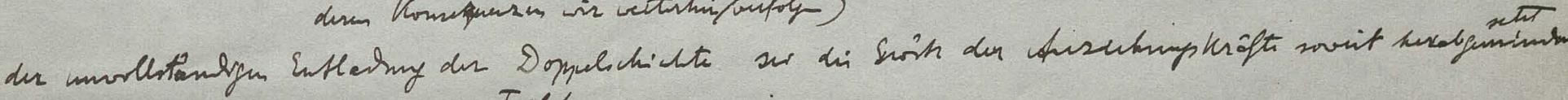

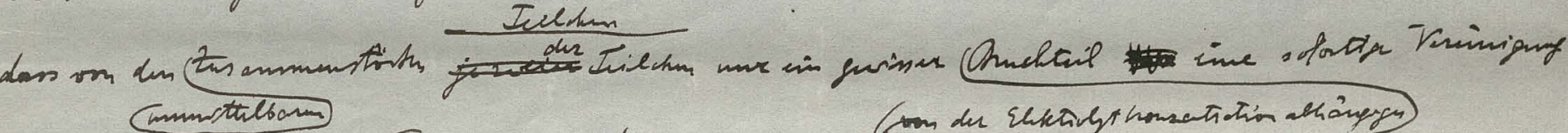

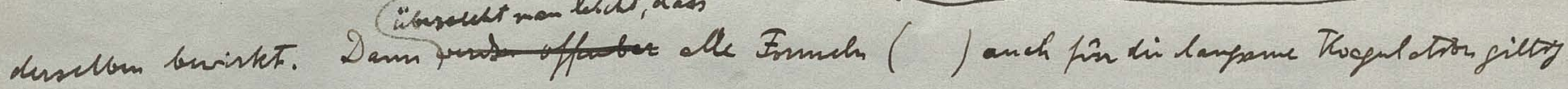

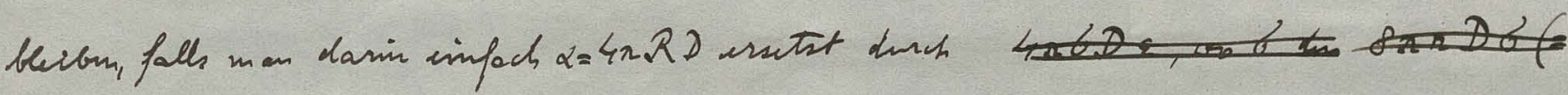

$$
\alpha=\sin D \varepsilon=\frac{4}{3} \frac{H \theta}{N} \frac{\varepsilon}{\mu}
$$

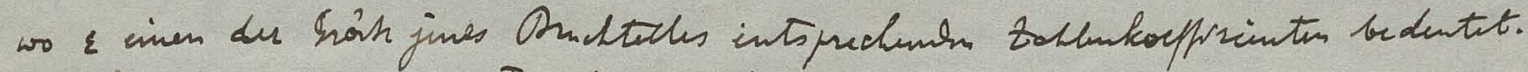

oer quantititive Vulat du Togelotion vân

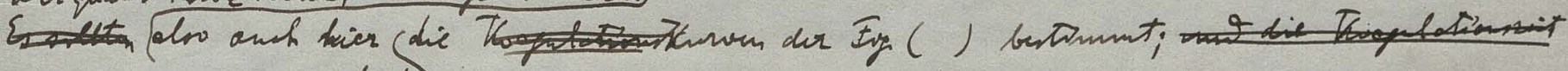
dunch.

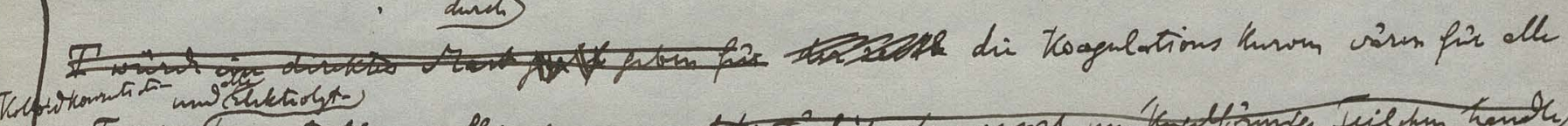

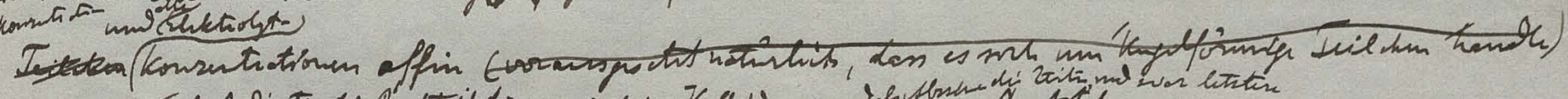

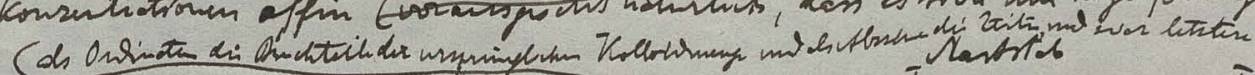

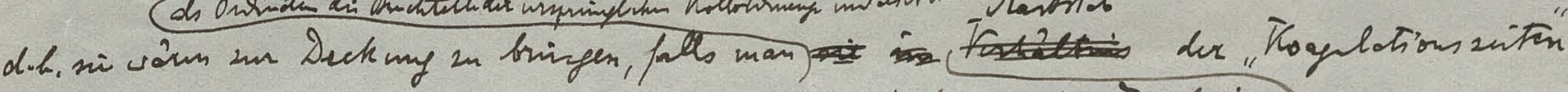
$T=\frac{3}{4} \frac{N \mu}{J t \theta} \cdot \frac{1}{\varepsilon v_{0}}$

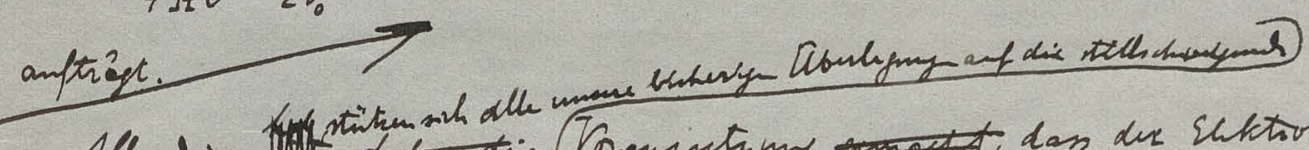

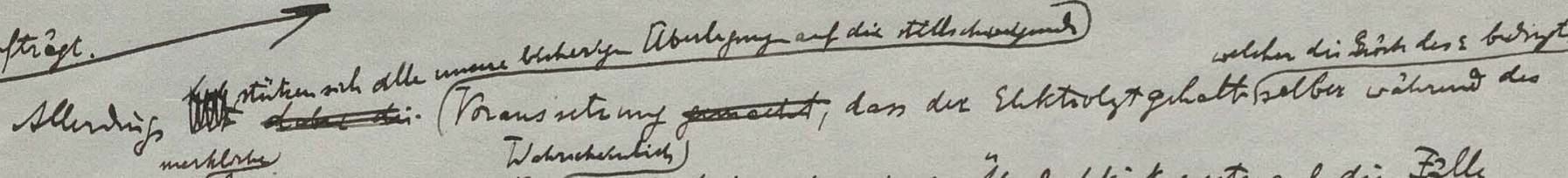

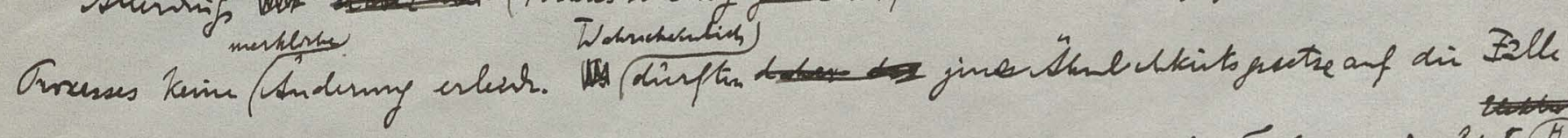

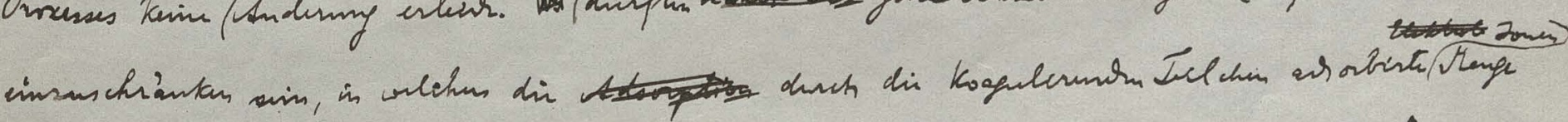

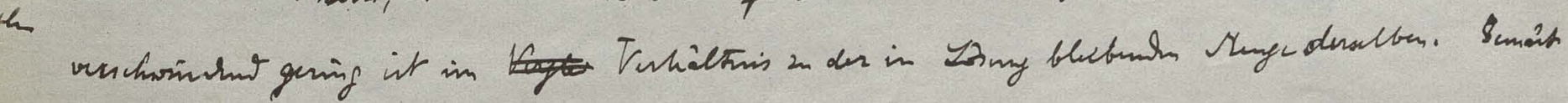

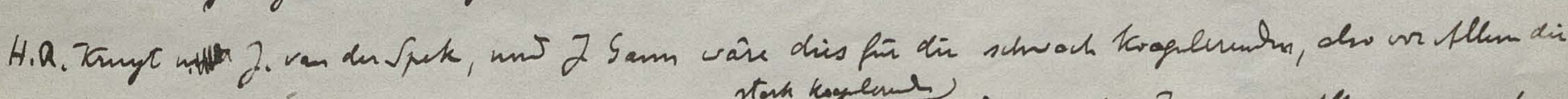

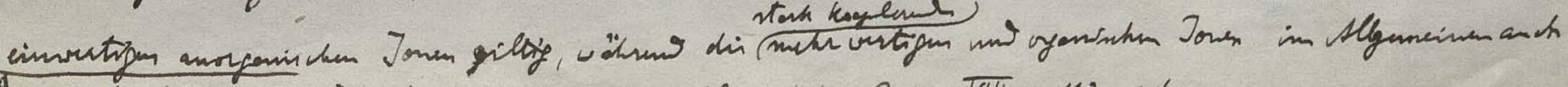

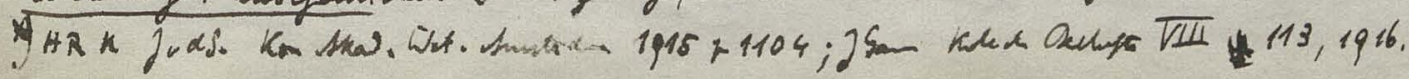




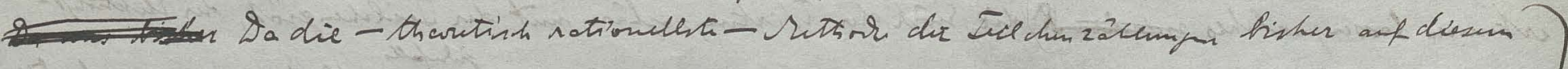

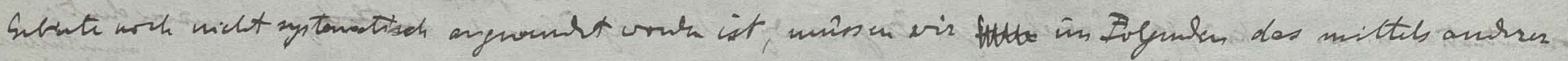

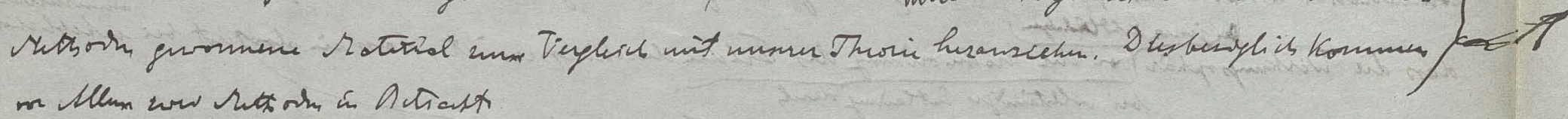

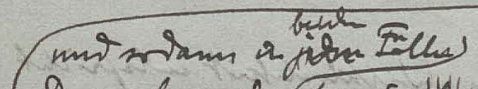

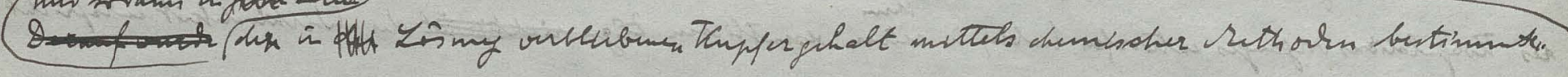
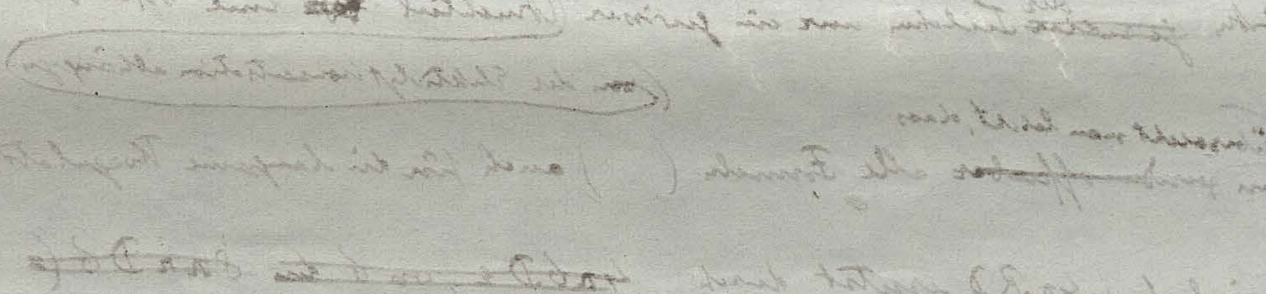

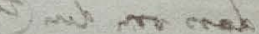

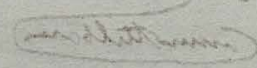

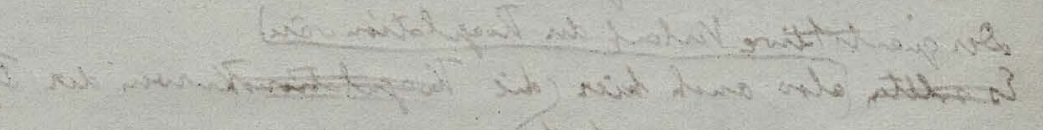

2utitise
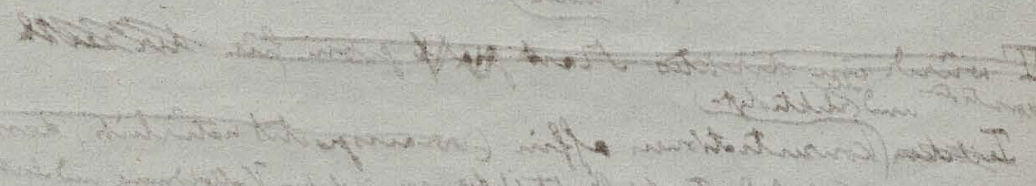

and 4 in

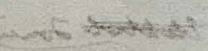
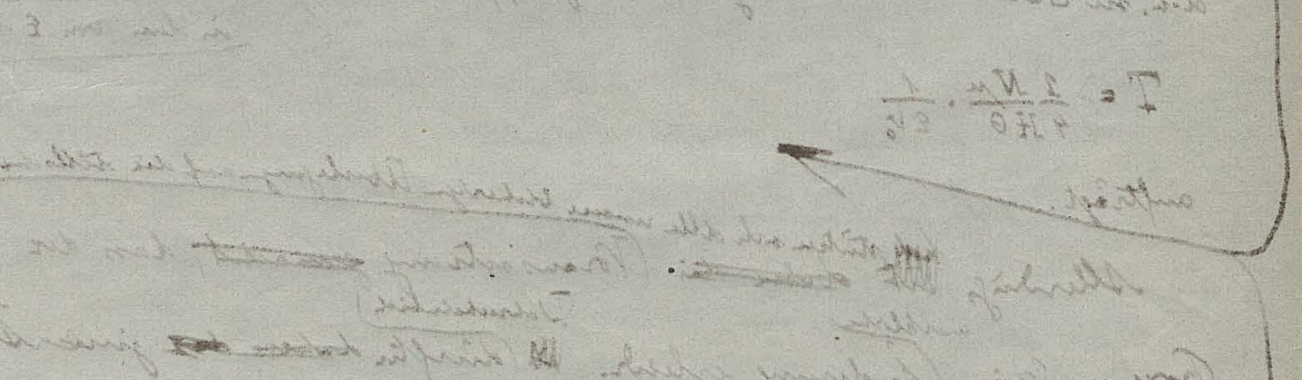

Ans 2

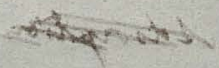




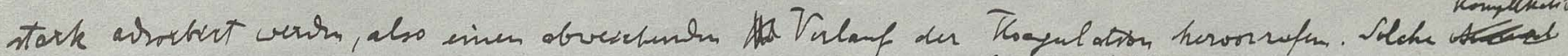

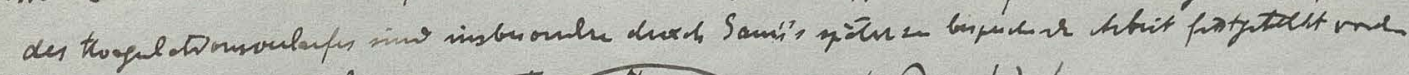

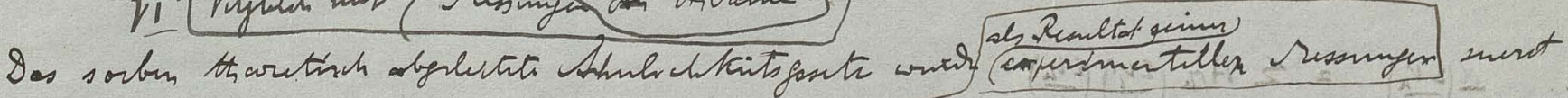

If If

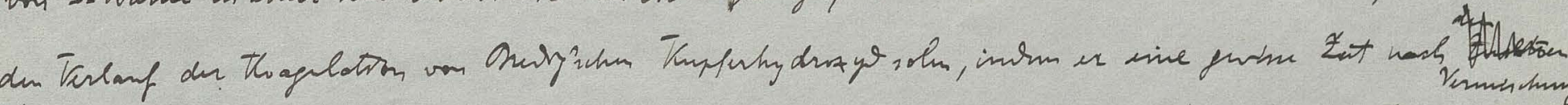

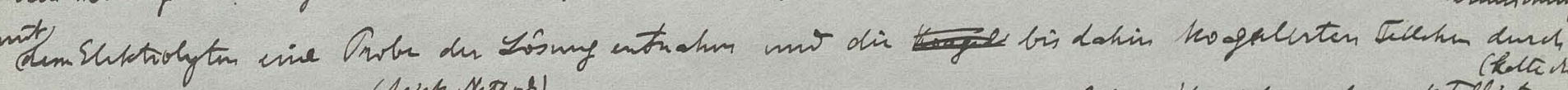

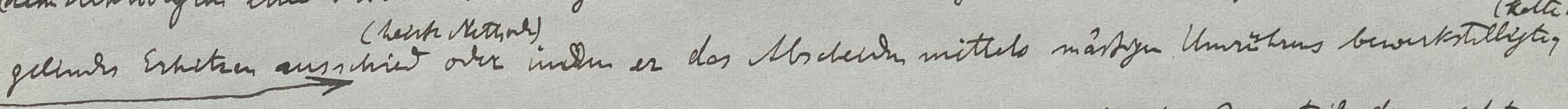

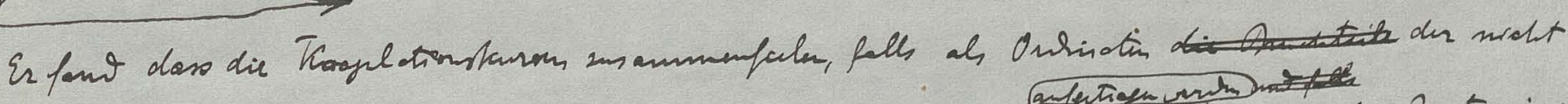

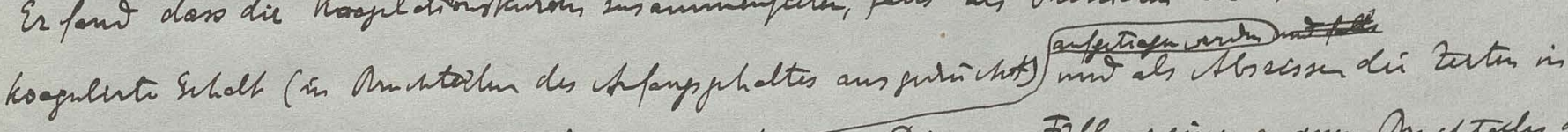

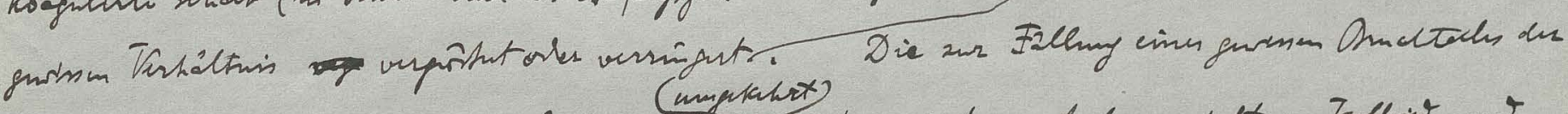

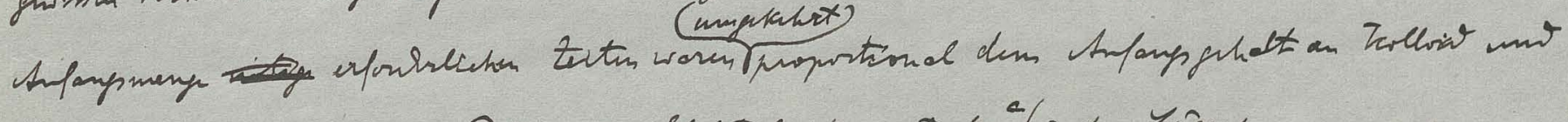

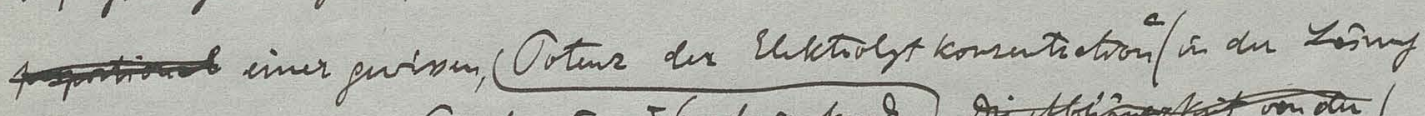

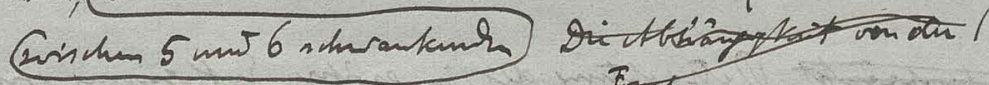

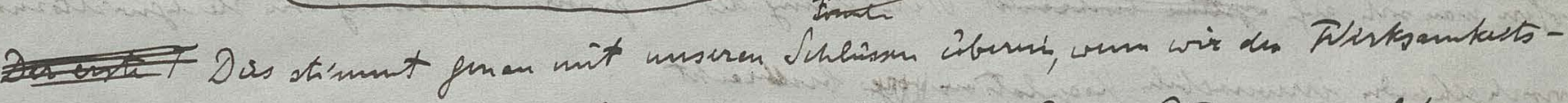

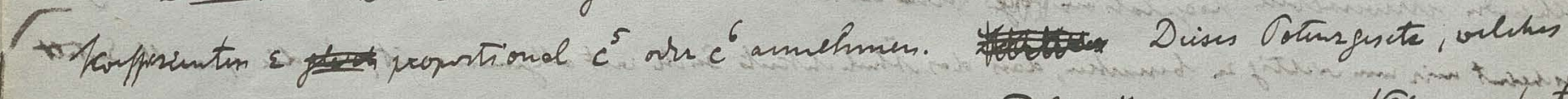

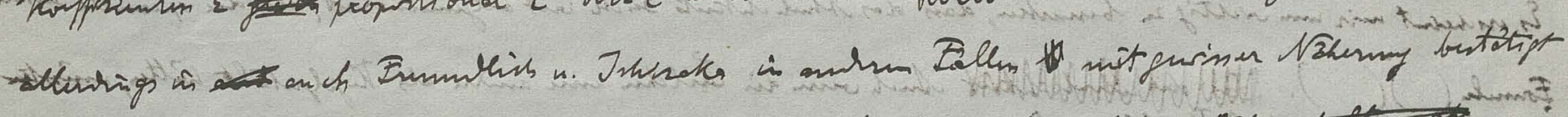

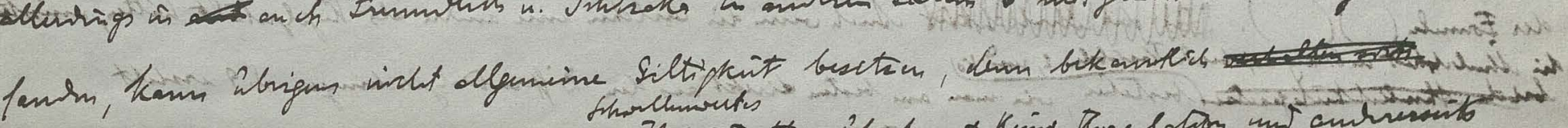

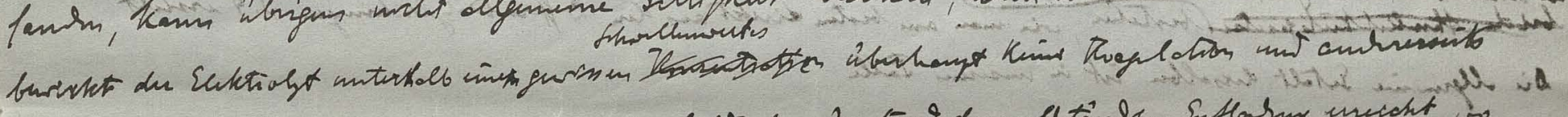

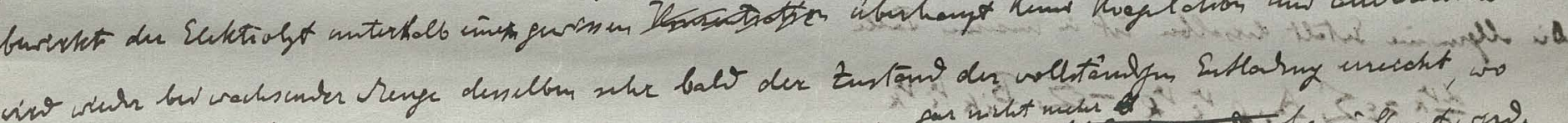

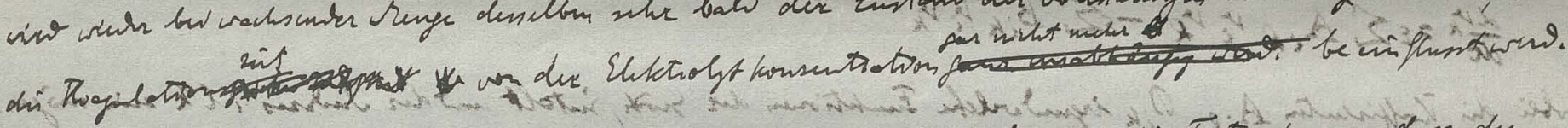

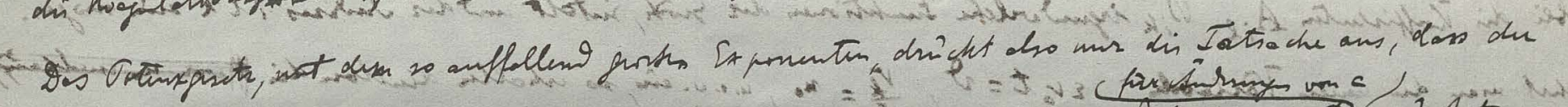

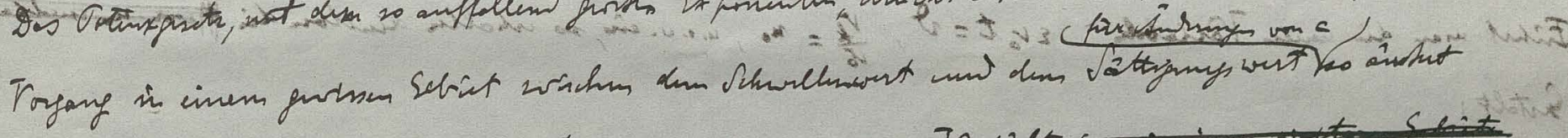

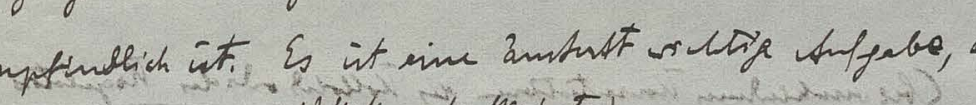

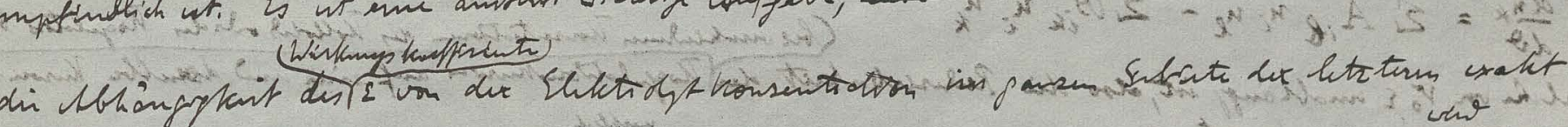

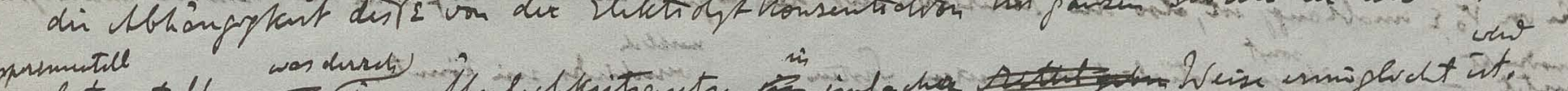

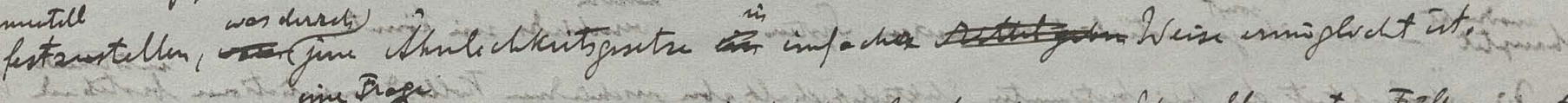

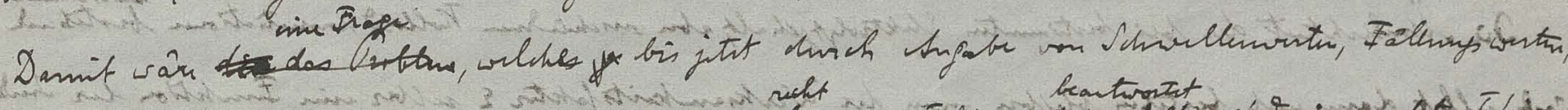

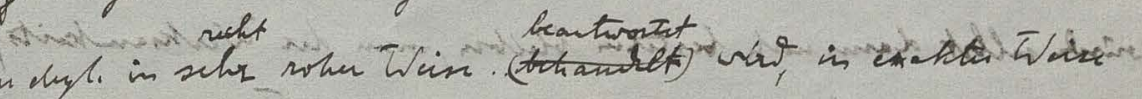
gutnt.

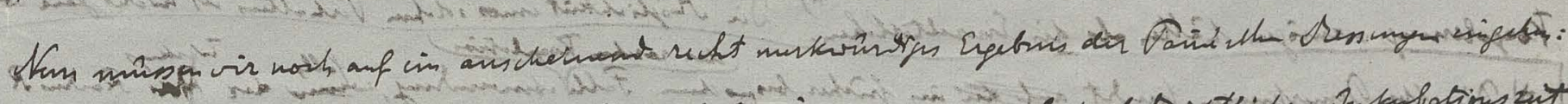

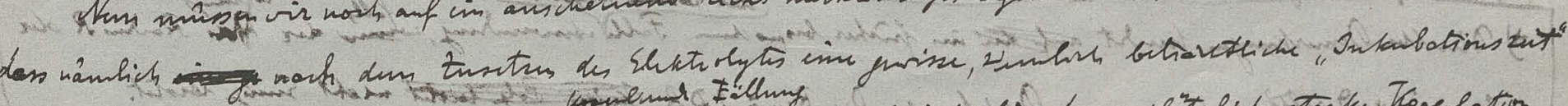

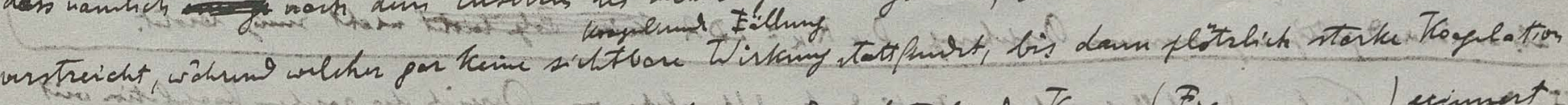

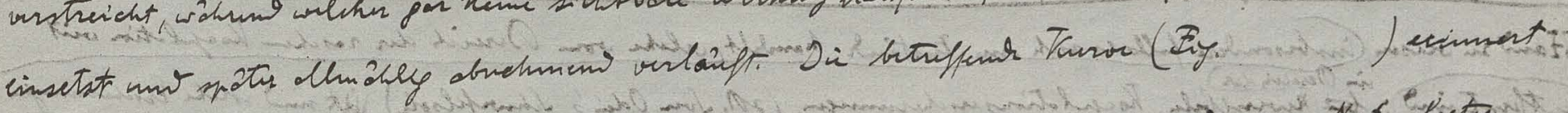

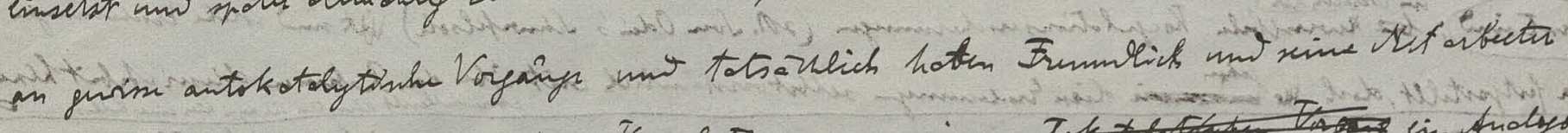

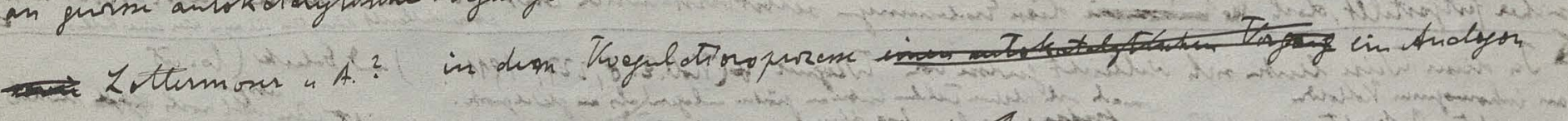

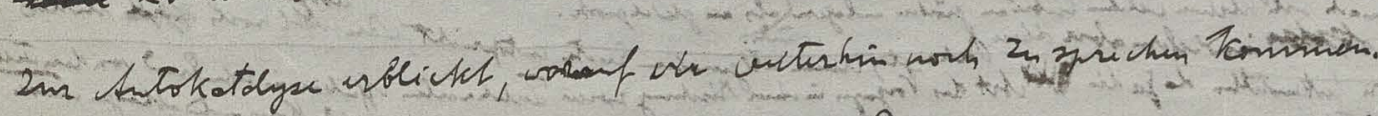

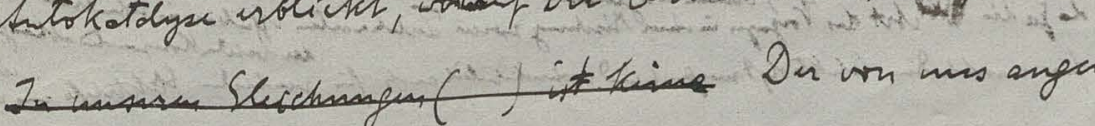

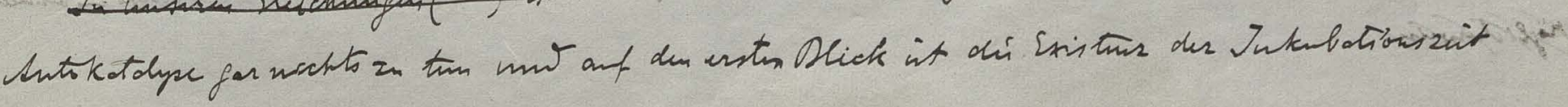
nit nurerm Eomeh nicht en verembarm 


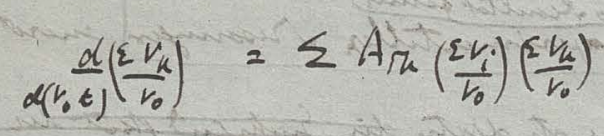

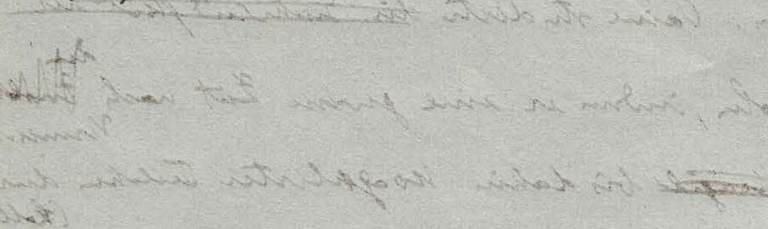

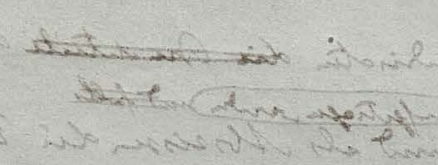

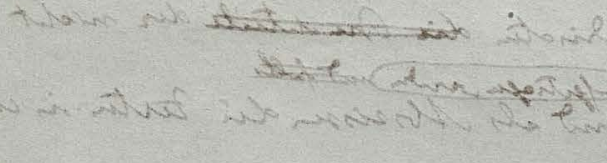

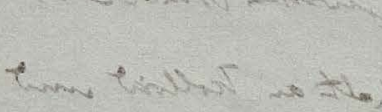

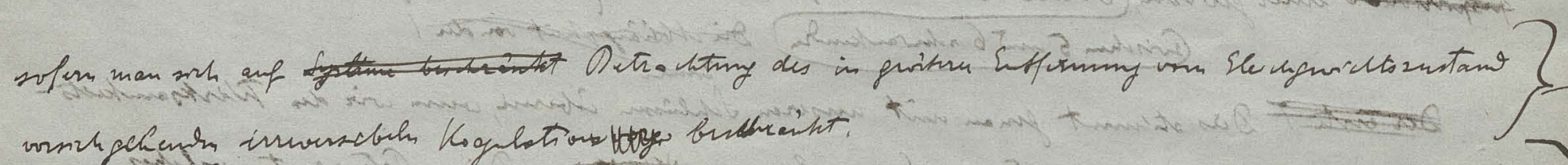

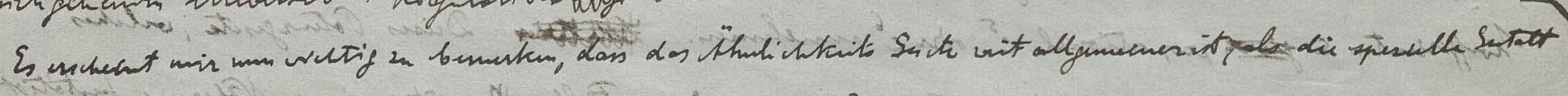

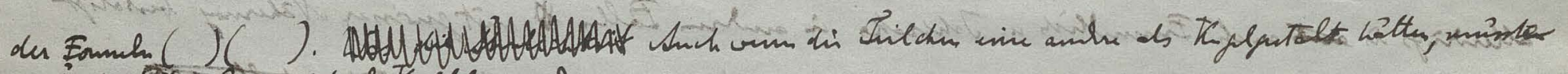

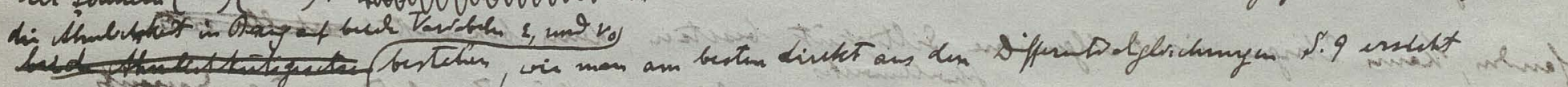

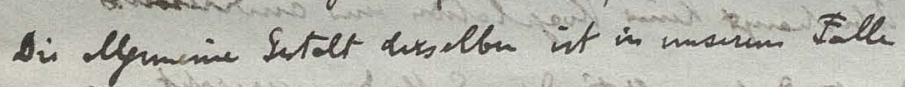

$$
\frac{d \nu_{k}}{d t}=\sum \sum A_{i} v_{i} v_{R}-\sum \sum B_{i k} v_{i} \nu_{k}
$$

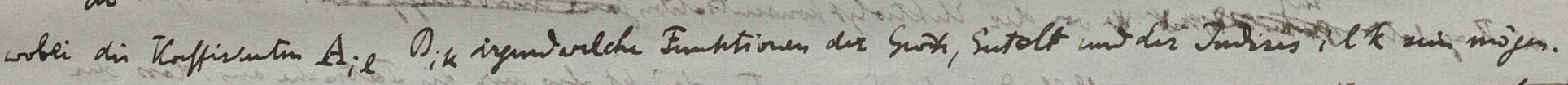

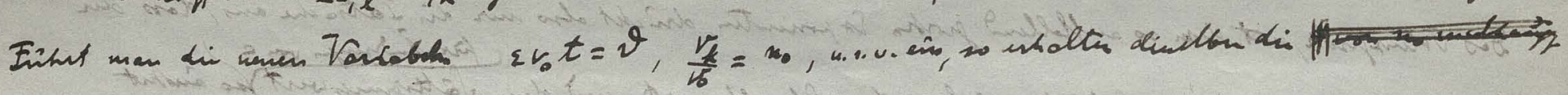
Suttel:

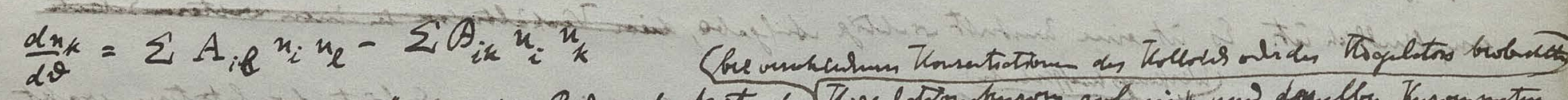

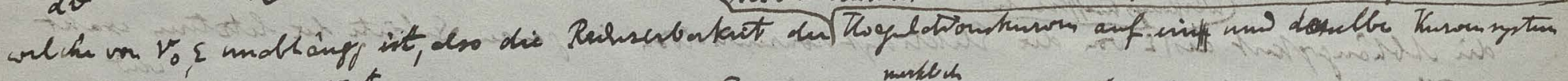

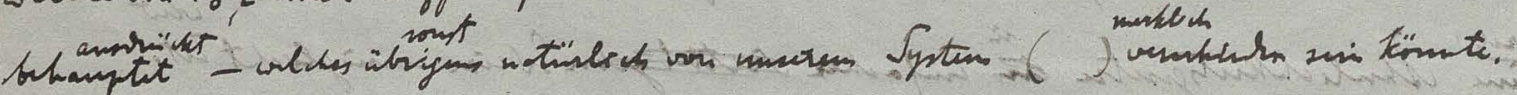

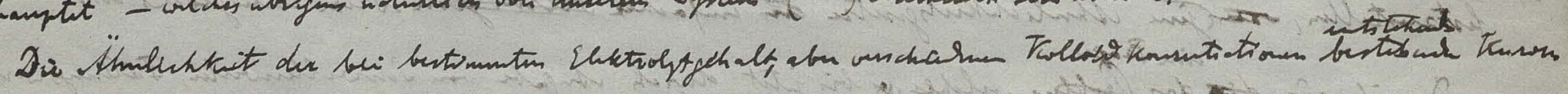

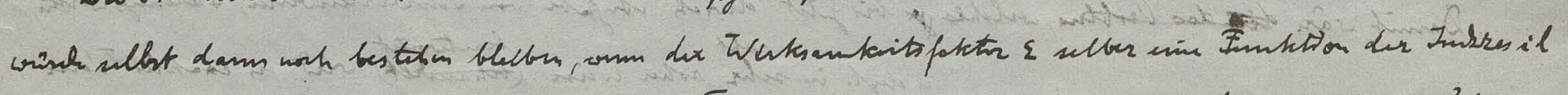

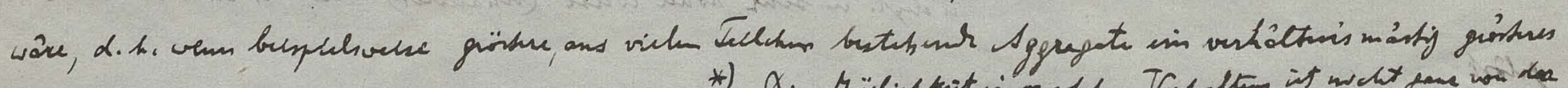

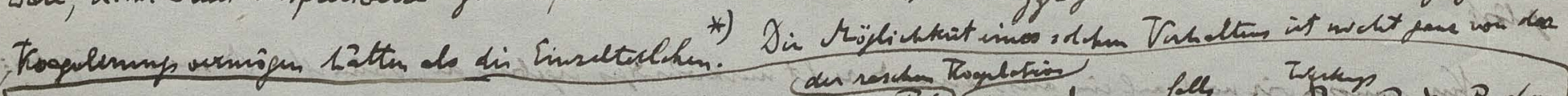

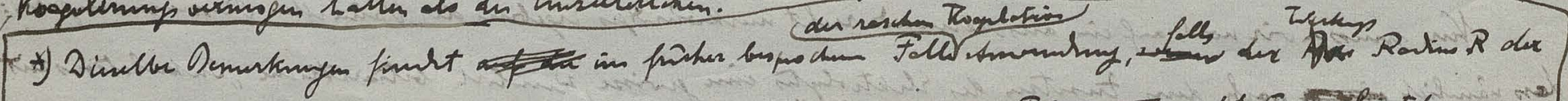

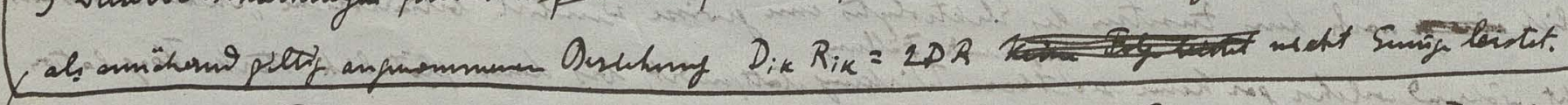

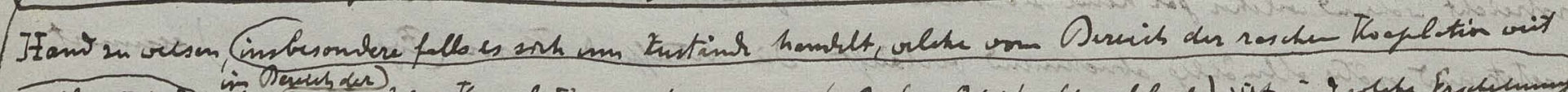

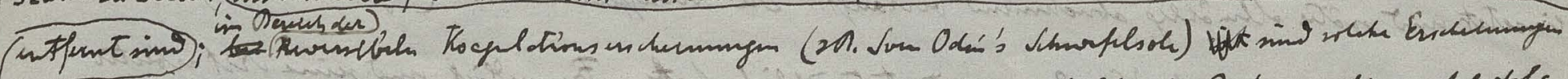

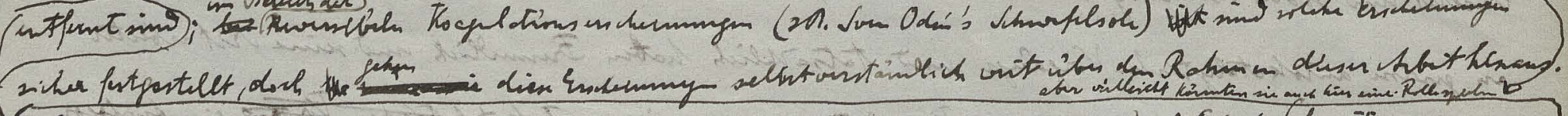

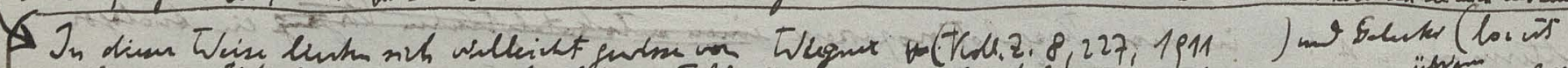

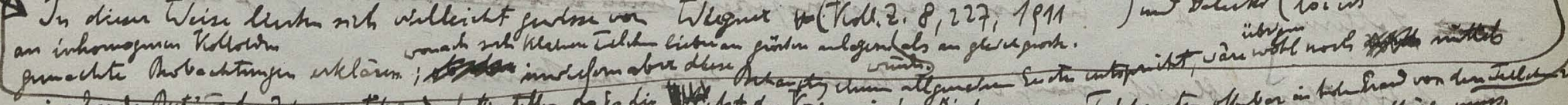

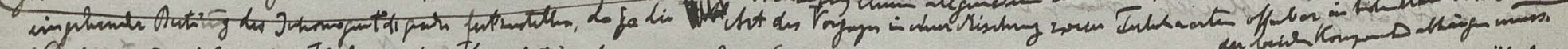

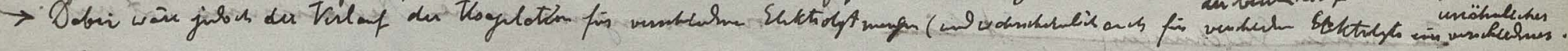
सugeraingary 


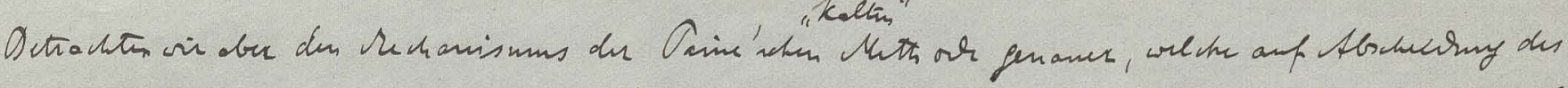

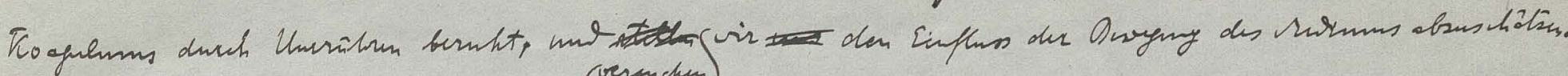

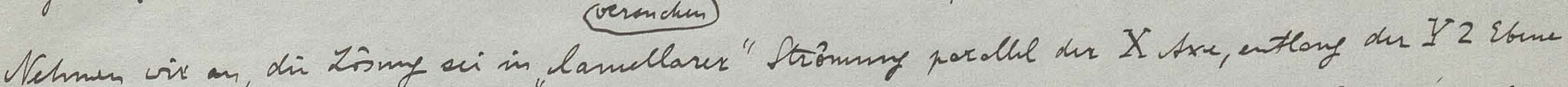

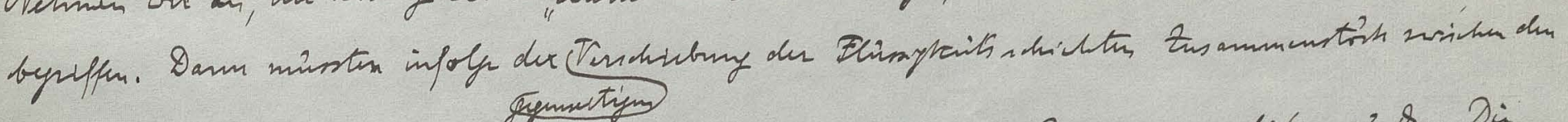

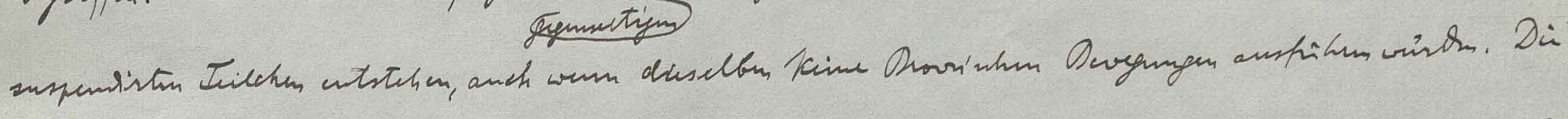

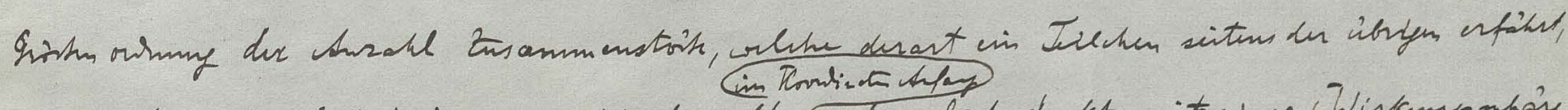

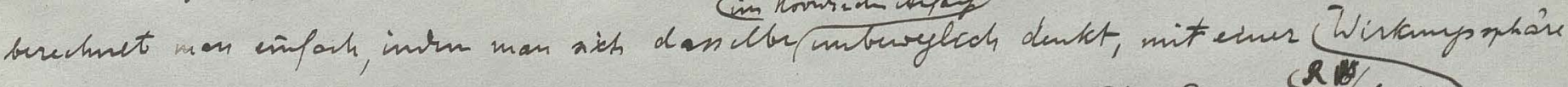

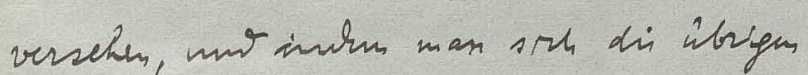

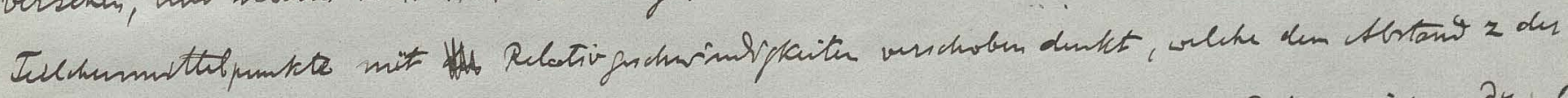

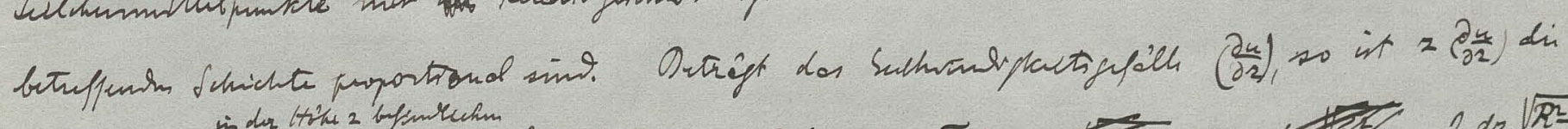

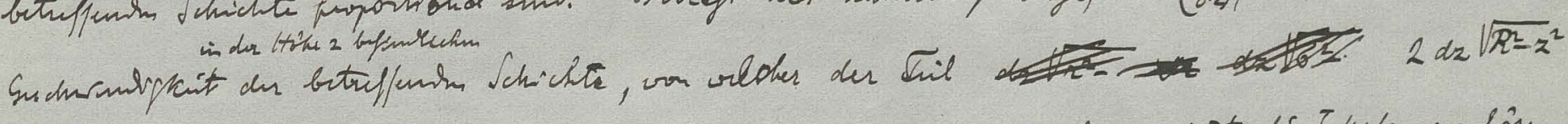

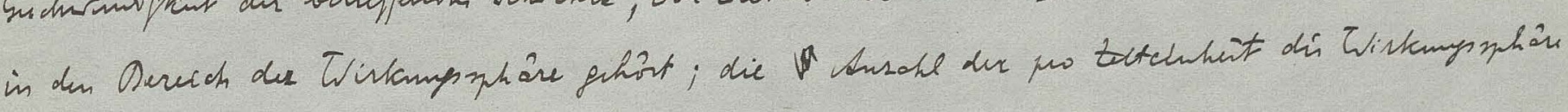

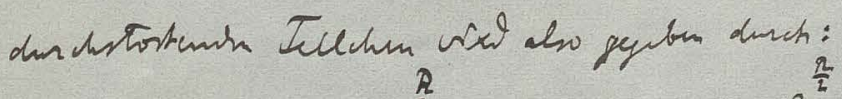

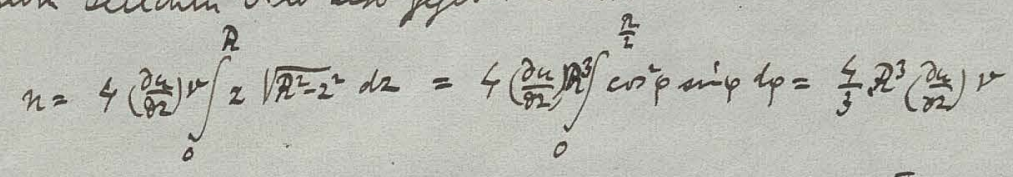

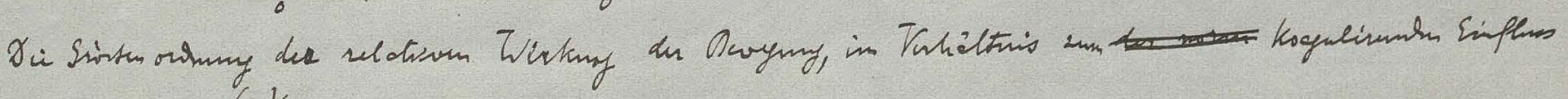

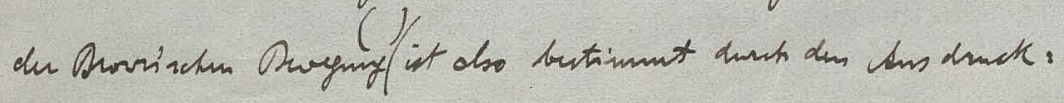

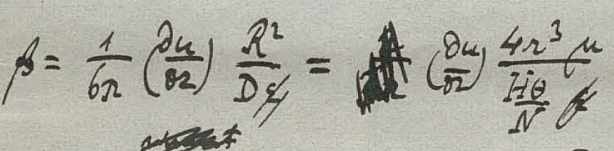

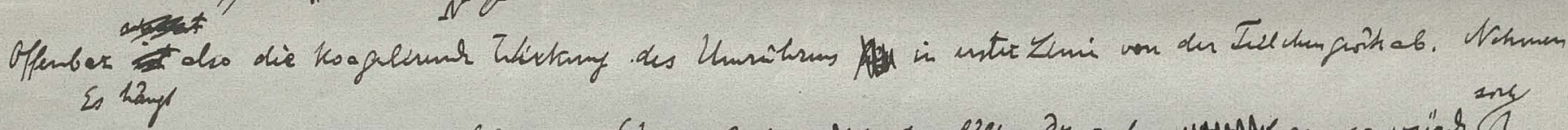

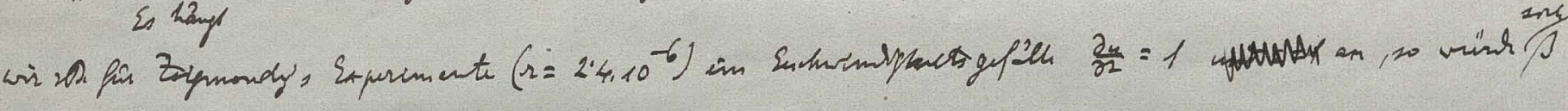

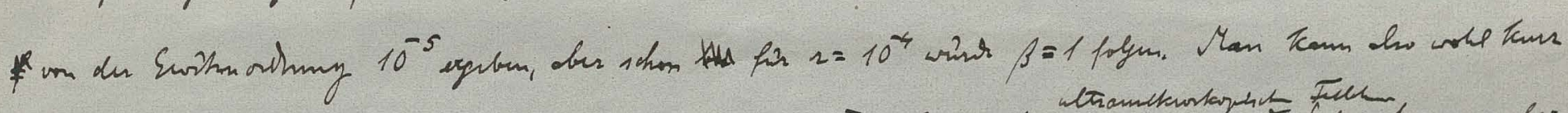

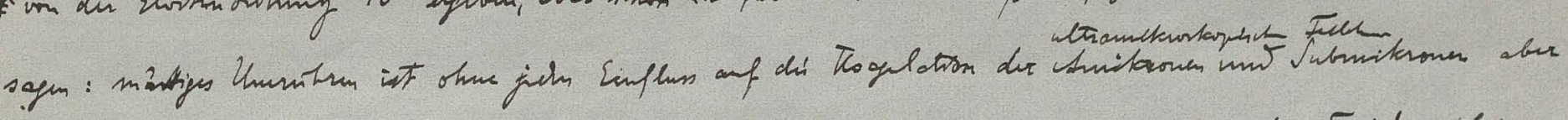

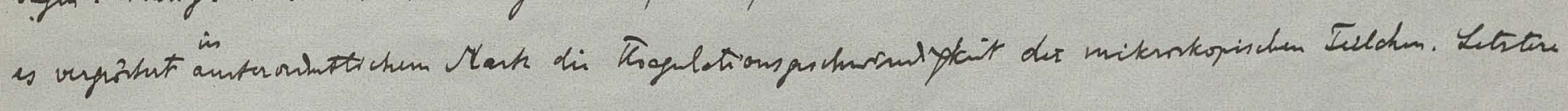

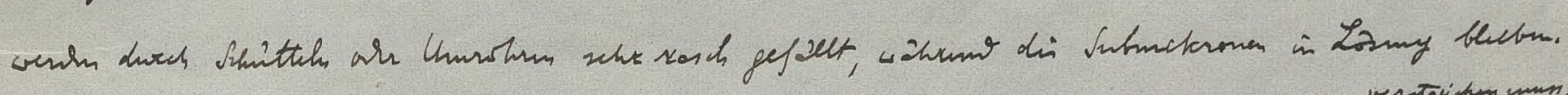

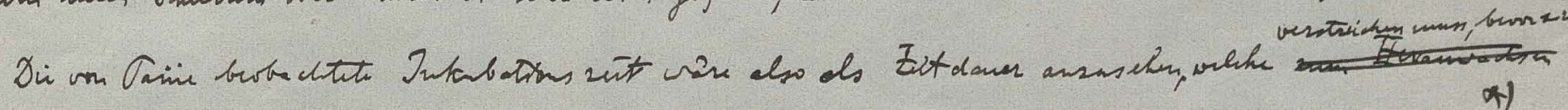

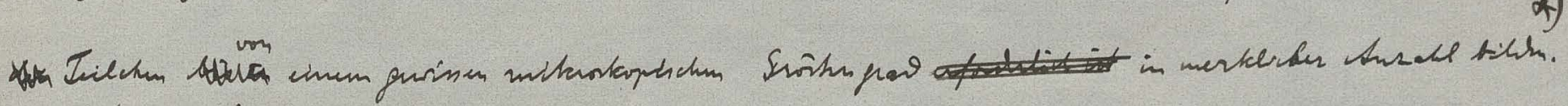
(rem anch rote)

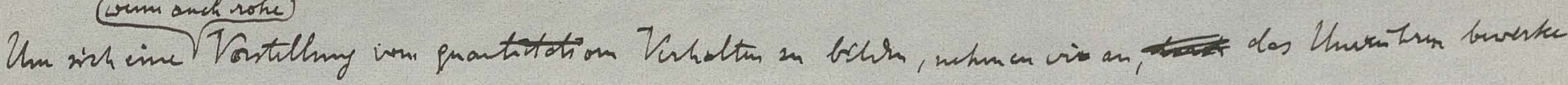

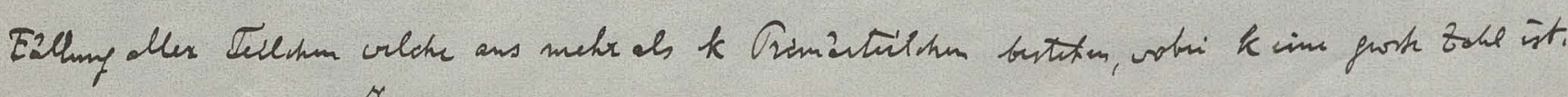

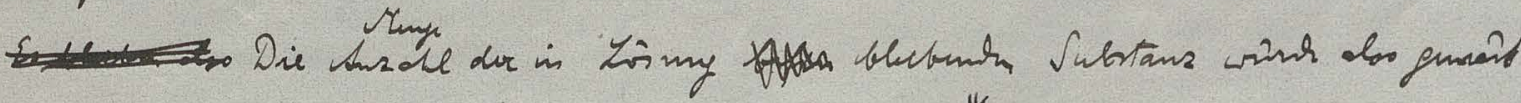
Porming ( )

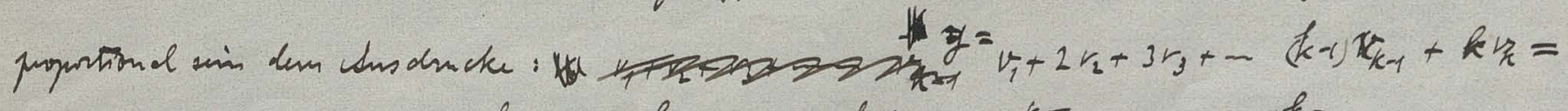

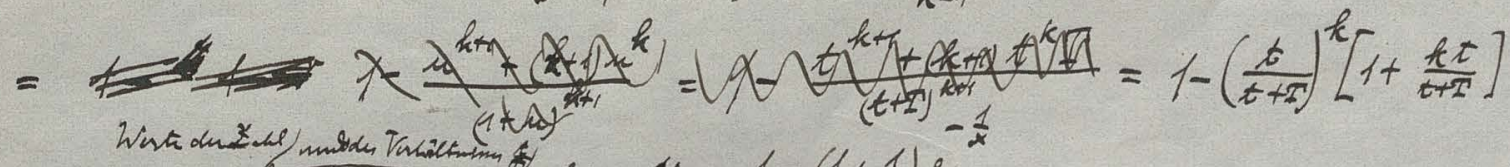

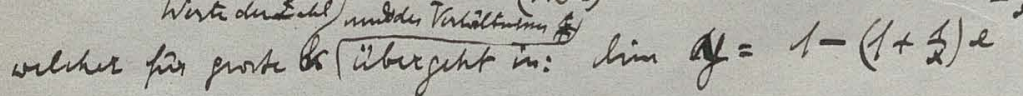

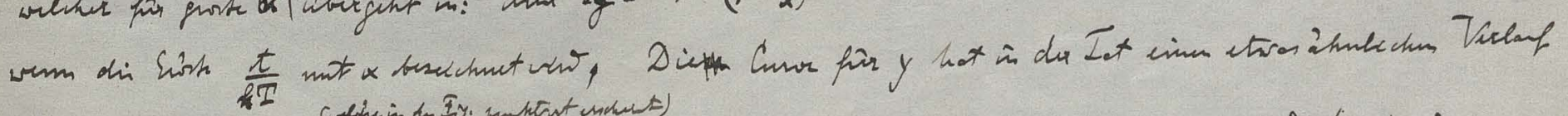

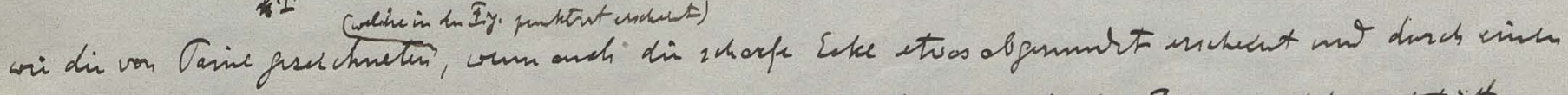




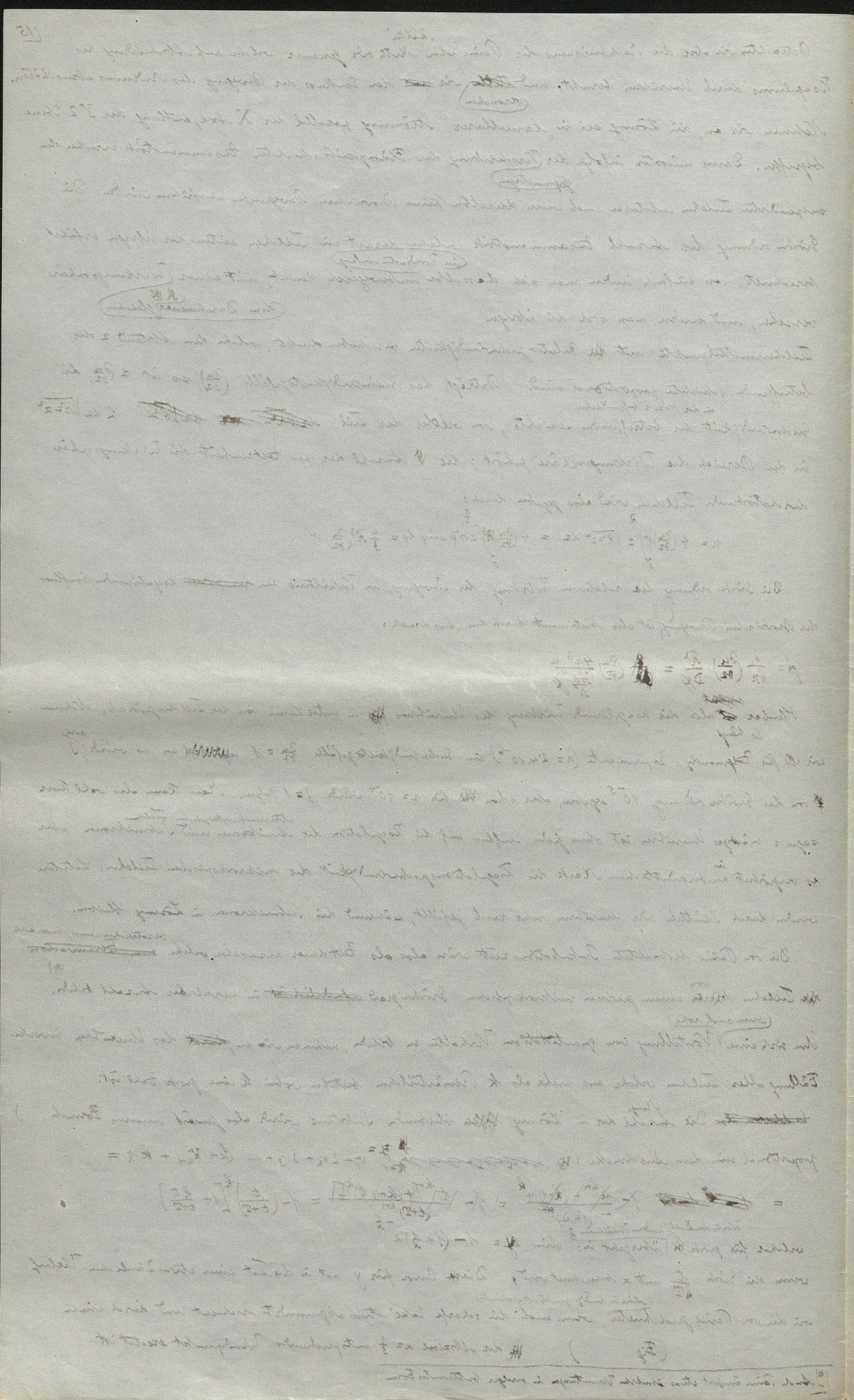


5216

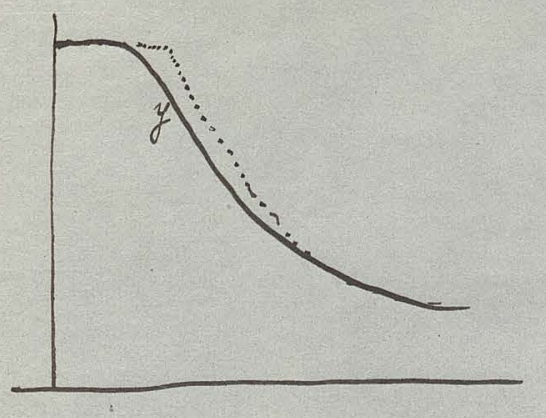

in algunerne Churessm

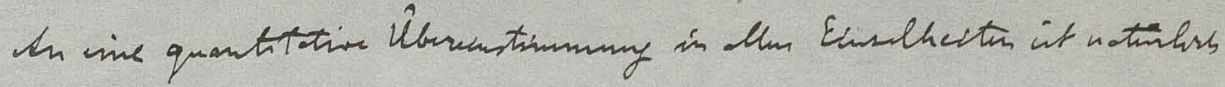
wichtis dentem, doer sovel Kam mon voll rgem, dass dir Existurs cines

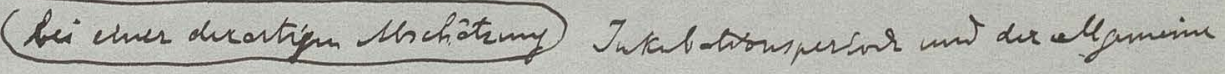

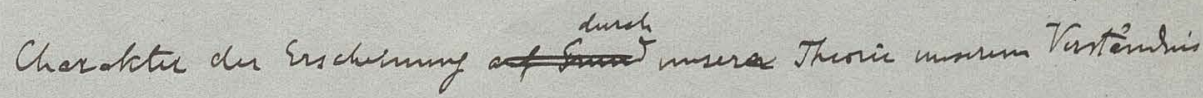
aklait enebent.

VI Vugeuh not Visterortsh mumpen

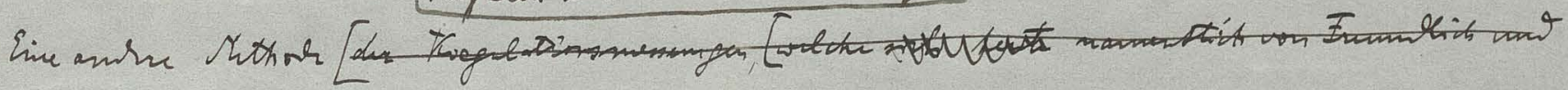

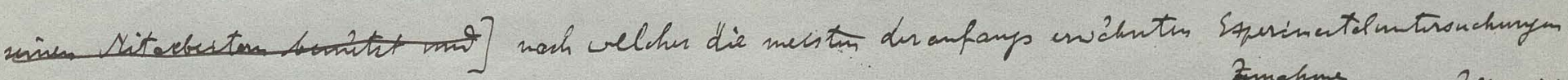
Znatume der Zothrgteix

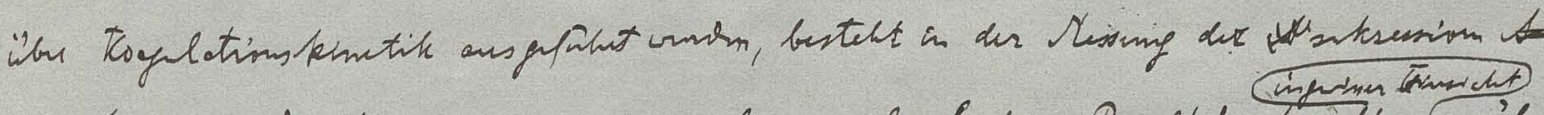

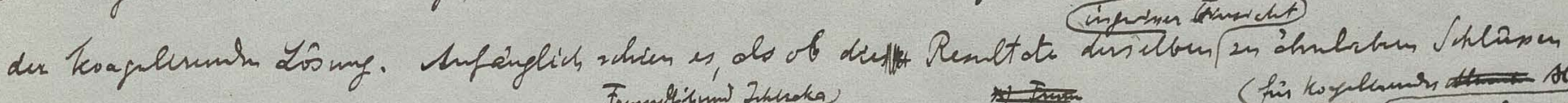

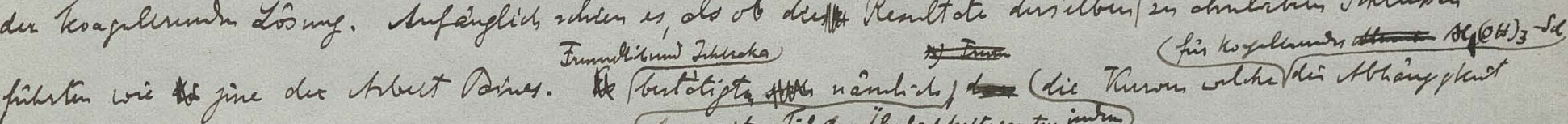

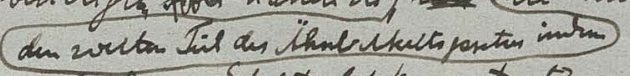

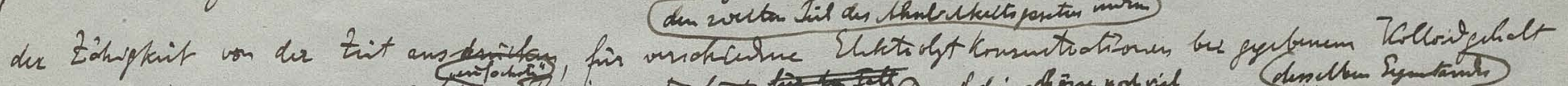

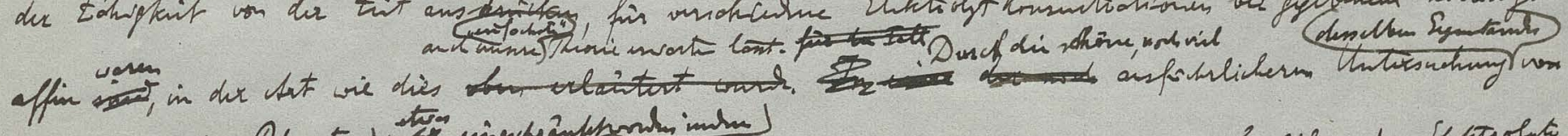

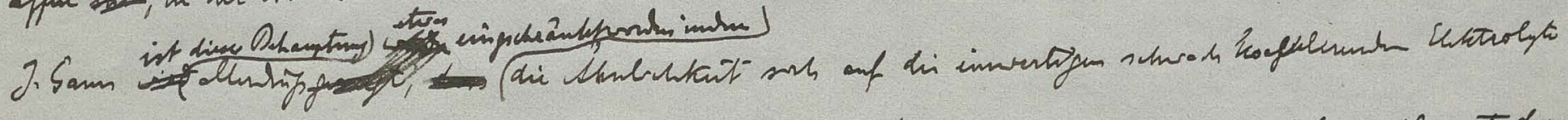

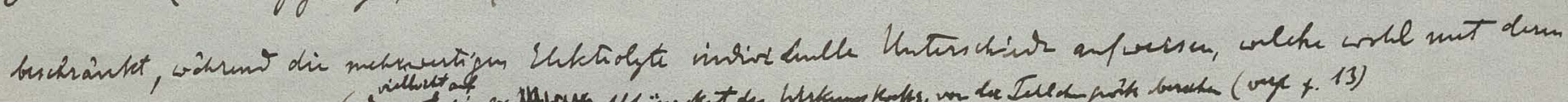

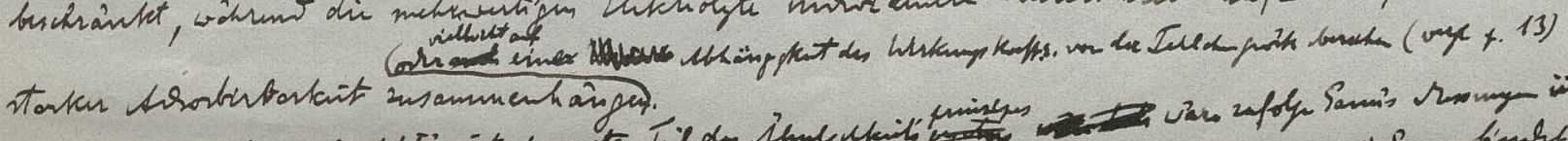

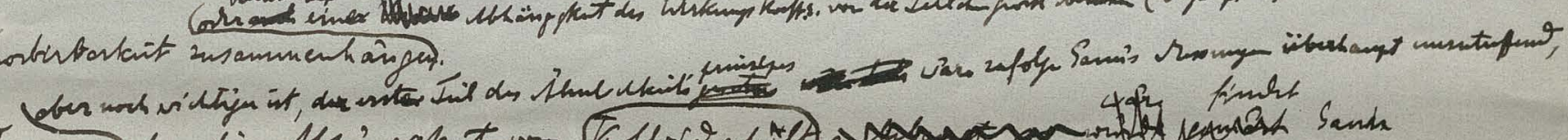

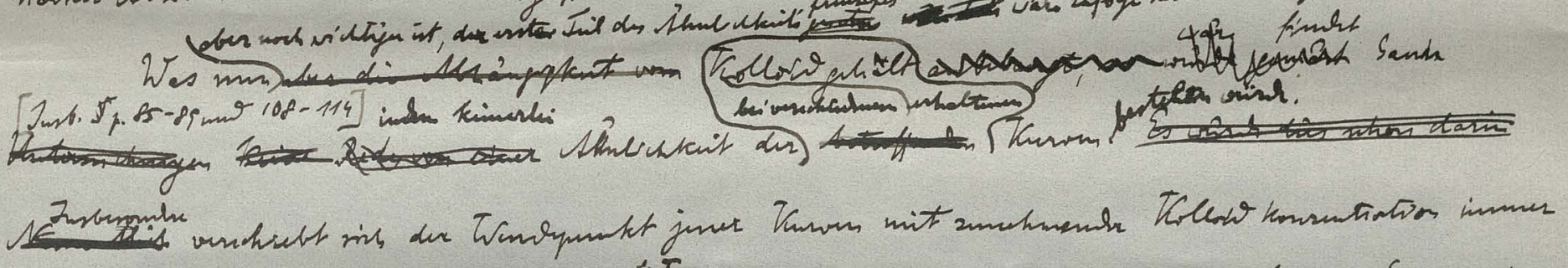

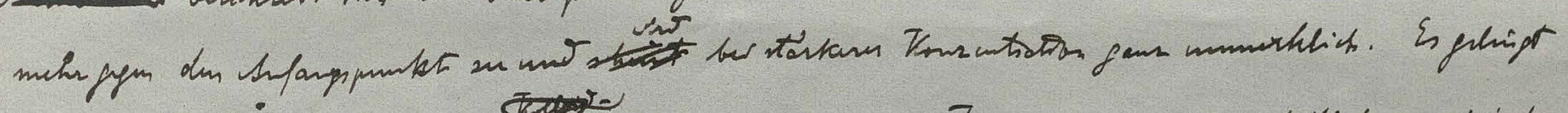

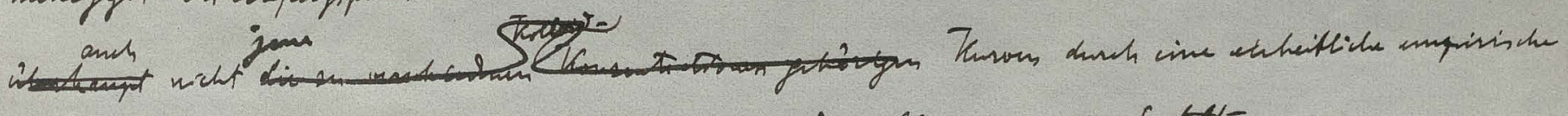

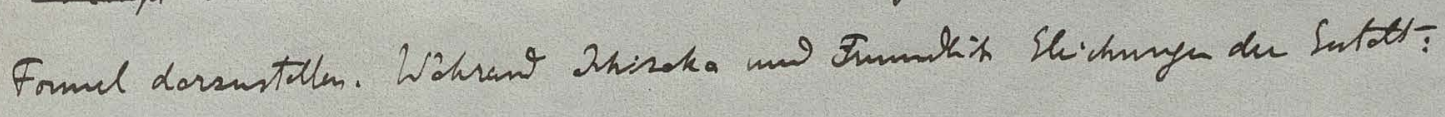

$$
\begin{aligned}
& \frac{d x}{d t}=k t(1+b x)(1-x)^{2} \\
& \text { ose } \frac{d x}{d t}=k^{\prime} t\left(1+b^{\prime} x\right)(1-x)
\end{aligned}
$$

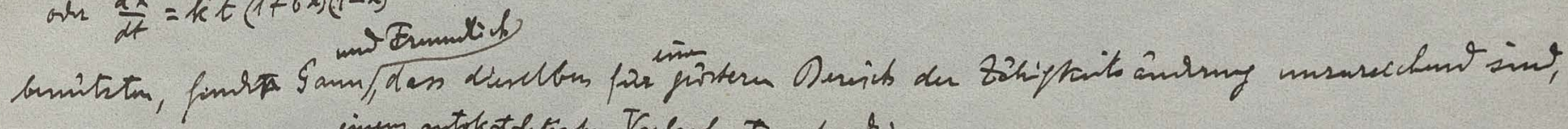

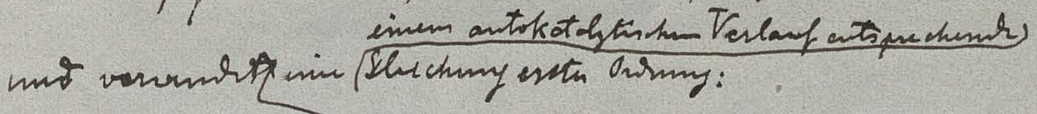

$$
\left.\frac{d x}{d t}=k_{1}\left(1+b_{1} x\right)(1-x)\right)
$$

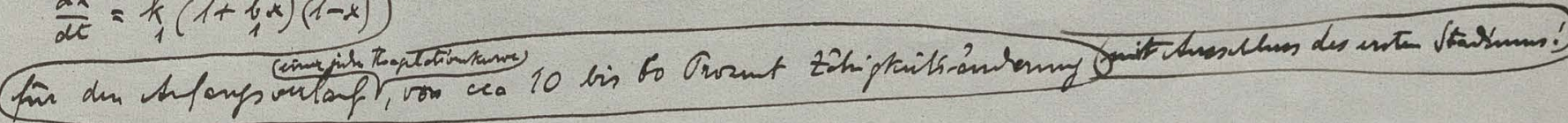

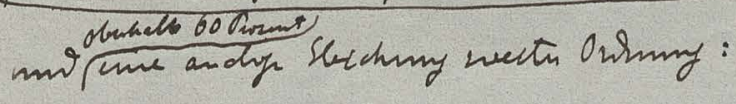

$$
\frac{d x}{d t^{t}}=k_{2}\left(1+b_{2} x\right)(1-x)^{2}
$$

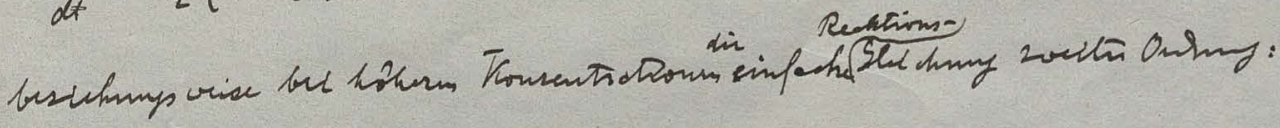

$$
\frac{d x}{d t}=k^{4}(1-x)^{2}
$$

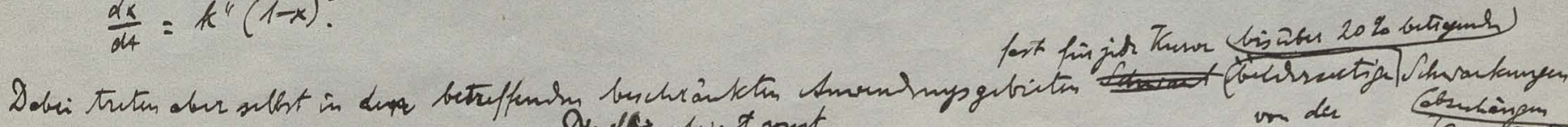

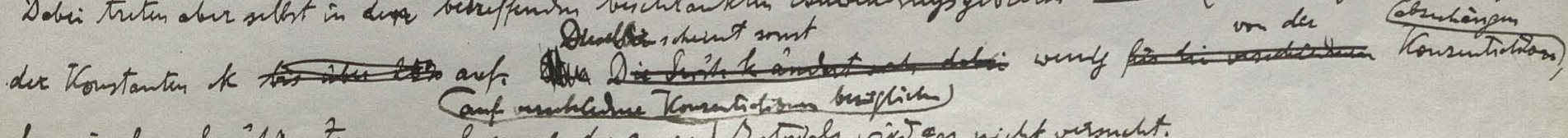

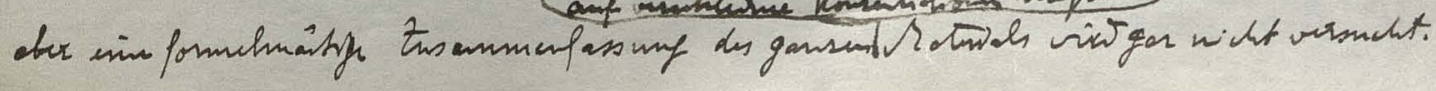





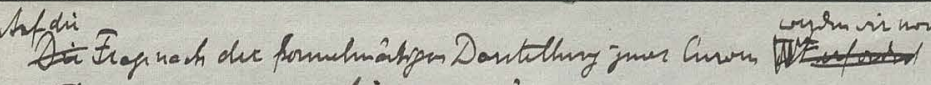

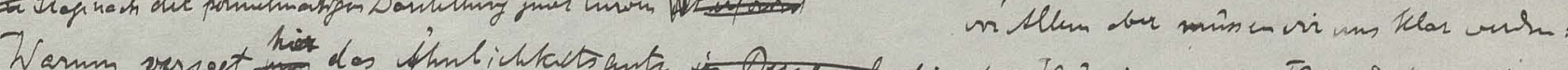

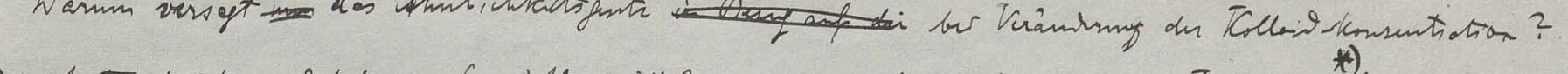

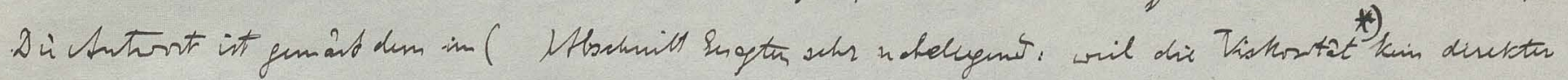

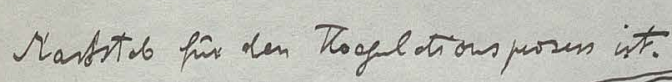

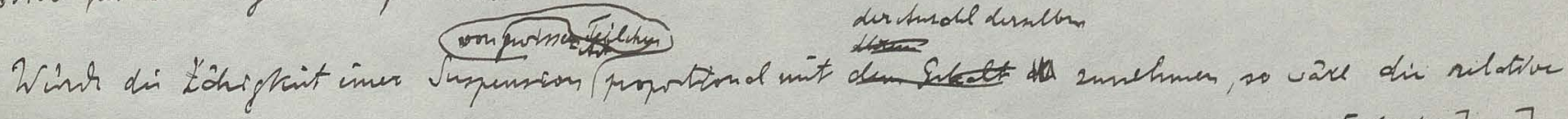

dersterobl dernetar

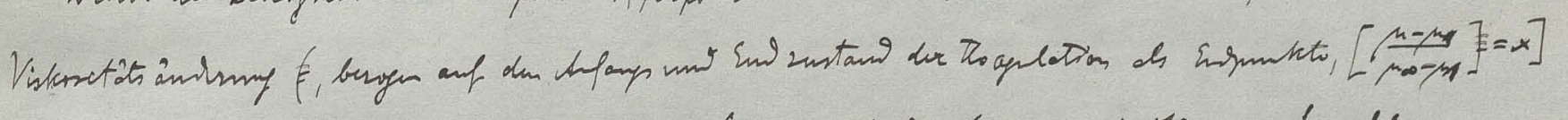

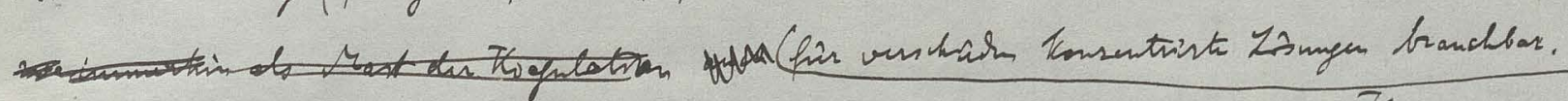

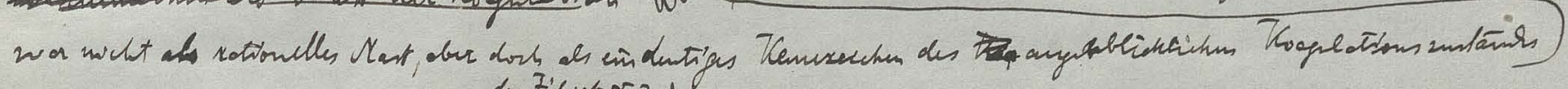

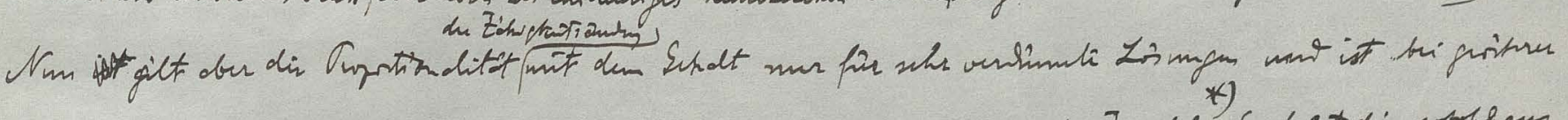

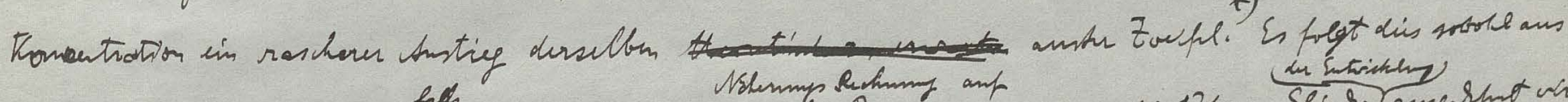

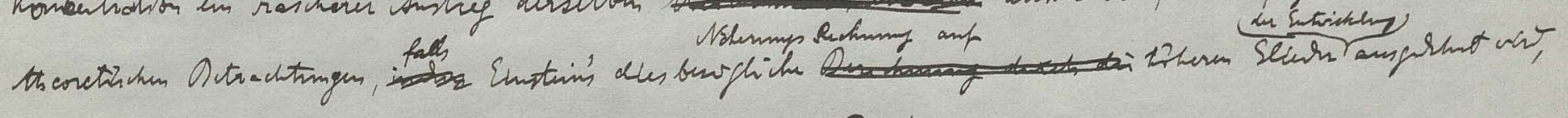

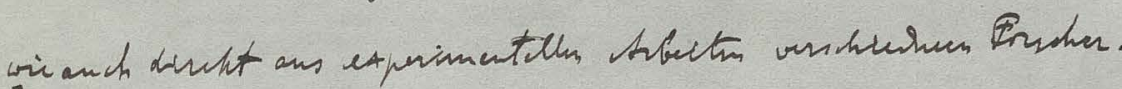

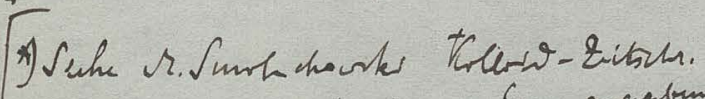

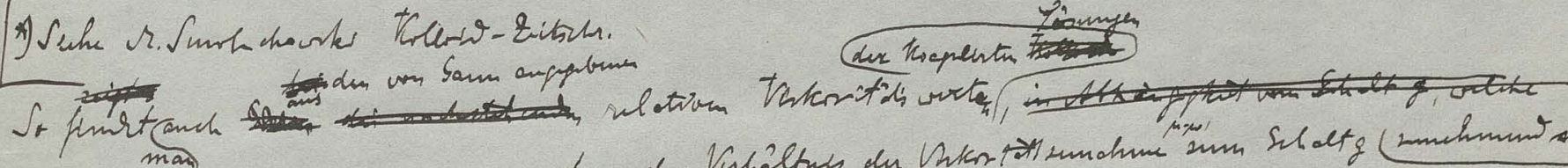

\begin{tabular}{|c|c|c|c|}
\hline & $\frac{m}{\mu^{00}}$ & $\underline{\mu-\mu_{0}}$ & \\
\hline $111^{\circ}$ & ioro & $2 \div 600$ & 0.23 \\
\hline 1.5 & & $28 k 5$ & 0286 \\
\hline 20 & 11420 & & 0.287 \\
\hline 25 & & & 0.395 \\
\hline 30 & $\begin{array}{l}1.837 \\
2175\end{array}$ & C.tes & \\
\hline 40 & 2.807 & & \\
\hline
\end{tabular}

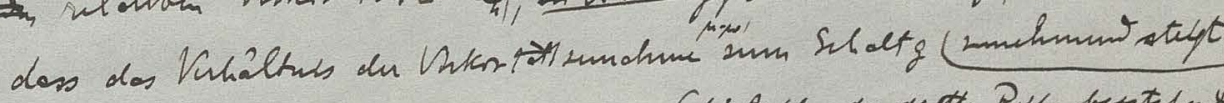

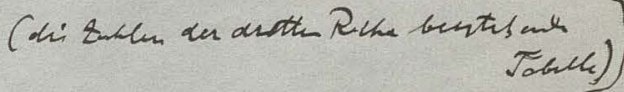

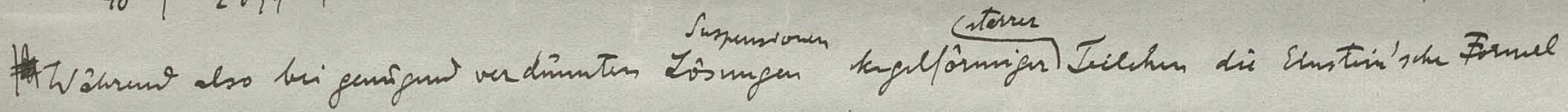

$$
\mu=\mu_{0}\left[1+\frac{5}{2} \phi\right]
$$

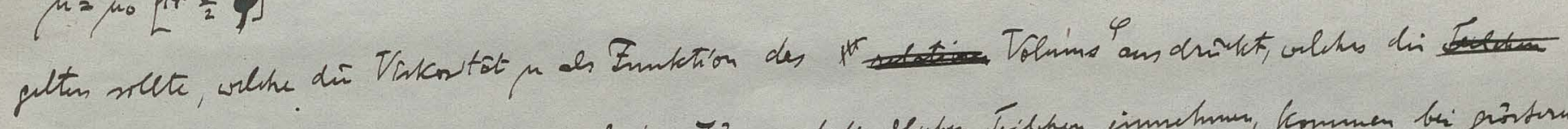

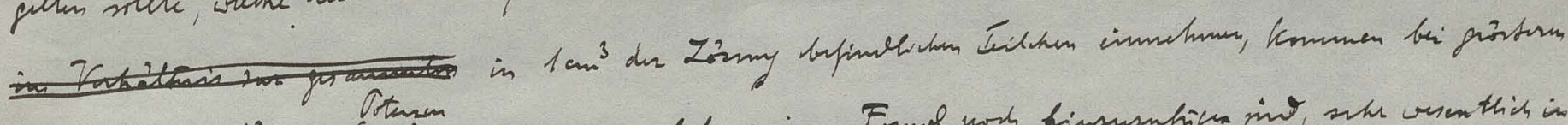

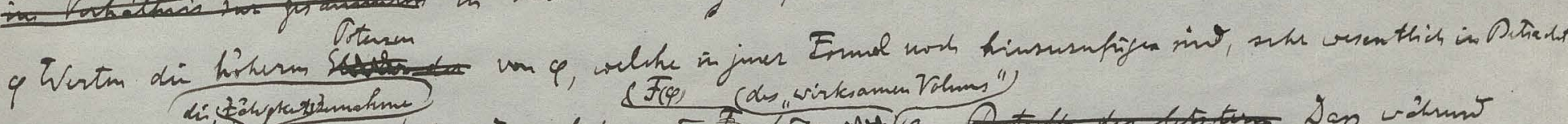
Es ist also int ins

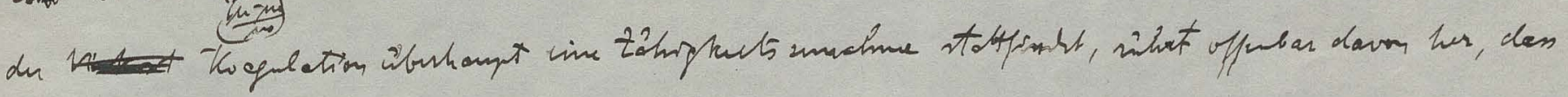

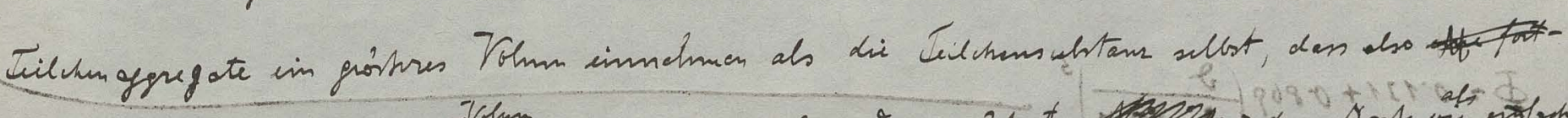

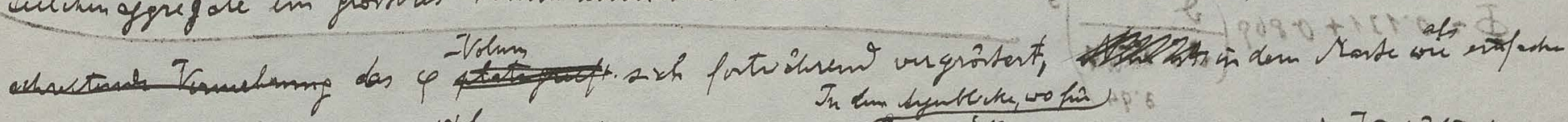

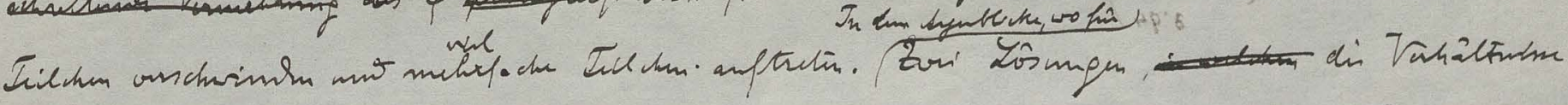

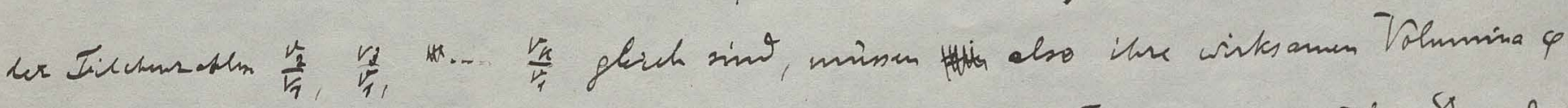

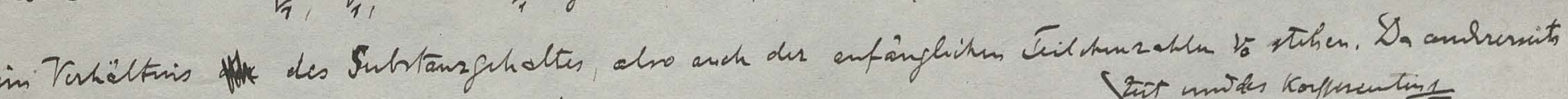

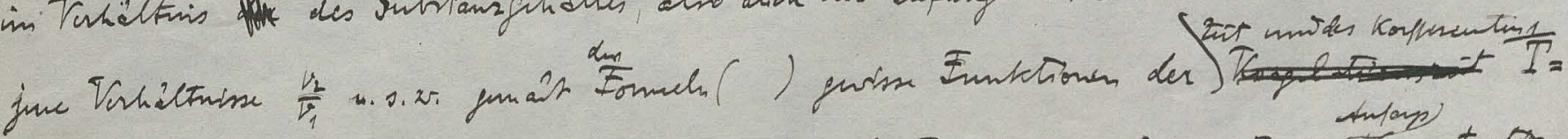

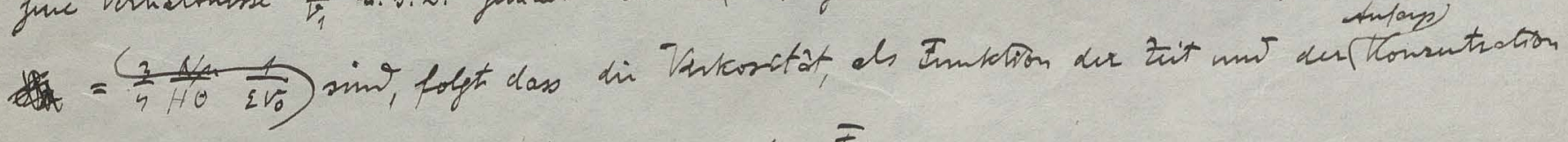

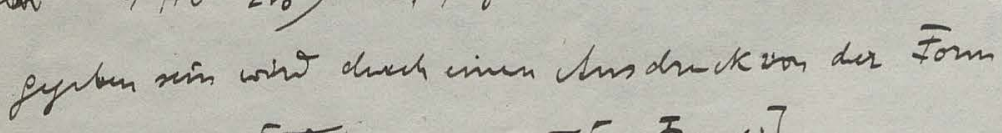

$\mu=I\left[r_{0} \Phi\left(v_{0} t\right)\right]$

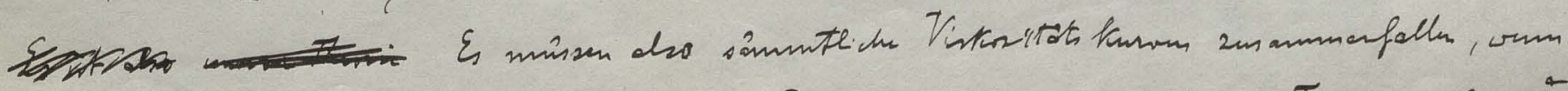

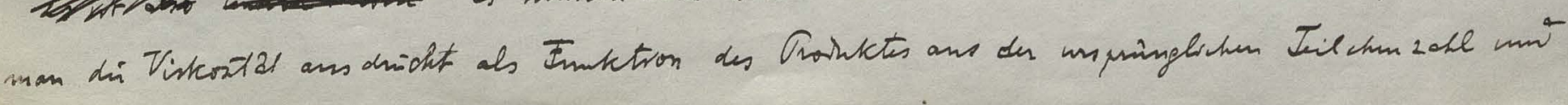




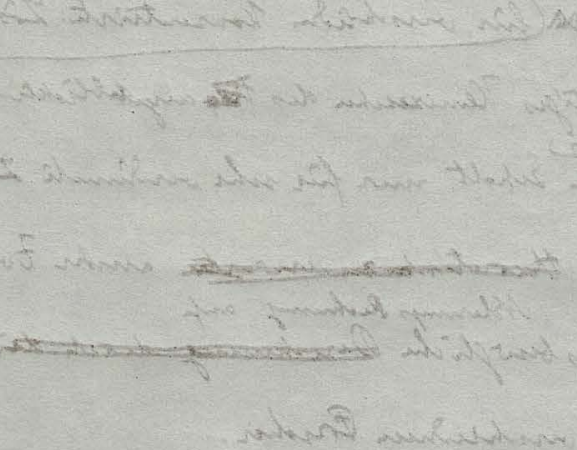

tent
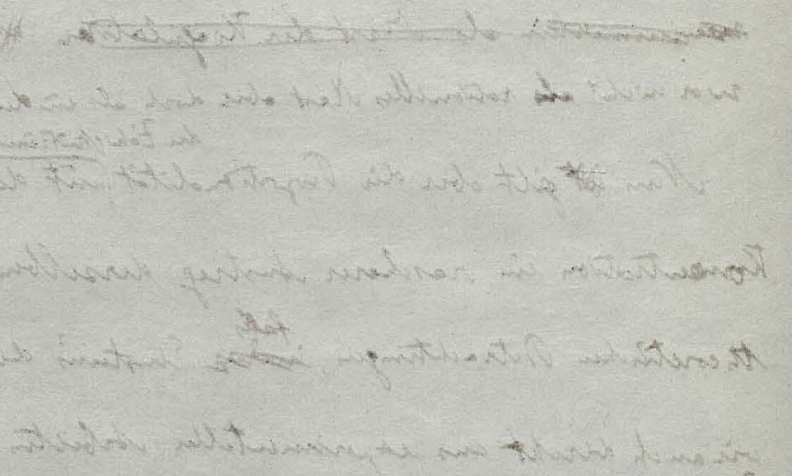

संज्या
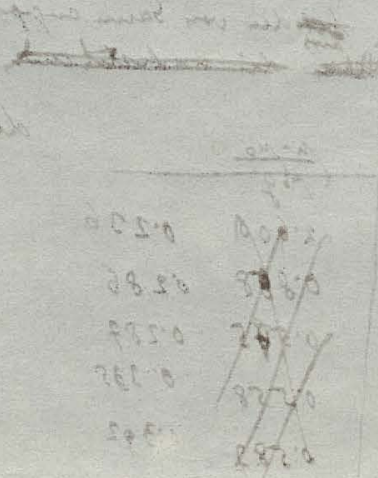

we
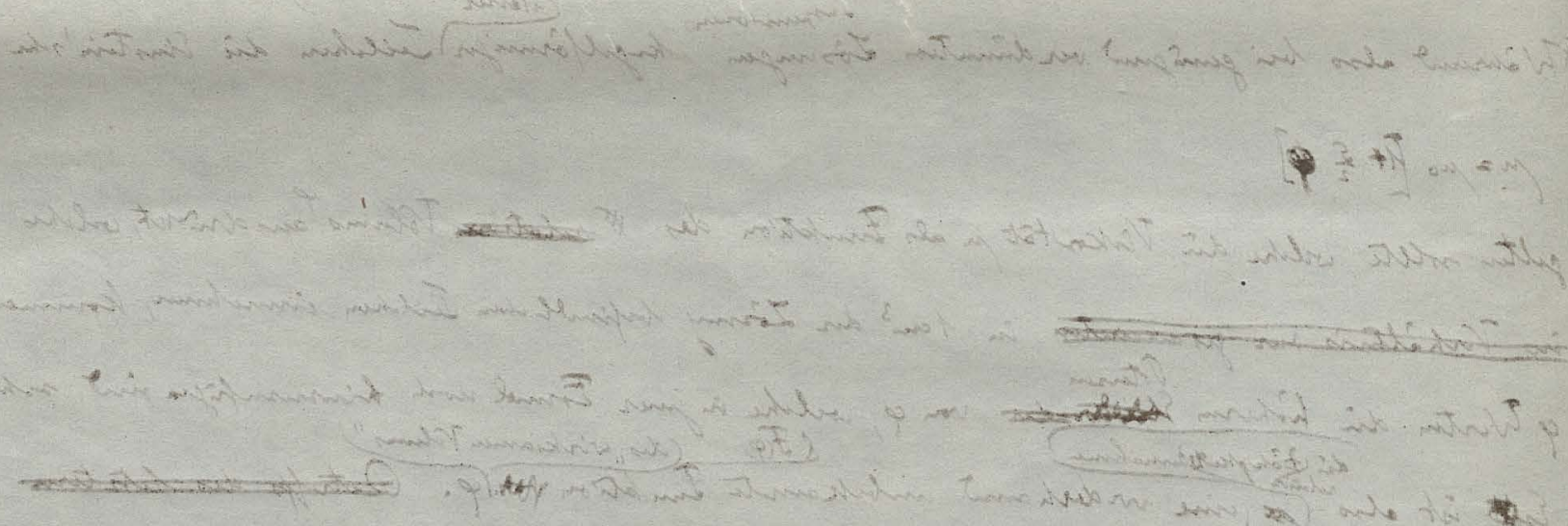

$\Phi=0.131+0.869\left(\frac{\vartheta}{2+1.9 \cdot 14}\right)^{3}$

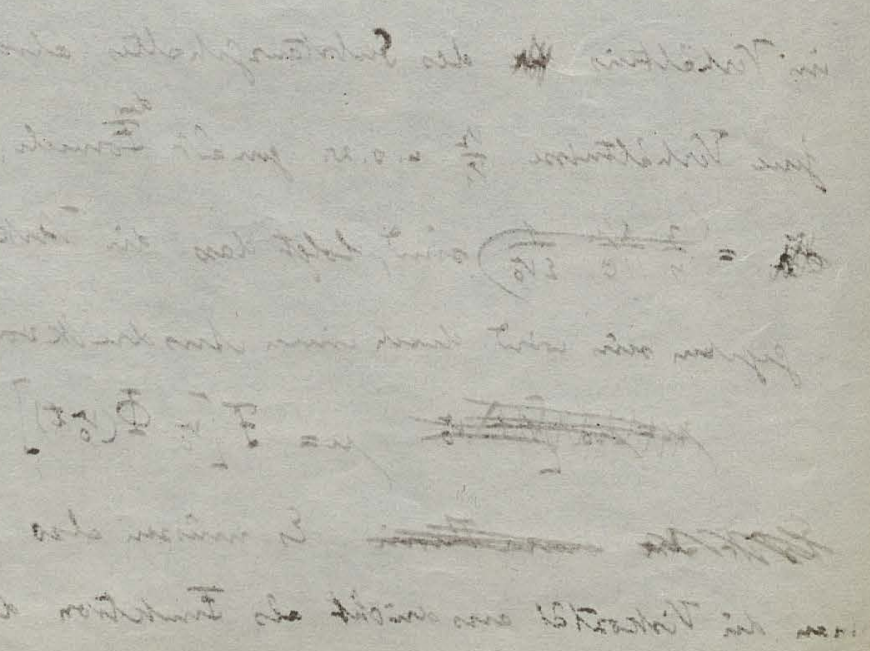




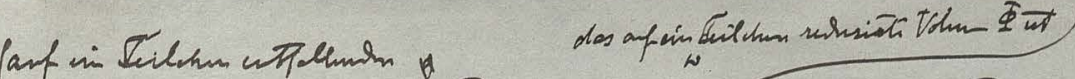

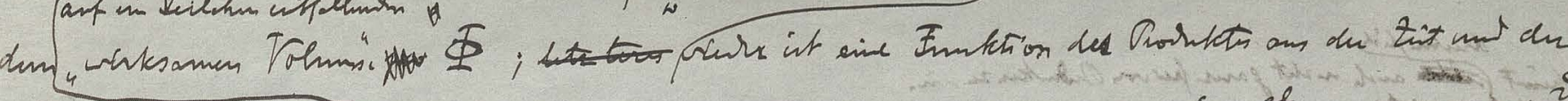

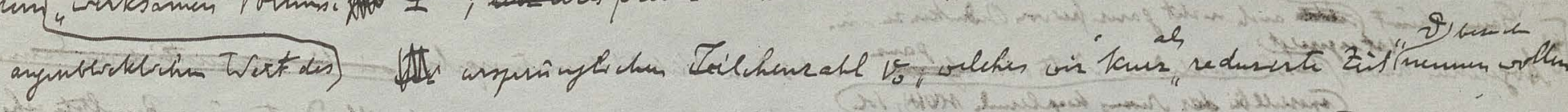

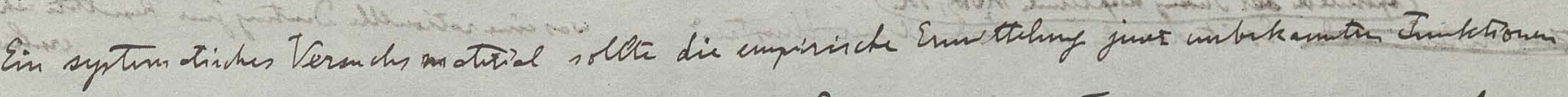

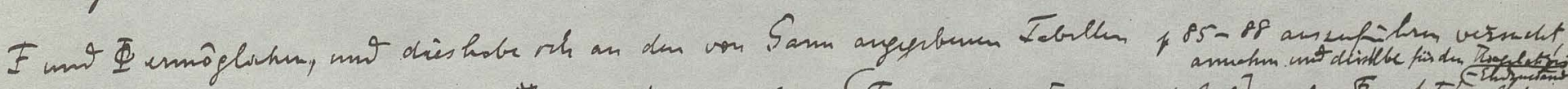

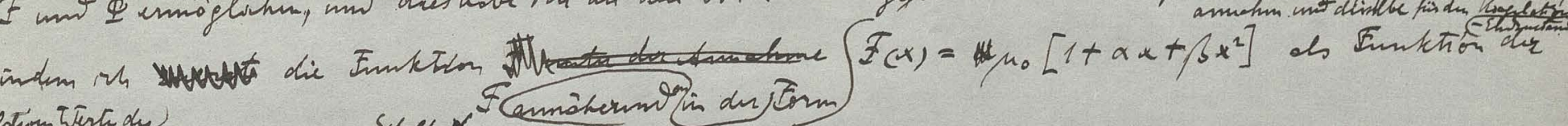

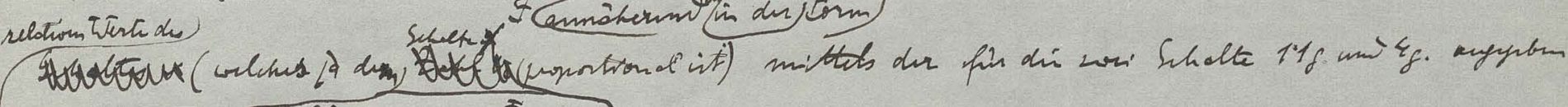

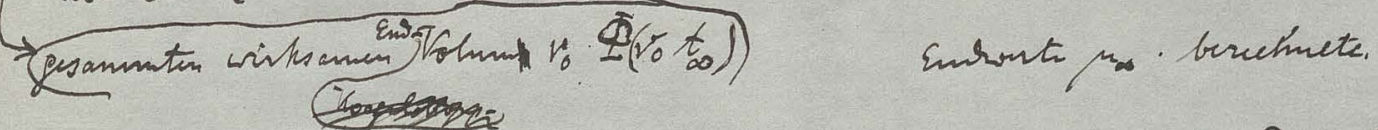

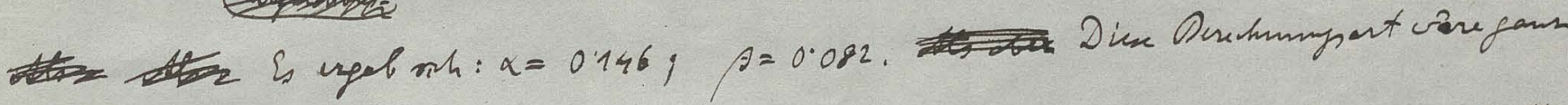

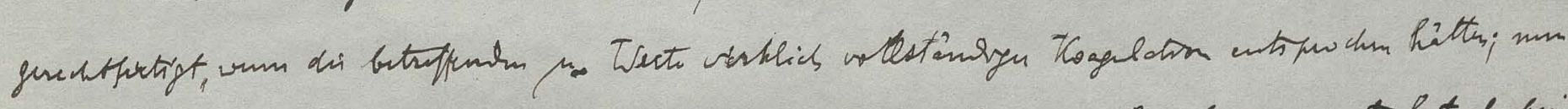

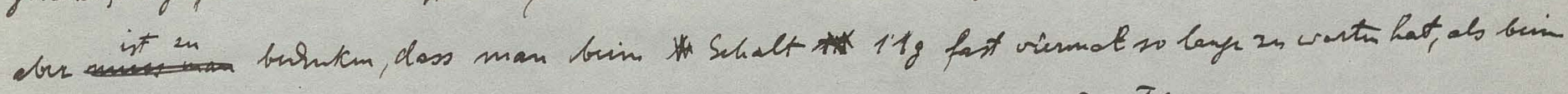

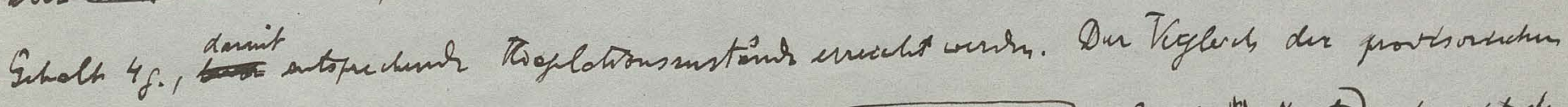

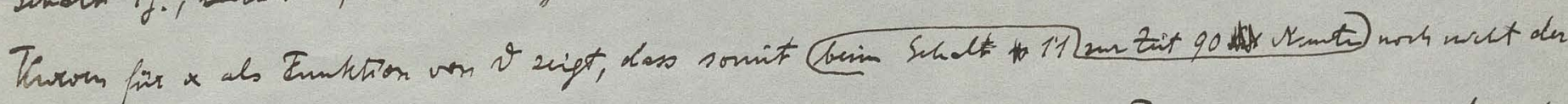

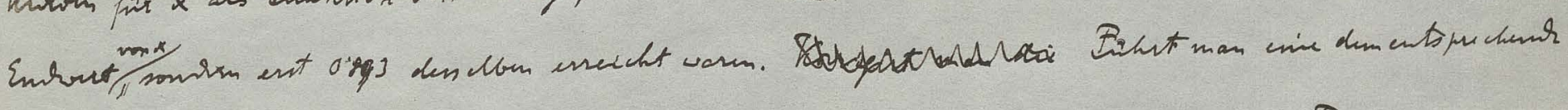

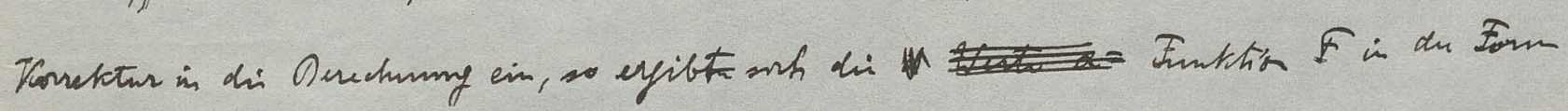
$F=c_{1}=\mu_{0}\left[1+0.198 x_{n}+0.069 \nu^{2}\right]$

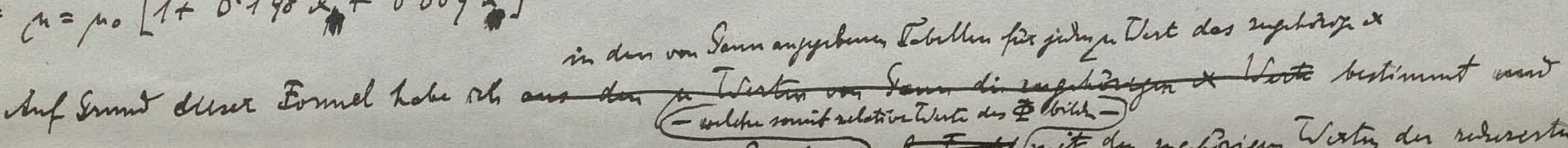

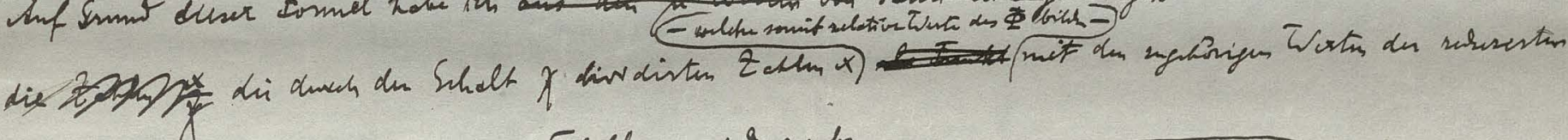

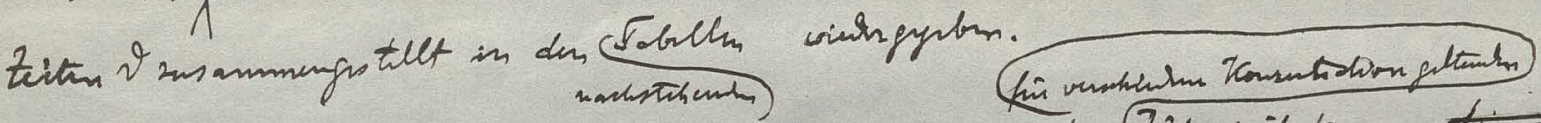

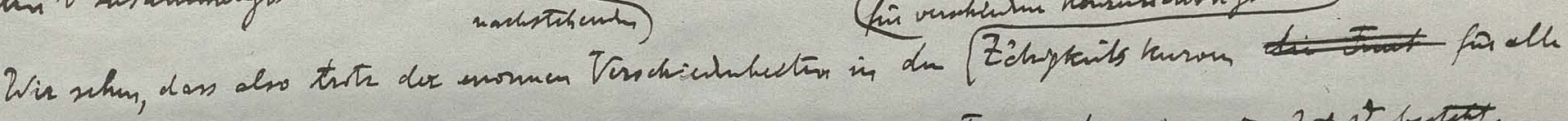

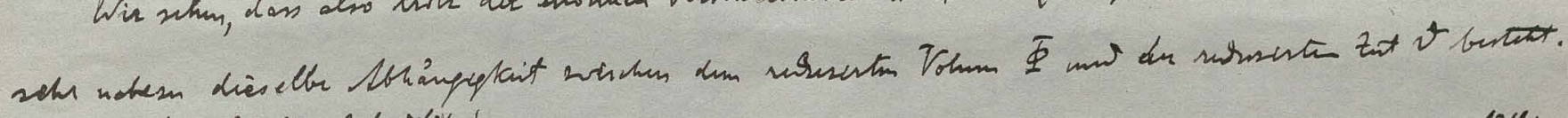

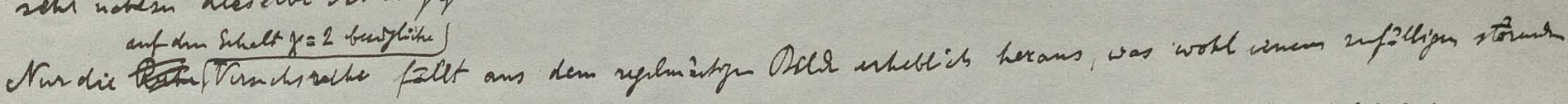

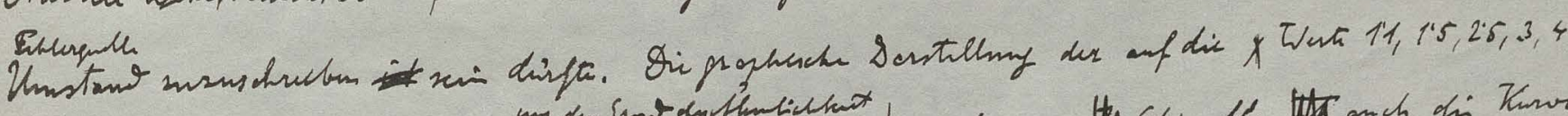

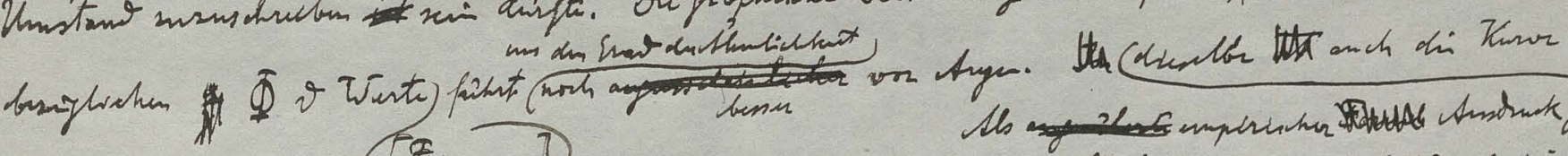

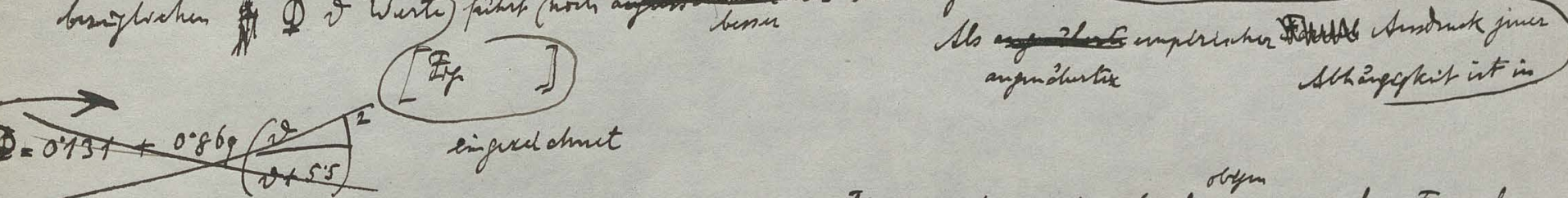

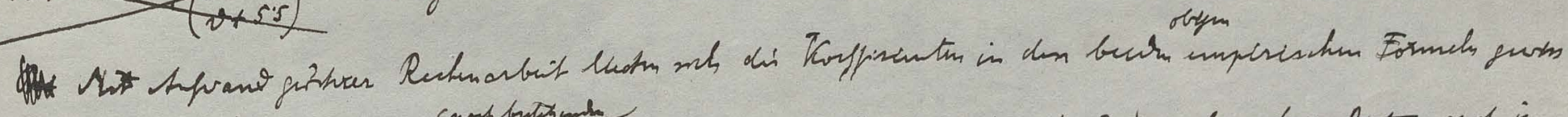

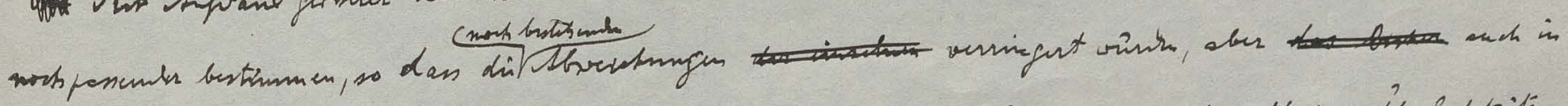

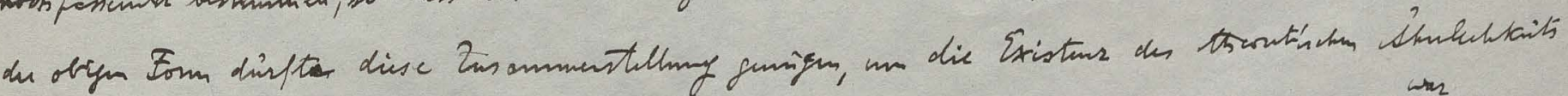

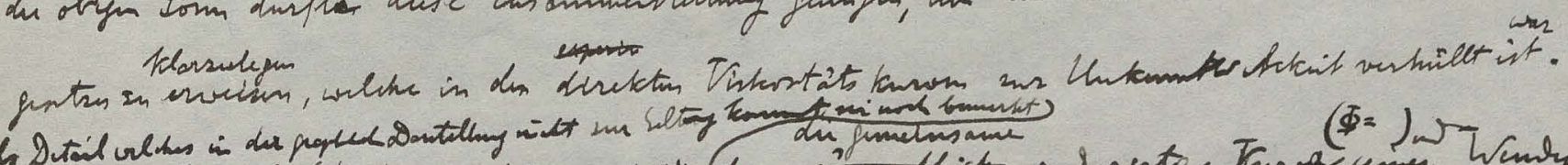

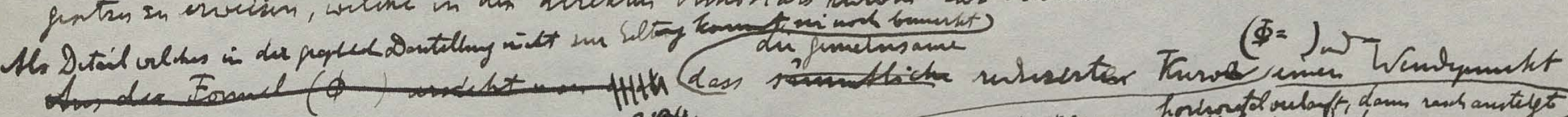

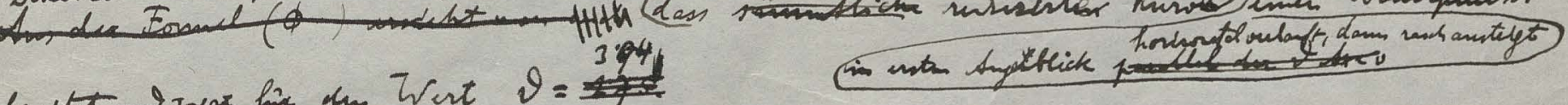
bust unistar fin den tort $\vartheta=$

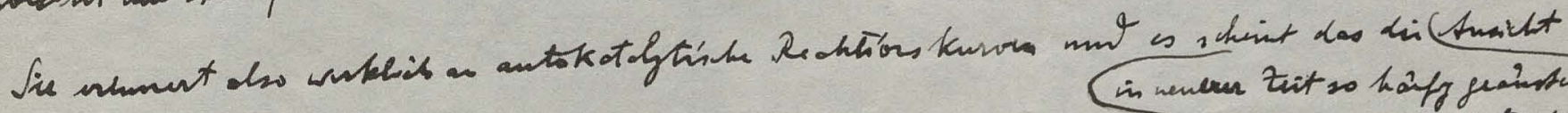

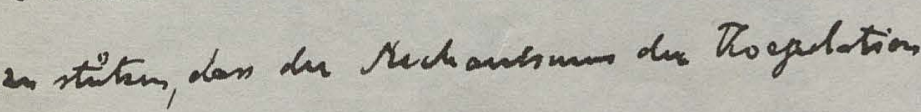

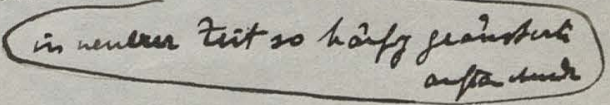
Ftrochich antsketolytiche Notris it. 


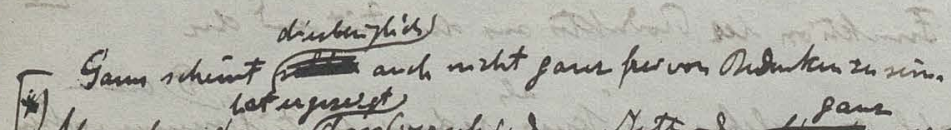

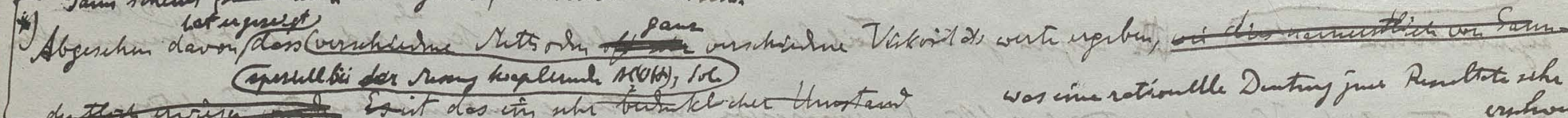

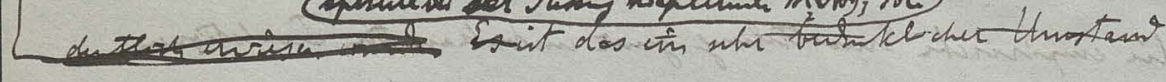




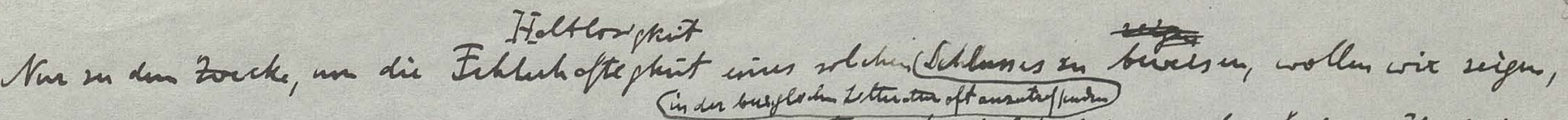

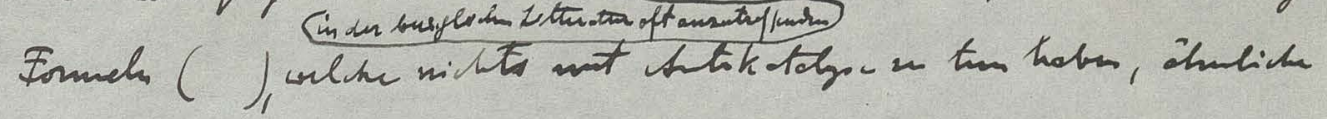

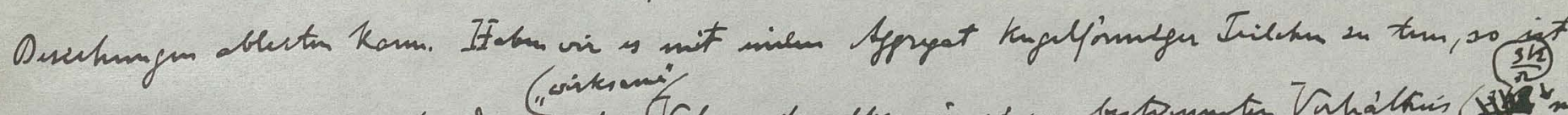

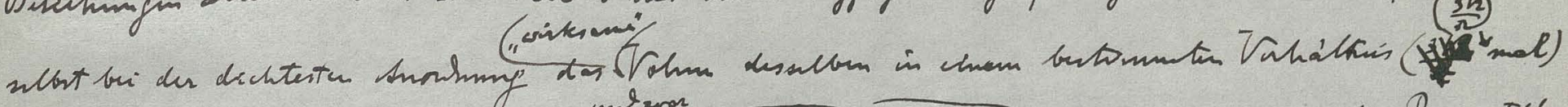

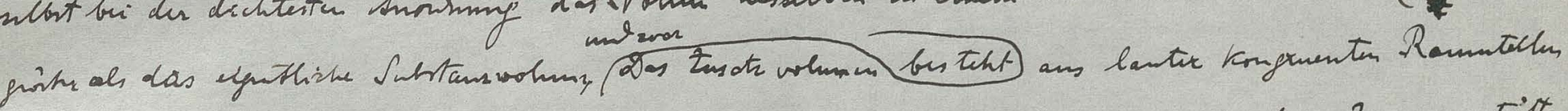

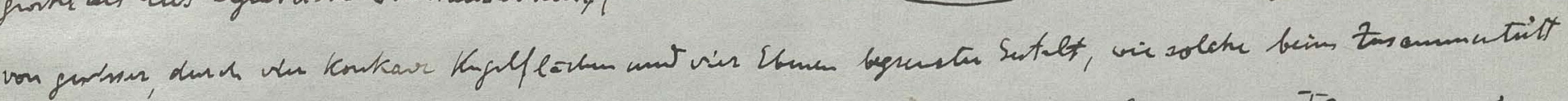

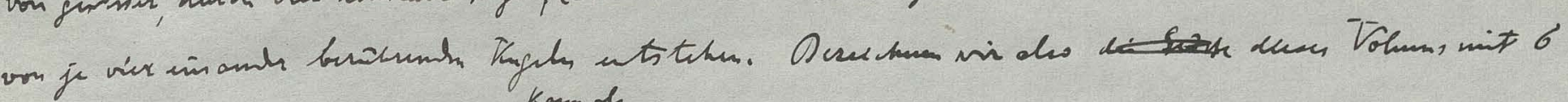

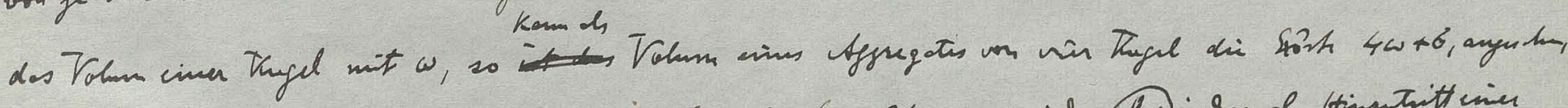

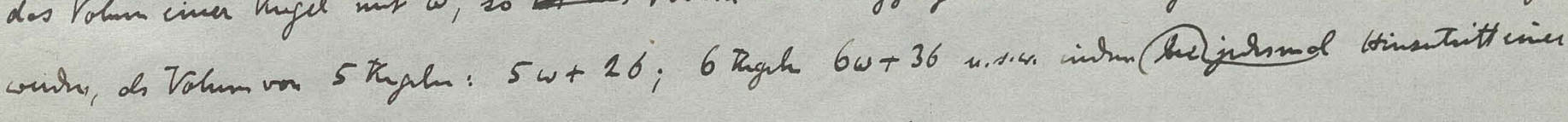

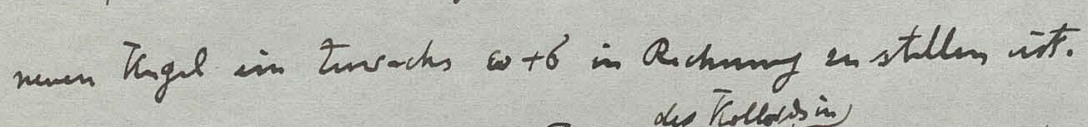

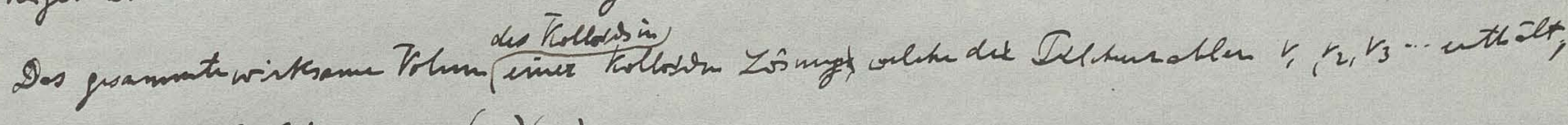

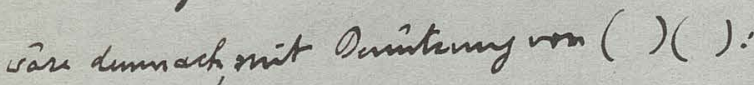

\section{a}

$x=\left(v_{1}+8 r_{2}+3 r_{3}\right) u+v_{4}(4 v+6)+v_{5}(5 v+26)+r_{6}(6 w+36)+$ $=\omega \Sigma k v_{k}+\sigma \sum k v_{k+3}=\omega v_{0}+\sigma v_{0}\left(\frac{t}{a T}\right)^{3}$

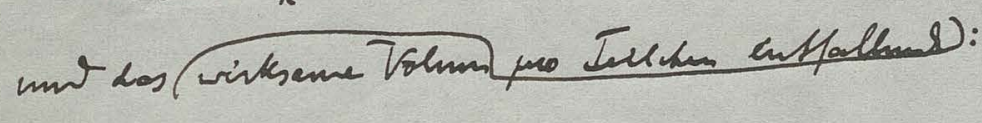

$\Phi=\omega+b\left(\frac{t}{t+T}\right)^{3}$

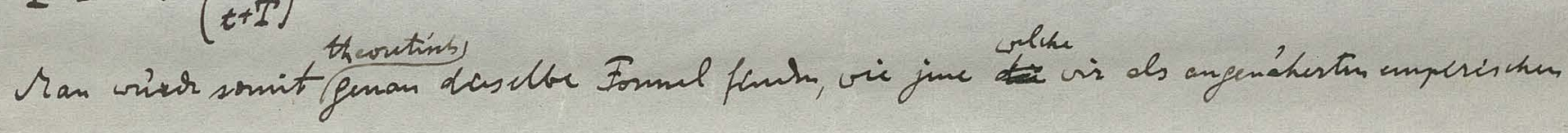
Susduck der Susumgen Samis dblectim.

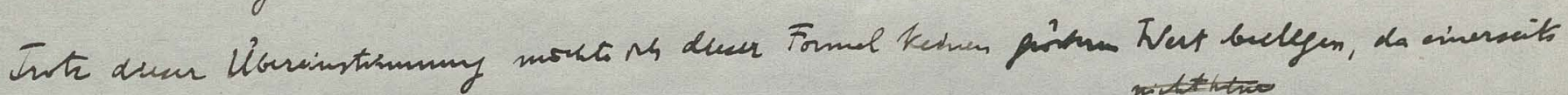

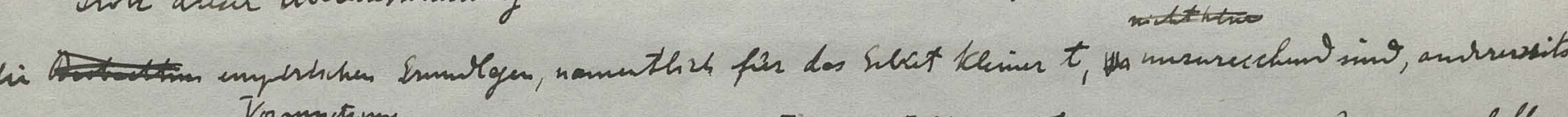

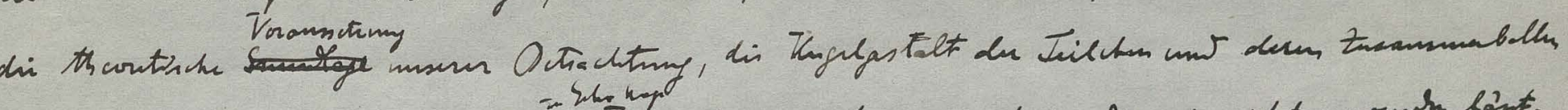

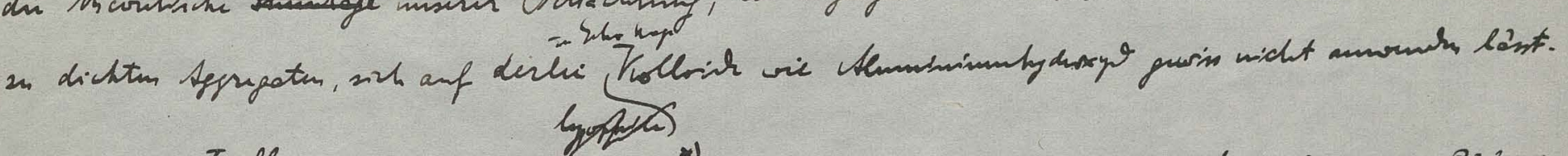

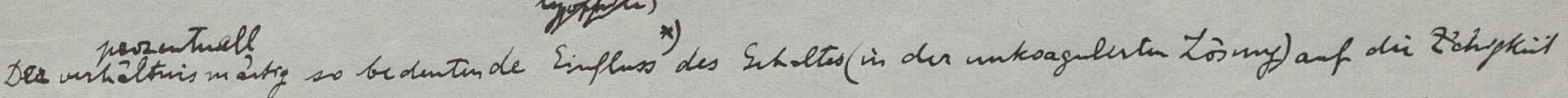

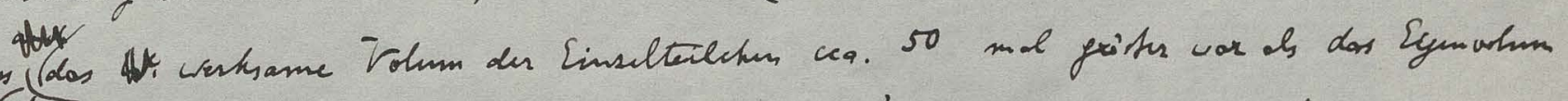

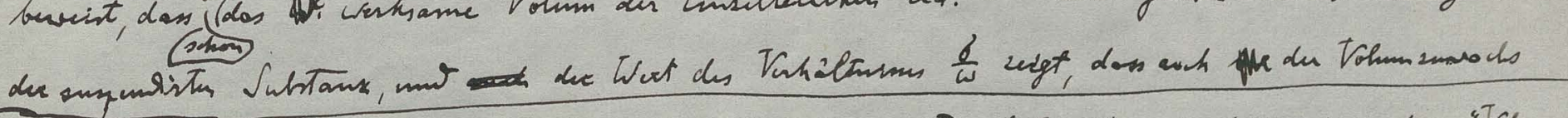

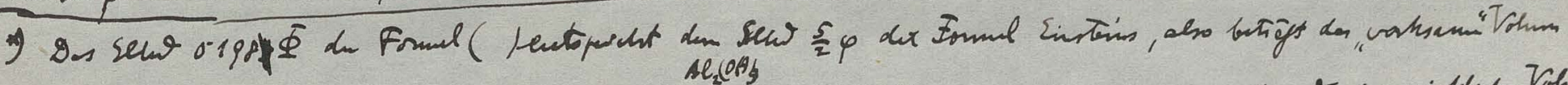

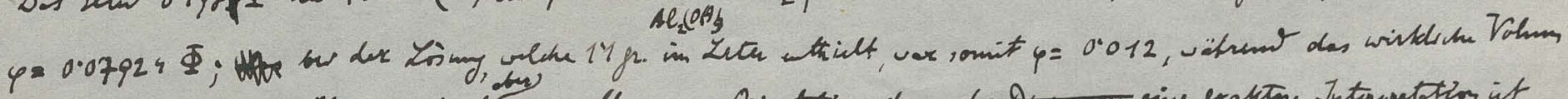

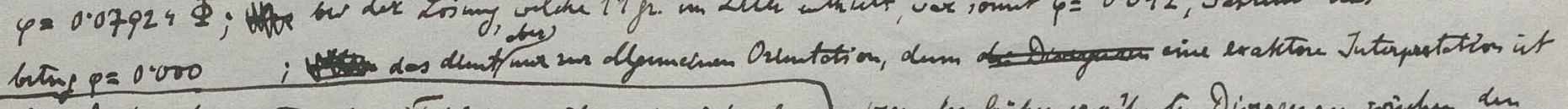

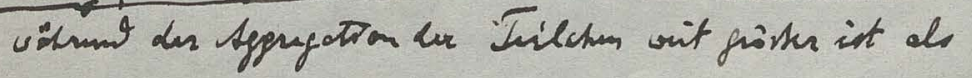
des ofign Taplthosin utopurds.

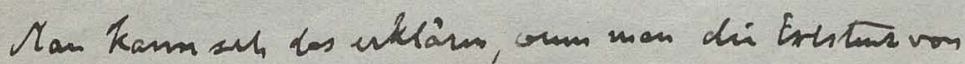

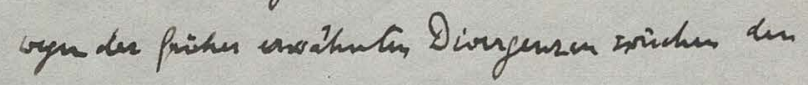

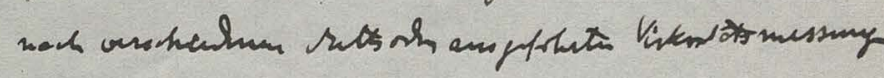
most molesis. tanem

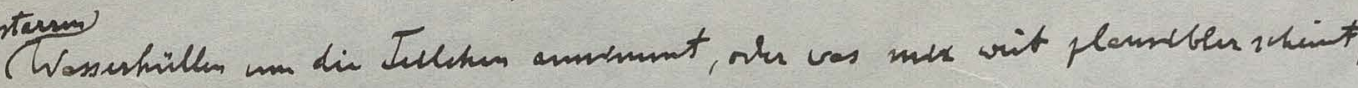

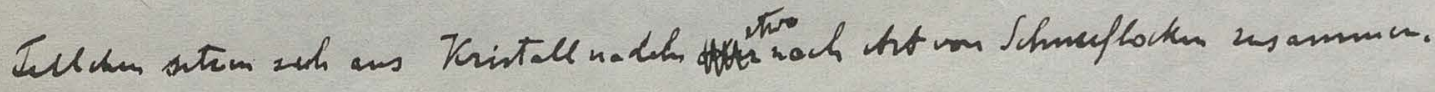

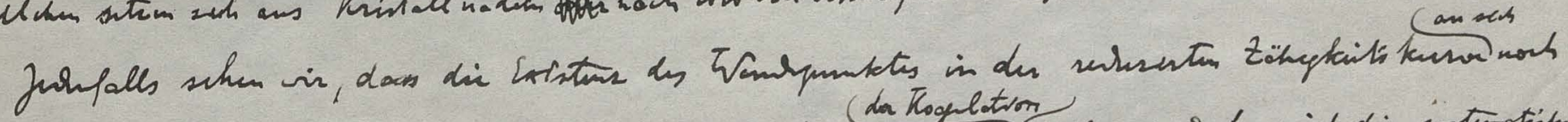

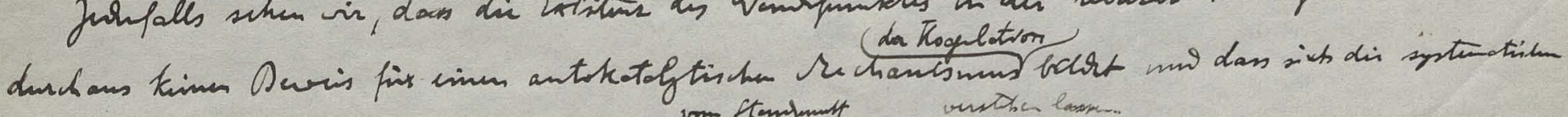

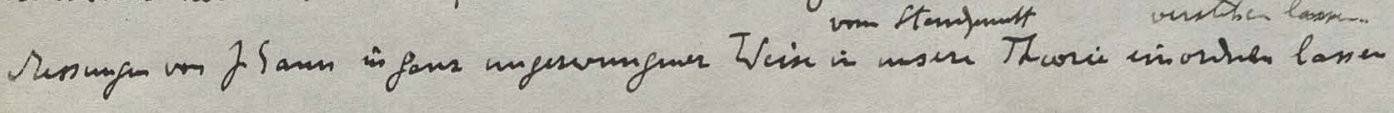




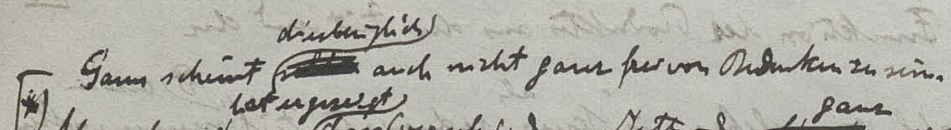

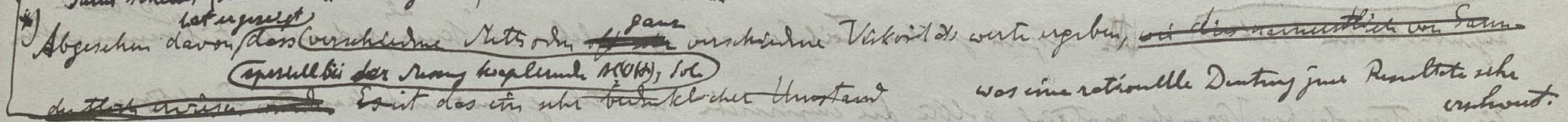

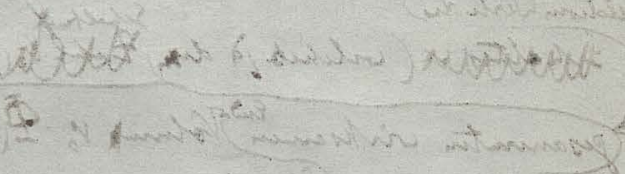


(b)

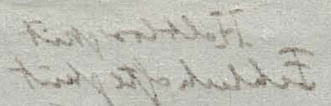

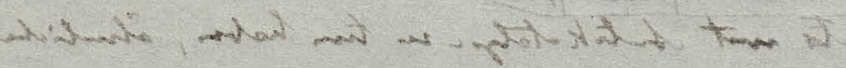

$(9-)^{2}+4$

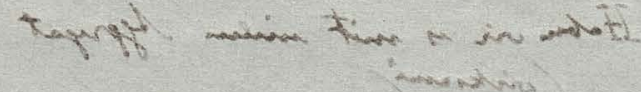

Wiat

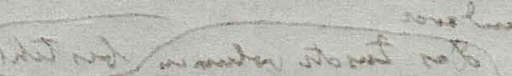

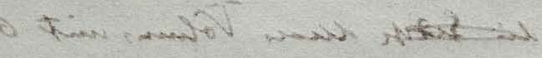

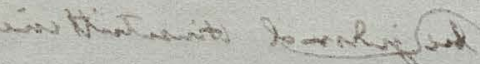

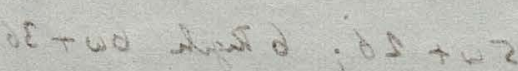

Fine mulato

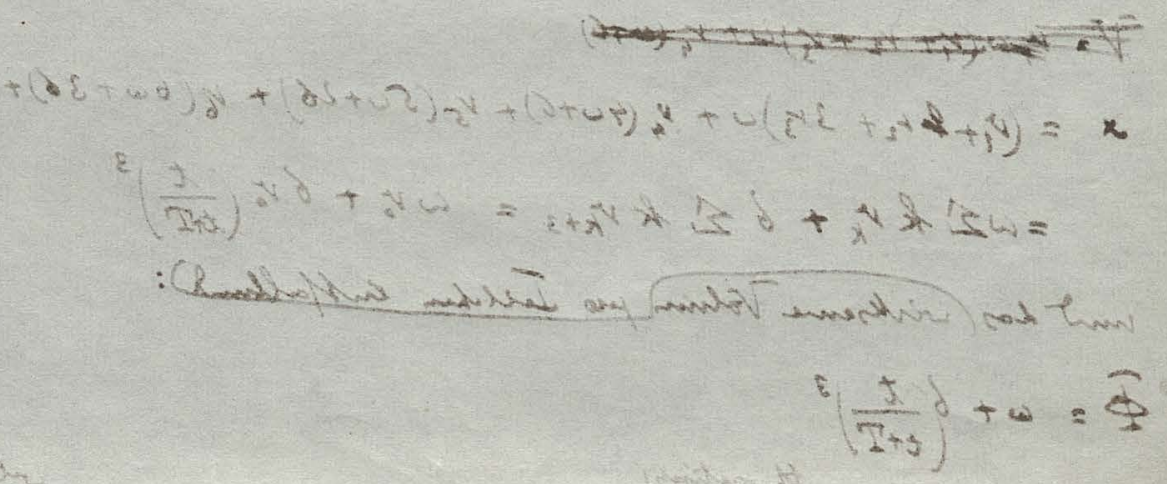

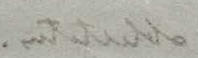

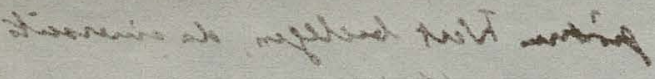

Dintermans *

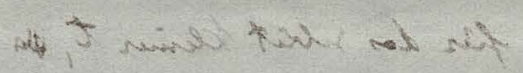

$(x+2)$

समत्रा

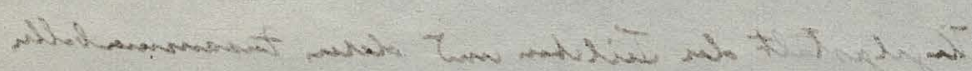

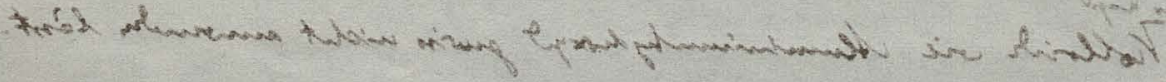

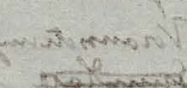

in $\log +x^{-1}$

trawe

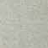


in slly

56

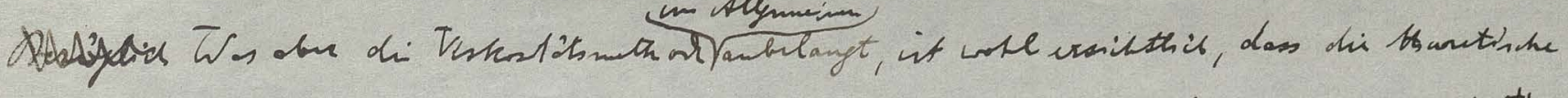

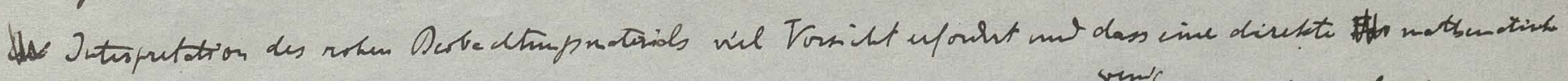

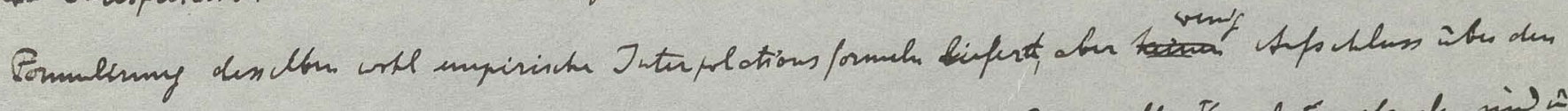

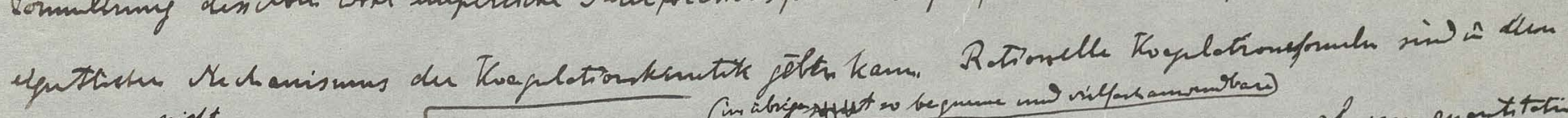

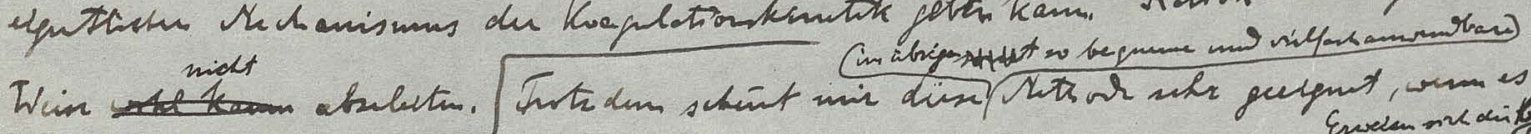

Hetersushng der Wuks

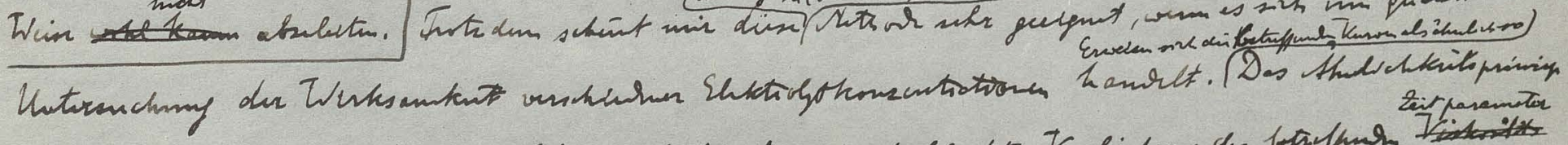

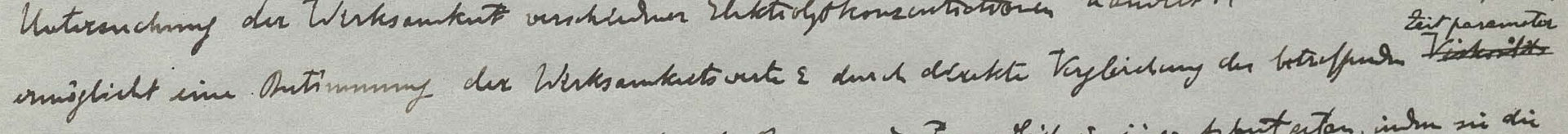

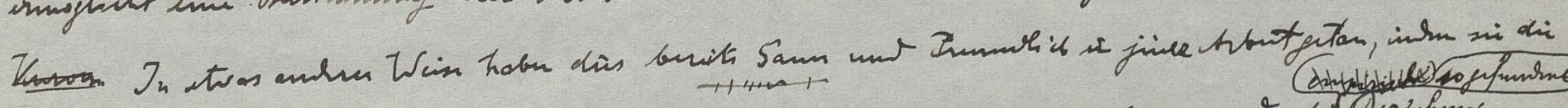

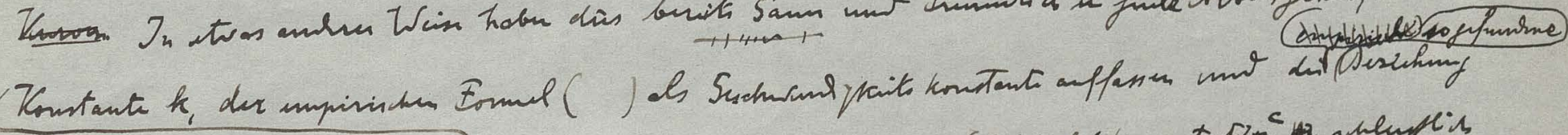

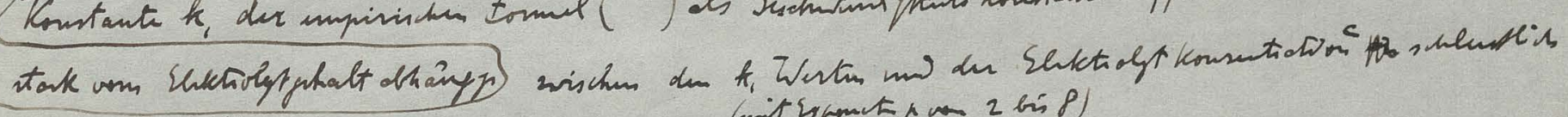

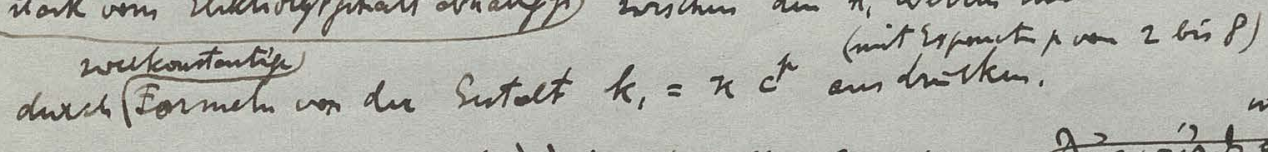

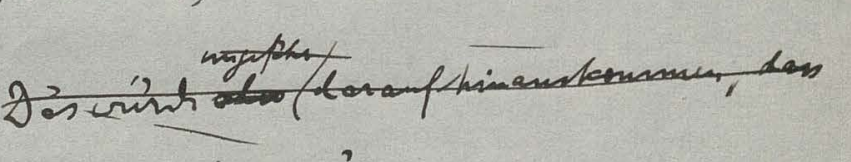

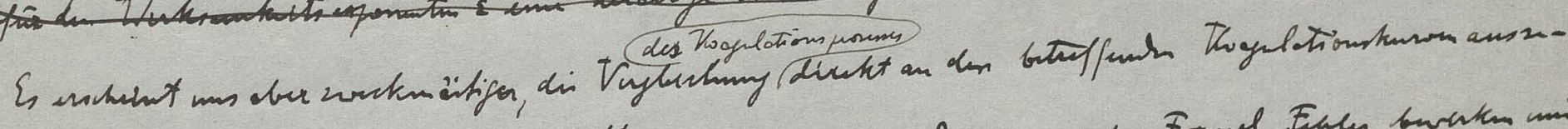

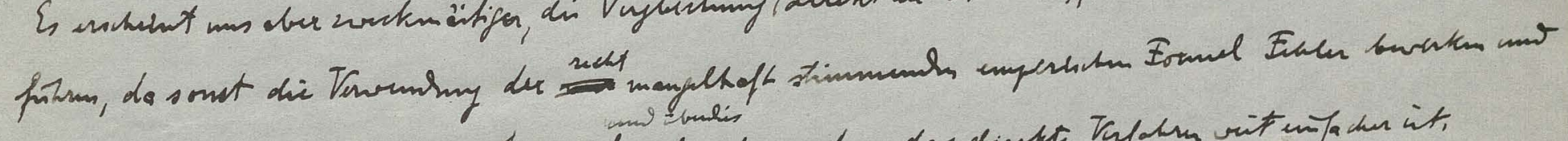

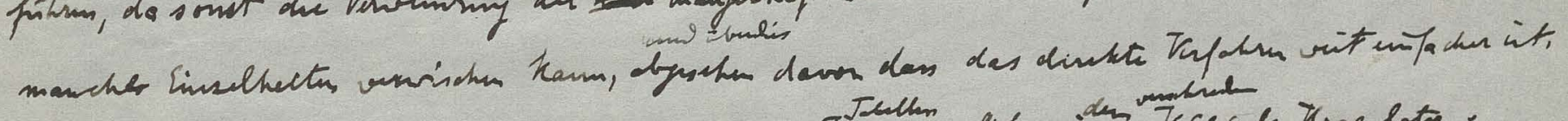

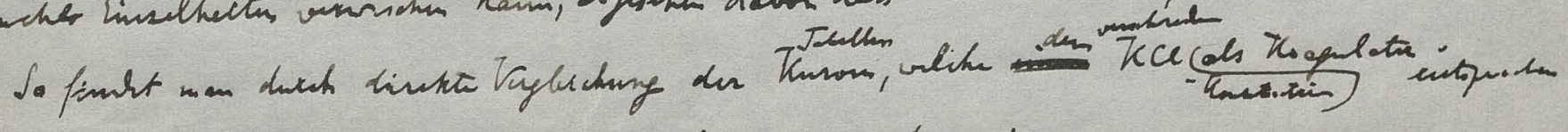

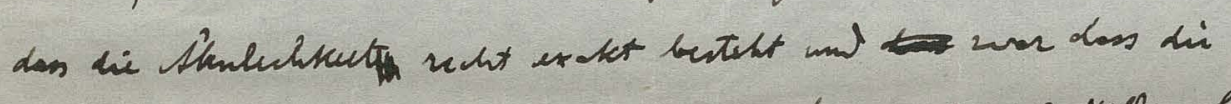

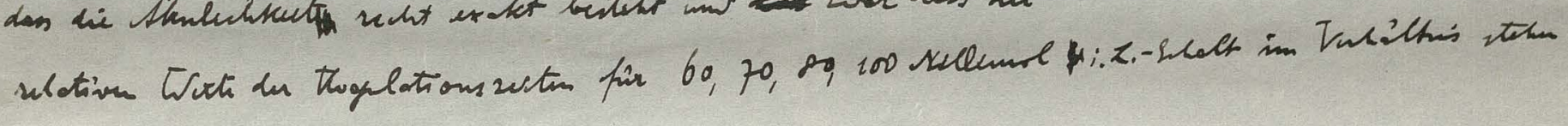

$$
T_{100}: T_{80}: T_{70}: T_{60}=132.75: 5.92: 12 \cdot 7
$$

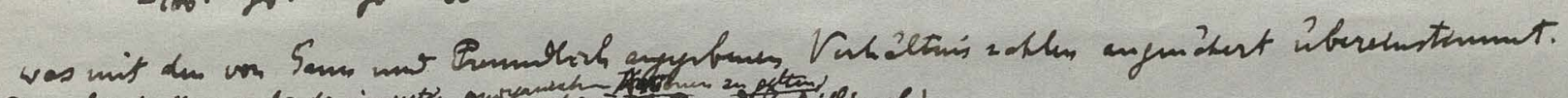

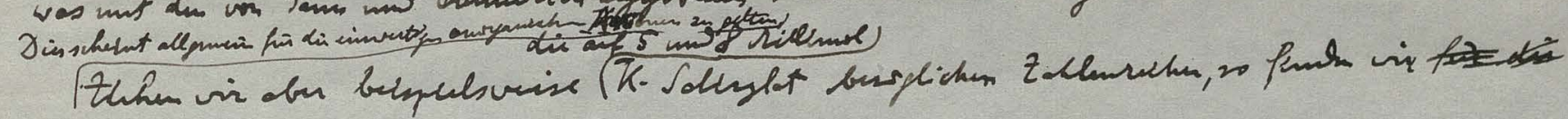

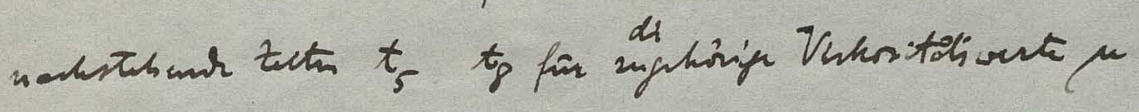

$$
\begin{array}{llllllllllllll}
\mu & 52.4 & 5.25 & 52.9 & 53.8 & 550 & 57.7 & 62.0 & 65.7 & 67.4 & 68.7 & 70.1 \\
t_{5} & 012.5 & 30 & 56 & 78 & 107 & 135 & 168 & 186 & 206 & 240 \\
t_{8} & 0 & 2 & 5 & 10 & 15 & 22 & 30 & 40 & 50 & 60 & 75 \\
t_{g} t_{t g} & 6.3 & 6.0 & 5.6 & 5.2 & 4.9 & 4.5 & 4.2 & 3.7 & 3.5 & 3.0
\end{array}
$$

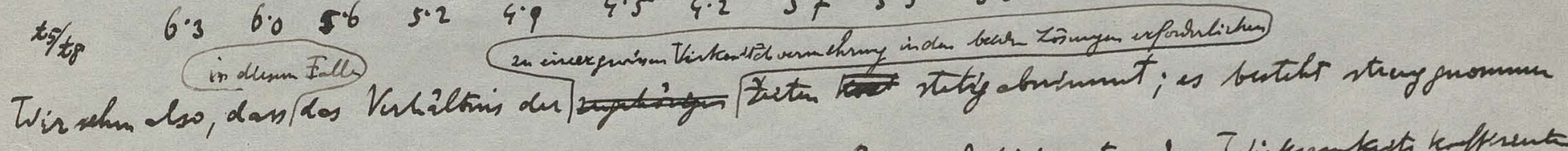

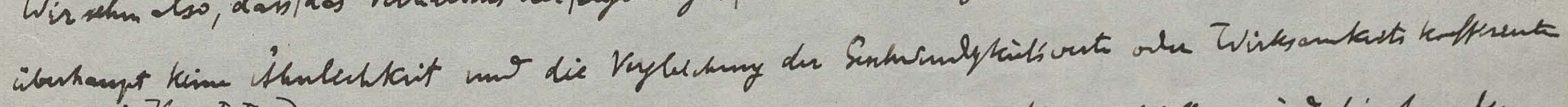

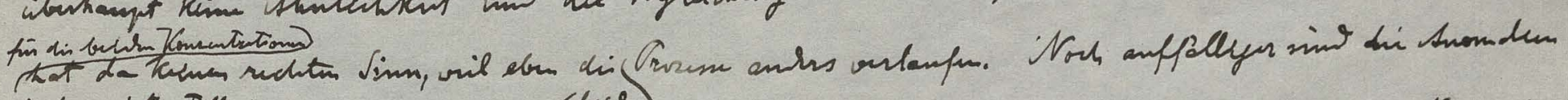

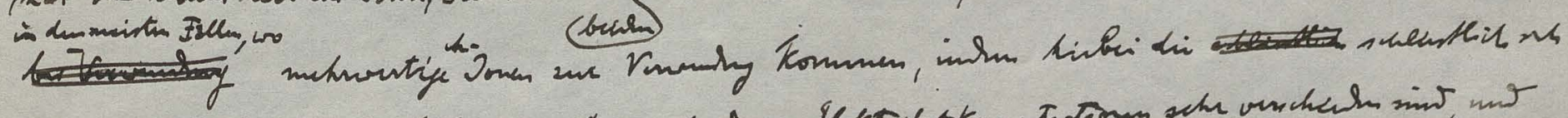

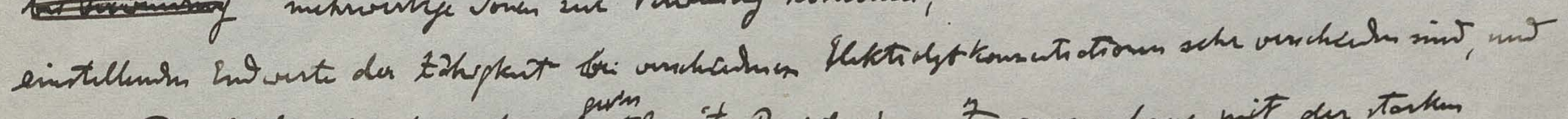

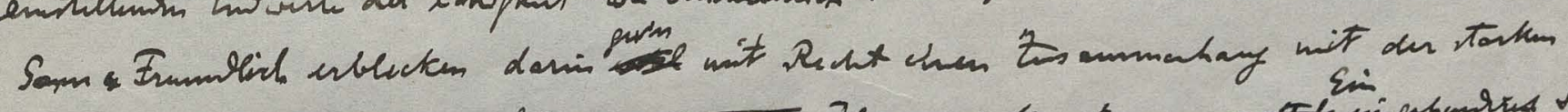

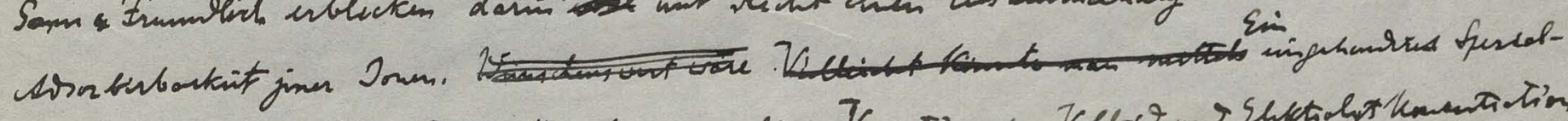

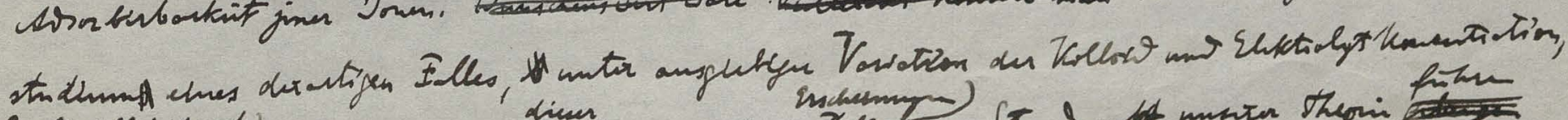

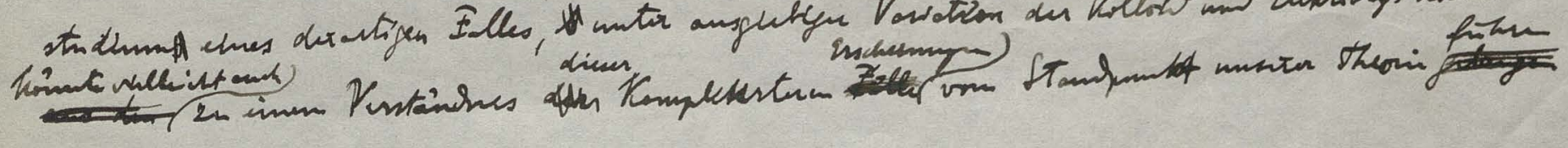




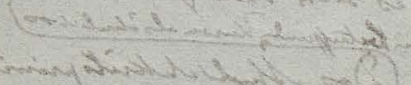

Hentivi 2

$i \rightarrow \infty$

nis

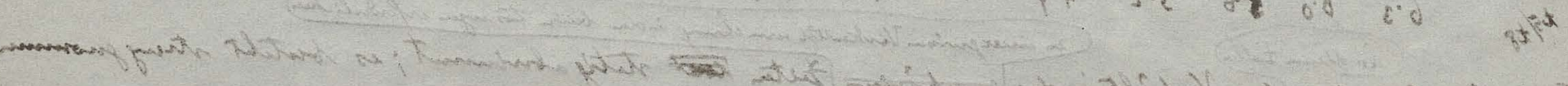

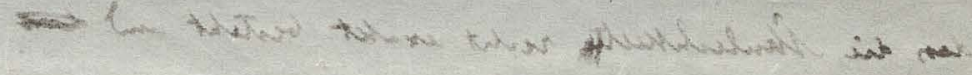

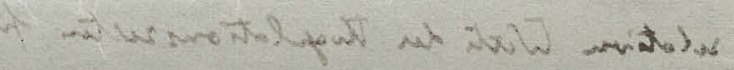

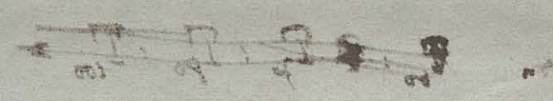

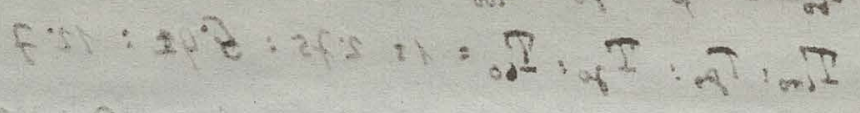

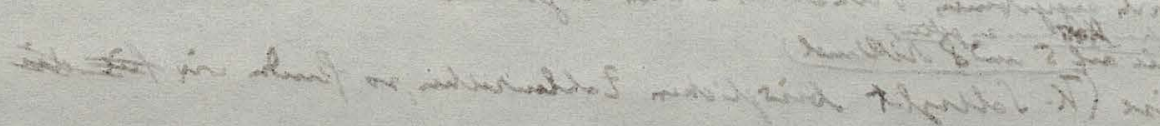

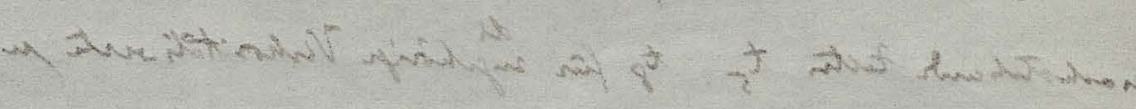
स.

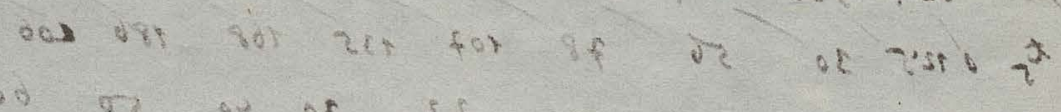

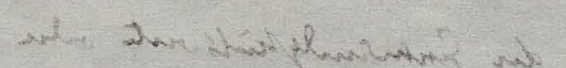

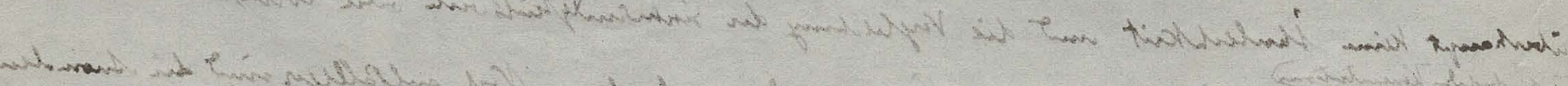

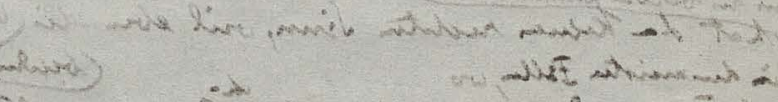

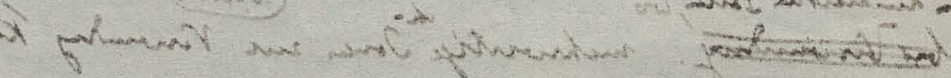

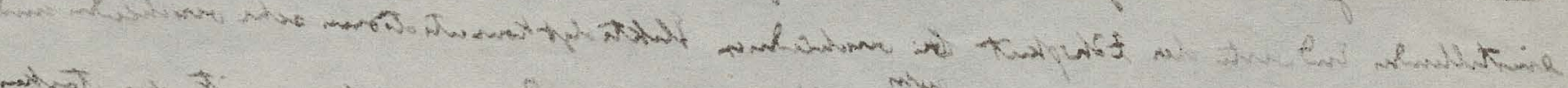

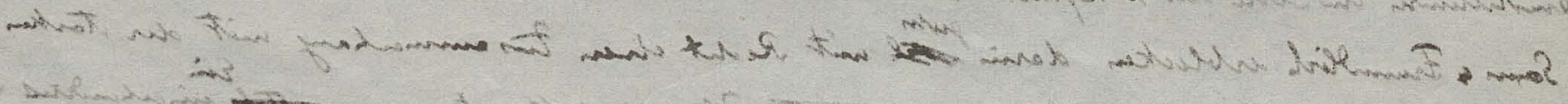

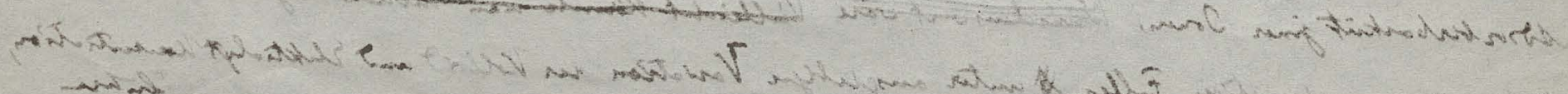

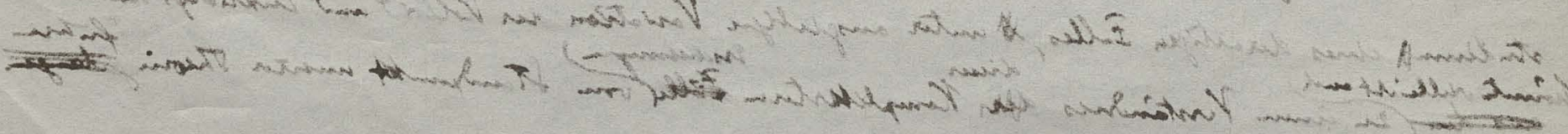




\section{Vugleech mot humencher Temitis.}

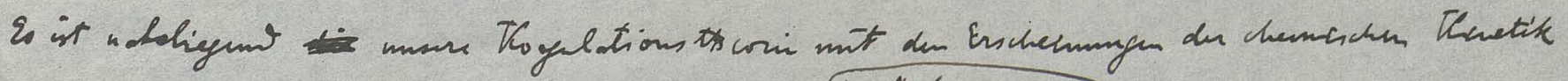

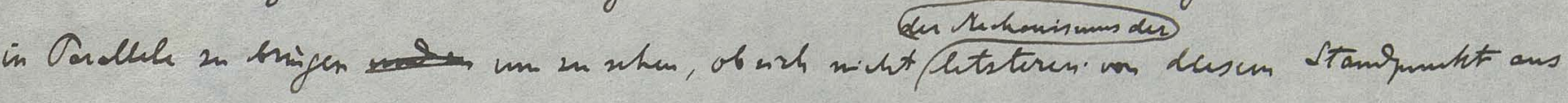
numum Fastambis ratues bingen lasst.

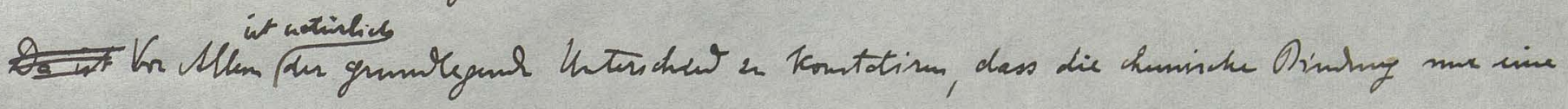

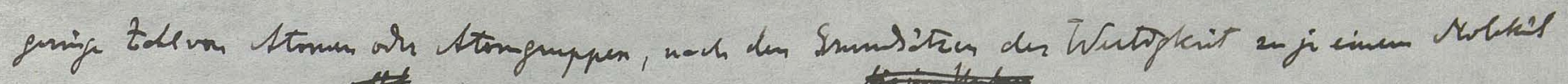

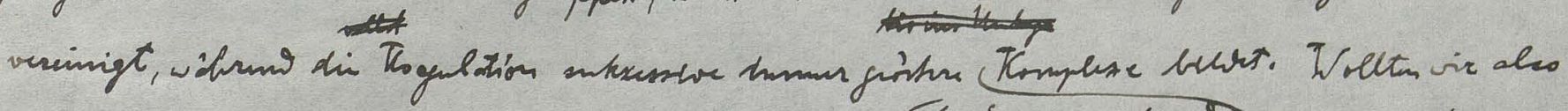

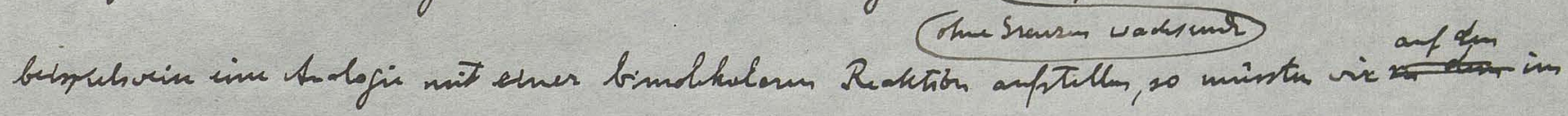

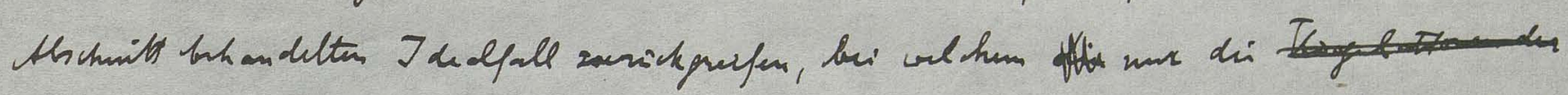

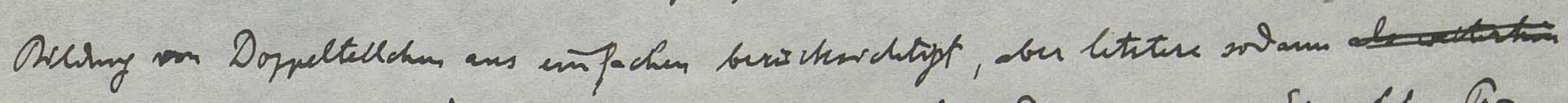

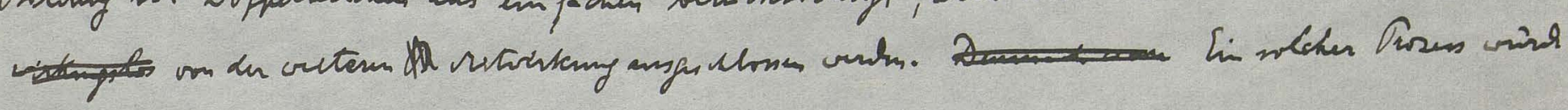
due Rothionsglechuny gunigu:

$$
v_{1}=\frac{v_{0}}{1+\frac{t}{T}}=\frac{v_{0}}{1+8 \Omega D R u_{0} t}=\frac{v_{0}}{1+}
$$

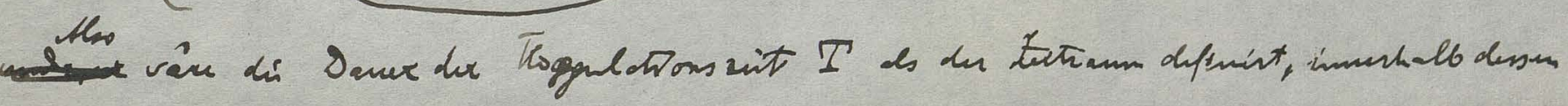

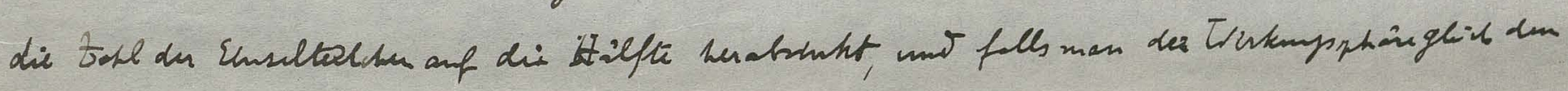

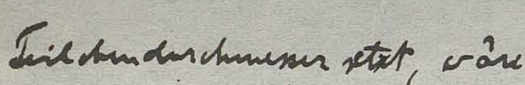

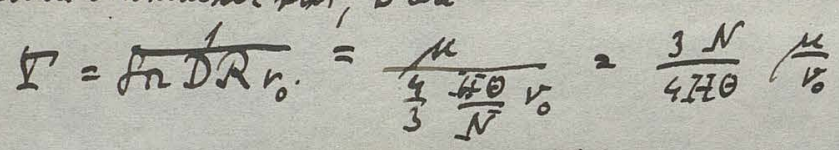

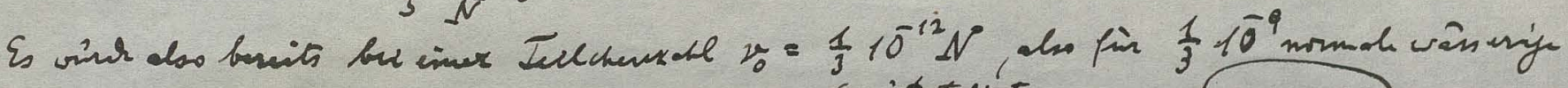

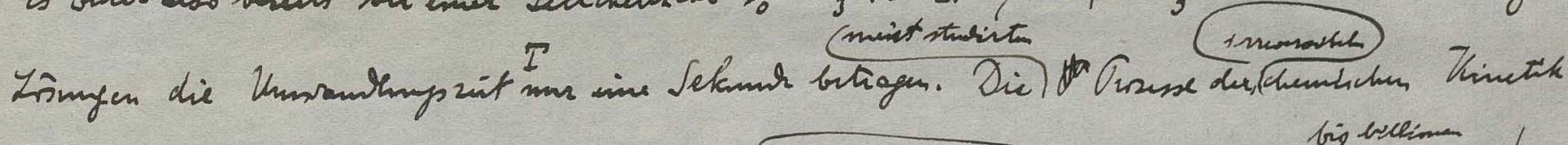

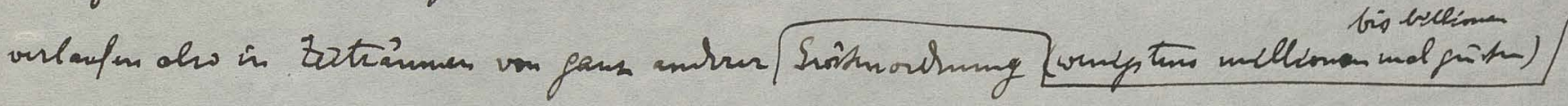

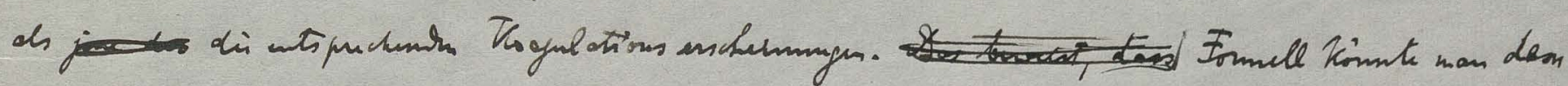

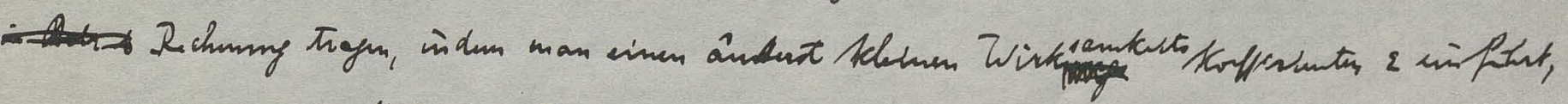

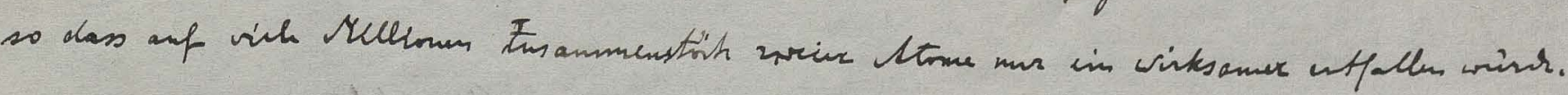

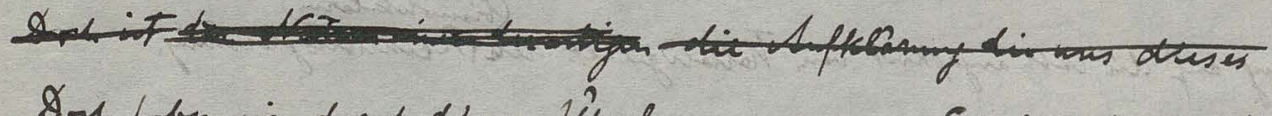

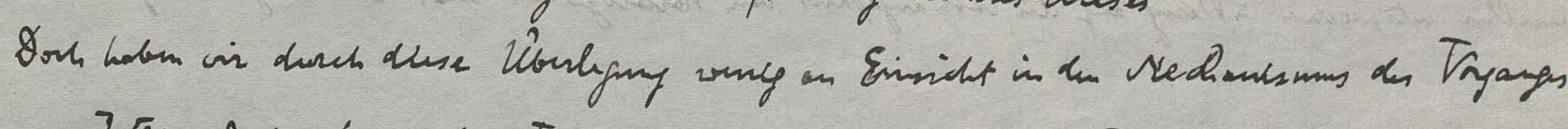

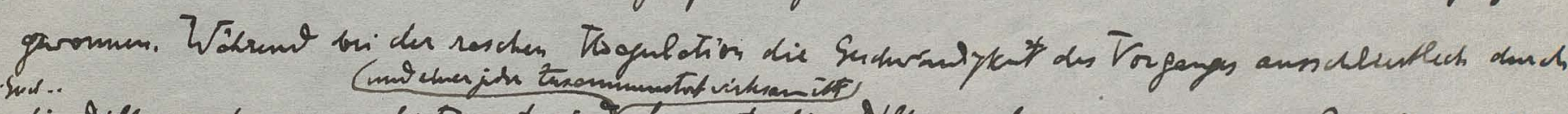

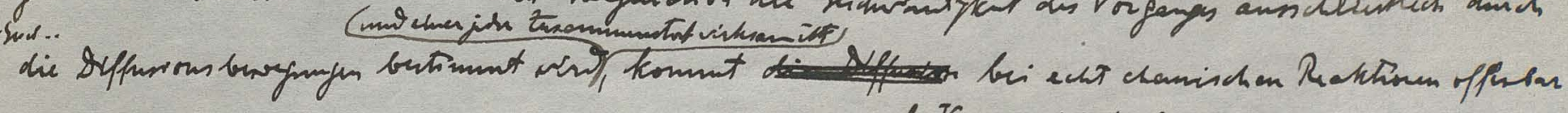

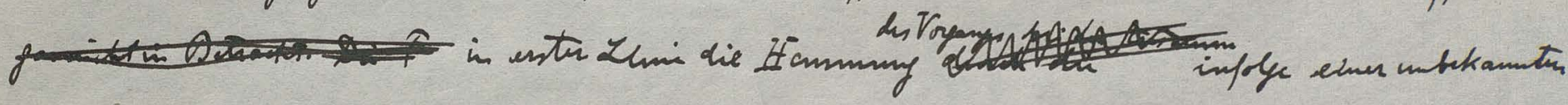

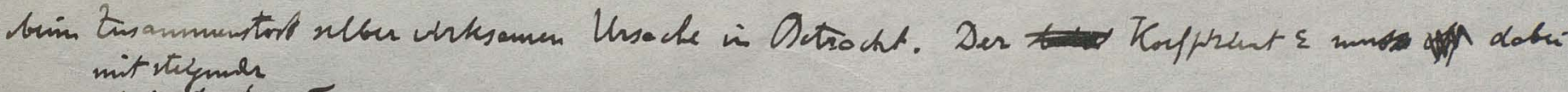

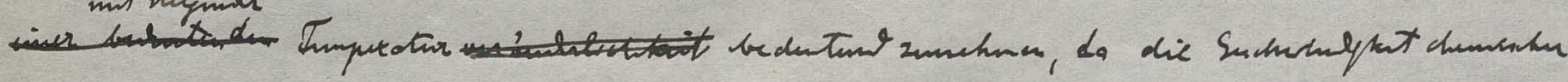

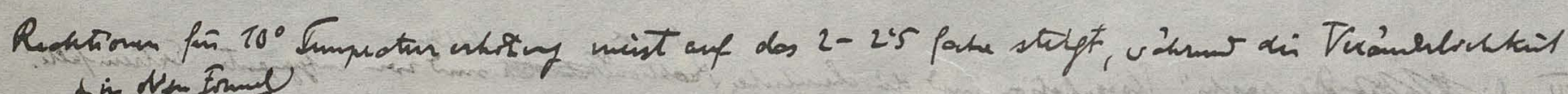

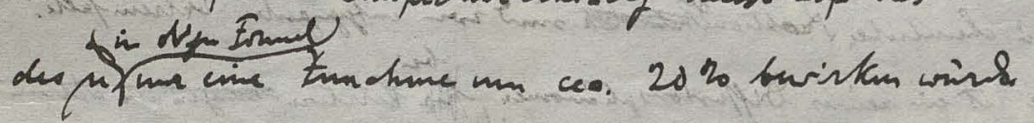


Shathen

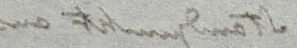

Wh

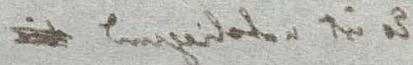

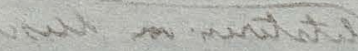

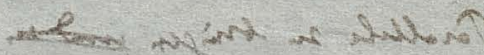
intos? whes whimativer

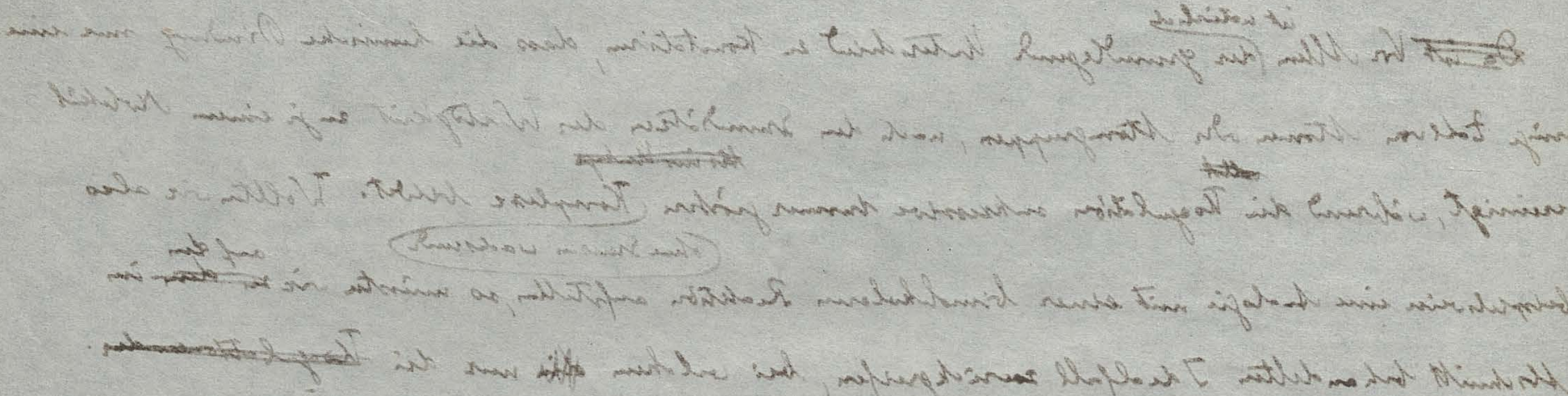

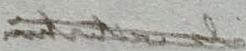

Rिं कारी लो

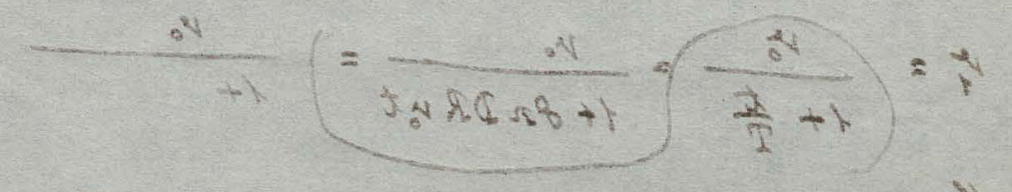

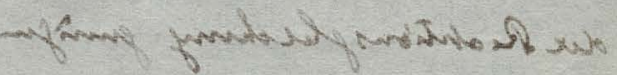

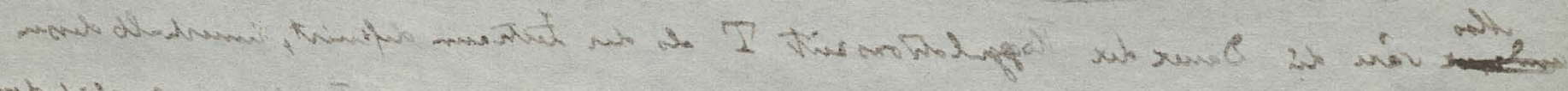

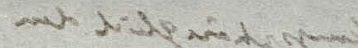

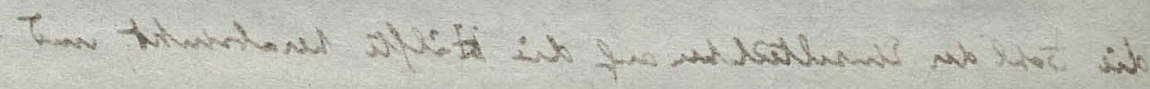

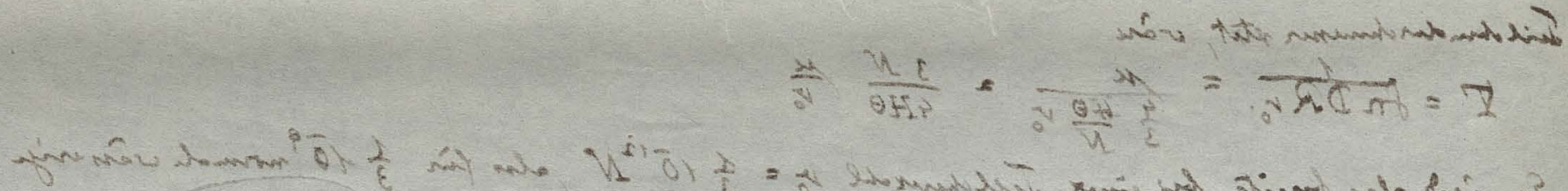

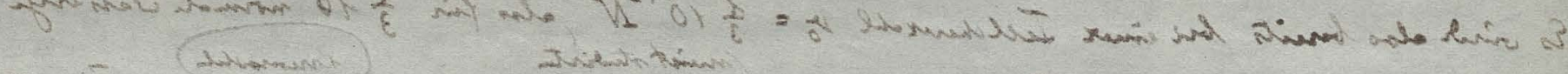
Xitar

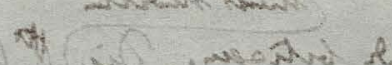

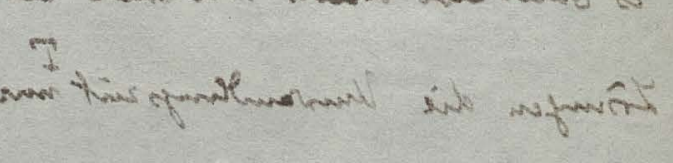

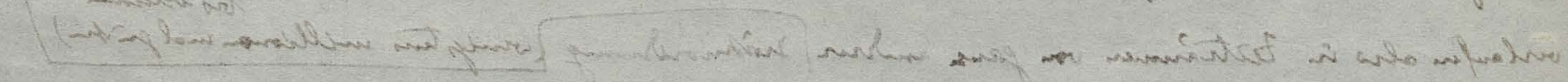

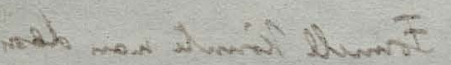

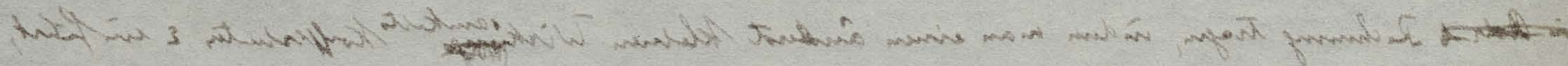

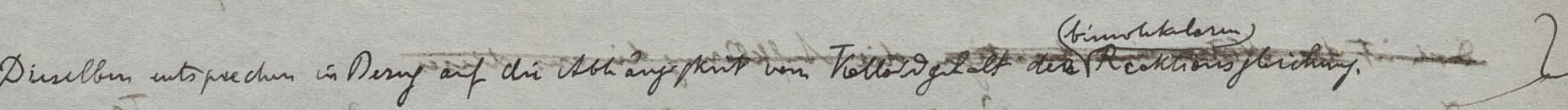

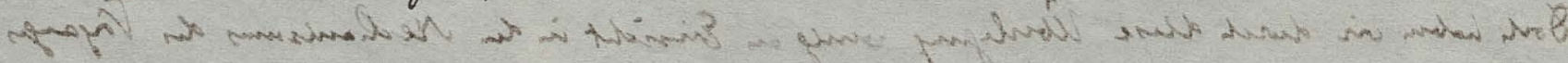

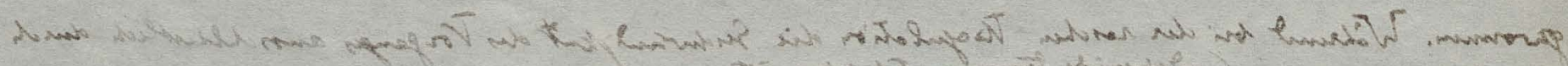

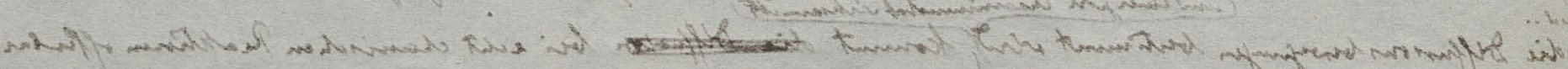

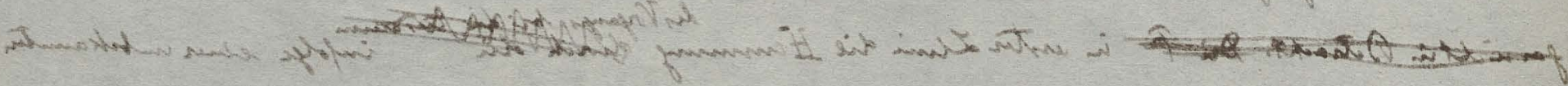

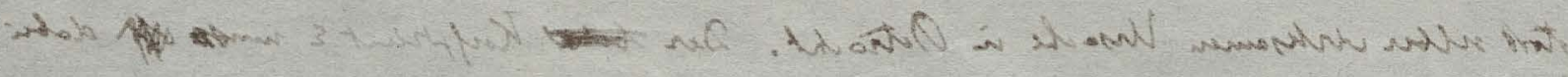

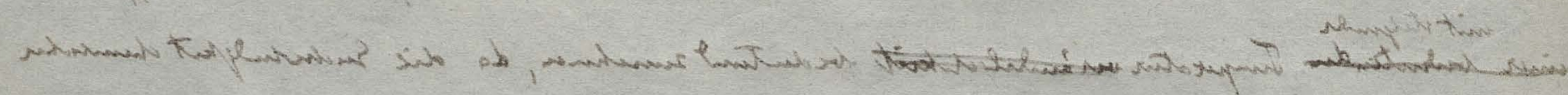

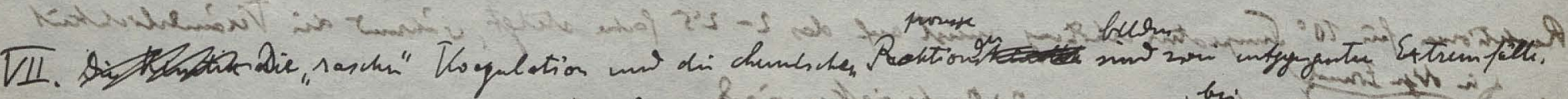

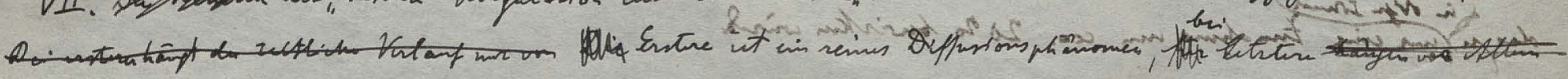

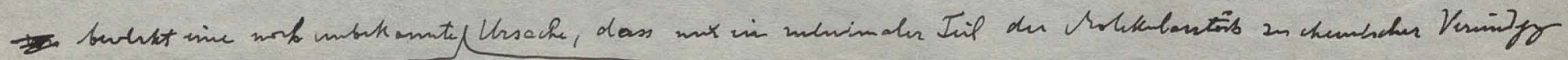

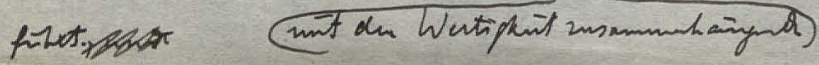




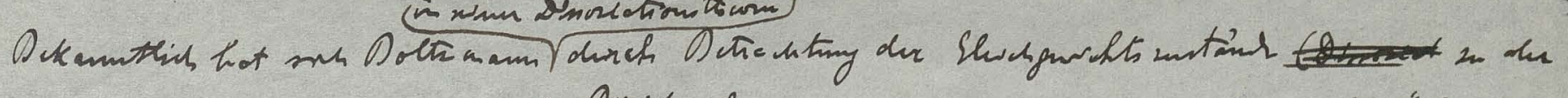

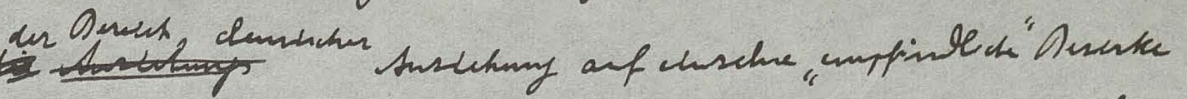

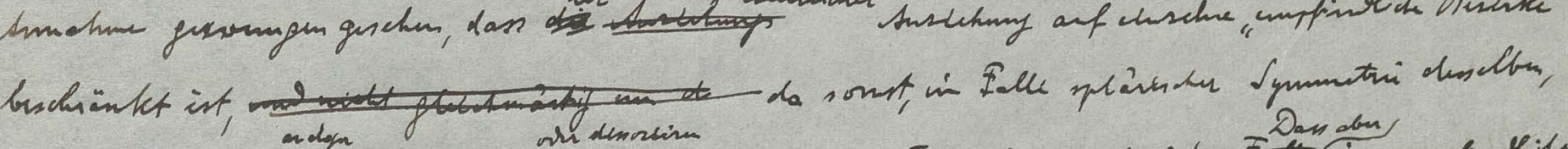

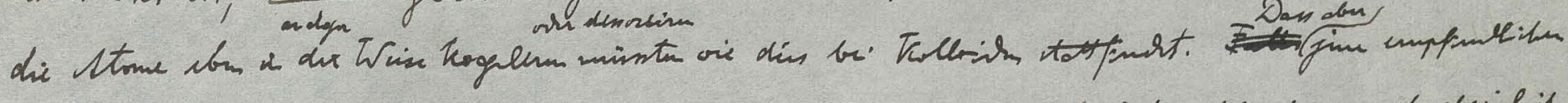

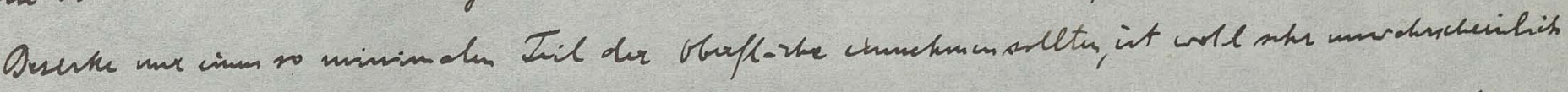

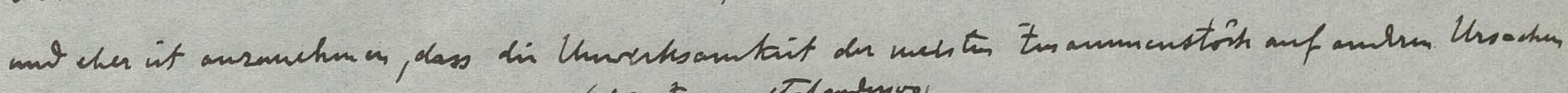

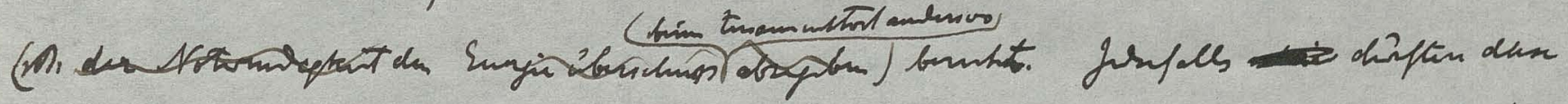

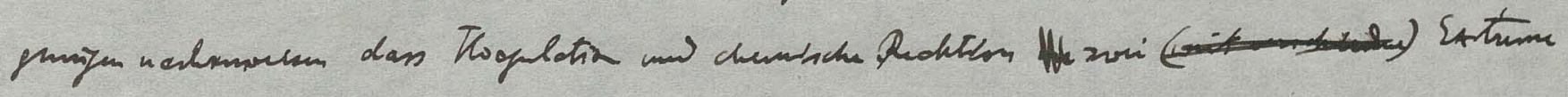

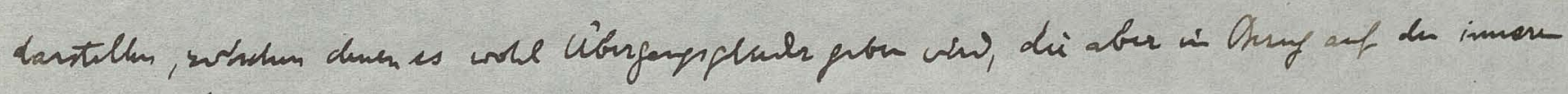

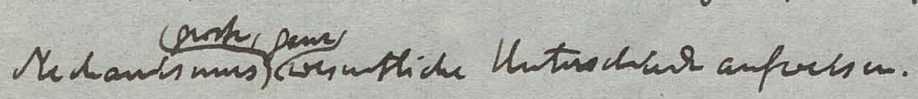

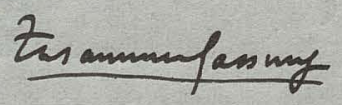

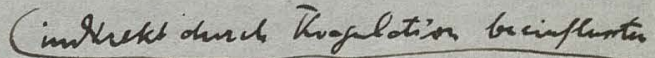

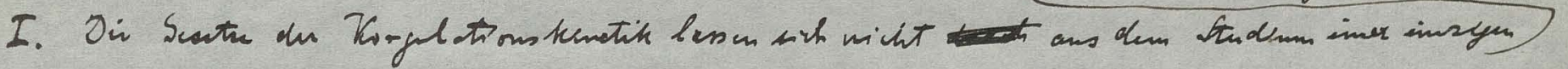

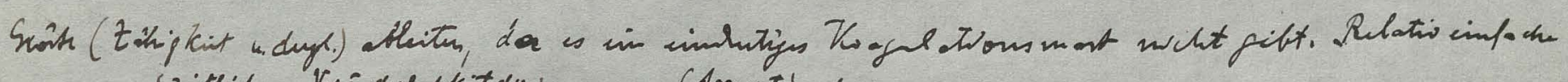

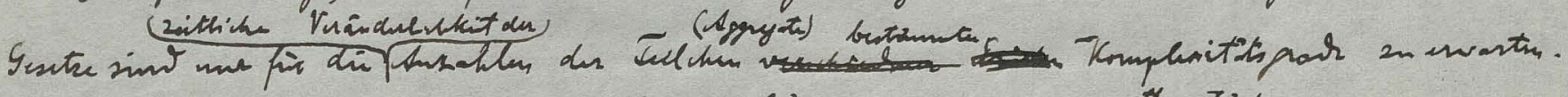

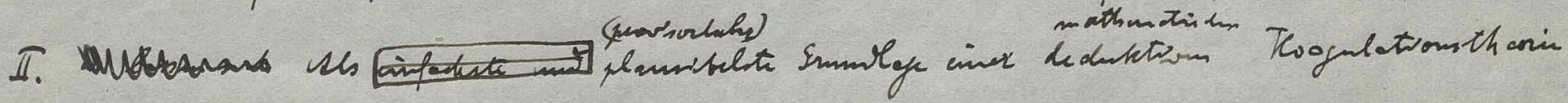

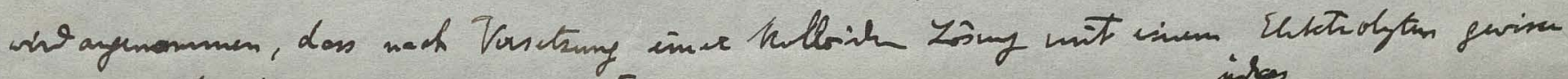

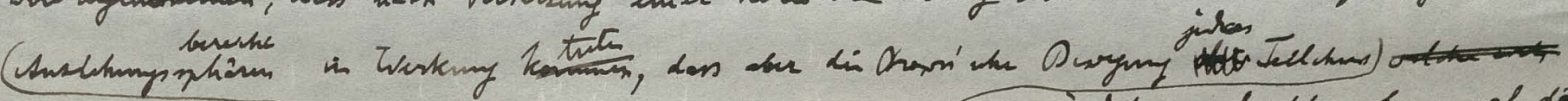

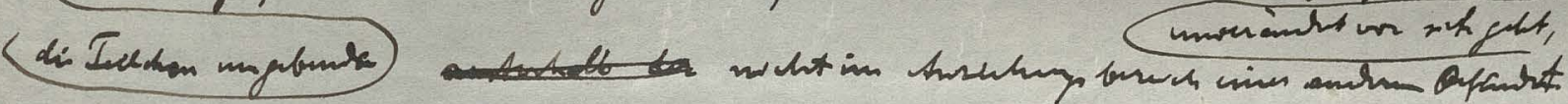

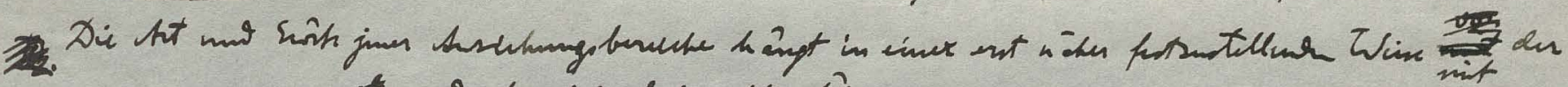

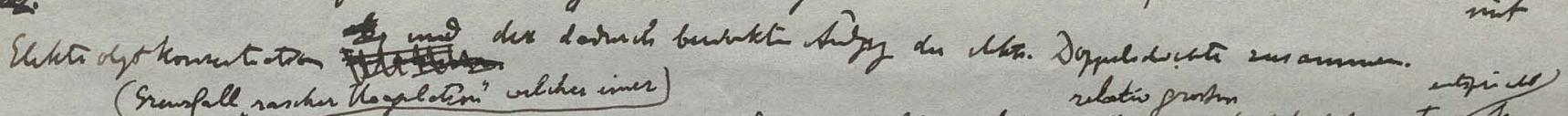

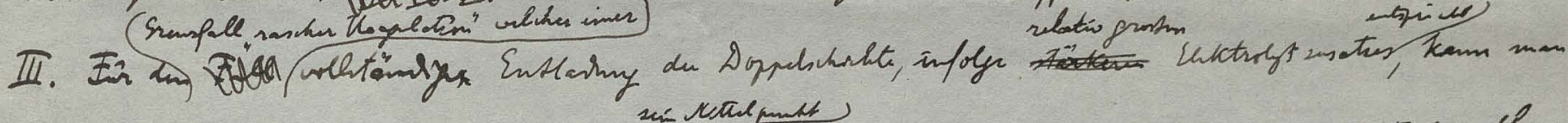

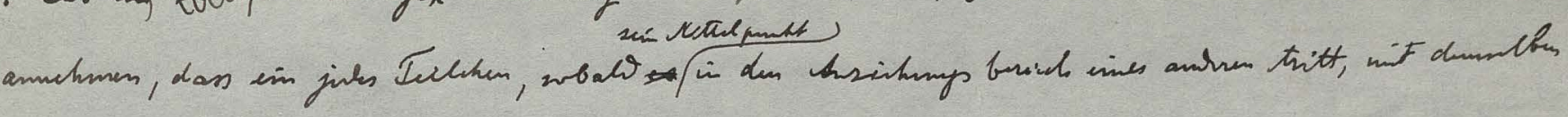

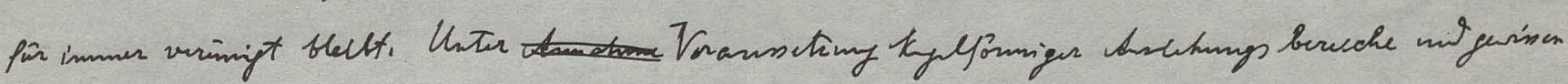

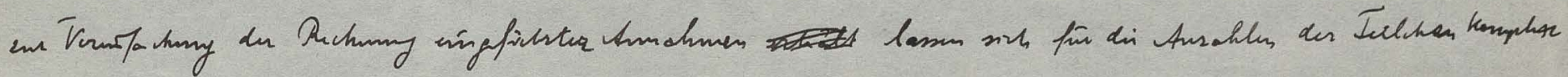

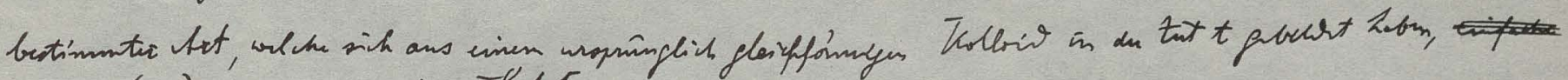

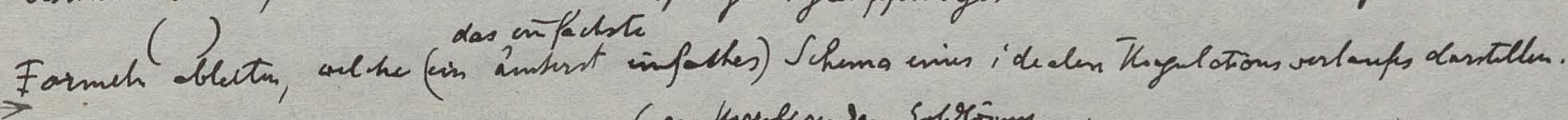

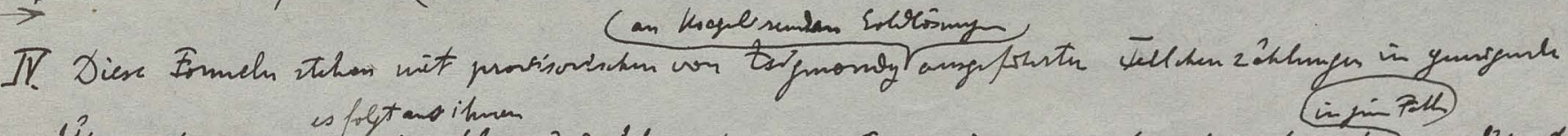

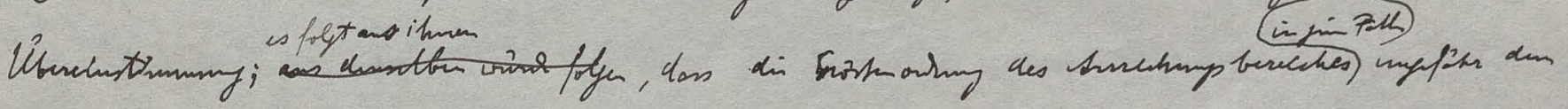
Tul henden chmener ents pucult. trated

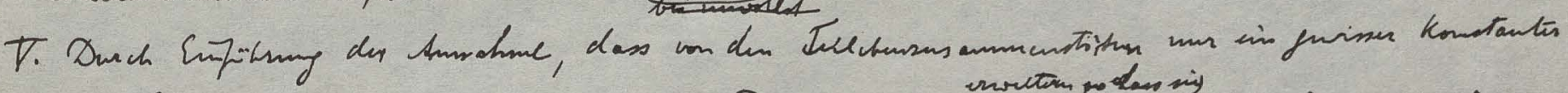

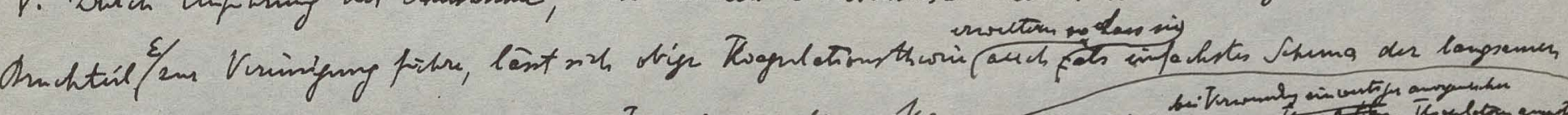

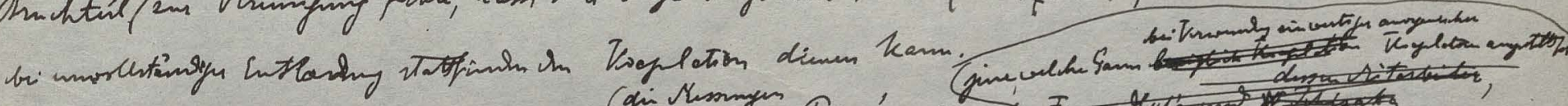

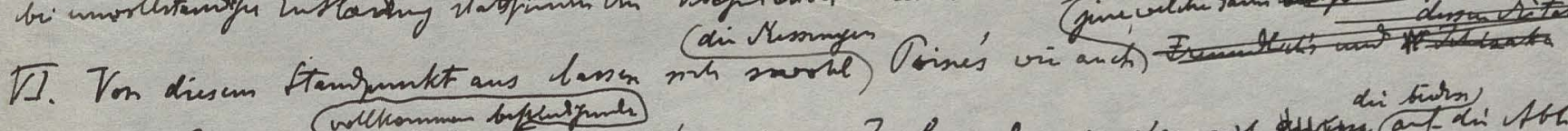

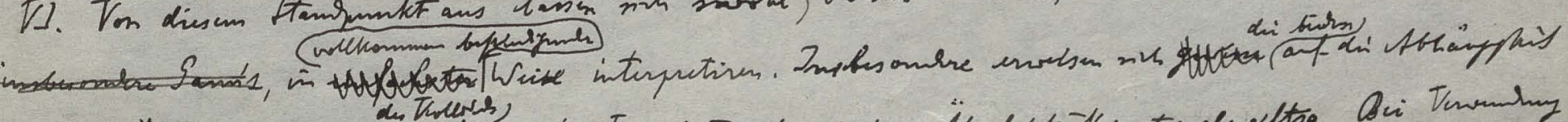

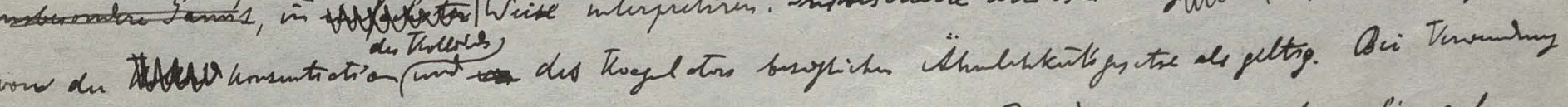

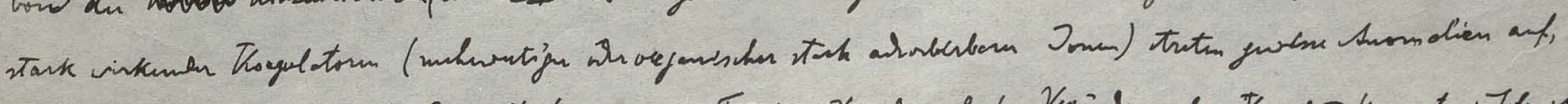

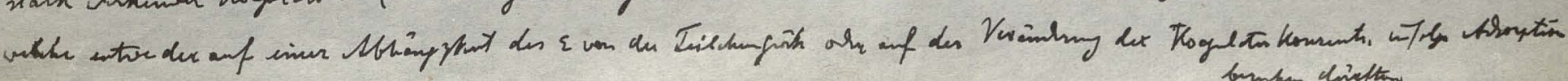





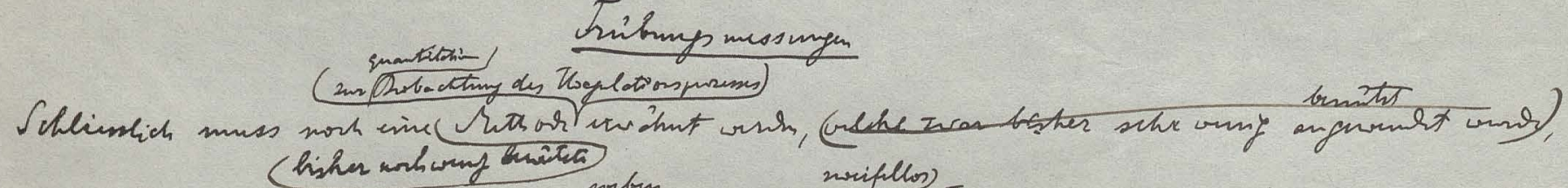

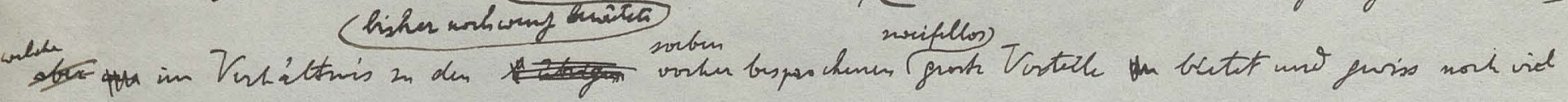

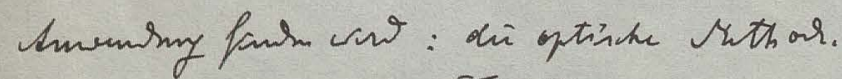

What dir I lant Raglepi, Jome

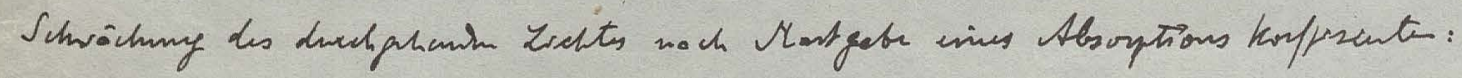

$h=\frac{32}{3} \frac{\left.n^{3} V^{2}+\frac{n^{2}-1}{x^{2}+2}\right)^{2}}{\lambda^{2}}$

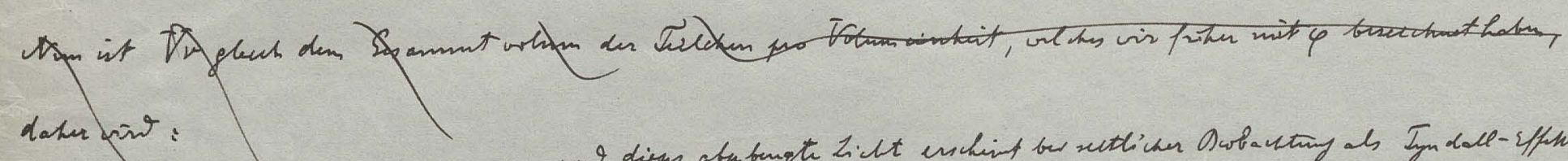

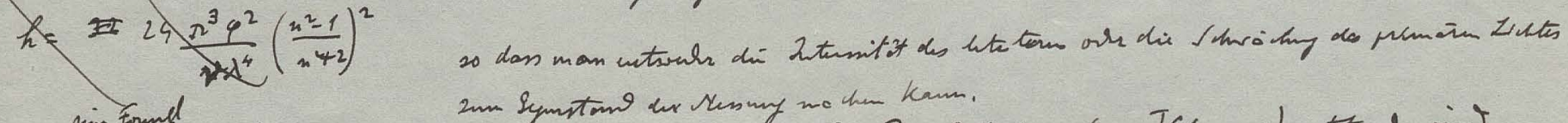

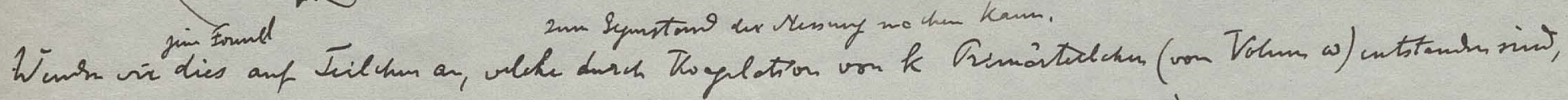

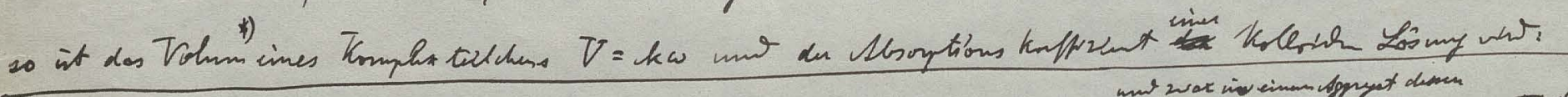

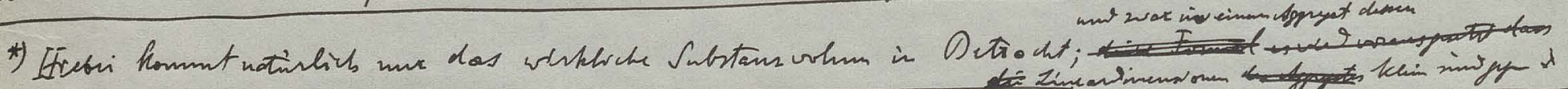

$$
h=24 \frac{n^{3} \omega^{2}}{\lambda^{4}}\left(\frac{n^{2}-1}{n^{2}+2}\right)^{2} \sum k^{2} \frac{1}{k}
$$

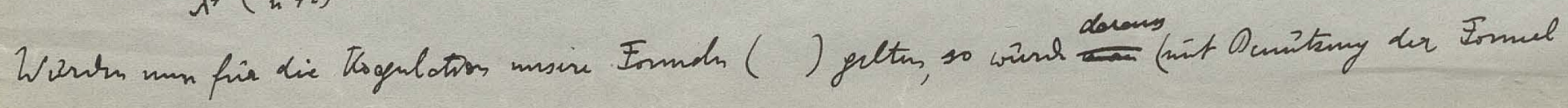
$\sum k^{2} x^{k-1}=\frac{1+x}{(1-x)^{3}}$

fofm:
$h=24 \frac{n^{3} u^{2}}{h^{4}}\left(\frac{n^{2}-1}{n^{2}+h}\right)^{2} v_{0}\left(1+\frac{2 t}{T}\right)$

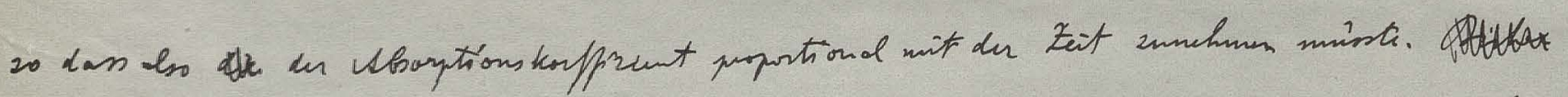

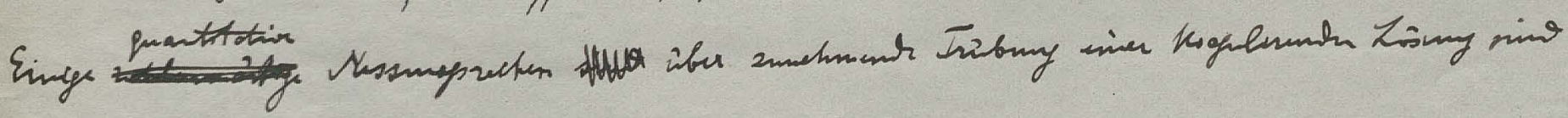
un Lotermour an Notrimolliam 


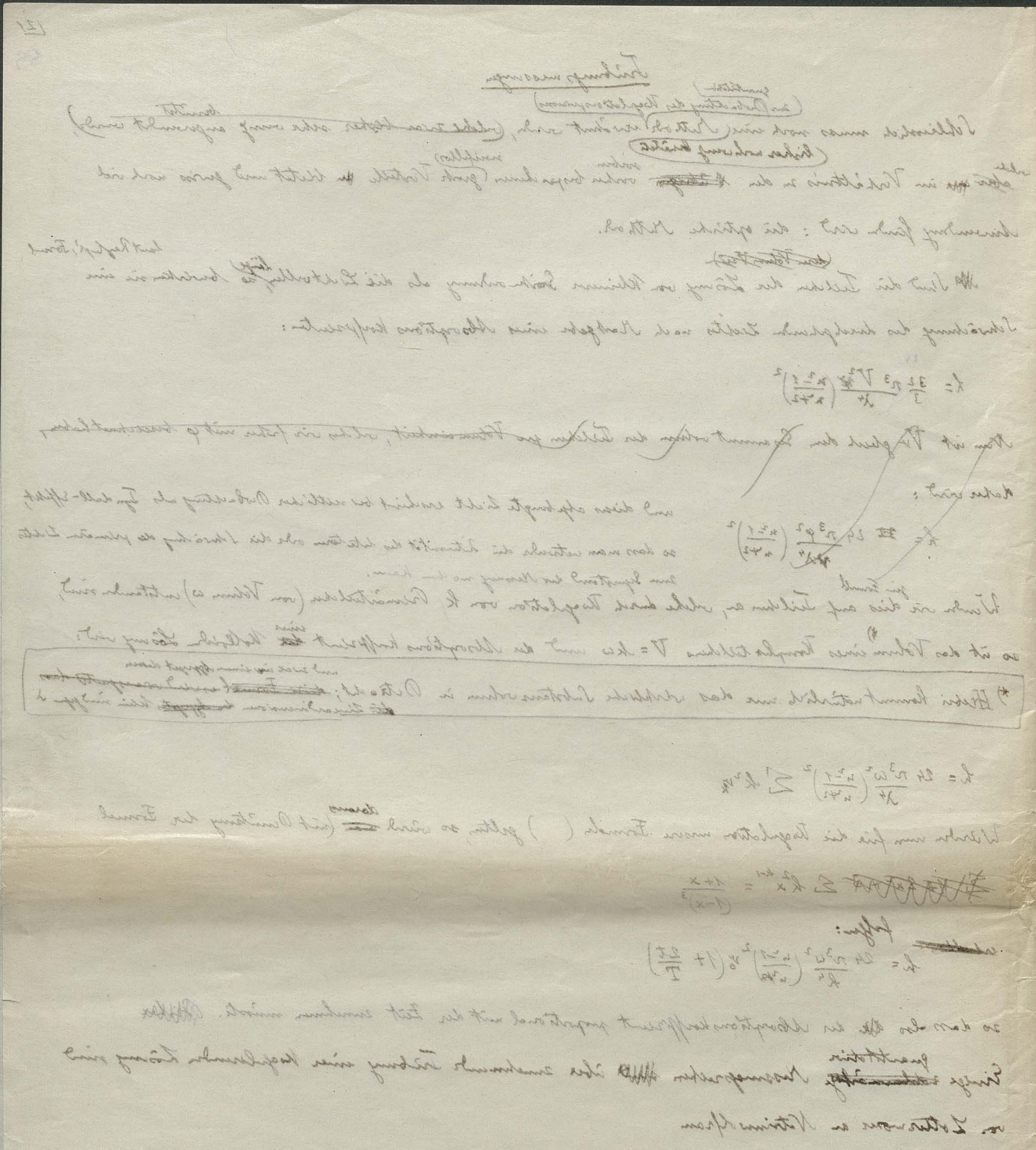




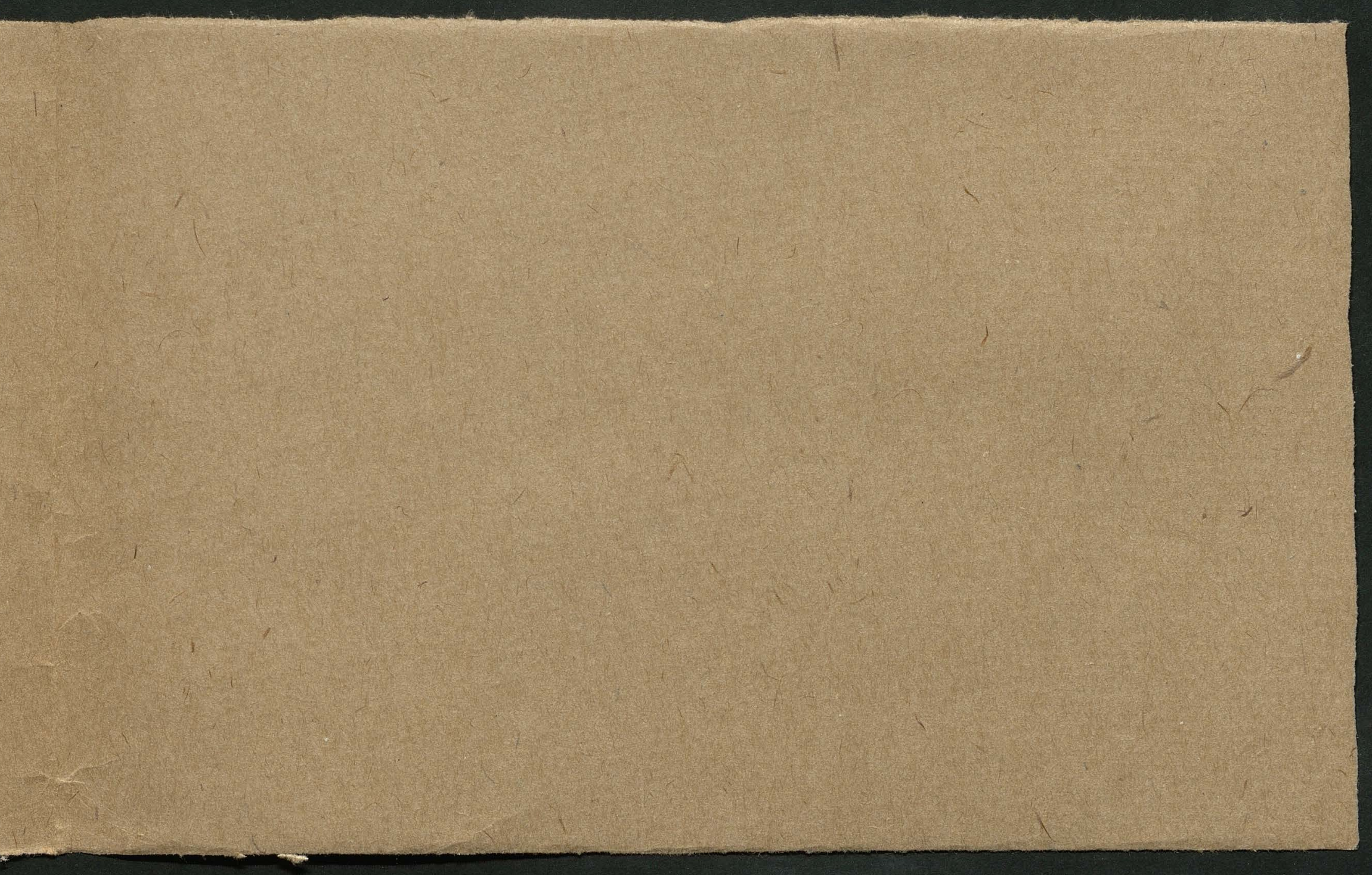




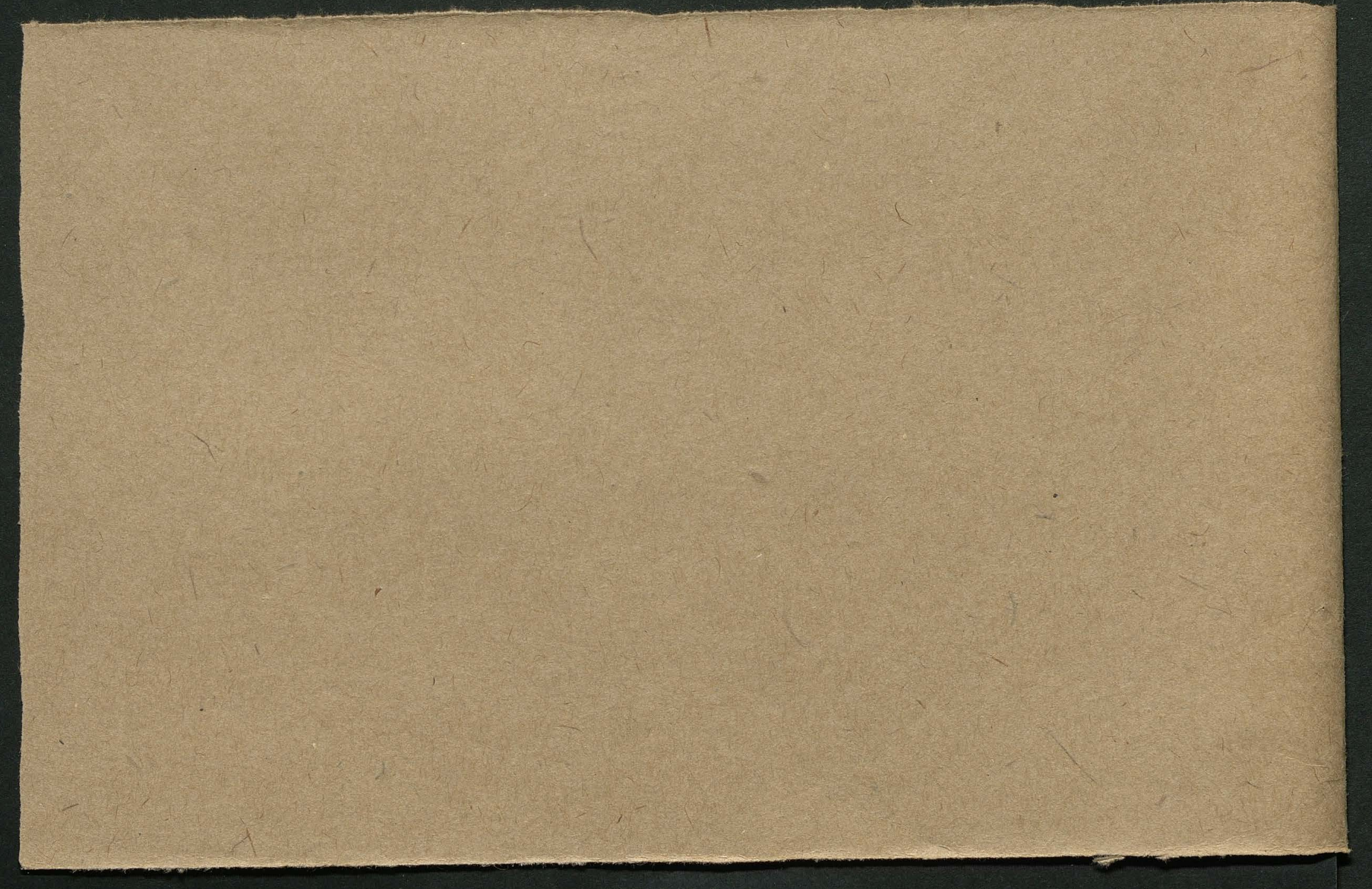




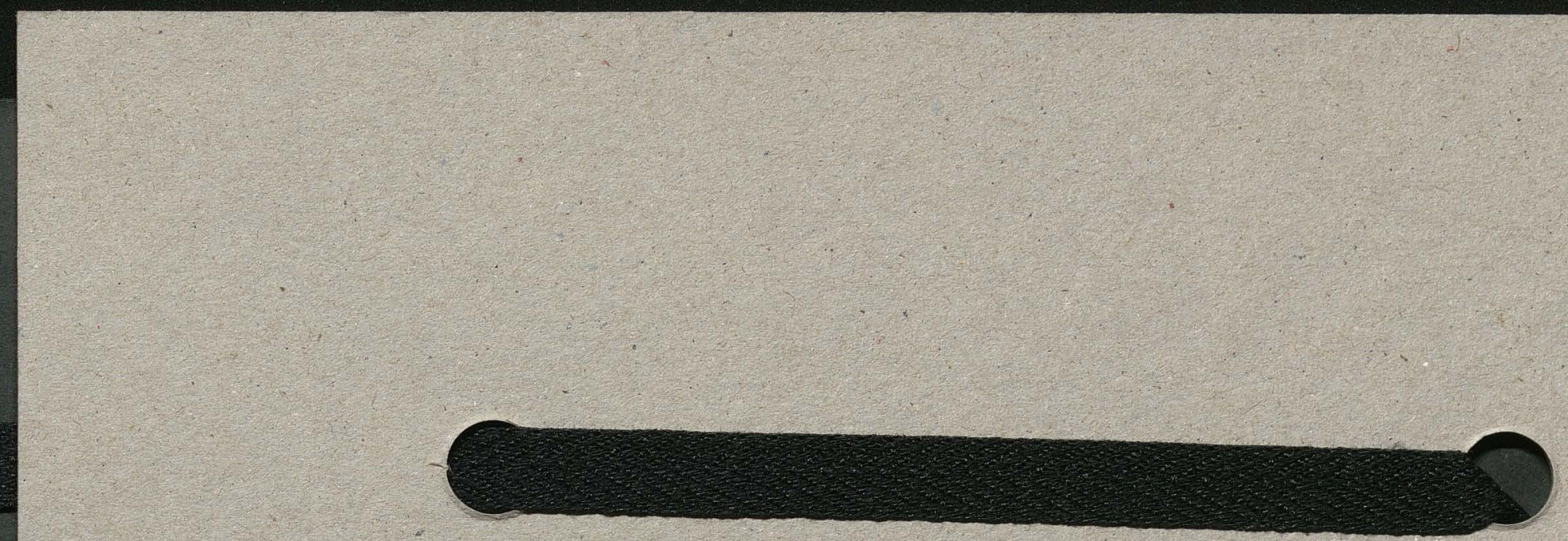


